WILEY-VCH

\title{
Oxidative Fluorination of Cyclopropylamides via Organic Photoredox Catalysis
}

\author{
Ming-Ming Wang and Jérôme Waser ${ }^{*[a]}$
}

\begin{abstract}
We report an oxidative ring-opening strategy to transform cyclopropylamides and cyclobutylamides into fluorinated imines. The imines can be isolated in their more stable hemiaminal form, with the fluorine atom installed selectively at the $\gamma$ or $\delta$ position. Both cheap benzophenone with UV A light or organic and inorganic dyes with blue light could be used as photoredox catalysts to promote this process. Various fluorinated amines were then obtained by nucleophilic attack on the hemiaminals in one pot, giving access to a broad range of useful building blocks for medicinal chemistry.
\end{abstract}

Tremendous efforts have been devoted to the development of site-selective fluorination methods, given the significance of fluorinated compounds in medicine and agrochemistry. ${ }^{[1]}$ Among these methods, the formation of $\mathrm{C}\left(\mathrm{sp}^{3}\right)-\mathrm{F}$ bonds via ring opening fluorination of carbocycles is an attractive route. ${ }^{[2]}$ While success has been achieved in ring opening fluorination of arylcyclopropanes (Scheme 1A), ${ }^{[3]}$ cyclopropanols ${ }^{[4]}$ and cyclobutanols $^{[4 a, b]}$ (Scheme 1B), their nitrogen-substituted counterparts were not studied yet, despite the importance of nitrogen-containing fluorinated drugs and agrochemicals.

Previous studies on ring-opening of aminocyclopropanes and aminocyclobutanes focused on Donor-Acceptor systems, which are more reactive..$^{[5]}$ Simple systems lacking electron-withdrawing groups have been less exploited, with most approaches using transition-metal catalyst for $\mathrm{C}-\mathrm{C}$ activation. ${ }^{[6]}$ Oxidative methods proceeding via radical pathways constitute an interesting alternative. ${ }^{[7]}$ Zheng and co-workers demonstrated that cyclopropylanilines and cyclobutylanilines can be oxidized to a radical cation species I using photoredox catalysis (Scheme 1C). ${ }^{[8]}$ After ring opening, the resulting iminium radical II underwent $(3+2)$ annulation with alkenes or alkynes. Our group later extended this approach to cyclopropenes ${ }^{[9]}$ and Stephenson and co-workers applied it to the synthesis of 1aminonorbornanes. ${ }^{[10]}$ They also developed an alternative strategy involving the activation of cyclopropylimines through their triplet excited state. ${ }^{[11]}$ Our group exploited another approach based on a Hofmann-Löffler-Freytag (HLF)-inspired reaction to

[a] Ming-Ming Wang and Prof. Dr. Jérôme Waser Laboratory of Catalysis and Organic Synthesis Institute of Chemical Sciences and Engineering Ecole Polytechnique Fédérale de Lausanne EPFL SB ISIC LCSO, BCH 4306, 1015 Lausanne (CH)

Fax: (+)41216939700

E-mail: jerome.waser@epfl.ch

Homepage: http://lcso.epfl.ch/

Supporting information for this article is given via a link at the end of the document. Raw data for NMR, IR and MS is available at zenodo.org, DOI: 10.5281/zenodo.3895605 generate a nitrogen-centered radical from $N$-halogen aminocyclopropane III (Scheme 1D). ${ }^{[12]}$ The $\mathrm{N}$ radical then underwent ring-opening and radical recombination to generate $\gamma$ halogenated imines isolable in their $\mathrm{N}, \mathrm{O}$-acetal form IV. However, this approach failed in the case of fluorination.

A. Fluorination of cyclopropanes B. Fluorination of cyclic tertiary alcohols

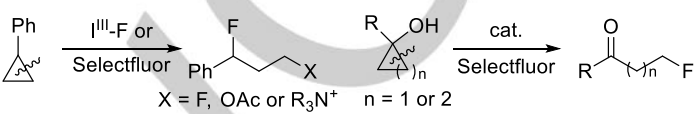

C. (3+2) annulation of cyclopropylanilines

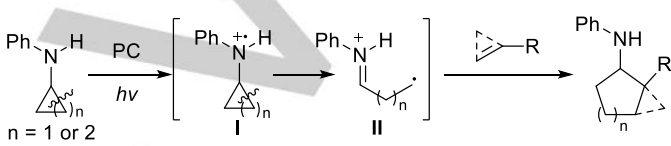

D. HLF reaction inspired 1,3-difunctionalization of aminocyclopropanes

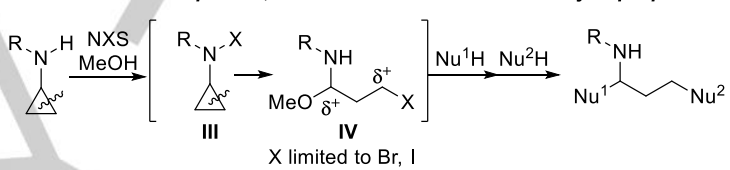

E. This work: ring-opening fluorination of aminocyclopropanes

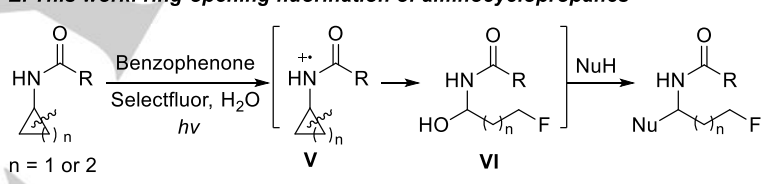

Scheme 1. Oxidative ring-opening fluorination of cyclopropanes (A) Fluorination of cyclopropanols and cyclobutanols (B). Photoredox catalysis enabled $(3+2)$ annulation of cyclopropylanilines (C). 1,3-Difunctionalization of aminocyclopropanes (D). This work: oxidative fluorination of cyclopropylamides (E)

We envisioned that a highly oxidizing excited photocatalyst should be able to activate cyclopropylamides via single electron transfer (SET) oxidation to give amidium radical $\mathbf{V}$ (Scheme $1 \mathrm{E}) .^{[13]}$ After ring-opening, the formed alkyl radical could be trapped by a fluorination reagent, and the imine stabilized as a hemiaminal VI. Herein, we report the successful implementation of this strategy, using either cheap benzophenone with black light (365 nm) or organic/inorganic dyes with blue LEDs, and Selectfluor acting as both oxidant and fluorination reagent. The reaction is complementary to the method of Lectka for the synthesis of fluorinated amines (Scheme $1 \mathrm{~A}, \mathrm{R}=\mathrm{NR}_{3}{ }^{+}$), ${ }^{\left[{ }^{[a]}\right]}$ as a reversed regioselectivity is observed for fluorination compared with arylcyclopropanes. The obtained hemiaminals were easily converted into diverse products by reaction with nucleophiles.

With benzamide cyclopropane 1a as substrate, the combination of benzophenone and Selectfluor under irradiation at $365 \mathrm{~nm}$ in $\mathrm{MeCN} / \mathrm{H}_{2} \mathrm{O}$ was optimal, yielding 3-fluorinated hemiaminal $2 \mathrm{a}$ in $74 \%$ yield (Table 1 , entry 1 ). ${ }^{[14]}$ Control experiments showed no 
conversion in the absence of benzophenone or irradiation (Table 1 , entries 2 and 3 ). Only $10 \%$ of product was observed when using $\mathrm{NFSI}$ as fluorinating reagent (Table 1, entry 4). 9-Fluorenone, which is efficient for the ring-opening fluorination of arylcyclopropanes, ${ }^{\text {[3a] }}$ failed in this case (Table 1, entry 5$)$. Other photocatalysts such as $\left[\mathrm{Ir}\left(\mathrm{dF}-\mathrm{CF}_{3} \mathrm{ppy}\right)_{2}(\mathrm{dtbbpy})\right] \mathrm{PF}_{6}$ and Mes-Acr ${ }^{+}$ can also be utilized to achieve similar results using visible light (Table 1, entries 6 and 7). Lower yields were obtained with substrates containing less electron-donating substituents on the benzene ring of the amide (Table 1, entries 8 and 9). No conversion was observed with a strong electron-withdrawing substituent such as a nitrobenzoyl (Table 1, entry 10) or a tosyl (Ts) group (Table 1, entry 11). With a Boc group, no product was isolated due to fast decomposition of the hemiaminal (Table 1 , entry 12). With a pivaloyl group, the yield dropped to $29 \%$ with incomplete conversion (Table 1, entry 13). When mixing cyclopropyl aniline $\mathbf{1 h}$ with Selectfluor, fast degradation was observed even prior to UV irradiation (Table 1, entry 14).
We then developed one pot protocols to replace the hydroxy group by adding nucleophiles to the reaction mixture (Scheme 2). We focused on benzophenone as catalyst, because of its broad availability and low prize. N,O-, N,S- or $N, N$ - acetals can be accessed in $39-73 \%$ yield (products $3 a-f)$. The hemiaminal can also be reduced by $\mathrm{NaBH}_{3} \mathrm{CN}$, affording $3 \mathbf{g}$ in $83 \%$ yield. $\mathrm{A}$ Petasis reaction ${ }^{[15]}$ gave allylic amine $3 \mathrm{~h}$ in $42 \%$ yield. $1,3,5-$ Trimethoxybenzene afforded $3 \mathbf{i}$ in $69 \%$ yield while only $22 \%$ yield of $\mathbf{3 j}$ was observed when using 1,3-dimethoxybenzene. With pyrrole, C2 addition product $\mathbf{3 k}$ was isolated in $46 \%$ yield.

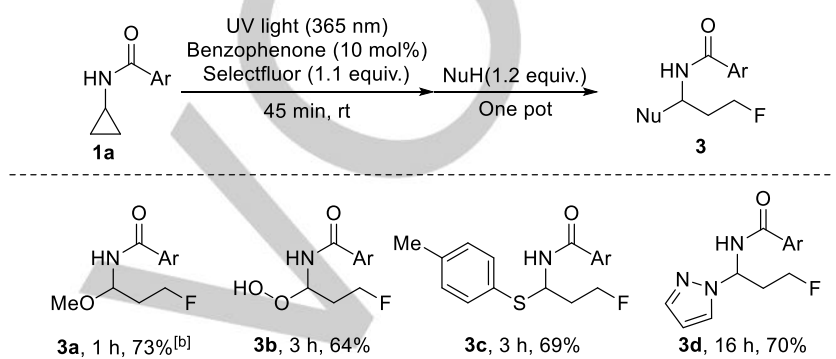

Table 1. Optimization of the reaction conditions ${ }^{[a]}$

$$
\triangle_{1(0.3 \mathrm{mmol})} \frac{\begin{array}{c}
\text { UV light }(365 \mathrm{~nm}) \\
\text { Benzophenone (10 mol\%) } \\
\text { Selectfluor (1.1 equiv.) }
\end{array}}{\text { MeCN:H } \mathrm{H}_{2} \mathrm{O}(4: 6,0.4 \mathrm{M}), \mathrm{rt}}
$$
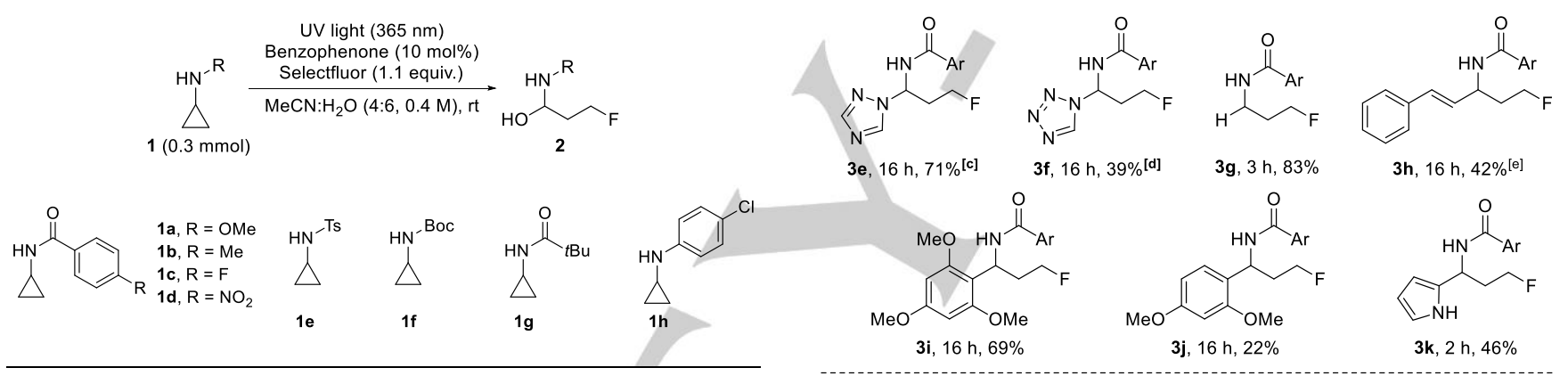

\begin{tabular}{|c|c|c|}
\hline Entry & Deviation from standard condition & Yield $\left[b^{b]}\right.$ \\
\hline 1 & none & $74 \%$ \\
\hline 2 & no benzophenone & 0 \\
\hline 3 & no irradiation & 0 \\
\hline 4 & NFSI instead of Selectfluor & $10 \%[c]$ \\
\hline 5 & 9-fluorenone instead of benzophenone & $6 \%[c]$ \\
\hline 6 & {$\left[\operatorname{lr}\left(\mathrm{dF}-\mathrm{CF}_{3} \mathrm{ppy}\right)_{2}(\mathrm{dtbbpy})\right] \mathrm{PF}_{6}$ with blue LED ${ }^{[\mathrm{d}]}$} & $76 \%[c]$ \\
\hline 7 & Mes-Acr ${ }^{+}$with blue LED ${ }^{[e]}$ & $75 \%[c]$ \\
\hline 8 & $\mathbf{1 b}$ as substrate $\mathrm{e}^{[\mathrm{f}]}$ & \\
\hline 9 & 1c as substrate ${ }^{[f]}$ & $43 \%$ \\
\hline 10 & 1d as substrate & 0 \\
\hline 11 & $1 e$ as substrate & 0 \\
\hline 12 & $1 f$ as substrate & decomposed \\
\hline 13 & $1 \mathrm{~g}$ as substrate & $29 \%[9]$ \\
\hline 14 & 1h as substrate & decomposed \\
\hline
\end{tabular}

${ }^{[a]}$ Reaction conditions: $0.30 \mathrm{mmol}$ scale for $45 \mathrm{~min}$. ${ }^{[\mathrm{b}]}$ Yield of isolated product. [c]Yield determined by ${ }^{1} \mathrm{H}$ NMR using $\mathrm{CH}_{2} \mathrm{Br}_{2}$ as internal standard. [d]With $1 \mathrm{~mol} \%$ $\left[\operatorname{Ir}\left(\mathrm{dF}-\mathrm{CF}_{3} \mathrm{ppy}_{2}\right)_{2}(\mathrm{dtbbpy})\right]_{\mathrm{PF}} 6$ and 1.5 equiv. Selectfluor for $1 \mathrm{~h}$. ${ }^{[\mathrm{e}] \text { With } 2 \mathrm{~mol} \%}$ 9-

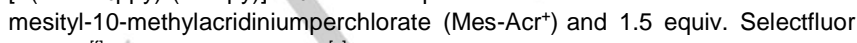
for 3 h. ${ }^{[f]}$ Reaction run for $4 \mathrm{~h}$. ${ }^{\left[{ }^{[9]}\right.}$ Reaction run for $10 \mathrm{~h}$ and product $2 \mathrm{~g}$ converted to an indole adduct to simplify purification.

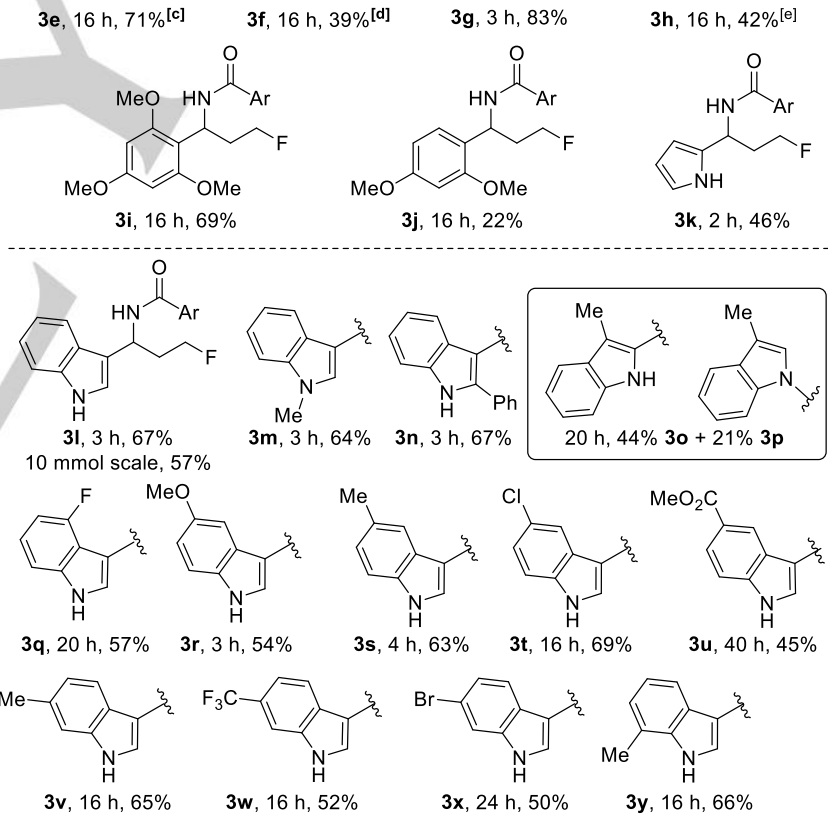

Scheme 2. Scope of nucleophiles in the one-pot ring-opening fluorination reaction. [a]Reactions were run at a $0.30 \mathrm{mmol}$ scale for $45 \mathrm{~min}$, then a solution of nucleophile (1.2 equiv.) in $0.5 \mathrm{~mL} \mathrm{MeCN}$ was added and the mixture was kept stirring at room temperature for the indicated time, $\mathrm{Ar}=p-\mathrm{MeO}-\mathrm{Ph} .{ }^{[b]} \mathrm{MeOH}(1.0$ $\mathrm{mL}, 82$ equiv.) was used. ${ }^{[c]} 1,2,4-1 H$-Triazole (2.0 equiv.) was used. ${ }^{[d]} 1,2,3,4$ $1 \mathrm{H}$-Tetrazole (2.0 equiv.) was used. [e]Potassium trans-styryltrifluoroborate $(2.0$ equiv) was used.

The addition of indole provided $3 \mathbf{I}$ in $67 \%$ yield. Upon scaling up this reaction to $10 \mathrm{mmol}, 1.86$ grams (57\% yield) of 31 were 
obtained. Indoles bearing a $\mathrm{N}$-methyl or a 2-phenyl group provided the corresponding products $3 \mathrm{~m}$ and $3 \mathrm{n}$ in 64 and $67 \%$ yield. With 3-methyl indole, 30 and $3 p$ resulting from $C$ and $N$ alkylation were isolated in $65 \%$ yield in 2:1 ratio. 4-Fluoro indole was well tolerated, giving $\mathbf{3 q}$ in $57 \%$ yield. Indoles bearing electron-donating substituents like methoxy (3r) and methyl (3s), as well as electron-withdrawing substituents like a chloro (3t) and an ester groups $(\mathbf{3 u})$ at the $\mathrm{C} 5$ position gave yields ranging from $45 \%$ to $69 \%$. A methyl (3v), a $\mathrm{CF}_{3}(\mathbf{3 w})$ and a bromo (3x) group at $\mathrm{C} 6$ or a methyl at $\mathrm{C} 7$ position (3y) led to product formation in 50 $66 \%$ yield.

We then examined the scope of aminocyclopropanes and aminocyclobutanes (Scheme 3). When using 2-methyl substituted aminocyclopropane $4 a$ (d.r.= 4:1), 5a was isolated as a mixture of two diastereoisomers in a 1:1 ratio. With 2-phenyl substituted aminocyclopropane $\mathbf{4 b}$, $\mathbf{5} \mathbf{b}$ was obtained in $90 \%$ yield after reduction by $\mathrm{NaBH}_{3} \mathrm{CN}$. When using bicyclic compound 4c, a mixture of two diastereoisomers in a ratio of 1.4:1 was isolated in $60 \%$ yield. 2,2-Difluoro aminocyclopropane $4 \mathrm{~d}$ afforded trifluoromethyl hemiaminal $\mathbf{5 d}$ in $68 \%$ yield.

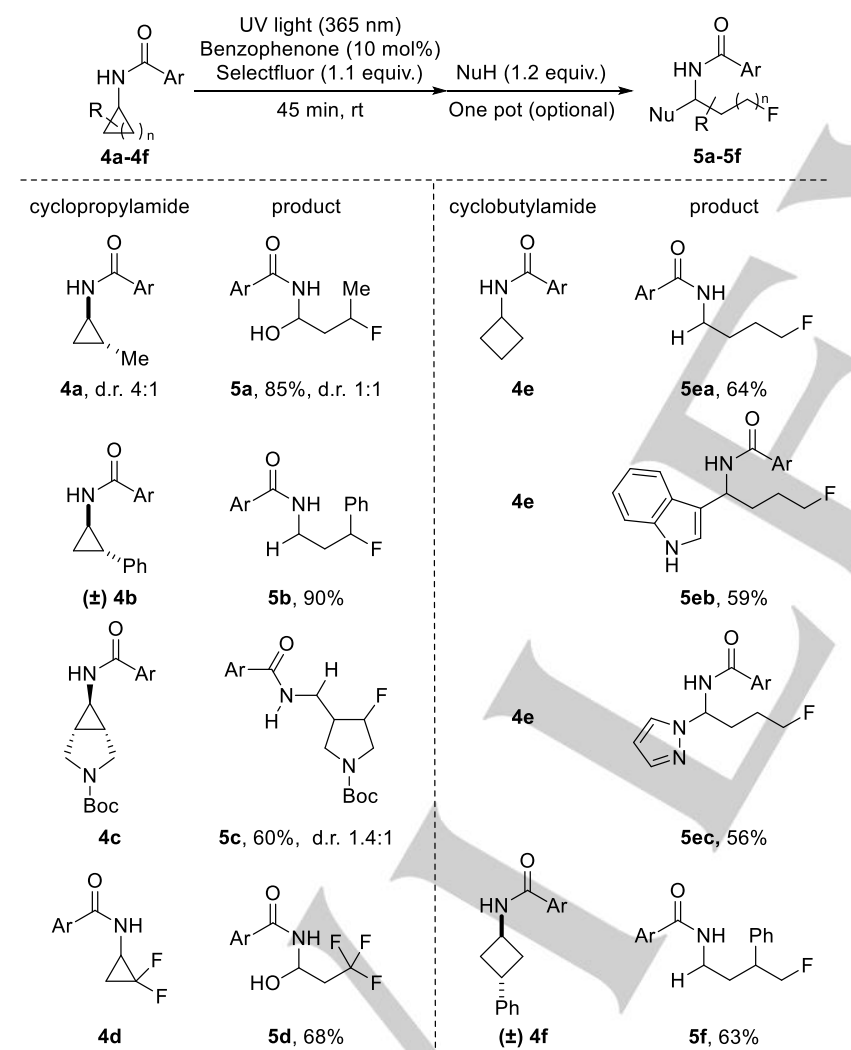

Scheme 3. Scope of multi-substituted aminocyclopropanes and aminocyclobutanes in the ring-opening fluorination reaction. $\mathrm{Ar}=\mathrm{p}-\mathrm{MeO}-\mathrm{Ph}$ See supporting Information for detailed reaction conditions.

With aminocyclobutane $4 \mathbf{e}$, a series of products 5 ea-5ec was obtained in $56-64 \%$ yield, by simply adding different nucleophiles (hydride, indole or pyrazole) for the second step. When using 3 phenyl aminocyclobutane $\mathbf{4 f}$, $5 \mathbf{f}$ resulting from selective $\mathrm{C} 1-\mathrm{C} 2$ bond cleavage next to nitrogen was obtained in $63 \%$ yield, while C3-C4 bond cleavage was observed only in traces by ${ }^{19} \mathrm{~F}$ NMR in the reaction mixture. With cyclopropylbenzene (6) oxyfluorinated product 7 was isolated in $63 \%$ yield, with the same regioselectivity as previously observed for aminofluorination (Scheme 4). ${ }^{[3]}$ This shows that selectivity for ring-opening is substrate controlled and not originating from our different reaction conditions.

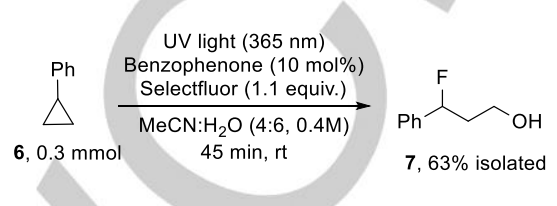

Scheme 4. Oxy-fluorination of cyclopropylbenzene (6).

In order to better understand the mechanism, we performed several control experiments (Scheme 5). When the trans and the cis isomers of $\mathbf{4 a}$ were submitted separately to the reaction conditions, the same diastereomeric ratio was observed for product $\mathbf{5 a}$, supporting the formation of a ring-opened intermediate (Scheme 5, equation 1). When adding TEMPO, we obtained product 8 in $13 \%$ yield and recovered $84 \%$ 1a suggesting that a primary alkyl radical was formed (Scheme 5 , equation 2). In order to exclude initiation by hydrogen atom transfer (HAT) to form a neutral amidyl radical, we tested the reaction of $\mathrm{N}$-cyclopropyl-4-methoxy- $\mathrm{N}$-methylbenzamide (9). Products 11 and 12 were obtained, resulting probably from the hydrolysis of unstable hemiaminal intermediate $\mathbf{1 0}$ (Scheme 5 equation 3). Attempts to synthesize a N-fluorinated aminocyclopropane corresponding to 1a were unsuccessful, ${ }^{[16]}$ and an independently synthesized $\mathrm{N}$-fluoro amide did not react under our reaction conditions (See Supporting Information for details). ${ }^{[17]}$ This makes $\mathrm{N}-\mathrm{H}$ fluorination as a first step highly improbable.

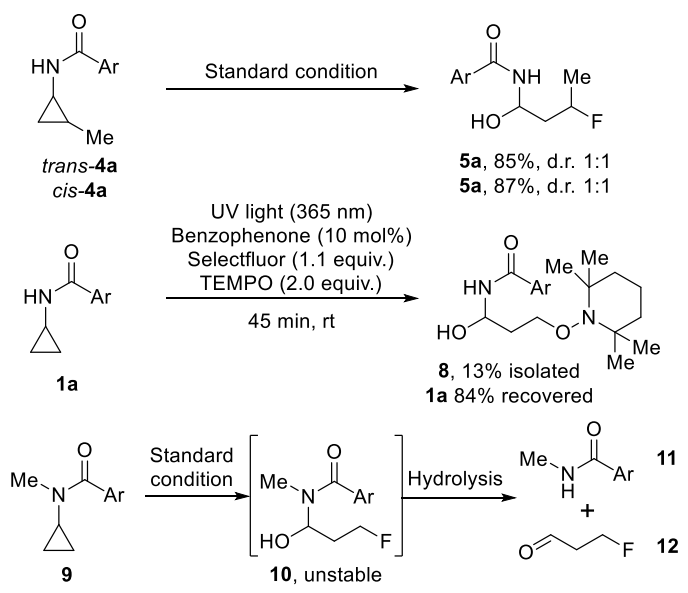

Scheme 5. Mechanistic investigations. 
Based on these results and literature precedence, a first speculative reaction mechanism can be proposed (Scheme 6). Photo-excited ketones such as benzophenone (BP) in their triplet state can initiate processes such as hydrogen atom transfer $(\mathrm{HAT}),{ }^{[18]}$ triplet energy transfer $(\mathrm{EnT})^{[19]}$ and single electron transfer (SET). ${ }^{[20]}$ By comparing the reduction potentials of cyclopropylamide 1a, Selectfluor and triplet state benzophenone $\left(\mathbf{B P}^{3^{*}}\right)$, neither Selectfluor $\left(E_{1 / 2}{ }^{\text {red }}=+0.33 \mathrm{~V}\right)^{[21,22]}$ nor triplet state benzophenone $\left(\mathrm{E}_{1 / 2}{ }^{\mathrm{BP} / \mathrm{BP}-}=+1.27 \mathrm{~V}\right)^{[23]}$ are able to oxidize cyclopropylamide 1a (measured $E_{1 / 2}^{\text {red }}=+1.67 \mathrm{~V}$ ). However, Selectfluor or the Selectfluor-derived radical cation $\left(\mathbf{I}, \mathrm{E}_{1 / 2}{ }^{\text {red }}=\right.$ $+0.79 \mathrm{~V})^{[24]}$ could oxidize triplet state benzophenone $\left(\mathbf{B P}^{3^{*}}, \mathrm{E}_{1 / 2}\right.$ $\left.\mathrm{BP}^{2} / \mathrm{BP}^{*}=-0.62 \mathrm{~V}\right)$ to the benzophenone radical cation $\mathrm{BP}^{+}$, which is oxidizing enough to convert $\mathbf{1 a}$ to radical cation intermediate III $\left(\mathrm{E}_{1 / 2}{ }^{\mathrm{BP}+/ \mathrm{BP}}=+2.37 \mathrm{~V}\right)$. For $\left[\operatorname{Ir}\left(\mathrm{dF}-\mathrm{CF}_{3} \mathrm{ppy}\right)_{2}(\mathrm{dtbbpy})\right] P F_{6}$, a similar catalytic cycle could be proposed when considering its redox properties $\left(\mathrm{E}_{1 / 2}{ }^{\operatorname{Ir}(\mathrm{III})^{*} / \mathrm{Ir}(\mathrm{II})}=+1.21 \mathrm{~V}, \mathrm{E}_{1 / 2} \operatorname{lr(II)^{*}/\mathrm {Ir}(\mathrm {IV})}=-0.89 \mathrm{~V}\right.$ and $\mathrm{E}_{1 / 2}$ $\operatorname{Ir}(\mathrm{IV}) / \mathrm{Ir}(\mathrm{III})=+1.69 \mathrm{~V}){ }^{[21]}$ For the stronger oxidizing Mes-Acr ${ }^{+}$dye $\left(E_{1 / 2}{ }^{\text {red }}=+2.06 \mathrm{~V}\right),{ }^{[25]}$ another mechanism may be operative. After SET oxidation, III would undergo ring opening to form IV, followed by radical fluorination with Selectfluor and nucleophilic addition of water to the iminium to yield $\mathbf{2 a}$. The reversal of regiochemistry observed compared to the work of Lectka can be tentatively attributed to the low stability of III leading to ring-opening, whereas the radical cation obtained from arylcyclopropanes is more stable and undergoes first nucleophilic addition.

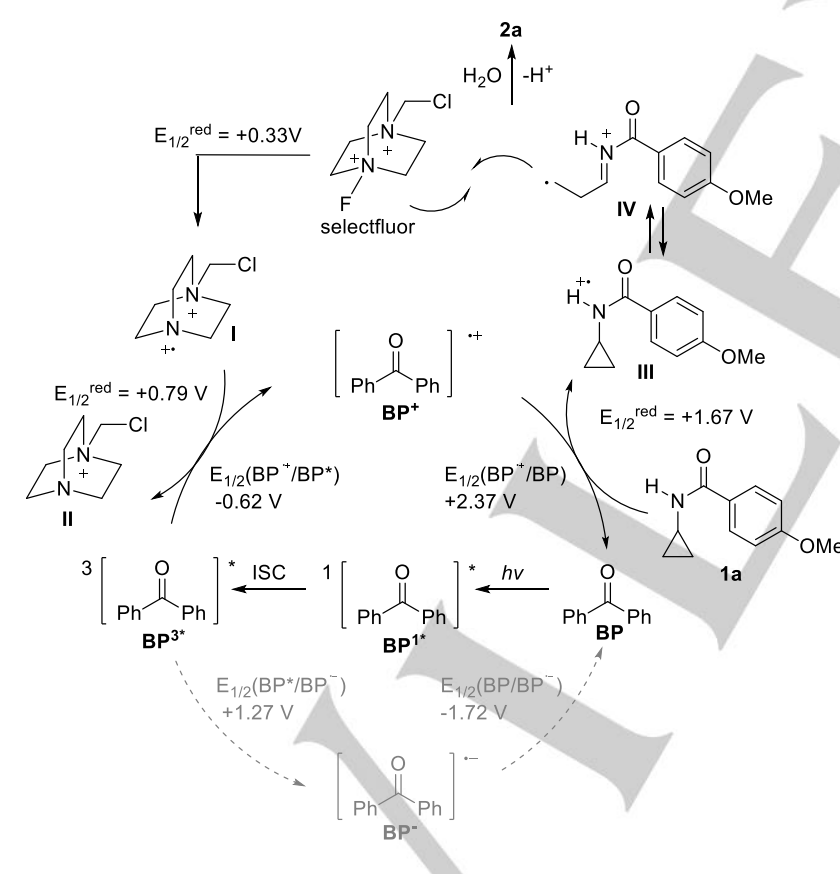

Scheme 6. Speculative reaction mechanism.

In summary, we have developed a strategy for the ring-opening fluorination of cyclopropylamides and cyclobutylamides using cheap benzophenone as organophotoredox catalyst. The hemiaminal products can be converted to other building blocks by substituting the hydroxy group with diverse nucleophiles. Based on the simple reaction procedure and the structural diversity of nitrogen- and fluorine-containing building blocks obtained, we believe that this methodology will be useful in synthetic and medicinal chemistry.

\section{Acknowledgements}

We thank the Swiss National Science Foundation (SNSF, grant nos. 200021_165788 and 200020_182798) and EPFL for financial support. We thank Dr. Luca Buzzetti, Dr. Zhikun Zhang and Ms. Stephanie Amos from ISIC EPFL for helpful discussion. We thank Mr. Bastian Muriel from our laboratory for the synthesis of compound $\mathbf{1 h}$.

Keywords: aminocyclopropanes • ring-opening • photoreaction • fluorination • benzophenone

[1] a) Organofluorine chemistry (Eds.: R. E. Banks, B. E. Smart, J. C Tatlow) Springer US: Boston, MA, 1994. b) K. Müller, C. Faeh, F Diederich, Science 2007, 317, 1881; c) P. A. Champagne, J. Desroches, J.-D. Hamel, M. Vandamme, J.-F. Paquin, Chem. Rev. 2015, 115, 9073 d) Q. Cheng, T. Ritter, Trends Chem. 2019, 1, 461; e) R. Szpera, D. F. J. Moseley, L. B. Smith, A. J. Sterling, V. Gouverneur, Angew. Chem., Int. Ed. 2019, 58, 14824.

[2] a) H. Yan, C. Zhu, Sci. China Chem. 2017, 60, 214; b) X. Wu, C. Zhu Chem. Rec. 2018, 18, 587; c) Y. Liu, Q.-L. Wang, Z. Chen, C.-S. Zhou B.-Q. Xiong, P.-L. Zhang, C.-A. Yang, Q. Zhou, Beilstein J. Org. Chem 2019, 15, 256; d) S. P. Morcillo, Angew. Chem., Int. Ed. 2019, 58, 14044

[3] a) C. R. Pitts, B. Ling, J. A. Snyder, A. E. Bragg, T. Lectka, J. Am. Chem. Soc. 2016, 138, 6598; b) S. M. Banik, K. M. Mennie, E. N. Jacobsen, J. Am. Chem. Soc. 2017, 139, 9152; c) N. O. Ilchenko, M. Hedberg, K. J. Szabó, Chem. Sci. 2017, 8, 1056.

[4] a) H. Zhao, X. Fan, J. Yu, C. Zhu, J. Am. Chem. Soc. 2015, 137, 3490 b) N. Ishida, S. Okumura, Y. Nakanishi, M. Murakami, Chem. Lett. 2015 44, 821; c) S. Ren, C. Feng, T.-P. Loh, Org. Biomol. Chem. 2015, 13, 5105 ; d) S. Bloom, D. D. Bume, C. R. Pitts, T. Lectka, Chem. Eur.J. 2015 21, 8060; e) Y. Deng, N. I. Kauser, S. M. Islam, J. T. Mohr, Eur. J. Org Chem. 2017, 2017, 5872; f) Y. A. Konik, M. Kudrjashova, N. Konrad, S. Kaabel, I. Järving, M. Lopp, D. G. Kananovich, Org. Biomol. Chem. 2017, $15,4635$.

[5] General reviews on donor-acceptor cyclopropanes: a) H.-U. Reissig, R Zimmer, Chem. Rev. 2003, 103, 1151; b) T. F. Schneider, J. Kaschel, D. B. Werz, Angew. Chem., Int. Ed. 2014, 53, 5504; Selected examples of aminocyclopropanes: c) F. de Nanteuil, J. Waser, Angew. Chem., Int. Ed. 2011, 50, 12075; d) A. R. Rivero, I. Fernández, M. Á. Sierra, Org. Lett. 2013, 15, 4928; e) F. de Nanteuil, E. Serrano, D. Perrotta, J. Waser, J. Am. Chem. Soc. 2014, 136, 6239; f) S. Racine, F. de Nanteuil, E. Serrano, J. Waser, Angew. Chem., Int. Ed. 2014, 53, 8484; g) D. Perrotta, M.-M. Wang, J. Waser, Angew. Chem., Int. Ed. 2018, 57, 5120; h) M. Zhu, D.-C. Wang, M.-S. Xie, G.-R. Qu, H.-M. Guo, Chem. Eur. J. 2018, 24, 15512; i) E.-J. Hao, D.-D. Fu, D.-C. Wang, T. Zhang, G.-R. Qu, G.X. Li, Y. Lan, H.-M. Guo, Org. Chem. Front. 2019, 6, 863; j) T. Liang, M.S. Xie, G.-R. Qu, H.-M. Guo, Asian J. Org. Chem. 2019, 8, 1405; k) M. C. Zhang, D.-C. Wang, M.-S. Xie, G.-R. Qu, H.-M. Guo, S.-L. You, Chem 2019, 5, 156; For a comparison of the reactivity of different donoracceptor cyclopropanes, see: I) A. Kreft, A. Lücht, J. Grunenberg, P. G. Jones, D. B. Werz, Angew. Chem., Int. Ed. 2019, 58, 1955. 
[6] a) S. Rousseaux, B. Liégault, K. Fagnou, Chem. Sci. 2012, 3, 244; b) M. H. Shaw, E. Y. Melikhova, D. P. Kloer, W. G. Whittingham, J. F. Bower, J. Am. Chem. Soc. 2013, 135, 4992; c) M. H. Shaw, R. A. Croft, W. G. Whittingham, J. F. Bower, J. Am. Chem. Soc. 2015, 137, 8054; d) M. H. Shaw, N. G. McCreanor, W. G. Whittingham, J. F. Bower, J. Am. Chem. Soc. 2015, 137, 463; e) N. G. McCreanor, S. Stanton, J. F. Bower, J. Am. Chem. Soc. 2016, 138, 11465; f) M. H. Shaw, W. G. Whittingham, J. F. Bower, Tetrahedron 2016, 72, 2731; g) H. Kondo, K. Itami, J. Yamaguchi, Chem. Sci. 2017, 8, 3799; h) G.-W. Wang, J. F. Bower, J. Am. Chem. Soc. 2018, 140, 2743; i) Y.-L. Zhang, R.-T. Guo, J.-H. He, X.-C. Wang, Org. Lett. 2019, 21, 4239; j) G.-W. Wang, O. Boyd, T. A. Young, S. M. Bertrand, J. F. Bower, J. Am. Chem. Soc. 2020, 142, 1740.

[7] a) J. Lee, J. S. U, S. C. Blackstock, J. K. Cha, J. Am. Chem. Soc. 1997, 119,10241 ; b) J. D. Ha, J. Lee, S. C. Blackstock, J. K. Cha, J. Org. Chem 1998, 63, 8510; c) Y. Takemoto, S. Yamagata, S.-i. Furuse, C. Iwata, Chem. Commun. 1998, 651; d) C. Madelaine, Y. Six, O. Buriez, Angew. Chem., Int. Ed. 2007, 46, 8046 .

[8] a) S. Maity, M. Zhu, R. S. Shinabery, N. Zheng, Angew. Chem., Int. Ed. 2012, 51, 222; b) T. H. Nguyen, S. Maity, N. Zheng, Beilstein J. Org. Chem. 2014, 10, 975; c) T. H. Nguyen, S. A. Morris, N. Zheng, Adv Synth. Catal. 2014, 356, 2831; d) J. Wang, N. Zheng, Angew. Chem., Int Ed. 2015, 54, 11424

[9] B. Muriel, A. Gagnebin, J. Waser, Chem. Sci. 2019, 10, 10716.

[10] D. Staveness, T. M. Sadano, K. Li, E. A. Burnham, K. D. Jackson, C. R. J. Stephenson. Chem 2019, 5, 215

[11] D. Staveness, J. L. Collins, R. C. McAtee, C. R. J. Stephenson. Angew. Chem., Int. Ed. 2019, 58, 19000.

[12] a) N. C. Mancey, N. Sandon, A.-L. Auvinet, R. J. Butlin, W. Czechtizky, J. P. A. Harrity, Chem. Commun. 2011, 47, 9804; b) M.-M. Wang, J. Waser, Angew. Chem., Int. Ed. 2019, 58, 13880; c) Q. Wang, N. Zheng, Org. Lett. 2019, 21, 9999.

[13] J. B. McManus, N. P. R. Onuska, D. A. Nicewicz, J. Am. Chem. Soc. 2018, 140, 9056.

[14] See Supporting Information for details of tested reaction conditions. It is interesting to note that standard borosilicate glass tubes can be used, as less than $10 \%$ light is absorbed at $365 \mathrm{~nm}$, see: https://www.pgoonline.com/int/borofloat.html.

[15] D. E. Carrera, Chem. Commun. 2017, 53, 11185.

[16] B. J. Groendyke, D. I. AbuSalim, S. P. Cook, J. Am. Chem. Soc. 2016 138,12771

[17] In addition, light on/off and Stern-Volmer experiments were performed, but they did not allow clear cut conclusions, see Supporting Information for details.

[18] a) S. Kamijo, T. Hoshikawa, M. Inoue, Org. Lett. 2011, 13, 5928; b) T. Hoshikawa, S. Kamijo, M. Inoue, Org. Biomol. Chem. 2013, 11, 164; c) Y. Masuda, N. Ishida, M. Murakami, J. Am. Chem. Soc. 2015, 137, $14063 ;$ d) N. Ishida, Y. Masuda, S. Uemoto, M. Murakami, Chem. Eur. J. 2016, 22, 6524; e) Y. Shen, Y. Gu, R. Martin, J. Am. Chem. Soc. 2018 140, 12200; f) N. Ishida, Y. Masuda, Y. Imamura, K. Yamazaki, M. Murakami, J. Am. Chem. Soc. 2019, 141, 19611.

[19] a) E. Arceo, E. Montroni, P. Melchiorre, Angew. Chem. Int. Ed. 2014, 53 , 12064; b) A. Tröster, R. Alonso, A. Bauer, T. Bach, J. Am. Chem. Soc. 2016, 138, 7808 .

[20] Photoexcited triplet state ketones are known to be oxidants, see: a) C. G. Schaefer, K. S. Peters, J. Am. Chem. Soc. 1980, 102, 7566; b) L. Li, X. Mu, W. Liu, Y. Wang, Z. Mi, C.-J. Li, J. Am. Chem. Soc. 2016, 138, 5809; Their application as reductants is much less frequent. For a rare example, see: c) C. B. Tripathi, T. Ohtani, M. T. Corbett, T. Ooi, Chem. Sci. 2017, 8, 5622. For other possible pathways to generate benzophenone radical cations, see: d) X. Cai, Z. Han, S. Yao, N. Lin, Sci China Ser B 2001, 44, 582; e) K. Ohkubo, T. Nanjo, S. Fukuzumi, Org. Lett. 2005, 7, 4265.

[21] S. Ventre, F. R. Petronijevic, D. W. C. MacMillan, J. Am. Chem. Soc. 2015, 137, 5654
[22] All redox values indicated in this manuscript are vs SCE in MeCN.

[23] W. L. Wallace, R. P. Van Duyne, F. D. Lewis, J. Am. Chem. Soc. 1976 98, 5319.

[24] D.-M. Yan, Q.-Q. Zhao, L. Rao, J.-R. Chen, W.-J. Xiao, Chem. Eur. J. 2018, 24, 16895.

[25] K. A. Margrey, D. A. Nicewicz, Acc. Chem. Res. 2016, 49, 1997. 


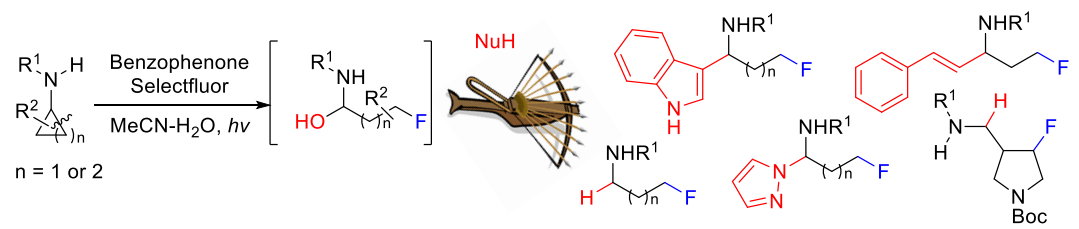

Make it shine: We report a photocatalyzed ring-opening fluorination of cyclopropylamides and cyclobutylamides. Both cheap benzophenone with UV A light or organic and inorganic dyes with blue light could be used to promote this process. Various fluorinated amines were then obtained by nucleophilic attack on the formed hemiaminals in one pot, giving access to a broad range of useful building blocks for medicinal chemistry.
Ming-Ming Wang and Jérôme Waser*

Page No. - Page No.

Oxidative

Fluorination

Cyclopropylamides

via Organic Photoredox Catalysis 


\section{Table of Contents}

$\begin{array}{ll}\text { 1. General methods } & \text { S2 }\end{array}$

2. Optimisation of the ring-opening fluorination reaction $\quad$ S3

3. Preparation of starting materials $\quad$ S4

4. Scope of ring-opening fluorination of mono-substituted cyclopropanes $\quad$ S13

5. Scope of the nucleophiles for elimination-addition of hemiaminals $\quad$ S17

6. Scope of ring-opening fluorination for muti-substituted aminocyclopropanes $\begin{array}{ll}\text { or aminocyclobutanes } & \text { S31 }\end{array}$

7. Scale-up synthesis of compound $31 \quad$ S37

$\begin{array}{ll}\text { 8. Mechanistic studies } & \text { S38 }\end{array}$

9. Spectra for new compounds $\quad$ S46 


\section{General Methods}

For quantitative flash chromatography, distilled technical grade solvents were used. THF, $\mathrm{Et}_{2} \mathrm{O}$, toluene, hexane and $\mathrm{CH}_{2} \mathrm{Cl}_{2}$ were dried by passage over activated alumina under nitrogen atmosphere $\left(\mathrm{H}_{2} \mathrm{O}\right.$ content $<7 \mathrm{ppm}$, Karl-Fischer titration). All chemicals were purchased and used as received unless stated otherwise. Chromatographic purification was performed as flash chromatography using Macherey-Nagel silica 40-63, $60 \AA$, using the solvents indicated as eluent with 0.1-0.5 bar pressure. TLC was performed on Merck silica gel 60 F254 TLC plastic or aluminium plates and visualized with UV light, permanganate CAN or p-anisaldehyde stains. Melting points were measured on a calibrated Büchi B-540 melting point apparatus using open glass capillaries. ${ }^{1} \mathrm{H}-\mathrm{NMR}$ spectra were recorded at room temperature on a Brucker DPX-400 $400 \mathrm{MHz}$ spectrometer in $\mathrm{CDCl}_{3}$, Acetone- $d_{6}, \mathrm{CD}_{3} \mathrm{CN}$ or $\mathrm{CD}_{3} \mathrm{OD}$, all signals are reported in ppm with the internal chloroform signal at $7.26 \mathrm{ppm}$, the internal acetone signal at $2.09 \mathrm{ppm}$, the internal acetonitrile signal at $1.94 \mathrm{ppm}$ and the internal methanol signal at $3.34 \mathrm{ppm}$ as standard. The data is being reported as $(\mathrm{s}=$ singlet, $\mathrm{d}=$ doublet, $\mathrm{t}=$ triplet, $\mathrm{q}=$ quadruplet, $\mathrm{p}=$ quintet, $\mathrm{m}=$ multiplet or unresolved, $\mathrm{br}=$ broad signal, integration, coupling constant $(\mathrm{s})$ in $\mathrm{Hz}$, interpretation). ${ }^{13} \mathrm{C}-\mathrm{NMR}$ spectra were recorded with $1 \mathrm{H}$-decoupling on a Brucker DPX-400 $100 \mathrm{MHz}$ spectrometer in $\mathrm{CDCl}_{3}$, Acetone- $d_{6}$ or $\mathrm{CD}_{3} \mathrm{CN}$, all signals are reported in ppm with the internal chloroform signal at $77.0 \mathrm{ppm}$, Acetone- $d_{6}$ signal at $29.8 \mathrm{ppm}$ or $\mathrm{CD}_{3} \mathrm{CN}$ signal at $1.3 \mathrm{ppm}$ as standard. Infrared spectra were recorded on a JASCO FT-IR B4100 or a Bruker Alpha-P spectrophotometer with an ATR device and a ZnSe prism and are reported as $\mathrm{cm}-1$ ( $\mathrm{w}=$ weak, $\mathrm{m}=$ medium, $\mathrm{s}=$ strong). High resolution mass spectrometric measurements were performed by the mass spectrometry service of ISIC at the EPFL on a MICROMASS (ESI) Q-TOF Ultima API. Fluorescence quenching experiment was conducted on a Varian Cary Eclipse machine. UV/Vis spectroscopy was performed on an Agilent Cary $60 \mathrm{UV}-\mathrm{Vis}$ machine. Cyclic voltammetry was performed with a Biologic SP-150 Potentiostat.

For reactions under the irradiation of UV light $(365 \mathrm{~nm})$, reactions were performed in $12 * 75 \mathrm{~mm}$ borosilicate glass tubes which were placed around $7 \mathrm{~cm}$ far away from lamps (CAMAG UV Lamp 4, long-wave UV light $365 \mathrm{~nm}$ ) in Rayonet RPR-100 photochemical reactor. For reactions under the irradiation of blue light, reactions were performed in $12 * 75 \mathrm{~mm}$ borosilicate glass tubes which were hold using a rack for test tubes placed at the center of a crystallization flask. On this flask were attached the blue LEDs (Ruban LED avec câble à extrémités ouvertes Barthelme Y51516414 18240524 V 502 $\mathrm{cm}$ bleu $1 \mathrm{pc}(\mathrm{s})$, bought directly on www.conrad.ch/fr). The distance between the LEDs and the test tubes was approximatively 3 to $4 \mathrm{~cm}$. Long irradiation for more than $2 \mathrm{~h}$ resulted in temperature increasing up to $34^{\circ} \mathrm{C}$. 


\section{Optimisation of the ring-opening fluorination reaction}

\subsection{Screening of protecting group}

For substrates 1a-k, reactions were carried out by following General Procedure B (GP B). It is worth attention that $\mathbf{1 h}$ underwent quick degradation as soon as solvent was added, before UV irradiation.

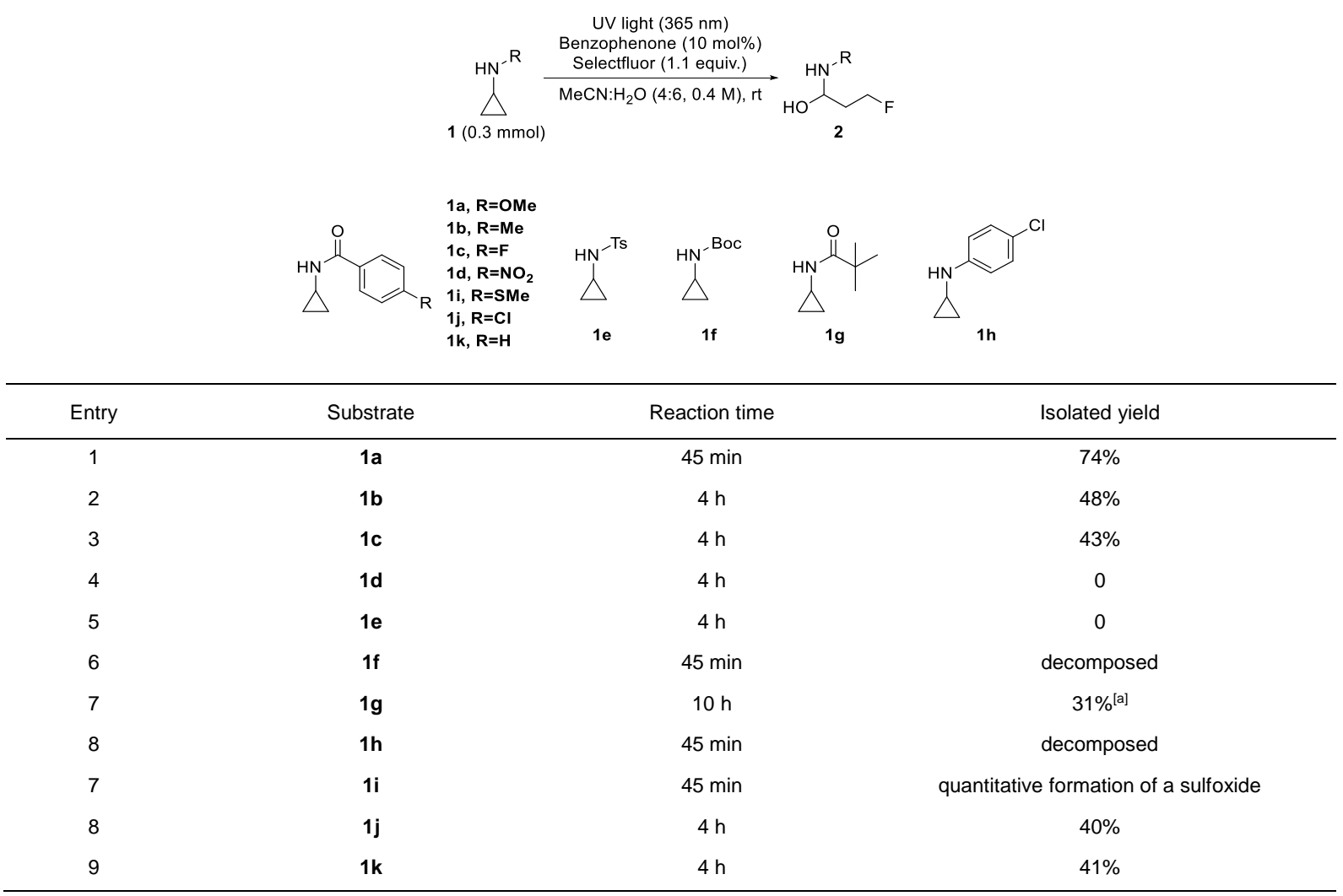

[a] ${ }^{1} \mathrm{H}$ NMR yield using fluorobenzene as internal standard. Product $\mathbf{2 g}$ was converted to an indole adduct $\mathbf{2 g}$-indole by following General Procedure $\mathrm{C}$, in order to simplify purification.

\subsection{Catalyst screening}

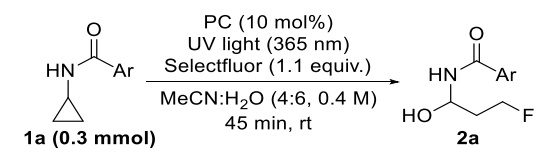

\begin{tabular}{|c|c|c|c|}
\hline Entry & Catalyst & Deviation & Isolated yield \\
\hline 1 & Benzophenone & - & $74 \%$ \\
\hline 2 & 9-Fluorenone & - & $6 \%[a]$ \\
\hline 3 & 9-Fluorenone & No water & 0 \\
\hline 4 & Benzophenone & No water & 0 \\
\hline 5 & {$\left[\mathrm{Ir}\left(\mathrm{dF}-\mathrm{CF}_{3} \mathrm{ppy}\right)_{2}(\mathrm{dtbbpy}) \mathrm{PF}_{6}(1 \mathrm{~mol} \%)\right.$} & Selectfluor (1.5 equiv), $1.5 \mathrm{~h}$, blue LED & $76 \%{ }^{[a]}$ \\
\hline 6 & 9-Mesityl-10-methylacridinium perchlorate (2 mol\%) & Selectfluor (1.5 equiv), $3 \mathrm{~h}$, blue LED & $75 \%$ [a] \\
\hline
\end{tabular}

[a] ${ }^{1} \mathrm{H}$ NMR yield using $\mathrm{CH}_{2} \mathrm{Br}_{2}$ or fluorobenzene as internal standard.

\subsection{Solvent screening}




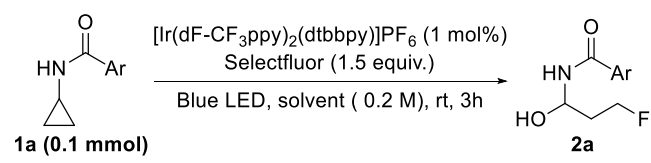

\begin{tabular}{ccc}
\hline Entry & Solvent & NMR yield ${ }^{[a]}$ \\
\hline 1 & $\mathrm{MeCN}$ & $<5 \%$ \\
2 & $\mathrm{CD}_{2} \mathrm{Cl}_{2}$ & 0 \\
3 & $\mathrm{Acetone}-d_{6}$ & 10 \\
4 & $\mathrm{DMSO}-d_{6}$ & 0 \\
5 & $\mathrm{Methanol}-d_{4}$ & 0 \\
6 & $\mathrm{MeCN}^{-} \mathrm{H}_{2} \mathrm{O}(4: 6)$ & $76 \%$ \\
7 & $\mathrm{CD}_{2} \mathrm{Cl}_{2}-\mathrm{D}_{2} \mathrm{O}(4: 6)$ & $<5 \%$ \\
8 & $\mathrm{Acetone}_{2} \mathrm{O}(4: 6)$ & $53 \%$ \\
9 & $\mathrm{DMSO}-\mathrm{H}_{2} \mathrm{O}(4: 6)$ & 0 \\
\hline
\end{tabular}

[a] ${ }^{1} \mathrm{H}$ NMR yield using fluorobenzene as internal standard.

\section{Preparation of starting materials}

\subsection{Synthesis of the aminocyclopropanes}

\section{General Procedure A (GP A):}<smiles>[R]C(=O)N[C@H](C)[C@@H](C)NC([R])=O</smiles>

Following a modified version of a reported procedure,,$^{[1]}$ to a solution of cyclopropylamine $(0.70 \mathrm{~mL}$, $10 \mathrm{mmol}, 1.1$ equiv.) and triethylamine $(1.40 \mathrm{~mL}, 10.0 \mathrm{mmol}, 1.1$ equiv.) in dichloromethane $(10 \mathrm{~mL})$ was slowly added a solution of acyl chloride $(9.09 \mathrm{mmol}, 1.0$ equiv.) in dichloromethane $(10 \mathrm{~mL})$ at 0 ${ }^{\circ} \mathrm{C}$. The reaction mixture was stirred at room temperature usually for 16 hours. Upon completion, the mixture was quenched by the addition of $1 \mathrm{~N} \mathrm{HCl}(10 \mathrm{~mL})$. The aqueous layer was then extracted with dichloromethane. The organic extract was washed with $1 \mathrm{M} \mathrm{NaOH}(10 \mathrm{~mL})$ and brine $(10 \mathrm{~mL})$, dried over $\mathrm{Na}_{2} \mathrm{SO}_{4}$, filtered and concentrated in vacuo. In most cases, the crude product was pure enough to be used as such, without further purification.

The synthesis of $\mathbf{1 a}-\mathbf{1} \mathbf{i}$ has already been described by our group. The procedures are taken from the indicated publications to facilitate reproduction of the results by having all data in the same file.

\footnotetext{
${ }^{1}$ Baburajan, P.; Elango, K. P. Tetrahedron Lett. 2014, 55, 1006-1010.
} 
<smiles>COc1ccc(C(=O)NC2CC2)cc1</smiles>

Following GP A, using 4-methoxybenzoyl chloride (1.55 g, $9.09 \mathrm{mmol}), \quad \mathrm{N}$-cyclopropyl-4methoxybenzamide 1a was obtained as a white solid (1.90 g, $8.99 \mathrm{mmol}, 99 \%)$.

${ }^{1} \mathrm{H}$ NMR $\left(400 \mathrm{MHz}, \mathrm{CDCl}_{3}\right) \delta 7.74-7.66(\mathrm{~d}, J=8.8 \mathrm{~Hz}, 2 \mathrm{H}, \mathrm{ArH}), 6.94-6.85(\mathrm{~d}, J=8.8 \mathrm{~Hz}, 2 \mathrm{H}, \operatorname{ArH}), 6.21$ $(\mathrm{s}, 1 \mathrm{H}, \mathrm{NH}), 3.84\left(\mathrm{~s}, 3 \mathrm{H}, \mathrm{OCH}_{3}\right), 2.88(\mathrm{tq}, J=7.1,3.6 \mathrm{~Hz}, 1 \mathrm{H}, \mathrm{CH}), 0.85\left(\mathrm{td}, J=7.0,5.3 \mathrm{~Hz}, 2 \mathrm{H}, \mathrm{CH}_{2}\right), 0.65$ $-0.55\left(\mathrm{~m}, 2 \mathrm{H}, \mathrm{CH}_{2}\right)$.

${ }^{1} \mathrm{H}$ NMR data correspond to the reported values. ${ }^{[1]}$

N-cyclopropyl-4-methylbenzamide (1b).<smiles>Cc1ccc(C(=O)NC2CC2)cc1</smiles>

Following GP A, using 4-methylbenzoyl chloride (1.41 g, $9.09 \mathrm{mmol}), \quad N$-cyclopropyl-4methylbenzamide $1 \mathrm{~b}$ was obtained as a white solid $(1.51 \mathrm{~g}, 8.62 \mathrm{mmol}, 95 \%)$.

${ }^{1} \mathrm{H}$ NMR $\left(400 \mathrm{MHz}, \mathrm{CDCl}_{3}\right): \delta=7.72-7.55(\mathrm{~m}, 2 \mathrm{H}, \mathrm{ArH}), 7.23-7.11(\mathrm{~m}, 2 \mathrm{H}, \operatorname{ArH}), 6.33(\mathrm{~d}, J=39.2 \mathrm{~Hz}$, $1 \mathrm{H}, \mathrm{NH}), 2.88(\mathrm{tt}, J=7.2,3.5 \mathrm{~Hz}, 1 \mathrm{H}, \mathrm{CH}), 2.37\left(\mathrm{~d}, J=3.1 \mathrm{~Hz}, 3 \mathrm{H}, \mathrm{CH}_{3}\right), 0.92-0.75\left(\mathrm{~m}, 2 \mathrm{H}, \mathrm{CH}_{2}\right), 0.68-$ $0.54\left(\mathrm{~m}, 2 \mathrm{H}, \mathrm{CH}_{2}\right)$.

${ }^{1} \mathrm{H}$ NMR data correspond to the reported values. ${ }^{[2]}$

N-Cyclopropyl-4-fluorobenzamide (1c).

${ }^{2}$ Zhang, Y.; Liu, B.; Gou, Z.; Li, Y.; Zhang, X.; Wang, Y.; Yu, S.; Li, Y.; Sun, D. Bioorg. Med. Chem. Lett. 2015, 25, 791-794. 


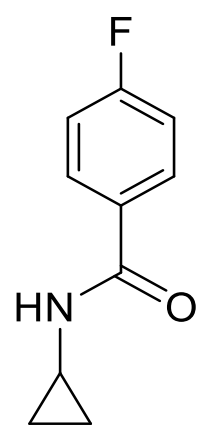

Following GP A, using 4-fluorobenzoyl chloride (1.44 g, $9.09 \mathrm{mmol}$ ), $\mathrm{N}$-cyclopropyl-4-fluorobenzamide $1 \mathrm{c}$ was obtained as a white solid $(1.50 \mathrm{~g}, 8.36 \mathrm{mmol}, 92 \%)$.

${ }^{1} \mathbf{H}$ NMR $\left(400 \mathrm{MHz}, \mathrm{CDCl}_{3}\right): \delta=7.79-7.70(\mathrm{~m}, 2 \mathrm{H}, \mathrm{ArH}), 7.12-7.01(\mathrm{~m}, 2 \mathrm{H}, \mathrm{ArH}), 6.37(\mathrm{~s}, 1 \mathrm{H}, \mathrm{NH}), 2.87$ (tq, $J=7.1,3.6 \mathrm{~Hz}, 1 \mathrm{H}, \mathrm{CH}), 0.84\left(\mathrm{td}, J=7.0,5.3 \mathrm{~Hz}, 2 \mathrm{H}, \mathrm{CH}_{2}\right), 0.65-0.56\left(\mathrm{~m}, 2 \mathrm{H}, \mathrm{CH}_{2}\right)$.

${ }^{1} \mathrm{H}$ NMR data correspond to the reported values. ${ }^{[3]}$

N-Cyclopropyl-4-nitrobenzamide (1d).<smiles>O=C(NC1CC1)c1ccc([N+](=O)[O-])cc1</smiles>

Following GP A, using 4-nitrobenzoyl chloride (1.69 g, $9.09 \mathrm{mmol}), \mathrm{N}$-cyclopropyl-4-nitrobenzamide $1 \mathrm{~d}$ was obtained as a pale yellow solid $(1.71 \mathrm{~g}, 8.27 \mathrm{mmol}, 91 \%)$.

$\mathbf{R}_{\mathbf{f}}$ : 0.31 (silica, pentanes:ethyl acetate 1:1);

Mp: $176-177^{\circ} \mathrm{C}$;

${ }^{1} \mathrm{H}$ NMR $\left(400 \mathrm{MHz}, \mathrm{CDCl}_{3}\right): \delta=8.30-8.22(\mathrm{~m}, 2 \mathrm{H}, \mathrm{ArH}), 7.94-7.86(\mathrm{~m}, 2 \mathrm{H}, \mathrm{ArH}), 6.41(\mathrm{~s}, 1 \mathrm{H}, \mathrm{NH}), 2.92$ (tq, $J=7.2,3.7 \mathrm{~Hz}, 1 \mathrm{H}, \mathrm{CH}), 0.91\left(\mathrm{td}, J=7.1,5.4 \mathrm{~Hz}, 2 \mathrm{H}, \mathrm{CH}_{2}\right), 0.70-0.59\left(\mathrm{~m}, 2 \mathrm{H}, \mathrm{CH}_{2}\right)$;

${ }^{13} \mathrm{C}$ NMR $\left(101 \mathrm{MHz}, \mathrm{CDCl}_{3}\right): \delta=166.8,149.6,139.9,128.0,123.8,23.4,6.8$;

IR (film): $\tilde{v}=3280$ (m), 1639 (s), 1597 (w), 1533 (m), 1514 (s), 1350 (m), 1308 (m);

HRMS (ESI) calcd. for $\mathrm{C}_{10} \mathrm{H}_{11} \mathrm{~N}_{2} \mathrm{O}_{3}{ }^{+}[\mathrm{M}+\mathrm{H}]^{+}$207.0764; Found 207.0761 .

$N$-(1-Cyano-3-iodopropyl)-4-methylbenzenesulfonamide (1e).<smiles>Cc1ccc(S(=O)(=O)NC2CC2)cc1</smiles>

\footnotetext{
${ }^{3}$ Kondo, H.; Itami, K.; Yamaguchi, J. Chem. Sci. 2017, 8, 3799-3803.
} 
Following GP A, using tosyl chloride (1.73 g, $9.09 \mathrm{mmol}), \quad \mathrm{N}$-(1-Cyano-3-iodopropyl)-4methylbenzenesulfonamide $1 \mathrm{e}$ was obtained as a colorless solid (1.90 g, $9.00 \mathrm{mmol}, 99 \%)$.

${ }^{1} \mathrm{H}$ NMR $\left(400 \mathrm{MHz}, \mathrm{CDCl}_{3}\right): \delta=7.79(\mathrm{~d}, J=8.4 \mathrm{~Hz}, 2 \mathrm{H}, \operatorname{ArH}), 7.32(\mathrm{~d}, J=8.0 \mathrm{~Hz}, 2 \mathrm{H}, \operatorname{ArH}), 4.94(\mathrm{~s}, 1 \mathrm{H}, \mathrm{NH})$, $2.43\left(\mathrm{~s}, 3 \mathrm{H}, \mathrm{CH}_{3}\right), 2.23(\mathrm{tt}, J=6.6,3.7 \mathrm{~Hz}, 1 \mathrm{H}, \mathrm{CH}), 0.65-0.52\left(\mathrm{~m}, 4 \mathrm{H}, \mathrm{CH}_{2}\right)$.

${ }^{1} \mathrm{H}$ NMR data correspond to the reported values. ${ }^{[4]}$

tert-Butyl cyclopropylcarbamate (1f).

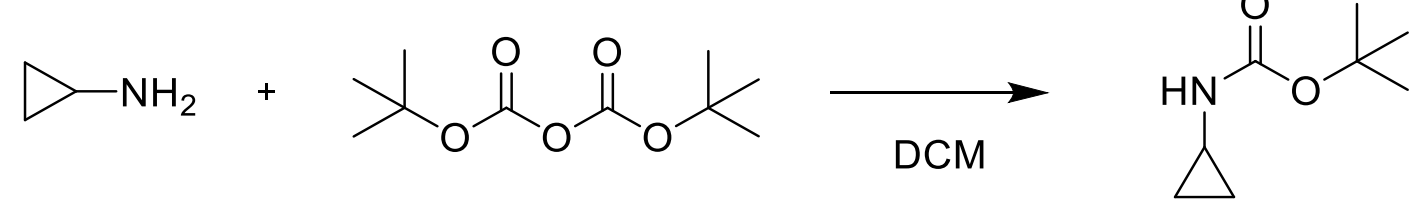

Following a modified version of a reported procedure ${ }^{[5]}$ to a solution of cyclopropylamine $(1.40 \mathrm{~mL}$, $20.0 \mathrm{mmol}, 1.0$ equiv.) in dichloromethane $(20 \mathrm{~mL})$ was slowly added a solution of di-tert-butyl dicarbonate $(4.85 \mathrm{~g}, 22.0 \mathrm{mmol}, 1.1$ equiv. $)$ in dichloromethane $(20 \mathrm{~mL})$ at $0{ }^{\circ} \mathrm{C}$. The reaction mixture was stirred at for 16 hours room temperature. Upon completion, the mixture was quenched by addition of $1 \mathrm{~N} \mathrm{HCl}(10 \mathrm{~mL})$. The aqueous layer was then extracted with dichloromethane. The organic layer was washed with $1 \mathrm{M} \mathrm{NaOH}(10 \mathrm{~mL})$ and brine $(10 \mathrm{~mL})$, and dried over $\mathrm{Na}_{2} \mathrm{SO}_{4}$, filtered and concentrated in vacuo. tert-Butyl cyclopropylcarbamate $1 \mathrm{f}$ was obtained as a white solid $(3.11 \mathrm{~g}, 19.8$ $\mathrm{mmol}, 99 \%)$, which was pure enough to be used without further purification.

${ }^{1} \mathrm{H}$ NMR $\left(400 \mathrm{MHz}, \mathrm{CDCl}_{3}\right): \delta=4.70(\mathrm{brs}, 1 \mathrm{H}, \mathrm{NH}), 2.57-2.47(\mathrm{~m}, 1 \mathrm{H}, \mathrm{CH}), 1.44\left(\mathrm{~s}, 9 \mathrm{H}, \mathrm{CH}_{3}\right), 0.72-0.63$ $\left(\mathrm{m}, 2 \mathrm{H}, \mathrm{CH}_{2}\right), 0.53-0.39\left(\mathrm{~m}, 2 \mathrm{H}, \mathrm{CH}_{2}\right)$.

${ }^{1} \mathrm{H}$ NMR data correspond to the reported values. ${ }^{[5]}$

\section{$\mathrm{N}$-Cyclopropylpivalamide (1g).}

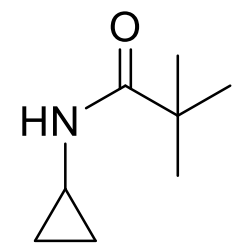

Following GP A, using pivaloyl chloride (1.32 g, $11.0 \mathrm{mmol}), \mathrm{N}$-cyclopropylpivalamide $1 \mathrm{~g}$ was obtained as a white solid $(0.90 \mathrm{~g}, 6.37 \mathrm{mmol}, 64 \%)$.

${ }^{1} \mathrm{H}$ NMR $\left(400 \mathrm{MHz}, \mathrm{CDCl}_{3}\right): \delta=5.71(\mathrm{~s}, 1 \mathrm{H}, \mathrm{NH}), 2.76-2.60(\mathrm{~m}, 1 \mathrm{H}, \mathrm{CH}), 1.16\left(\mathrm{~s}, 9 \mathrm{H}, \mathrm{CH}_{3}\right), 0.86-0.71$ $\left(\mathrm{m}, 2 \mathrm{H}, \mathrm{CH}_{2}\right), 0.57-0.36\left(\mathrm{~m}, 2 \mathrm{H}, \mathrm{CH}_{2}\right)$.

${ }^{1} \mathrm{H}$ NMR data correspond to the reported values. ${ }^{[6]}$

4-Chloro-N-cyclopropylaniline (1h).

\footnotetext{
${ }^{4}$ O'Sullivan, S.; Doni, E.; Tuttle, T.; Murphy. J. A. Angew. Chem. Int. Ed. 2014, 53, 474-478.

${ }^{5}$ Tars, K.; Leitan, J.; Kazaks, A.; Zelencova, D.; Liepinsh, E.; Kuka, J.; Makrecka, M.; Lola, D.; Andrianovs, V.; Gustina, D.; Grinberga, S.; Liepinsh, E.; Kalvinsh, I.; Dambrova, M.; Loza, E.; Pugovics, O. J. Med. Chem. 2014, 57, 2213-2236.

${ }^{6}$ Miyamura, S.; Araki, M.; Suzuki, T.; Yamaguchi, J.; Itami. K. Angew. Chem. Int. Ed. 2015, 54, 846-851.
} 
<smiles>Clc1ccc(NC2CC2)cc1</smiles>

This compound was synthesized for another project in our group. For its synthesis and characterization, please refer to: Chem. Sci. 2019, 10, 10716, SI page 17.

\section{N-Cyclopropyl-4-(methylthio)benzamide (1i).}<smiles>CSc1ccc(C(=O)NC2CC2)cc1</smiles>

Following GP A, using 4-(methylthio)benzoyl chloride (1.70 g, $9.09 \mathrm{mmol}), \quad \mathrm{N}$-cyclopropyl-4(methylthio)benzamide 1i was obtained as an off-white solid (1.41 g, $6.80 \mathrm{mmol}, 75 \%$ ).

$\mathbf{R}_{\mathbf{f}}: 0.33$ (silica, pentanes:ethyl acetate 1:1);

Mp: $158-160^{\circ} \mathrm{C}$;

${ }^{1} \mathrm{H}$ NMR $\left(400 \mathrm{MHz}, \mathrm{CDCl}_{3}\right): \delta=7.68-7.61(\mathrm{~m}, 2 \mathrm{H}, \mathrm{ArH}), 7.25-7.20(\mathrm{~m}, 2 \mathrm{H}, \mathrm{ArH}), 6.24(\mathrm{~s}, 1 \mathrm{H}, \mathrm{NH}), 2.89$ (dq, J = 7.0, 3.4 Hz, 1H, CH), $2.50\left(\mathrm{~s}, 3 \mathrm{H}, \mathrm{SCH}_{3}\right), 0.91-0.80\left(\mathrm{~m}, 2 \mathrm{H}, \mathrm{CH}_{2}\right), 0.66-0.54\left(\mathrm{~m}, 2 \mathrm{H}, \mathrm{CH}_{2}\right)$; ${ }^{13} \mathrm{C}$ NMR $\left(101 \mathrm{MHz}, \mathrm{CDCl}_{3}\right): \delta=168.3,143.4,130.4,127.2,125.3,23.1,15.0,6.8$;

IR (film): $\tilde{v}=3271$ (m), 3006 (w), 1622 (s), 1556 (s), 1486 (m), 1315 (m), 1121 (w), $840(\mathrm{~m}), 761$ (m); HRMS (ESI) calcd. for $\mathrm{C}_{11} \mathrm{H}_{13} \mathrm{NNaOS}^{+}[\mathrm{M}+\mathrm{Na}]^{+}$230.0610; Found 230.0613 .

\section{4-Chloro-N-cyclopropylbenzamide (1j).}<smiles>O=C(NC1CC1)c1ccc(Cl)cc1</smiles>

Following GP A, using 4-chlorobenzoyl chloride (1.59 g, $9.09 \mathrm{mmol})$, 4-chloro- $N$-cyclopropylbenzamide 1j was obtained as a white solid (1.65 g, $8.43 \mathrm{mmol}, 93 \%)$.

$\mathbf{R}_{\mathbf{f}}: 0.59$ (silica, pentanes:ethyl acetate 2:3);

Mp: $133-135^{\circ} \mathrm{C}$;

${ }^{1} \mathrm{H}$ NMR $\left(400 \mathrm{MHz}, \mathrm{CDCl}_{3}\right): \delta=7.70-7.64(\mathrm{~m}, 2 \mathrm{H}, \mathrm{ArH}), 7.41-7.35(\mathrm{~m}, 2 \mathrm{H}, \operatorname{ArH}), 6.27(\mathrm{~s}, 1 \mathrm{H}, \mathrm{NH}), 2.88$ (tt, $J=7.1,3.5 \mathrm{~Hz}, 1 \mathrm{H}, \mathrm{CH}), 0.90-0.83\left(\mathrm{~m}, 2 \mathrm{H}, \mathrm{CH}_{2}\right), 0.66-0.55\left(\mathrm{~m}, 2 \mathrm{H}, \mathrm{CH}_{2}\right)$;

${ }^{13} \mathrm{C}$ NMR $\left(101 \mathrm{MHz}, \mathrm{CDCl}_{3}\right): \delta=167.8,137.7,132.7,128.8,128.3,23.2,6.8$; 
IR (film): $\tilde{v}=3309$ (m), 1639 (s), 1528 (m), 1484 (m), 1312 (m), 1093 (m), 847 (m);

HRMS (ESI) calcd. for $\mathrm{C}_{10} \mathrm{H}_{10} \mathrm{CINNaO}+[\mathrm{M}+\mathrm{Na}]^{+} 218.0343$; Found 218.0344 .

\section{$N$-cyclopropylbenzamide (1k).}<smiles>O=C(NC1CC1)c1ccccc1</smiles>

Following GP A, using benzoyl chloride $(1.28 \mathrm{~g}, 9.09 \mathrm{mmol}), N$-cyclopropylbenzamide $1 \mathbf{k}$ was obtained as a white solid (1.38 g, $8.57 \mathrm{mmol}, 94 \%)$.

${ }^{1} \mathrm{H}$ NMR $\left(400 \mathrm{MHz}, \mathrm{CDCl}_{3}\right): \delta=7.76-7.67(\mathrm{~m}, 2 \mathrm{H}, \mathrm{ArH}), 7.49-7.43(\mathrm{~m}, 1 \mathrm{H}, \mathrm{ArH}), 7.41-7.34(\mathrm{~m}, 2 \mathrm{H}$, $\operatorname{ArH}), 6.46(\mathrm{~s}, 1 \mathrm{H}, \mathrm{NH}), 2.88(\mathrm{tq}, J=7.1,3.7 \mathrm{~Hz}, 1 \mathrm{H}, \mathrm{CH}), 0.87-0.78\left(\mathrm{~m}, 2 \mathrm{H}, \mathrm{CH}_{2}\right), 0.66-0.50(\mathrm{~m}, 2 \mathrm{H}$, $\mathrm{CH}_{2}$ ).

${ }^{1} \mathrm{H}$ NMR data correspond to the reported values. ${ }^{[7]}$

4-Methoxy-N-2-methylcyclopropyl)benzamide (4a).

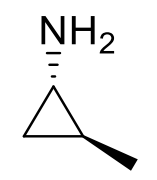

$4: 1$ d.r.<smiles>COc1ccc(C(=O)N[C@@H]2C[C@H]2C)cc1</smiles>

trans<smiles>COc1ccc(C(=O)N[C@@H]2C[C@@H]2C)cc1</smiles>

cis

Following a modified version of a reported procedure, ${ }^{[1]}$ to a solution of 2-methylcyclopropan-1-amine (250 mg, $3.52 \mathrm{mmol}, 4: 1$ d.r., ordered from Fluorochem) and $\mathrm{Et}_{3} \mathrm{~N}(0.54 \mathrm{~mL}, 3.9 \mathrm{mmol}, 1.1$ equiv.) in dichloromethane $(10 \mathrm{~mL}$ ) was slowly added a solution of 4-methoxybenzoyl chloride $(658 \mathrm{mg}, 3.87$ mmol, 1.1 equiv.) in dichloromethane $(5 \mathrm{~mL})$ at $0{ }^{\circ} \mathrm{C}$. The reaction mixture was stirred at room temperature usually for 16 hours. Upon completion, the mixture was quenched by the addition of $1 \mathrm{~N}$ $\mathrm{HCl}(10 \mathrm{~mL})$. The aqueous layer was then extracted with dichloromethane. The organic extract was washed with brine $(10 \mathrm{~mL})$, dried over $\mathrm{Na}_{2} \mathrm{SO}_{4}$, filtered and concentrated in vacuo. 4-Methoxy-N-2methylcyclopropyl)benzamide 4 a was obtained as a white solid (655 mg, $3.20 \mathrm{mmol}, 4: 1$ d.r., 91\%) after first purification by column chromatography on silica using 3:2 pentanes:ethyl acetate as eluent. Second purification was performed by column chromatography on silica using 3:2 pentanes:ethyl acetate as eluent, trans-4a $(272 \mathrm{mg})$ and cis-4a $(79 \mathrm{mg})$ were obtained separately, together with the rest of product 4 a recovered as a mixture of diastereomers.

\section{4-Methoxy-N-trans-2-methylcyclopropyl)benzamide (trans-4a)}

$\mathbf{R}_{\mathbf{f}}: 0.37$ (silica, pentanes:ethyl acetate 1:1);

Mp: $94-96^{\circ} \mathrm{C}$;

\footnotetext{
${ }^{7}$ Sureshbabu, P.; Sadaf, A.; Chaudhary, P.; Kandasamy, J. Org. Biomol. Chem. 2019, 17, 845-850.
} 
${ }^{1}$ H NMR $\left(400 \mathrm{MHz}, \mathrm{CDCl}_{3}\right): \delta=7.75-7.62(\mathrm{~m}, 2 \mathrm{H}, \mathrm{ArH}), 6.94-6.83(\mathrm{~m}, 2 \mathrm{H}, \mathrm{ArH}), 6.20(\mathrm{~s}, 1 \mathrm{H}, \mathrm{NH}), 3.83$ $\left(\mathrm{s}, 3 \mathrm{H}, \mathrm{OCH}_{3}\right), 2.56(\mathrm{dq}, J=6.9,3.4 \mathrm{~Hz}, 1 \mathrm{H}, \mathrm{NCH}), 1.13\left(\mathrm{~d}, J=6.1 \mathrm{~Hz}, 3 \mathrm{H}, \mathrm{CH}_{3}\right), 0.95$ (ddt, J = 12.2, 6.2, $3.2 \mathrm{~Hz}, 1 \mathrm{H}, \mathrm{CHCH}_{3}$ ), 0.73 (ddd, $\left.J=9.2,5.4,3.8 \mathrm{~Hz}, 1 \mathrm{H}, \mathrm{CH}_{2}\right), 0.61$ (dt, $J=7.2,5.7 \mathrm{~Hz}, 1 \mathrm{H}, \mathrm{CH}_{2}$ ).

${ }^{13} \mathrm{C} \mathrm{NMR}\left(101 \mathrm{MHz}, \mathrm{CDCl}_{3}\right): \delta=168.1,162.1,128.6,126.7,113.6,55.4,30.4,17.2,14.9$ (signals of $\mathrm{C} 2$ and $\mathrm{C} 3$ are overlapped).

IR (film): $\tilde{v}$ = 3274 (m), 3003 (w), 2952 (w), 1624 (s), 1606 (s), 1574 (m), 1541 (s), 1254 (s), 1031 (m), $843(\mathrm{~m})$;

HRMS (APCl) calcd. for $\mathrm{C}_{12} \mathrm{H}_{15} \mathrm{NNaO}_{2}{ }^{+}[\mathrm{M}+\mathrm{Na}]^{+} 228.0995$; Found 228.0993.

4-Methoxy-N-cis-2-methylcyclopropyl)benzamide (cis-4a)

$\mathbf{R}_{\mathbf{f}}: 0.26$ (silica, pentanes:ethyl acetate $1: 1$ );

Mp: $89-91^{\circ} \mathrm{C}$;

${ }^{1} \mathrm{H}$ NMR $\left(400 \mathrm{MHz}, \mathrm{CDCl}_{3}\right): \delta=7.76-7.69(\mathrm{~m}, 2 \mathrm{H}, \mathrm{ArH}), 6.92-6.87(\mathrm{~m}, 2 \mathrm{H}, \mathrm{ArH}), 6.07(\mathrm{~s}, 1 \mathrm{H}, \mathrm{NH}), 3.84$ (s, 3H, OCH 3 ), 2.90 (dddd, $J=9.9,7.0,4.0,3.0 \mathrm{~Hz}, 1 \mathrm{H}, \mathrm{NCH}), 1.15-1.07\left(\mathrm{~m}, 4 \mathrm{H}, \mathrm{CH}_{3}+\mathrm{CH}_{2}\right.$ ), 1.03 (dddd, $\left.J=8.8,5.4,3.2,1.2 \mathrm{~Hz}, 1 \mathrm{H}, \mathrm{CH}_{2}\right), 0.26-0.15\left(\mathrm{~m}, 1 \mathrm{H}, \mathrm{CHCH}_{3}\right)$.

${ }^{13} \mathrm{C}$ NMR $\left(101 \mathrm{MHz}, \mathrm{CDCl}_{3}\right): \delta=168.7,162.1,128.6,126.9,113.7,55.4,27.5,13.2,12.5,11.7$.

IR (film): $\tilde{v}=3292$ (m), 2958 (w), 1631 (s), 1606 (s), 1499 (s), 1252 (s), 1178 (m), 1028 (m), 844 (m);

HRMS (APCl) calcd. for $\mathrm{C}_{12} \mathrm{H}_{15} \mathrm{NNaO}_{2}{ }^{+}[\mathrm{M}+\mathrm{Na}]^{+}$228.0995; Found 228.0993.

\section{4-Methoxy- $N$-(trans-2-phenylcyclopropyl)benzamide (4b).}

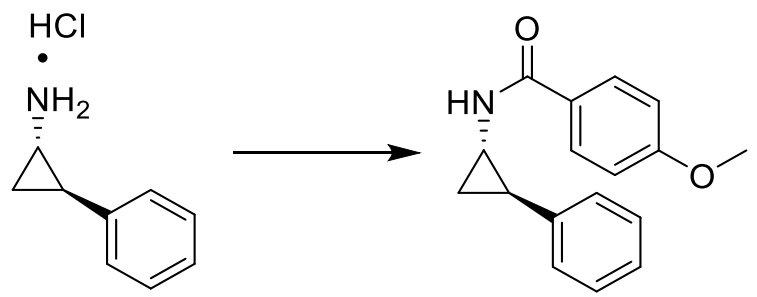

Following a modified version of a reported procedure, ${ }^{[1]}$ to a solution of trans-2phenylcyclopropylamine hydrochloride $(635 \mathrm{mg}, 3.74 \mathrm{mmol}, 1.1$ equiv., ordered from Acros) and triethylamine (1.0 mL, $7.2 \mathrm{mmol}, 2.0$ equiv.) in dichloromethane $(10 \mathrm{~mL})$ was slowly added a solution of 4-methoxybenzoyl chloride $\left(607 \mathrm{mg}, 3.56 \mathrm{mmol}, 1.0\right.$ equiv.) in dichloromethane $(10 \mathrm{~mL})$ at $0{ }^{\circ} \mathrm{C}$. The reaction mixture was stirred at room temperature for 16 hours. Upon completion, the mixture was quenched by the addition of $1 \mathrm{~N} \mathrm{HCl}(10 \mathrm{~mL})$. The aqueous layer was then extracted with dichloromethane. The organic extract was washed with $1 \mathrm{M} \mathrm{NaOH}(10 \mathrm{~mL})$ and brine $(10 \mathrm{~mL})$, dried over $\mathrm{Na}_{2} \mathrm{SO}_{4}$, filtered and concentrated in vacuo. 4-Methoxy- $N$-(trans-2-phenylcyclopropyl)benzamide 4b was obtained as a white solid $(0.92 \mathrm{~g}, 3.4 \mathrm{mmol}, 97 \%)$.

$\mathbf{R}_{\mathbf{f}}: 0.50$ (silica, pentanes:ethyl acetate 1:1);

Mp: $153-155^{\circ} \mathrm{C}$;

${ }^{1} \mathrm{H}$ NMR $\left(400 \mathrm{MHz}, \mathrm{CDCl}_{3}\right): \delta=7.81-7.65(\mathrm{~m}, 2 \mathrm{H}, \mathrm{ArH}), 7.31-7.26(\mathrm{~m}, 2 \mathrm{H}, \mathrm{ArH}), 7.24-7.15(\mathrm{~m}, 3 \mathrm{H}$, $\operatorname{ArH}), 6.97-6.83(\mathrm{~m}, 2 \mathrm{H}, \mathrm{ArH}), 6.39(\mathrm{~s}, 1 \mathrm{H}, \mathrm{NH}), 3.84\left(\mathrm{~s}, 3 \mathrm{H}, \mathrm{OCH}_{3}\right), 3.06(\mathrm{tt}, J=7.4,3.4 \mathrm{~Hz}, 1 \mathrm{H}, \mathrm{NCH})$, 2.16 (ddd, $J=9.8,6.3,3.4 \mathrm{~Hz}, 1 \mathrm{H}, \mathrm{PhCH}$ ), 1.29 (dddd, $J=27.6,10.0,6.0,4.4 \mathrm{~Hz}, 2 \mathrm{H}, \mathrm{CH}_{2}$ );

${ }^{13} \mathrm{C}$ NMR $\left(101 \mathrm{MHz}, \mathrm{CDCl}_{3}\right): \delta=168.1,162.2,140.4,128.7,128.4,126.6,126.5,126.1,113.7,55.4,32.5$, 24.9, 16.3;

IR (film): $\tilde{v}=3291$ (w), 1632 (s), 1606 (s), 1500 (s), 1255 (s), 1029 (m), 845 (m);

HRMS (ESI) calcd. for $\mathrm{C}_{17} \mathrm{H}_{17} \mathrm{NNaO}_{2}{ }^{+}[\mathrm{M}+\mathrm{Na}]^{+}$290.1151; Found 290.1151 .

(1R,5S,6S)-tert-Butyl 6-(4-methoxybenzamido)-3-azabicyclo[3.1.0]hexane-3-carboxylate (4c). 
<smiles>CC(C)(C)OC(=O)N1C[C@H]2[C@H](N)[C@H]2C1</smiles><smiles>COc1ccc(C(=O)Cl)cc1</smiles><smiles>CCN(C)CC(=O)O</smiles><smiles>COc1ccc(C(=O)NC2[C@H]3CN(C(=O)OC(C)(C)C)C[C@H]23)cc1</smiles>

Following a modified version of a reported procedure, ${ }^{[1]}$ to a solution of $(1 R, 5 S, 6 S)$-tert-butyl 6 -amino)3-azabicyclo[3.1.0] hexane-3-carboxylate $(495 \mathrm{mg}, 2.50 \mathrm{mmol}, 1.0$ equiv., ordered from Spirochem) and triethylamine $(0.40 \mathrm{~mL}, 2.9 \mathrm{mmol}, 1.1$ equiv. $)$ in dichloromethane $(10 \mathrm{~mL})$ was slowly added a solution of 4-methoxybenzoyl chloride $(470 \mathrm{mg}, 2.76 \mathrm{mmol}, 1.1$ equiv.) in dichloromethane $(5 \mathrm{~mL})$ at $0{ }^{\circ} \mathrm{C}$. The reaction mixture was stirred at room temperature usually for 16 hours. Upon completion, the mixture was quenched by the addition of $1 \mathrm{~N} \mathrm{HCl}(10 \mathrm{~mL})$. The aqueous layer was then extracted with dichloromethane. The organic extract was washed with brine $(10 \mathrm{~mL})$, dried over $\mathrm{Na}_{2} \mathrm{SO}_{4}$, filtered and concentrated in vacuo. (1R,5S,6S)-tert-butyl 6-(4-methoxybenzamido)-3-azabicyclo[3.1.0]hexane3-carboxylate (4c) was obtained as a beige solid (740 mg, $2.23 \mathrm{mmol}, 89 \%)$ after purification by column chromatography on silica using 2:3 pentanes:ethyl acetate as eluent.

$\mathbf{R}_{\mathbf{f}}: 0.29$ (silica, pentanes:ethyl acetate 2:3);

Mp: $160-162^{\circ} \mathrm{C}$;

${ }^{1} \mathrm{H}$ NMR $\left(400 \mathrm{MHz}, \mathrm{CDCl}_{3}\right): \delta=7.74-7.64(\mathrm{~m}, 2 \mathrm{H}, \mathrm{ArH}), 6.93-6.82(\mathrm{~m}, 2 \mathrm{H}, \mathrm{ArH}), 6.41-6.24(\mathrm{~m}, 1 \mathrm{H}$, $\mathrm{NH}), 3.82\left(\mathrm{~d}, J=1.9 \mathrm{~Hz}, 3 \mathrm{H}, \mathrm{OCH}_{3}\right), 3.77-3.65\left(\mathrm{~m}, 2 \mathrm{H}, \mathrm{CH}_{2}\right), 3.45-3.34\left(\mathrm{~m}, 2 \mathrm{H}, \mathrm{CH}_{2}\right), 2.60-2.55(\mathrm{~m}$, $1 \mathrm{H}, \mathrm{NCH}), 1.79-1.68(\mathrm{~m}, 2 \mathrm{H}, \mathrm{CH}), 1.42\left(\mathrm{~d}, \mathrm{~J}=1.4 \mathrm{~Hz}, 9 \mathrm{H}, \mathrm{CH}_{3}\right)$; Mixture of 2 rotamers with almost 1:1 ratio. They are not completely resolved.

${ }^{13} \mathrm{C} \mathrm{NMR}\left(101 \mathrm{MHz}, \mathrm{CDCl}_{3}\right): \delta=168.0,162.2,154.5,128.7,126.3,113.7,79.5,55.4,47.8,47.5,33.1$, 28.4, 25.2, 23.7; Mixture of 2 rotamers with almost 1:1 ratio. They are not completely resolved.

IR (film): $\tilde{v}=3302$ (w), 2974 (w), 2931 (w), 2873 (w), 1694 (s), 1606 (s), 1502 (s), 1393 (s), 1253 (s), $1172(\mathrm{~s}), 1115(\mathrm{~s}), 1029(\mathrm{~m}), 844(\mathrm{w}), 769(\mathrm{w}), 730(\mathrm{w})$;

HRMS (ESI) calcd. for $\mathrm{C}_{18} \mathrm{H}_{24} \mathrm{~N}_{2} \mathrm{NaO}_{4}{ }^{+}[\mathrm{M}+\mathrm{Na}]^{+}$355.1628; Found 355.1631.

\section{$N$-(2,2-Difluorocyclopropyl)-4-methoxybenzamide (4d).}

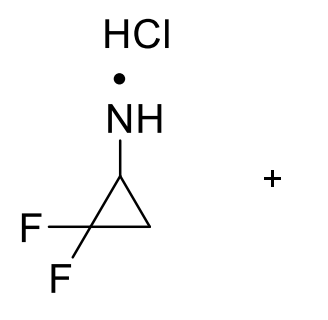<smiles>COc1ccc(C(=O)Cl)cc1</smiles>
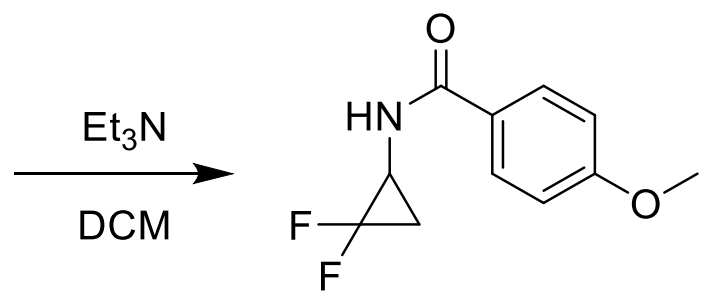

Following a modified version of a reported procedure, ${ }^{[1]}$ to a solution of 2,2-difluorocyclopropylamine hydrochloride ( $250 \mathrm{mg}, 1.93 \mathrm{mmol}, 1.0$ equiv., ordered from Fluorochem) and triethylamine $(0.60 \mathrm{~mL}$, $4.3 \mathrm{mmol}, 2.2$ equiv.) in dichloromethane $(10 \mathrm{~mL}$ ) was slowly added a solution of 4-methoxybenzoyl chloride (370 mg, $2.17 \mathrm{mmol}, 1.1$ equiv.) in dichloromethane $(5 \mathrm{~mL})$ at $0{ }^{\circ} \mathrm{C}$. The reaction mixture was stirred at room temperature usually for 16 hours. Upon completion, the mixture was quenched by the addition of $1 \mathrm{~N} \mathrm{HCl}(10 \mathrm{~mL})$. The aqueous layer was then extracted with dichloromethane. The organic extract was washed with brine $(10 \mathrm{~mL})$, dried over $\mathrm{Na}_{2} \mathrm{SO}_{4}$, filtered and concentrated in vacuo. $\mathrm{N}-(2,2-$ 
difluorocyclopropyl)-4-methoxybenzamide (4d) was obtained as a white solid (320 mg, $1.41 \mathrm{mmol}$, 73\%) after purification by column chromatography on silica using 2:1 pentanes:ethyl acetate as eluent.

$\mathbf{R}_{\mathbf{f}}: 0.25$ (silica, pentanes:ethyl acetate 2:1);

Mp: $128-129^{\circ} \mathrm{C}$;

${ }^{1} \mathrm{H}$ NMR $\left(400 \mathrm{MHz}, \mathrm{CDCl}_{3}\right): \delta=7.73(\mathrm{~d}, J=8.7 \mathrm{~Hz}, 2 \mathrm{H}, \operatorname{ArH}), 6.91(\mathrm{~d}, J=8.7 \mathrm{~Hz}, 2 \mathrm{H}, \operatorname{ArH}), 6.39(\mathrm{~s}, 1 \mathrm{H}$, $\mathrm{NH}), 3.84\left(\mathrm{~s}, 3 \mathrm{H}, \mathrm{OCH}_{3}\right), 3.51(\mathrm{dtq}, J=12.1,5.9,3.4,3.0 \mathrm{~Hz}, 1 \mathrm{H}, \mathrm{CH}), 1.87$ (dtd, $J=13.5,9.3,6.4 \mathrm{~Hz}, 1 \mathrm{H}$, $\left.\mathrm{CH}_{2}\right), 1.50-1.35\left(\mathrm{~m}, 1 \mathrm{H}, \mathrm{CH}_{2}\right)$;

${ }^{13} \mathrm{C}$ NMR (101 MHz, CDCl $): \delta={ }^{13} \mathrm{C}$ NMR (101 MHz, Chloroform-d) $\delta 167.8,162.6,128.9,125.5,113.8$, 111.1 (dd, $J=291.4,284.3 \mathrm{~Hz}), 55.4,30.8(\mathrm{dd}, J=15.0,9.4 \mathrm{~Hz}), 19.3(\mathrm{t}, J=9.9 \mathrm{~Hz}$ );

${ }^{19} \mathrm{~F} \mathrm{NMR}\left(376 \mathrm{MHz}, \mathrm{CDCl}_{3}\right): \delta=-131.2(\mathrm{~d}, J=162.2 \mathrm{~Hz}, 1 \mathrm{~F}),-143.6(\mathrm{~d}, J=162.2 \mathrm{~Hz}, 1 \mathrm{~F})$;

IR (film): $\tilde{v}=3307$ (m), 1638 (s), 1608 (m), 1500 (s), 1471 (m), 1257 (s), $1222(\mathrm{~s}), 1014(\mathrm{~m}), 845(\mathrm{~m})$;

HRMS (ESI) calcd. for $\mathrm{C}_{11} \mathrm{H}_{12} \mathrm{~F}_{2} \mathrm{NO}_{2}{ }^{+}[\mathrm{M}+\mathrm{H}]^{+}$228.0831; Found 228.0843.

$N$-Cyclobutyl-4-methoxybenzamide (4e)

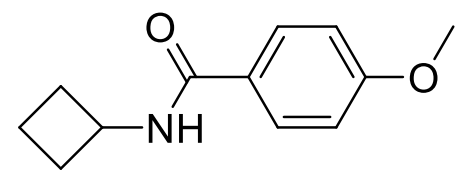

Following GP A, using cyclobutylamine ( $523 \mathrm{mg}, 7.40 \mathrm{mmol}, 1.1$ equiv.) and 4-methoxybenzoyl chloride (1.19 g, $7.00 \mathrm{mmol}, 1.0$ equiv.), $\mathrm{N}$-cyclobutyl-4-methoxybenzamide $4 \mathrm{e}$ was obtained as a white solid (1.08 g, $5.27 \mathrm{mmol}, 75 \%)$.

$\mathbf{R}_{\mathbf{f}}: 0.44$ (silica, pentanes:ethyl acetate 1:1);

Mp: $126-128^{\circ} \mathrm{C}$;

${ }^{1} \mathrm{H}$ NMR $\left(400 \mathrm{MHz}, \mathrm{CDCl}_{3}\right) \delta=7.84-7.61(\mathrm{~m}, 2 \mathrm{H}, \mathrm{ArH}), 7.00-6.82(\mathrm{~m}, 2 \mathrm{H}, \mathrm{ArH}), 6.16(\mathrm{~s}, 1 \mathrm{H}, \mathrm{NH}), 4.58$ $(\mathrm{h}, J=8.1 \mathrm{~Hz}, 1 \mathrm{H}, \mathrm{CH}), 3.84\left(\mathrm{~s}, 3 \mathrm{H}, \mathrm{OCH}_{3}\right), 2.53-2.32\left(\mathrm{~m}, 2 \mathrm{H}, \mathrm{CH}_{2} \mathrm{CH}_{2} \mathrm{CH}_{2}\right), 2.02-1.86\left(\mathrm{~m}, 2 \mathrm{H}, \mathrm{CH}_{2}\right), 1.76$ (tt, $J=11.4,6.5 \mathrm{~Hz}, 2 \mathrm{H}, \mathrm{CH}_{2}$ );

${ }^{13} \mathrm{C}$ NMR $\left(101 \mathrm{MHz}, \mathrm{CDCl}_{3}\right): \delta=166.0,162.1,128.6,126.8,113.7,55.4,45.1,31.4,15.2$;

IR (film): $\tilde{v}=3305$ (w), 2941 (w), 1628 (s), 1607 (s), 1503 (s), 1253 (s), 1030 (m), 844 (m);

HRMS (ESI) calcd. for $\mathrm{C}_{12} \mathrm{H}_{16} \mathrm{NO}_{2}{ }^{+}[\mathrm{M}+\mathrm{H}]^{+}$206.1176; Found 206.1175.

4-Methoxy- $N$-(trans-3-phenylcyclobutyl)benzamide (4f).<smiles>NC1CC(c2ccccc2)C1</smiles><smiles>COc1ccc(C(=O)Cl)cc1</smiles><smiles>CCN(CC)CC(=O)O</smiles><smiles>COc1ccc(C(=O)NC2CC(c3ccccc3)C2)cc1</smiles>

Following a modified version of a reported procedure, ${ }^{[1]}$ to a solution of trans-3-phenylcyclobutan-1amine $(250 \mathrm{mg}, 1.70 \mathrm{mmol}, 1.0$ equiv., ordered from Fluorochem) and triethylamine $(0.27 \mathrm{~mL}, 1.9$ mmol, 1.1 equiv.) in dichloromethane $(10 \mathrm{~mL})$ was slowly added a solution of 4-methoxybenzoyl chloride (320 mg, $1.88 \mathrm{mmol}, 1.1$ equiv.) in dichloromethane $(5 \mathrm{~mL})$ at $0{ }^{\circ} \mathrm{C}$. The reaction mixture was stirred at room temperature usually for 16 hours. Upon completion, the mixture was quenched by the addition of $1 \mathrm{~N} \mathrm{HCl}(10 \mathrm{~mL})$. The aqueous layer was then extracted with dichloromethane. The organic extract was washed with brine $(10 \mathrm{~mL})$, dried over $\mathrm{Na}_{2} \mathrm{SO}_{4}$, filtered and concentrated in vacuo. 4- 
Methoxy- $N$-(trans-3-phenylcyclobutyl)benzamide $\mathbf{4 f}$ was obtained as a white solid (448 $\mathrm{mg}, 1.59 \mathrm{mmol}$, $94 \%$ ) after purification by column chromatography on silica using 1:1 pentanes:ethyl acetate as eluent.

$\mathbf{R}_{\mathbf{f}}: 0.50$ (silica, pentanes:ethyl acetate 1:1);

Mp: $164-166^{\circ} \mathrm{C}$;

${ }^{1}$ H NMR $\left(400 \mathrm{MHz}, \mathrm{CDCl}_{3}\right): \delta=7.83-7.71(\mathrm{~m}, 2 \mathrm{H}, \mathrm{ArH}), 7.39-7.26(\mathrm{~m}, 4 \mathrm{H}, \mathrm{ArH}), 7.26-7.17(\mathrm{~m}, 1 \mathrm{H}$, ArH), $6.98-6.87(\mathrm{~m}, 2 \mathrm{H}, \mathrm{ArH}), 6.36(\mathrm{~d}, J=6.9 \mathrm{~Hz}, 1 \mathrm{H}, \mathrm{NH}), 4.71(\mathrm{ddtd}, J=14.2,7.9,6.3,1.3 \mathrm{~Hz}, 1 \mathrm{H}$, $\mathrm{NCH}), 3.86\left(\mathrm{~s}, 3 \mathrm{H}, \mathrm{OCH}_{3}\right), 3.71-3.57(\mathrm{~m}, 1 \mathrm{H}, \mathrm{PhCH}), 2.74-2.61\left(\mathrm{~m}, 2 \mathrm{H}, \mathrm{CH}_{2}\right), 2.55-2.43\left(\mathrm{~m}, 2 \mathrm{H}, \mathrm{CH}_{2}\right)$; ${ }^{13} \mathrm{C}$ NMR $\left(101 \mathrm{MHz}, \mathrm{CDCl}_{3}\right): \delta 166.5,162.1,144.8,128.7,128.5,126.8,126.5,126.0,113.7,55.4,43.7$, 36.9, 34.6;

IR (film): $\tilde{v}=3338$ (m), 2938 (w), 1628 (s), 1605 (m), 1499 (s), 1251 (s), 1031 (m);

HRMS (ESI) calcd. for $\mathrm{C}_{18} \mathrm{H}_{20} \mathrm{NO}_{2}{ }^{+}[\mathrm{M}+\mathrm{H}]^{+}$282.1489; Found 282.1480.

$N$-Cyclopropyl-4-methoxy- $N$-methylbenzamide (9).<smiles>COc1ccc(C(=O)N(C)C2CC2)cc1</smiles>

Following GP D, $N$-cyclopropyl-methylamine hydrochloride $(323 \mathrm{mg}, 3.00 \mathrm{mmol}, 1.1$ equiv.) was used as starting material. $\mathrm{N}$-cyclopropyl-4-methoxy- $\mathrm{N}$-methylbenzamide 9 (560 mg, $2.73 \mathrm{mmol}, 97 \%$ ) was obtained as a yellow oil which solidified during storage.

$\mathbf{R}_{\mathbf{f}}: 0.40$ (silica, pentanes:ethyl acetate 2:3);

Mp: $59-61^{\circ} \mathrm{C}$;

${ }^{1} \mathrm{H}$ NMR (400 MHz, CDCl 3 ): $\delta=7.55-7.43(\mathrm{~m}, 2 \mathrm{H}, \mathrm{ArH}), 6.94-6.82(\mathrm{~m}, 2 \mathrm{H}, \mathrm{ArH}), 3.83\left(\mathrm{~s}, 3 \mathrm{H}, \mathrm{OCH}_{3}\right)$, $3.07\left(\mathrm{~s}, 3 \mathrm{H}, \mathrm{NCH}_{3}\right), 2.82(\mathrm{tt}, J=7.0,3.9 \mathrm{~Hz}, 1 \mathrm{H}, \mathrm{CH}), 0.63\left(\mathrm{~d}, J=6.7 \mathrm{~Hz}, 2 \mathrm{H}, \mathrm{CH}_{2}\right), 0.47(\mathrm{p}, J=5.8,5.0 \mathrm{~Hz}$, $\left.2 \mathrm{H}, \mathrm{CH}_{2}\right)$;

${ }^{13} \mathrm{C}$ NMR $\left(101 \mathrm{MHz}, \mathrm{CDCl}_{3}\right): \delta=172.2,160.6,129.4,129.4,113.1,55.2,35.6,33.1,9.4$;

IR (film): $\tilde{v}=3010$ (w), 2936(w), 1626 (s), 1607 (s), 1381 (s), 1250 (s), $1172(\mathrm{~m}), 1027$ (m), $842(\mathrm{~m})$;

HRMS (ESI) calcd. for $\mathrm{C}_{12} \mathrm{H}_{15} \mathrm{NNaO}_{2}^{+}[\mathrm{M}+\mathrm{Na}]^{+}$228.0995; Found 228.0999.

\section{Scope of ring-opening fluorination of mono-substituted cyclopropanes}

\section{General Procedure B (GP B):}

In a $12 * 75 \mathrm{~mm}$ Borosilicate glass tube, the corresponding aminocyclopropane $(0.300 \mathrm{mmol}, 1.0$ equiv.), selectfluor (117 mg, $0.330 \mathrm{mmol}, 1.1$ equiv.) and benzophenone ( $5.4 \mathrm{mg}, 0.030 \mathrm{mmol}, 0.10$ equiv.) were dissolved in $0.75 \mathrm{~mL}$ of $\mathrm{MeCN}-\mathrm{H}_{2} \mathrm{O}(\mathrm{v}: \mathrm{v}$ 4:6, $0.40 \mathrm{M})$. The reaction mixture was degassed by three freeze-pump-thaw cycles and backfilled with $\mathrm{N}_{2}$. The mixture was then stirred at room temperature under $365 \mathrm{~nm}$ irradiation in Rayonet Reactor for 45 minutes, if not specified otherwise. The distance between glass tube and lamp was $7 \mathrm{~cm}$. After the completion of the reaction, the crude product was directly submitted to column chromatography on silica using pentanes:ethyl acetate as eluent.

$N$-(3-Fluoro-1-hydroxypropyl)-4-methoxybenzamide (2a). 
<smiles>COc1ccc(C(=O)NC(O)CCF)cc1</smiles>

Following GP B, starting from N-cyclopropyl-4-methoxybenzamide 1 a $(57.3 \mathrm{mg}, 0.300 \mathrm{mmol}), \mathrm{N}$-(3fluoro-1-hydroxypropyl)-4-methoxybenzamide $2 \mathrm{a}(50.5 \mathrm{mg}, 0.222 \mathrm{mmol}, 74 \%)$ was obtained as a white solid after purification by column chromatography on silica using 3:2 pentanes:ethyl acetate as eluent.

$\mathbf{R}_{\mathbf{f}}: 0.27$ (silica, pentanes:ethyl acetate 1:1);

Mp: $119-121^{\circ} \mathrm{C}$;

${ }^{1} \mathrm{H}$ NMR $\left(400 \mathrm{MHz}, \mathrm{CDCl}_{3}\right): \delta=7.78-7.69(\mathrm{~m}, 2 \mathrm{H}, \mathrm{ArH}), 7.19-7.05(\mathrm{~m}, 1 \mathrm{H}, \mathrm{NH}), 6.96-6.87(\mathrm{~m}, 2 \mathrm{H}$, $\operatorname{ArH}), 5.71(\mathrm{dt}, J=7.3,5.3 \mathrm{~Hz}, 1 \mathrm{H}, \mathrm{CH}), 4.96-4.58\left(\mathrm{~m}, 2 \mathrm{H}, \mathrm{FCH}_{2}\right), 4.27(\mathrm{~s}, 1 \mathrm{H}, \mathrm{OH}$, peak was not splitted), $3.85\left(\mathrm{~s}, 3 \mathrm{H}, \mathrm{OCH}_{3}\right), 2.27-2.06\left(\mathrm{~m}, 2 \mathrm{H}, \mathrm{CH}_{2}\right)$;

${ }^{13} \mathrm{C}$ NMR $\left(101 \mathrm{MHz}, \mathrm{CDCl}_{3}\right): \delta=168.1,162.7,128.9,125.5,113.8,80.7(\mathrm{~d}, J=161.6 \mathrm{~Hz}), 72.8(\mathrm{~d}, J=2.1$ $\mathrm{Hz}), 55.4,35.1(\mathrm{~d}, J=18.8 \mathrm{~Hz})$;

${ }^{19}$ F NMR $\left(376 \mathrm{MHz}, \mathrm{CDCl}_{3}\right): \delta=-218.0$;

IR (film): $\tilde{v}=3318(\mathrm{br}, \mathrm{s}), 2968(\mathrm{w}), 1640(\mathrm{~m}), 1606$ (s), 1503 (s), 1256 (s), $1179(\mathrm{~m}), 1029(\mathrm{~m}), 846(\mathrm{~m})$; HRMS (APPI) calcd. for $\mathrm{C}_{11} \mathrm{H}_{14} \mathrm{FNO}_{3}{ }^{+}[\mathrm{M}]^{+}$227.0952; Found 227.0949.

$N$-(3-Fluoro-1-hydroxypropyl)-4-methylbenzamide (2b).<smiles>Cc1ccc(C(=O)NC(O)CCF)cc1</smiles>

Following GP B, starting from $\mathrm{N}$-cyclopropyl-4-methylbenzamide $1 \mathrm{~b}(52.5 \mathrm{mg}, 0.300 \mathrm{mmol})$ and after stirring for 4 hours, $\mathrm{N}$-(3-fluoro-1-hydroxypropyl)-4-methylbenzamide $\mathbf{2 b}$ ( $30.3 \mathrm{mg}, 0.144 \mathrm{mmol}, 48 \%$ ) was obtained as a white solid after purification by column chromatography on silica using $3: 2$ pentanes:ethyl acetate as eluent.

$\mathbf{R}_{\mathbf{f}}: 0.32$ (silica, pentanes:ethyl acetate 1:1);

Mp: $103-105^{\circ} \mathrm{C}$;

${ }^{1} \mathrm{H}$ NMR (400 MHz, Acetone- $\left.d_{6}\right): \delta=8.04(\mathrm{~s}, 1 \mathrm{H}, \mathrm{NH}), 7.85-7.75(\mathrm{~m}, 2 \mathrm{H}, \operatorname{ArH}), 7.26(\mathrm{~d}, J=8.0 \mathrm{~Hz}, 2 \mathrm{H}$, $\operatorname{ArH}), 5.72(\mathrm{dtd}, J=8.3,6.5,4.3 \mathrm{~Hz}, 1 \mathrm{H}, \mathrm{CH}), 4.99(\mathrm{~d}, J=4.3 \mathrm{~Hz}, 1 \mathrm{H}, \mathrm{OH}), 4.74-4.50\left(\mathrm{~m}, 2 \mathrm{H}, \mathrm{FCH}_{2}\right), 2.37$ $\left(\mathrm{s}, 3 \mathrm{H}, \mathrm{CH}_{3}\right), 2.18-2.07\left(\mathrm{~m}, 2 \mathrm{H}, \mathrm{CH}_{2}\right)$;

${ }^{13} \mathrm{C}$ NMR $\left(101 \mathrm{MHz}\right.$, Acetone- $\left.d_{6}\right): \delta=167.4,142.6,132.7,129.7,128.2,81.5(\mathrm{~d}, J=161.8 \mathrm{~Hz}), 71.9$ (d, $J$ $=6.6 \mathrm{~Hz}), 37.5(\mathrm{~d}, J=19.5 \mathrm{~Hz}), 21.3$;

${ }^{19} \mathrm{~F} \mathrm{NMR}\left(376 \mathrm{MHz}\right.$, Acetone- $\left.d_{6}\right): \delta=-222.2$;

IR (film): $\tilde{v}=3301$ (br, s) 1643 (s), 1532 (m), 1504 (m), 1281 (w), 756 (m);

HRMS (APCl) calcd. for $\mathrm{C}_{11} \mathrm{H}_{14} \mathrm{FNNaO}_{2}{ }^{+}[\mathrm{M}+\mathrm{Na}]^{+} 234.0901$; Found 234.0899 .

4-Fluoro-N-(3-fluoro-1-hydroxypropyl)benzamide (2c).<smiles>O=C(NC(O)CCF)c1ccc(F)cc1</smiles> 
Following GP B, starting from $\mathrm{N}$-cyclopropyl-4-fluorobenzamide $1 \mathrm{c}(62.1 \mathrm{mg}, 0.300 \mathrm{mmol})$ and after stirring for 4 hours, 4-fluoro- $N$-(3-fluoro-1-hydroxypropyl)benzamide $2 \mathrm{c}(27.6 \mathrm{mg}, 0.128 \mathrm{mmol}, 43 \%)$ was obtained as a white solid after purification by column chromatography on silica using 3:2 pentanes:ethyl acetate as eluent.

$\mathbf{R}_{\mathbf{f}}: 0.42$ (silica, pentanes:ethyl acetate 1:1);

Mp: $80-82^{\circ} \mathrm{C}$;

${ }^{1} \mathrm{H}$ NMR $\left(400 \mathrm{MHz}, \mathrm{CDCl}_{3}\right): \delta=7.78(\mathrm{dd}, J=8.9,5.2 \mathrm{~Hz}, 2 \mathrm{H}, \mathrm{ArH}), 7.20-7.08(\mathrm{~m}, 3 \mathrm{H}, \mathrm{ArH}+\mathrm{NH}), 5.72$ $(\mathrm{dtd}, J=7.1,5.2,3.2 \mathrm{~Hz}, 1 \mathrm{H}, \mathrm{CH}), 5.11-4.43(\mathrm{~m}, 2 \mathrm{H}, \mathrm{FCH}$ ), $4.17(\mathrm{~d}, J=3.2 \mathrm{~Hz}, 1 \mathrm{H}, \mathrm{OH}), 2.44-1.92$ $\left(\mathrm{m}, 2 \mathrm{H}, \mathrm{CH}_{2}\right)$;

${ }^{13} \mathrm{C}$ NMR $\left(101 \mathrm{MHz}, \mathrm{CDCl}_{3}\right): \delta=167.5,165.1(\mathrm{~d}, J=253.0 \mathrm{~Hz}), 129.5,129.5(\mathrm{~d}, J=9.0 \mathrm{~Hz}), 115.8(\mathrm{~d}, J=$ $21.9 \mathrm{~Hz}), 80.7(\mathrm{~d}, J=161.4 \mathrm{~Hz}), 72.9(\mathrm{~d}, J=8.9 \mathrm{~Hz}), 35.0(\mathrm{~d}, J=18.6 \mathrm{~Hz})$;

${ }^{19} \mathrm{~F}$ NMR $\left(376 \mathrm{MHz}, \mathrm{CDCl}_{3}\right)$ : $\delta=-107.0,-217.8$;

IR (film): $\tilde{v}=3315$ (br, s) 1644 (s), 1604 (s), 1501 (s), 1236 (m), 852 (m);

HRMS (APCl) calcd. for $\mathrm{C}_{10} \mathrm{H}_{11} \mathrm{~F}_{2} \mathrm{NNaO}_{2}{ }^{+}[\mathrm{M}+\mathrm{Na}]^{+}$238.0650; Found 238.0648.

$N$-(3-Fluoro-1-(1H-indol-3-yl)propyl)pivalamide (2g-indole).<smiles>CC(C)(C)C(=O)NC(CCF)c1c[nH]c2ccccc12</smiles>

Following GP B, starting from $N$-cyclopropylpivalamide $1 \mathrm{~g}(42.3 \mathrm{mg}, 0.300 \mathrm{mmol})$ and after stirring for 10 hours for the first step, $1 \mathrm{H}$-indole $(42.2 \mathrm{mg}, 0.360 \mathrm{mmol}, 1.2$ equiv.) in $0.5 \mathrm{~mL} \mathrm{MeCN}$ was then added to the reaction crude. After the reaction mixture was stirred for another 16 hours, $\mathrm{N}$-(3-fluoro-1-(1Hindol-3-yl)propyl)pivalamide $\mathbf{2 g}$-indole $(8.0 \mathrm{mg}, 0.029 \mathrm{mmol}, 29 \%)$ was obtained as a yellow solid after purification by column chromatography on silica using 1:1 pentanes:ethyl acetate as eluent.

$\mathbf{R}_{\mathbf{f}}: 0.47$ (silica, pentanes:ethyl acetate 1:1);

Mp: $76-79{ }^{\circ} \mathrm{C}$;

${ }^{1} \mathrm{H}$ NMR $\left(400 \mathrm{MHz}, \mathrm{CDCl}_{3}\right): \delta=8.23(\mathrm{~s}, 1 \mathrm{H}$, indole $\mathrm{NH}), 7.59(\mathrm{~d}, J=7.9 \mathrm{~Hz}, 1 \mathrm{H}, \operatorname{ArH}), 7.38(\mathrm{~d}, J=8.2 \mathrm{~Hz}$, $1 \mathrm{H}, \operatorname{ArH}), 7.22(\mathrm{ddd}, J=8.2,7.1,1.2 \mathrm{~Hz}, 1 \mathrm{H}, \operatorname{ArH}), 7.15-7.10(\mathrm{~m}, 2 \mathrm{H}, \mathrm{ArH}), 6.04(\mathrm{~d}, J=7.8 \mathrm{~Hz}, 1 \mathrm{H}, \mathrm{NH})$, $5.51(\mathrm{q}, J=7.2 \mathrm{~Hz}, 1 \mathrm{H}, \mathrm{CH}), 4.67-4.41\left(\mathrm{~m}, 2 \mathrm{H}, \mathrm{FCH}_{2}\right), 2.48-2.32\left(\mathrm{~m}, 2 \mathrm{H}, \mathrm{CH}_{2}\right), 1.20\left(\mathrm{~s}, 9 \mathrm{H}, \mathrm{CH}_{3}\right)$;

${ }^{13} \mathrm{C}$ NMR $\left(101 \mathrm{MHz}, \mathrm{CDCl}_{3}\right): \delta=177.8,136.6,125.7,122.5,121.7,119.9,119.1,116.2,111.5,82.0$ (d, J $=163.9 \mathrm{~Hz}), 43.8(\mathrm{~d}, J=5.7 \mathrm{~Hz}), 38.7,35.4(\mathrm{~d}, J=19.2 \mathrm{~Hz}), 27.5$;

${ }^{19} \mathrm{~F}$ NMR $\left(376 \mathrm{MHz}, \mathrm{CDCl}_{3}\right): \delta=-218.0$;

IR (film): $\tilde{v}=3411$ (w), 3287 (m), 2965 (m), 1640 (s), 1510 (s), 1197 (w), 1011 (w), 743 (s);

HRMS (ESI) calcd. for $\mathrm{C}_{16} \mathrm{H}_{21} \mathrm{FN}_{2} \mathrm{NaO}^{+}[\mathrm{M}+\mathrm{Na}]^{+}$299.1530; Found 299.1536.

4-Chloro-N-(3-fluoro-1-hydroxypropyl)benzamide (2j).<smiles>O=C(NC(O)CCF)c1ccc(Cl)cc1</smiles>

Following GP B, starting from 4-chloro- $N$-cyclopropylbenzamide $1 \mathbf{j}(58.5 \mathrm{mg}, 0.300 \mathrm{mmol}$ ) and after stirring for 4 hours, 4-chloro- $N$-(3-fluoro-1-hydroxypropyl)benzamide $2 \mathrm{j}$ ( $27.7 \mathrm{mg}, 0.120 \mathrm{mmol}, 40 \%$ ) 
was obtained as a white solid after purification by column chromatography on silica using 3:2 pentanes:ethyl acetate as eluent.

$\mathbf{R}_{\mathbf{f}}: 0.32$ (silica, pentanes:ethyl acetate 1:1);

Mp: $114-116^{\circ} \mathrm{C}$;

${ }^{1} \mathrm{H}$ NMR $\left(400 \mathrm{MHz}, \mathrm{CDCl}_{3}\right): \delta=7.75-7.64(\mathrm{~m}, 2 \mathrm{H}, \mathrm{ArH}), 7.45-7.34(\mathrm{~m}, 2 \mathrm{H}, \mathrm{ArH}), 7.21(\mathrm{~s}, 1 \mathrm{H}, \mathrm{NH}), 5.72$ $\left.(\mathrm{td}, J=6.9,4.8 \mathrm{~Hz}, 1 \mathrm{H}, \mathrm{CH}), 5.00-4.55(\mathrm{~m}, 2 \mathrm{H}, \mathrm{FCH})_{2}\right), 4.30(\mathrm{~d}, J=3.0 \mathrm{~Hz}, 1 \mathrm{H}, \mathrm{OH}), 2.30-2.01(\mathrm{~m}, 2 \mathrm{H}$, $\left.\mathrm{CH}_{2}\right)$;

${ }^{13} \mathrm{C}$ NMR $\left(101 \mathrm{MHz}, \mathrm{CDCl}_{3}\right): \delta=167.4,138.5,131.7,129.0,128.5,80.7(\mathrm{~d}, J=161.6 \mathrm{~Hz}), 72.8(\mathrm{~d}, J=2.0$ $\mathrm{Hz}), 35.1(\mathrm{~d}, \mathrm{~J}=18.6 \mathrm{~Hz})$;

${ }^{19} \mathrm{~F}$ NMR $\left(376 \mathrm{MHz}, \mathrm{CDCl}_{3}\right): \delta=-218.1$;

IR (film): $\tilde{v}=3299$ (m) 1644 (s), 1532 (s), 1487 (m), 1094 (m), 847 (w);

HRMS (APCI) calcd. for $\mathrm{C}_{10} \mathrm{H}_{11} \mathrm{ClFNNaO}_{2}{ }^{+}[\mathrm{M}+\mathrm{Na}]^{+} 254.0355$; Found 254.0352 .

N-(3-Fluoro-1-hydroxypropyl)benzamide (2k).<smiles>O=C(NC(O)CCF)c1ccccc1</smiles>

Following GP B, starting from $\mathrm{N}$-cyclopropylbenzamide $\mathbf{1 k}(48.3 \mathrm{mg}, 0.300 \mathrm{mmol})$ and after stirring for 4 hours, $\mathrm{N}$-(3-fluoro-1-hydroxypropyl)benzamide $2 \mathrm{k}(24.2 \mathrm{mg}, 0.123 \mathrm{mmol}, 41 \%)$ was obtained as a yellow solid after purification by column chromatography on silica using 3:2 pentanes:ethyl acetate as eluent.

$\mathbf{R}_{\mathbf{f}}: 0.30$ (silica, pentanes:ethyl acetate 1:1);

Mp: $114-116^{\circ} \mathrm{C}$;

${ }^{1} \mathrm{H}$ NMR $\left(400 \mathrm{MHz}\right.$, Acetone- $\left.d_{6}\right): \delta=8.14(\mathrm{~s}, 1 \mathrm{H}, \mathrm{NH}), 7.94-7.87(\mathrm{~m}, 2 \mathrm{H}, \operatorname{Ar} H), 7.56-7.50(\mathrm{~m}, 1 \mathrm{H}, \operatorname{Ar} H)$, $7.49-7.42(\mathrm{~m}, 2 \mathrm{H}, \mathrm{ArH}), 5.81-5.66(\mathrm{~m}, 1 \mathrm{H}, \mathrm{CH}), 5.07(\mathrm{~d}, J=4.2 \mathrm{~Hz}, 1 \mathrm{H}, \mathrm{OH}), 4.73-4.52\left(\mathrm{~m}, 2 \mathrm{H}, \mathrm{FCH}_{2}\right)$, $2.19-2.08\left(\mathrm{~m}, 2 \mathrm{H}, \mathrm{CH}_{2}\right)$;

${ }^{13} \mathrm{C}$ NMR $\left(101 \mathrm{MHz}\right.$, Acetone- $\left.d_{6}\right): \delta=167.5,135.5,132.3,129.1,128.2,81.5(\mathrm{~d}, J=161.8 \mathrm{~Hz}), 71.9$ (dd, $J=10.0,6.7 \mathrm{~Hz}), 37.5(\mathrm{~d}, J=19.5 \mathrm{~Hz})$;

${ }^{19}$ F NMR $\left(376 \mathrm{MHz}\right.$, Acetone- $\left.d_{6}\right): \delta=-222.2$;

IR (film): $\tilde{v}=3315$ (br, s) 1644 (s), 1530 (s), 1489 (m), 1283 (m), 1035 (m), 713 (m);

HRMS (APCl) calcd. for $\mathrm{C}_{10} \mathrm{H}_{12} \mathrm{FNNaO}_{2}{ }^{+}[\mathrm{M}+\mathrm{Na}]^{+} 220.0744$; Found 220.0742.

$\mathrm{N}$-Cyclopropyl-4-(methylsulfinyl)benzamide (2i).<smiles>CS(=O)c1ccc(C(=O)NC2CC2)cc1</smiles>

Following GP B, starting from $\mathrm{N}$-cyclopropyl-4-(methylthio)benzamide $1 \mathbf{i}(62.1 \mathrm{mg}, 0.300 \mathrm{mmol}$ ) and after stirring for 3 hours, $\mathrm{N}$-cyclopropyl-4-(methylsulfinyl)benzamide $\mathbf{2 i}$ ( $63.5 \mathrm{mg}, 0.285 \mathrm{mmol}, 95 \%$ ) was obtained as a white solid after purification by column chromatography on silica using 10:1 dichloromethane:methanol as eluent.

$\mathbf{R}_{\mathbf{f}}: 0.42$ (silica, dichloromethane:methanol 10:1); 
Mp: $175-177^{\circ} \mathrm{C}$;

${ }^{1} \mathrm{H}$ NMR $\left(400 \mathrm{MHz}, \mathrm{CDCl}_{3}\right): \delta=7.92-7.85(\mathrm{~m}, 2 \mathrm{H}, \mathrm{ArH}), 7.66-7.59(\mathrm{~m}, 2 \mathrm{H}, \mathrm{ArH}), 6.71(\mathrm{~s}, 1 \mathrm{H}, \mathrm{NH}), 2.92$ (tq, $J=6.4,3.3 \mathrm{~Hz}, 1 \mathrm{H}, \mathrm{CH}), 2.71\left(\mathrm{~s}, 3 \mathrm{H}, \mathrm{CH}_{3}\right), 0.87\left(\mathrm{td}, J=7.0,5.3 \mathrm{~Hz}, 2 \mathrm{H}, \mathrm{CH}_{2}\right), 0.69-0.60\left(\mathrm{~m}, 2 \mathrm{H}, \mathrm{CH}_{2}\right)$; ${ }^{13} \mathrm{C}$ NMR $\left(101 \mathrm{MHz}, \mathrm{CDCl}_{3}\right): \delta=167.7,148.9,137.2,128.0,123.6,43.8,23.3,6.7$;

IR (film): $\tilde{v}=3269$ (m), 2994 (w), 2918 (w), 1638 (s), 1535 (s), 1303 (m), 1044 (s), $856(\mathrm{~m})$;

HRMS (ESI) calcd. for $\mathrm{C}_{11} \mathrm{H}_{14} \mathrm{NO}_{2} \mathrm{~S}^{+}[\mathrm{M}+\mathrm{H}]^{+} 224.0740$; Found 224.0740.

\section{Scope of the nucleophiles for elimination-addition of hemiaminals}

\section{General Procedure C (GP C):}

In a $12 * 75 \mathrm{~mm}$ borosilicate glass tube, $\mathrm{N}$-cyclopropyl-4-methoxybenzamide $1 \mathrm{a}(57.3 \mathrm{mg}, 0.300 \mathrm{mmol})$, selectfluor (117 mg, $0.330 \mathrm{mmol}, 1.1$ equiv.) and benzophenone $(5.4 \mathrm{mg}, 0.030 \mathrm{mmol}, 0.10$ equiv.) were dissolved in $0.75 \mathrm{~mL}$ of $\mathrm{MeCN}-\mathrm{H}_{2} \mathrm{O}(\mathrm{v}: \mathrm{v}$ 4:6, $0.40 \mathrm{M}$ ). The reaction mixture was degassed by three freeze-pump-thaw cycles and backfilled with $\mathrm{N}_{2}$. The mixture was then stirred at room temperature under $365 \mathrm{~nm}$ irradiation in Rayonet Reactor for 45 minutes. Then the tube was taken out from the Rayonet Reactor and a solution of nucleophile $(0.360 \mathrm{mmol}, 1.2$ equiv.) in $0.50 \mathrm{~mL} \mathrm{MeCN}$ was added dropwise. The reaction mixture was stirred at room temperature for 3 hours, if not specified otherwise. After the completion of the reaction, the crude product was directly submitted to column chromatography on silica using pentanes:ethyl acetate as eluent.

$N$-(3-Fluoro-1-methoxypropyl)-4-methoxybenzamide (3a).<smiles>COc1ccc(C(=O)NC(CCF)OC)cc1</smiles>

Following GP C, methanol (1.0 mL, $25 \mathrm{mmol}, 82$ equiv.) was used as nucleophile. After the reaction mixture was stirred for 1 hours, $\mathrm{N}$-(3-fluoro-1-methoxypropyl)-4-methoxybenzamide (3a) $(53.0 \mathrm{mg}$, $0.220 \mathrm{mmol}, 73 \%$ ) was obtained as a white solid after purification by column chromatography on silica using 3:2 pentanes:ethyl acetate as eluent.

$\mathbf{R}_{\mathbf{f}}: 0.46$ (silica, pentanes:ethyl acetate 1:1);

Mp: $110-112^{\circ} \mathrm{C}$;

${ }^{1} \mathrm{H}$ NMR $\left(400 \mathrm{MHz}, \mathrm{CDCl}_{3}\right): \delta=7.82-7.68(\mathrm{~m}, 2 \mathrm{H}, \operatorname{ArH}), 7.01-6.85(\mathrm{~m}, 2 \mathrm{H}, \operatorname{ArH}), 6.56(\mathrm{~d}, J=8.8 \mathrm{~Hz}$, $1 \mathrm{H}, \mathrm{NH}), 5.55(\mathrm{dt}, \mathrm{J}=10.1,5.3 \mathrm{~Hz}, 1 \mathrm{H}, \mathrm{CH}), 4.85-4.52\left(\mathrm{~m}, 2 \mathrm{H}, \mathrm{FCH}_{2}\right), 3.85\left(\mathrm{~s}, 3 \mathrm{H}, \mathrm{ArOCH}_{3}\right), 3.42(\mathrm{~s}, 3 \mathrm{H}$, $\left.\mathrm{OCH}_{3}\right), 2.24-2.01\left(\mathrm{~m}, 2 \mathrm{H}, \mathrm{CH}_{2}\right)$;

${ }^{13} \mathrm{C}$ NMR $\left(101 \mathrm{MHz}, \mathrm{CDCl}_{3}\right): \delta=166.8,162.5,128.9,125.9,113.8,80.6(\mathrm{~d}, J=162.9 \mathrm{~Hz}), 78.4(\mathrm{~d}, J=3.4$ $\mathrm{Hz}), 56.0,55.4,35.9(\mathrm{~d}, J=19.0 \mathrm{~Hz})$;

${ }^{19}$ F NMR $\left(376 \mathrm{MHz}, \mathrm{CDCl}_{3}\right): \delta=-219.3$;

IR (film): $\tilde{v}=3304$ (w), 2937 (w), 2839 (w), 1642 (s), 1606 (s), 1531 (m), 1503 (s), 1257 (s), 1178 (m), $845(\mathrm{~m})$;

HRMS (ESI) calcd. for $\mathrm{C}_{12} \mathrm{H}_{16} \mathrm{FNNaO}_{3}{ }^{+}[\mathrm{M}+\mathrm{Na}]^{+}$264.1006; Found 264.1014.

$N$-(3-Fluoro-1-hydroperoxypropyl)-4-methoxybenzamide (3b). 
<smiles>COc1ccc(C(=O)NC(CCF)OO)cc1</smiles>

Following GP C, hydrogen peroxide solution $30 \%(\mathrm{w} / \mathrm{w})$ in water (37 uL, $0.36 \mathrm{mmol}, 1.2$ equiv.) was used as nucleophile. After the reaction mixture was stirred for 3 hours, $\mathrm{N}$-(3-fluoro-1hydroperoxypropyl)-4-methoxybenzamide (3b) $(46.5 \mathrm{mg}, 0.191 \mathrm{mmol}, 64 \%)$ was obtained as a white solid after purification by column chromatography on silica using 1:1 pentanes:ethyl acetate as eluent.

$\mathbf{R}_{\mathbf{f}}: 0.41$ (silica, pentanes:ethyl acetate 2:3);

Mp: $93-95^{\circ} \mathrm{C}$;

${ }^{1} \mathrm{H}$ NMR $\left(400 \mathrm{MHz}\right.$, Acetone- $\left.d_{6}\right): \delta=10.95(\mathrm{~s}, 1 \mathrm{H}, \mathrm{OOH}), 8.09(\mathrm{~d}, J=7.7 \mathrm{~Hz}, 1 \mathrm{H}, \mathrm{NH}), 7.94-7.91(\mathrm{~m}, 2 \mathrm{H}$, $\operatorname{ArH}), 7.01-6.97(\mathrm{~m}, 2 \mathrm{H}, \mathrm{ArH}), 5.95(\mathrm{dt}, J=8.9,6.8 \mathrm{~Hz}, 1 \mathrm{H}, \mathrm{CH}), 4.69-4.51(\mathrm{~m}, 2 \mathrm{H}, \mathrm{FCH})_{2}, 3.86(\mathrm{~s}, 3 \mathrm{H}$, $\left.\mathrm{OCH}_{3}\right), 2.39-2.09\left(\mathrm{~m}, 2 \mathrm{H}, \mathrm{CH}_{2}\right)$;

${ }^{13} \mathrm{C}$ NMR $\left(101 \mathrm{MHz}\right.$, Acetone- $\left.d_{6}\right): \delta=167.0,163.3,130.2,127.4,114.3,82.3(\mathrm{~d}, J=5.9 \mathrm{~Hz}), 81.2(\mathrm{~d}, J=$ $163.0 \mathrm{~Hz}), 55.8,33.7(\mathrm{~d}, J=20.0 \mathrm{~Hz})$;

${ }^{19} \mathrm{~F} \mathrm{NMR}\left(376 \mathrm{MHz}\right.$, Acetone- $\left.d_{6}\right): \delta=-221.6$;

IR (film): $\tilde{v}=3305$ (br, s), 2971 (w), 1644 (s), 1606 (s), 1503 (s), 1258 (s), 1178 (m), 1028 (m), 845 (m);

HRMS (ESI) calcd. for $\mathrm{C}_{11} \mathrm{H}_{14} \mathrm{FNNaO}_{4}{ }^{+}[\mathrm{M}+\mathrm{Na}]^{+}$266.0799; Found 266.0798 .

\section{$N$-(3-Fluoro-1-(p-tolylthio)propyl)-4-methoxybenzamide (3c).}<smiles>COc1ccc(C(=O)NC(CCF)Sc2ccc(C)cc2)cc1</smiles>

Following GP C, 4-methylthiophenol ( $44.6 \mathrm{mg}, 0.360 \mathrm{mmol}, 1.2$ equiv.) was used as nucleophile. After the reaction mixture was stirred for 3 hours, $N$-(3-fluoro-1-( $p$-tolylthio)propyl)-4-methoxybenzamide (3c) $(68.5 \mathrm{mg}, 0.206 \mathrm{mmol}, 69 \%)$ was obtained as a white solid after purification by column chromatography on silica using 3:1 pentanes:ethyl acetate as eluent.

$\mathbf{R}_{\mathbf{f}}: 0.33$ (silica, pentanes:ethyl acetate 3:1);

Mp: $97-99^{\circ} \mathrm{C}$;

${ }^{1} \mathrm{H}$ NMR $\left(400 \mathrm{MHz}, \mathrm{CDCl}_{3}\right): \delta=7.66-7.59(\mathrm{~m}, 2 \mathrm{H}, \mathrm{ArH}), 7.40-7.33(\mathrm{~m}, 2 \mathrm{H}, \mathrm{ArH}), 7.12-7.06(\mathrm{~m}, 2 \mathrm{H}$, $\operatorname{ArH}), 6.90-6.86(\mathrm{~m}, 2 \mathrm{H}, \mathrm{ArH}), 6.45(\mathrm{~d}, J=9.4 \mathrm{~Hz}, 1 \mathrm{H}, \mathrm{NH}), 5.69$ (ddd, $J=9.4,7.4,5.7 \mathrm{~Hz}, 1 \mathrm{H}, \mathrm{CH}), 4.86$ $-4.52\left(\mathrm{~m}, 2 \mathrm{H}, \mathrm{FCH}_{2}\right), 3.83\left(\mathrm{~s}, 3 \mathrm{H}, \mathrm{OCH}_{3}\right), 2.39-2.09\left(\mathrm{~m}, 5 \mathrm{H}, \mathrm{CH}_{3}+\mathrm{CH}_{2}\right)$;

${ }^{13} \mathrm{C}$ NMR $\left(101 \mathrm{MHz}, \mathrm{CDCl}_{3}\right): \delta=165.8,162.3,138.4,133.9,129.9,128.7,128.2,126.0,113.7,81.2$ (d, J $=165.6 \mathrm{~Hz}$ ), 55.4, $55.1(\mathrm{~d}, J=3.9 \mathrm{~Hz}), 36.1(\mathrm{~d}, J=19.5 \mathrm{~Hz}), 21.1$;

${ }^{19}$ F NMR (376 MHz, $\left.\mathrm{CDCl}_{3}\right): \delta=-218.7$;

IR (film): $\tilde{v}=3293$ (w), 2963 (w), 1636 (s), 1605 (s), 1500 (s), 1293 (s), 1028 (m), 843 (m), 810 (m);

HRMS (ESI) calcd. for $\mathrm{C}_{18} \mathrm{H}_{20} \mathrm{FNNaO}_{2} \mathrm{~S}^{+}[\mathrm{M}+\mathrm{Na}]^{+}$356.1091; Found 356.1095.

N-(3-Fluoro-1-(1H-pyrazol-1-yl)propyl)-4-methoxybenzamide (3d). 


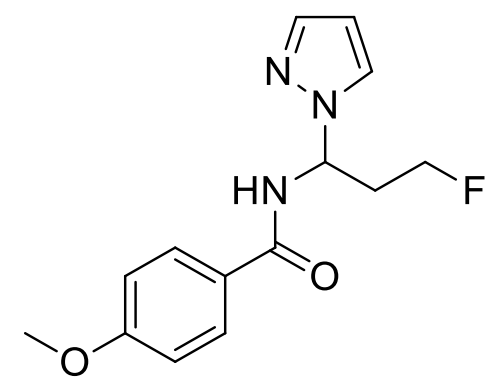

Following GP C, $1 H$-pyrazole ( $24.5 \mathrm{mg}, 0.360 \mathrm{mmol}, 1.2$ equiv.) was used as nucleophile. After the reaction mixture was stirred for 16 hours, $N$-(3-fluoro-1-(1H-pyrazol-1-yl)propyl)-4methoxybenzamide (3d) $(58.2 \mathrm{mg}, 0.210 \mathrm{mmol}, 70 \%)$ was obtained as a white solid after purification by column chromatography on silica using 1:1 pentanes:ethyl acetate as eluent.

$\mathbf{R}_{\mathbf{f}}: 0.26$ (silica, pentanes:ethyl acetate 1:1);

Mp: $146-148^{\circ} \mathrm{C}$;

${ }^{1} \mathrm{H}$ NMR $\left(400 \mathrm{MHz}, \mathrm{CDCl}_{3}\right): \delta=7.77-7.71(\mathrm{~m}, 2 \mathrm{H}, \mathrm{ArH}+\mathrm{NH}), 7.71-7.66(\mathrm{~m}, 2 \mathrm{H}, \operatorname{ArH}), 7.56(\mathrm{~d}, J=1.7$ $\mathrm{Hz}, 1 \mathrm{H}, \operatorname{ArH}), 6.84-6.79(\mathrm{~m}, 2 \mathrm{H}, \mathrm{ArH}), 6.57(\mathrm{q}, J=7.8 \mathrm{~Hz}, 1 \mathrm{H}, \mathrm{CH}), 6.25(\mathrm{t}, J=2.1 \mathrm{~Hz}, 1 \mathrm{H}, \operatorname{ArH}), 4.63-$ $4.07\left(\mathrm{~m}, 2 \mathrm{H}, \mathrm{FCH}_{2}\right), 3.80\left(\mathrm{~s}, 3 \mathrm{H}, \mathrm{OCH}_{3}\right), 2.72-2.54\left(\mathrm{~m}, 2 \mathrm{H}, \mathrm{CH}_{2}\right)$;

${ }^{13} \mathrm{C}$ NMR $\left(101 \mathrm{MHz}, \mathrm{CDCl}_{3}\right): \delta=166.4,162.5,140.4,130.8,129.1,125.2,113.6,105.2,79.9$ (d, $J=165.7$ $\mathrm{Hz}), 62.3(\mathrm{~d}, J=3.9 \mathrm{~Hz}), 55.4,35.0(\mathrm{~d}, J=19.8 \mathrm{~Hz})$;

${ }^{19} \mathrm{~F}$ NMR (376 $\left.\mathrm{MHz}, \mathrm{CDCl}_{3}\right): \delta=-221.8$;

IR (film): $\tilde{v}=3304$ (w), 2970 (w), 1645 (m), 1606 (m), 1502 (s), 1252 (s), 1027 (m), 845 (m), 767 (m);

HRMS (ESI) calcd. for $\mathrm{C}_{14} \mathrm{H}_{16} \mathrm{FN}_{3} \mathrm{NaO}_{2}{ }^{+}[\mathrm{M}+\mathrm{Na}]^{+} 300.1119$; Found 300.1124 .

N-(3-Fluoro-1-(1H-1,2,4-triazol-1-yl)propyl)-4-methoxybenzamide (3e).<smiles>COc1ccc(C(=O)NC(CCF)n2cncn2)cc1</smiles>

Following GP C, 1,2,4-1 $\mathrm{H}$-triazole (41.4 $\mathrm{mg}, 0.600 \mathrm{mmol}, 2.0$ equiv.) was used as nucleophile. After the reaction mixture was stirred for 16 hours, $N$-(3-fluoro-1-(1H-1,2,4-triazol-1-yl)propyl)-4methoxybenzamide (3e) $(59.1 \mathrm{mg}, 0.213 \mathrm{mmol}, 71 \%)$ was obtained as a white solid after purification by column chromatography on silica using $100 \%$ ethyl acetate as eluent.

$\mathbf{R}_{\mathbf{f}}: 0.26$ (silica, $100 \%$ ethyl acetate);

Mp: $156-158^{\circ} \mathrm{C}$;

${ }^{1} \mathrm{H}$ NMR $\left(400 \mathrm{MHz}\right.$, Acetone- $\left.d_{6}\right): \delta=8.60(\mathrm{~d}, J=8.4 \mathrm{~Hz}, 1 \mathrm{H}, \mathrm{NH}), 8.55(\mathrm{~s}, 1 \mathrm{H}, \mathrm{ArH}), 7.94-7.86(\mathrm{~m}, 3 \mathrm{H}$, $\mathrm{ArH}), 7.02-6.93(\mathrm{~m}, 2 \mathrm{H}, \mathrm{ArH}), 6.61(\mathrm{q}, J=7.7 \mathrm{~Hz}, 1 \mathrm{H}, \mathrm{CH}), 4.75-4.33(\mathrm{~m}, 2 \mathrm{H}, \mathrm{FCH})_{2}, 3.85\left(\mathrm{~s}, 3 \mathrm{H}, \mathrm{OCH}_{3}\right)$, 2.69 (ddt, $J=25.7,7.5,5.7 \mathrm{~Hz}, 2 \mathrm{H}, \mathrm{CH}_{2}$ );

${ }^{13} \mathrm{C}$ NMR $\left(101 \mathrm{MHz}\right.$, Acetone- $\left.d_{6}\right): \delta=167.1,163.7,152.6,144.9,130.3,126.4,114.4,80.8(\mathrm{~d}, J=163.7$ $\mathrm{Hz}), 61.8(\mathrm{~d}, J=5.7 \mathrm{~Hz}), 55.8,35.0(\mathrm{~d}, J=20.0 \mathrm{~Hz})$;

${ }^{19} \mathrm{~F}$ NMR $\left(376 \mathrm{MHz}, \mathrm{CDCl}_{3}\right): \delta=-221.2$;

IR (film): $\tilde{v}=3303$ (w), 1653 (m), 1606 (m), 1503 (s), 1256 (s), $1178(\mathrm{~m}), 1025$ (m), $846(\mathrm{~m})$;

HRMS (ESI) calcd. for $\mathrm{C}_{13} \mathrm{H}_{15} \mathrm{FN}_{4} \mathrm{NaO}_{2}{ }^{+}[\mathrm{M}+\mathrm{Na}]^{+}$301.1071; Found 301.1064. 


\section{$N$-(3-Fluoro-1-(1H-tetrazol-1-yl)propyl)-4-methoxybenzamide (3f).}<smiles>COc1ccc(C(=O)NC(CCF)n2cnnn2)cc1</smiles>

Following GP C, $1 \mathrm{H}$-tetrazole ( $42.0 \mathrm{mg}, 0.600 \mathrm{mmol}, 2.0$ equiv.) was used as nucleophile. After the reaction mixture was stirred for 16 hours, $\mathrm{N}$-(3-fluoro-1-(1H-tetrazol-1-yl)propyl)-4methoxybenzamide (3f) $(33.0 \mathrm{mg}, 0.118 \mathrm{mmol}, 39 \%)$ was obtained as a white solid after purification by column chromatography on silica using $2: 3$ pentanes:ethyl acetate as eluent.

$\mathbf{R}_{\mathbf{f}}: 0.26$ (silica, pentanes:ethyl acetate 2:3);

Mp: $157-159^{\circ} \mathrm{C}$;

${ }^{1} \mathrm{H}$ NMR $\left(400 \mathrm{MHz}\right.$, Acetone- $\left.d_{6}\right): \delta=9.30(\mathrm{~s}, 1 \mathrm{H}, \mathrm{ArH}), 8.86(\mathrm{~d}, J=8.4 \mathrm{~Hz}, 1 \mathrm{H}, \mathrm{NH}), 7.93-7.87(\mathrm{~m}, 2 \mathrm{H}$, $\mathrm{ArH}), 7.03-6.97(\mathrm{~m}, 2 \mathrm{H}, \mathrm{ArH}), 6.87(\mathrm{q}, \mathrm{J}=8.1 \mathrm{~Hz}, 1 \mathrm{H}, \mathrm{CH}), 4.85-4.49(\mathrm{~m}, 2 \mathrm{H}, \mathrm{FCH})_{2}, 3.85\left(\mathrm{~s}, 3 \mathrm{H}, \mathrm{OCH}_{3}\right)$, $2.89-2.79\left(\mathrm{~m}, 2 \mathrm{H}, \mathrm{CH}_{2}\right)$;

${ }^{13} \mathrm{C}$ NMR $\left(101 \mathrm{MHz}\right.$, Acetone- $\left.d_{6}\right): \delta=167.3,163.9,144.1,130.4,126.0,114.5,80.7(\mathrm{~d}, J=164.3 \mathrm{~Hz})$, $62.5(\mathrm{~d}, J=5.2 \mathrm{~Hz}), 55.9,35.0(\mathrm{~d}, J=20.1 \mathrm{~Hz})$;

${ }^{19}$ F NMR $\left(376 \mathrm{MHz}\right.$, Acetone- $\left.d_{6}\right): \delta=-223.2$;

IR (film): $\tilde{v}=3294$ (w), 2971 (w), 1650 (m), 1605 (s), 1503 (s), 1256 (s), 1177 (m), 1025 (m), 845 (m);

HRMS (ESI) calcd. for $\mathrm{C}_{12} \mathrm{H}_{14} \mathrm{FN}_{5} \mathrm{NaO}_{2}{ }^{+}[\mathrm{M}+\mathrm{Na}]^{+} 302.1024$; Found 302.1017.

\section{$\mathrm{N}$-(3-Fluoropropyl)-4-methoxybenzamide (3g).}<smiles>COc1ccc(C(=O)NCCCF)cc1</smiles>

Following GP C, $\mathrm{NaBH}_{3} \mathrm{CN}(22.6 \mathrm{mg}, 0.360 \mathrm{mmol}, 1.2$ equiv.) was used as nucleophile. After the reaction mixture was stirred for 3 hours, $\mathrm{N}$-(3-fluoropropyl)-4-methoxybenzamide ( $3 \mathrm{~g})(52.4 \mathrm{mg}, 0.248 \mathrm{mmol}$, $83 \%$ ) was obtained as a beige solid after purification by column chromatography on silica using $3: 2$ pentanes:ethyl acetate as eluent.

$\mathbf{R}_{\mathbf{f}}$ : 0. (silica, pentanes:ethyl acetate 1:1);

Mp: $71-73^{\circ} \mathrm{C}$;

${ }^{1}$ H NMR $\left(400 \mathrm{MHz}, \mathrm{CDCl}_{3}\right): \delta=7.79-7.64(\mathrm{~m}, 2 \mathrm{H}, \mathrm{ArH}), 6.97-6.85(\mathrm{~m}, 2 \mathrm{H}, \mathrm{ArH}), 6.38(\mathrm{~s}, 1 \mathrm{H}, \mathrm{NH}), 4.59$ (dt, $J=47.3,5.6 \mathrm{~Hz}, 2 \mathrm{H}, \mathrm{FCH}_{2}$ ), $3.84\left(\mathrm{~s}, 3 \mathrm{H}, \mathrm{OCH}_{3}\right), 3.60$ (q, $\left.J=6.3 \mathrm{~Hz}, 2 \mathrm{H}, \mathrm{NCH}_{2}\right), 2.03$ (dddd, $J=28.2$, $12.1,6.5,5.5 \mathrm{~Hz}, 2 \mathrm{H}, \mathrm{CH}_{2}$ );

${ }^{13} \mathrm{C}$ NMR $\left(101 \mathrm{MHz}, \mathrm{CDCl}_{3}\right): \delta=167.1,162.1,128.6,126.7,113.7,82.9(\mathrm{~d}, J=163.8 \mathrm{~Hz}), 55.4,37.1(\mathrm{~d}, J$ $=4.2 \mathrm{~Hz}), 30.2(\mathrm{~d}, J=19.1 \mathrm{~Hz})$;

${ }^{19} \mathrm{~F} \mathrm{NMR}\left(376 \mathrm{MHz}, \mathrm{CDCl}_{3}\right): \delta=-219.9$;

IR (film): $\tilde{v}=3298$ (m), 2967 (w), 1630 (s), 1607 (s), 1542 (m), 1505 (s), 1299 (m), 1256 (s), 1179 (m), $848(\mathrm{~m})$;

HRMS (ESI) calcd. for $\mathrm{C}_{11} \mathrm{H}_{15} \mathrm{FNO}_{2}{ }^{+}[\mathrm{M}+\mathrm{H}]^{+} 212.1081$; Found 212.1083. 
<smiles>COc1ccc(C(=O)NC(/C=C/c2ccccc2)CCF)cc1</smiles>

Following GP C, potassium trans-styryltrifluoroborate $(126 \mathrm{mg}, 0.600 \mathrm{mmol}, 2.0$ equiv.) was used as nucleophile. After the reaction mixture was stirred for 16 hours, (E)- $\mathrm{N}$-(5-fluoro-1-phenylpent-1-en-3yl)-4-methoxybenzamide (3h) $(39.0 \mathrm{mg}, 0.125 \mathrm{mmol}, 42 \%)$ was obtained as a white solid after purification by column chromatography on silica using 3:1 pentanes:ethyl acetate as eluent.

$\mathbf{R}_{\mathbf{f}}: 0.29$ (silica, pentanes:ethyl acetate 2:1);

Mp: $121-123^{\circ} \mathrm{C}$;

${ }^{1} \mathrm{H}$ NMR $\left(400 \mathrm{MHz}\right.$, Acetone- $\left.d_{6}\right): \delta=7.96-7.86(\mathrm{~m}, 2 \mathrm{H}, \operatorname{ArH}), 7.70(\mathrm{~d}, J=8.4 \mathrm{~Hz}, 1 \mathrm{H}, \mathrm{NH}), 7.47-7.39$ $(\mathrm{m}, 2 \mathrm{H}, \mathrm{ArH}), 7.36-7.27(\mathrm{~m}, 2 \mathrm{H}, \mathrm{ArH}), 7.26-7.19(\mathrm{~m}, 1 \mathrm{H}, \mathrm{ArH}), 7.01-6.93(\mathrm{~m}, 2 \mathrm{H}, \mathrm{ArH}), 6.65(\mathrm{dd}, J=$ 16.0, $1.2 \mathrm{~Hz}, 1 \mathrm{H}$, vinyl $\mathrm{CH}), 6.40(\mathrm{dd}, J=16.0,6.7 \mathrm{~Hz}, 1 \mathrm{H}$, vinyl $\mathrm{CH}), 4.99(\mathrm{~h}, J=7.3,6.8 \mathrm{~Hz}, 1 \mathrm{H}, \mathrm{CH}), 4.73$ $-4.48(\mathrm{~m}, 2 \mathrm{H}, \mathrm{FCH} 2), 3.84\left(\mathrm{~s}, 3 \mathrm{H}, \mathrm{OCH}_{3}\right), 2.18\left(\mathrm{ddt}, J=24.8,7.2,6.0 \mathrm{~Hz}, 2 \mathrm{H}, \mathrm{CH}_{2}\right)$;

${ }^{13} \mathrm{C}$ NMR $\left(101 \mathrm{MHz}\right.$, Acetone- $\left.d_{6}\right): \delta=165.4,162.1,137.1,130.2,130.1,129.0,128.5,127.4,127.3$, $126.3,113.4,81.1(\mathrm{~d}, J=163.3 \mathrm{~Hz}), 54.9,48.2(\mathrm{~d}, J=5.7 \mathrm{~Hz}), 35.6(\mathrm{~d}, J=19.6 \mathrm{~Hz})$;

${ }^{19} \mathrm{~F}$ NMR $\left(376 \mathrm{MHz}, \mathrm{CDCl}_{3}\right): \delta=-220.4$;

IR (film): $\tilde{v}=3316$ (w), 2963 (w), 1630 (s), 1606 (s), 1538 (m), 1504 (s), 1255 (s), 1178 (m), 1030 (m), $749(\mathrm{~m})$;

HRMS (ESI) calcd. for $\mathrm{C}_{19} \mathrm{H}_{21} \mathrm{FNO}_{2}{ }^{+}[\mathrm{M}+\mathrm{H}]^{+}$314.1551; Found 314.1539.

$N$-(3-Fluoro-1-(2,4,6-trimethoxyphenyl)propyl)-4-methoxybenzamide (3i).<smiles>COc1ccc(C(=O)NC(CCF)c2c(OC)cc(OC)cc2OC)cc1</smiles>

Following GP C, 1,3,5-trimethoxybenzene ( $60.5 \mathrm{mg}, 0.360 \mathrm{mmol}, 1.2$ equiv.) was used as nucleophile. After the reaction mixture was stirred for 16 hours, $\mathrm{N}$-(3-fluoro-1-(2,4,6-trimethoxyphenyl)propyl)-4methoxybenzamide (3i) $(77.8 \mathrm{mg}, 0.206 \mathrm{mmol}, 69 \%)$ was obtained as a white solid after purification by column chromatography on silica using 3:2 pentanes:ethyl acetate as eluent.

$\mathbf{R}_{\mathbf{f}}: 0.44$ (silica, pentanes:ethyl acetate $3: 2$ );

Mp: $133-135^{\circ} \mathrm{C}$;

${ }^{1} \mathrm{H}$ NMR $\left(400 \mathrm{MHz}, \mathrm{CDCl}_{3}\right): \delta=7.75-7.66(\mathrm{~m}, 2 \mathrm{H}, \mathrm{ArH}), 7.55(\mathrm{~d}, J=9.6 \mathrm{~Hz}, 1 \mathrm{H}, \mathrm{NH}), 6.95-6.85(\mathrm{~m}, 2 \mathrm{H}$, $\operatorname{ArH}), 6.16(\mathrm{~s}, 2 \mathrm{H}, \mathrm{ArH}), 5.98(\mathrm{dt}, J=9.6,7.4 \mathrm{~Hz}, 1 \mathrm{H}, \mathrm{CH}), 4.47(\mathrm{dt}, J=47.1,6.6 \mathrm{~Hz}, 2 \mathrm{H}, \mathrm{FCH}$ ) , $3.88(\mathrm{~s}, 6 \mathrm{H}$, $\left.\mathrm{OCH}_{3}\right), 3.84\left(\mathrm{~s}, 3 \mathrm{H}, \mathrm{OCH}_{3}\right), 3.81\left(\mathrm{~s}, 3 \mathrm{H}, \mathrm{OCH}_{3}\right), 2.39-2.11\left(\mathrm{~m}, 2 \mathrm{H}, \mathrm{CH}_{2}\right)$; 
${ }^{13} \mathrm{C}$ NMR $\left(101 \mathrm{MHz}, \mathrm{CDCl}_{3}\right): \delta=165.6,161.9,160.5,158.6,128.6,127.4,113.6,109.7,91.1,82.2(\mathrm{~d}, J=$ $164.2 \mathrm{~Hz}$ ), 55.9, 55.4 (there are two ${ }^{13} \mathrm{C}$ signals for four methoxy groups, meaning signals for the two para methoxy groups are overlapped), $41.7(\mathrm{~d}, J=8.1 \mathrm{~Hz}), 36.1(\mathrm{~d}, J=19.0 \mathrm{~Hz})$;

${ }^{19} \mathrm{~F}$ NMR $\left(376 \mathrm{MHz}, \mathrm{CDCl}_{3}\right): \delta=-218.4$;

IR (film): $\tilde{v}$ = 3445 (w), 2962 (w), 2839 (w), 1653 (m), 1606 (s), 1493 (s), 1252 (s), 1124 (s), 1030 (m), $844(w), 814(w)$;

HRMS (ESI) calcd. for $\mathrm{C}_{20} \mathrm{H}_{24} \mathrm{FNNaO}_{5}{ }^{+}[\mathrm{M}+\mathrm{Na}]^{+} 400.1531$; Found 400.1522 .

N-(1-(2,4-Dimethoxyphenyl)-3-fluoropropyl)-4-methoxybenzamide (3j).<smiles>COc1ccc(C(=O)NC(CCF)c2ccc(OC)cc2OC)cc1</smiles>

Following GP C, 1,3-dimethoxybenzene ( $49.7 \mathrm{mg}, 0.360 \mathrm{mmol}, 1.2$ equiv.) was used as nucleophile. After the reaction mixture was stirred for 16 hours, $\mathrm{N}$-(1-(2,4-dimethoxyphenyl)-3-fluoropropyl)-4methoxybenzamide (3j) $(22.4 \mathrm{mg}, 0.0646 \mathrm{mmol}, 22 \%)$ was obtained as a white solid after purification by column chromatography on silica using 3:2 pentanes:ethyl acetate as eluent.

$\mathbf{R}_{\mathbf{f}}: 0.43$ (silica, pentanes:ethyl acetate 1:1);

Mp: $133-134{ }^{\circ} \mathrm{C}$;

${ }^{1} \mathrm{H}$ NMR (400 MHz, Acetone- $\left.d_{6}\right): \delta=7.90-7.83(\mathrm{~m}, 2 \mathrm{H}, \mathrm{ArH}), 7.74(\mathrm{~d}, J=8.8 \mathrm{~Hz}, 1 \mathrm{H}, \mathrm{NH}), 7.27(\mathrm{~d}, J=$ $8.4 \mathrm{~Hz}, 1 \mathrm{H}, \mathrm{ArH}), 7.01-6.91(\mathrm{~m}, 2 \mathrm{H}, \mathrm{ArH}), 6.58(\mathrm{~d}, J=2.4 \mathrm{~Hz}, 1 \mathrm{H}, \operatorname{ArH}), 6.48(\mathrm{dd}, J=8.4,2.4 \mathrm{~Hz}, 1 \mathrm{H}$, $\operatorname{ArH}), 5.52(\mathrm{td}, J=8.6,6.2 \mathrm{~Hz}, 1 \mathrm{H}, \mathrm{CH}), 4.62-4.36(\mathrm{~m}, 2 \mathrm{H}, \mathrm{FCH})_{2}, 3.90\left(\mathrm{~s}, 3 \mathrm{H}, \mathrm{OCH}_{3}\right), 3.84\left(\mathrm{~s}, 3 \mathrm{H}, \mathrm{OCH}_{3}\right)$, $3.78\left(\mathrm{~s}, 3 \mathrm{H}, \mathrm{OCH}_{3}\right), 2.33-2.18\left(\mathrm{~m}, 2 \mathrm{H}, \mathrm{CH}_{2}\right)$;

${ }^{13} \mathrm{C}$ NMR (101 MHz, Acetone- $\left.d_{6}\right): \delta=166.0,163.0,161.2,159.0,129.8,129.2,128.4,123.7,114.3,105.3$, 99.6, $82.4(\mathrm{~d}, J=163.3 \mathrm{~Hz}), 55.9,55.8,55.6,47.3(\mathrm{~d}, J=6.1 \mathrm{~Hz}), 37.0(\mathrm{~d}, J=19.4 \mathrm{~Hz}$ );

${ }^{19} \mathrm{~F}$ NMR $\left(376 \mathrm{MHz}\right.$, Acetone- $\left.d_{6}\right): \delta=-220.2$;

IR (film): $\tilde{v}=3325$ (w), 2962 (w), 1632 (s), 1607 (s), 1504 (s), 1254 (s), 1031 (m), 843 (m);

HRMS (ESI) calcd. for $\mathrm{C}_{19} \mathrm{H}_{23} \mathrm{FNO}_{4}{ }^{+}[\mathrm{M}+\mathrm{H}]^{+} 348.1606$; Found 348.1603 .

$N$-(3-Fluoro-1-(1H-pyrrol-2-yl)propyl)-4-methoxybenzamide (3k).<smiles>COc1ccc(C(=O)NC(CCF)c2ccc[nH]2)cc1</smiles>

Following GP C, $1 \mathrm{H}$-pyrrole $(24.1 \mathrm{mg}, 0.360 \mathrm{mmol}, 1.2$ equiv.) was used as nucleophile. After the reaction mixture was stirred for 2 hours, $N$-(3-fluoro-1-(1H-pyrrol-2-yl)propyl)-4-methoxybenzamide 
(3k) (38.2 $\mathrm{mg}, 0.138 \mathrm{mmol}, 46 \%$ ) was obtained as a red solid after purification by column chromatography on silica using 3:1 pentanes:ethyl acetate as eluent.

$\mathbf{R}_{\mathbf{f}}: 0.30$ (silica, pentanes:ethyl acetate 2:1);

Mp: $150-152^{\circ} \mathrm{C}$;

${ }^{1} \mathrm{H}$ NMR $\left(400 \mathrm{MHz}, \mathrm{CDCl}_{3}\right): \delta=9.37(\mathrm{~s}, 1 \mathrm{H}$, pyrrole $\mathrm{NH}), 7.78-7.61(\mathrm{~m}, 2 \mathrm{H}, \mathrm{ArH}), 6.98-6.82(\mathrm{~m}, 2 \mathrm{H}$, $\operatorname{ArH}), 6.74(\mathrm{td}, J=2.6,1.5 \mathrm{~Hz}, 1 \mathrm{H}, \mathrm{ArH}), 6.56(\mathrm{~d}, J=7.6 \mathrm{~Hz}, 1 \mathrm{H}, \mathrm{NH}), 6.12(\mathrm{q}, J=2.9 \mathrm{~Hz}, 1 \mathrm{H}, \operatorname{ArH}), 6.05$ $(\mathrm{qd}, J=2.9,1.8 \mathrm{~Hz}, 1 \mathrm{H}, \mathrm{ArH}), 5.31(\mathrm{dt}, J=8.3,6.2 \mathrm{~Hz}, 1 \mathrm{H}, \mathrm{CH}), 4.87-4.52(\mathrm{~m}, 2 \mathrm{H}, \mathrm{FCH}), 3.84(\mathrm{~s}, 3 \mathrm{H}$, $\left.\mathrm{OCH}_{3}\right), 2.65-2.31\left(\mathrm{~m}, 2 \mathrm{H}, \mathrm{CH}_{2}\right)$;

${ }^{13} \mathrm{C}$ NMR $\left(101 \mathrm{MHz}, \mathrm{CDCl}_{3}\right): \delta=167.9,162.4,132.6,128.8,126.1,118.0,113.8,107.5,104.4,82.0$ (d, J $=164.2 \mathrm{~Hz}), 55.4,45.5(\mathrm{~d}, J=3.5 \mathrm{~Hz}), 33.3(\mathrm{~d}, J=19.5 \mathrm{~Hz})$;

${ }^{19}$ F NMR $\left(376 \mathrm{MHz}, \mathrm{CDCl}_{3}\right): \delta=-217.2$;

IR (film): $\tilde{v}=3320$ (br,s), 2965 (w), 1621 (s), 1606 (s), $1533(\mathrm{~m}), 1503$ (s), 1255 (s), 1178 (m), 1027 (m), $728(w)$;

HRMS (ESI) calcd. for $\mathrm{C}_{15} \mathrm{H}_{17} \mathrm{FN}_{2} \mathrm{NaO}_{2}{ }^{+}[\mathrm{M}+\mathrm{Na}]^{+}$299.1166; Found 299.1174.

$N$-(3-Fluoro-1-(1H-indol-3-yl)propyl)-4-methoxybenzamide (3I).<smiles>COc1ccc(C(=O)NC(CCF)c2c[nH]c3ccccc23)cc1</smiles>

Following GP C, $1 \mathrm{H}$-indole ( $42.2 \mathrm{mg}, 0.360 \mathrm{mmol}, 1.2$ equiv.) was used as nucleophile. After the reaction mixture was stirred for 3 hours, $\mathrm{N}$-(3-fluoro-1-(1H-indol-3-yl)propyl)-4-methoxybenzamide (3I) $(65.2$ $\mathrm{mg}, 0.200 \mathrm{mmol}, 67 \%$ ) was obtained as a dark solid after purification by column chromatography on silica using 1:1 pentanes:ethyl acetate as eluent.

$\mathbf{R}_{\mathbf{f}}: 0.46$ (silica, pentanes:ethyl acetate 1:1);

Mp: $63-66^{\circ} \mathrm{C}$;

${ }^{1} \mathrm{H}$ NMR $\left(400 \mathrm{MHz}, \mathrm{CDCl}_{3}\right): \delta=8.28(\mathrm{~s}, 1 \mathrm{H}$, indole $\mathrm{NH}), 7.74-7.69(\mathrm{~m}, 2 \mathrm{H}, \mathrm{ArH}), 7.67(\mathrm{dd}, J=8.0,1.0$ $\mathrm{Hz}, 1 \mathrm{H}, \mathrm{ArH}), 7.38(\mathrm{dt}, J=8.2,1.0 \mathrm{~Hz}, 1 \mathrm{H}, \mathrm{ArH}), 7.21$ (ddd, $J=8.2,7.0,1.2 \mathrm{~Hz}, 1 \mathrm{H}, \operatorname{ArH}), 7.16$ (dd, $J=$ 2.6, $0.7 \mathrm{~Hz}, 1 \mathrm{H}, \mathrm{ArH}), 7.12$ (ddd, $J=8.0,7.0,1.1 \mathrm{~Hz}, 1 \mathrm{H}, \operatorname{ArH}), 6.92-6.85(\mathrm{~m}, 2 \mathrm{H}, \operatorname{ArH}), 6.48(\mathrm{~d}, J=8.0$ $\mathrm{Hz}, 1 \mathrm{H}, \mathrm{NH}), 5.72(\mathrm{q}, J=7.1 \mathrm{~Hz}, 1 \mathrm{H}, \mathrm{CH}), 4.75-4.46\left(\mathrm{~m}, 2 \mathrm{H}, \mathrm{FCH}_{2}\right), 3.82\left(\mathrm{~s}, 3 \mathrm{H}, \mathrm{OCH}_{3}\right), 2.59-2.42(\mathrm{~m}$, $\left.2 \mathrm{H}, \mathrm{CH}_{2}\right)$;

${ }^{13} \mathrm{C}$ NMR $\left(101 \mathrm{MHz}, \mathrm{CDCl}_{3}\right): \delta=166.3,162.2,136.7,128.7,126.7,125.7,122.5,122.0,120.0,119.1$, $116.0,113.7,111.5,82.0$ (d, $J=163.9 \mathrm{~Hz}), 55.4,44.3(\mathrm{~d}, J=5.7 \mathrm{~Hz}), 35.5$ (d, J = 19.2 Hz);

${ }^{19} \mathrm{~F}$ NMR $\left(376 \mathrm{MHz}, \mathrm{CDCl}_{3}\right): \delta=-218.2$;

IR (film): $\tilde{v}$ = 3408 (w), 3292 (m), 2965(w), 1631 (s), 1606 (s), 1499 (s), 1254 (s), 1178 (m), 1029 (m), $745(\mathrm{~m})$;

HRMS (ESI) calcd. for $\mathrm{C}_{19} \mathrm{H}_{19} \mathrm{FN}_{2} \mathrm{NaO}_{2}{ }^{+}[\mathrm{M}+\mathrm{Na}]^{+}$349.1323; Found 349.1322.

$\mathrm{N}$-(3-Fluoro-1-(1-methyl-1H-indol-3-yl)propyl)-4-methoxybenzamide (3m). 


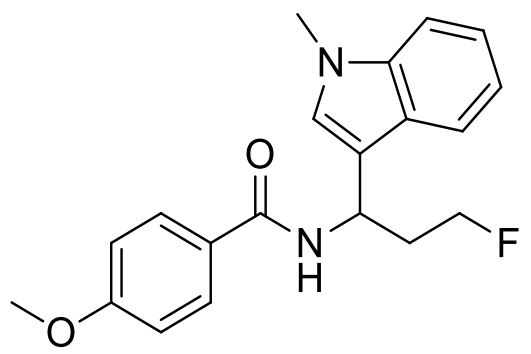

Following GP C, 1-methylindole ( $47.2 \mathrm{mg}, 0.360 \mathrm{mmol}, 1.2$ equiv.) was used as nucleophile. After the reaction mixture was stirred for 3 hours, $N$-(3-fluoro-1-(1-methyl-1H-indol-3-yl)propyl)-4methoxybenzamide $(3 \mathrm{~m})(65.5 \mathrm{mg}, 0.193 \mathrm{mmol}, 64 \%)$ was obtained as a beige solid after purification by column chromatography on silica using 1:1 pentanes:ethyl acetate as eluent.

$\mathbf{R}_{\mathbf{f}}: 0.40$ (silica, pentanes:ethyl acetate $1: 1$ );

Mp: $168-169^{\circ} \mathrm{C}$;

${ }^{1}$ H NMR $\left(400 \mathrm{MHz}\right.$, Acetone- $\left.d_{6}\right): \delta=7.90-7.84(\mathrm{~m}, 2 \mathrm{H}, \mathrm{ArH}), 7.72(\mathrm{dt}, J=7.9,1.0 \mathrm{~Hz}, 2 \mathrm{H}, \mathrm{ArH}+\mathrm{NH})$, $7.35(\mathrm{dt}, J=8.3,0.9 \mathrm{~Hz}, 1 \mathrm{H}, \operatorname{ArH}), 7.27(\mathrm{~d}, J=0.8 \mathrm{~Hz}, 1 \mathrm{H}, \operatorname{ArH}), 7.16$ (ddd, $J=8.3,7.0,1.1 \mathrm{~Hz}, 1 \mathrm{H}, \operatorname{ArH})$, 7.01 (ddd, J = 8.0, 7.0, 1.0 Hz, 1H, ArH), $6.95-6.89(\mathrm{~m}, 2 \mathrm{H}, \mathrm{ArH}), 5.80-5.70(\mathrm{~m}, 1 \mathrm{H}, \mathrm{CH}), 4.74-4.46$ $(\mathrm{m}, 2 \mathrm{H}, \mathrm{FCH} 2), 3.81\left(\mathrm{~s}, 3 \mathrm{H}, \mathrm{OCH}_{3}\right), 3.79\left(\mathrm{~s}, 3 \mathrm{H}, \mathrm{NCH}_{3}\right), 2.63-2.30\left(\mathrm{~m}, 2 \mathrm{H}, \mathrm{CH}_{2}\right)$;

${ }^{13} \mathrm{C}$ NMR $\left(101 \mathrm{MHz}\right.$, Acetone- $\left.d_{6}\right): \delta=166.2,162.9,138.2,129.9,128.2,127.8,127.5,122.4,120.2$, $119.7,116.7,114.2,110.3,82.4(\mathrm{~d}, J=163.0 \mathrm{~Hz}$ ), 55.7, 43.3 (d, $J=6.5 \mathrm{~Hz}), 36.6(\mathrm{~d}, J=19.5 \mathrm{~Hz}$ ), 32.8 ;

${ }^{19} \mathrm{~F}$ NMR $\left(376 \mathrm{MHz}\right.$, Acetone- $\left.d_{6}\right): \delta=-220.0$;

IR (film): $\tilde{v}=3306$ (w), 2961 (m), 1626 (s), 1606 (s), 1502 (s), 1253 (s), $1178(\mathrm{~m}), 1030$ (m), 743 (m);

HRMS (ESI) calcd. for $\mathrm{C}_{20} \mathrm{H}_{21} \mathrm{FN}_{2} \mathrm{NaO}_{2}{ }^{+}[\mathrm{M}+\mathrm{Na}]^{+} 363.1479$; Found 363.1481 .

$N$-(3-Fluoro-1-(2-phenyl-1H-indol-3-yl)propyl)-4-methoxybenzamide (3n).<smiles>COc1ccc(C(=O)NC(CCF)c2c(-c3ccccc3)[nH]c3ccccc23)cc1</smiles>

Following GP C, 2-phenylindole ( $69.5 \mathrm{mg}, 0.360 \mathrm{mmol}, 1.2$ equiv.) was used as nucleophile. After the reaction mixture was stirred for 3 hours, $N$-(3-fluoro-1-(2-phenyl-1H-indol-3-yl)propyl)-4methoxybenzamide (3n) $(80.6 \mathrm{mg}, 0.200 \mathrm{mmol}, 67 \%)$ was obtained as a pink solid after purification by column chromatography on silica using 2:1 pentanes:ethyl acetate as eluent.

$\mathbf{R}_{\mathbf{f}}: 0.31$ (silica, pentanes:ethyl acetate 2:1);

Mp: $105-107^{\circ} \mathrm{C}$;

${ }^{1}$ H NMR $\left(400 \mathrm{MHz}, \mathrm{CDCl}_{3}\right): \delta=8.32(\mathrm{~s}, 1 \mathrm{H}$, indole $\mathrm{NH}), 7.81-7.77(\mathrm{~m}, 1 \mathrm{H}, \mathrm{ArH}), 7.69-7.61(\mathrm{~m}, 4 \mathrm{H}$, ArH), $7.50-7.44(\mathrm{~m}, 2 \mathrm{H}, \mathrm{ArH}), 7.44-7.37(\mathrm{~m}, 2 \mathrm{H}, \mathrm{ArH}), 7.25(\mathrm{ddd}, J=8.1,7.1,1.3 \mathrm{~Hz}, 1 \mathrm{H}, \mathrm{ArH}), 7.20$ $(\mathrm{td}, J=7.4,1.2 \mathrm{~Hz}, 1 \mathrm{H}, \operatorname{ArH}), 6.90-6.83(\mathrm{~m}, 2 \mathrm{H}, \operatorname{ArH}), 6.74(\mathrm{~d}, J=7.1 \mathrm{~Hz}, 1 \mathrm{H}, \mathrm{NH}), 5.75(\mathrm{q}, J=7.2 \mathrm{~Hz}$, $1 \mathrm{H}, \mathrm{CH}), 4.61-4.32\left(\mathrm{~m}, 2 \mathrm{H}, \mathrm{FCH}_{2}\right), 3.82\left(\mathrm{~s}, 3 \mathrm{H}, \mathrm{OCH}_{3}\right), 2.76-2.35\left(\mathrm{~m}, 2 \mathrm{H}, \mathrm{CH}_{2}\right)$;

${ }^{13} \mathrm{C}$ NMR $\left(101 \mathrm{MHz}, \mathrm{CDCl}_{3}\right): \delta=166.1,162.1,136.2,136.1,132.2,129.1,128.8,128.7,128.5,126.7$, 126.6, 122.5, 120.3, 119.1, 113.7, 111.6, 111.5, 82.0 (d, $J=164.3 \mathrm{~Hz}$ ), 55.4, 45.1 (d, J = 5.7 Hz), 36.6 (d, $J=19.4 \mathrm{~Hz})$;

${ }^{19} \mathrm{~F}$ NMR $\left(376 \mathrm{MHz}, \mathrm{CDCl}_{3}\right): \delta=-218.2$; 
IR (film): $\tilde{v}=3428$ (w), 3274 (w), 2962 (w), 1637 (s), 1605 (s), 1491 (s), 1253 (s), 1177 (m), 1029 (m), $843(\mathrm{~m}), 745(\mathrm{~m})$;

HRMS (ESI) calcd. for $\mathrm{C}_{25} \mathrm{H}_{23} \mathrm{FN}_{2} \mathrm{NaO}_{2}^{+}[\mathrm{M}+\mathrm{Na}]^{+}$425.1636; Found 425.1631.

$N$-(3-Fluoro-1-(3-methyl-1H-indol-2-yl)propyl)-4-methoxybenzamide (30).<smiles>COc1ccc(C(=O)NC(CCF)c2[nH]c3ccccc3c2C)cc1</smiles>

Following GP C, 3-methylindole ( $47.2 \mathrm{mg}, 0.360 \mathrm{mmol}, 1.2$ equiv.) was used as nucleophile. After the reaction mixture was stirred for 20 hours, $N$-(3-fluoro-1-(3-methyl-1H-indol-2-yl)propyl)-4methoxybenzamide (3o) $(44.8 \mathrm{mg}, 0.132 \mathrm{mmol}, 44 \%)$ and $\mathrm{N}$-(3-fluoro-1-(3-methyl-1H-indol-1yl)propyl)-4-methoxybenzamide (3p) $(21.4 \mathrm{mg}, 0.063 \mathrm{mmol}, 21 \%)$ were obtained as beige solids after purification by column chromatography on silica using 3:1 pentanes:ethyl acetate as eluent.

$\mathbf{R}_{\mathbf{f}}: 0.33$ (silica, pentanes:ethyl acetate 2:1);

Mp: $170-172{ }^{\circ} \mathrm{C}$;

${ }^{1} \mathrm{H}$ NMR $\left(400 \mathrm{MHz}, \mathrm{CDCl}_{3}\right): \delta=9.17(\mathrm{~s}, 1 \mathrm{H}$, indole NH), $7.76-7.68(\mathrm{~m}, 2 \mathrm{H}, \mathrm{ArH}), 7.53(\mathrm{~d}, J=7.9 \mathrm{~Hz}, 1 \mathrm{H}$, $\operatorname{ArH}), 7.31(\mathrm{~d}, J=8.0 \mathrm{~Hz}, 1 \mathrm{H}, \operatorname{ArH}), 7.16(\mathrm{ddd}, J=8.1,7.0,1.2 \mathrm{~Hz}, 1 \mathrm{H}, \mathrm{ArH}), 7.09(\mathrm{td}, J=7.4,6.8,1.1 \mathrm{~Hz}$, $1 \mathrm{H}, \mathrm{ArH}), 6.93-6.86(\mathrm{~m}, 2 \mathrm{H}, \mathrm{ArH}), 6.81(\mathrm{~d}, J=7.1 \mathrm{~Hz}, 1 \mathrm{H}, \mathrm{NH}), 5.25(\mathrm{q}, J=7.2 \mathrm{~Hz}, 1 \mathrm{H}, \mathrm{CH}), 4.67-4.38$ $\left(\mathrm{m}, 2 \mathrm{H}, \mathrm{FCH}_{2}\right), 3.84\left(\mathrm{~s}, 3 \mathrm{H}, \mathrm{OCH}_{3}\right), 2.77-2.45\left(\mathrm{~m}, 2 \mathrm{H}, \mathrm{CH}_{2}\right), 2.33\left(\mathrm{~s}, 3 \mathrm{H}, \mathrm{CH}_{3}\right)$;

${ }^{13} \mathrm{C}$ NMR $\left(101 \mathrm{MHz}\right.$, Acetone- $\left.d_{6}\right): \delta=165.7,162.2,135.9,134.6,129.0,128.9,126.9,121.2,118.5$, $118.2,113.4,110.8,107.1,81.1$ (d, $J=163.6 \mathrm{~Hz}), 54.9,43.0$ (d, $J=5.5 \mathrm{~Hz}$ ), 35.3 (d, J=19.9 Hz), 7.7;

${ }^{19} \mathrm{~F}$ NMR $\left(376 \mathrm{MHz}, \mathrm{CDCl}_{3}\right): \delta=-220.1$;

IR (film): $\tilde{v}$ = 3312 (m), 2963 (w), 2919 (w), 1607 (s), 1504 (s), 1257 (s), 1028 (m), 844 (w), 740 (m);

HRMS (APCl) calcd. for $\mathrm{C}_{20} \mathrm{H}_{22} \mathrm{FN}_{2} \mathrm{O}_{2}^{+}[\mathrm{M}+\mathrm{H}]^{+} 341.1660$; Found 341.1654.

$N$-(3-Fluoro-1-(3-methyl-1H-indol-1-yl)propyl)-4-methoxybenzamide (3p).<smiles>COc1ccc(C(=O)NC(CCF)n2cc(C)c3ccccc32)cc1</smiles>

$\mathbf{R}_{\mathbf{f}}: 0.36$ (silica, pentanes:ethyl acetate $2: 1$ );

Mp: $278-280^{\circ} \mathrm{C}$;

${ }^{1} \mathrm{H}$ NMR $\left(400 \mathrm{MHz}, \mathrm{CDCl}_{3}\right): \delta=7.72-7.65(\mathrm{~m}, 2 \mathrm{H}, \mathrm{ArH}), 7.58-7.51(\mathrm{~m}, 2 \mathrm{H}, \mathrm{ArH}), 7.26-7.20(\mathrm{~m}, 1 \mathrm{H}$, $\operatorname{ArH}), 7.16-7.09(\mathrm{~m}, 1 \mathrm{H}, \operatorname{ArH}), 7.06(\mathrm{~d}, J=1.3 \mathrm{~Hz}, 1 \mathrm{H}, \operatorname{ArH}), 6.90-6.85(\mathrm{~m}, 2 \mathrm{H}, \operatorname{ArH}), 6.78(\mathrm{~d}, J=8.0 \mathrm{~Hz}$, $\left.1 \mathrm{H}, \mathrm{NH}), 6.71(\mathrm{q}, J=7.1 \mathrm{~Hz}, 1 \mathrm{H}, \mathrm{CH}), 4.66-4.35(\mathrm{~m}, 2 \mathrm{H}, \mathrm{FCH})_{2}\right), 3.82\left(\mathrm{~s}, 3 \mathrm{H}, \mathrm{OCH}_{3}\right), 2.61$ (ddtd, $J=26.5$, $\left.11.2,6.7,4.5 \mathrm{~Hz}, 2 \mathrm{H}, \mathrm{CH}_{2}\right), 2.31\left(\mathrm{~d}, J=1.1 \mathrm{~Hz}, 3 \mathrm{H}, \mathrm{CH}_{3}\right)$; 
${ }^{13} \mathrm{C}$ NMR $\left(101 \mathrm{MHz}, \mathrm{CDCl}_{3}\right): \delta=166.2,162.6,135.7,129.2,128.9,125.6,122.3,121.4,119.5,119.1$, $113.8,112.3,109.9,80.4$ (d, $J=165.7 \mathrm{~Hz}), 58.6(\mathrm{~d}, J=4.5 \mathrm{~Hz}), 55.4,35.4$ (d, $J=19.9 \mathrm{~Hz}), 9.7$;

${ }^{19} \mathrm{~F}$ NMR $\left(376 \mathrm{MHz}, \mathrm{CDCl}_{3}\right): \delta=-222.2$;

IR (film): $\tilde{v}=3282$ (w), 2967 (w), 1635 (s), 1606 (s), 1504 (s), 1257 (s), 1177 (m), 1030 (m), 745 (m);

HRMS (ESI) calcd. for $\mathrm{C}_{20} \mathrm{H}_{21} \mathrm{FN}_{2} \mathrm{NaO}_{2}{ }^{+}[\mathrm{M}+\mathrm{Na}]^{+}$363.1479; Found 363.1469.

$\mathrm{N}$-(3-Fluoro-1-(4-fluoro-1H-indol-3-yl)propyl)-4-methoxybenzamide (3q).<smiles>COc1ccc(C(=O)NC(CCF)c2c[nH]c3cccc(F)c23)cc1</smiles>

Following GP C, 4-fluoro- $1 \mathrm{H}$-indole ( $48.6 \mathrm{mg}, 0.360 \mathrm{mmol}, 1.2$ equiv.) was used as nucleophile. After the reaction mixture was stirred for 20 hours, $\mathrm{N}$-(3-fluoro-1-(4-fluoro- $1 H$-indol-3-yl)propyl)-4methoxybenzamide (3q) $(59.2 \mathrm{mg}, 0.172 \mathrm{mmol}, 57 \%)$ was obtained as a beige solid after purification by column chromatography on silica using 1:1 pentanes:ethyl acetate as eluent.

$\mathbf{R}_{\mathbf{f}}: 0.33$ (silica, pentanes:ethyl acetate 2:3);

Mp: $82-84^{\circ} \mathrm{C}$;

${ }^{1}$ H NMR (400 MHz, Acetone- $\left.d_{6}\right): \delta=10.45(\mathrm{~s}, 1 \mathrm{H}$, indole NH), $7.93-7.80(\mathrm{~m}, 2 \mathrm{H}, \operatorname{ArH}), 7.59(\mathrm{~d}, J=8.4$ $\mathrm{Hz}, 1 \mathrm{H}, \mathrm{NH}), 7.42(\mathrm{~s}, 1 \mathrm{H}, \mathrm{ArH}), 7.25(\mathrm{~d}, J=8.2 \mathrm{~Hz}, 1 \mathrm{H}, \mathrm{ArH}), 7.08(\mathrm{td}, J=8.0,5.2 \mathrm{~Hz}, 1 \mathrm{H}, \operatorname{ArH}), 6.99-6.92$ $(\mathrm{m}, 2 \mathrm{H}, \mathrm{ArH}), 6.75$ (ddd, J = 11.6, 7.8, 0.6 Hz, $1 \mathrm{H}, \mathrm{ArH}), 5.76(\mathrm{q}, J=7.8 \mathrm{~Hz}, 1 \mathrm{H}, \mathrm{CH}), 4.73-4.40(\mathrm{~m}, 2 \mathrm{H}$, $\left.\mathrm{FCH}_{2}\right), 3.83\left(\mathrm{~s}, 3 \mathrm{H}, \mathrm{OCH}_{3}\right), 2.52-2.34\left(\mathrm{~m}, 2 \mathrm{H}, \mathrm{CH}_{2}\right)$;

${ }^{13} \mathrm{C}$ NMR (101 MHz, Acetone- $\left.d_{6}\right): \delta=166.1,162.9,157.3(\mathrm{~d}, J=242.8 \mathrm{~Hz}), 140.8(\mathrm{dd}, J=15.5,12.2 \mathrm{~Hz})$, $129.8,128.3,124.1(\mathrm{~d}, J=15.9 \mathrm{~Hz}), 122.9(\mathrm{~d}, J=8.1 \mathrm{~Hz}), 115.9(\mathrm{t}, J=4.1 \mathrm{~Hz}), 115.3(\mathrm{dd}, J=20.8,2.8 \mathrm{~Hz})$, 114.3, $108.9(\mathrm{t}, J=4.4 \mathrm{~Hz}), 104.8(\mathrm{~d}, J=20.2 \mathrm{~Hz}), 82.4(\mathrm{~d}, J=163.1 \mathrm{~Hz}), 55.7,44.8(\mathrm{~d}, J=6.4 \mathrm{~Hz}), 38.1$ (dd, $J=19.5,2.8 \mathrm{~Hz})$;

${ }^{19} \mathrm{~F}$ NMR (376 MHz, Acetone- $\left.d_{6}\right): \delta=-121.5,-220.2 ;$

IR (film): $\tilde{v}=3273$ (w), 2964 (w), 1634 (s), 1606 (s), 1499 (s), 1255 (s), 1032 (m), 848 (m), 738 (m);

HRMS (ESI) calcd. for $\mathrm{C}_{19} \mathrm{H}_{19} \mathrm{~F}_{2} \mathrm{~N}_{2} \mathrm{O}_{2}{ }^{+}[\mathrm{M}+\mathrm{H}]^{+} 345.1409$; Found 345.1406 .

$N$-(3-Fluoro-1-(5-methoxy-1H-indol-3-yl)propyl)-4-methoxybenzamide (3r).<smiles>COc1ccc(C(=O)NC(CCF)c2c[nH]c3ccc(OC)cc23)cc1</smiles>

Following GP C, 5 -methoxy- $1 H$-indole $(52.9 \mathrm{mg}, 0.360 \mathrm{mmol}, 1.2$ equiv.) was used as nucleophile. After the reaction mixture was stirred for 3 hours, $\mathrm{N}$-(3-fluoro-1-(5-methoxy-1H-indol-3-yl)propyl)-4methoxybenzamide $(3 \mathrm{r})(58.0 \mathrm{mg}, 0.163 \mathrm{mmol}, 54 \%)$ was obtained as a beige solid after purification by column chromatography on silica using 1:1 pentanes:ethyl acetate as eluent.

$\mathbf{R}_{\mathbf{f}}: 0.34$ (silica, pentanes:ethyl acetate 1:1); 
Mp: $133-136^{\circ} \mathrm{C}$;

${ }^{1} \mathrm{H}$ NMR (400 MHz, Acetone- $\left.d_{6}\right): \delta=10.01(\mathrm{~s}, 1 \mathrm{H}$, indole $\mathrm{NH}), 7.93-7.84(\mathrm{~m}, 2 \mathrm{H}, \operatorname{ArH}), 7.70(\mathrm{~d}, J=8.8$ $\mathrm{Hz}, 1 \mathrm{H}, \mathrm{NH}), 7.33(\mathrm{~d}, J=2.1 \mathrm{~Hz}, 1 \mathrm{H}, \mathrm{ArH}), 7.30-7.23(\mathrm{~m}, 2 \mathrm{H}, \mathrm{ArH}), 6.97-6.91(\mathrm{~m}, 2 \mathrm{H}, \mathrm{ArH}), 6.75(\mathrm{dd}, J$ $=8.7,2.5 \mathrm{~Hz}, 1 \mathrm{H}, \mathrm{ArH}), 5.75(\mathrm{td}, J=8.6,6.0 \mathrm{~Hz}, 1 \mathrm{H}, \mathrm{CH}), 4.74-4.50\left(\mathrm{~m}, 2 \mathrm{H}, \mathrm{FCH}_{2}\right), 3.81\left(\mathrm{~s}, 3 \mathrm{H}, \mathrm{OCH}_{3}\right)$, $3.73\left(\mathrm{~s}, 3 \mathrm{H}, \mathrm{OCH}_{3}\right), 2.61-2.39\left(\mathrm{~m}, 2 \mathrm{H}, \mathrm{CH}_{2}\right)$;

${ }^{13} \mathrm{C}$ NMR (101 MHz, Acetone- $d_{6}$, mixture of two rotamers, minor rotamer omitted for clarity): $\delta=166.4$, $162.9,154.8,132.9,129.9,128.3,127.8,123.7,117.4,114.2,112.9,112.6,101.8,82.4$ (d, $J=162.9 \mathrm{~Hz})$, $55.8,55.7,43.4(\mathrm{~d}, J=6.6 \mathrm{~Hz}), 36.3(\mathrm{~d}, J=19.8 \mathrm{~Hz})$;

${ }^{19} \mathrm{~F}$ NMR $\left(376 \mathrm{MHz}\right.$, Acetone- $\left.d_{6}\right): \delta=-219.9$;

IR (film): $\tilde{v}=3311$ (w), 2962 (w), 1625 (s), 1606 (s), 1500 (s), 1255 (s), 1174 (m), 1029 (m), 844 (m);

HRMS (ESI) calcd. for $\mathrm{C}_{20} \mathrm{H}_{21} \mathrm{FN}_{2} \mathrm{NaO}_{3}{ }^{+}[\mathrm{M}+\mathrm{Na}]^{+} 379.1428$; Found 379.1428.

$N$-(3-Fluoro-1-(5-methyl-1H-indol-3-yl)propyl)-4-methoxybenzamide (3s).<smiles>COc1ccc(C(=O)NC(CCF)c2c[nH]c3ccc(C)cc23)cc1</smiles>

Following GP C, 5-methyl-1H-indole ( $47.2 \mathrm{mg}, 0.360 \mathrm{mmol}, 1.2$ equiv.) was used as nucleophile. After the reaction mixture was stirred for 4 hours, $N$-(3-fluoro-1-(5-methyl-1H-indol-3-yl)propyl)-4methoxybenzamide (3s) $(64.0 \mathrm{mg}, 0.188 \mathrm{mmol}, 63 \%)$ was obtained as a pink solid after purification by column chromatography on silica using 1:1 pentanes:ethyl acetate as eluent.

$\mathbf{R}_{\mathbf{f}}: 0.34$ (silica, pentanes:ethyl acetate 1:1);

Mp: $140-142^{\circ} \mathrm{C}$;

${ }^{1} \mathrm{H}$ NMR $\left(400 \mathrm{MHz}, \mathrm{CDCl}_{3}\right): \delta=8.14(\mathrm{~s}, 1 \mathrm{H}$, indole NH), $7.77-7.66(\mathrm{~m}, 2 \mathrm{H}, \mathrm{ArH}), 7.44(\mathrm{~s}, 1 \mathrm{H}, \mathrm{ArH}), 7.27$ $(\mathrm{d}, J=6.7 \mathrm{~Hz}, 1 \mathrm{H}, \operatorname{ArH}), 7.13(\mathrm{~d}, J=2.5 \mathrm{~Hz}, 1 \mathrm{H}, \operatorname{ArH}), 7.04(\mathrm{dd}, J=8.3,1.6 \mathrm{~Hz}, 1 \mathrm{H}, \operatorname{ArH}), 6.92-6.85(\mathrm{~m}$, $2 \mathrm{H}, \mathrm{ArH}), 6.45(\mathrm{~d}, J=7.9 \mathrm{~Hz}, 1 \mathrm{H}, \mathrm{NH}), 5.67(\mathrm{q}, J=7.1 \mathrm{~Hz}, 1 \mathrm{H}, \mathrm{CH}), 4.72-4.47(\mathrm{~m}, 2 \mathrm{H}, \mathrm{FCH})_{2}, 3.83(\mathrm{~s}, 3 \mathrm{H}$, $\left.\mathrm{OCH}_{3}\right), 2.57-2.45\left(\mathrm{~m}, 2 \mathrm{H}, \mathrm{CH}_{2}\right), 2.43\left(\mathrm{~s}, 3 \mathrm{H}, \mathrm{CH}_{3}\right)$;

${ }^{13} \mathrm{C}$ NMR $\left(101 \mathrm{MHz}, \mathrm{CDCl}_{3}\right): \delta=166.3,162.1,135.0,129.3,128.7,126.8,125.9,124.2,122.1,118.7$, $115.4,113.7,111.2,82.0$ (d, $J=164.0 \mathrm{~Hz}), 55.4,44.3(\mathrm{~d}, J=5.9 \mathrm{~Hz}), 35.5(\mathrm{~d}, J=19.2 \mathrm{~Hz}), 21.5$;

${ }^{19} \mathrm{~F}$ NMR $\left(376 \mathrm{MHz}, \mathrm{CDCl}_{3}\right): \delta=-218.2$

IR (film): $\tilde{v}$ = 3407 (w), 3307 (br, m), 2962 (w), 2917 (w), 1630 (s), 1605 (s), 1497 (s), 1254 (s), 1177 (m), $1029(\mathrm{~m}), 731(\mathrm{~m})$;

HRMS (ESI) calcd. for $\mathrm{C}_{20} \mathrm{H}_{21} \mathrm{FN}_{2} \mathrm{NaO}_{2}^{+}[\mathrm{M}+\mathrm{Na}]^{+}$363.1479; Found 363.1473.

$N$-(1-(5-Chloro-1H-indol-3-yl)-3-fluoropropyl)-4-methoxybenzamide (3t).<smiles>COc1ccc(C(=O)NC(CCF)c2c[nH]c3ccc(Cl)cc23)cc1</smiles>

Following GP C, 5-chloro- $1 H$-indole $(54.4 \mathrm{mg}, 0.360 \mathrm{mmol}, 1.2$ equiv.) was used as nucleophile. After the reaction mixture was stirred for 16 hours, $\mathrm{N}$-(1-(5-chloro- $1 \mathrm{H}$-indol-3-yl)-3-fluoropropyl)-4- 
methoxybenzamide (3t) $(74.3 \mathrm{mg}, 0.206 \mathrm{mmol}, 69 \%)$ was obtained as a pink solid after purification by column chromatography on silica using 1:2 pentanes:ethyl acetate as eluent.

$\mathbf{R}_{\mathbf{f}}: 0.46$ (silica, pentanes:ethyl acetate 1:2);

Mp: $103-105^{\circ} \mathrm{C}$;

${ }^{1}$ H NMR $\left(400 \mathrm{MHz}, \mathrm{CDCl}_{3}\right): \delta=8.57(\mathrm{~s}, 1 \mathrm{H}$, indole NH), $7.75-7.69(\mathrm{~m}, 2 \mathrm{H}, \operatorname{ArH}), 7.60(\mathrm{~s}, 1 \mathrm{H}, \operatorname{ArH}), 7.23$ $(\mathrm{d}, J=8.7 \mathrm{~Hz}, 1 \mathrm{H}, \operatorname{ArH}), 7.11(\mathrm{dd}, J=8.6,2.0 \mathrm{~Hz}, 2 \mathrm{H}, \operatorname{ArH}), 6.88(\mathrm{~d}, J=8.6 \mathrm{~Hz}, 2 \mathrm{H}, \operatorname{ArH}), 6.59(\mathrm{~d}, J=7.8$ $\mathrm{Hz}, 1 \mathrm{H}, \mathrm{NH}), 5.64(\mathrm{q}, J=7.0 \mathrm{~Hz}, 1 \mathrm{H}, \mathrm{CH}), 4.71-4.44\left(\mathrm{~m}, 2 \mathrm{H}, \mathrm{FCH}_{2}\right), 3.82\left(\mathrm{~s}, 3 \mathrm{H}, \mathrm{OCH}_{3}\right), 2.45(\mathrm{dq}, J=26.2$, $\left.6.1 \mathrm{~Hz}, 2 \mathrm{H}, \mathrm{CH}_{2}\right)$;

${ }^{13} \mathrm{C}$ NMR $\left(101 \mathrm{MHz}, \mathrm{CDCl}_{3}\right): \delta=166.6,162.3,135.0,128.7,126.6,126.5,125.5,123.5,122.7,118.4$, $115.6,113.8,112.6,82.0(\mathrm{~d}, J=163.7 \mathrm{~Hz}), 55.4,44.5(\mathrm{~d}, J=4.6 \mathrm{~Hz}), 35.4(\mathrm{~d}, J=19.2 \mathrm{~Hz}$ );

${ }^{19} \mathrm{~F}$ NMR $\left(376 \mathrm{MHz}, \mathrm{CDCl}_{3}\right): \delta=-218.0$;

IR (film): $\tilde{v}=3430$ (w), 3271 (m), 2960 (w), 1630 (s), 1605 (s), 1498 (s), 1253 (s), 1177 (m), 1029 (m), $731(\mathrm{~m})$;

HRMS (ESI) calcd. for $\mathrm{C}_{19} \mathrm{H}_{18} \mathrm{ClFN}_{2} \mathrm{NaO}_{2}{ }^{+}[\mathrm{M}+\mathrm{Na}]^{+}$383.0933; Found 383.0930.

Methyl 3-(3-fluoro-1-(4-methoxybenzamido)propyl)-1H-indole-5-carboxylate (3u).<smiles>COC(=O)c1ccc2[nH]cc(C(CCF)NC(=O)c3ccc(OC)cc3)c2c1</smiles>

Following GP C, methyl- $1 \mathrm{H}$-indole-5-carboxylate $(63.0 \mathrm{mg}, 0.360 \mathrm{mmol}, 1.2$ equiv.) was used as nucleophile. After the reaction mixture was stirred for 40 hours, methyl 3-(3-fluoro-1-(4methoxybenzamido)propyl)-1H-indole-5-carboxylate (3u) $(51.4 \mathrm{mg}, 0.134 \mathrm{mmol}, 45 \%)$ was obtained as a yellow solid after purification by column chromatography on silica using 1:2 pentanes:ethyl acetate as eluent.

$\mathbf{R}_{\mathbf{f}}: 0.28$ (silica, pentanes:ethyl acetate 1:2);

Mp: $147-149^{\circ} \mathrm{C}$;

${ }^{1} \mathrm{H}$ NMR $\left(400 \mathrm{MHz}\right.$, Acetone- $\left.d_{6}\right): \delta=10.56(\mathrm{~s}, 1 \mathrm{H}$, indole $\mathrm{NH}), 8.54(\mathrm{dd}, J=1.6,0.7 \mathrm{~Hz}, 1 \mathrm{H}, \mathrm{ArH}), 7.96-$ $7.90(\mathrm{~m}, 1 \mathrm{H}, \mathrm{NH}), 7.90-7.86(\mathrm{~m}, 2 \mathrm{H}, \mathrm{ArH}), 7.81(\mathrm{dd}, J=8.6,1.6 \mathrm{~Hz}, 1 \mathrm{H}, \mathrm{ArH}), 7.53(\mathrm{~d}, J=0.9 \mathrm{~Hz}, 1 \mathrm{H}$, $\operatorname{ArH}), 7.47(\mathrm{dd}, J=8.7,0.7 \mathrm{~Hz}, 1 \mathrm{H}, \mathrm{ArH}), 6.96-6.90(\mathrm{~m}, 2 \mathrm{H}, \mathrm{ArH}), 5.82(\mathrm{q}, J=7.8 \mathrm{~Hz}, 1 \mathrm{H}, \mathrm{CH}), 4.63$ (dddt, $\left.J=47.1,37.7,9.3,6.0 \mathrm{~Hz}, 2 \mathrm{H}, \mathrm{FCH}_{2}\right), 3.84\left(\mathrm{~s}, 3 \mathrm{H}, \mathrm{OCH}_{3}\right), 3.81\left(\mathrm{~s}, 3 \mathrm{H}, \mathrm{OCH}_{3}\right), 2.63-2.47\left(\mathrm{~m}, 2 \mathrm{H}, \mathrm{CH}_{2}\right)$;

${ }^{13} \mathrm{C}$ NMR $\left(101 \mathrm{MHz}\right.$, Acetone- $d_{6}$, mixture of two rotamers, ratio $=$ ca. $\left.1: 1\right): \delta=168.3,166.6,162.9$, $140.3,140.1,129.9,128.2,126.9,126.9,125.2,125.0,123.6,122.9,122.1,119.1,119.1,114.2,112.1$, 112.0, $82.3(\mathrm{~d}, J=163.2 \mathrm{~Hz}), 55.7,51.9,43.4(\mathrm{~d}, J=6.2 \mathrm{~Hz}), 36.7(\mathrm{~d}, J=19.7 \mathrm{~Hz})$;

${ }^{13} \mathrm{C}$ NMR $\left(151 \mathrm{MHz}\right.$, Acetonitrile- $\left.d_{3}, 70{ }^{\circ} \mathrm{C}\right): \delta=169.0,167.5,163.6,140.6,130.2,128.6,127.2,125.4$, $124.2,123.0,122.9,119.5,115.0,112.6,83.1$ (d, $J=162.2 \mathrm{~Hz}$ ), 56.5, 52.5, 44.3 (d, $J=5.9 \mathrm{~Hz}$ ), 37.0 (d, $J$ $=19.6 \mathrm{~Hz})$;

${ }^{19} \mathrm{~F}$ NMR (376 MHz, Acetone- $\left.d_{6}\right): \delta=-220.1$;

IR (film): $\tilde{v}=3315$ (w), 2952 (w), 1695 (s), 1607 (s), 1502 (s), 1435 (m), 1252 (s), 1178 (m), $1112(\mathrm{~m})$, $770(\mathrm{~m})$;

HRMS (ESI) calcd. for $\mathrm{C}_{21} \mathrm{H}_{21} \mathrm{FN}_{2} \mathrm{NaO}_{4}{ }^{+}[\mathrm{M}+\mathrm{Na}]^{+}$407.1378; Found 407.1381.

$N$-(3-Fluoro-1-(6-methyl-1H-indol-3-yl)propyl)-4-methoxybenzamide (3v). 


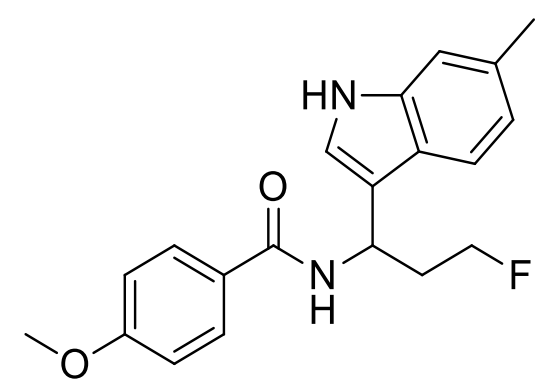

Following GP C, 6-methyl-1H-indole $(47.2 \mathrm{mg}, 0.360 \mathrm{mmol}, 1.2$ equiv.) was used as nucleophile. After the reaction mixture was stirred for 16 hours, $\mathrm{N}$-(3-fluoro-1-(6-methyl-1H-indol-3-yl)propyl)-4methoxybenzamide $3 \mathrm{v}(65.8 \mathrm{mg}, 0.194 \mathrm{mmol}, 65 \%)$ was obtained as a red solid after purification by column chromatography on silica using 1:1 pentanes:ethyl acetate as eluent.

$\mathbf{R}_{\mathbf{f}}: 0.35$ (silica, pentanes:ethyl acetate 1:1);

Mp: $153-156^{\circ} \mathrm{C}$;

${ }^{1} \mathrm{H}$ NMR $\left(400 \mathrm{MHz}\right.$, Acetone- $\left.d_{6}\right): \delta=10.01(\mathrm{~s}, 1 \mathrm{H}$, indole $\mathrm{NH}), 7.93-7.82(\mathrm{~m}, 2 \mathrm{H}, \operatorname{ArH}), 7.69(\mathrm{~d}, J=8.7$ $\mathrm{Hz}, 1 \mathrm{H}, \mathrm{NH}), 7.60(\mathrm{~d}, J=8.1 \mathrm{~Hz}, 1 \mathrm{H}, \mathrm{ArH}), 7.28(\mathrm{dd}, J=2.5,0.8 \mathrm{~Hz}, 1 \mathrm{H}, \mathrm{ArH}), 7.19(\mathrm{dt}, J=1.6,0.8 \mathrm{~Hz}, 1 \mathrm{H}$, $\operatorname{ArH}), 6.96-6.89(\mathrm{~m}, 2 \mathrm{H}, \mathrm{ArH}), 6.87-6.80(\mathrm{~m}, 1 \mathrm{H}, \mathrm{ArH}), 5.75(\mathrm{td}, J=8.6,6.2 \mathrm{~Hz}, 1 \mathrm{H}, \mathrm{CH}), 4.74-4.45$ $\left(\mathrm{m}, 2 \mathrm{H}, \mathrm{FCH}_{2}\right), 3.81\left(\mathrm{~s}, 3 \mathrm{H}, \mathrm{OCH}_{3}\right), 2.56-2.42\left(\mathrm{~m}, 2 \mathrm{H}, \mathrm{CH}_{2}\right), 2.38\left(\mathrm{~s}, 3 \mathrm{H}, \mathrm{CH}_{3}\right)$;

${ }^{13} \mathrm{C}$ NMR $\left(101 \mathrm{MHz}\right.$, Acetone- $\left.d_{6}\right): \delta=166.3,162.9,138.3,131.8,129.9,128.3,125.3,122.4,121.6$, 119.7, 117.4, 114.2, 112.1, 82.4 (d, $J=163.0 \mathrm{~Hz}$ ), 55.7, 43.5 (d, J = 6.4 Hz), 36.6 (d, J = 19.7 Hz), 21.7;

${ }^{19} \mathrm{~F}$ NMR $\left(376 \mathrm{MHz}, \mathrm{CDCl}_{3}\right): \delta=-219.9$;

IR (film): $\tilde{v}=3295$ (w), 2963 (w), 1629(s), 1606 (s), 1500 (s), 1254 (s), 1178 (m), 1030 (m), 802 (m);

HRMS (ESI) calcd. for $\mathrm{C}_{20} \mathrm{H}_{21} \mathrm{FN}_{2} \mathrm{NaO}_{2}{ }^{+}[\mathrm{M}+\mathrm{Na}]^{+}$363.1479; Found 363.1480.

$N$-(3-Fluoro-1-(6-(trifluoromethyl)-1H-indol-3-yl)propyl)-4-methoxybenzamide (3w).<smiles>COc1ccc(C(=O)NC(CCF)c2c[nH]c3cc(C(F)(F)F)ccc23)cc1</smiles>

Following GP C, 6-trifluoromethyl- $1 \mathrm{H}$-indole $(66.6 \mathrm{mg}, 0.360 \mathrm{mmol}, 1.2$ equiv.) was used as nucleophile. After the reaction mixture was stirred for 16 hours, $\mathrm{N}$-(3-fluoro-1-(6-(trifluoromethyl)-1H-indol-3yl)propyl)-4-methoxybenzamide $3 w(61.5 \mathrm{mg}, 0.156 \mathrm{mmol}, 52 \%)$ was obtained as a pink solid after purification by column chromatography on silica using 1:1 pentanes:ethyl acetate as eluent.

$\mathbf{R}_{\mathbf{f}}: 0.36$ (silica, pentanes:ethyl acetate 1:1);

Mp: $78-80^{\circ} \mathrm{C}$;

${ }^{1} \mathrm{H}$ NMR $\left(400 \mathrm{MHz}, \mathrm{CDCl}_{3}\right): \delta=8.91(\mathrm{~s}, 1 \mathrm{H}$, indole NH), $7.76-7.69(\mathrm{~m}, 3 \mathrm{H}, \operatorname{ArH}), 7.57(\mathrm{~s}, 1 \mathrm{H}, \operatorname{ArH}), 7.29$ $(\mathrm{d}, J=8.4 \mathrm{~Hz}, 1 \mathrm{H}, \operatorname{ArH}), 7.21(\mathrm{~d}, J=2.5 \mathrm{~Hz}, 1 \mathrm{H}, \operatorname{ArH}), 6.93-6.86(\mathrm{~m}, 2 \mathrm{H}, \operatorname{ArH}), 6.63(\mathrm{~d}, J=7.5 \mathrm{~Hz}, 1 \mathrm{H}$, $\mathrm{NH}), 5.73(\mathrm{q}, J=7.1 \mathrm{~Hz}, 1 \mathrm{H}, \mathrm{CH}), 4.74-4.44(\mathrm{~m}, 2 \mathrm{H}, \mathrm{FCH} 2), 3.82\left(\mathrm{~s}, 3 \mathrm{H}, \mathrm{OCH}_{3}\right), 2.56-2.37\left(\mathrm{~m}, 2 \mathrm{H}, \mathrm{CH}_{2}\right)$; ${ }^{13} \mathrm{C}$ NMR $\left(101 \mathrm{MHz}, \mathrm{CDCl}_{3}\right): \delta=166.7,162.4,135.5,128.7,127.9,126.3,125.0$ (q, $\left.J=271.7 \mathrm{~Hz}\right), 124.7$, $124.4(q, J=31.9 \mathrm{~Hz}), 119.3,116.5(q, J=3.5 \mathrm{~Hz}), 116.2,113.9,109.1(q, J=4.4 \mathrm{~Hz}), 81.9(\mathrm{~d}, J=164.0$ $\mathrm{Hz}), 55.4,44.5(\mathrm{~d}, J=4.4 \mathrm{~Hz}), 35.4(\mathrm{~d}, J=19.3 \mathrm{~Hz})$;

${ }^{19} \mathrm{~F}$ NMR $\left(376 \mathrm{MHz}, \mathrm{CDCl}_{3}\right): \delta=-60.7,-217.9$; 
IR (film): $\tilde{v}=3272$ (w), 2965 (w), 1628(s), 1606 (s), 1499 (s), 1355 (s), 1255 (s), 1111 (s), 734 (m);

HRMS (ESI) calcd. for $\mathrm{C}_{20} \mathrm{H}_{19} \mathrm{~F}_{4} \mathrm{~N}_{2} \mathrm{O}_{2}{ }^{+}[\mathrm{M}+\mathrm{H}]^{+} 395.1377$; Found 395.1372.

$N$-(1-(6-Bromo-1H-indol-3-yl)-3-fluoropropyl)-4-methoxybenzamide (3x).<smiles>COc1ccc(C(=O)NC(CCF)c2c[nH]c3cc(Br)ccc23)cc1</smiles>

Following GP C, 6-bromo- $1 H$-indole $(70.2 \mathrm{mg}, 0.360 \mathrm{mmol}, 1.2$ equiv.) was used as nucleophile. After the reaction mixture was stirred for 24 hours, $N$-(1-(6-bromo- $1 H$-indol-3-yl)-3-fluoropropyl)-4methoxybenzamide $3 \times(61.0 \mathrm{mg}, 0.151 \mathrm{mmol}, 50 \%)$ was obtained as a yellow solid after purification by column chromatography on silica using 1:1 pentanes:ethyl acetate as eluent.

$\mathbf{R}_{\mathbf{f}}: 0.33$ (silica, pentanes:ethyl acetate 1:1);

Mp: $175-178^{\circ} \mathrm{C}$;

${ }^{1} \mathrm{H}$ NMR $\left(400 \mathrm{MHz}, \mathrm{CDCl}_{3}\right): \delta=8.34(\mathrm{~s}, 1 \mathrm{H}$, indole $\mathrm{NH}), 7.73-7.68(\mathrm{~m}, 2 \mathrm{H}, \mathrm{ArH}), 7.53-7.45(\mathrm{~m}, 2 \mathrm{H}$, $\operatorname{ArH}), 7.18(\mathrm{dd}, J=8.4,1.7 \mathrm{~Hz}, 1 \mathrm{H}, \mathrm{ArH}), 7.09(\mathrm{~d}, J=2.4 \mathrm{~Hz}, 1 \mathrm{H}, \operatorname{ArH}), 6.92-6.87(\mathrm{~m}, 2 \mathrm{H}, \operatorname{ArH}), 6.50(\mathrm{~d}$, $J=8.1 \mathrm{~Hz}, 1 \mathrm{H}, \mathrm{NH}), 5.69(\mathrm{q}, J=7.1 \mathrm{~Hz}, 1 \mathrm{H}, \mathrm{CH}), 4.70-4.49\left(\mathrm{~m}, 2 \mathrm{H}, \mathrm{FCH}_{2}\right), 3.82\left(\mathrm{~s}, 3 \mathrm{H}, \mathrm{OCH}_{3}\right), 2.56-2.33$ (m, 2H, $\mathrm{CH}_{2}$ );

${ }^{13} \mathrm{C} \mathrm{NMR}\left(101 \mathrm{MHz}, \mathrm{CDCl}_{3}\right): \delta=166.5,162.3,137.4,128.7,126.4,124.6,123.2,122.4,120.3,116.3$, $116.1,114.4,113.8,81.9$ (d, J = 164.0 Hz), 55.4, 44.2 (d, J = 4.8 Hz), 35.4 (d, J = $19.1 \mathrm{~Hz}$ );

${ }^{19}$ F NMR $\left(376 \mathrm{MHz}, \mathrm{CDCl}_{3}\right): \delta=-217.9$;

IR (film): $\tilde{v}=3269$ (w), 2962 (w), 1630 (s), 1605 (s), 1497 (s), 1254 (s), 1177 (m), 1029 (m), 730 (m);

HRMS (ESI) calcd. for $\mathrm{C}_{19} \mathrm{H}_{18}{ }^{79} \mathrm{BrFN}_{2} \mathrm{NaO}_{2}{ }^{+}[\mathrm{M}+\mathrm{Na}]^{+} 427.0428$; Found 427.0424 .

$N$-(3-Fluoro-1-(7-methyl-1H-indol-3-yl)propyl)-4-methoxybenzamide (3y).<smiles>COc1ccc(C(=O)NC(CCF)c2c[nH]c3c(C)cccc23)cc1</smiles>

Following GP C, 7-methyl-1H-indole $(47.2 \mathrm{mg}, 0.360 \mathrm{mmol}, 1.2$ equiv.) was used as nucleophile. After the reaction mixture was stirred for 16 hours, $N$-(3-fluoro-1-(7-methyl-1H-indol-3-yl)propyl)-4methoxybenzamide (3y) $(67.4 \mathrm{mg}, 0.198 \mathrm{mmol}, 66 \%)$ was obtained as a beige solid after purification by column chromatography on silica using 1:1 pentanes:ethyl acetate as eluent.

$\mathbf{R}_{\mathbf{f}}: 0.37$ (silica, pentanes:ethyl acetate 1:1);

Mp: $175-176^{\circ} \mathrm{C}$;

${ }^{1}$ H NMR $\left(400 \mathrm{MHz}\right.$, Acetone- $\left.d_{6}\right): \delta=10.13(\mathrm{~s}, 1 \mathrm{H}$, indole $\mathrm{NH}), 7.93-7.81(\mathrm{~m}, 2 \mathrm{H}, \mathrm{ArH}), 7.70(\mathrm{~d}, J=8.7$ $\mathrm{Hz}, 1 \mathrm{H}, \mathrm{NH}), 7.57(\mathrm{dd}, J=5.7,3.5 \mathrm{~Hz}, 1 \mathrm{H}, \operatorname{ArH}), 7.36(\mathrm{~d}, J=2.6 \mathrm{~Hz}, 1 \mathrm{H}, \operatorname{ArH}), 6.96-6.85(\mathrm{~m}, 4 \mathrm{H}, \operatorname{Ar} H)$, 
$\left.5.77(\mathrm{td}, J=8.4,6.4 \mathrm{~Hz}, 1 \mathrm{H}, \mathrm{CH}), 4.76-4.46(\mathrm{~m}, 2 \mathrm{H}, \mathrm{FCH})_{2}\right), 3.81\left(\mathrm{~s}, 3 \mathrm{H}, \mathrm{OCH}_{3}\right), 2.58-2.42\left(\mathrm{~m}, 5 \mathrm{H}, \mathrm{CH}_{2}\right.$ $\left.+\mathrm{CH}_{3}\right)$

${ }^{13} \mathrm{C}$ NMR $\left(101 \mathrm{MHz}\right.$, Acetone- $\left.d_{6}\right): \delta=166.3,162.9,137.2,129.9,128.3,127.1,123.0,122.8,121.4$, $120.1,117.9,117.7,114.2,82.4(\mathrm{~d}, J=163.1 \mathrm{~Hz}), 55.7,43.5$ (d, $J=6.6 \mathrm{~Hz}$ ), 36.7 (d, J=19.6 Hz), 16.9;

${ }^{19} \mathrm{~F}$ NMR (376 MHz, Acetone- $\left.d_{6}\right): \delta=-220.0$;

IR (film): $\tilde{v}=3293$ (w), 2964 (w), 1632 (s), 1606 (s), 1499 (s), 1254 (s), 1177 (m), 1029 (m), 844 (m);

HRMS (ESI) calcd. for $\mathrm{C}_{20} \mathrm{H}_{21} \mathrm{FN}_{2} \mathrm{NaO}_{2}{ }^{+}[\mathrm{M}+\mathrm{Na}]^{+}$363.1479; Found 363.1480.

\section{Scope of ring-opening fluorination for muti-substituted aminocyclopropanes or aminocyclobutanes}

\section{General Procedure D (GP D):}

In a $12 * 75 \mathrm{~mm}$ borosilicate glass tube, substituted cyclopropylamides or cyclobutylamides $(0.100$ mmol, 1.0 equiv.), selectfluor ( $39.0 \mathrm{mg}, 0.110 \mathrm{mmol}, 1.1$ equiv.) and benzophenone (1.8 $\mathrm{mg}, 0.010$ mmol, 0.10 equiv.) were dissolved in $0.50 \mathrm{~mL}$ of $\mathrm{MeCN}-\mathrm{H}_{2} \mathrm{O}(\mathrm{v}: \mathrm{v}$ 4:6, $0.20 \mathrm{M})$. The reaction mixture was degassed by three freeze-pump-thaw cycles and backfilled with $\mathrm{N}_{2}$. Then the mixture was stirred at room temperature under $365 \mathrm{~nm}$ irradiation in Rayonet Reactor until the reaction was complete. The tube was taken out from the Rayonet Reactor and a solution of nucleophile $(0.120 \mathrm{mmol}, 1.2$ equiv. $)$ in $0.20 \mathrm{~mL}$ MeCN was added dropwise. The reaction mixture was stirred at room temperature for 3 hours, if not specified otherwise. After the completion of the reaction, the crude product was directly submitted to column chromatography on silica using pentanes:ethyl acetate as eluent.

\section{N-(3-Fluoro-1-hydroxybutyl)-4-methoxybenzamide (5a).}<smiles>COc1ccc(C(=O)NC(O)CC(C)F)cc1</smiles>

Following GP D, 4-methoxy- $N$-(2-methylcyclopropyl)benzamide (4a) (20.5 mg, 0.100 mmol, 1.0 equiv.) was used as starting material and the reaction was kept stirring under irradiation for 45 minutes. $\mathbf{N}$-(3fluoro-1-hydroxybutyl)-4-methoxybenzamide (5a) $(20.6 \mathrm{mg}, 0.0855 \mathrm{mmol}, 85 \%, \quad 1: 1$ d.r., diastereomeric value was determined by integration of the two peaks in ${ }^{19} \mathrm{~F}$ NMR) was obtained as a white solid after purification by column chromatography on silica using 1:1 pentanes:ethyl acetate as eluent.

$\mathbf{R}_{\mathbf{f}}: 0.29$ (silica, pentanes:ethyl acetate 1:1);

Mp: $136-138^{\circ} \mathrm{C}$;

${ }^{1} \mathrm{H}$ NMR $\left(400 \mathrm{MHz}\right.$, Acetone- $d_{6}$; mixture of diastereoisomers in a 1:1 ratio: the signals corresponding to the two diastereoisomers are partially resolved): $\delta=7.99(\mathrm{~s}, 1 \mathrm{H}, \mathrm{NH}), 7.93-7.81(\mathrm{~m}, 2 \mathrm{H}, \mathrm{ArH}), 7.01$ $-6.91(\mathrm{~m}, 2 \mathrm{H}, \mathrm{ArH}), 5.76-5.63(\mathrm{~m}, 1 \mathrm{H}, \mathrm{NCH}), 5.06-4.77(\mathrm{~m}, 2 \mathrm{H}, \mathrm{FCH}+\mathrm{OH}), 3.85\left(\mathrm{~s}, 3 \mathrm{H}, \mathrm{OCH}_{3}\right), 2.20-$ $2.07\left(\mathrm{~m}, 1 \mathrm{H}, \mathrm{CH}_{2}\right), 1.99-1.88\left(\mathrm{~m}, 1 \mathrm{H}, \mathrm{CH}_{2}\right), 1.35$ (ddd, $\left.\mathrm{J}=23.8,6.2,4.9 \mathrm{~Hz}, 3 \mathrm{H}, \mathrm{CH}_{3}\right)$;

${ }^{13} \mathrm{C}$ NMR (101 MHz, Acetone- $d_{6}$; mixture of diastereoisomers in a 1:1 ratio: the signals corresponding to the two diastereoisomers are partially resolved): $\delta=167.1,166.9,163.2,163.2,130.0,130.0,127.6$, 114.3, 114.3, 88.8 (d, $J=162.5 \mathrm{~Hz}$ ), $88.5(\mathrm{~d}, J=163.2 \mathrm{~Hz}), 72.2(\mathrm{~d}, J=6.4 \mathrm{~Hz}), 72.1$ (d, J = 5.5 Hz), 55.8, $44.0(\mathrm{~d}, J=20.6 \mathrm{~Hz}), 43.5(\mathrm{~d}, J=20.6 \mathrm{~Hz}), 21.5(\mathrm{~d}, J=22.4 \mathrm{~Hz}), 21.2(\mathrm{~d}, J=22.4 \mathrm{~Hz})$;

${ }^{19} \mathrm{~F}$ NMR $\left(376 \mathrm{MHz}\right.$, Acetone- $\left.d_{6}\right): \delta=-173.3,-175.4$;

IR (film): $\tilde{v}=3317$ (br, s), 2979 (w), $1638(\mathrm{~m}), 1606$ (s), 1503 (s), 1255 (s), 1029 (m), $846(\mathrm{w})$;

HRMS (APCl) calcd. for $\mathrm{C}_{12} \mathrm{H}_{16} \mathrm{FNNaO}_{3}{ }^{+}[\mathrm{M}+\mathrm{Na}]^{+}$264.1006; Found 264.1001. 
$N$-(3-Fluoro-3-phenylpropyl)-4-methoxybenzamide (5b).<smiles>COc1ccc(C(=O)NCCC(F)c2ccccc2)cc1</smiles>

Following GP D, 4-methoxy- $N$-(trans-2-phenylcyclopropyl)benzamide (4b) $(26.7 \mathrm{mg}, 0.100 \mathrm{mmol}, 1.0$ equiv.) was used as starting material and the reaction was kept stirring under irradiation for 1 hour. Then $\mathrm{NaBH}_{3} \mathrm{CN}$ (7.6 mg, $0.12 \mathrm{mmol}, 1.2$ equiv.) was added as nucleophile. After the reaction mixture was stirred for 3 hours, $\mathrm{N}$-(3-fluoro-3-phenylpropyl)-4-methoxybenzamide (5b) ( $25.8 \mathrm{mg}, 0.0900 \mathrm{mmol}$, $90 \%$ ) was obtained as a white solid after purification by column chromatography on silica using $1: 1$ pentanes:ethyl acetate as eluent.

$\mathbf{R}_{\mathbf{f}}: 0.50$ (silica, pentanes:ethyl acetate 1:1);

Mp: $71-73^{\circ} \mathrm{C}$;

${ }^{1} \mathrm{H}$ NMR $\left(400 \mathrm{MHz}, \mathrm{CDCl}_{3}\right): \delta=7.72-7.63(\mathrm{~m}, 2 \mathrm{H}, \mathrm{ArH}), 7.42-7.28(\mathrm{~m}, 5 \mathrm{H}, \mathrm{ArH}), 6.94-6.84(\mathrm{~m}, 2 \mathrm{H}$, $\operatorname{ArH}), 6.45(\mathrm{t}, J=5.8 \mathrm{~Hz}, 1 \mathrm{H}, \mathrm{NH}), 5.61(\mathrm{ddd}, J=47.9,7.3,5.2 \mathrm{~Hz}, 1 \mathrm{H}, \mathrm{CH}), 3.83(\mathrm{~s}, 3 \mathrm{H}, \mathrm{OCH})_{3}, 3.71-3.55$ (m, $\left.2 \mathrm{H}, \mathrm{NCH}_{2}\right), 2.30-2.16\left(\mathrm{~m}, 2 \mathrm{H}, \mathrm{NCH}_{2} \mathrm{CH}_{2}\right)$;

${ }^{13} \mathrm{C}$ NMR $\left(101 \mathrm{MHz}, \mathrm{CDCl}_{3}\right): \delta=167.0,162.1,139.5(\mathrm{~d}, J=19.7 \mathrm{~Hz}), 128.6,128.6,128.4(\mathrm{~d}, J=2.0 \mathrm{~Hz})$, $126.7,125.3(\mathrm{~d}, J=7.0 \mathrm{~Hz}), 113.6,93.5(\mathrm{~d}, J=170.1 \mathrm{~Hz}), 55.3,36.7(\mathrm{~d}, J=24.8 \mathrm{~Hz}), 36.6(\mathrm{~d}, J=1.8 \mathrm{~Hz})$; ${ }^{19} \mathrm{~F}$ NMR $\left(376 \mathrm{MHz}, \mathrm{CDCl}_{3}\right): \delta=-176.3$;

IR (film): $\tilde{v}=3315$ (w), 2932(w), 1629 (m), 1605 (m), 1542 (m), 1502 (s), 1252 (s), 1178 (m), 1028 (m), $844(\mathrm{~m})$;

HRMS (ESI) calcd. for $\mathrm{C}_{17} \mathrm{H}_{18} \mathrm{FNNaO}_{2}^{+}[\mathrm{M}+\mathrm{Na}]^{+} 310.1214$; Found 310.1212.

tert-Butyl 3-fluoro-4-((4-methoxybenzamido)methyl)pyrrolidine-1-carboxylate (5c).<smiles>COc1ccc(C(=O)NCC2CN(C(=O)OC(C)(C)C)CC2F)cc1</smiles>

Following GP D, tert-Butyl (1R,5S,6S)-6-(4-methoxybenzamido)-3-azabicyclo[3.1.0] hexane-3carboxylate (4c) (33.2 mg, $0.100 \mathrm{mmol}, 1.0$ equiv.) was used as starting material and the reaction was kept stirring under irradiation for 45 minutes. Then $\mathrm{NaBH}_{3} \mathrm{CN}(7.6 \mathrm{mg}, 0.12 \mathrm{mmol}, 1.2$ equiv.) was added as nucleophile. After the reaction mixture was stirred for 3 hours, tert-butyl 3-fluoro-4-((4methoxybenzamido)methyl)pyrrolidine-1-carboxylate (5c) $(21.1 \mathrm{mg}, 0.0599 \mathrm{mmol}, 60 \%, 1.4: 1 \mathrm{~d} . r$. , diastereomeric value was determined by integration of $\mathrm{FCH}$ signals from ${ }^{1} \mathrm{H} \mathrm{NMR}$ at $70{ }^{\circ} \mathrm{C}$ in $\mathrm{CD}_{3} \mathrm{CN}$ ) was obtained as a colorless gel which solidified during storage after purification by column chromatography on silica using 1:1 pentanes:ethyl acetate as eluent.

$\mathbf{R}_{\mathbf{f}}: 0.30$ (silica, pentanes:ethyl acetate 2:3);

Mp: $56-59^{\circ} \mathrm{C}$; 
${ }^{1} \mathbf{H}$ NMR (400 MHz, $\mathrm{CDCl}_{3}$; mixture of diastereoisomers and rotamers: the signals corresponding to the two diastereoisomers and two rotamers are partially resolved): $\delta=7.86-7.63(\mathrm{~m}, 2 \mathrm{H}, \operatorname{ArH}), 7.04-$ $6.80(\mathrm{~m}, 2 \mathrm{H}, \mathrm{ArH}), 6.35(\mathrm{~s}, 1 \mathrm{H}, \mathrm{NH}), 5.10(\mathrm{dd}, J=53.0,37.7 \mathrm{~Hz}, 1 \mathrm{H}, \mathrm{FCH}), 3.85\left(\mathrm{~s}, 3 \mathrm{H}, \mathrm{OCH}_{3}\right), 3.80-3.10$ $\left(\mathrm{m}, 6 \mathrm{H}, 3 \times \mathrm{NCH}_{2}\right), 2.69(\mathrm{t}, J=27.2 \mathrm{~Hz}, 1 \mathrm{H}, \mathrm{FCHCH}), 1.46\left(\mathrm{~d}, J=2.9 \mathrm{~Hz}, 9 \mathrm{H}, \mathrm{C}\left(\mathrm{CH}_{3}\right)_{3}\right)$;

${ }^{1} \mathrm{H}$ NMR $\left(400 \mathrm{MHz}\right.$, Acetonitrile- $d_{3}, 70{ }^{\circ} \mathrm{C}$; mixture of two diastereoisomers: the signals corresponding to the two diastereoisomers are partially resolved, diastereomeric ratio was determined by integration of FCH signals): $\delta=7.81-7.73(\mathrm{~m}, 4 \mathrm{H}, \mathrm{ArH}$, major + minor $), 7.02-6.97(\mathrm{~m}, 4 \mathrm{H}, \mathrm{ArH}$, major + minor), $6.94(\mathrm{~s}, 2 \mathrm{H}, \mathrm{NH}$, major + minor), 5.17 (dt, $J=53.6,3.3 \mathrm{~Hz}, 1 \mathrm{H}, \mathrm{FCH}$, major), 5.07 (ddd, $J=50.9,4.5,2.4$ $\mathrm{Hz}, 1 \mathrm{H}, \mathrm{FCH}$, minor), $3.85\left(\mathrm{~s}, 6 \mathrm{H}, \mathrm{OCH}_{3}\right.$, major + minor), $3.69-3.46\left(\mathrm{~m}, 8 \mathrm{H}, 2^{*} \mathrm{NCH}_{2}\right.$, major + minor), $3.41-3.27\left(\mathrm{~m}, 2 \mathrm{H}, \mathrm{NCH}_{2}\right.$, major), $3.15\left(\mathrm{t}, J=10.7 \mathrm{~Hz}, 2 \mathrm{H}, \mathrm{NCH}_{2}\right.$, minor), $2.74-2.55(\mathrm{~m}, 2 \mathrm{H}, \mathrm{FCHCH}$, major + minor), $1.45\left(\mathrm{~s}, 18 \mathrm{H}, 3^{*} \mathrm{CH}_{3}\right.$, major + minor $)$;

${ }^{13} \mathrm{C}$ NMR (101 MHz, $\mathrm{CDCl}_{3}$; mixture of diastereoisomers in a 1.4:1 ratio: the signals corresponding to the two diastereoisomers are partially resolved): $\delta=167.4,167.3,162.4,162.3,154.3,154.2,128.7$, $128.7,126.3,126.1,113.9,113.8,93.6(\mathrm{~d}, J=178.8 \mathrm{~Hz}), 93.0(\mathrm{~d}, J=177.8 \mathrm{~Hz}), 79.9,79.8,55.4,52.9(\mathrm{~d}$, $J=23.3 \mathrm{~Hz}$ ), $52.5(\mathrm{~d}, J=22.3 \mathrm{~Hz}), 46.8,46.6,43.3(\mathrm{~d}, J=19.4 \mathrm{~Hz}), 42.5(\mathrm{~d}, J=19.1 \mathrm{~Hz}), 37.5(\mathrm{~d}, J=6.8$ $\mathrm{Hz}), 37.2(\mathrm{~d}, J=6.5 \mathrm{~Hz}), 28.4$;

${ }^{19} \mathrm{~F}$ NMR $\left(376 \mathrm{MHz}, \mathrm{CDCl}_{3}\right): \delta=-176.1,-177.5,-193.4,-193.5$;

IR (film): $\tilde{v}=3339$ (w), 2974 (w), 1695 (s), 1606 (s), 1505 (s), 1411 (s), 1255 (s), 1175 (s), 845 (m);

HRMS (ESI) calcd. for $\mathrm{C}_{18} \mathrm{H}_{25} \mathrm{FN}_{2} \mathrm{NaO}_{4}^{+}[\mathrm{M}+\mathrm{Na}]^{+} 375.1691$; Found 375.1698.

\section{4-Methoxy-N-(3,3,3-trifluoro-1-hydroxypropyl)benzamide (5d).}<smiles>COc1ccc(C(=O)NC(O)CC(F)(F)F)cc1</smiles>

Following GP D, N-(2,2-difluorocyclopropyl)-4-methoxybenzamide (4d) $(22.7 \mathrm{mg}, 0.100 \mathrm{mmol}, 1.0$ equiv.) was used as starting material and the reaction was kept stirring under irradiation for 6 hours. 4-methoxy- $N$-(3,3,3-trifluoro-1-hydroxypropyl)benzamide (5d) $(18.0 \mathrm{mg}, 0.0684 \mathrm{mmol}, 68 \%$ ) was obtained as a white solid after purification by column chromatography on silica using 1:1 pentanes:ethyl acetate as eluent.

$\mathbf{R}_{\mathbf{f}}: 0.26$ (silica, pentanes:ethyl acetate 3:2);

Mp: $104-106^{\circ} \mathrm{C}$;

${ }^{1} \mathrm{H}$ NMR $\left(400 \mathrm{MHz}\right.$, Acetone- $\left.d_{6}\right): \delta=8.20(\mathrm{~d}, J=8.3 \mathrm{~Hz}, 1 \mathrm{H}, \mathrm{NH}), 7.91-7.83(\mathrm{~m}, 2 \mathrm{H}, \mathrm{ArH}), 7.02-6.95$ $(\mathrm{m}, 2 \mathrm{H}, \mathrm{ArH}), 5.93(\mathrm{dtd}, \mathrm{J}=8.4,6.2,4.6 \mathrm{~Hz}, 1 \mathrm{H}, \mathrm{CH}), 5.33(\mathrm{~d}, \mathrm{~J}=4.7 \mathrm{~Hz}, 1 \mathrm{H}, \mathrm{OH}), 3.85\left(\mathrm{~s}, 3 \mathrm{H}, \mathrm{OCH}_{3}\right), 2.73$ (qdd, $J=10.9,6.3,1.5 \mathrm{~Hz}, 2 \mathrm{H}, \mathrm{CH}_{2}$ );

${ }^{13} \mathrm{C}$ NMR (101 MHz, Acetone- $\left.d_{6}\right): \delta=166.6,163.4,130.0,127.3,126.8$ (q, $\left.J=277.1 \mathrm{~Hz}\right), 114.4,70.1$ (q, $J=4.4 \mathrm{~Hz}), 55.8,40.5(\mathrm{q}, J=27.1 \mathrm{~Hz})$;

${ }^{19} \mathrm{~F}$ NMR (376 MHz, Acetone- $\left.d_{6}\right): \delta=-64.2$;

IR (film): $\tilde{v}=3304$ (br, s), 1642 (s), 1607 (s), 1505 (s), 1254 (s), 1140 (s), 1029 (m), 843 (m);

HRMS (ESI) calcd. for $\mathrm{C}_{11} \mathrm{H}_{12} \mathrm{~F}_{3} \mathrm{NNaO}_{3}{ }^{+}[\mathrm{M}+\mathrm{Na}]^{+}$286.0661; Found 286.0653 .

$N$-(4-Fluorobutyl)-4-methoxybenzamide (5ea).<smiles>COc1ccc(C(=O)NCCCCF)cc1</smiles> 
Following GP D, N-cyclobutyl-4-methoxybenzamide (4e) (20.5 mg, $0.100 \mathrm{mmol}, 1.0$ equiv.) was used as starting material and the reaction was kept stirring under irradiation for 45 minutes. Then $\mathrm{NaBH}_{3} \mathrm{CN}$ ( $7.6 \mathrm{mg}, 0.12 \mathrm{mmol}, 1.2$ equiv.) was added as nucleophile. After the reaction mixture was stirred for 3 hours, $\mathrm{N}$-(4-fluorobutyl)-4-methoxybenzamide (5ea) $(14.3 \mathrm{mg}, 0.0636 \mathrm{mmol}, 64 \%)$ was obtained as a colorless oil after purification by column chromatography on silica using 1:1 pentanes:ethyl acetate as eluent.

$\mathbf{R}_{\mathbf{f}}: 0.28$ (silica, pentanes:ethyl acetate 1:1);

${ }^{1} \mathrm{H}$ NMR $\left(400 \mathrm{MHz}, \mathrm{CDCl}_{3}\right): \delta=7.79-7.65(\mathrm{~m}, 2 \mathrm{H}, \mathrm{ArH}), 6.98-6.83(\mathrm{~m}, 2 \mathrm{H}, \mathrm{ArH}), 6.20(\mathrm{~s}, 1 \mathrm{H}, \mathrm{NH}), 4.55$ $\left.\left(\mathrm{t}, J=5.7 \mathrm{~Hz}, 1 \mathrm{H}, \mathrm{FCH}_{2}\right), 4.43(\mathrm{td}, J=5.6,4.7,2.5 \mathrm{~Hz}, 1 \mathrm{H}, \mathrm{FCH})_{2}\right), 3.84\left(\mathrm{~s}, 3 \mathrm{H}, \mathrm{OCH}_{3}\right), 3.49(\mathrm{q}, J=6.7 \mathrm{~Hz}$, $\left.2 \mathrm{H}, \mathrm{NCH}_{2}\right), 1.85-1.72\left(\mathrm{~m}, 4 \mathrm{H}, \mathrm{CH}_{2}\right)$;

${ }^{13} \mathrm{C}$ NMR $\left(101 \mathrm{MHz}, \mathrm{CDCl}_{3}\right): \delta$ 167.1, 162.1, 128.6, 126.8, 113.7, 83.8 (d, $\left.J=164.4 \mathrm{~Hz}\right), 55.4,39.4,27.8$ (d, $J=19.9 \mathrm{~Hz}), 25.8(\mathrm{~d}, J=4.3 \mathrm{~Hz})$;

${ }^{19} \mathrm{~F}$ NMR $\left(376 \mathrm{MHz}, \mathrm{CDCl}_{3}\right): \delta=-218.2$;

IR (film): $\tilde{v}=3317$ (w), 2962 (w), 1632 (s), 1606 (s), 1504 (m), 1254 (s), 1179 (m), 1030 (m), 845 (m);

HRMS (ESI) calcd. for $\mathrm{C}_{12} \mathrm{H}_{17} \mathrm{FNO}_{2}{ }^{+}[\mathrm{M}+\mathrm{H}]^{+}$226.1238; Found 226.1232.

\section{$N$-(4-Fluoro-1-(1H-indol-3-yl)butyl)-4-methoxybenzamide (5eb).}<smiles>COc1ccc(C(=O)NC(CCCF)c2c[nH]c3ccccc23)cc1</smiles>

Following GP D, N-cyclobutyl-4-methoxybenzamide (4e) (20.5 mg, $0.100 \mathrm{mmol}, 1.0$ equiv.) was used as starting material and the reaction was kept stirring under irradiation for 45 minutes. Then $1 \mathrm{H}$-indole (14.0 mg, $0.120 \mathrm{mmol}, 1.2$ equiv.) was added as nucleophile. After the reaction mixture was stirred for 3 hours, $\mathrm{N}$-(4-fluoro-1-(1H-indol-3-yl)butyl)-4-methoxybenzamide (5eb) $(20.1 \mathrm{mg}, 0.0591 \mathrm{mmol}, 59 \%)$ was obtained as a orange oil after purification by column chromatography on silica using 1:1 pentanes:ethyl acetate as eluent.

$\mathbf{R}_{\mathbf{f}}: 0.43$ (silica, pentanes:ethyl acetate $1: 1$ );

${ }^{1}$ H NMR $\left(400 \mathrm{MHz}, \mathrm{CDCl}_{3}\right): \delta=8.39(\mathrm{~s}, 1 \mathrm{H}$, indole NH), 7.73- $7.65(\mathrm{~m}, 3 \mathrm{H}, \mathrm{ArH}), 7.38(\mathrm{dt}, J=8.2,0.9 \mathrm{~Hz}$, $1 \mathrm{H}, \operatorname{ArH}), 7.21$ (ddd, $J=8.3,7.1,1.2 \mathrm{~Hz}, 1 \mathrm{H}, \operatorname{ArH}), 7.17-7.14(\mathrm{~m}, 1 \mathrm{H}, \mathrm{ArH}), 7.11$ (ddd, $J=8.0,7.0,1.1$ $\mathrm{Hz}, 1 \mathrm{H}, \operatorname{ArH}), 6.90-6.83(\mathrm{~m}, 2 \mathrm{H}, \mathrm{ArH}), 6.31(\mathrm{~d}, J=8.2 \mathrm{~Hz}, 1 \mathrm{H}, \mathrm{NH}), 5.56(\mathrm{q}, J=7.6 \mathrm{~Hz}, 1 \mathrm{H}, \mathrm{CH}), 4.62-$ $4.38\left(\mathrm{~m}, 2 \mathrm{H}, \mathrm{FCH}_{2}\right), 3.81\left(\mathrm{~s}, 3 \mathrm{H}, \mathrm{OCH}_{3}\right), 2.23$ (dddd, $\left.\mathrm{J}=15.3,14.2,8.6,4.4 \mathrm{~Hz}, 2 \mathrm{H}, \mathrm{CH}_{2}\right), 1.93-1.77(\mathrm{~m}$, $\left.2 \mathrm{H}, \mathrm{CH}_{2} \mathrm{CH}_{2} \mathrm{~F}\right)$;

${ }^{13} \mathrm{C} \mathrm{NMR}\left(101 \mathrm{MHz}, \mathrm{CDCl}_{3}\right): \delta=166.4,162.1,136.6,128.7,126.7,126.0,122.5,121.8,119.9,119.2$, 116.5, 113.7, 111.4, 83.9 (d, $J=164.6 \mathrm{~Hz}$ ), 55.4, 46.4, 30.7 (d, $J=4.2 \mathrm{~Hz}), 27.6$ (d, $J=19.7 \mathrm{~Hz}$ );

${ }^{19} \mathrm{~F}$ NMR $\left(376 \mathrm{MHz}, \mathrm{CDCl}_{3}\right): \delta=-218.4$;

IR (film): $\tilde{v}$ = 3413 (w), 3273 (w), 2960 (w), 1630 (s), 1606 (s), 1498 (s), 1253 (s), 1178 (m), 1030 (m), $745(\mathrm{~m})$;

HRMS (ESI) calcd. for $\mathrm{C}_{20} \mathrm{H}_{21} \mathrm{FN}_{2} \mathrm{NaO}_{2}{ }^{+}[\mathrm{M}+\mathrm{Na}]^{+}$363.1479; Found 363.1477.

N-(4-Fluoro-1-(1H-pyrazol-1-yl)butyl)-4-methoxybenzamide (5ec). 
<smiles>COc1ccc(C(=O)NC(CCCF)n2cccn2)cc1</smiles>

Following GP D, N-cyclobutyl-4-methoxybenzamide (4e) $(20.5 \mathrm{mg}, 0.100 \mathrm{mmol}, 1.0$ equiv.) was used as starting material and the reaction was kept stirring under irradiation for 45 minutes. Then $1 \mathrm{H}$ pyrazole $(8.2 \mathrm{mg}, 0.12 \mathrm{mmol}, 1.2$ equiv.) was added as nucleophile. After the reaction mixture was stirred for 16 hours, $\mathrm{N}$-(4-fluoro-1-(1H-pyrazol-1-yl)butyl)-4-methoxybenzamide (5ec) (16.2 mg, 0.0556 $\mathrm{mmol}, 56 \%$ ) was obtained as a white solid after purification by column chromatography on silica using 1:1 pentanes:ethyl acetate as eluent.

$\mathbf{R}_{\mathbf{f}}: 0.36$ (silica, pentanes:ethyl acetate 1:1);

Mp: $157-158^{\circ} \mathrm{C}$;

${ }^{1} \mathrm{H}$ NMR $\left(400 \mathrm{MHz}\right.$, Acetone- $\left.d_{6}\right): \delta=8.37(\mathrm{~d}, J=8.7 \mathrm{~Hz}, 1 \mathrm{H}, \mathrm{NH}), 7.96-7.84(\mathrm{~m}, 2 \mathrm{H}, \operatorname{ArH}), 7.81(\mathrm{dd}, J=$ 2.3, 0.7 Hz, 1H, ArH), $7.45(\mathrm{~d}, J=1.7 \mathrm{~Hz}, 1 \mathrm{H}, \operatorname{ArH}), 7.01-6.91(\mathrm{~m}, 2 \mathrm{H}, \operatorname{ArH}), 6.37(\mathrm{q}, J=7.9 \mathrm{~Hz}, 1 \mathrm{H}, \mathrm{CH})$, $6.20(\mathrm{dd}, J=2.3,1.7 \mathrm{~Hz}, 1 \mathrm{H}, \mathrm{ArH}), 4.47(\mathrm{dtd}, J=47.4,6.0,0.9 \mathrm{~Hz}, 2 \mathrm{H}, \mathrm{FCH} 2), 3.84\left(\mathrm{~s}, 3 \mathrm{H}, \mathrm{OCH}_{3}\right), 2.46-$ $2.19\left(\mathrm{~m}, 2 \mathrm{H}, \mathrm{CH}_{2}\right), 1.87-1.53\left(\mathrm{~m}, 2 \mathrm{H}, \mathrm{CH}_{2}\right)$;

${ }^{13} \mathrm{C}$ NMR $\left(101 \mathrm{MHz}\right.$, Acetone- $\left.d_{6}\right): \delta=166.7,163.4,139.9,130.2,130.1,126.9,114.3,105.3,84.0$ (d, $J=$ $163.6 \mathrm{~Hz}$ ), 66.2, 55.8, 31.0 (d, J = 5.2 Hz), 27.3 (d, J = 20.0 Hz);

${ }^{19} \mathrm{~F}$ NMR $\left(376 \mathrm{MHz}\right.$, Acetone- $\left.d_{6}\right): \delta=-219.5$;

IR (film): $\tilde{v}=3302$ (w), 2965 (w), 1644 (m), 1606 (s), 1503 (s), 1254 (s), 1176 (m), 1031 (m), 762 (m);

HRMS (ESI) calcd. for $\mathrm{C}_{15} \mathrm{H}_{18} \mathrm{FN}_{3} \mathrm{NaO}_{2}{ }^{+}[\mathrm{M}+\mathrm{Na}]^{+} 314.1275$; Found 314.1270.

\section{$\mathrm{N}$-(4-Fluoro-3-phenylbutyl)-4-methoxybenzamide (5f).}<smiles>COc1ccc(C(=O)NCCC(CF)c2ccccc2)cc1</smiles>

Following GP D, 4-methoxy- $N$-(3-phenylcyclobutyl)benzamide (6f) (28.1 mg, $0.100 \mathrm{mmol}, 1.0$ equiv.) was used as starting material and the reaction was kept stirring under irradiation for 2 hours. Then $\mathrm{NaBH}_{3} \mathrm{CN}$ (7.6 mg, $0.12 \mathrm{mmol}, 1.2$ equiv.) was added as nucleophile. After the reaction mixture was stirred for 3 hours, $\mathrm{N}$-(4-fluoro-3-phenylbutyl)-4-methoxybenzamide (5f) $(19.0 \mathrm{mg}, 0.0631 \mathrm{mmol}, 63 \%)$ was obtained as a colorless oil after purification by column chromatography on silica using 1:1 pentanes:ethyl acetate as eluent.

$\mathbf{R}_{\mathbf{f}}: 0.48$ (silica, pentanes:ethyl acetate 1:1);

${ }^{1} \mathrm{H}$ NMR $\left(400 \mathrm{MHz}, \mathrm{CDCl}_{3}\right): \delta=7.60-7.52(\mathrm{~m}, 2 \mathrm{H}, \mathrm{ArH}), 7.38-7.32(\mathrm{~m}, 2 \mathrm{H}, \mathrm{ArH}), 7.27(\mathrm{tt}, J=7.8,1.3$ $\left.\mathrm{Hz}, 3 \mathrm{H}, \mathrm{ArH}), 6.93-6.83(\mathrm{~m}, 2 \mathrm{H}, \mathrm{ArH}), 5.95(\mathrm{~s}, 1 \mathrm{H}, \mathrm{NH}), 4.66-4.40(\mathrm{~m}, 2 \mathrm{H}, \mathrm{FCH})_{2}\right), 3.83\left(\mathrm{~s}, 3 \mathrm{H}, \mathrm{OCH}_{3}\right)$, $3.55-3.31\left(\mathrm{~m}, 2 \mathrm{H}, \mathrm{NCH}_{2}\right), 3.06$ (dddd, $\left.J=24.0,10.3,6.8,5.3 \mathrm{~Hz}, 1 \mathrm{H}, \mathrm{PhCH}\right), 2.26-1.92\left(\mathrm{~m}, 2 \mathrm{H}, \mathrm{CH}_{2}\right)$; ${ }^{13} \mathrm{C}$ NMR $\left(101 \mathrm{MHz}, \mathrm{CDCl}_{3}\right): \delta=166.8,162.0,140.1(\mathrm{~d}, J=6.1 \mathrm{~Hz}), 129.0,128.5,127.9,127.3,126.7$, 113.6, $86.9(\mathrm{~d}, J=173.8 \mathrm{~Hz}), 55.4,44.9(\mathrm{~d}, J=18.7 \mathrm{~Hz}), 38.4,31.4(\mathrm{~d}, J=4.0 \mathrm{~Hz})$; 
${ }^{19} \mathrm{~F}$ NMR $\left(376 \mathrm{MHz}, \mathrm{CDCl}_{3}\right): \delta=-217.8$;

IR (film): $\tilde{v}=3315$ (m), 2960 (m), 1630 (s), 1605 (s), 1502 (s), 1253 (s), 1026 (s), 700 (m);

HRMS (ESI) calcd. for $\mathrm{C}_{18} \mathrm{H}_{21} \mathrm{FNO}_{2}{ }^{+}[\mathrm{M}+\mathrm{H}]^{+} 302.1551$; Found 302.1556 .

\section{3-Fluoro-3-phenylpropan-1-ol (7).}<smiles>OCCC(F)c1ccccc1</smiles>

Following GP B, starting from Cyclopropylbenzene $6(35.4 \mathrm{mg}, 0.300 \mathrm{mmol})$ and after stirring for 45 minutes, 3-fluoro-3-phenylpropan-1-ol $7(29.1 \mathrm{mg}, 0.189 \mathrm{mmol}, 63 \%)$ was obtained as a colorless oil after purification by column chromatography on silica using 2:1 pentanes:ethyl acetate as eluent.

$\mathbf{R}_{\mathbf{f}}: 0.44$ (silica, pentanes:ethyl acetate 2:1, $\mathrm{KMnO}_{4}$ );

${ }^{1} \mathrm{H}$ NMR $\left(400 \mathrm{MHz}, \mathrm{CDCl}_{3}\right): \delta=7.49-7.30(\mathrm{~m}, 5 \mathrm{H}, \mathrm{ArH}), 5.68(\mathrm{ddd}, J=48.1,9.1,4.0 \mathrm{~Hz}, 1 \mathrm{H}, \mathrm{FCH}), 3.94$ - $3.74\left(\mathrm{~m}, 2 \mathrm{H}, \mathrm{OCH}_{2}\right), 2.32-1.97\left(\mathrm{~m}, 2 \mathrm{H}, \mathrm{CH}_{2}\right), 1.53(\mathrm{t}, J=5.5 \mathrm{~Hz}, 1 \mathrm{H}, \mathrm{OH})$;

${ }^{13} \mathrm{C}$ NMR $\left(101 \mathrm{MHz}, \mathrm{CDCl}_{3}\right): \delta=139.9(\mathrm{~d}, J=19.6 \mathrm{~Hz}), 128.5,128.4(\mathrm{~d}, J=1.3 \mathrm{~Hz}), 125.5(\mathrm{~d}, J=6.9 \mathrm{~Hz})$, $92.4(\mathrm{~d}, J=169.3 \mathrm{~Hz}), 59.1(\mathrm{~d}, J=4.3 \mathrm{~Hz}), 39.9(\mathrm{~d}, J=23.1 \mathrm{~Hz})$;

${ }^{19} \mathrm{~F}$ NMR $\left(376 \mathrm{MHz}, \mathrm{CDCl}_{3}\right): \delta=-177.5$;

IR (film): $\tilde{v}=3339$ (br,s), 2953 (w), 2926 (w), 1454 (w), 1048 (s), 759 (m), 698 (s).

${ }^{1} \mathrm{H} /{ }^{19} \mathrm{~F}$ NMR data correspond to the reported values. ${ }^{[8]}$

\footnotetext{
8 Remli, M.; Ayi, A. I.; Condom, R.; Guedj, R. Bull. Soc. Chim. Fr. 1986, 6, 864-867.
} 


\section{Scale-up synthesis of compound 31}

\section{Procedure:}

In a 30*200 mm borosilicate glass tube, $N$-cyclopropyl-4-methoxybenzamide $1 \mathrm{a}$ ( $1.91 \mathrm{~g}, 10.0 \mathrm{mmol}$ ), selectfluor ( $3.89 \mathrm{~g}, 11.0 \mathrm{mmol}, 1.1$ equiv.) and benzophenone (182 mg, $1.00 \mathrm{mmol}, 0.10$ equiv.) were dissolved in $12.5 \mathrm{~mL}$ of $\mathrm{MeCN}-\mathrm{H}_{2} \mathrm{O}$ ( $(\mathrm{v}: \mathrm{v}$ 4:6, $0.40 \mathrm{M}$ ). The reaction mixture was degassed by three freeze-pump-thaw cycles and backfilled with $\mathrm{N}_{2}$. The mixture was then stirred at room temperature under $365 \mathrm{~nm}$ irradiation in Rayonet Reactor for 45 minutes. Then the tube was taken out from the Rayonet Reactor and a solution of $1 \mathrm{H}$-indole $(1.40 \mathrm{~g}, 12.0 \mathrm{mmol}, 1.2$ equiv.) in $7.50 \mathrm{~mL} \mathrm{MeCN}$ was added dropwise. The reaction mixture was stirred at room temperature for 3 hours. After the completion of the reaction, the crude product was washed with water $(30 \mathrm{~mL})$ and extracted with dichloromethane $(30 \mathrm{~mL} \times 3)$. Then the organic phases were combined, dried over $\mathrm{Na}_{2} \mathrm{SO}_{4}$, concentrated. After purification by column chromatography on silica using pentanes:ethyl acetate as eluent, 31 (1.86 g, $5.71 \mathrm{mmol}, 57 \%)$ was obtained as product.

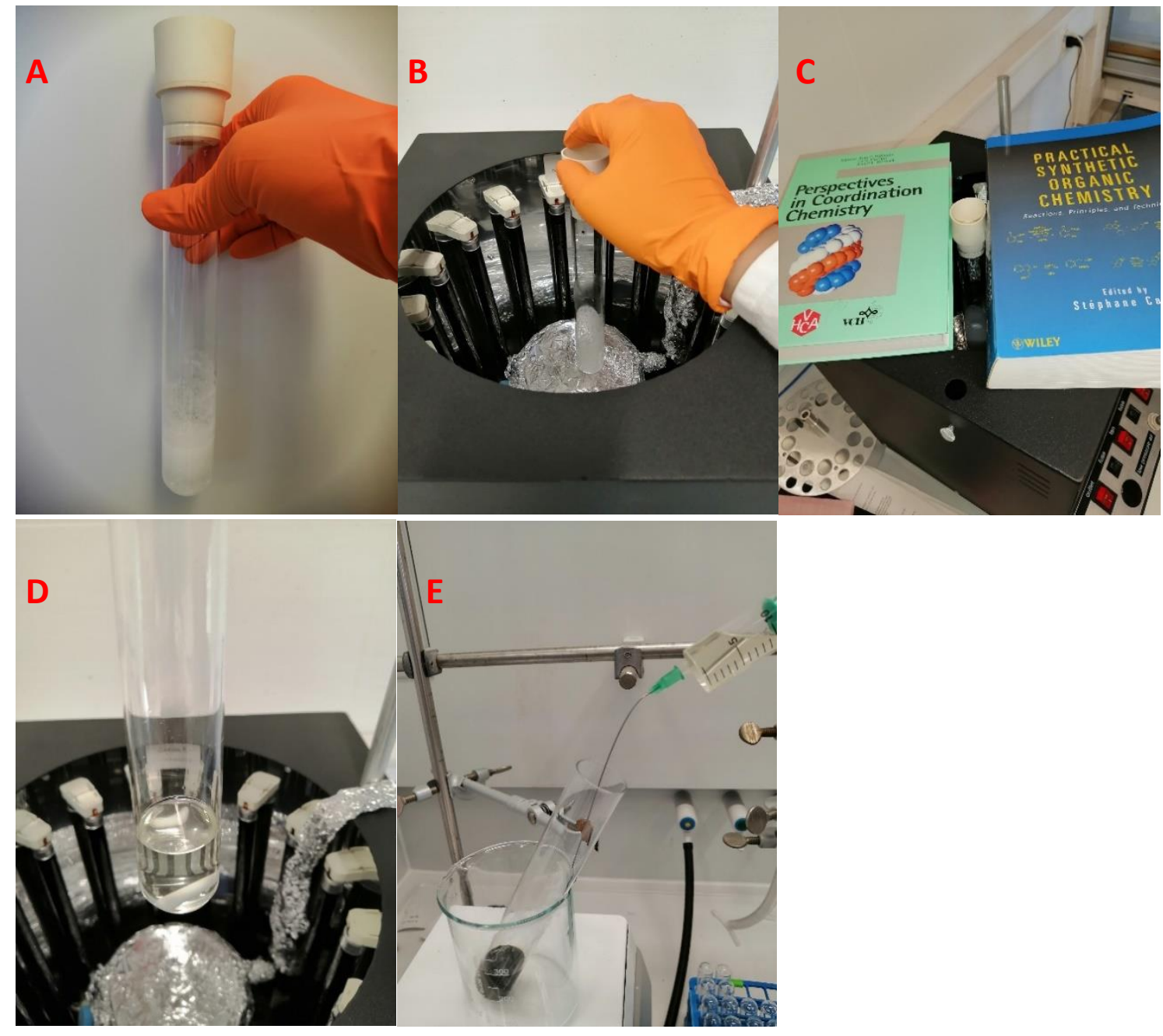
A. After freeze-pump-thaw cycles
B. Put the reaction tube into Rayonet Reactor
C. Fix the reaction tube (with books)
D. After irradiation
E. Add a solution of indole dropwise 


\section{Mechanistic studies}

\subsection{TEMPO experiment}

In a $12 * 75 \mathrm{~mm}$ borosilicate glass tube, $N$-cyclopropyl-4-methoxybenzamide (1a) $(19.1 \mathrm{mg}, 0.100 \mathrm{mmol}$, 1.0 equiv.), selectfluor ( $39.0 \mathrm{mg}, 0.110 \mathrm{mmol}, 1.1$ equiv.), benzophenone (1.8 $\mathrm{mg}, 0.010 \mathrm{mmol}, 0.10$ equiv.) and TEMPO (31.2 mg, $0.200 \mathrm{mmol}, 2.0$ equiv.) were dissolved in $0.50 \mathrm{~mL}$ of $\mathrm{MeCN}-\mathrm{H}_{2} \mathrm{O}$ (v:v 4:6, $0.20 \mathrm{M}$ ). The reaction mixture was degassed by three freeze-pump-thaw cycles and backfilled with $\mathrm{N}_{2}$. The mixture was then stirred at room temperature under $365 \mathrm{~nm}$ irradiation in Rayonet Reactor for 45 minutes. After the completion of the reaction, the crude product was directly submitted to column chromatography on silica using 3:2 pentanes:ethyl acetate as eluent and $\mathrm{N}$-(1-Hydroxy-3-((2,2,6,6tetramethylpiperidin-1-yl)oxy)propyl)-4-methoxybenzamide (8) $(4.7 \mathrm{mg}, 0.013 \mathrm{mmol}, 13 \%)$ was obtained as a white solid. $\mathrm{N}$-cyclopropyl-4-methoxybenzamide (1a) $(16.0 \mathrm{mg}, 0.084 \mathrm{mmol}, 84 \%)$ was recovered using 1:1 pentanes:ethyl acetate as eluent.

\section{$N$-(1-Hydroxy-3-((2,2,6,6-tetramethylpiperidin-1-yl)oxy)propyl)-4-methoxybenzamide (8).}<smiles>COc1ccc(C(=O)NC(O)CCON2C(C)(C)CCCC2(C)C)cc1</smiles>

$\mathbf{R}_{\mathbf{f}}: 0.39$ (silica, pentanes:ethyl acetate 1:1);

Mp: $118-121^{\circ} \mathrm{C}$;

${ }^{1} \mathrm{H}$ NMR $\left(400 \mathrm{MHz}, \mathrm{CDCl}_{3}\right): \delta=7.81-7.71(\mathrm{~m}, 2 \mathrm{H}, \mathrm{ArH}), 7.58(\mathrm{~d}, J=6.5 \mathrm{~Hz}, 1 \mathrm{H}, \mathrm{NH}), 6.95-6.86(\mathrm{~m}, 2 \mathrm{H}$, $\operatorname{ArH}), 5.65(\mathrm{q}, J=5.5 \mathrm{~Hz}, 1 \mathrm{H}, \mathrm{CH}), 4.21$ (ddd, $\left.J=9.6,8.3,3.6 \mathrm{~Hz}, 1 \mathrm{H}, \mathrm{OCH})_{2}\right), 4.12(\mathrm{~d}, J=2.8 \mathrm{~Hz}, 1 \mathrm{H}, \mathrm{OH}$ ), $3.93\left(\mathrm{ddd}, \mathrm{J}=9.8,6.1,3.7 \mathrm{~Hz}, 1 \mathrm{H}, \mathrm{OCH}_{2}\right), 3.85\left(\mathrm{~s}, 3 \mathrm{H}, \mathrm{OCH}_{3}\right), 2.10-1.95\left(\mathrm{~m}, 2 \mathrm{H}, \mathrm{OCH}_{2} \mathrm{CH}_{2}\right), 1.49-1.40$ (m, $\left.4 \mathrm{H}, \mathrm{CH}_{2}\right), 1.33-1.24\left(\mathrm{~m}, 2 \mathrm{H}, \mathrm{CH}_{2} \mathrm{CH}_{2} \mathrm{CH}_{2}\right), 1.23-0.99\left(\mathrm{~m}, 12 \mathrm{H}, \mathrm{CH}_{3}\right)$;

${ }^{13} \mathrm{C}$ NMR $\left(101 \mathrm{MHz}, \mathrm{CDCl}_{3}\right): \delta=168.2,162.5,129.0,126.0,113.7,74.2,72.6,59.8,55.4,39.7,39.6,33.5$, 33.0, 32.9, 20.5, 20.3, 17.0;

IR (film): $\tilde{v}=3341(\mathrm{~m}), 2930(\mathrm{~m}), 1643(\mathrm{~m}), 1606(\mathrm{~m}), 1503(\mathrm{~s}), 1255(\mathrm{~s}), 1177(\mathrm{~m}), 987(\mathrm{~m}), 845(\mathrm{w})$;

HRMS (ESI) calcd. for $\mathrm{C}_{20} \mathrm{H}_{33} \mathrm{~N}_{2} \mathrm{O}_{4}{ }^{+}[\mathrm{M}+\mathrm{H}]^{+}$365.2435; Found 365.2418.

\subsection{Light-dark interval experiment}

Procedure: The reaction was run similar with the standard procedure, except that fluorobenzene (28.8 $\mathrm{mg}, 0.300 \mathrm{mmol}, 1.0$ equiv.) was added as internal standard at the begining. After 10 minutes, $20 \mu \mathrm{L}$ of the reaction mixture was extracted by micro syringe under $\mathrm{N}_{2}$ flow. The aliquot was then diluted in $0.4 \mathrm{mLCDCl}_{3}$ and was then measured the substrate recovery rate (yield of product was not accounted as the hemiaminal could undergo hydrolysis slowly over time) by crude ${ }^{1} \mathrm{H}$ NMR. The reaction mixture was stirred under ambient light for 1 hour and an aliquot was prepared and analyzed by the above mentioned methods. Then the reaction mixture was kept stirring under irradiation in the Rayonet reactor for a second 10 minutes and an aliquot was prepared and analyzed. The reaction mixture was stirred under ambient light for a second hour and an aliquot was prepared and analyzed. Next the reaction mixture was kept stirring under irradiation in the Rayonet reactor for a third 10 minutes and an aliquot was prepared and analyzed. The reaction mixture was stirred under ambient light for a third hour and an aliquot was prepared and analyzed. The reaction mixture was kept stirring under irradiation in the Rayonet reactor for a forth 10 minutes and an aliquot was prepared and analyzed. 
Finally the reaction mixture was stirred under ambient light for a forth hour and an aliquot was prepared and analyzed.

Discussion: The light-dark interval experiments cannot exclude the chain process totally, but from the above figure, we can see the light is essential to the generation of product. If there is some chain propagation after stopping of irradiation, its contribution to the product should be small.

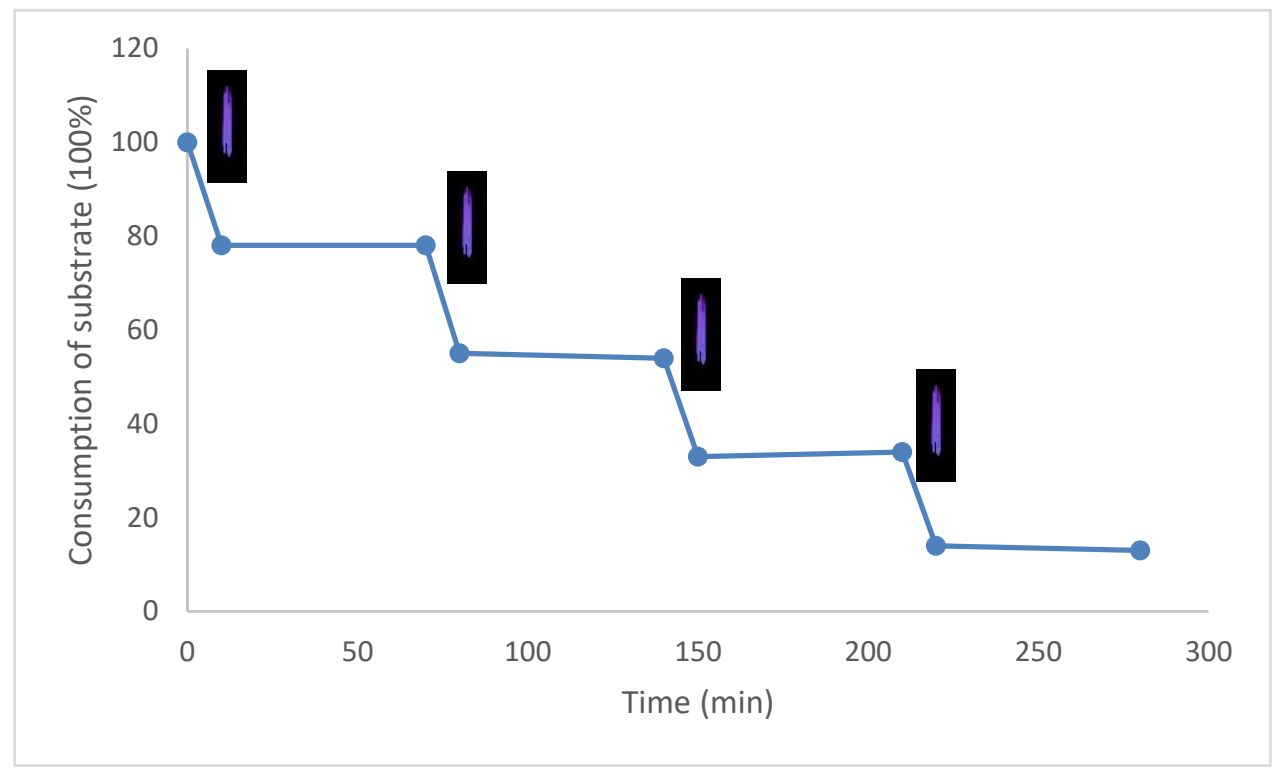

\subsection{UV-vis spectra}

The UV-vis spectra was measured by using the solution of corresponding compound in $\mathrm{MeCN}$-water (v:v 4:6).

UV-vis of substrate $1 \mathrm{a}\left(2 * 10^{-5} \mathrm{M}\right)$

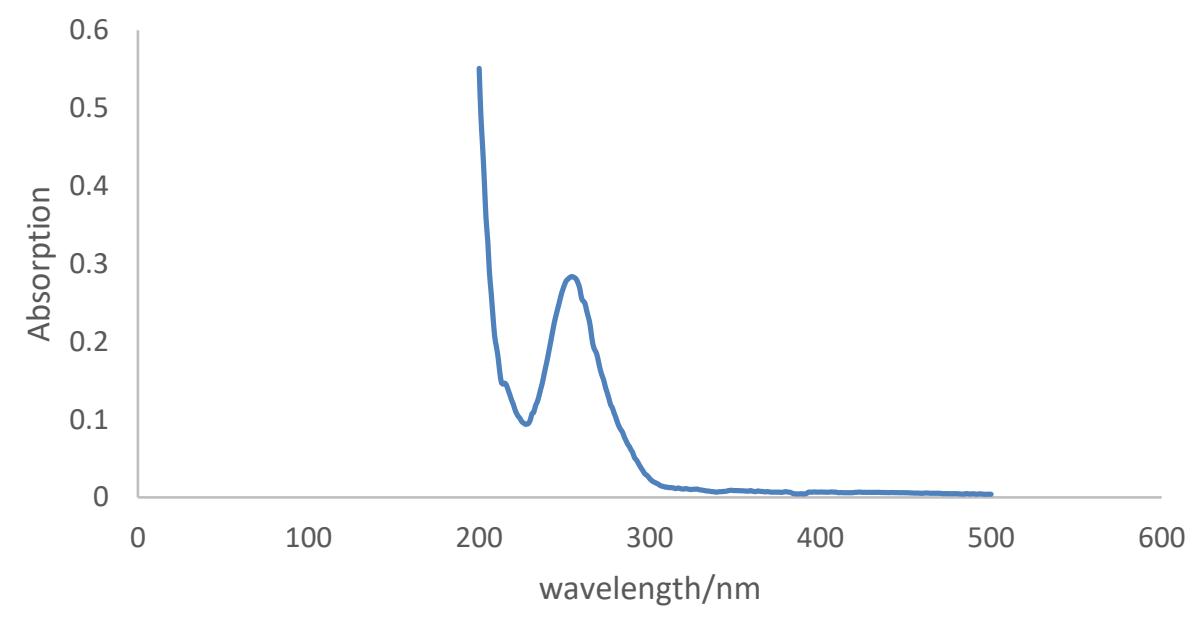




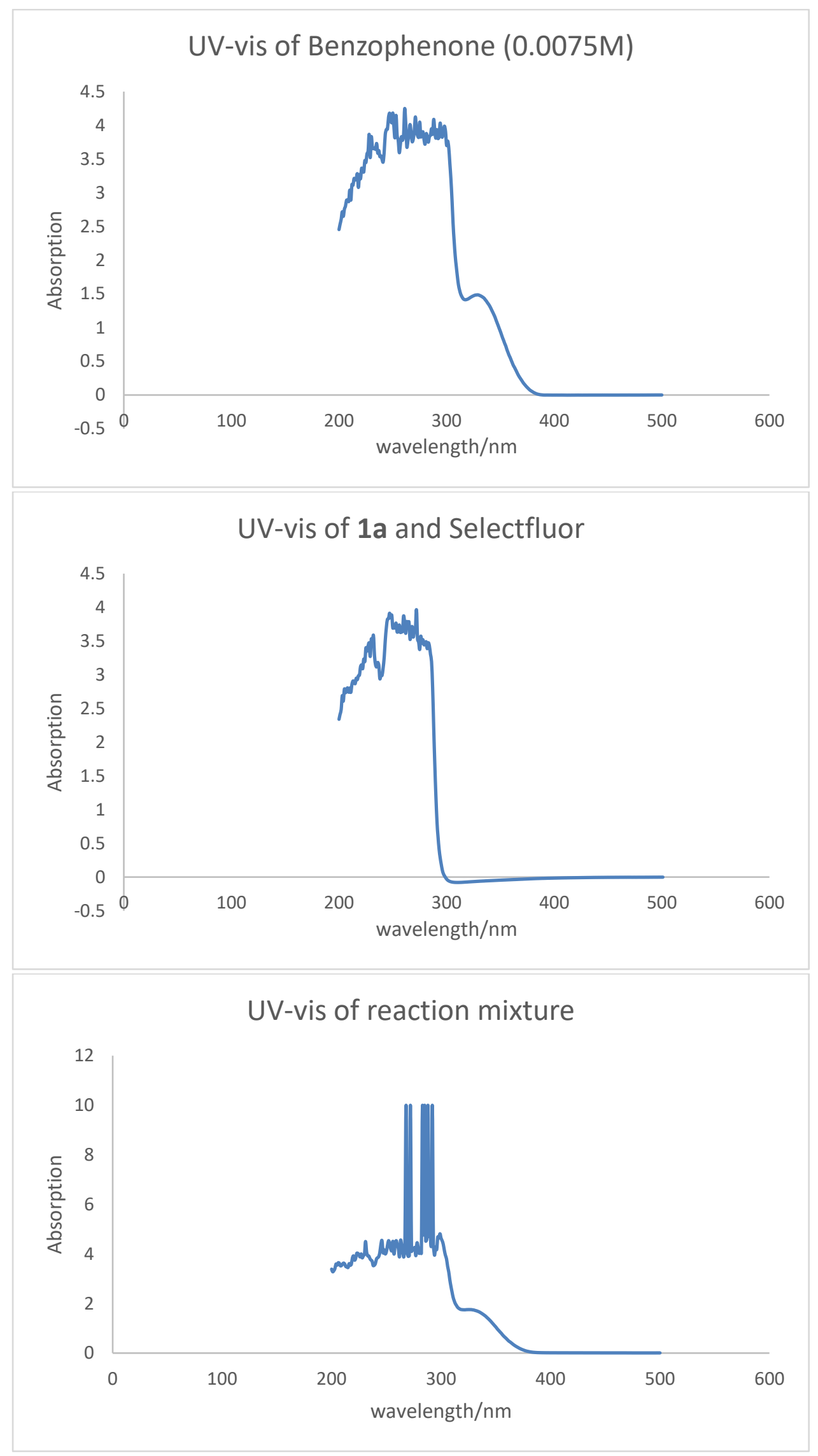




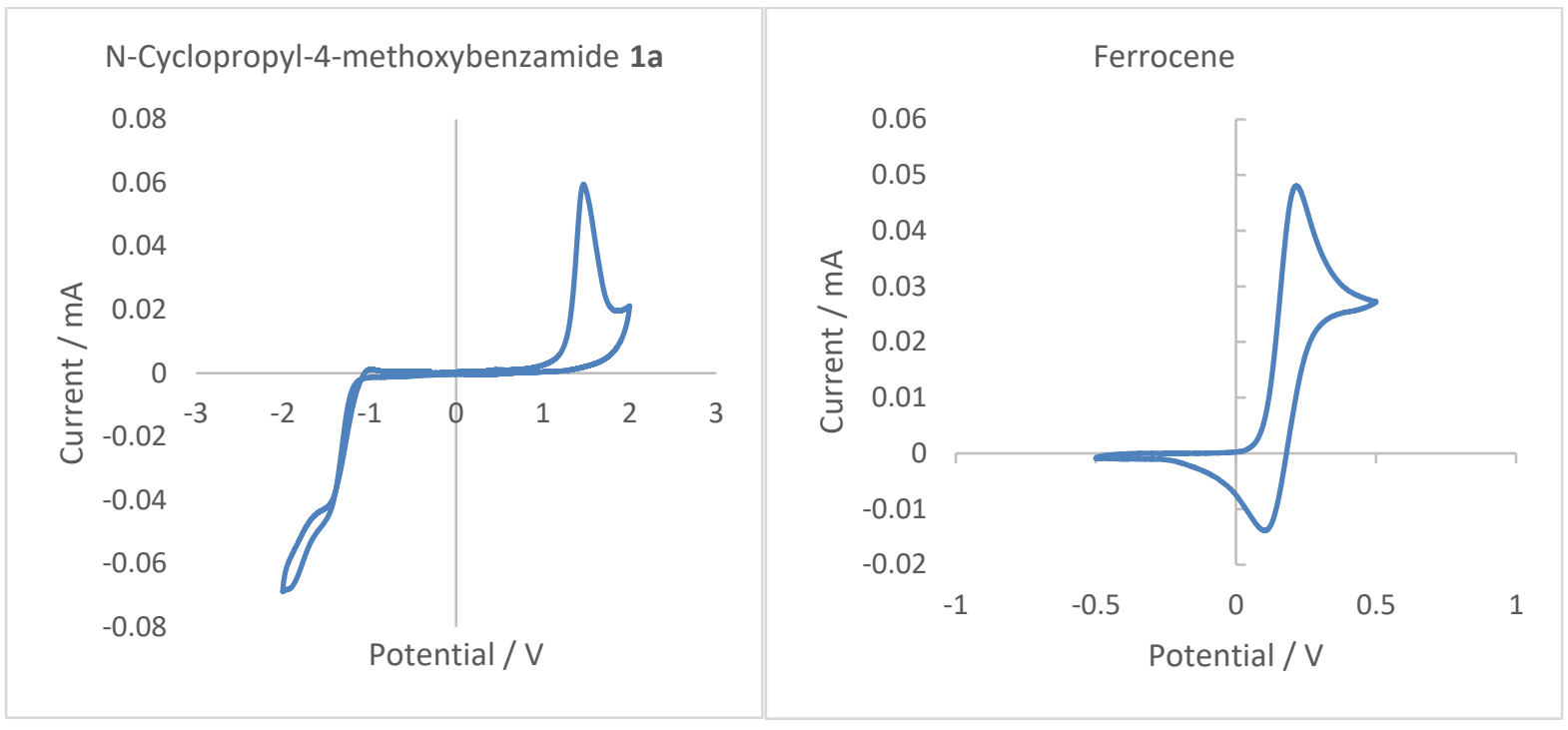

Procedure: Cyclic voltammetry was performed with a Biologic SP-150 Potentiostat, with a threeelectrode cell configuration: a glassy carbon electrode as the working electrode, Pt wire as the counter electrode and a $\mathrm{Ag} / \mathrm{Ag}^{+}$quasi-reference electrode with $0.01 \mathrm{M} \mathrm{AgBF}$ in acetonitrile. $\mathrm{Bu}_{4} \mathrm{NPF}_{6}$ was employed as the electrolyte and acetonitrile was used as solvent.

Ferrocene was used to calibrate the potential of $\mathrm{Ag} / \mathrm{Ag}^{+}$quasi-reference electrode.

Result: $E_{1 / 2}{ }^{\text {red }}(\mathbf{1 a})=+1.67 \mathrm{~V}$ vs SCE in MeCN.

\subsection{Stern-Volmer quenching experiments}

Procedure: Stern-Volmer fluorescence quenching experiments were conducted on a Varian Cary Eclipse machine. Benzophenone was recrystallized from ethanol. Selectfluor was recrystallized from acetonitrile/diethyl ether. 1a was recrystallized from ethyl acetate.

\section{For Ir photocatalyst:}

Stock solution of [Ir(dF-CF 3 ppy $\left.)_{2}(\mathrm{dtbbpy})\right] \mathrm{PF}_{6}\left(0.45 \mathrm{mg}\right.$ in volumetric flask of $100 \mathrm{~mL}$ with $\mathrm{MeCN}-\mathrm{H}_{2} \mathrm{O}$

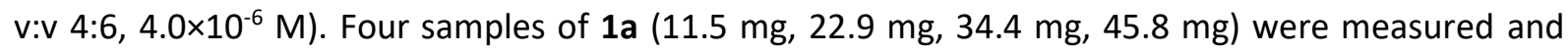
dissolved respectively with $3.0 \mathrm{~mL}$ stock solution of [Ir(dF-CF $\left.\left.\mathrm{CF}_{3} \mathrm{ppy}\right)_{2}(\mathrm{dtbbpy})\right] \mathrm{PF}_{6}$ to prepare solutions of 1a as $0.02 \mathrm{M}, 0.04 \mathrm{M}, 0.06 \mathrm{M}, 0.08 \mathrm{M}$. Similarly, four samples of selectfluor ( $21.2 \mathrm{mg}, 42.5 \mathrm{mg}, 63.7$ $\mathrm{mg}, 85.0 \mathrm{mg}$ ) were measured and dissolved respectively with $3.0 \mathrm{~mL}$ stock solution of [ $\mathrm{Ir}(\mathrm{dF}-$ $\left.\mathrm{CF}_{3} \mathrm{ppy}\right)_{2}$ (dtbbpy)]PF 6 to prepare solutions of selectfluor as $0.02 \mathrm{M}, 0.04 \mathrm{M}, 0.06 \mathrm{M}, 0.08 \mathrm{M}$.

The above solutions were transferred to quartz cuvettes and were bubbled with $\mathrm{N}_{2}$ for 2 minutes before analysis. The solution was excited at $365 \mathrm{~nm}$ and the emission intensity was recorded at 475 $\mathrm{nm}$. 


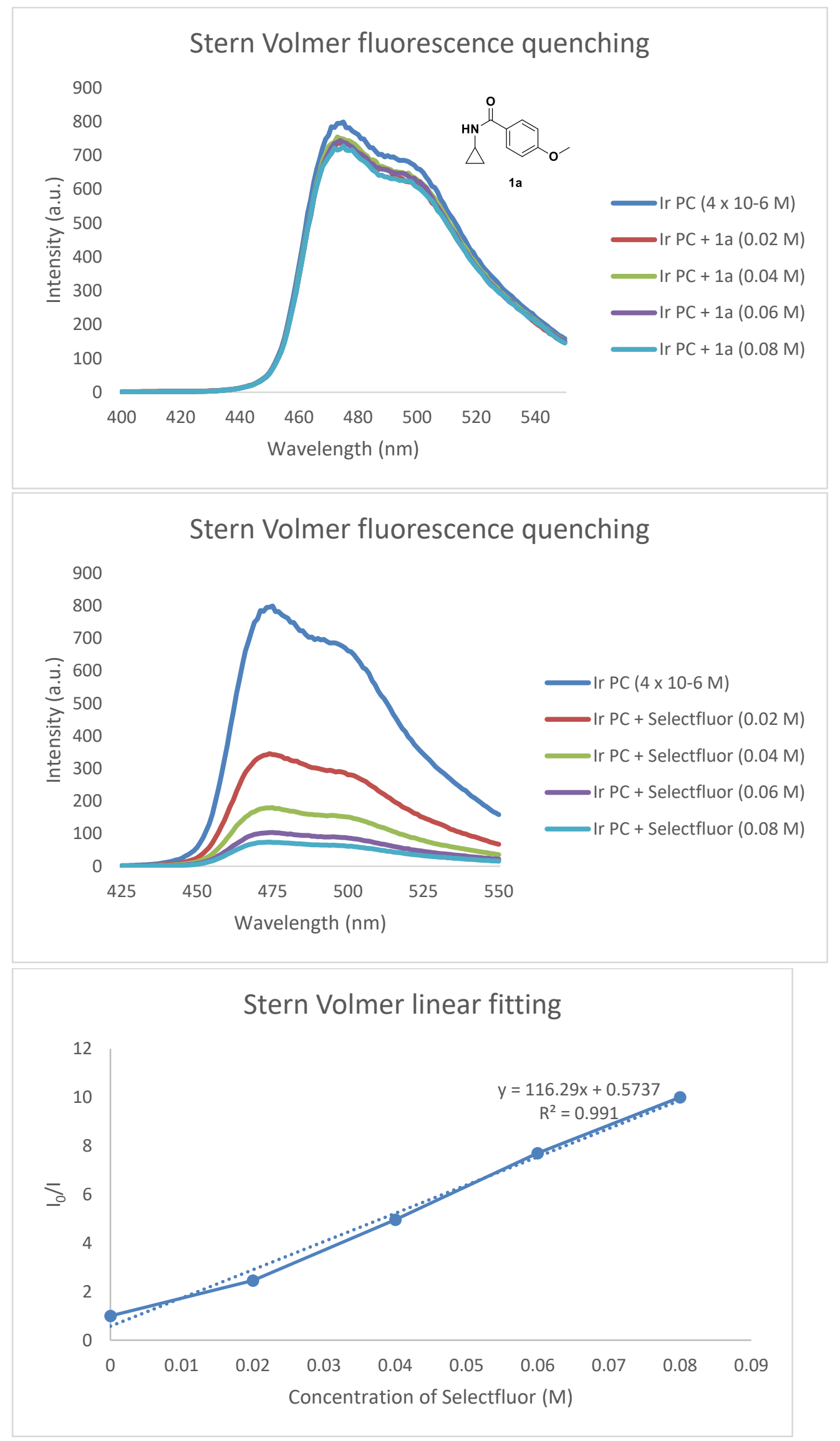




\section{For benzophenone photocatalyst:}

Stock solution of benzophenone (82.0 mg in volumetric flask of $50.0 \mathrm{~mL}$ with $\mathrm{MeCN}-\mathrm{H}_{2} \mathrm{O}$ v:v 4:6, 0.009 M). Four samples of $1 \mathrm{a}(1.33 \mathrm{mg}, 2.29 \mathrm{mg}, 3.44 \mathrm{mg}, 5.73 \mathrm{mg}$ ) were measured and dissolved respectively with $3.0 \mathrm{~mL}$ stock solution of benzophenone to prepare solutions of 1 a as $0.0023 \mathrm{M}, 0.004 \mathrm{M}, 0.006$ M, $0.01 \mathrm{M}$. Similarly, five samples of selectfluor (10.6 mg, $21.2 \mathrm{mg}, 42.5 \mathrm{mg}, 63.7 \mathrm{mg}, 85.0 \mathrm{mg}$ ) were measured and dissolved respectively with $3.0 \mathrm{~mL}$ stock solution of benzophenone to prepare solutions of selectfluor as $0.01 \mathrm{M}, 0.02 \mathrm{M}, 0.04 \mathrm{M}, 0.06 \mathrm{M}, 0.08 \mathrm{M}$.

The above solutions were transferred to quartz cuvettes and were bubbled with $\mathrm{N}_{2}$ for 2 minutes before analysis. The solution was excited at $365 \mathrm{~nm}$ and the emission intensity was recorded at 475 $\mathrm{nm}$.

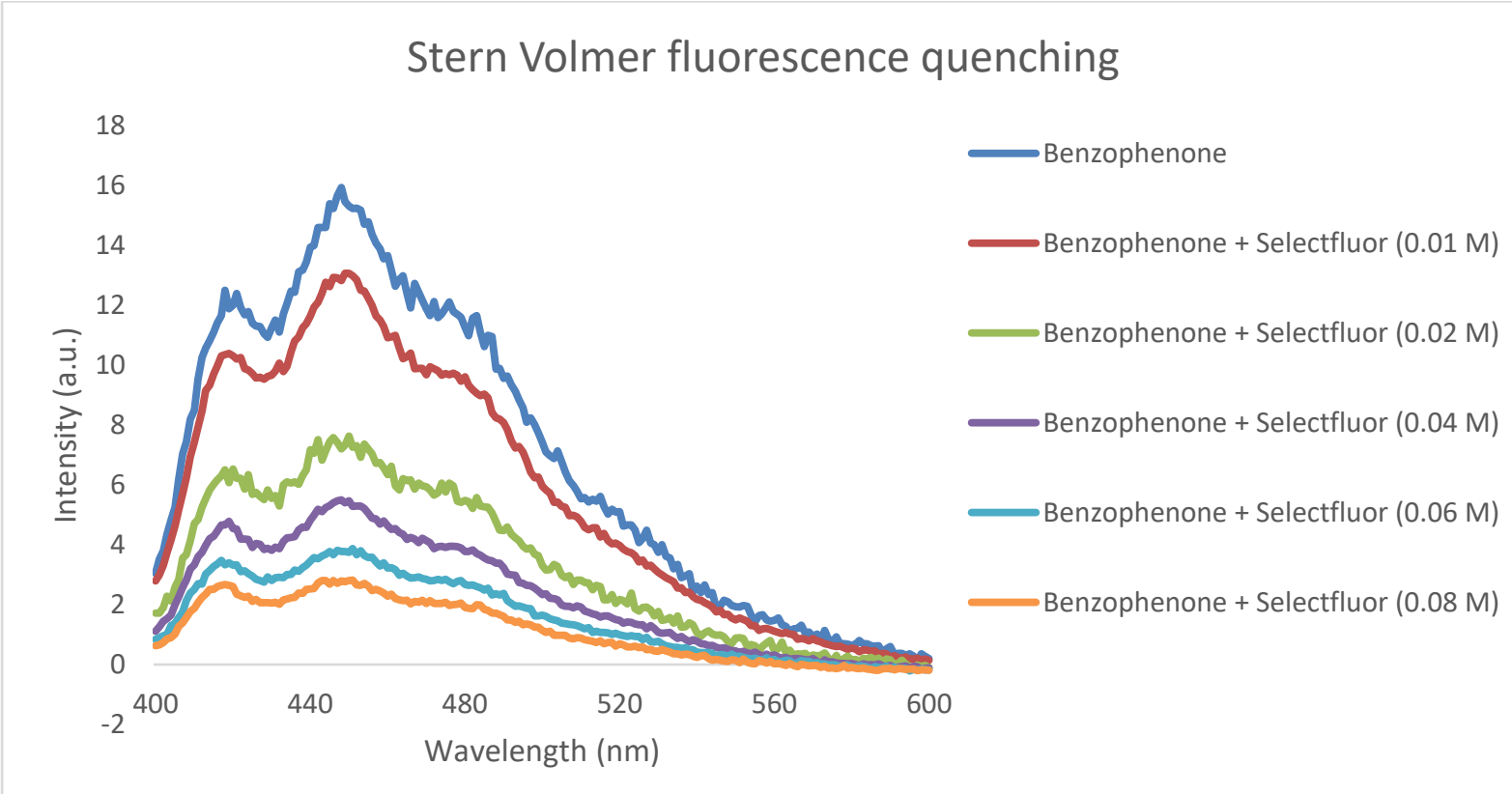

\section{Stern Volmer linear fitting}

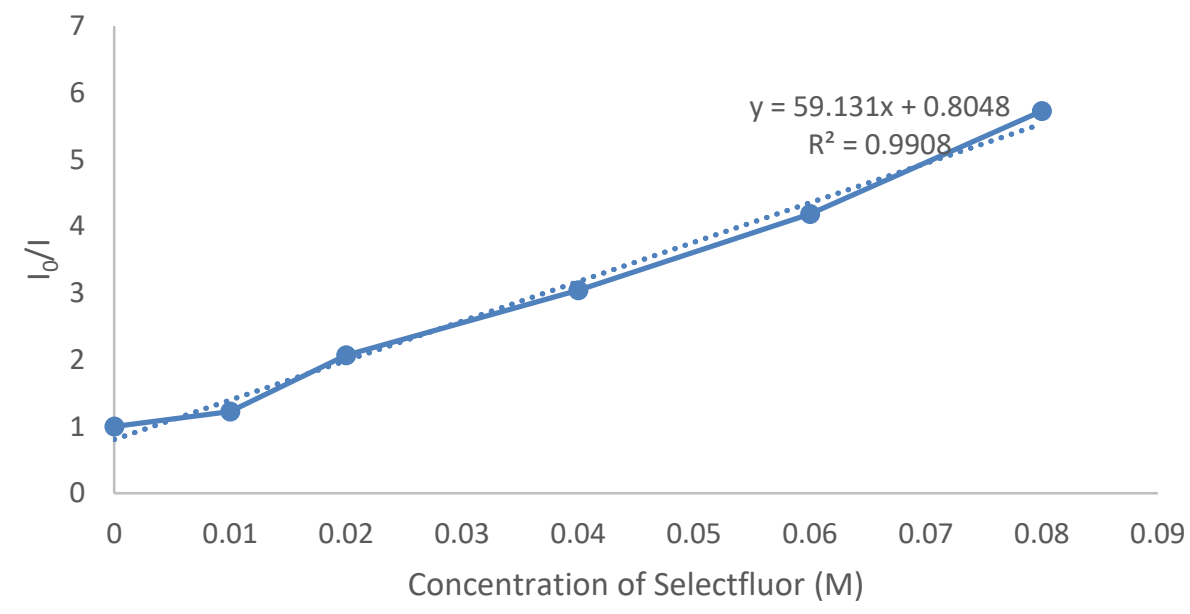




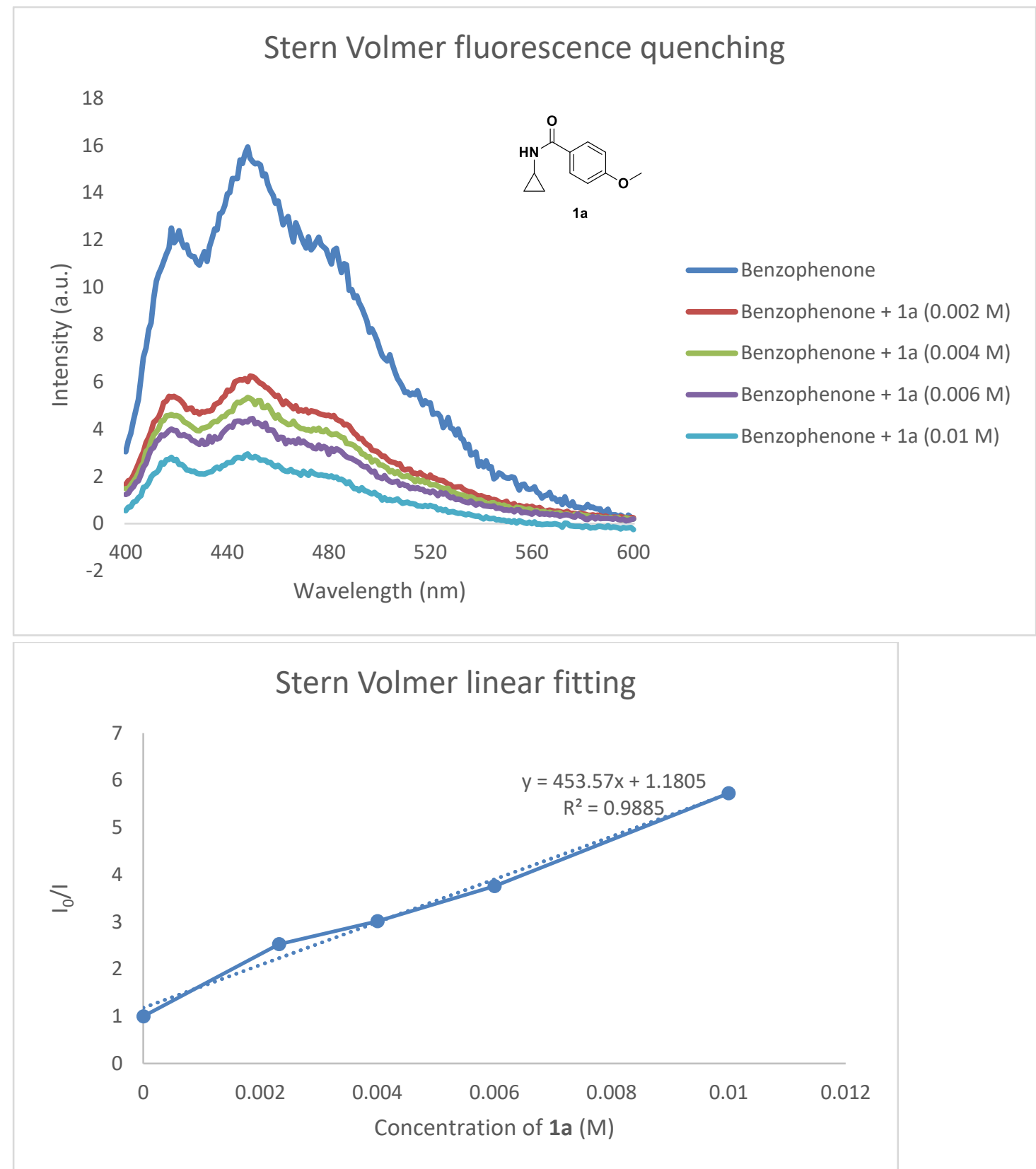

Note: It is well known that fluorescence of triplet state benzophenone can be quenched by multifunctional aromatics due to the formation of exciplexes. ${ }^{[9]}$

Therefore, we further performed Stern-Volmer quenching with substrate $\mathbf{1 g}$, results showed no obvious quenching of fluorescence of triplet state benzophenone by $\mathbf{1 g}$.

${ }^{9}$ Yamada, T. K. (1989) Photochemistry of aromatic ketones. I. Quenching of the benzophenone triplet state by multifunctional aromatics. II. Sensitized fluorescence of 9,10-dibromoanthracene and 1,3-dibromo-9,10-bis(phenylethynyl) anthracene by energy transfer from the triplet state of benzophenone and acetophenone. Doctoral dissertation. University of Southern California. 


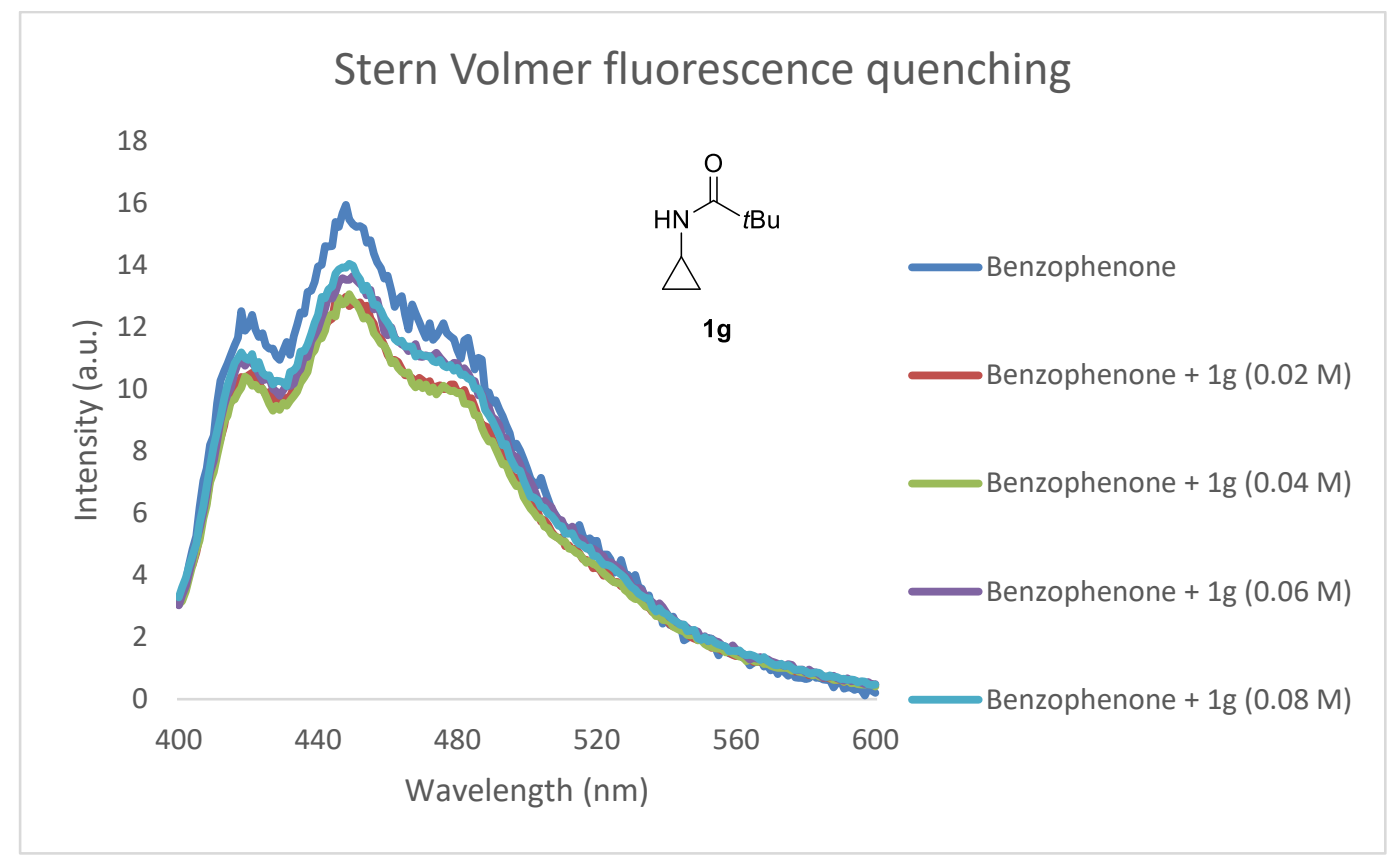

\subsection{Reaction of $N$-Cyclopropyl-4-methoxy- $N$-methylbenzamide (9)}

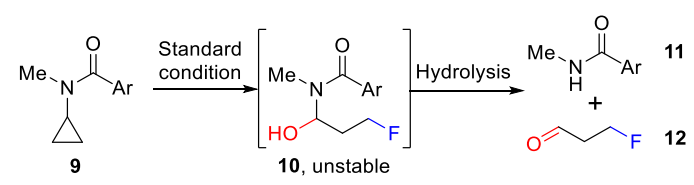

Following GP B, starting from $N$-Cyclopropyl-4-methoxy- $N$-methylbenzamide 9 ( $20.5 \mathrm{mg}, 0.100 \mathrm{mmol}$ ), 4-methoxy-N-methylbenzamide $11(12.0 \mathrm{mg}, 0.073 \mathrm{mmol}, 73 \%)$ was obtained as a white solid after purification by column chromatography on silica using 1:2 pentanes:ethyl acetate as eluent.

$\mathbf{R}_{\mathbf{f}}: 0.25$ (silica, pentanes:ethyl acetate $1: 2$ );

${ }^{1} \mathrm{H}$ NMR $\left(400 \mathrm{MHz}\right.$, Acetone- $\left.d_{6}\right): \delta=7.85(\mathrm{~d}, J=8.8 \mathrm{~Hz}, 2 \mathrm{H}, \operatorname{ArH}), 7.58(\mathrm{~s}, 1 \mathrm{H}, \mathrm{NH}), 7.12-6.79(\mathrm{~m}, 2 \mathrm{H}$, $\operatorname{ArH}), 3.84\left(\mathrm{~s}, 3 \mathrm{H}, \mathrm{OCH}_{3}\right), 2.88\left(\mathrm{~d}, J=2.9 \mathrm{~Hz}, 3 \mathrm{H}, \mathrm{NCH}_{3}\right)$.

${ }^{1} \mathrm{H}$ NMR data correspond to the reported values. ${ }^{[10]}$

3-Fluoropropanal 12 was observed from crude ${ }^{1} \mathrm{H}$ NMR and peaks correspond to the reported values. [11]

\subsection{N-fluoroamide synthesis}

Although we did not observe any $\mathrm{N}$-fluorination when mixing 1 a with Selectflluor, we still tried to synthesis $\mathrm{N}$-cyclopropyl- $\mathrm{N}$-fluoro-4-methoxybenzamide $\mathbf{1 3}$ in order to exclude the possibility of involving it as an intermediate. However, this goal was not achieved when we followed a reported protocol from Cook group. ${ }^{[12]}$ It is indicated in Cook's paper that their protocol for $\mathrm{N}$-fluorination is limited to $\mathrm{N}$-tert butyl amides since less-hindered amides undergo $\mathrm{N}$-sulfonation when treated with NFSI.

\footnotetext{
${ }^{10}$ Xia, Q.; Liu, X.; Zhang, Y.; Chen, C.; Chen, W. Org. Lett. 2013, 15, 3326-3329.

${ }^{11}$ Linclau, B.; Peron, F.; Bogdan, E.; Wells, N.; Wang, Z.; Compain, G.; Fontenelle, C.; Galland, N.; Le Questel, J.-

Y.; Graton, J. Chem. Eur. J. 2015, 21, 17808-17816.

12 Groendyke, B. J.; AbuSalim, D. I.; Cook, S. P. J. Am. Chem. Soc. 2016, 138, 12771-12774.
} 
<smiles>COc1ccc(C(=O)NC2CC2)cc1</smiles>

nBuLi, then NFSI

$1 a$
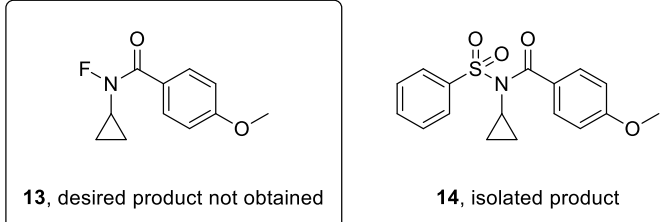

14 , isolated product

We were still curious about the stability of $\mathrm{N}$-fluoroamides under our reaction conditions. Therefore, we synthesized one of the substrates $\mathbf{1 5}$ reported reported by the Cook group by following their protocol and then test it under irradiation with stoichiometric amount of benzophenone. However, no conversion of the starting material 15 was observed based on crude ${ }^{1} \mathrm{H} /{ }^{19} \mathrm{~F} N M R$. As a result, involvement of a $\mathrm{N}$-fluoroamide intermediate can probably be ruled out.

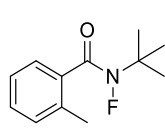

15

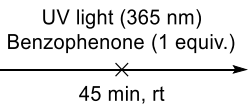

$45 \mathrm{~min}, \mathrm{rt}$

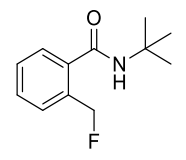

16, NR, RSM

\section{Spectra}

\section{4-Methoxy- $N$-(trans-2-methylcyclopropyl)benzamide (trans-4a) \\ ${ }^{1} \mathrm{H}-\mathrm{NMR}$ (400 MHz, CDCl 3$)$}

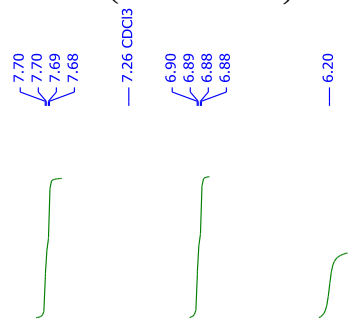<smiles>COc1ccc(C(=O)N[C@@H]2C[C@@H]2C)cc1</smiles>
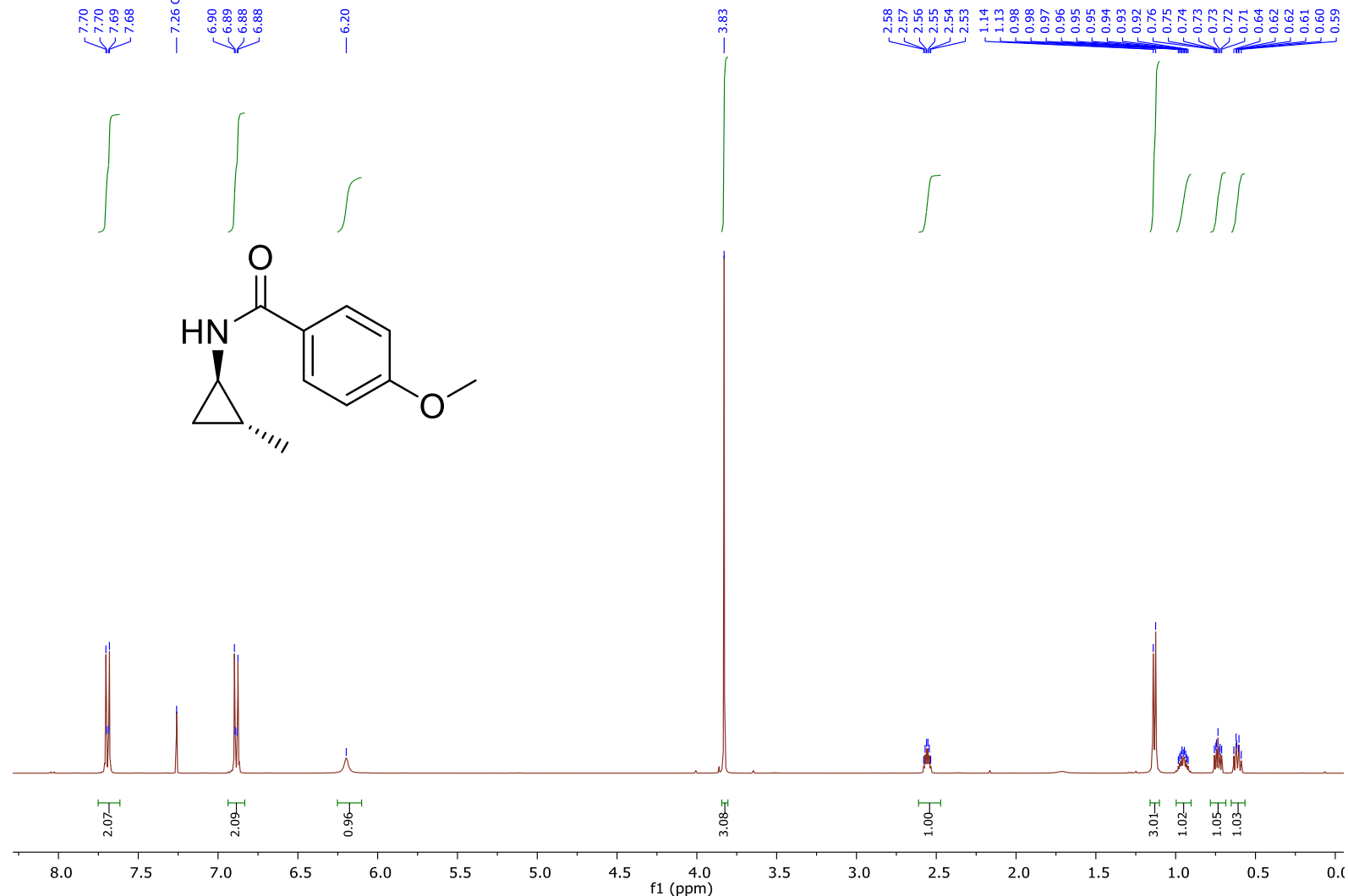

${ }^{13} \mathrm{C}-\mathrm{NMR}$ (101 MHz, $\left.\mathrm{CDCl}_{3}\right)$ 

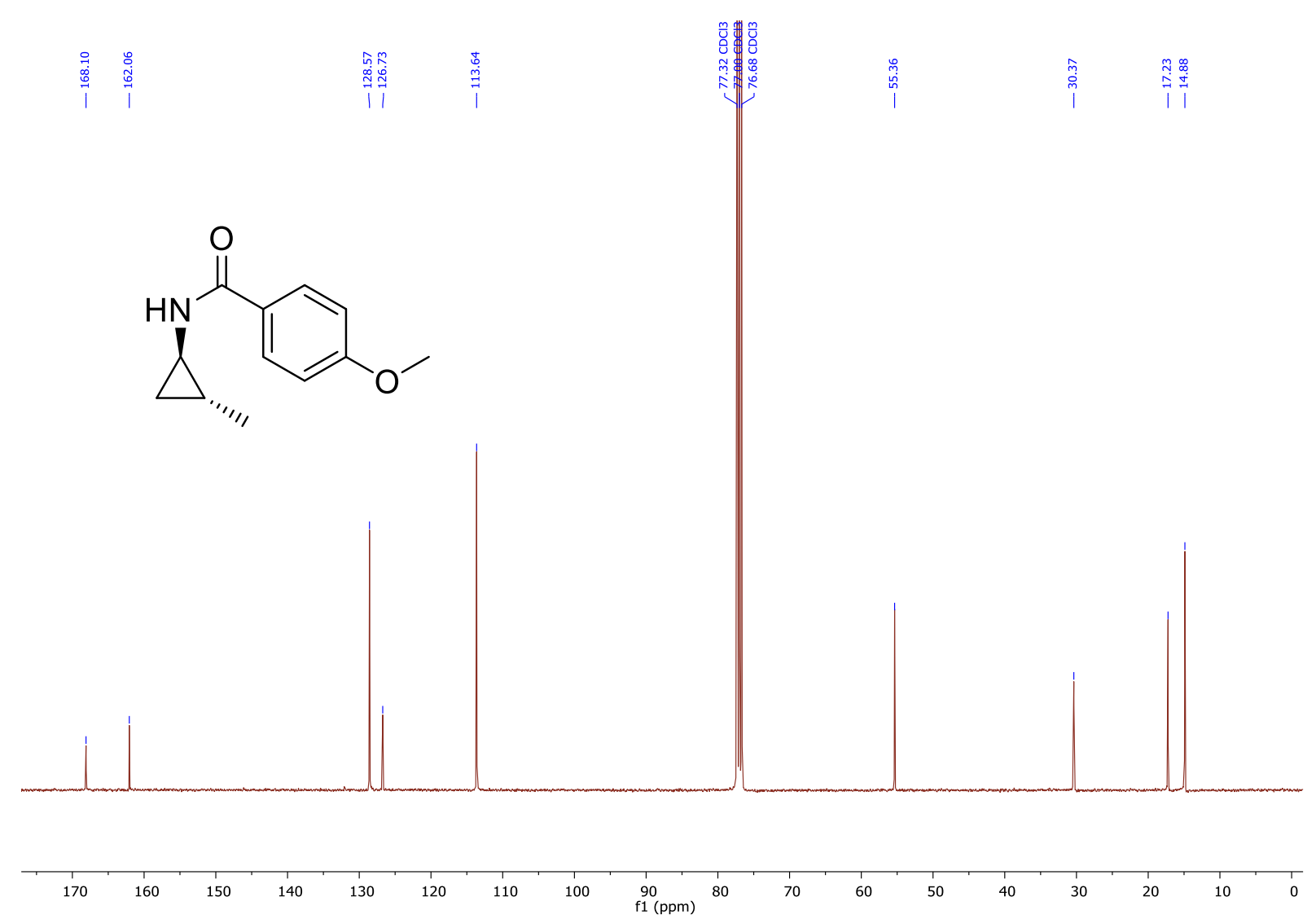

IR

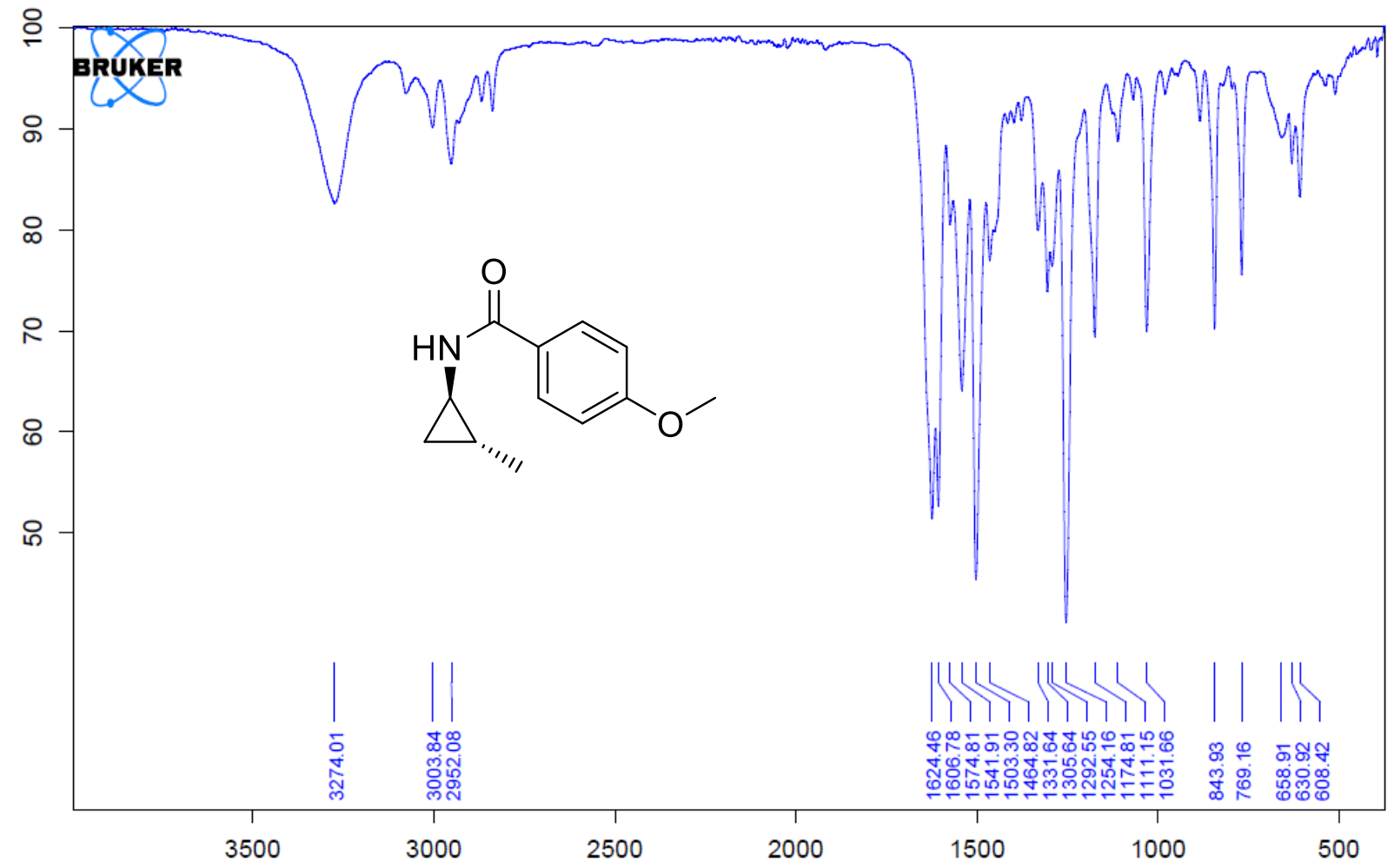

4-Methoxy- $N$-(cis-2-methylcyclopropyl)benzamide (cis-4a)

${ }^{1}$ H-NMR (400 MHz, $\left.\mathrm{CDCl}_{3}\right)$ 


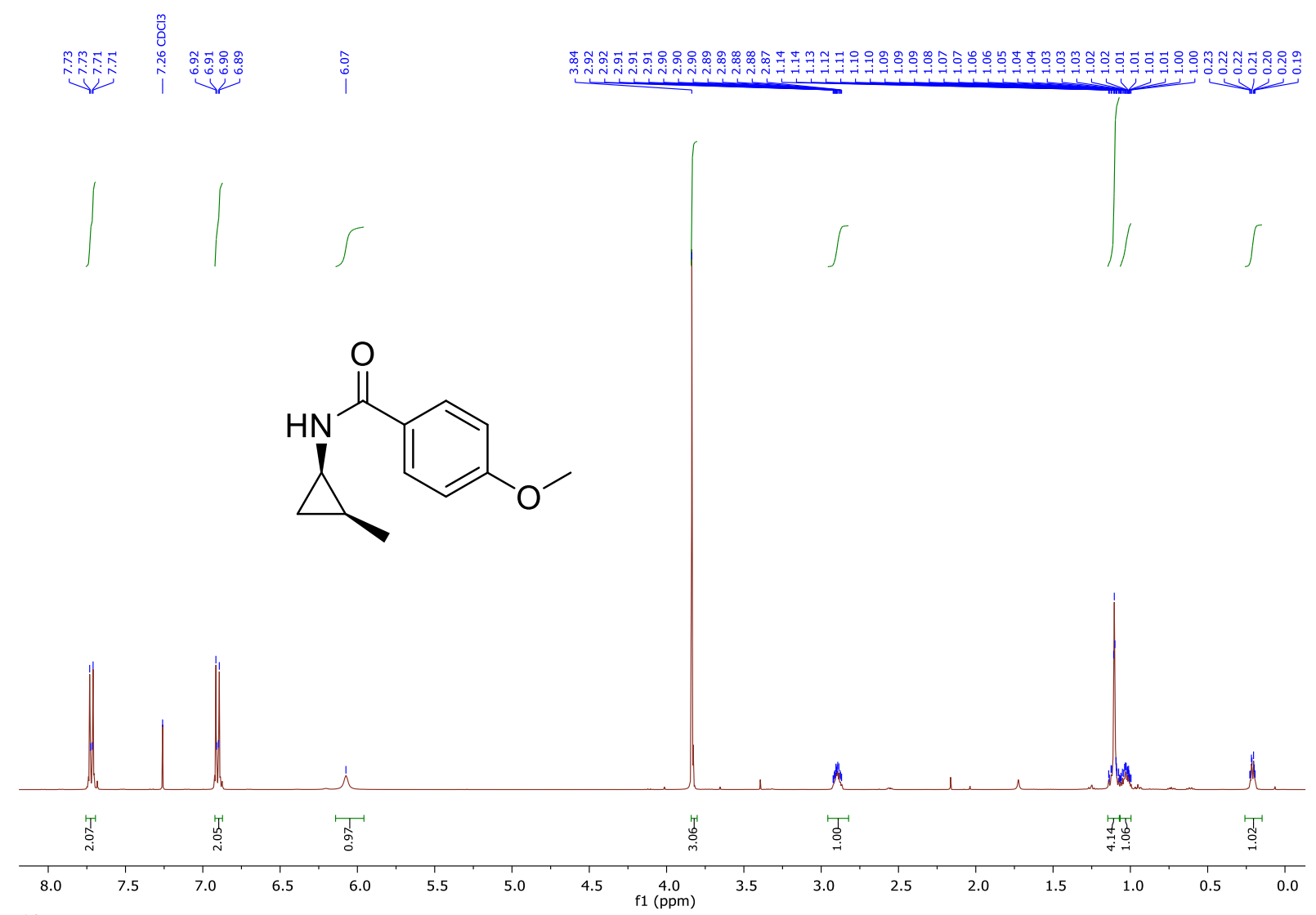

${ }^{13} \mathrm{C}$-NMR (101 MHz, CDCl$)$

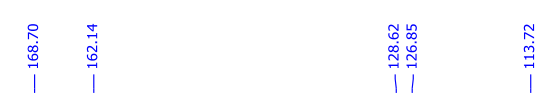
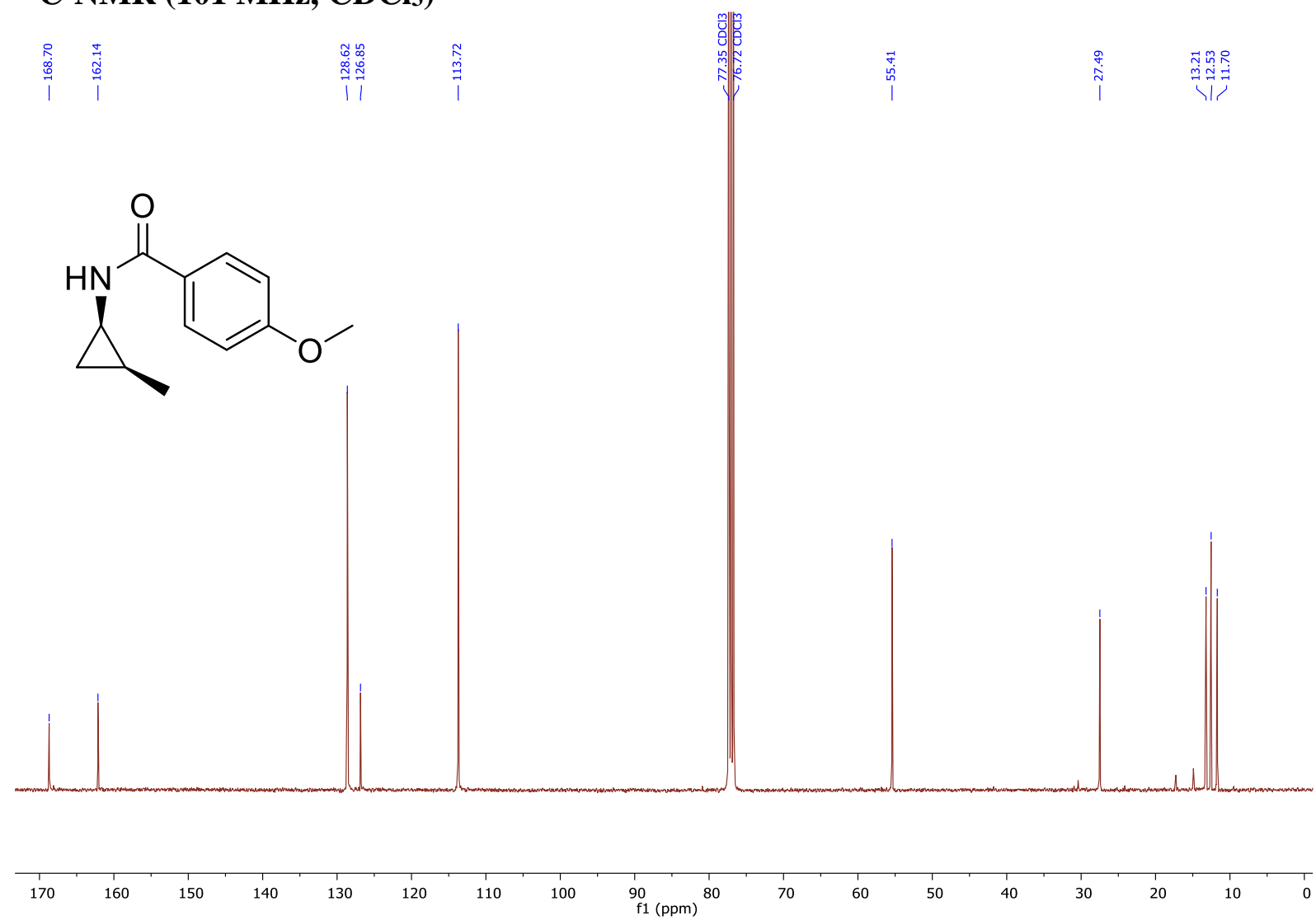

IR 


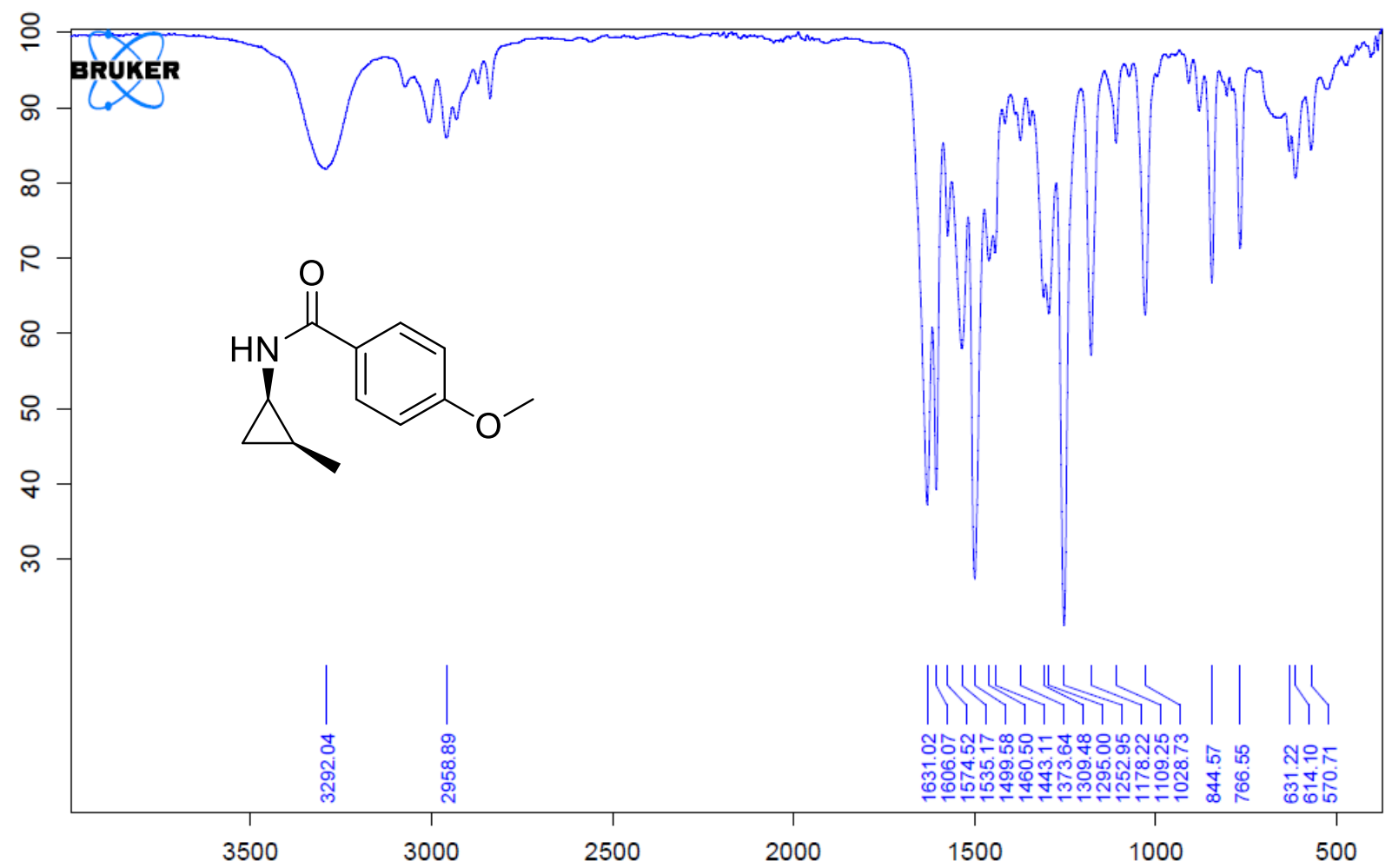

4-Methoxy- $N$-(trans-2-phenylcyclopropyl)benzamide (4b)

${ }^{1} \mathrm{H}-\mathrm{NMR}$ (400 MHz, $\mathrm{CDCl}_{3}$ )

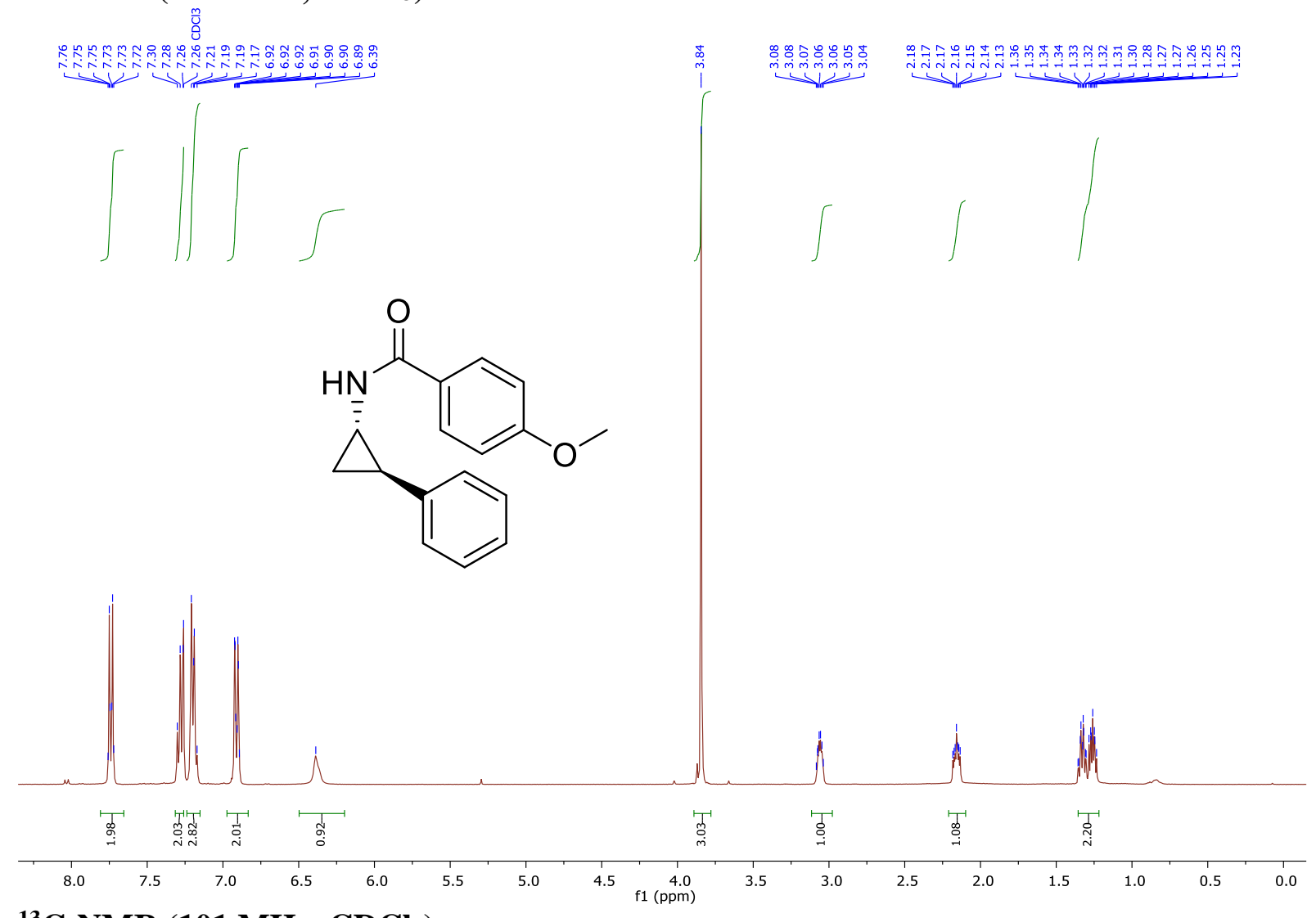

${ }^{13} \mathrm{C}-\mathrm{NMR}$ (101 MHz, CDCl 3$)$ 

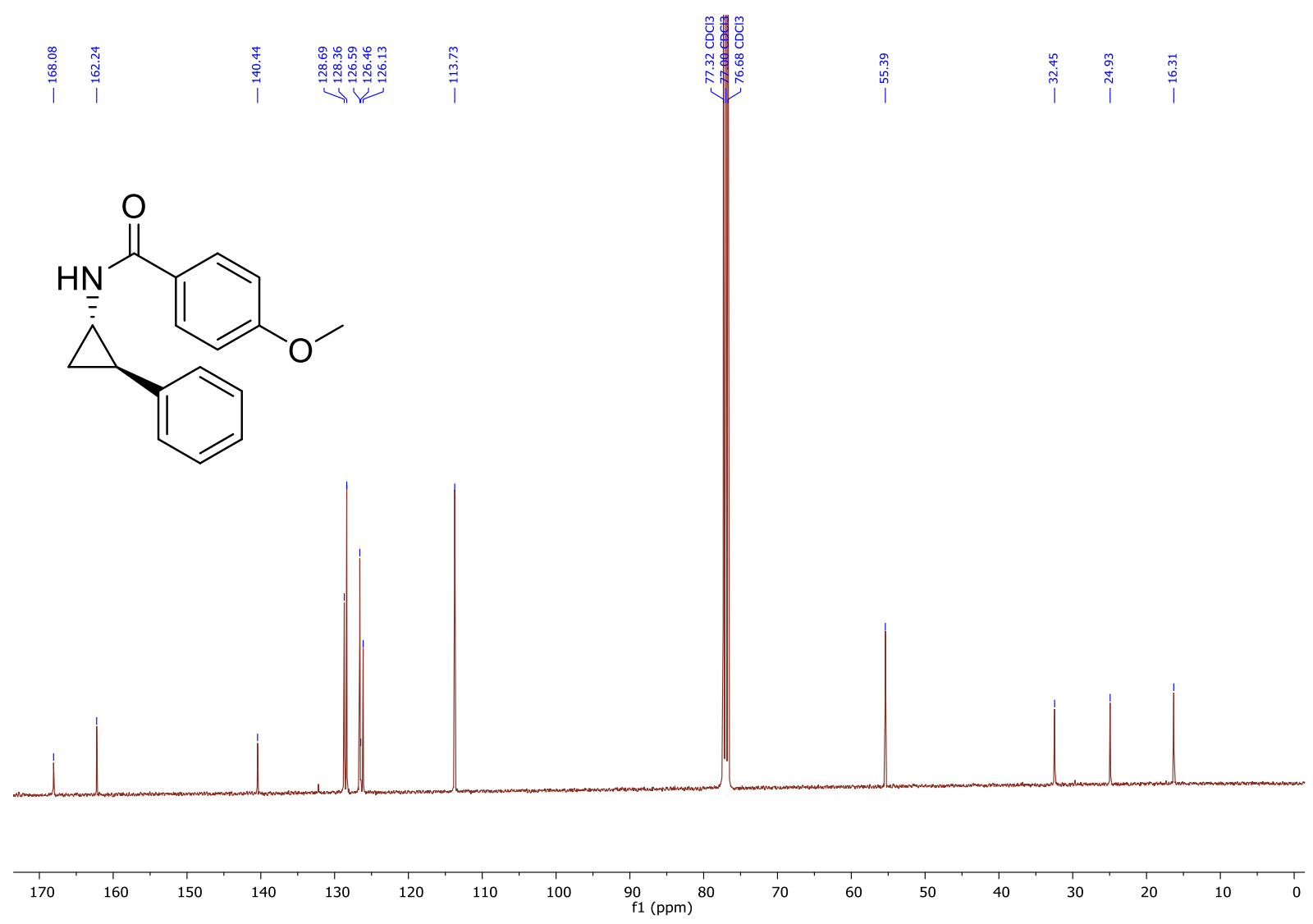

IR

$\stackrel{8}{\circ}$

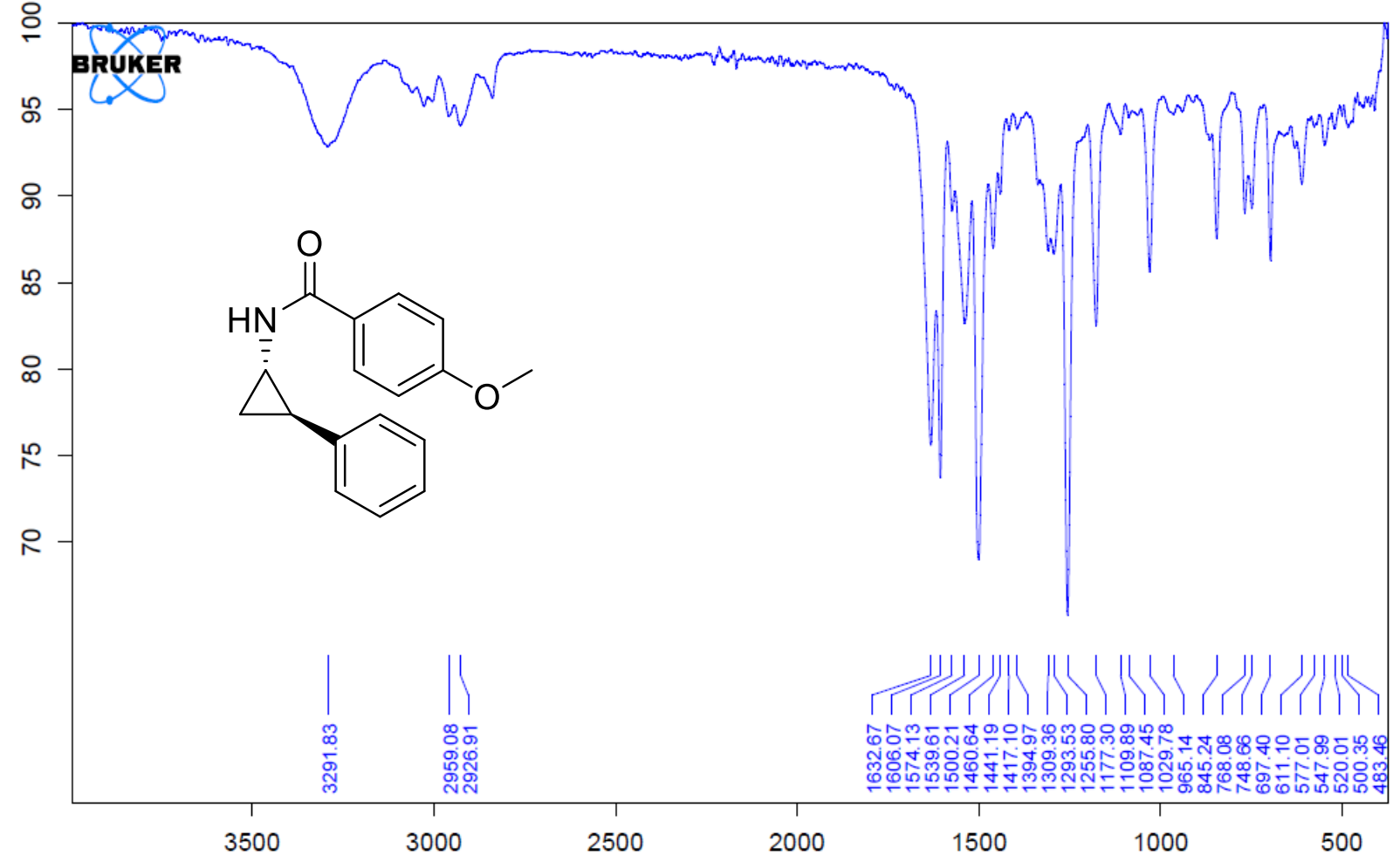

(1R,5S,6S)-tert-Butyl 6-(4-methoxybenzamido)-3-azabicyclo[3.1.0]hexane-3-carboxylate $(4 c)$

${ }^{1} \mathrm{H}-\mathrm{NMR}$ (400 MHz, $\mathrm{CDCl}_{3}$ ) 


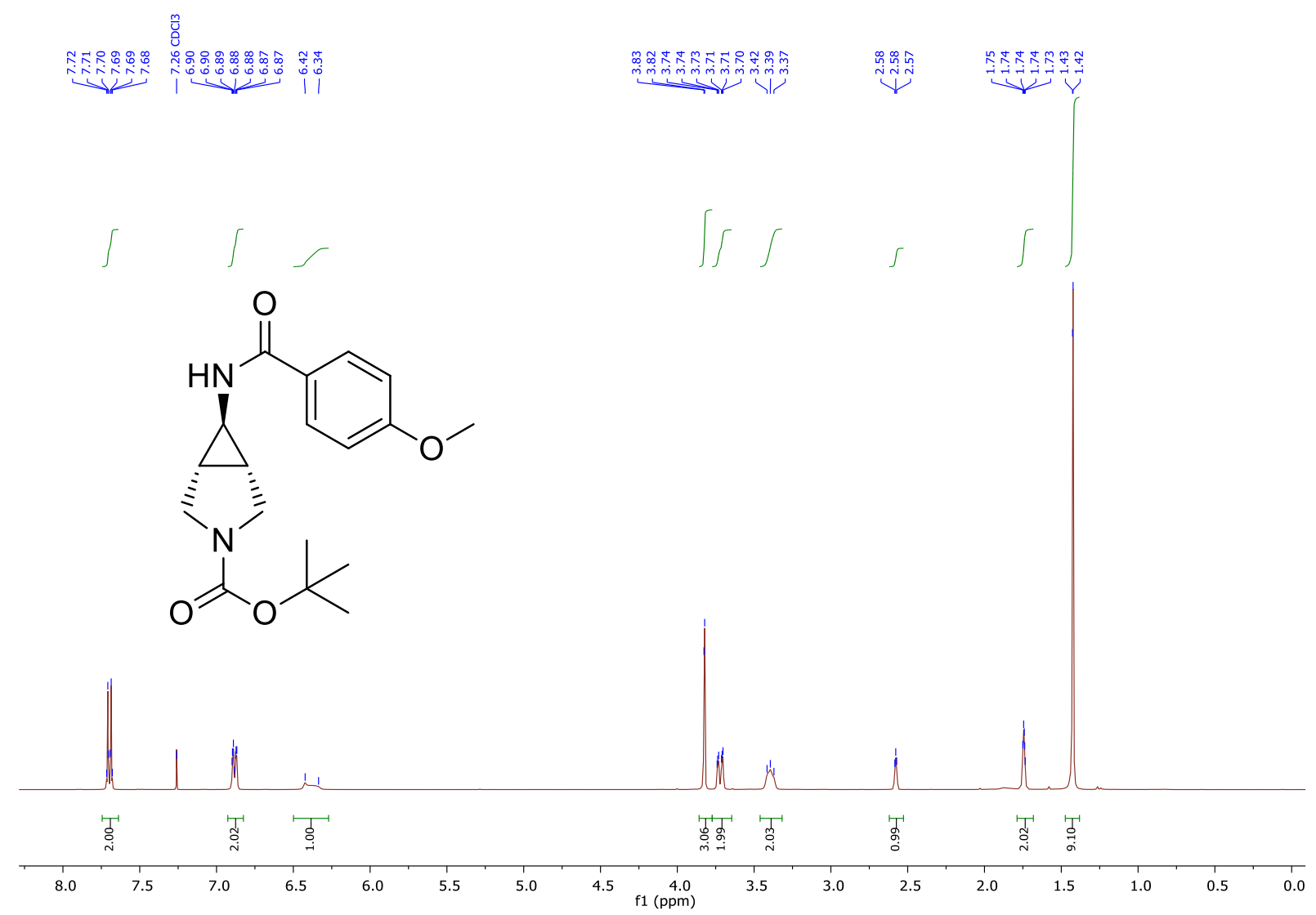

${ }^{13} \mathrm{C}-\mathrm{NMR}$ (101 MHz, CDCl3)

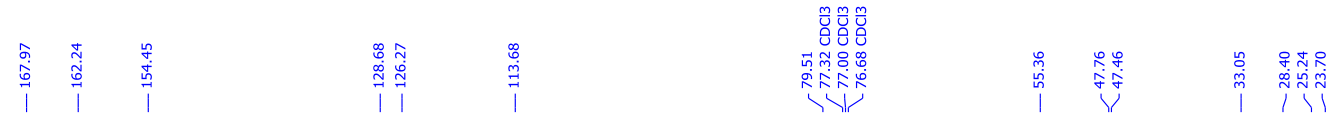
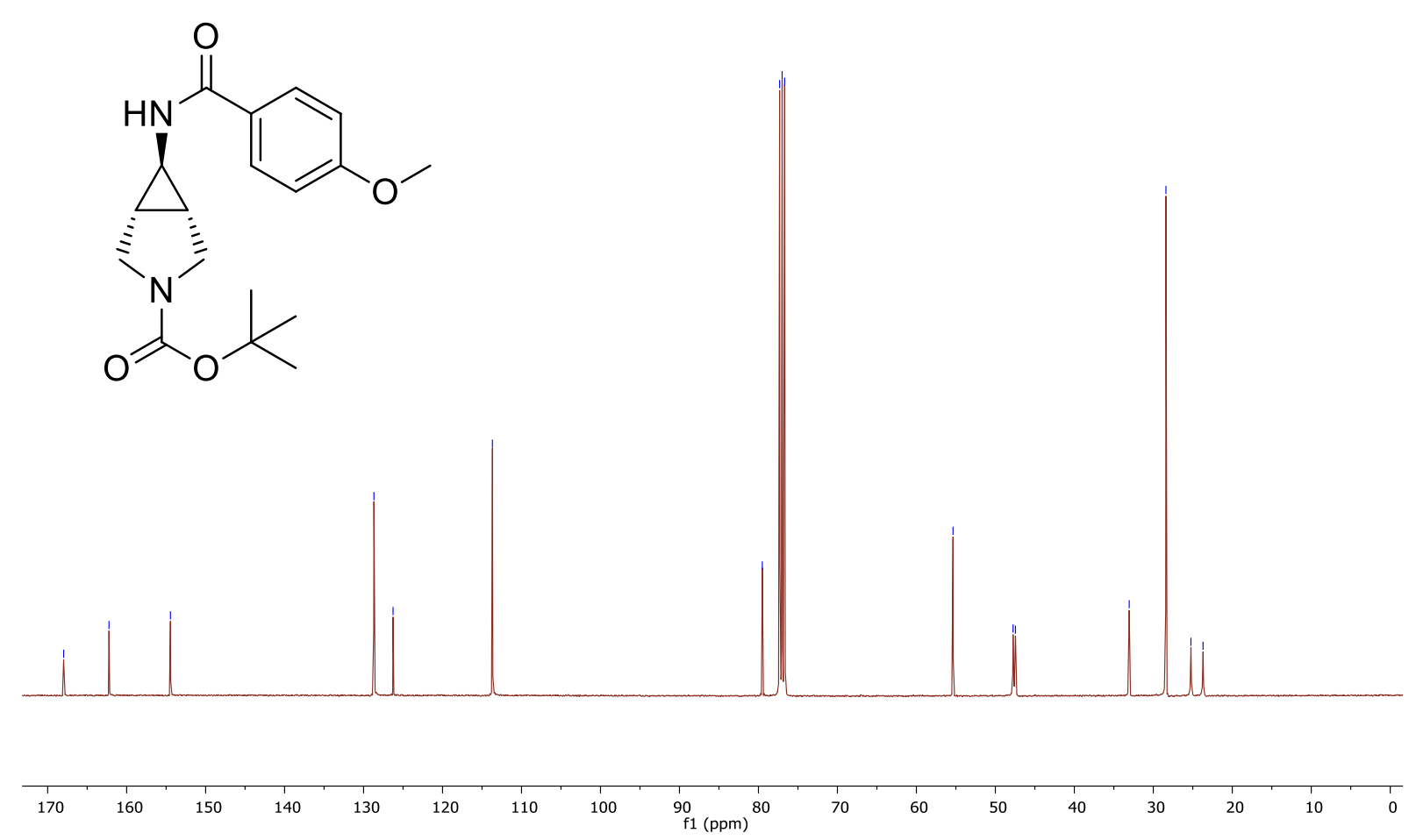

IR 


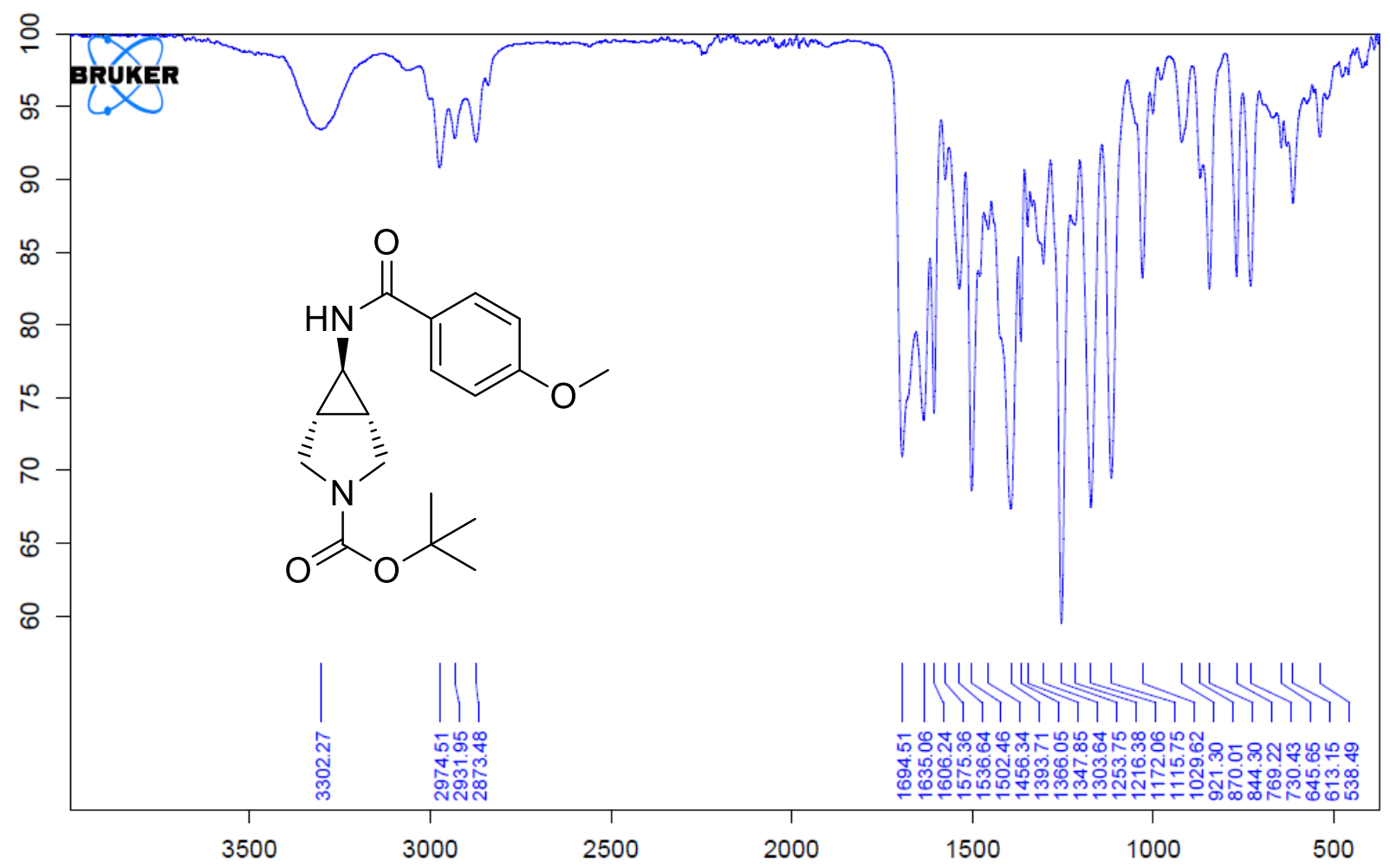

$N$-(2,2-Difluorocyclopropyl)-4-methoxybenzamide (4d)

${ }^{1} \mathrm{H}-\mathrm{NMR}$ (400 $\mathrm{MHz}, \mathrm{CDCl}_{3}$ )

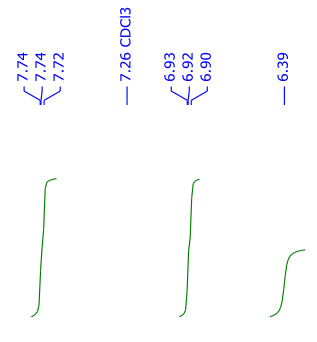

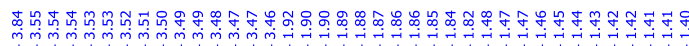<smiles>COc1ccc(C(=O)NC2CC2(F)F)cc1</smiles>

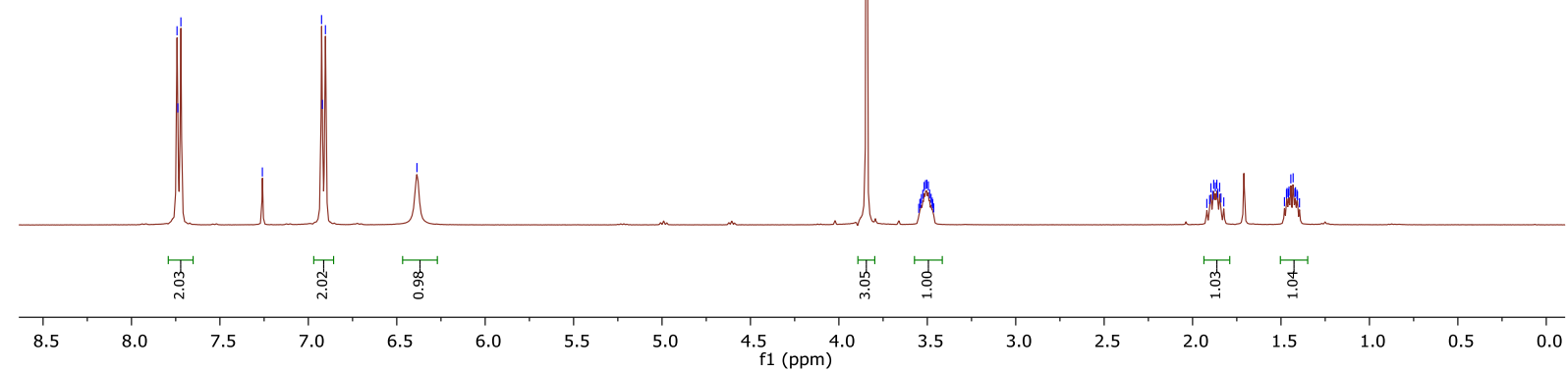

${ }^{13}$ C-NMR (101 MHz, $\left.\mathrm{CDCl}_{3}\right)$ 

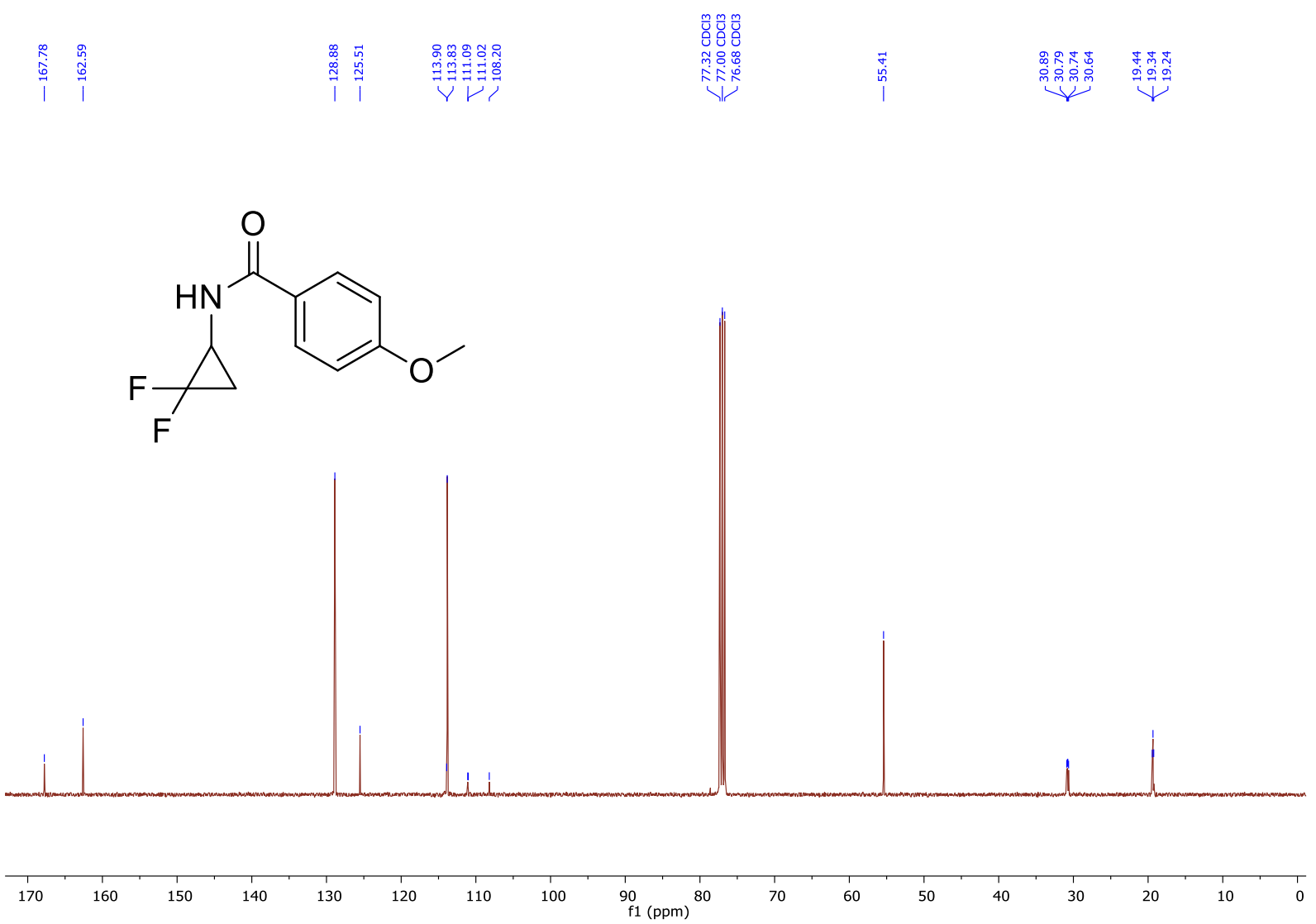

${ }^{19}$ F-NMR (376 MHz, CDCl3)<smiles>COc1ccc(C(=O)NC2CC2(F)F)cc1</smiles>

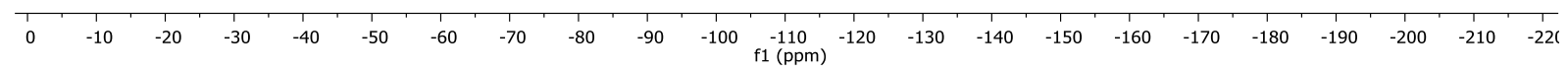
IR 


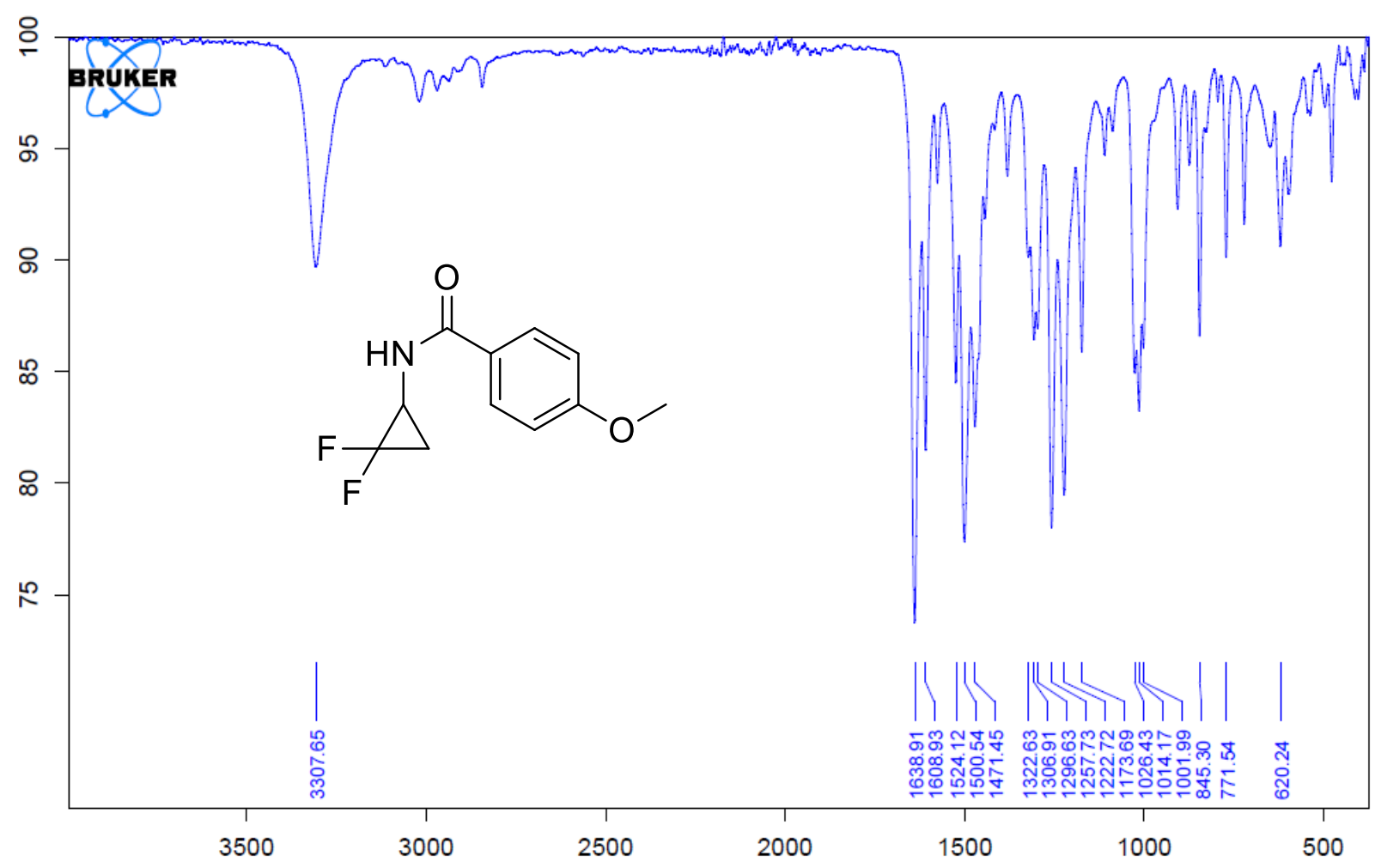

N-Cyclobutyl-4-methoxybenzamide (4e)

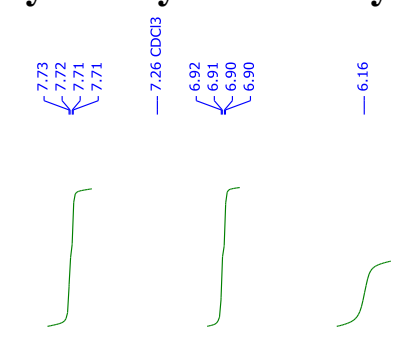

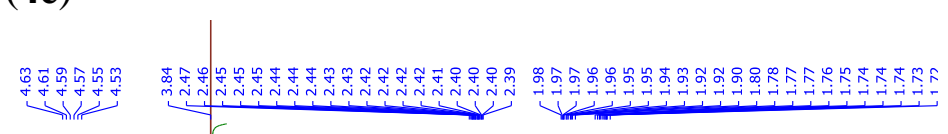

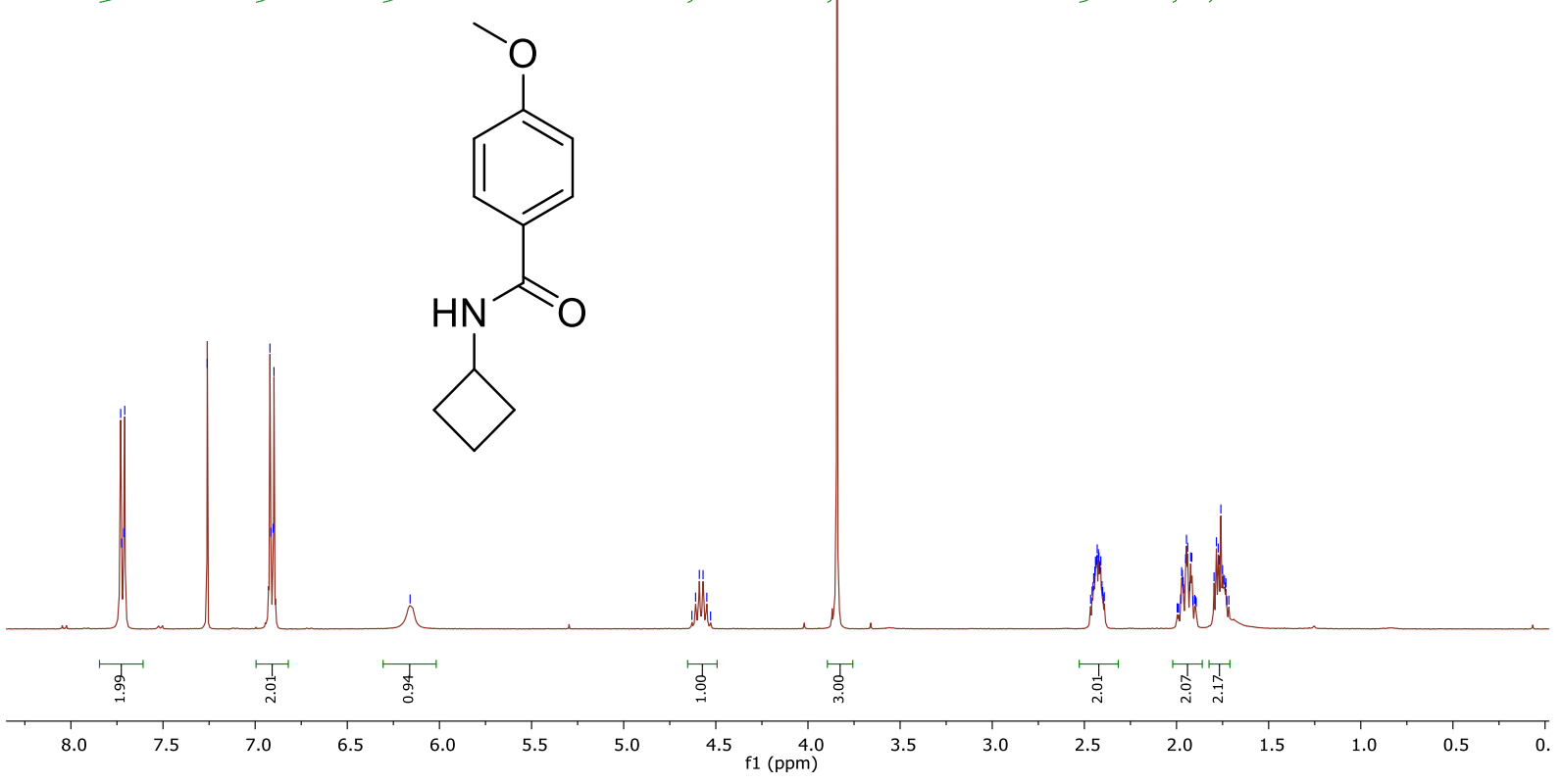



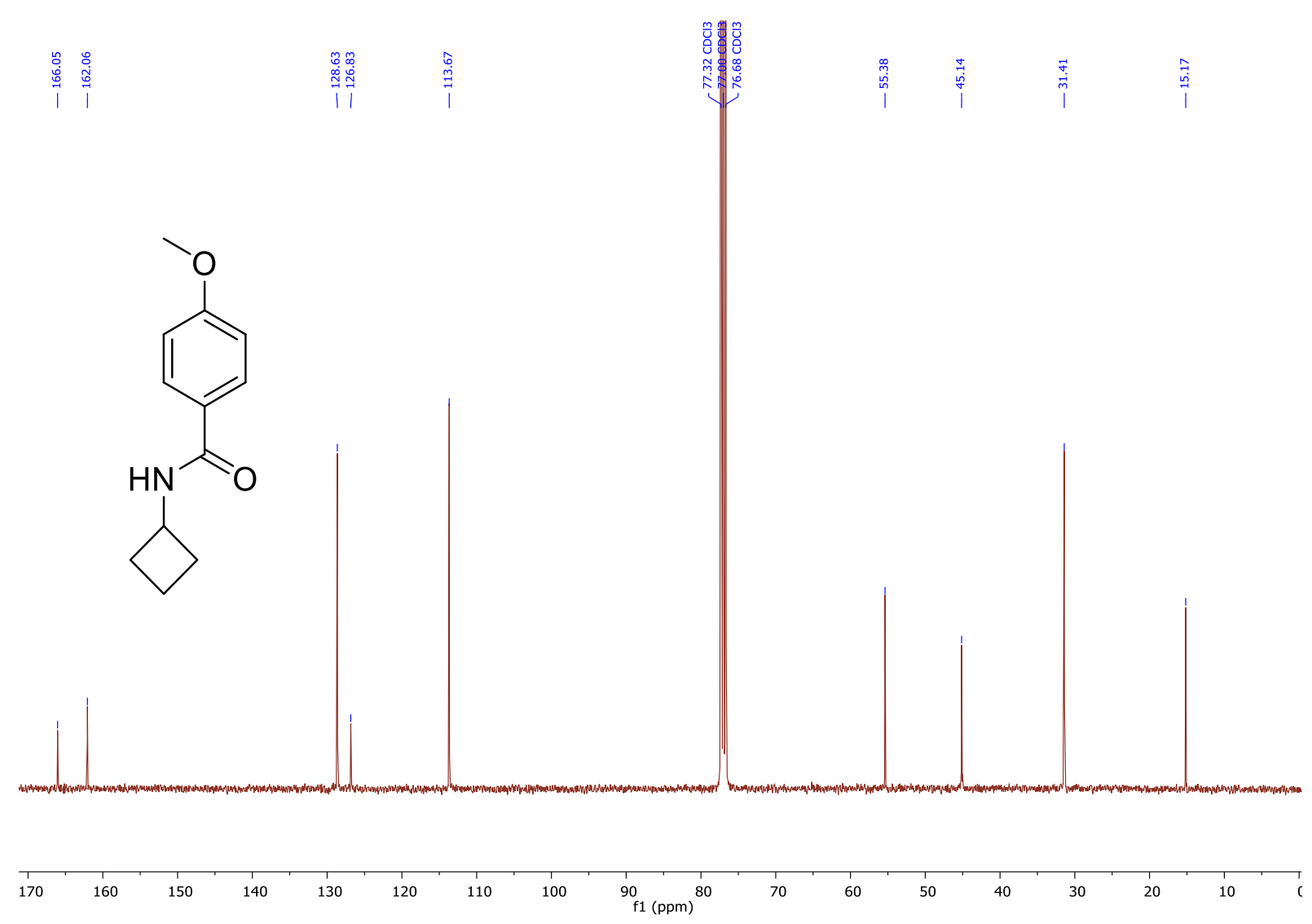

IR

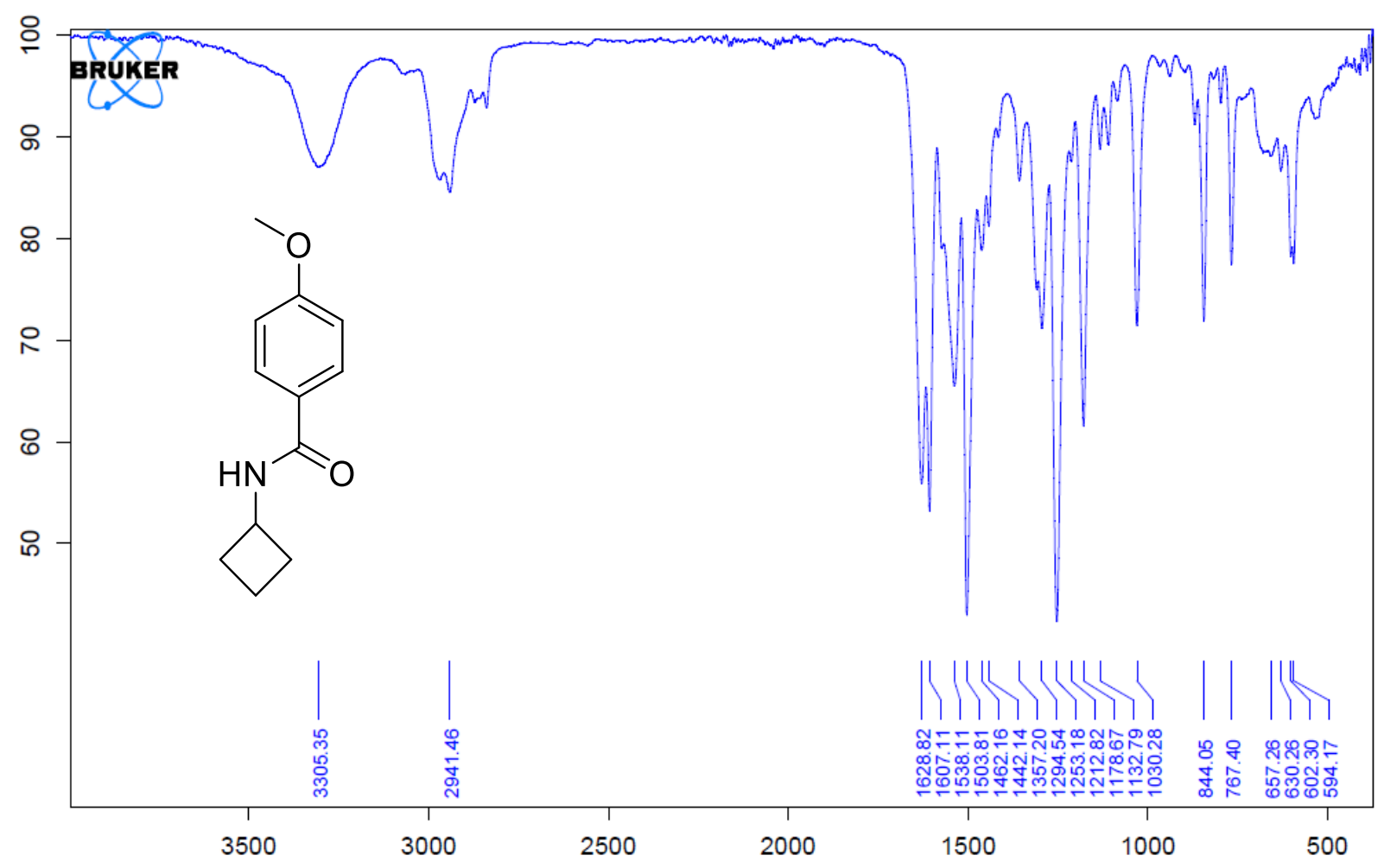

4-Methoxy- $N$-(trans-3-phenylcyclobutyl)benzamide (4f).

${ }^{1} \mathrm{H}-\mathrm{NMR}$ (400 MHz, $\mathrm{CDCl}_{3}$ ) 


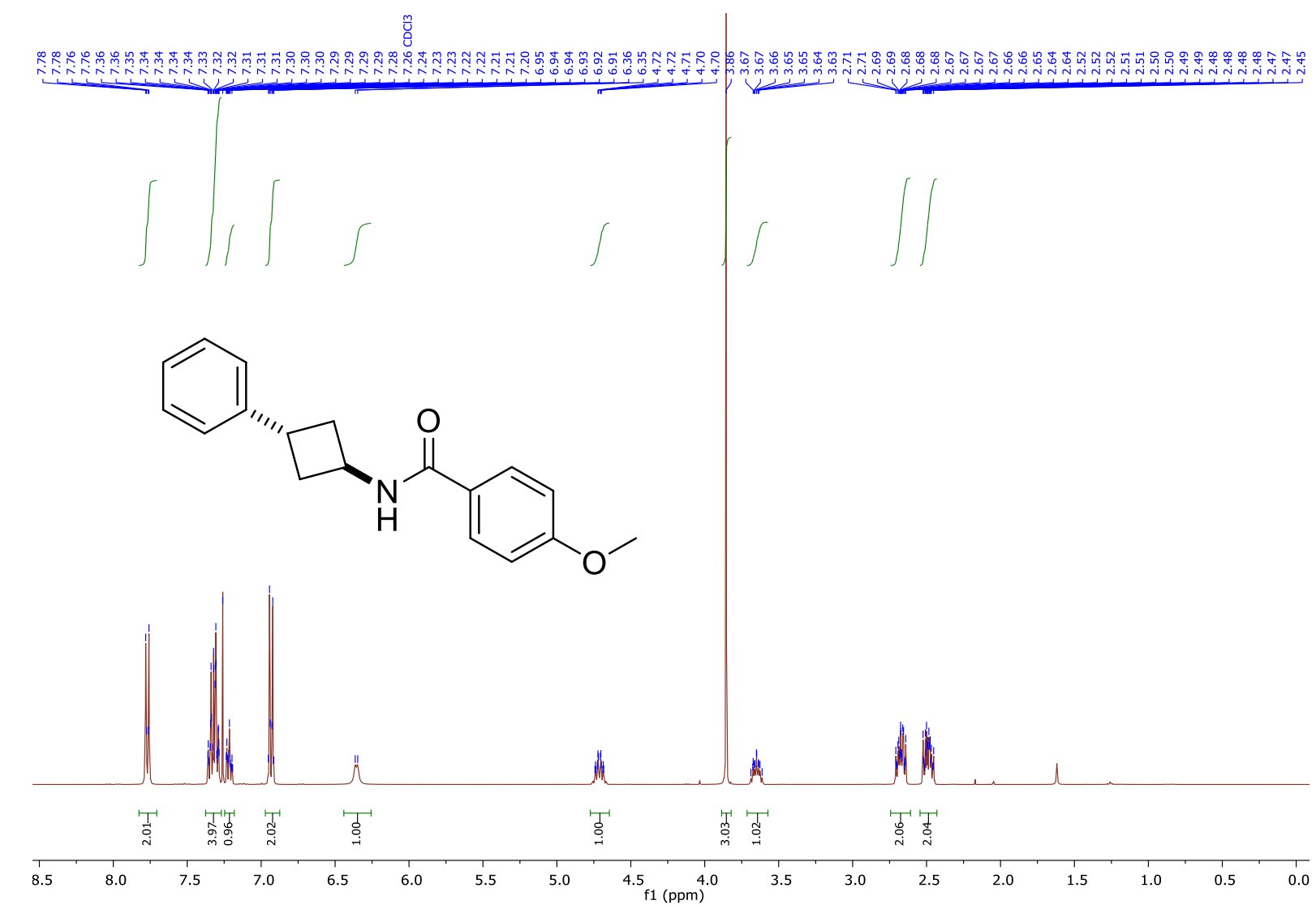

${ }^{13} \mathrm{C}-\mathrm{NMR}$ (101 MHz, CDCl3)

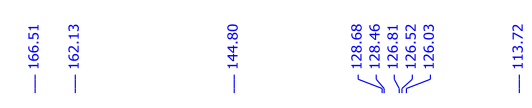
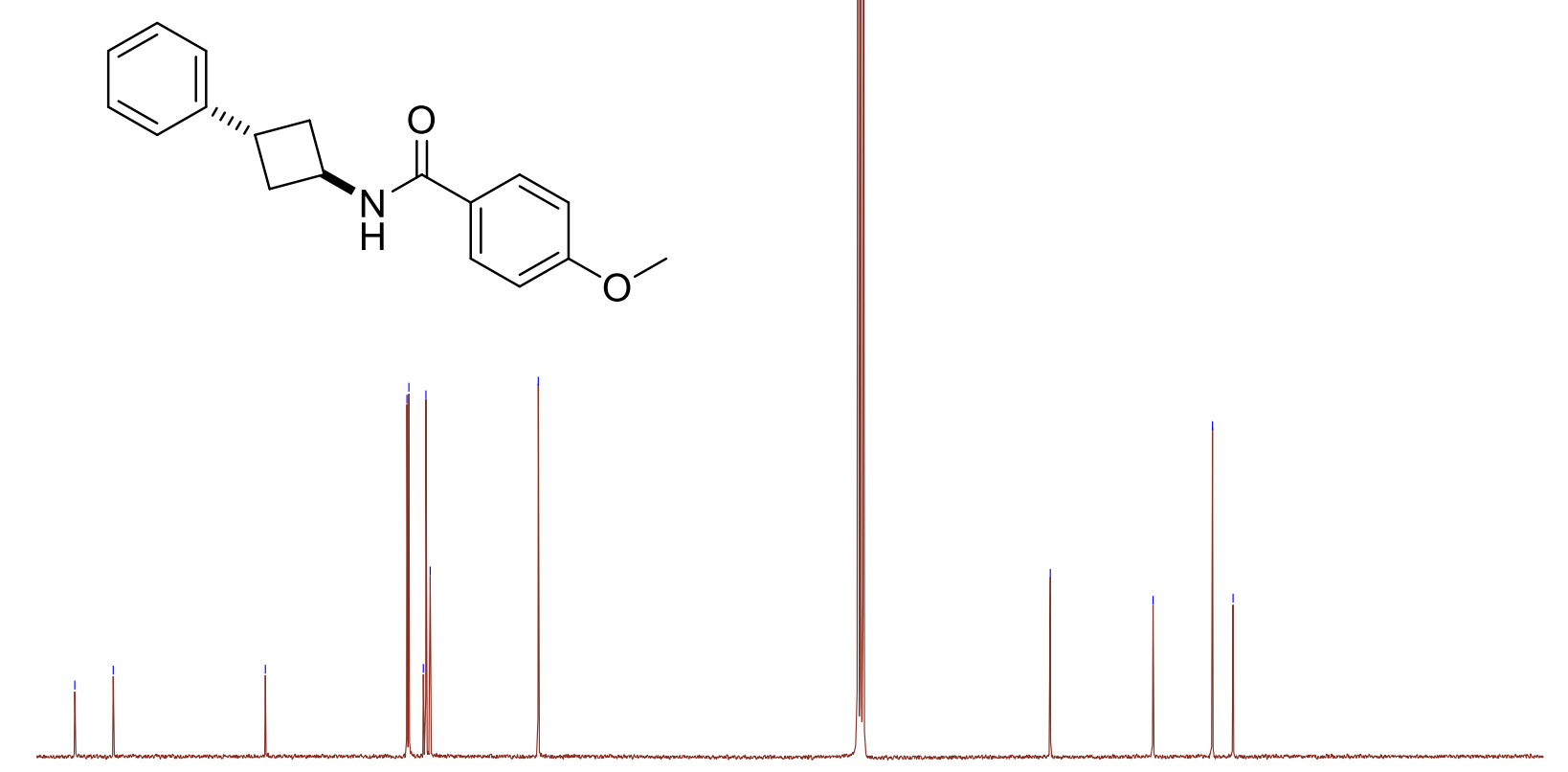

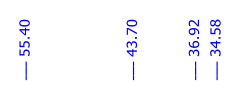

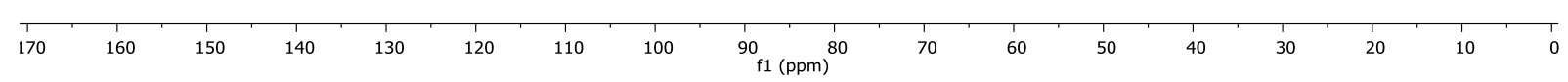

IR 


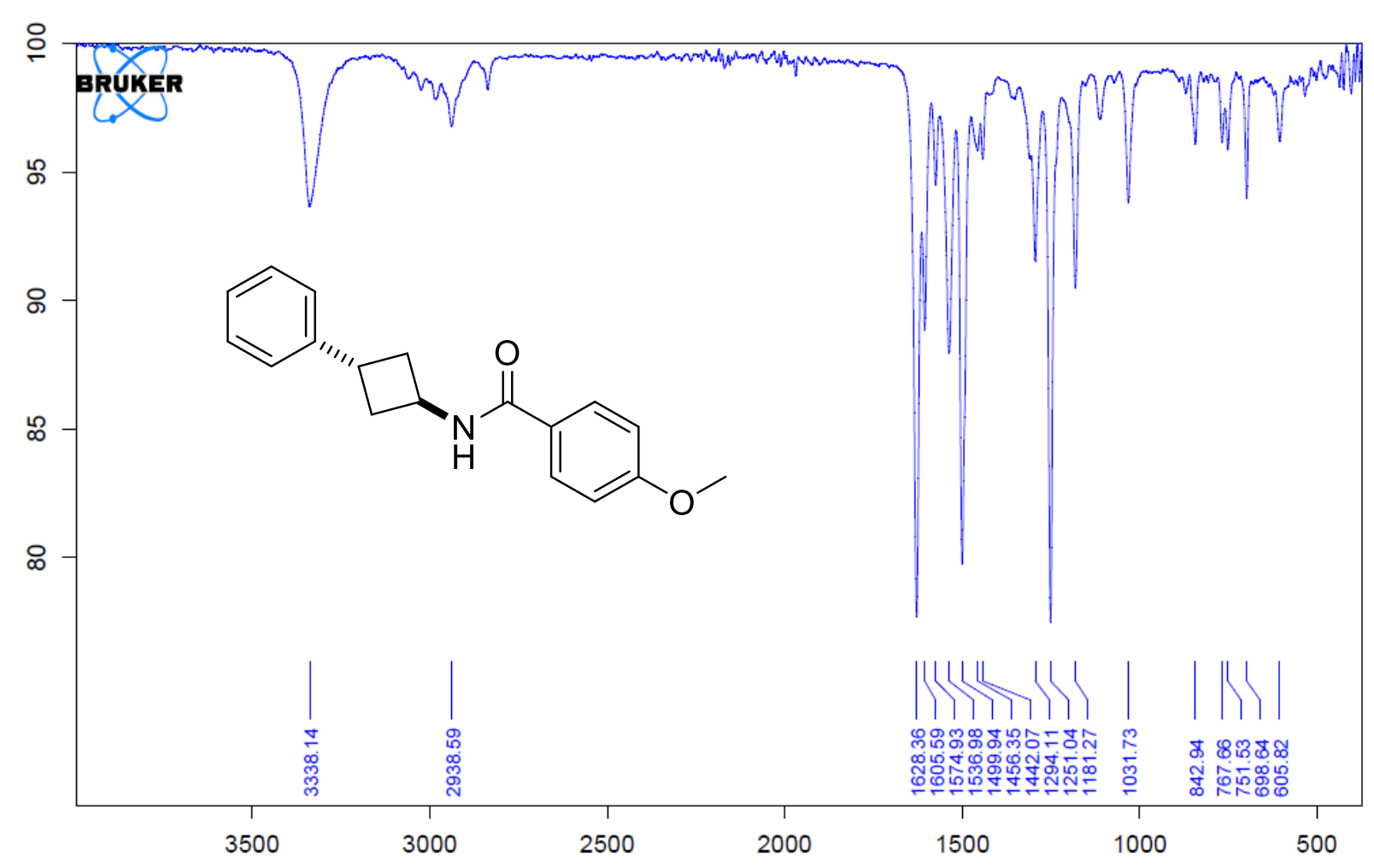

$N$-Cyclopropyl-4-methoxy- $N$-methylbenzamide (9)

${ }^{1} \mathrm{H}-\mathrm{NMR}$ (400 MHz, $\mathrm{CDCl}_{3}$ )

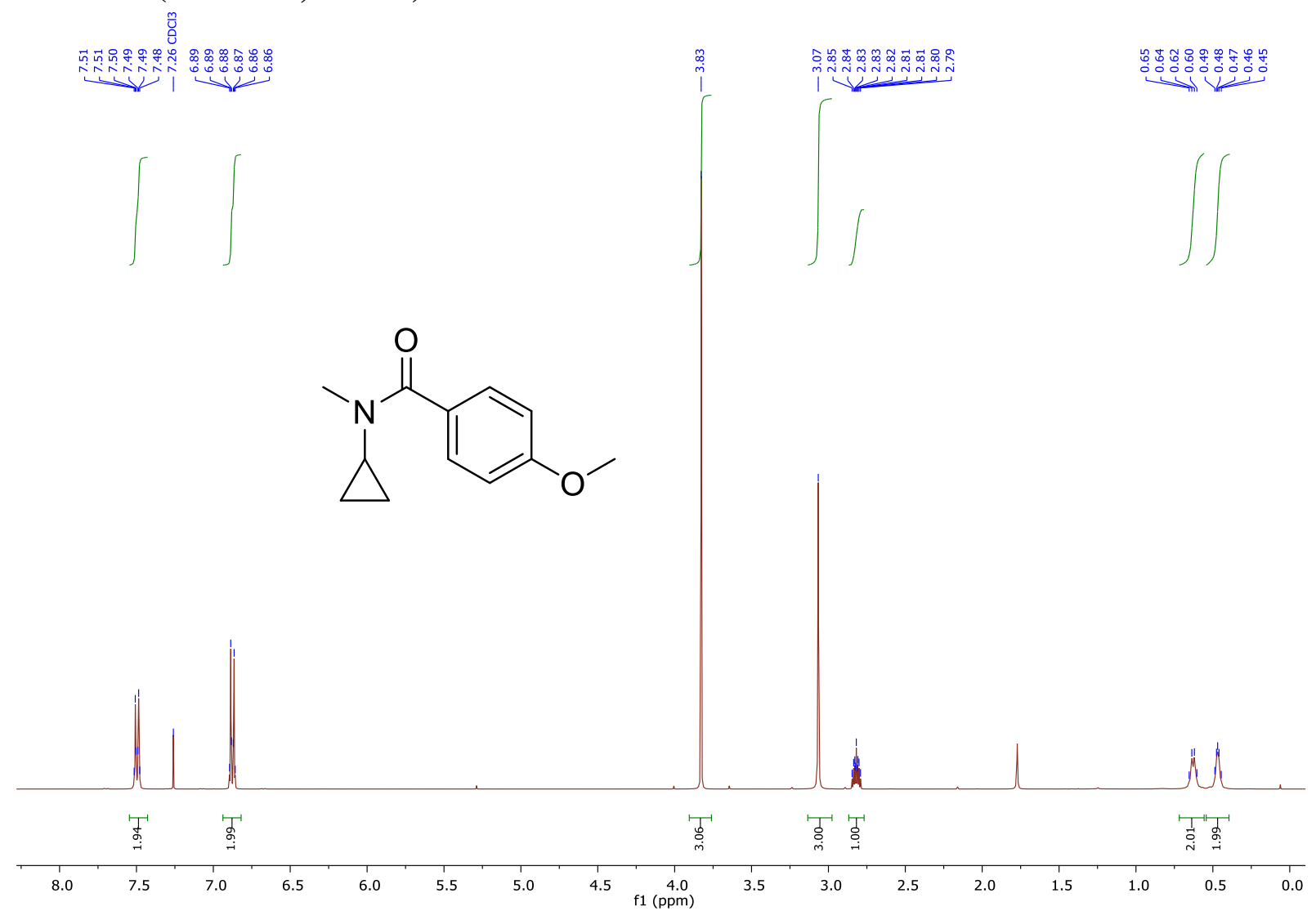

${ }^{13} \mathrm{C}-\mathrm{NMR}\left(101 \mathrm{MHz}, \mathrm{CDCl}_{3}\right)$ 

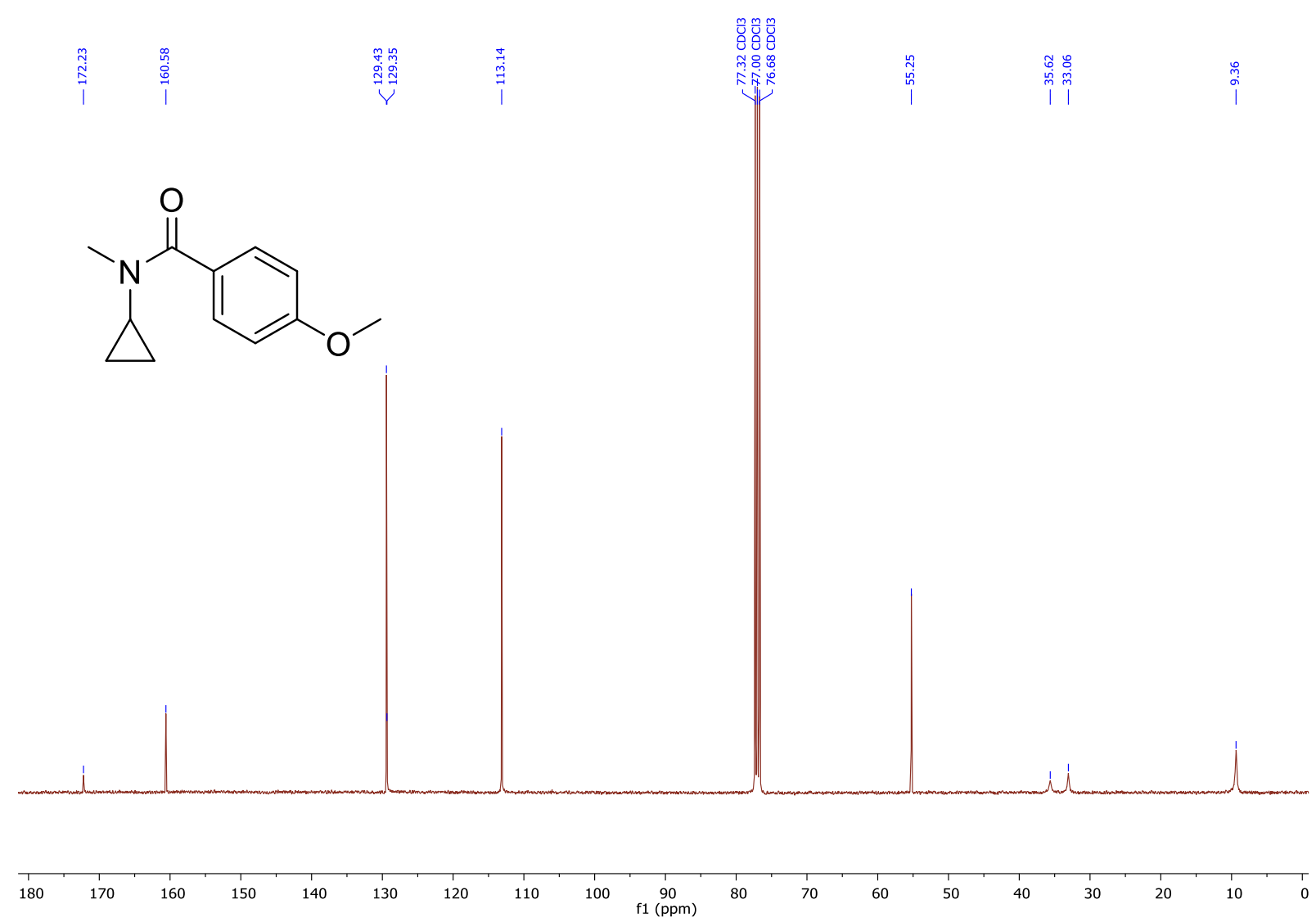

IR

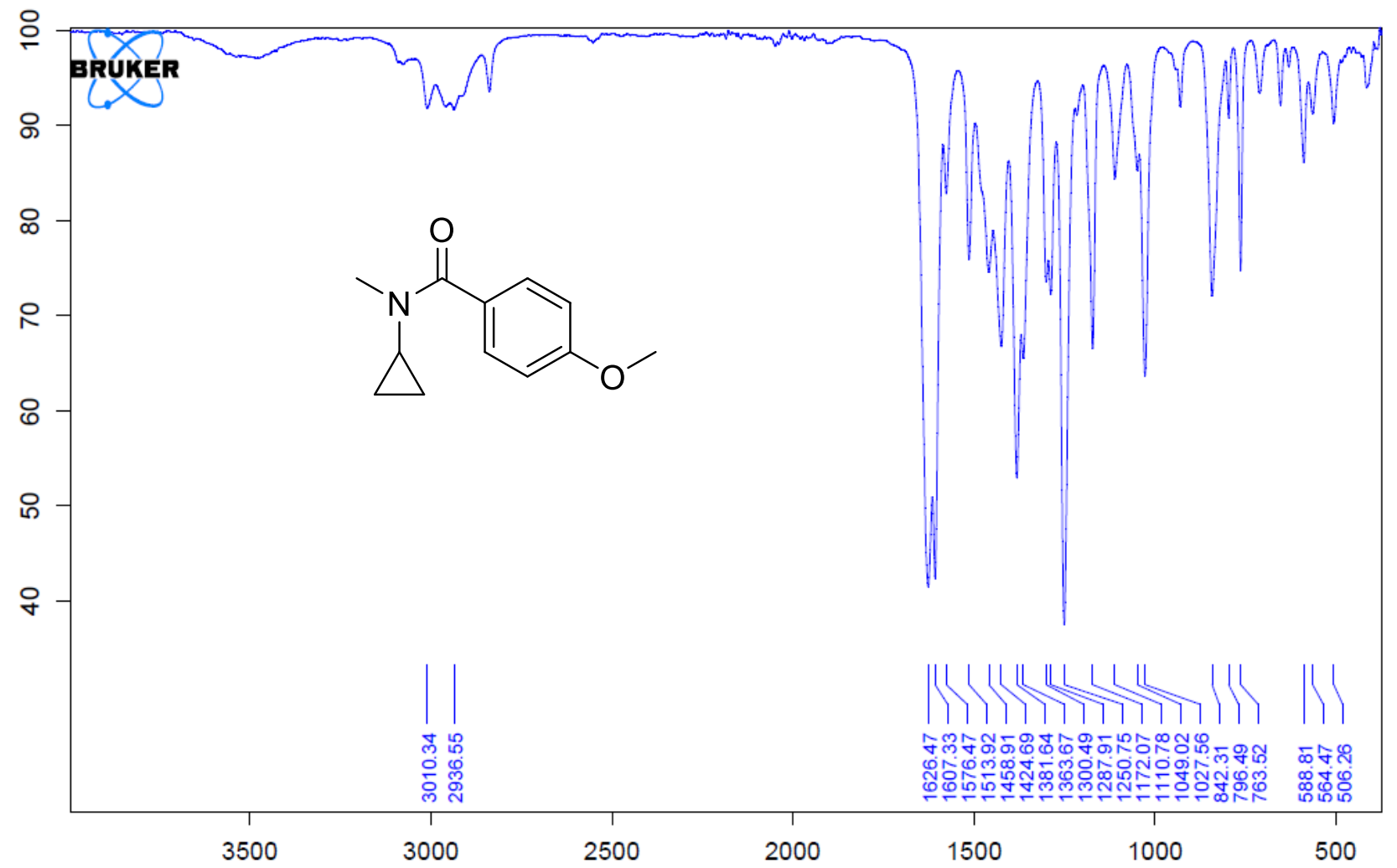

$N$-(3-Fluoro-1-hydroxypropyl)-4-methoxybenzamide (2a)

${ }^{1} \mathrm{H}-\mathrm{NMR}\left(400 \mathrm{MHz}, \mathrm{CDCl}_{3}\right)$ 


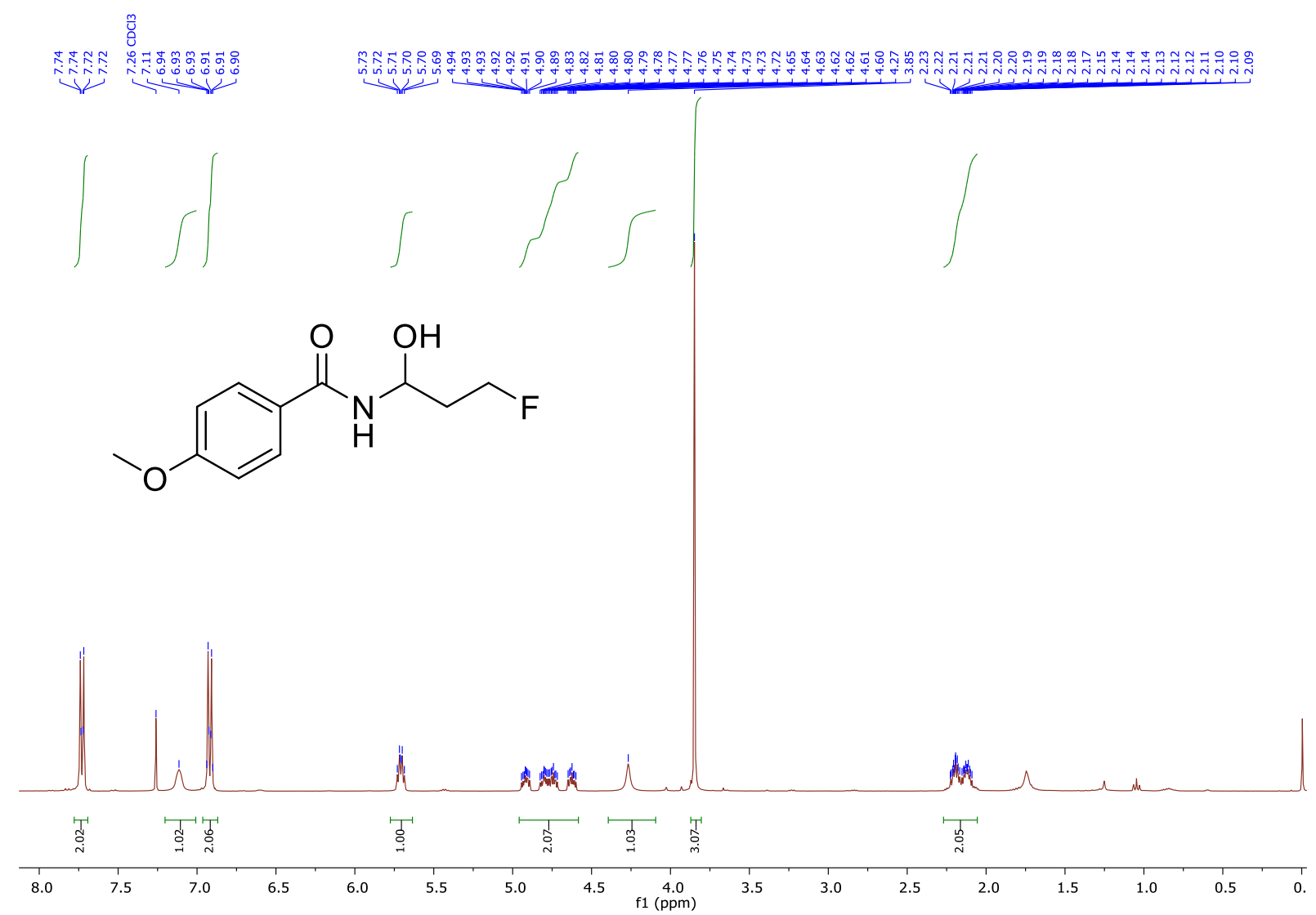

${ }^{13} \mathrm{C}-\mathrm{NMR}$ (101 MHz, CDCl3)
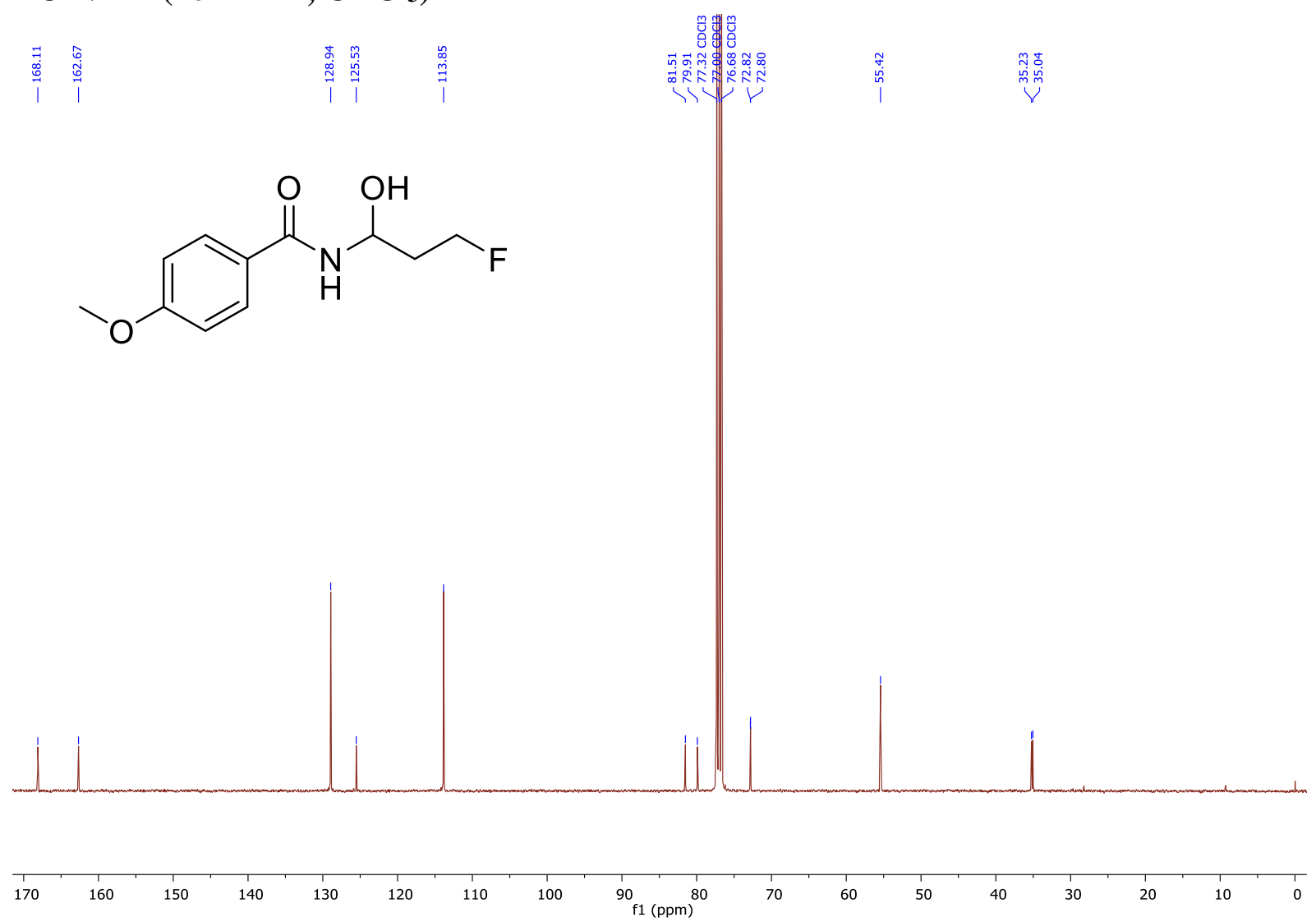

${ }^{19}$ F-NMR (376 MHz, CDCl 3 ) 
<smiles>COc1ccc(C(=O)NC(O)CCF)cc1</smiles>

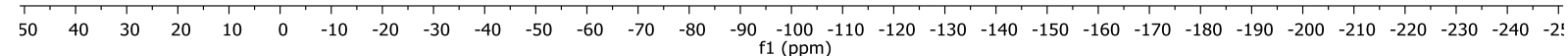

IR

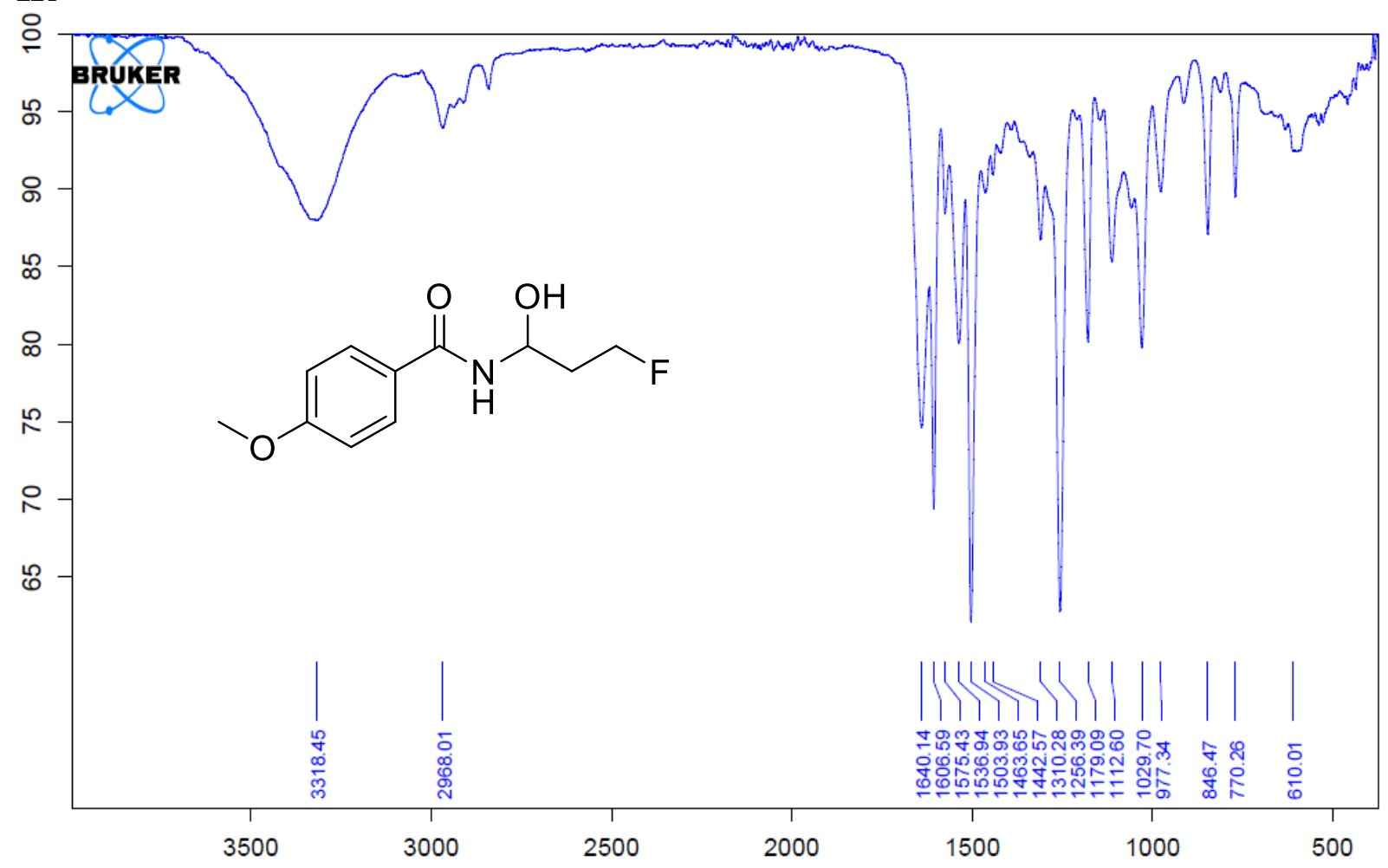

$N$-(3-Fluoro-1-hydroxypropyl)-4-methylbenzamide (2b)

${ }^{1} \mathrm{H}-\mathrm{NMR}$ (400 MHz, $\mathrm{CDCl}_{3}$ ) 


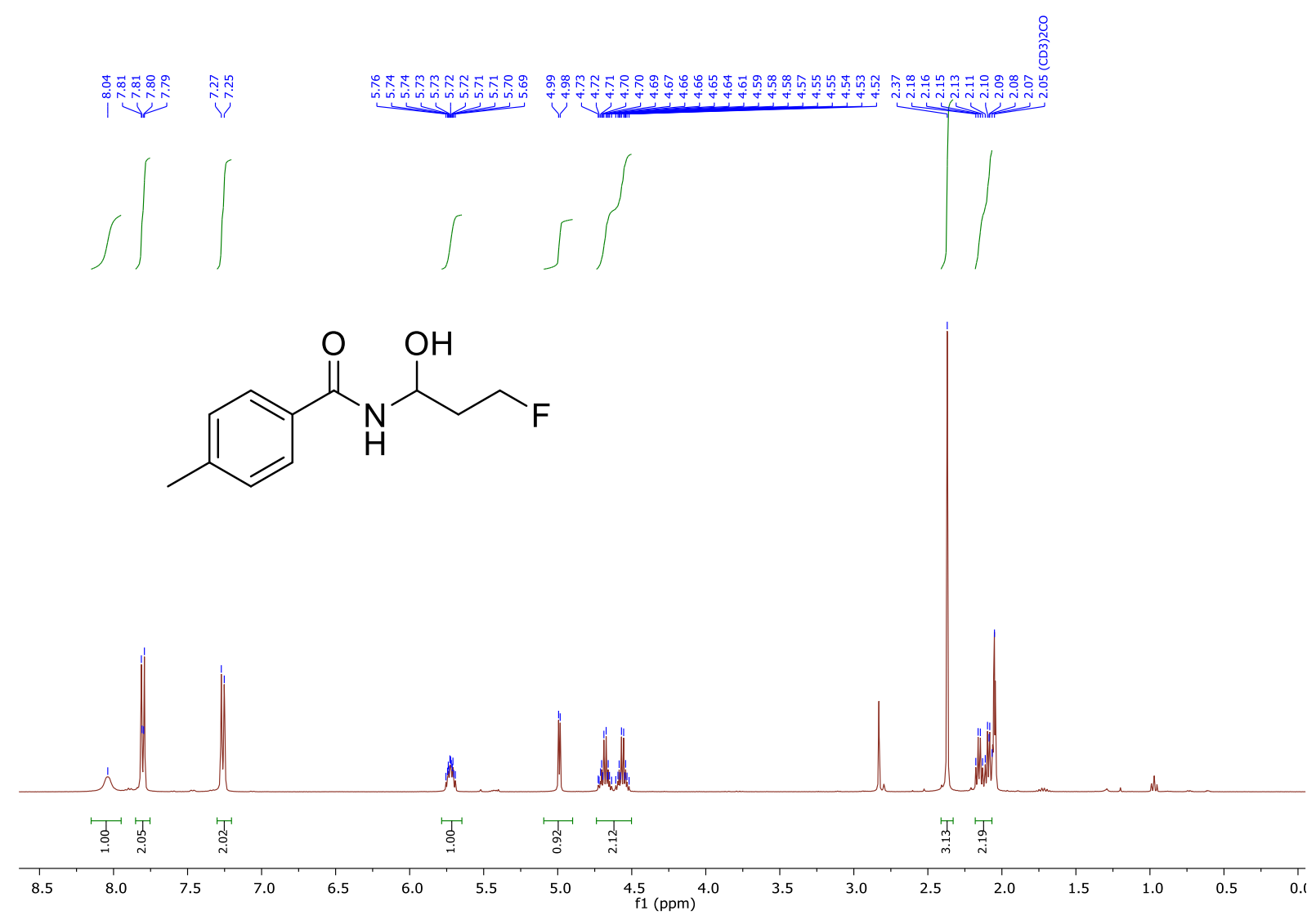

${ }^{13}$ C-NMR (101 MHz, CDCl3)
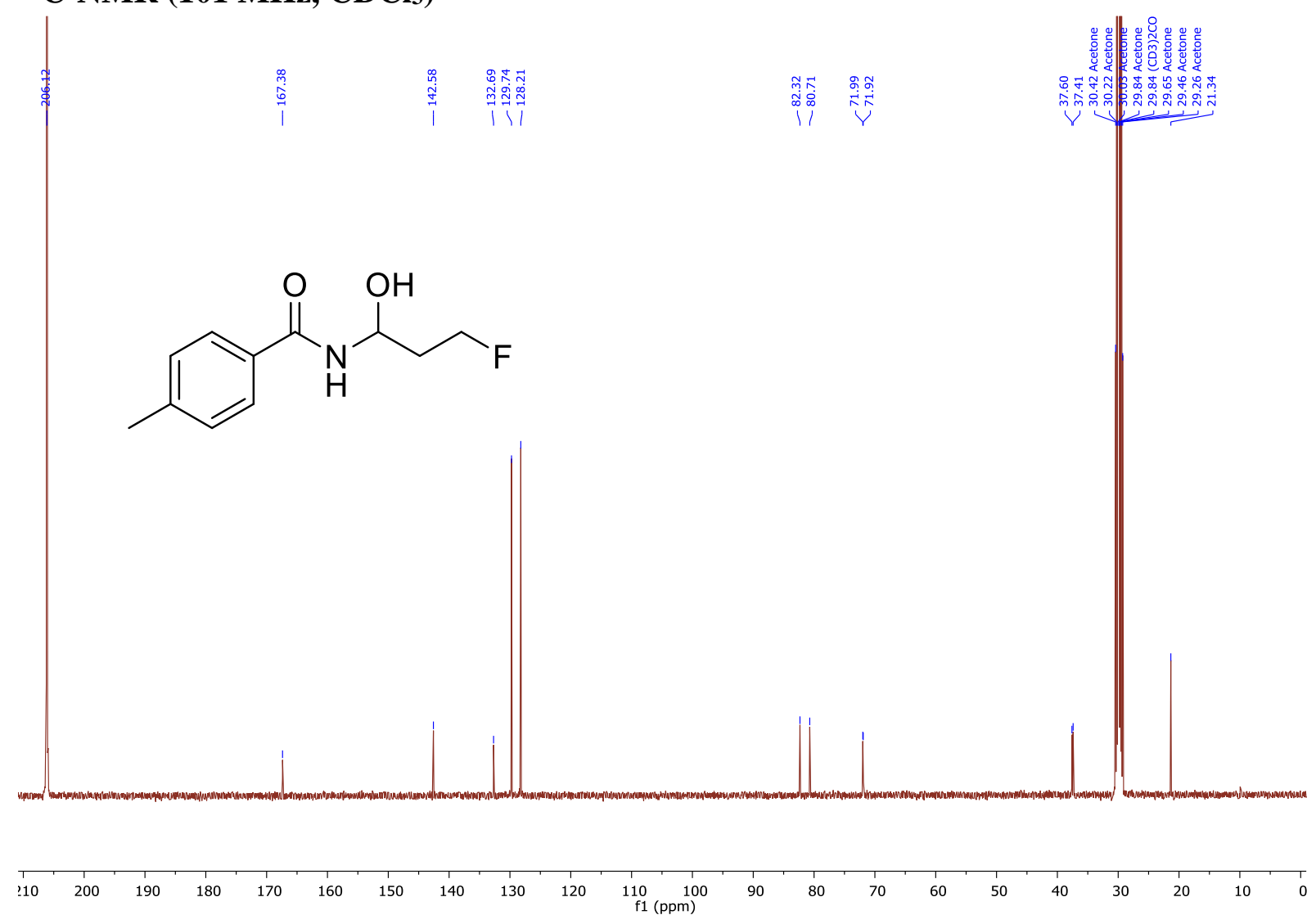

${ }^{19}$ F-NMR (376 MHz, Acetone-d6) 
<smiles>Cc1ccc(C(=O)NC(O)CCF)cc1</smiles>

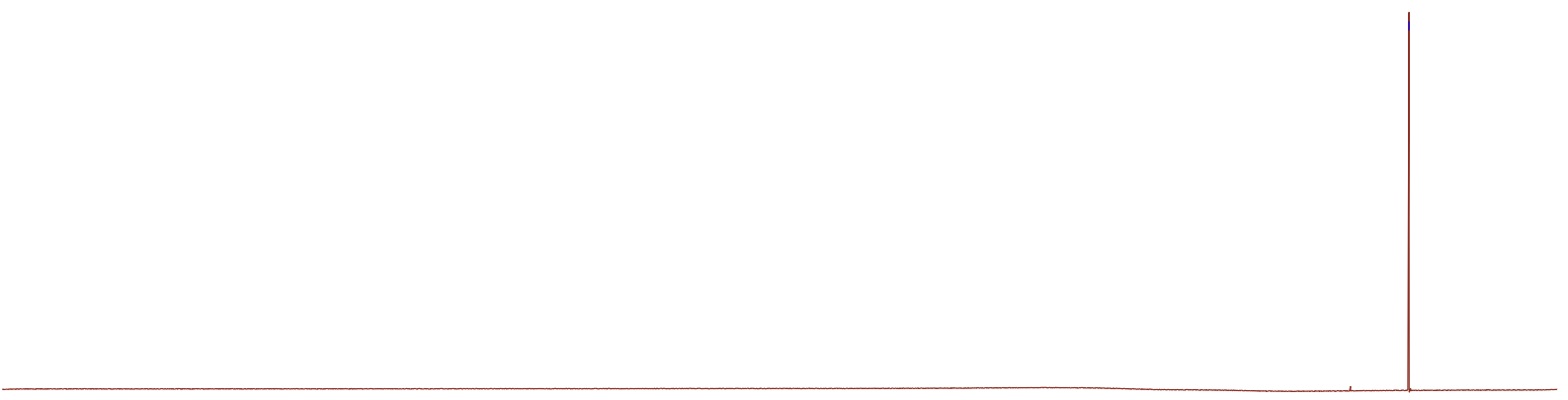

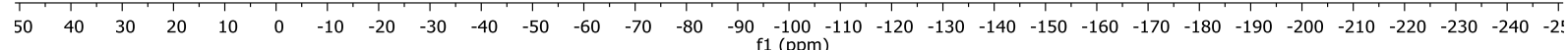

IR

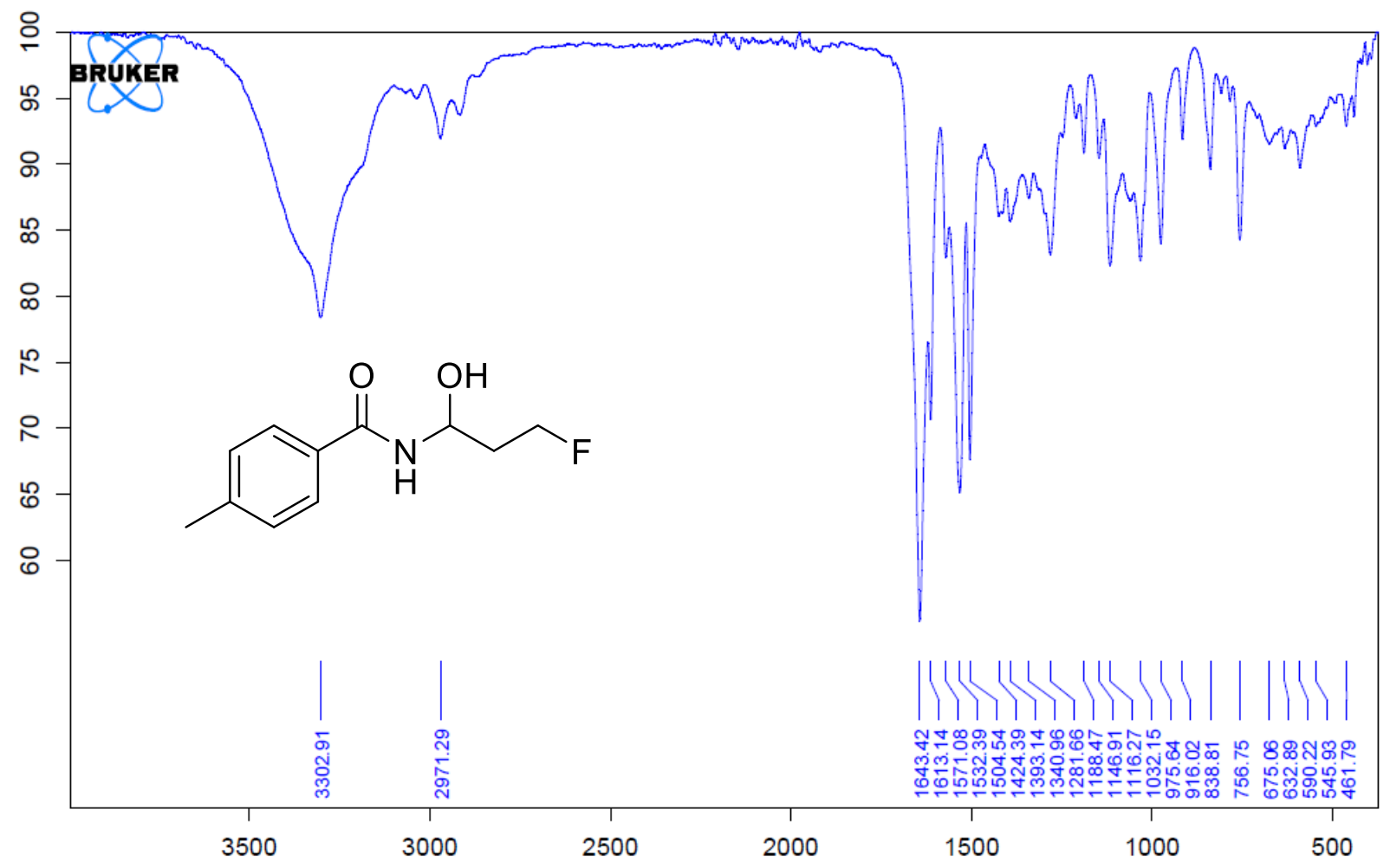

4-Fluoro- $N$-(3-fluoro-1-hydroxypropyl)benzamide (2c)

${ }^{1} \mathrm{H}-\mathrm{NMR}\left(400 \mathrm{MHz}, \mathrm{CDCl}_{3}\right)$ 


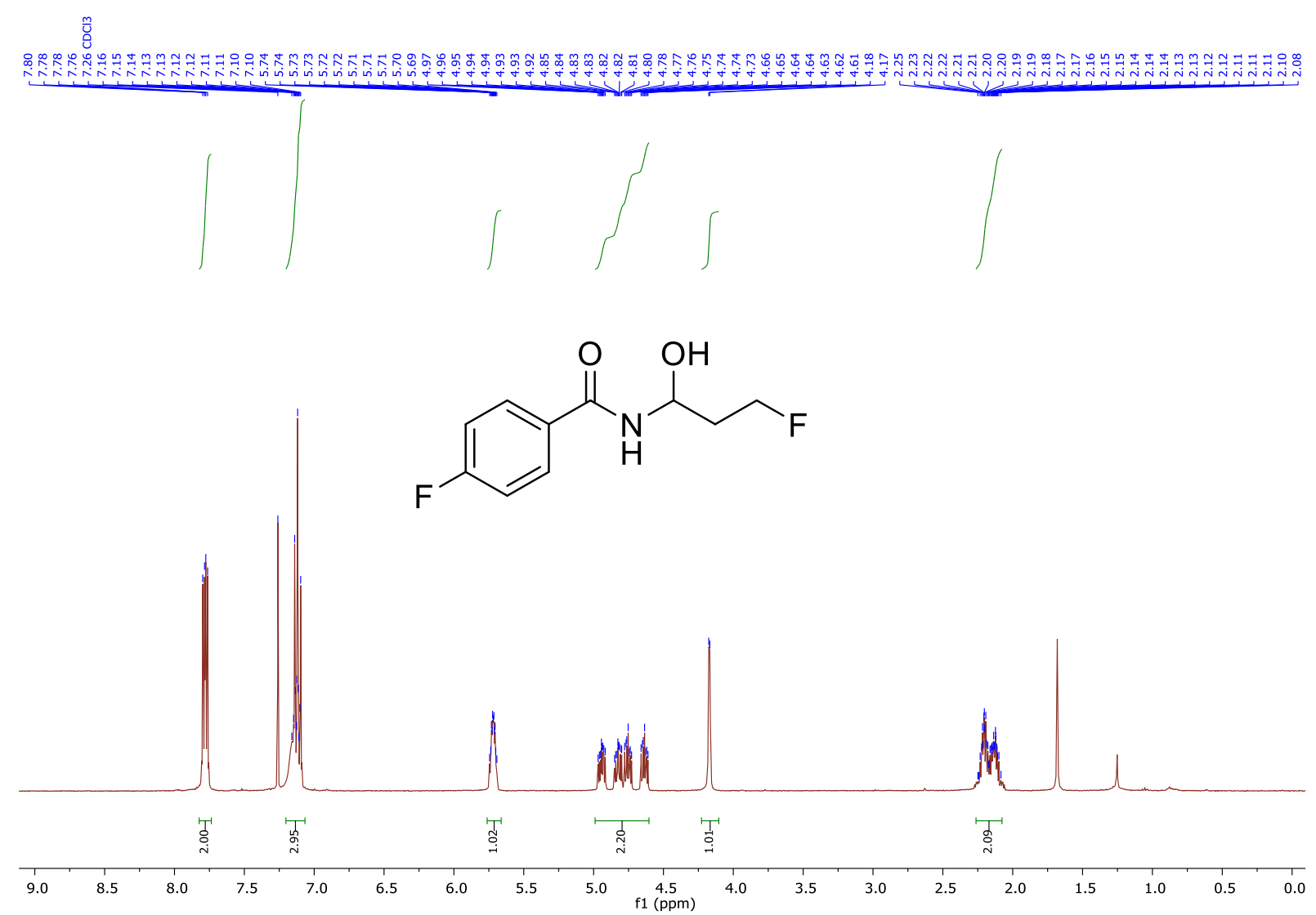

${ }^{13} \mathrm{C}$-NMR (101 MHz, CDCl3)

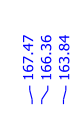

لَ

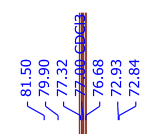

商热<smiles>O=C(NC(O)CCF)c1ccc(F)cc1</smiles>
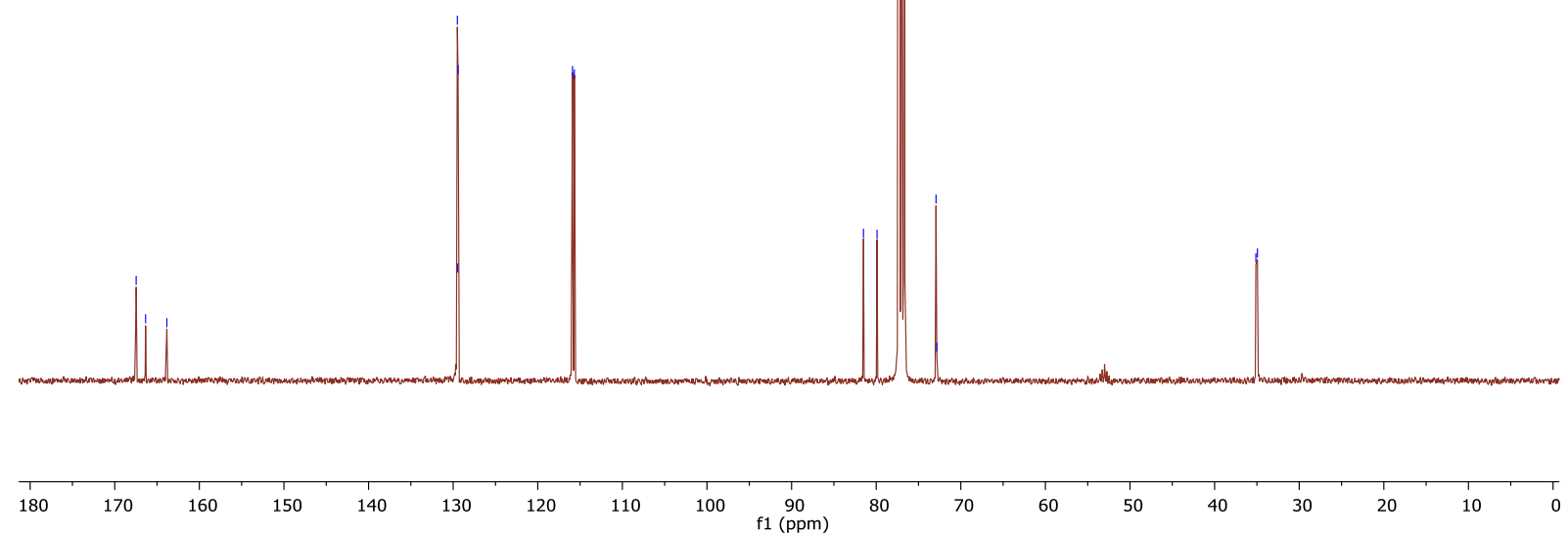

${ }^{19}$ F-NMR (376 MHz, CDCl3) 
<smiles>O=C(NC(O)CCF)c1ccc(F)cc1</smiles>

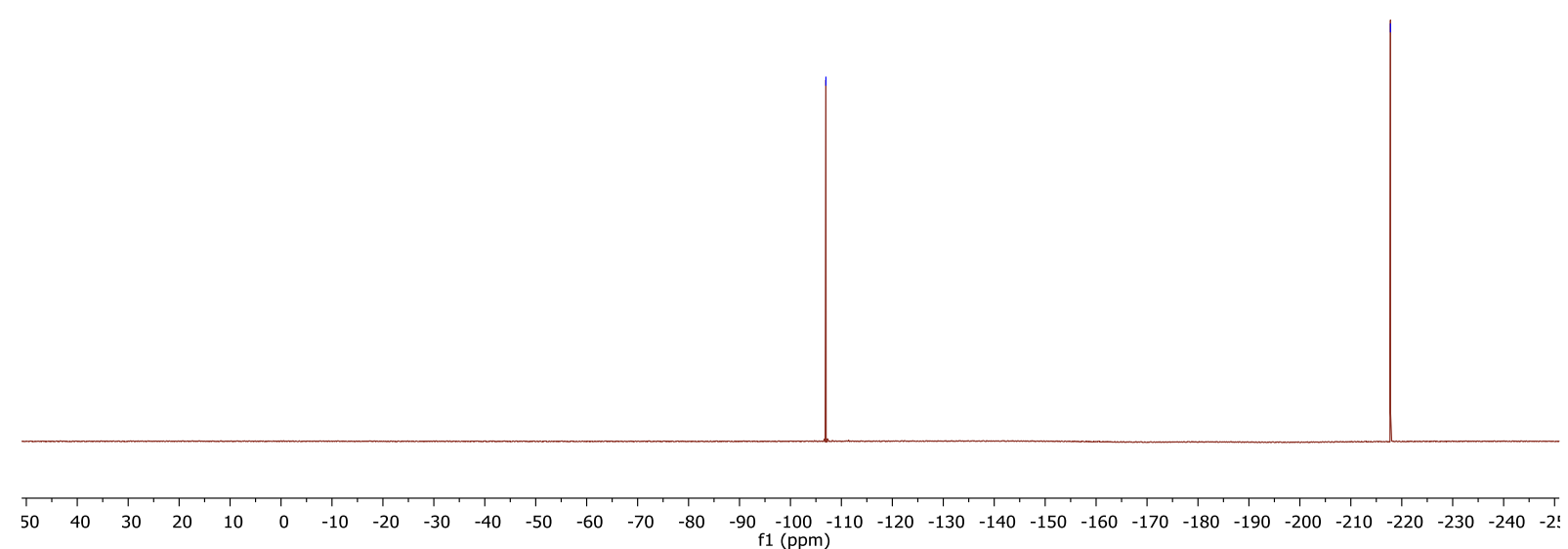

IR

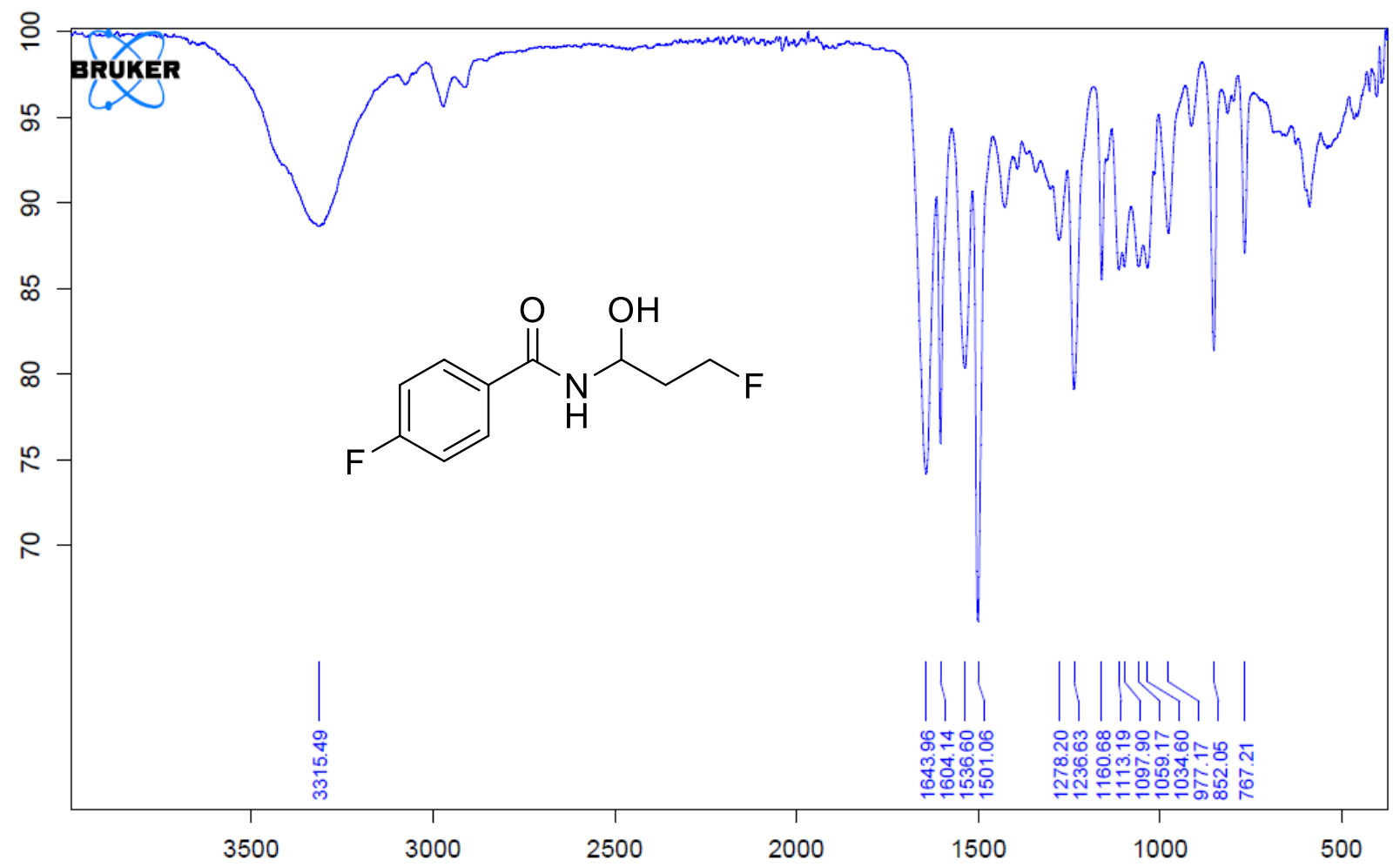

$N$-(3-Fluoro-1-(1H-indol-3-yl)propyl)pivalamide (2g-indole)

${ }^{1}$ H-NMR (400 MHz, $\mathrm{CDCl}_{3}$ ) 


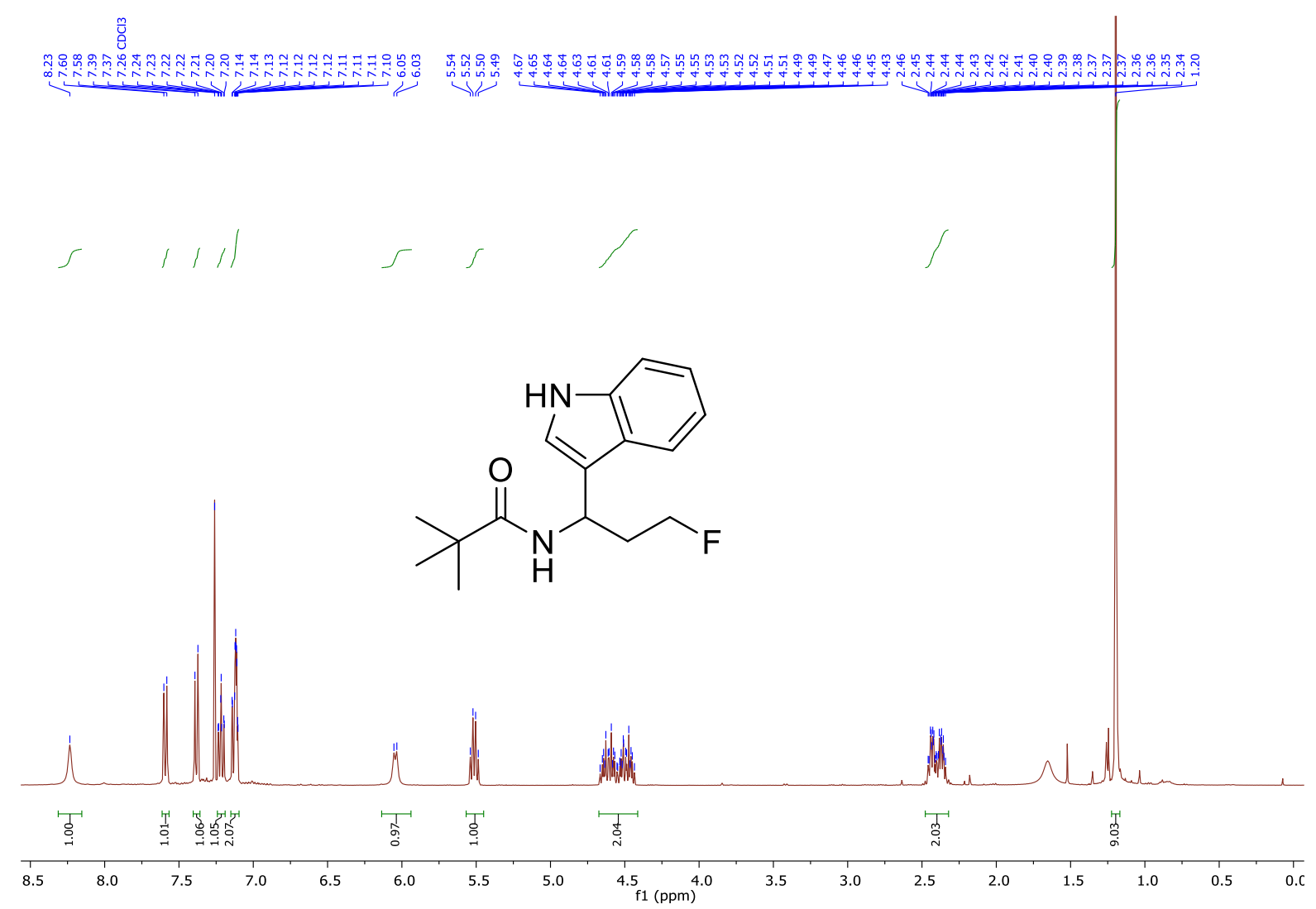

${ }^{13} \mathrm{C}-\mathrm{NMR}$ (101 MHz, CDCl 3$)$
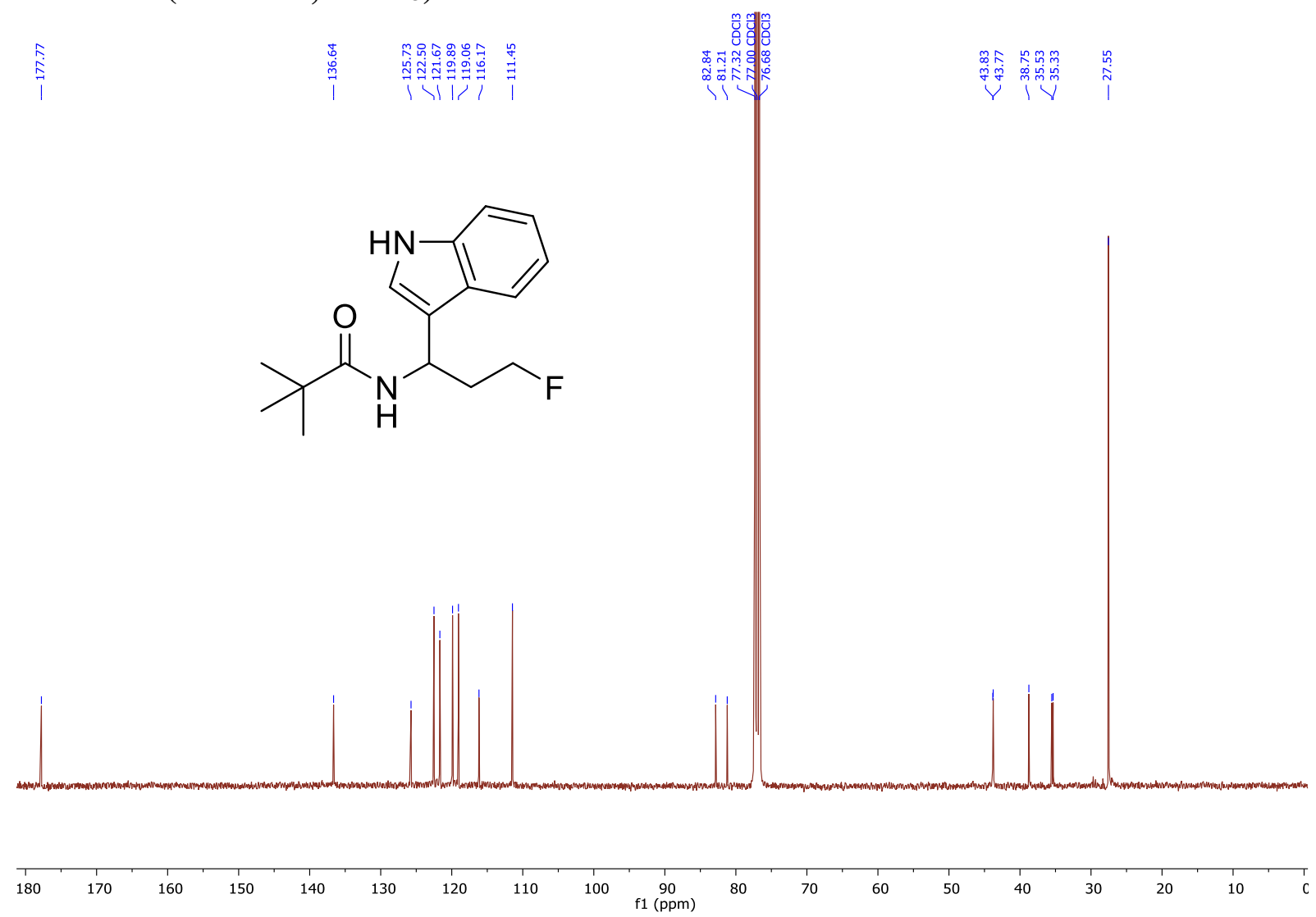

${ }^{19}$ F-NMR (376 MHz, $\left.\mathrm{CDCl}_{3}\right)$ 
<smiles>CC(C)(C)C(=O)NC(CCF)c1c[nH]c2ccccc12</smiles>

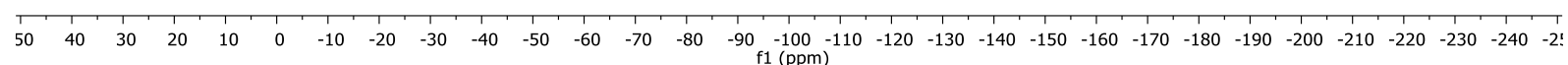

IR

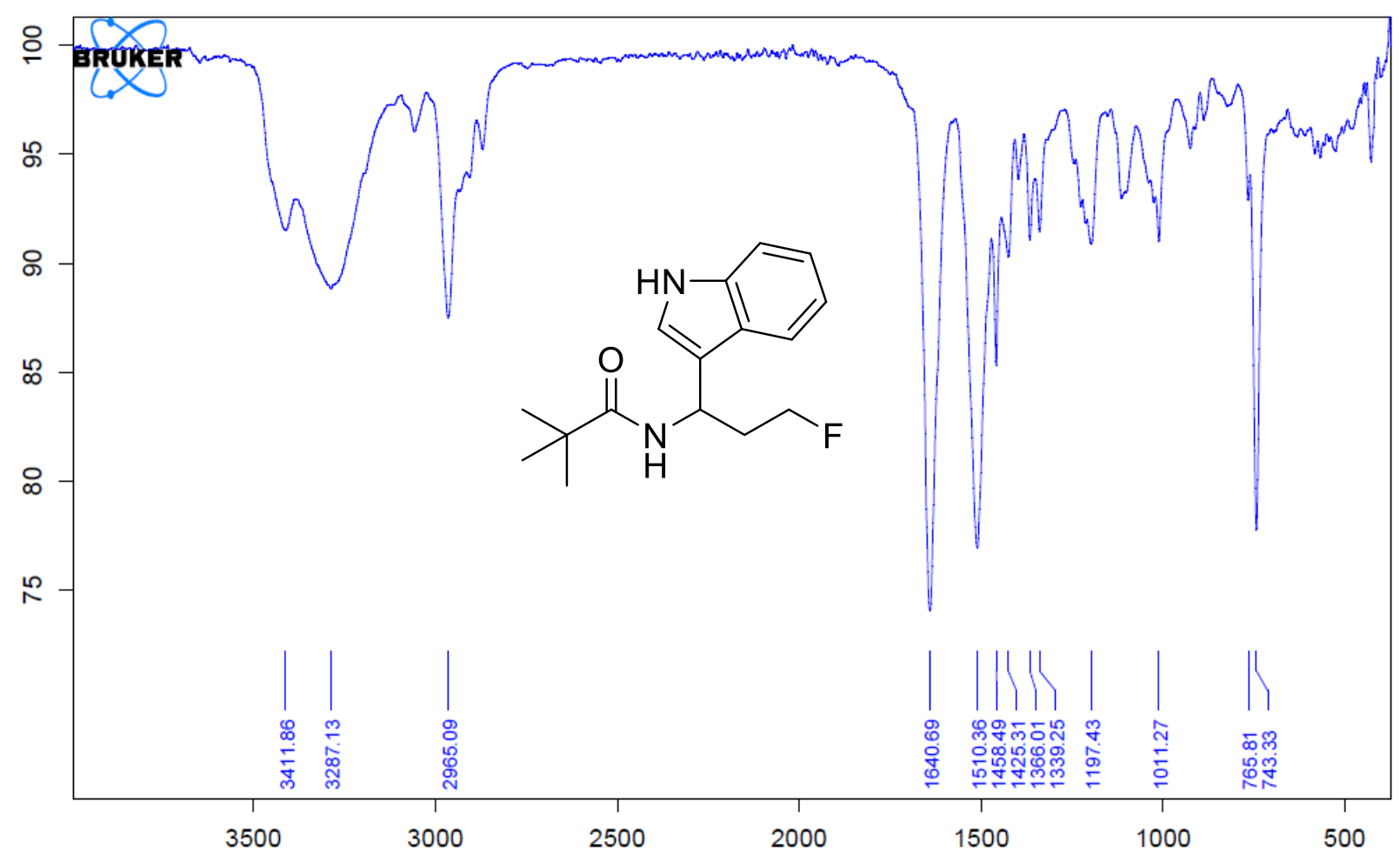

4-Chloro- $N$-(3-fluoro-1-hydroxypropyl)benzamide $(2 \mathbf{j})$

${ }^{1} \mathrm{H}-\mathrm{NMR}$ (400 MHz, $\mathrm{CDCl}_{3}$ ) 


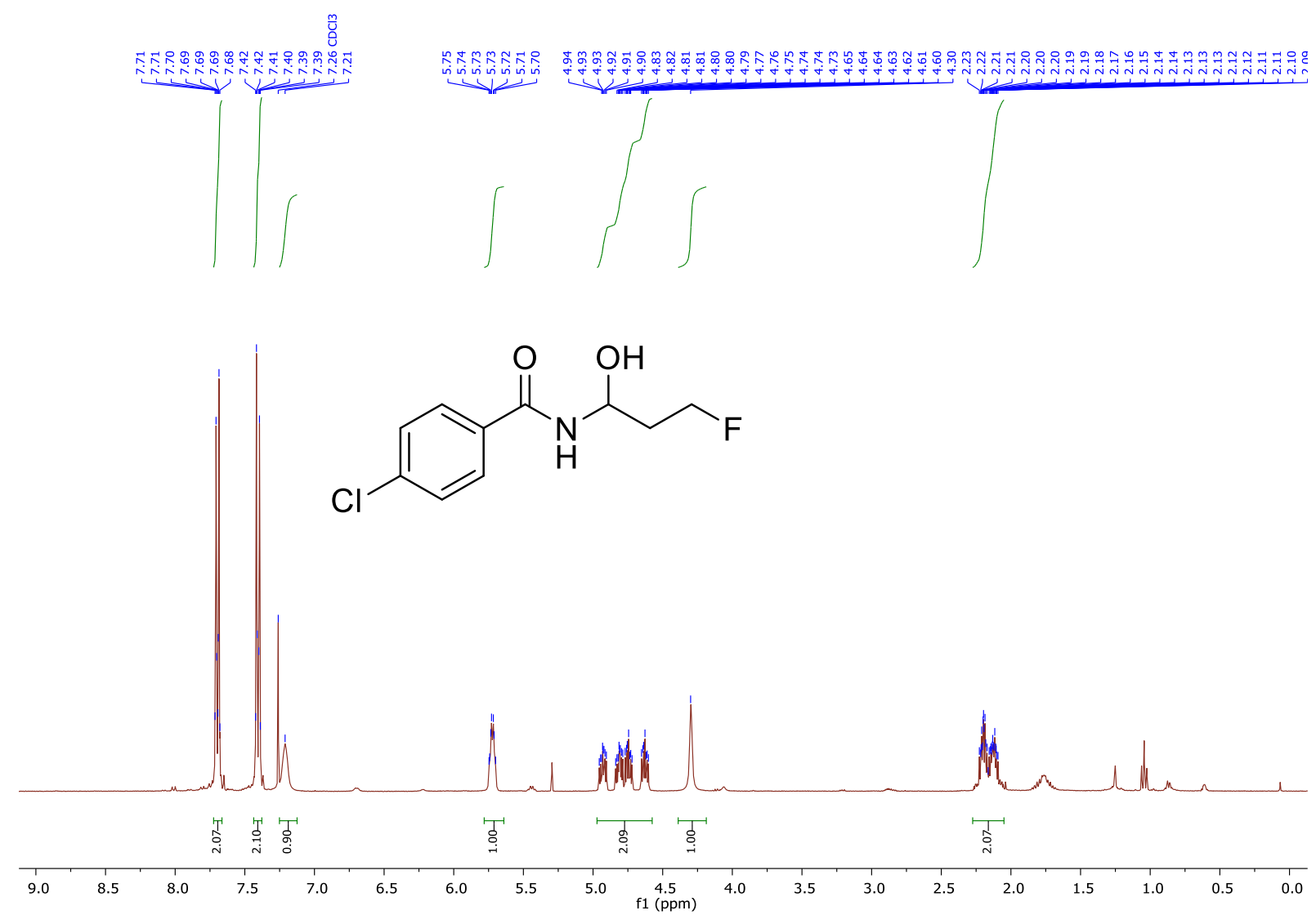

${ }^{13} \mathrm{C}-\mathrm{NMR}$ (101 MHz, $\left.\mathrm{CDCl}_{3}\right)$

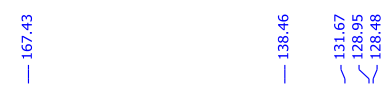

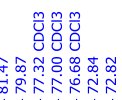

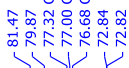

ทั่<smiles>O=C(NC(O)CCF)c1ccc(Cl)cc1</smiles>

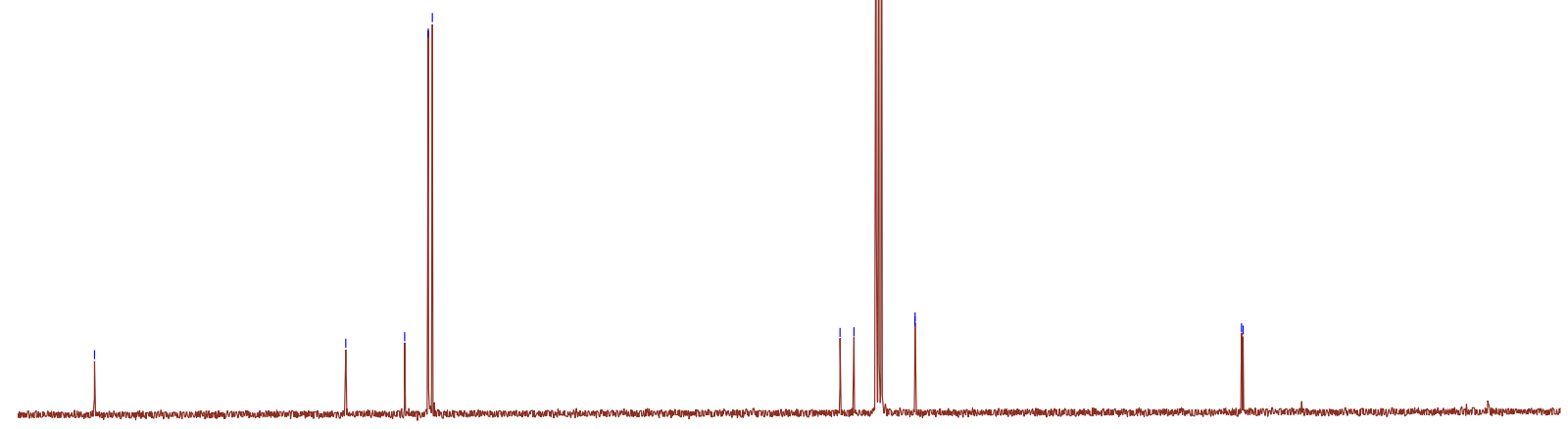

$\begin{array}{lllll}170 & 160 & 150 & 140 & 130\end{array}$

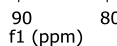

${ }^{19}$ F-NMR (376 MHz, CDCl3) 
<smiles>O=C(NC(O)CCF)c1ccc(Cl)cc1</smiles>

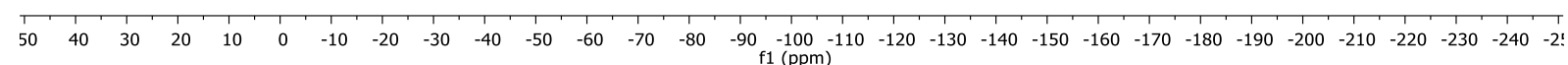

IR

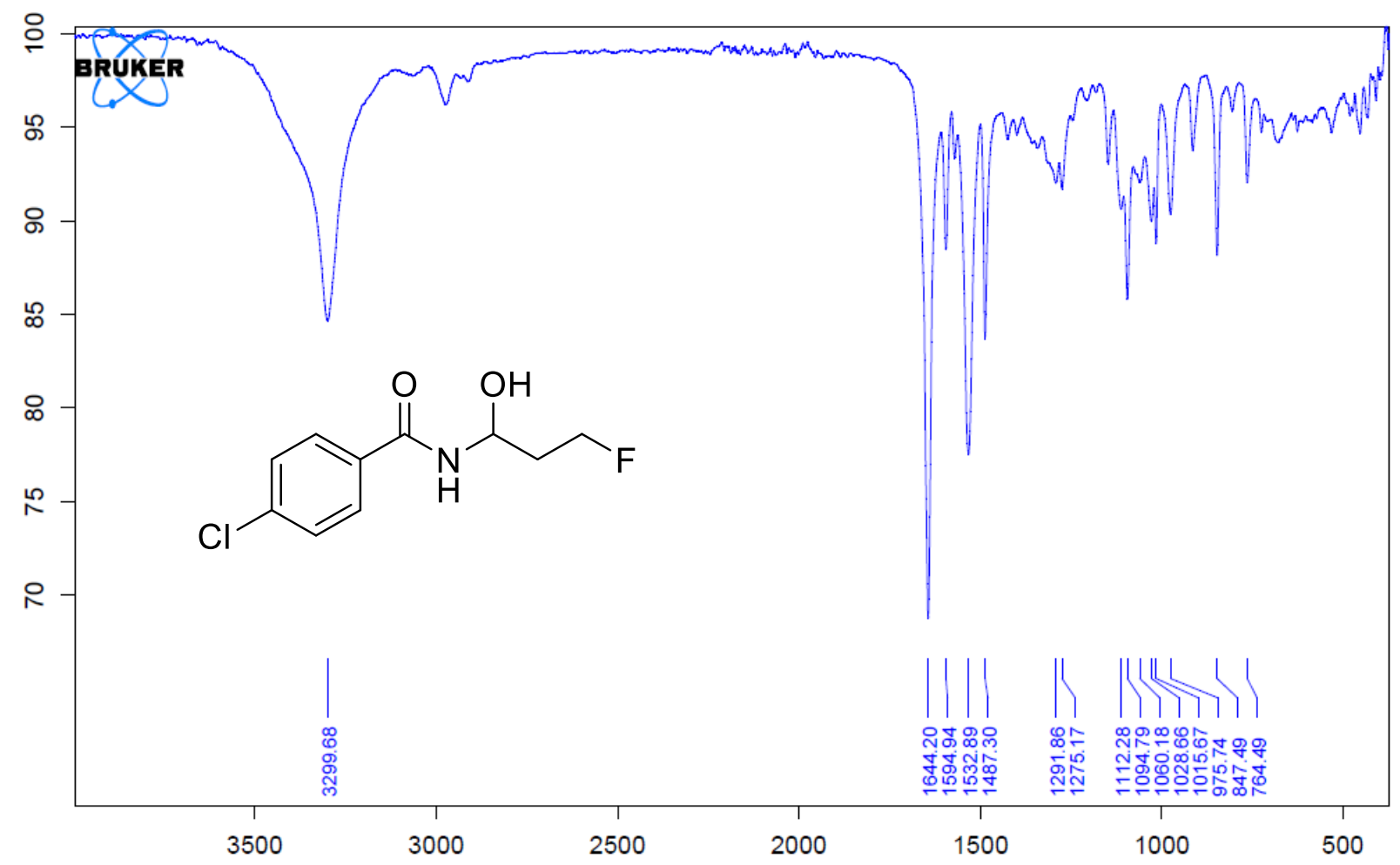

$N$-(3-Fluoro-1-hydroxypropyl)benzamide (2k)

${ }^{1}$ H-NMR (400 MHz, Acetone-d $d_{6}$ ) 


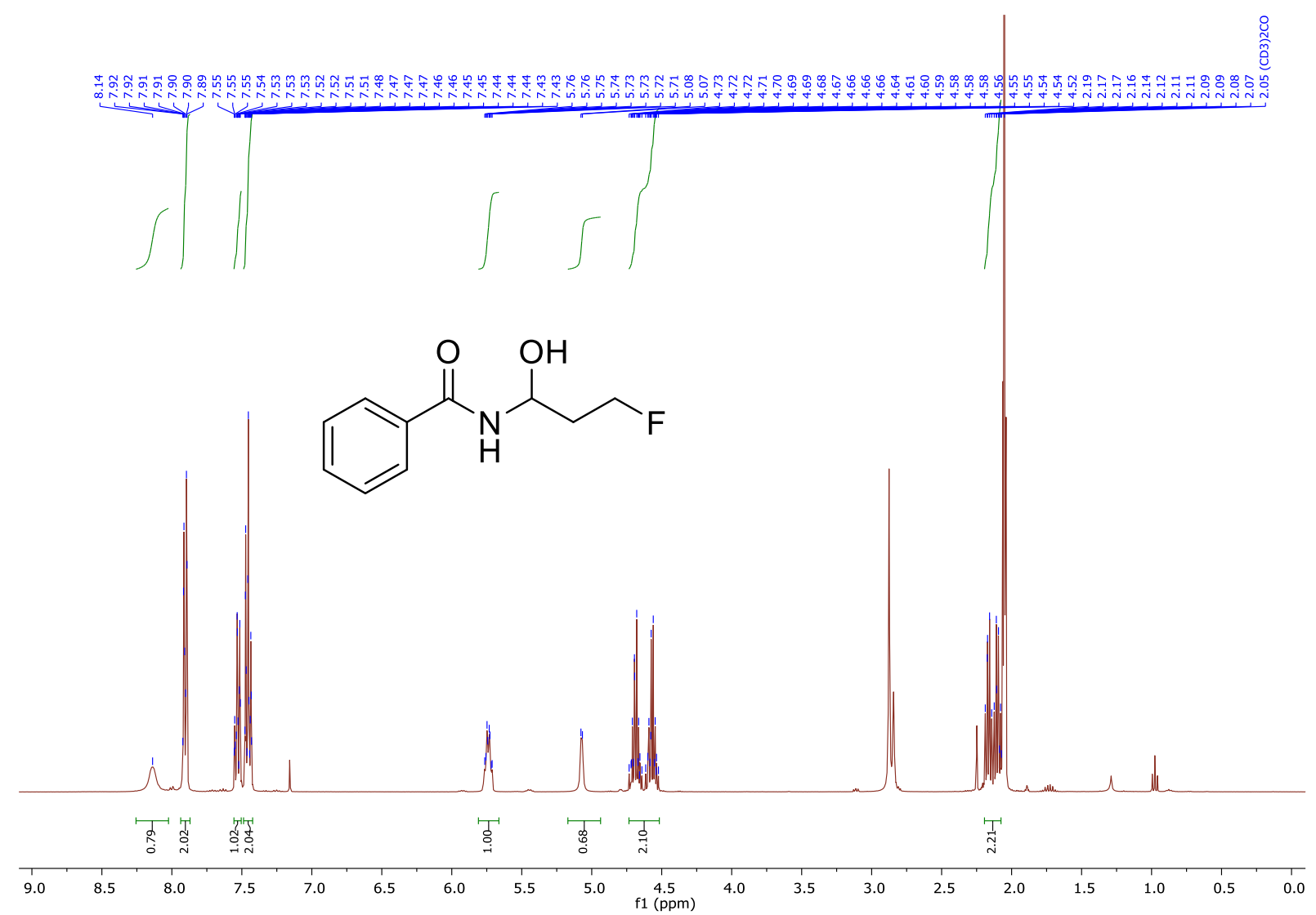

${ }^{13}$ C-NMR (101 MHz, Acetone-d6)
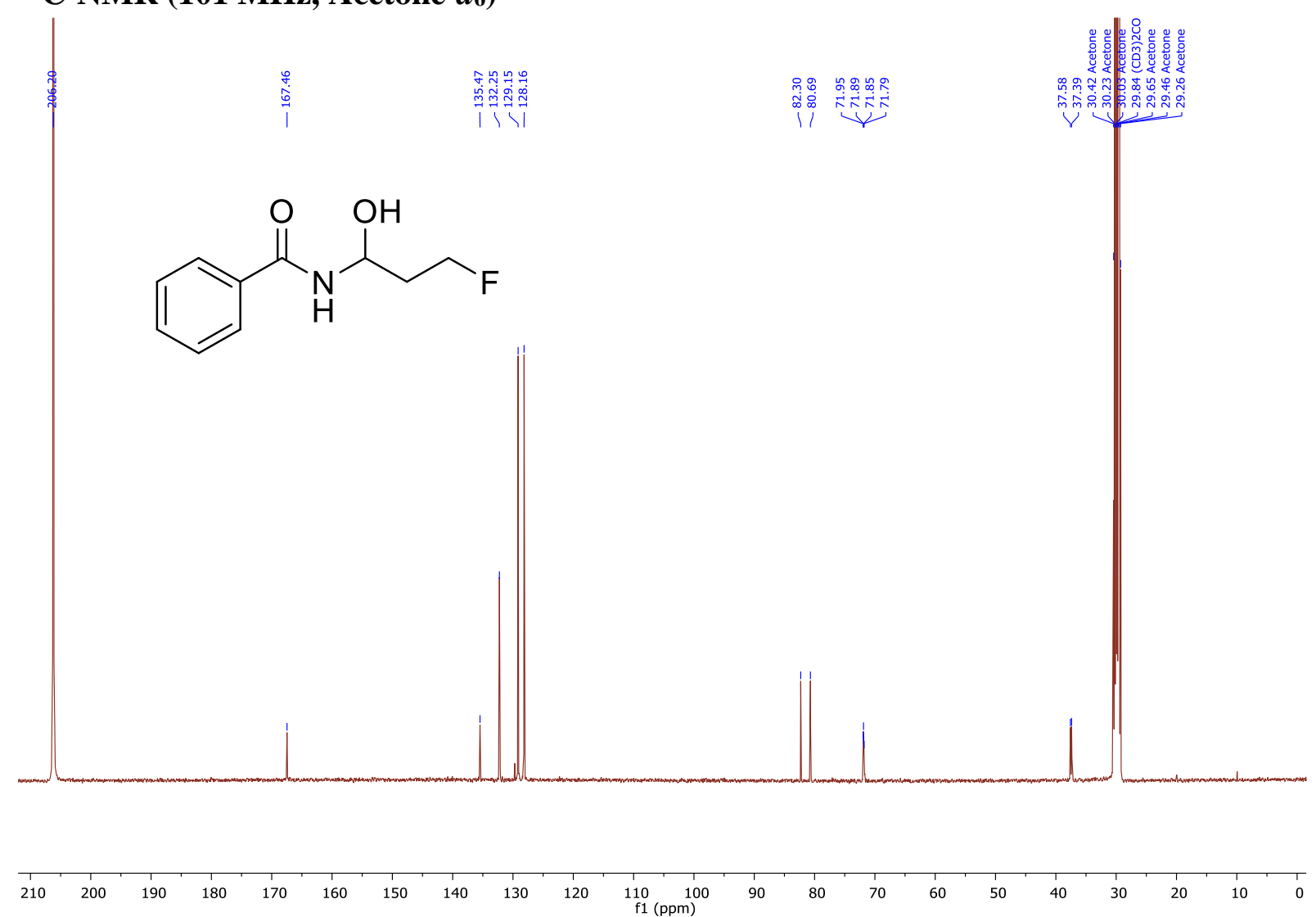

${ }^{19}$ F-NMR (376 MHz, Acetone-d6) 
<smiles>O=C(NC(O)CCF)c1ccccc1</smiles>

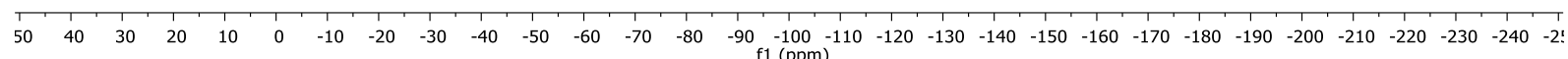

IR

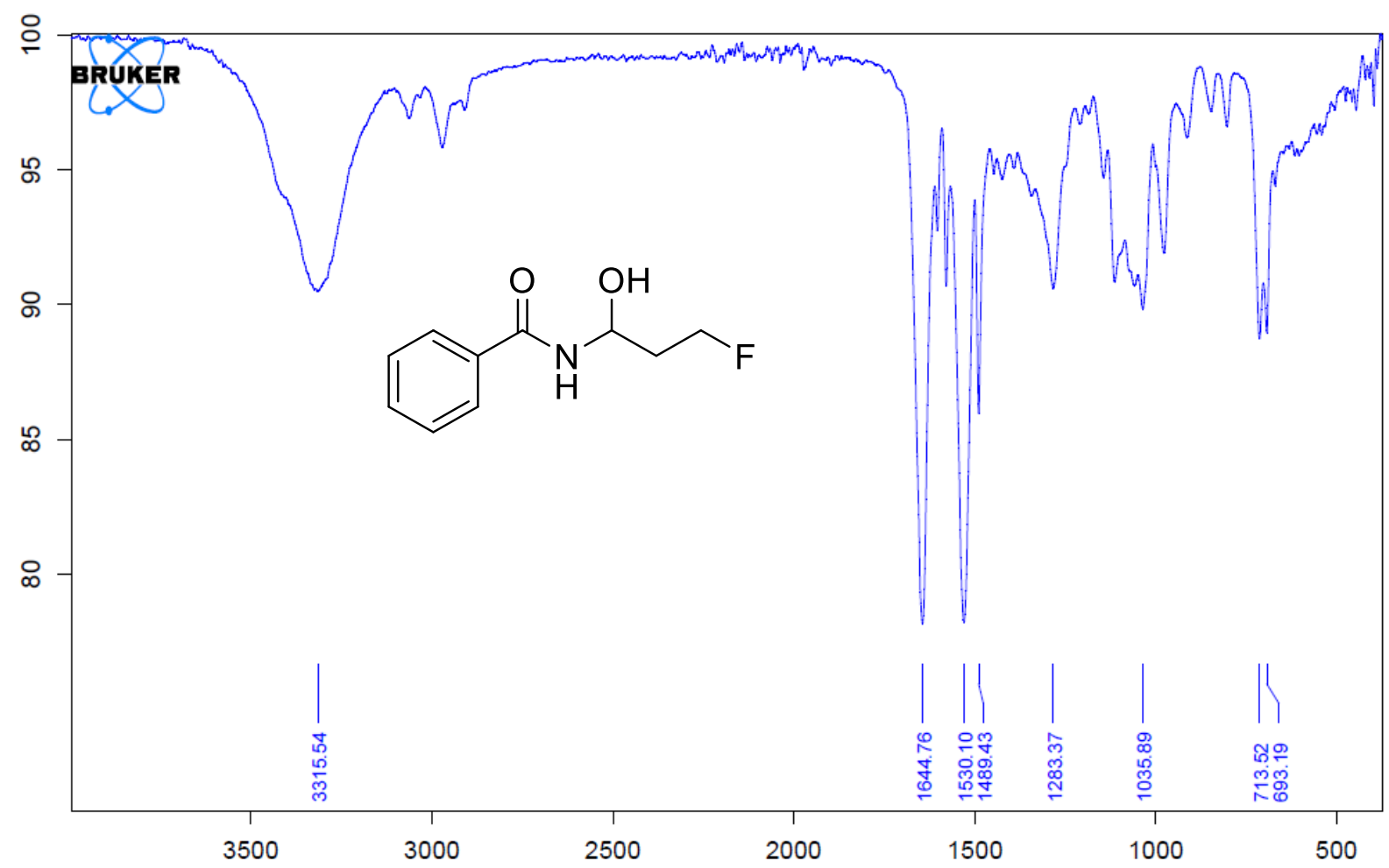

N-Cyclopropyl-4-(methylsulfinyl)benzamide (2i)

${ }^{1} \mathrm{H}-\mathrm{NMR}$ (400 MHz, $\mathrm{CDCl}_{3}$ ) 


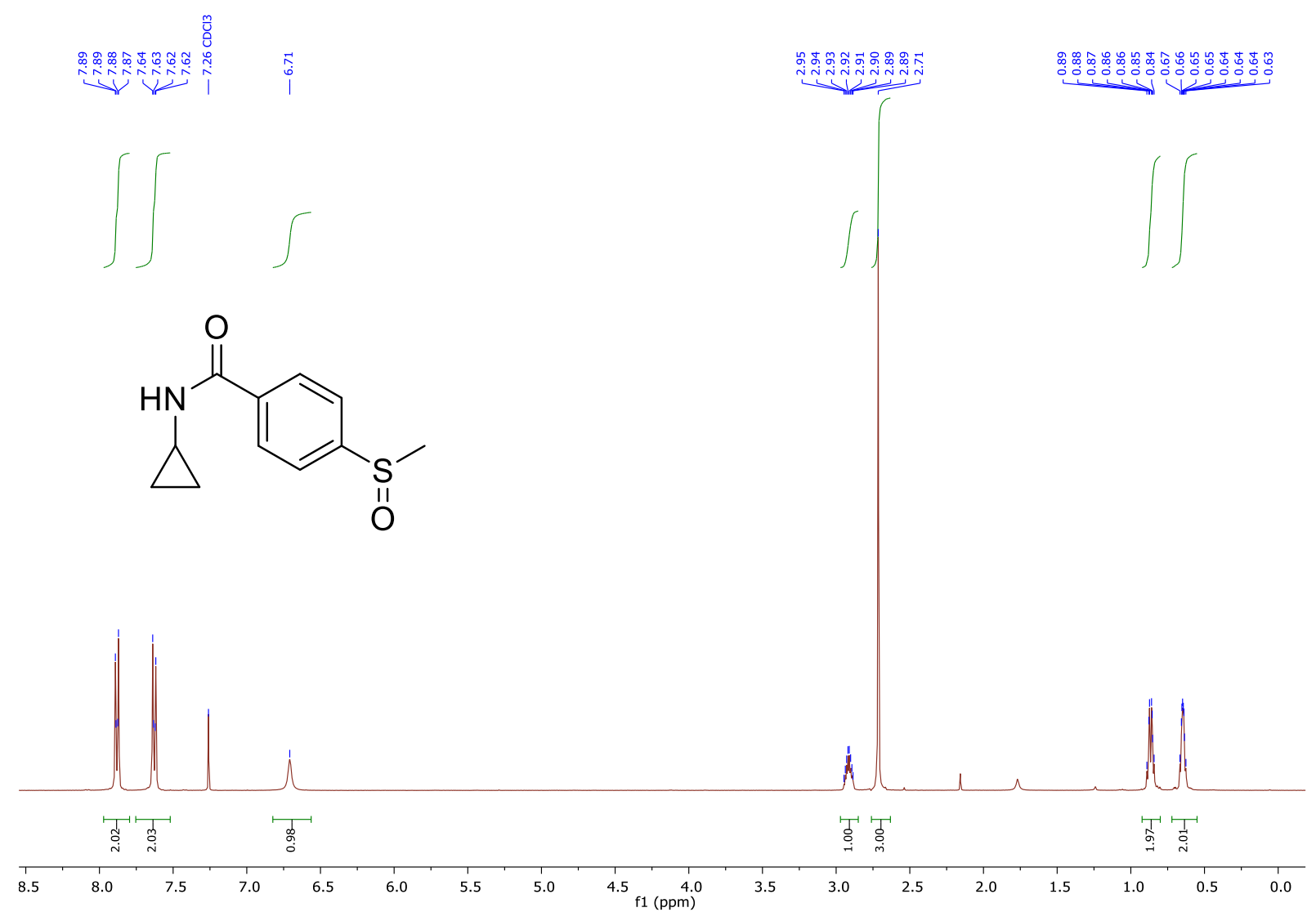

${ }^{13} \mathrm{C}-\mathrm{NMR}$ (101 MHz, CDCl3)<smiles>CS(=O)c1ccc(C(=O)NC2CC2)cc1</smiles>
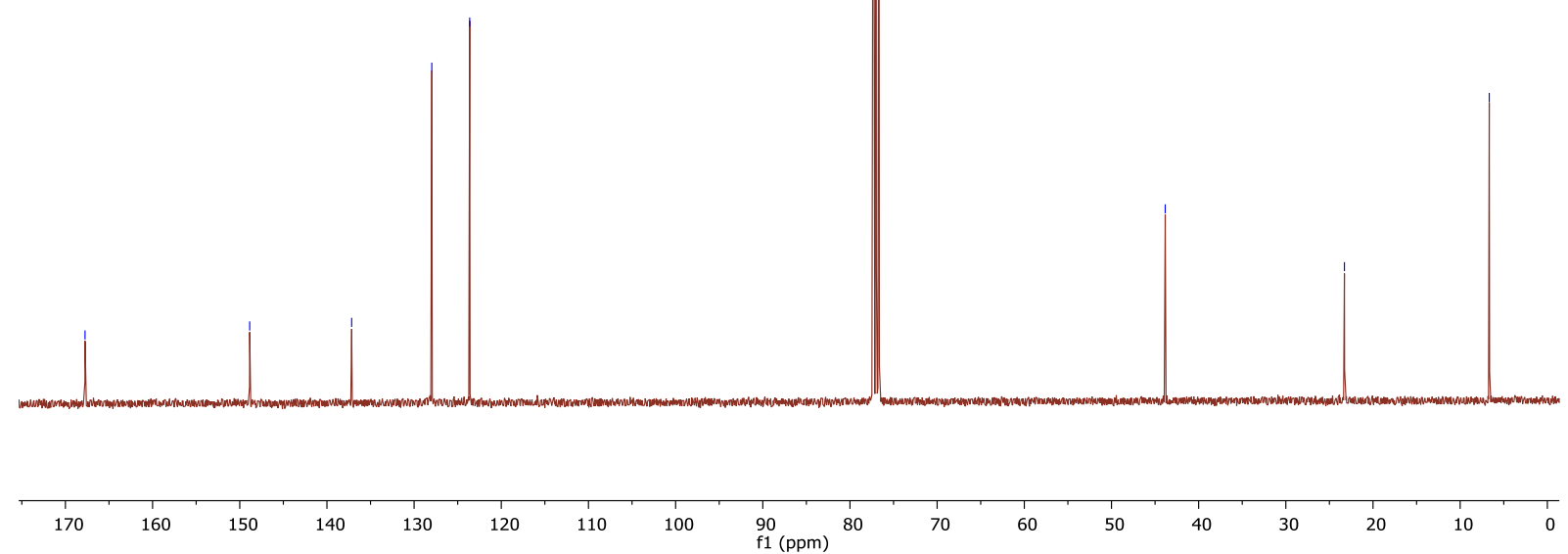

IR 


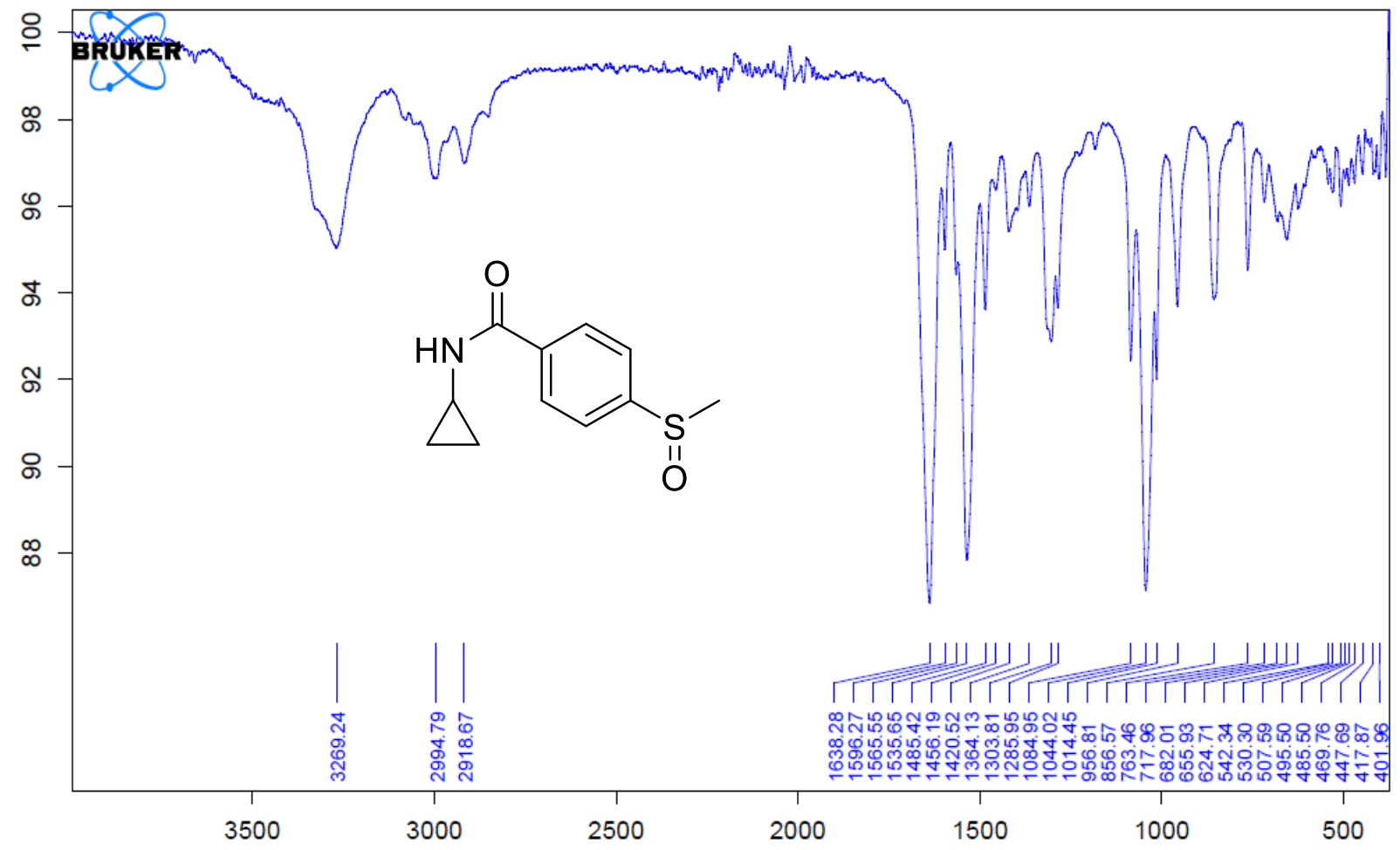

$N$-(3-Fluoro-1-methoxypropyl)-4-methoxybenzamide (3a)

${ }^{1} \mathrm{H}-\mathrm{NMR}$ (400 MHz, $\mathrm{CDCl}_{3}$ )

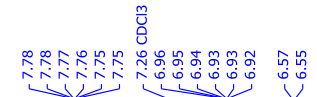

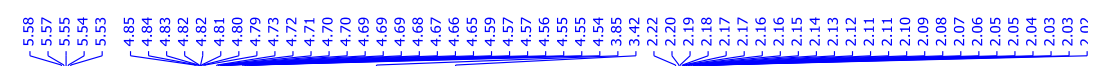<smiles>C1CC2CCC(C1)C2</smiles>
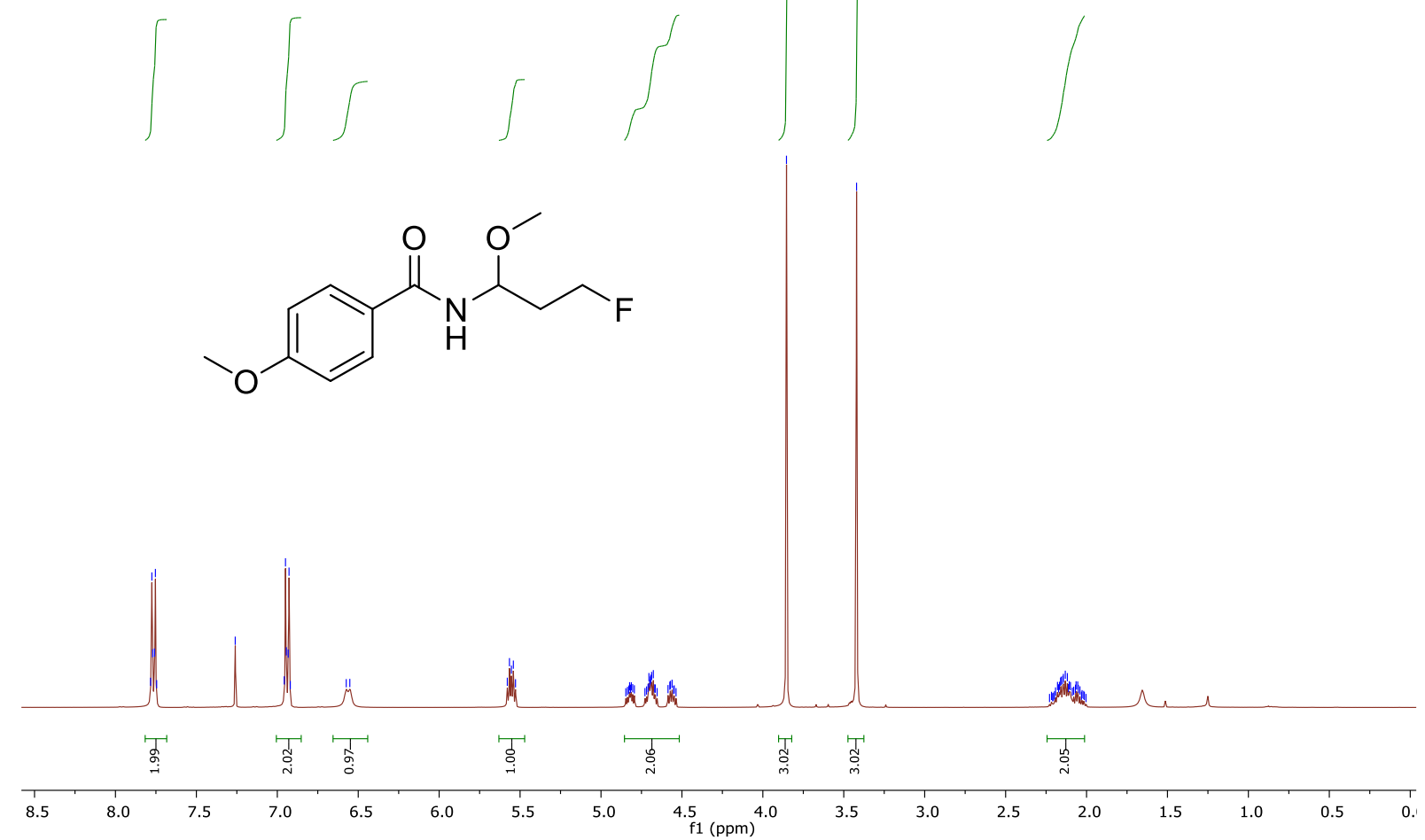

${ }^{13}$ C-NMR (101 MHz, $\left.\mathrm{CDCl}_{3}\right)$ 


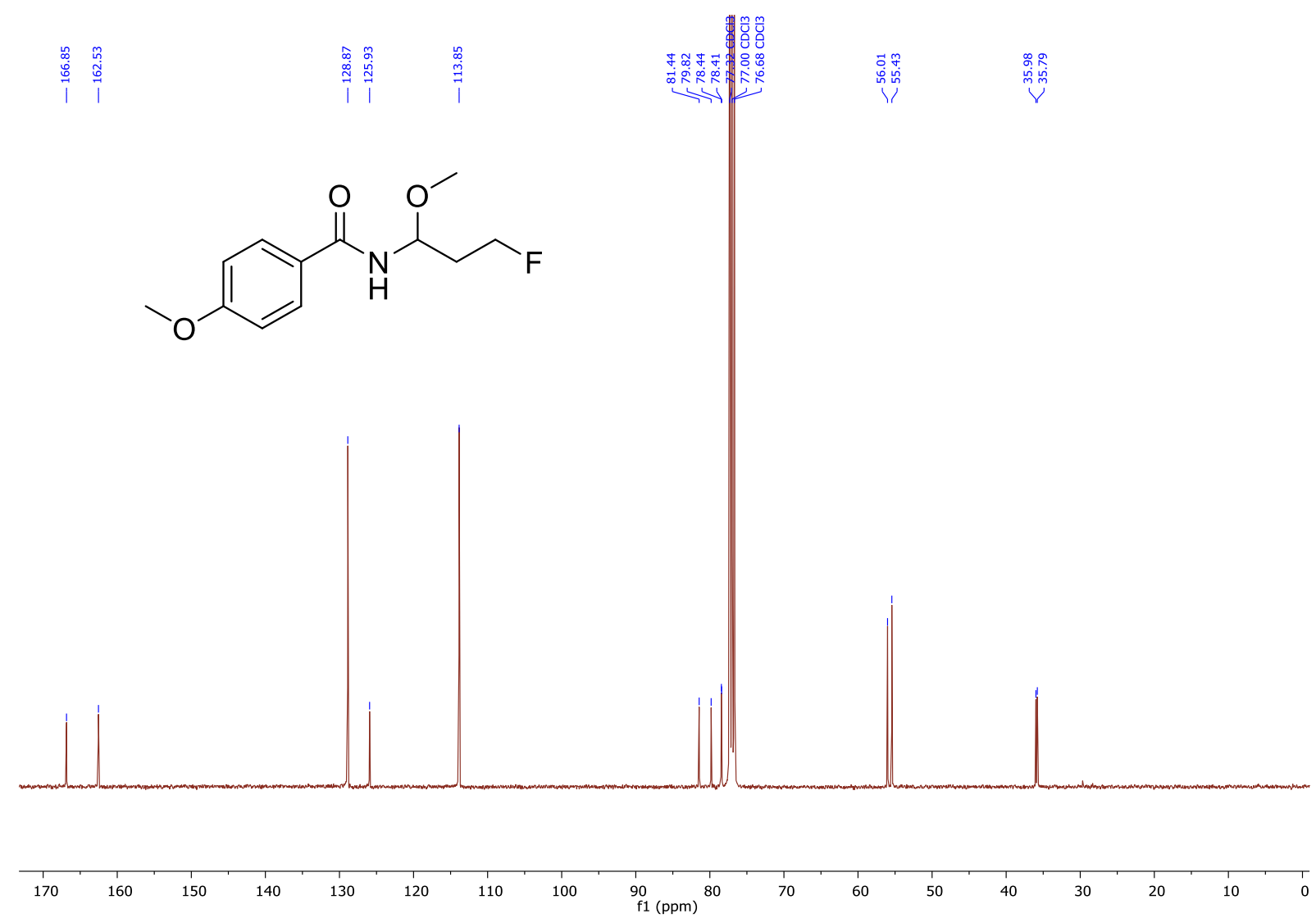

${ }^{19}$ F-NMR (376 MHz, CDCl3)<smiles>COc1ccc(C(=O)NC(CCF)OC)cc1</smiles>

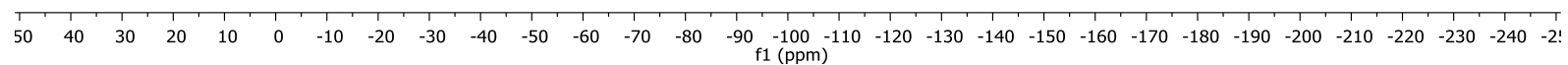
IR 


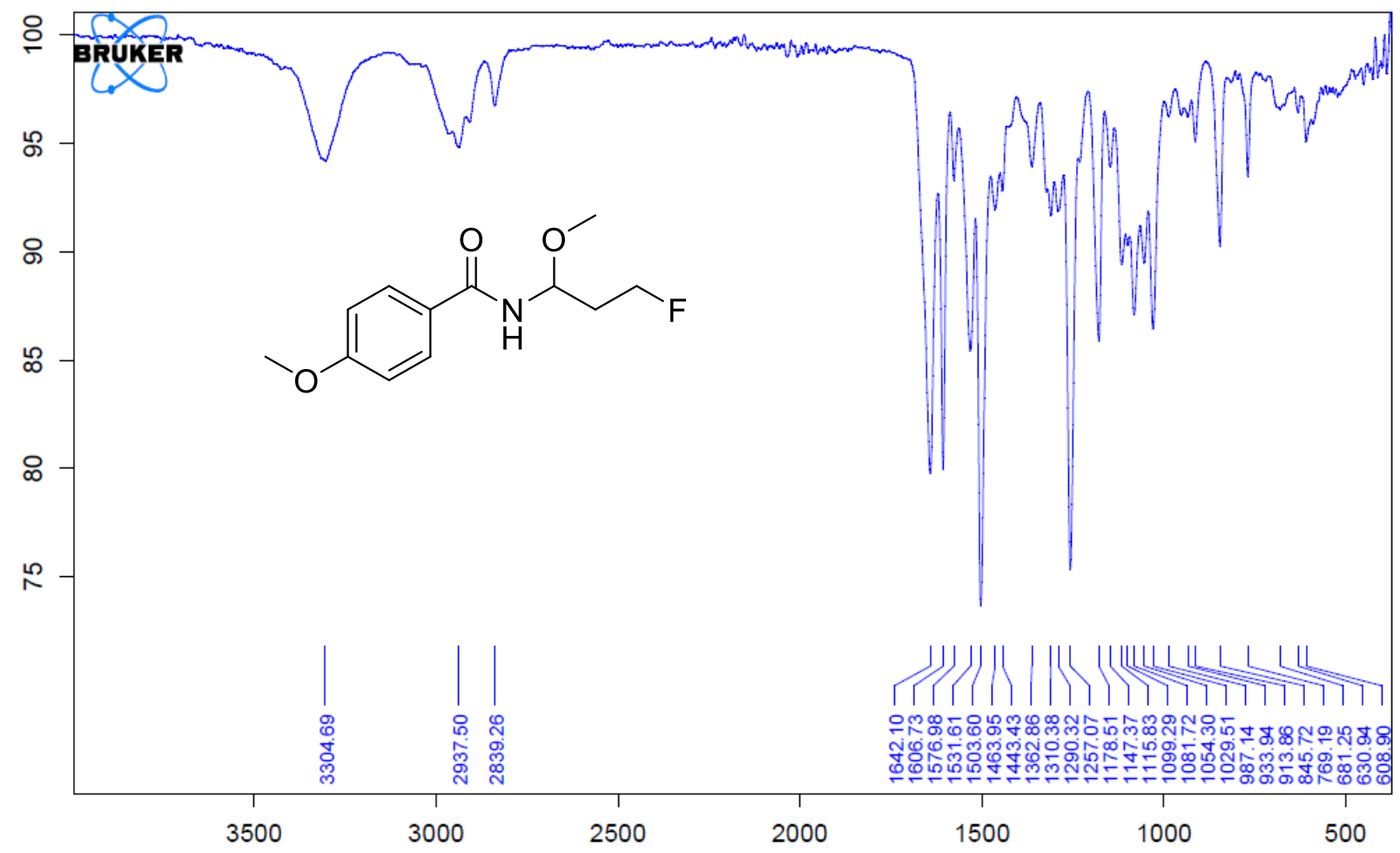

$N$-(3-Fluoro-1-hydroperoxypropyl)-4-methoxybenzamide (3b)

${ }^{1}$ H-NMR (400 MHz, Acetone-d6)

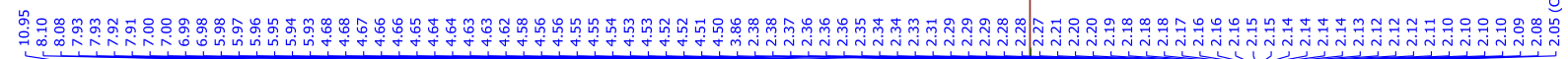

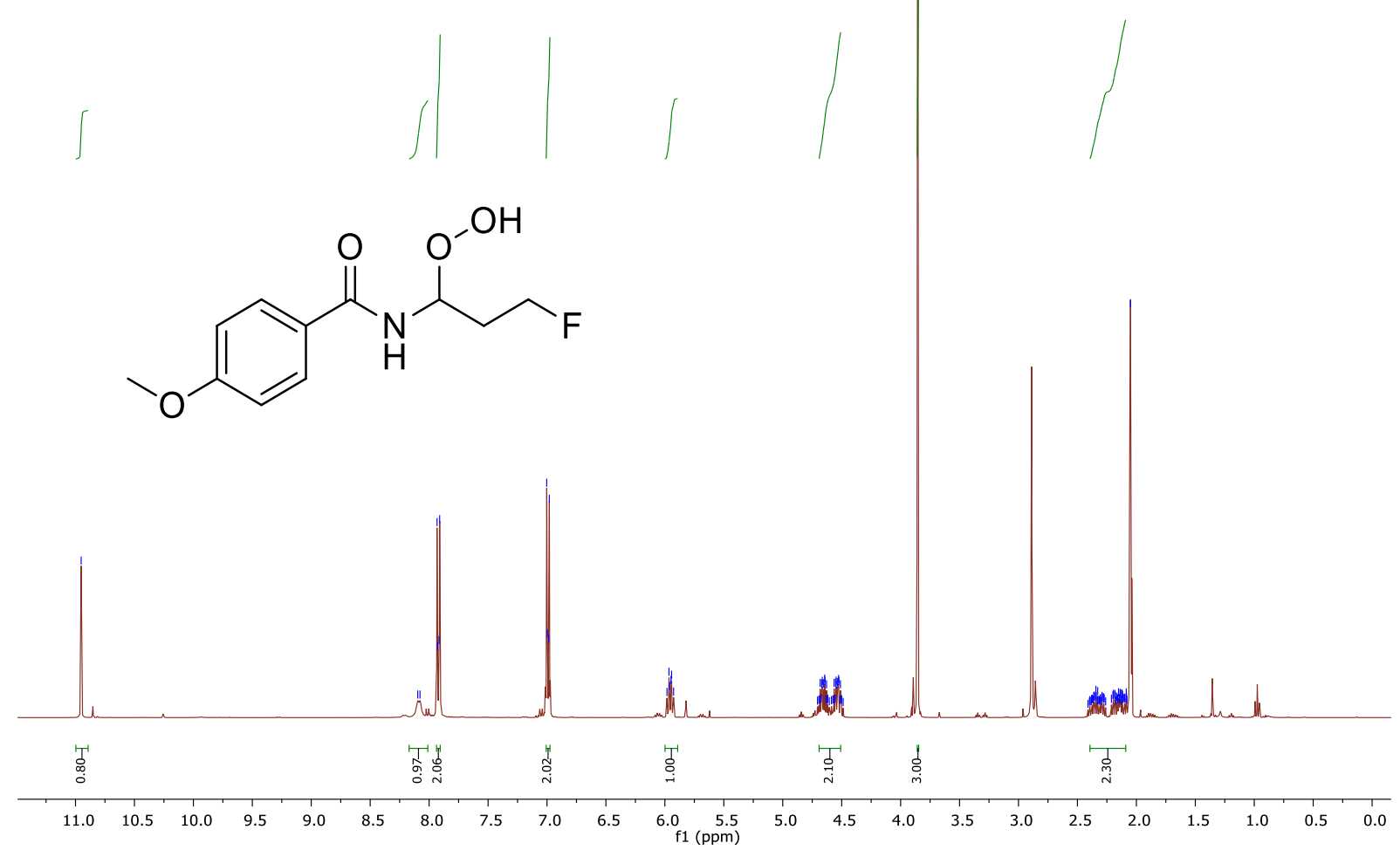

${ }^{13}$ C-NMR (101 MHz, Acetone- $\left.d_{6}\right)$ 

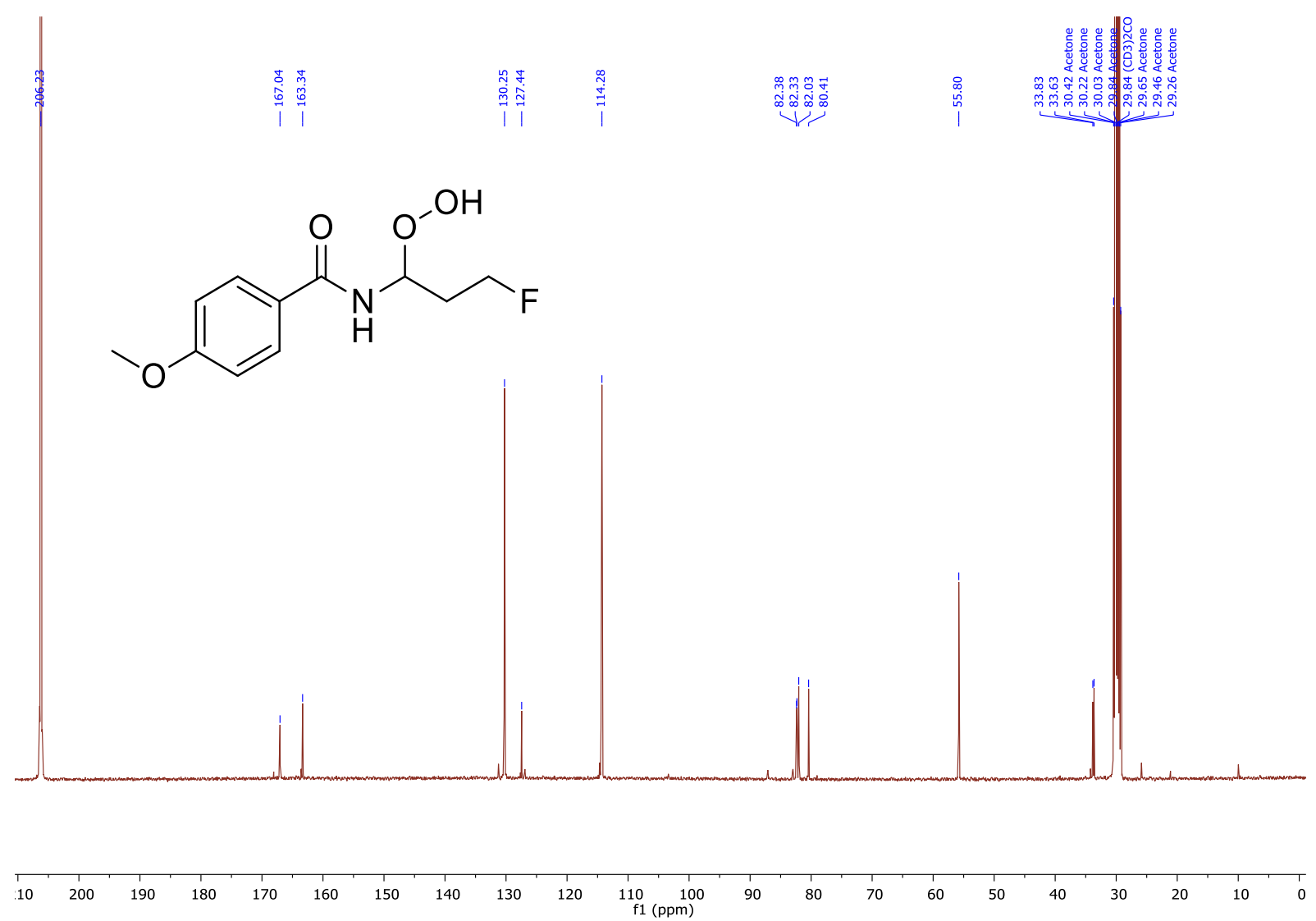

${ }^{19}$ F-NMR (376 MHz, Acetone-d6)<smiles>COc1ccc(C(=O)NC(CCF)OO)cc1</smiles>

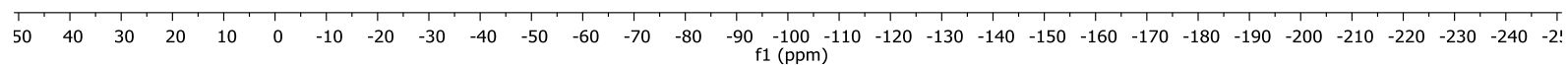
IR 


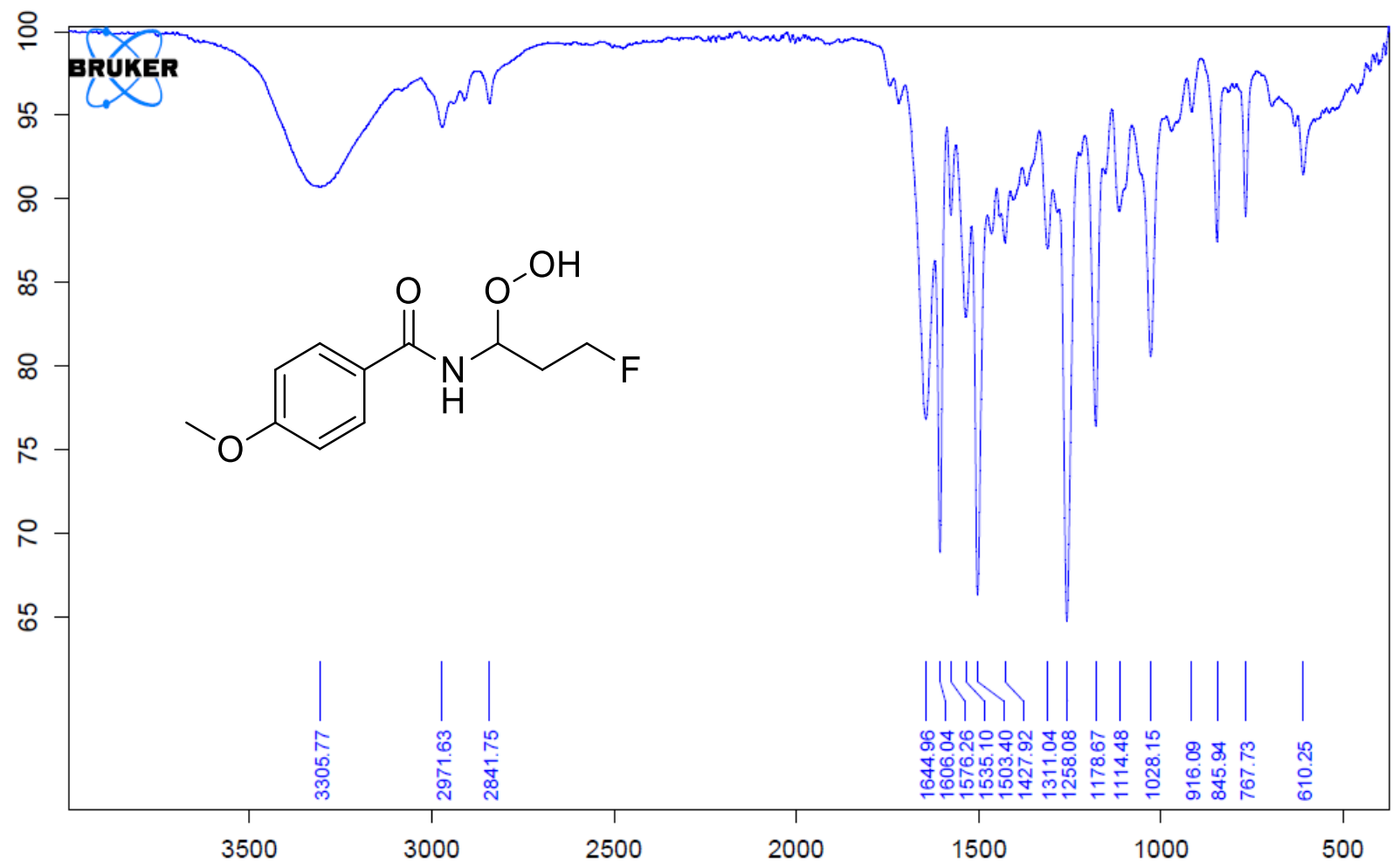

$N$-(3-Fluoro-1-(p-tolylthio)propyl)-4-methoxybenzamide (3c)

${ }^{1} \mathrm{H}-\mathrm{NMR}$ (400 MHz, $\mathrm{CDCl}_{3}$ )

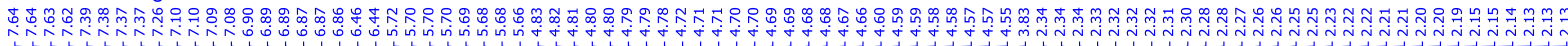

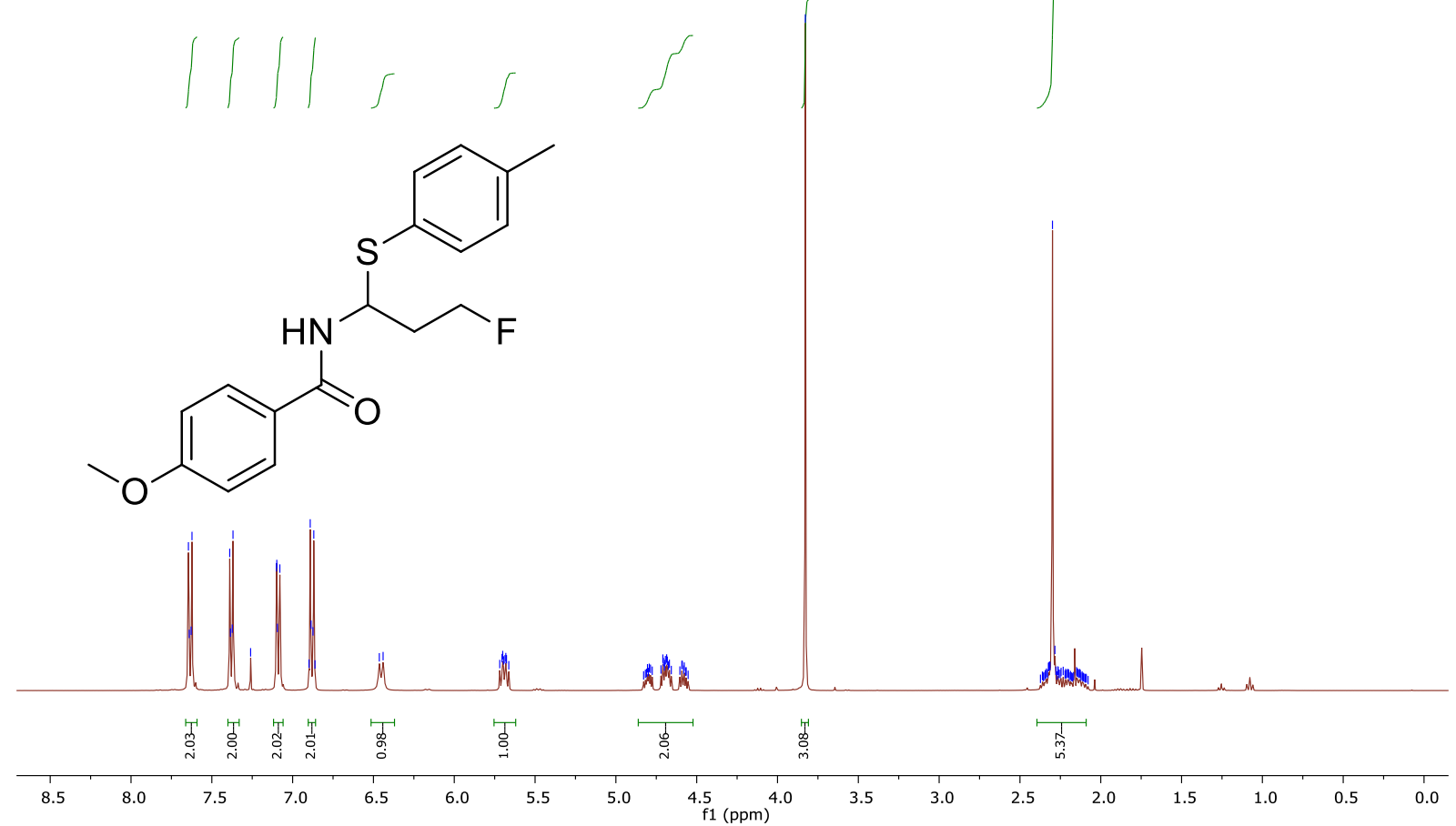

${ }^{13} \mathrm{C}$-NMR (101 MHz, $\left.\mathrm{CDCl}_{3}\right)$ 

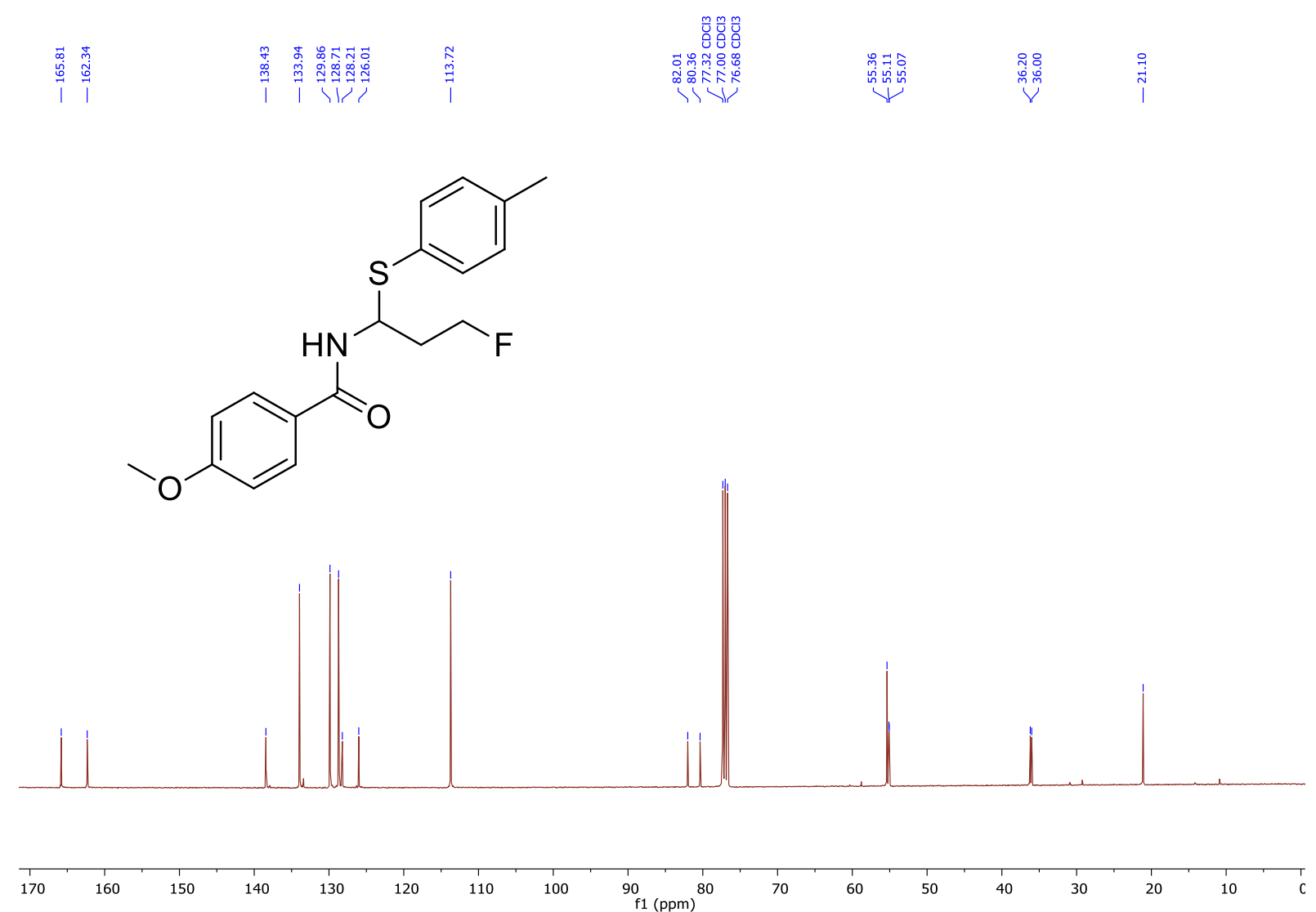

${ }^{19}$ F-NMR (376 MHz, CDCl3)<smiles>COc1ccc(C(=O)NC(CCF)Sc2ccc(C)cc2)cc1</smiles>

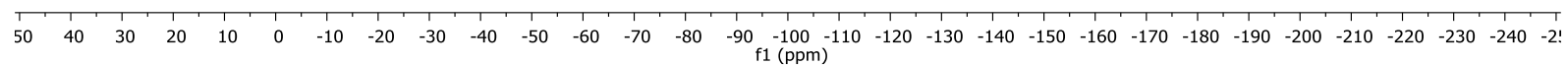
IR 


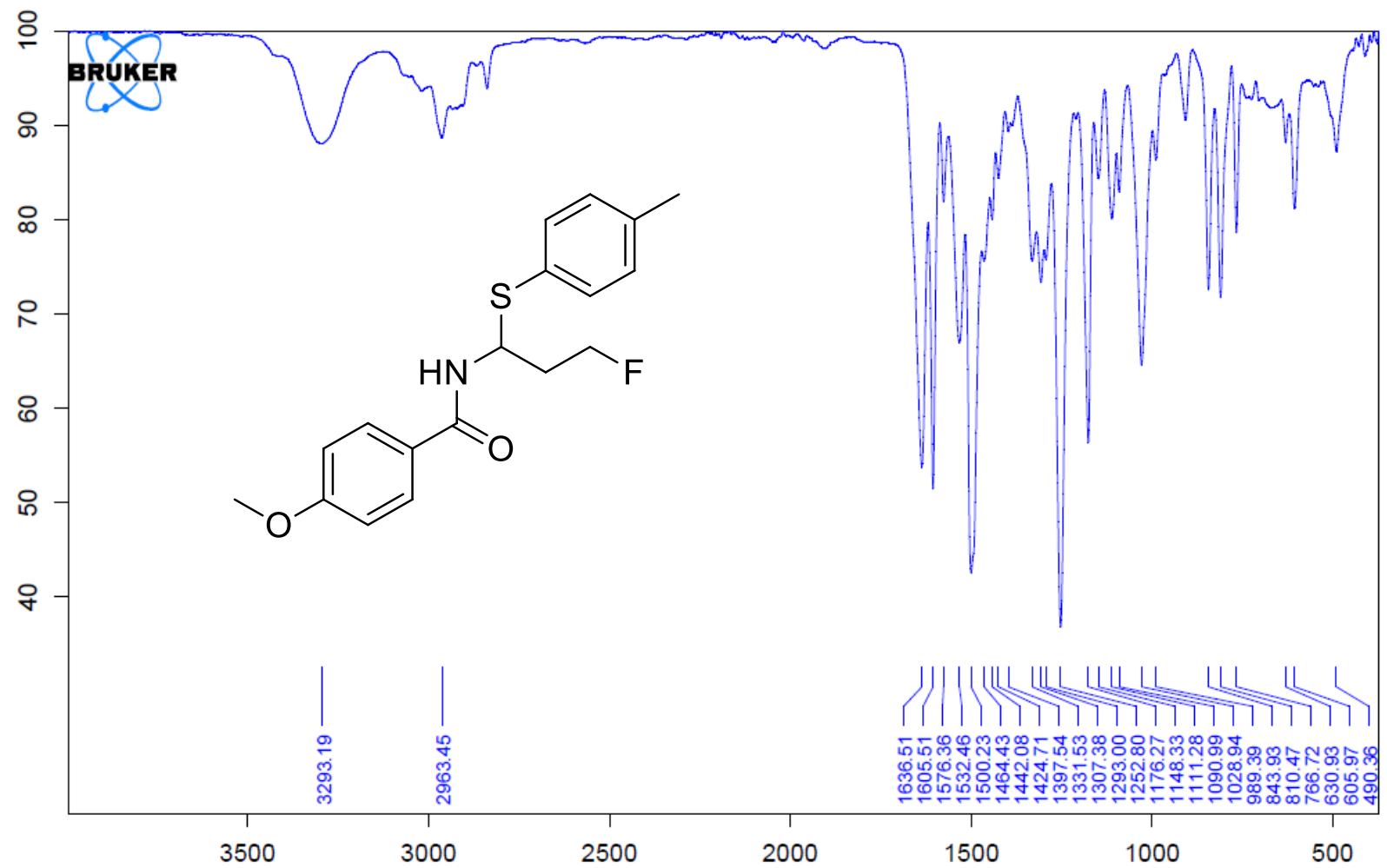

$N$-(3-Fluoro-1-(1H-pyrazol-1-yl)propyl)-4-methoxybenzamide (3d)

${ }^{1} \mathrm{H}-\mathrm{NMR}$ (400 MHz, $\mathrm{CDCl}_{3}$ )

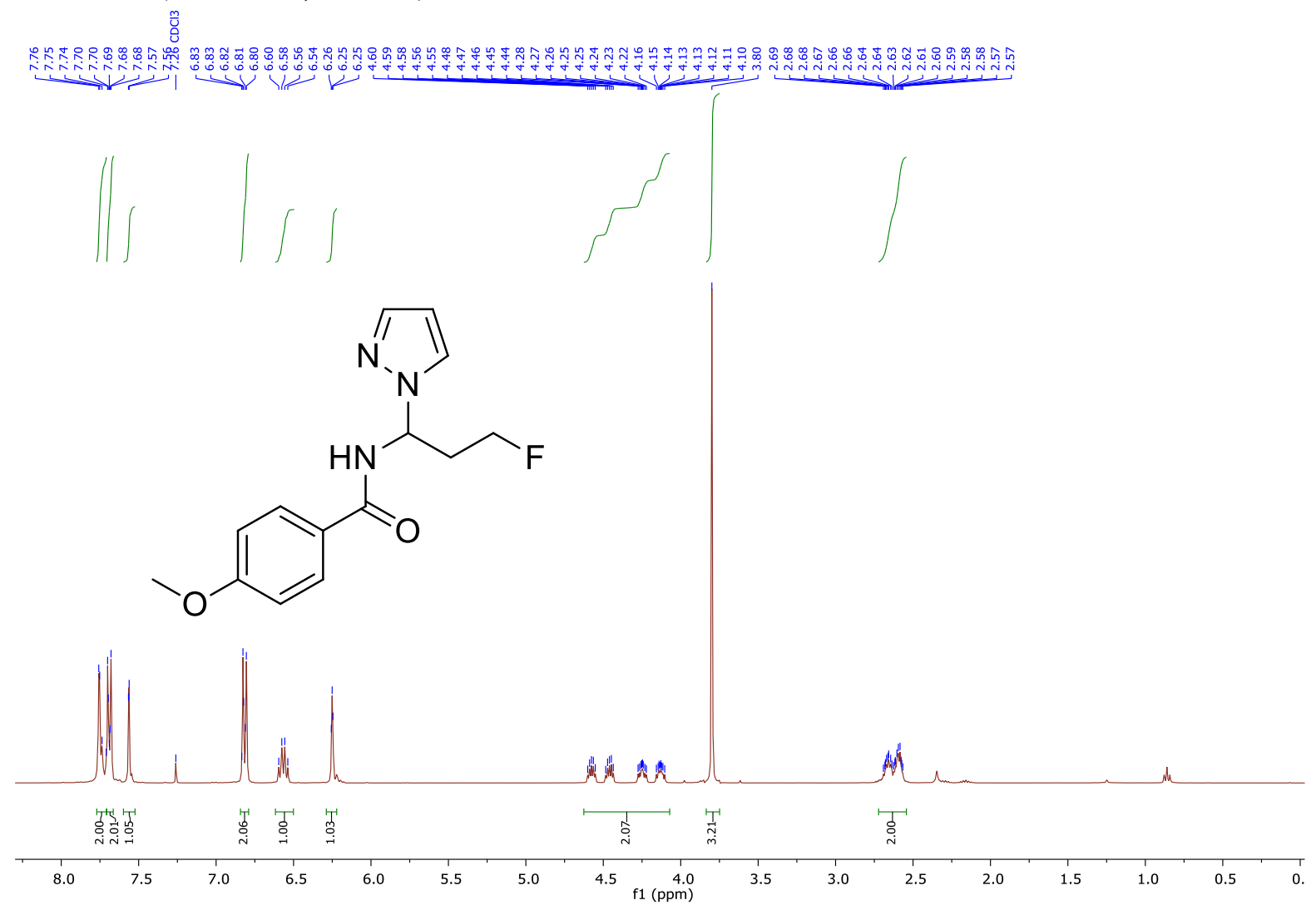

${ }^{13}$ C-NMR (101 MHz, CDCl3) 

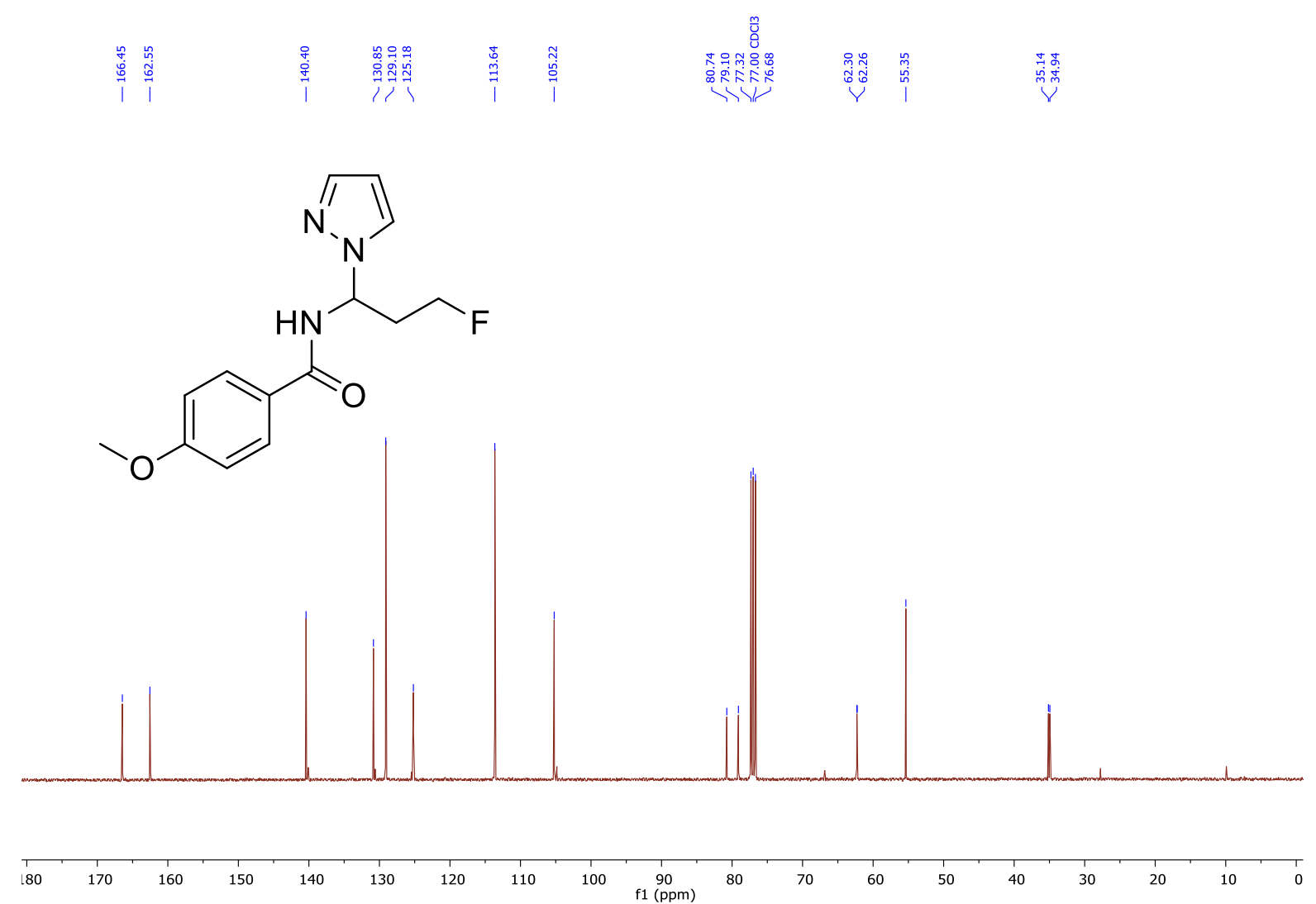

${ }^{19}$ F-NMR (376 MHz, CDCl 3 )

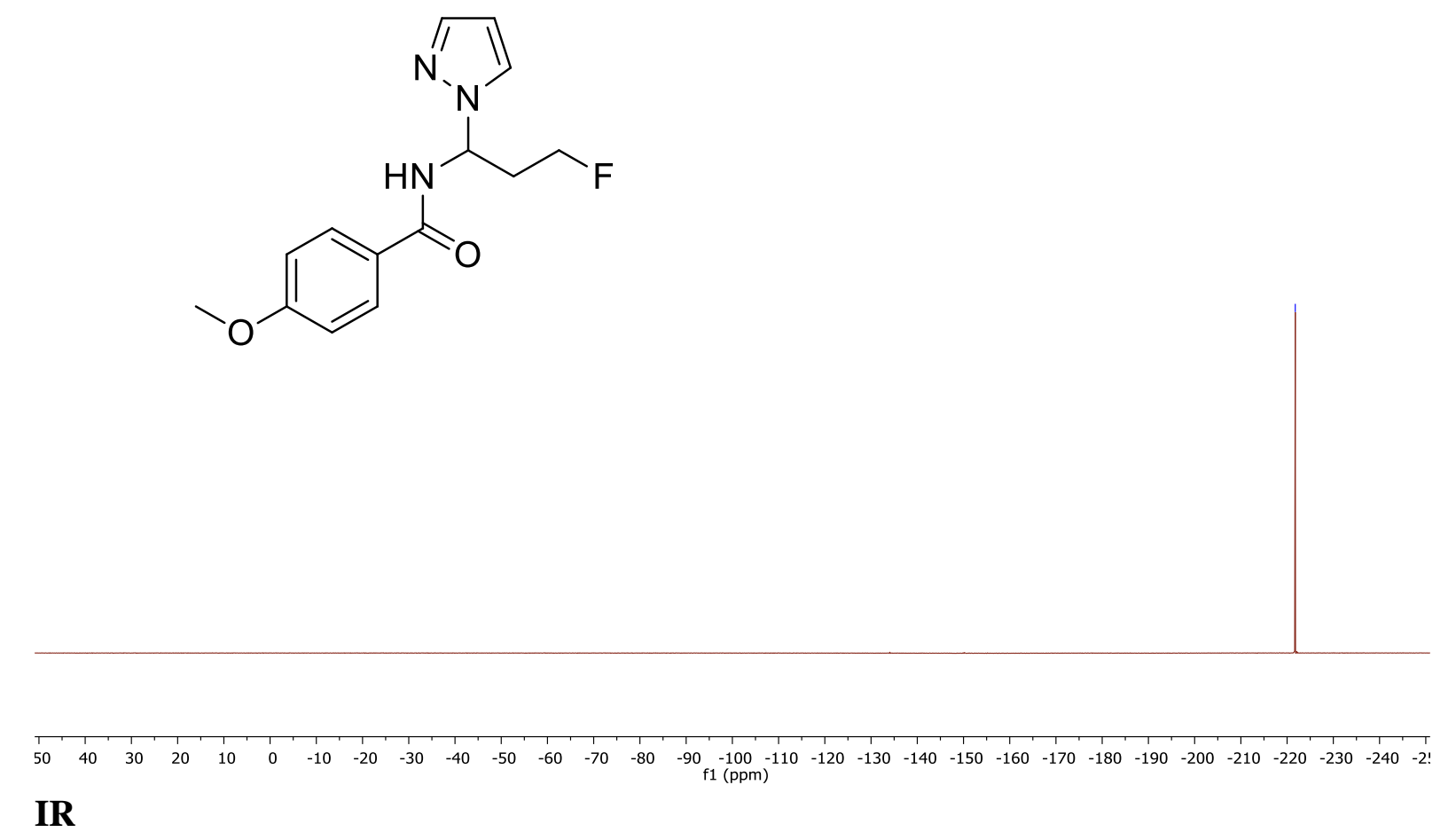

IR 


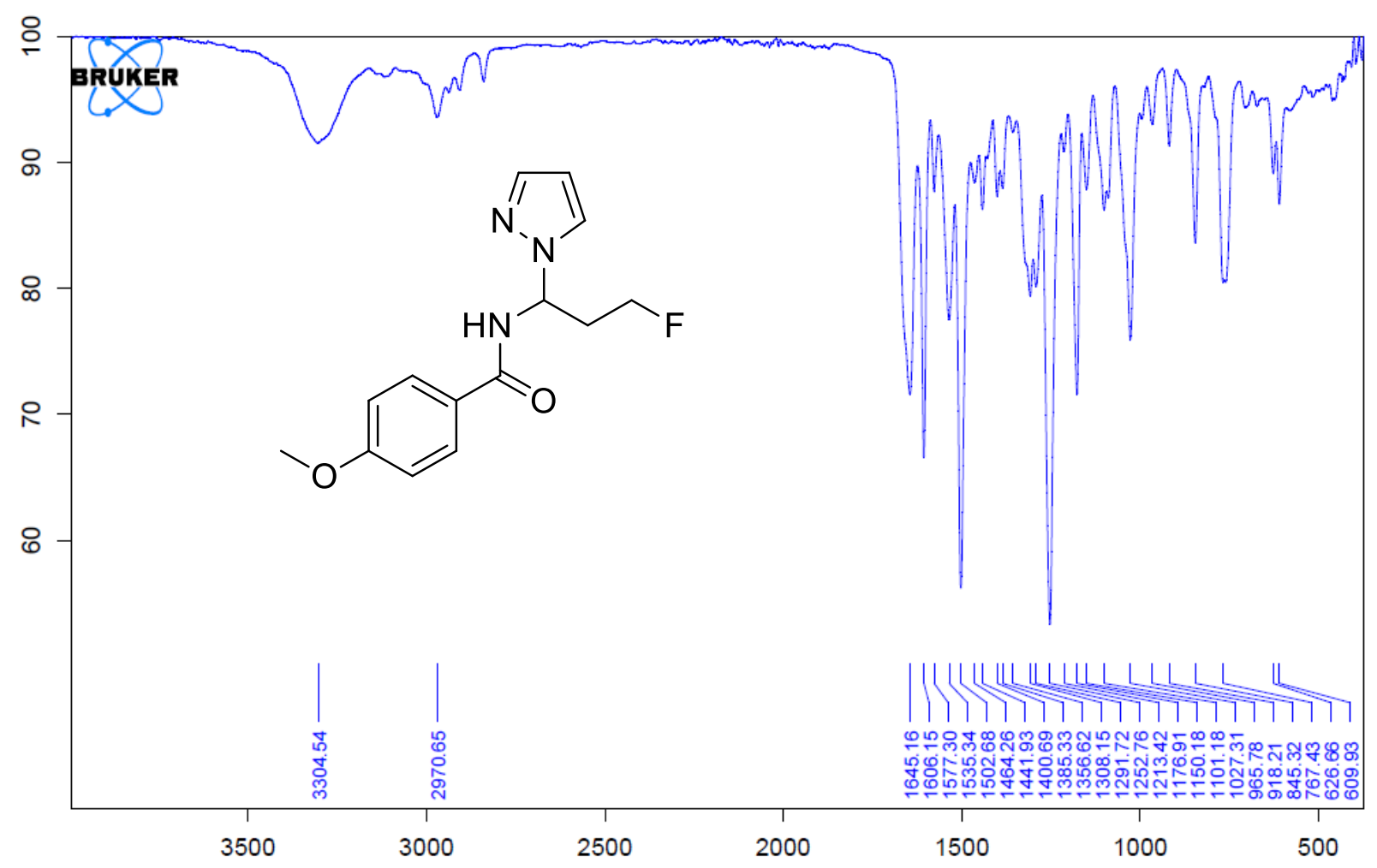

$N$-(3-Fluoro-1-(1H-1,2,4-triazol-1-yl)propyl)-4-methoxybenzamide (3e)

${ }^{1}$ H-NMR (400 MHz, Acetone-d6)

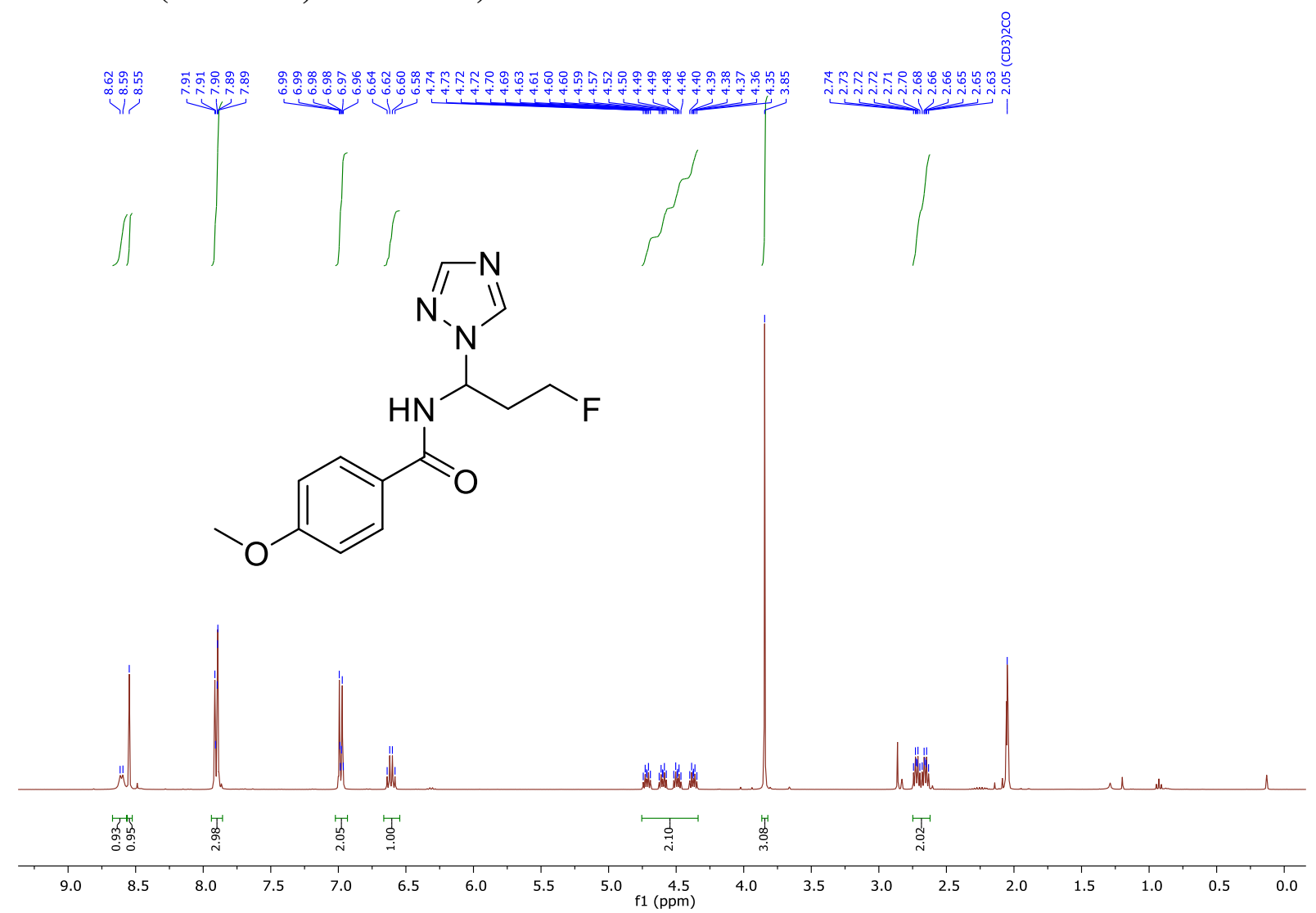

${ }^{13}$ C-NMR (101 MHz, Acetone-d6) 


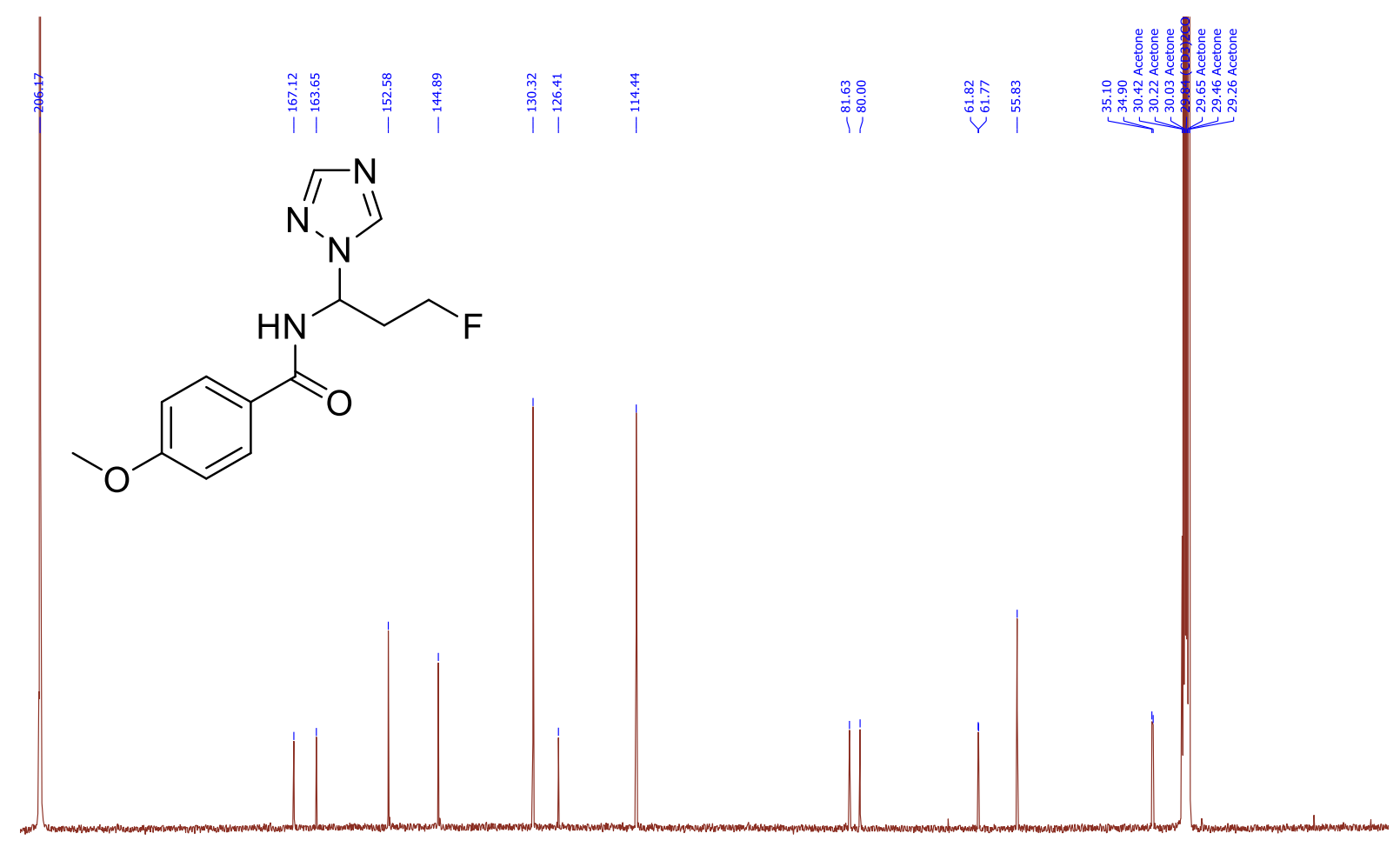

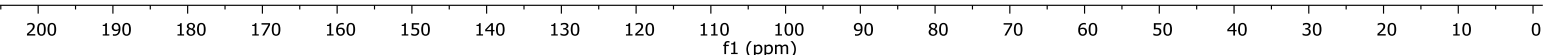

${ }^{19}$ F NMR (376 MHz, CDCl3)

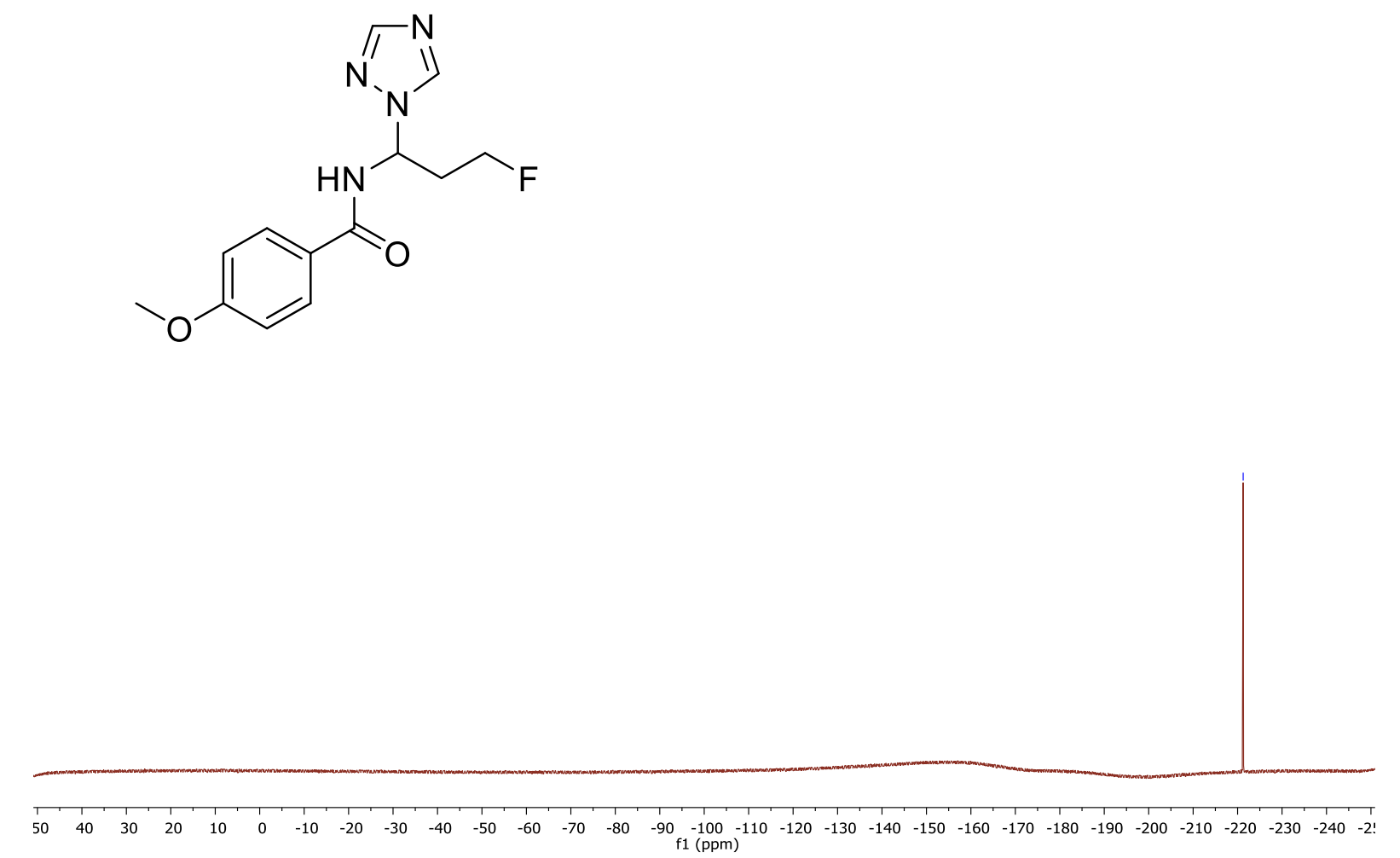

IR 


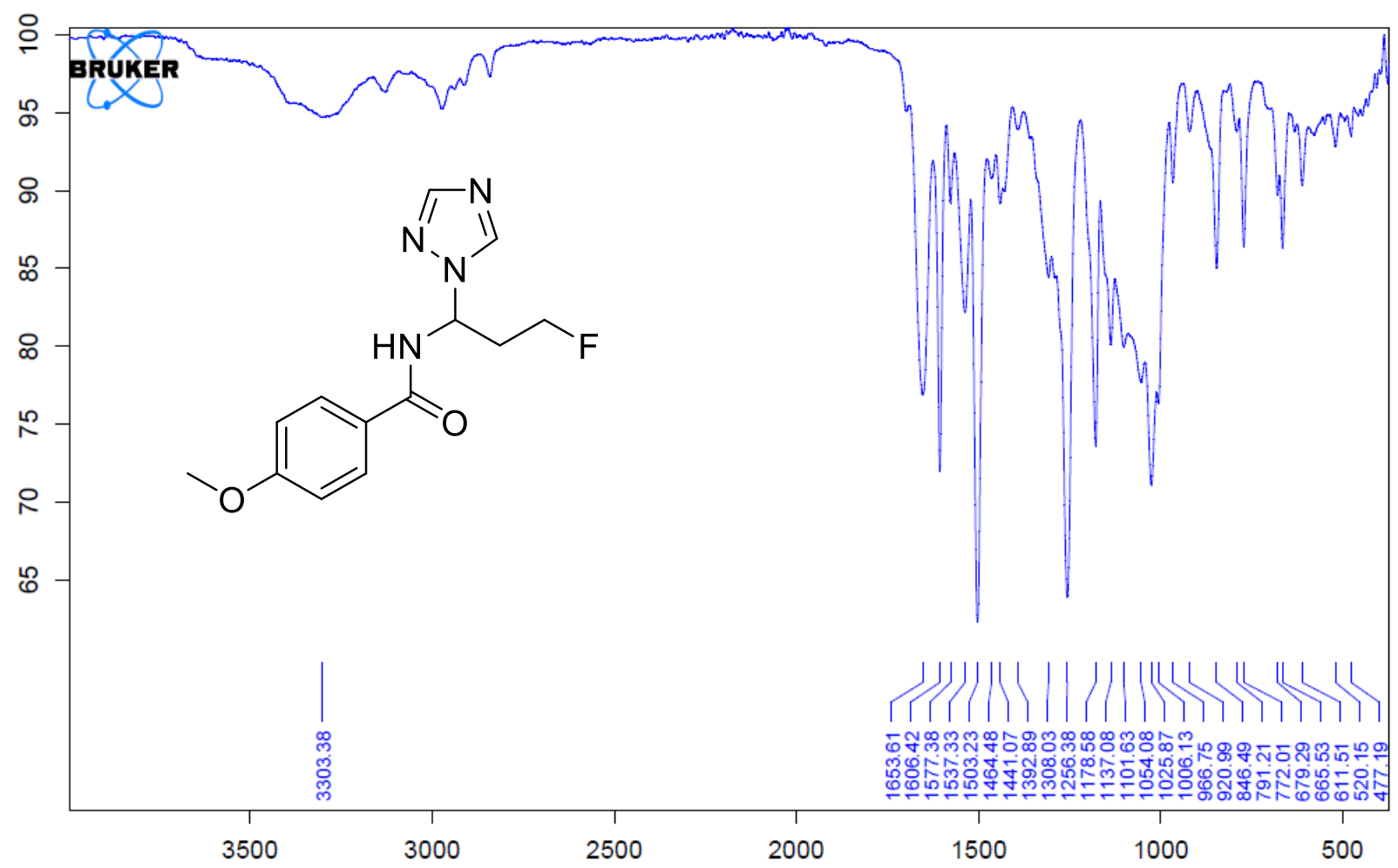

$N$-(3-Fluoro-1-(1H-tetrazol-1-yl)propyl)-4-methoxybenzamide (3f) ${ }^{1}$ H-NMR (400 MHz, Acetone-d $d_{6}$ )

每

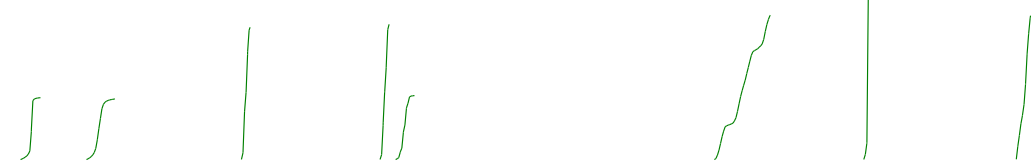<smiles>COc1ccc(C(=O)NC(CCF)n2cnnn2)cc1</smiles>

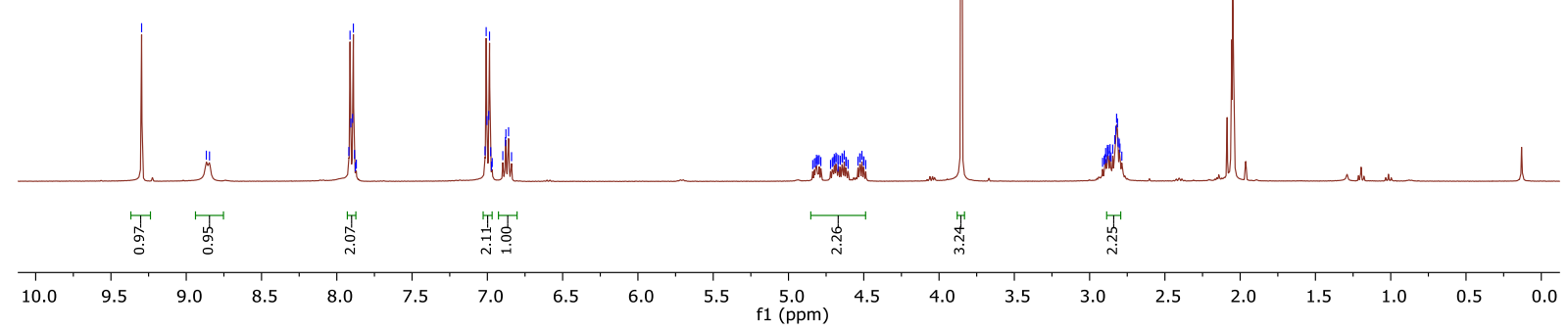

${ }^{13}$ C-NMR (101 MHz, Acetone-d6) 

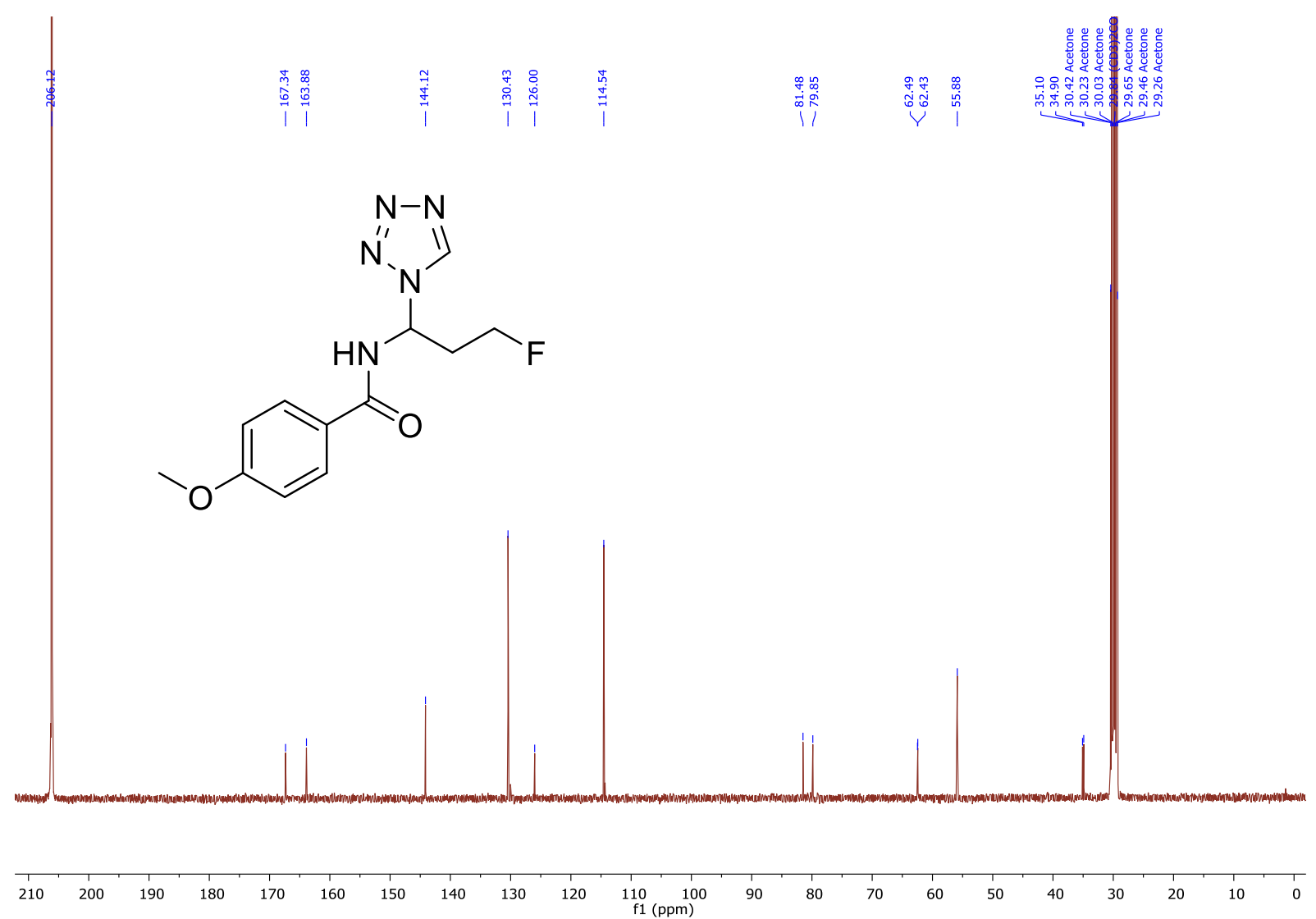

${ }^{19}$ F NMR (376 MHz, Acetone-d6)<smiles>COc1ccc(C(=O)NC(CCF)n2cnnn2)cc1</smiles>

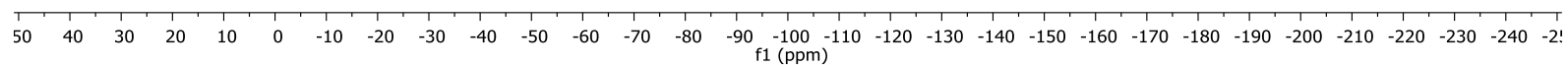
IR 


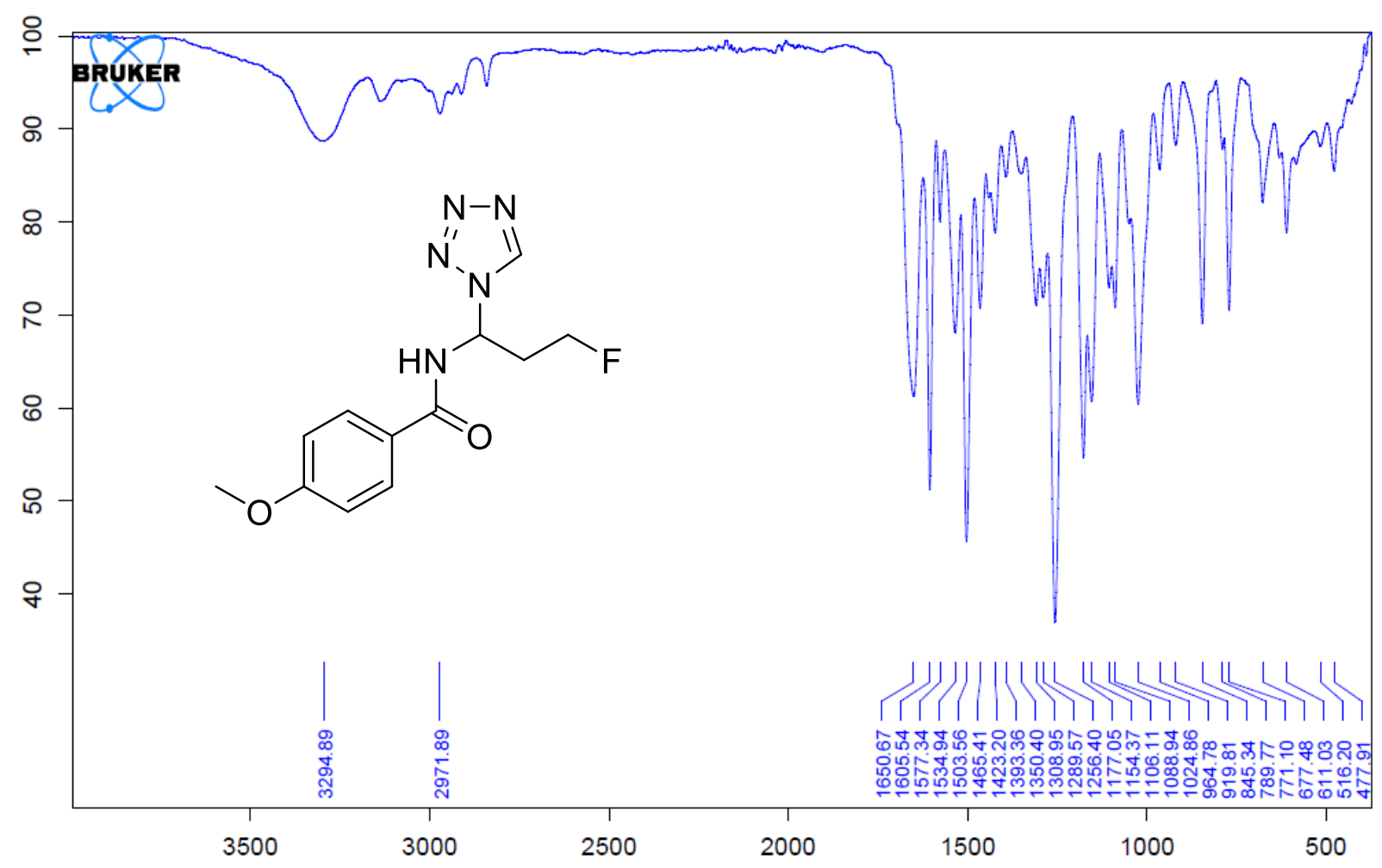

$N$-(3-Fluoropropyl)-4-methoxybenzamide (3g)

${ }^{1} \mathrm{H}-\mathrm{NMR}\left(400 \mathrm{MHz}, \mathrm{CDCl}_{3}\right)$

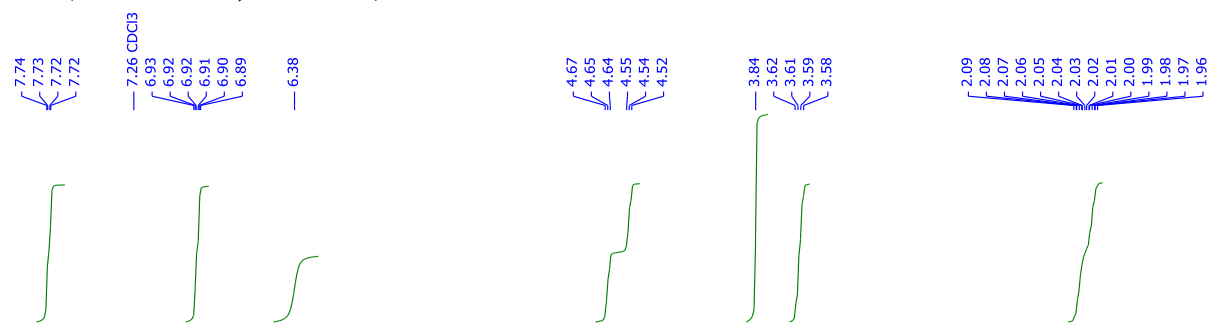<smiles>COc1ccc(C(=O)NCCCF)cc1</smiles>

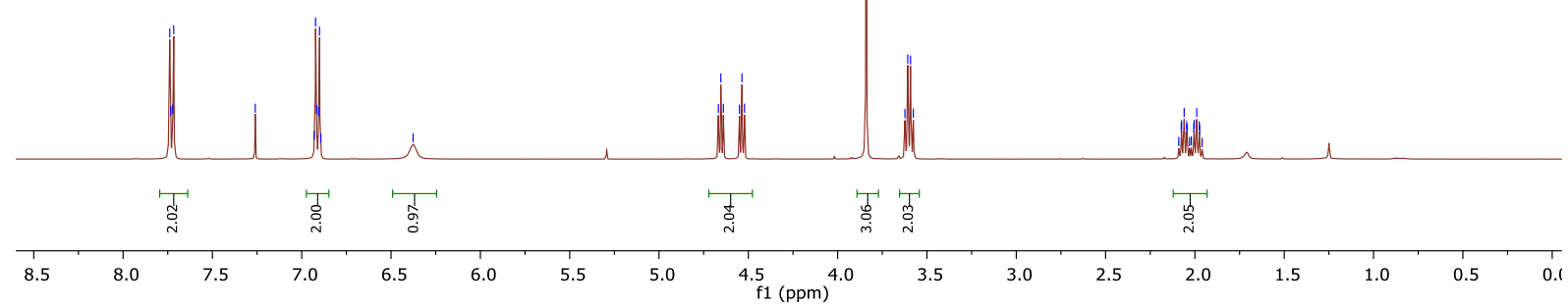

${ }^{13} \mathrm{C}-\mathrm{NMR}$ (101 MHz, CDCl3) 

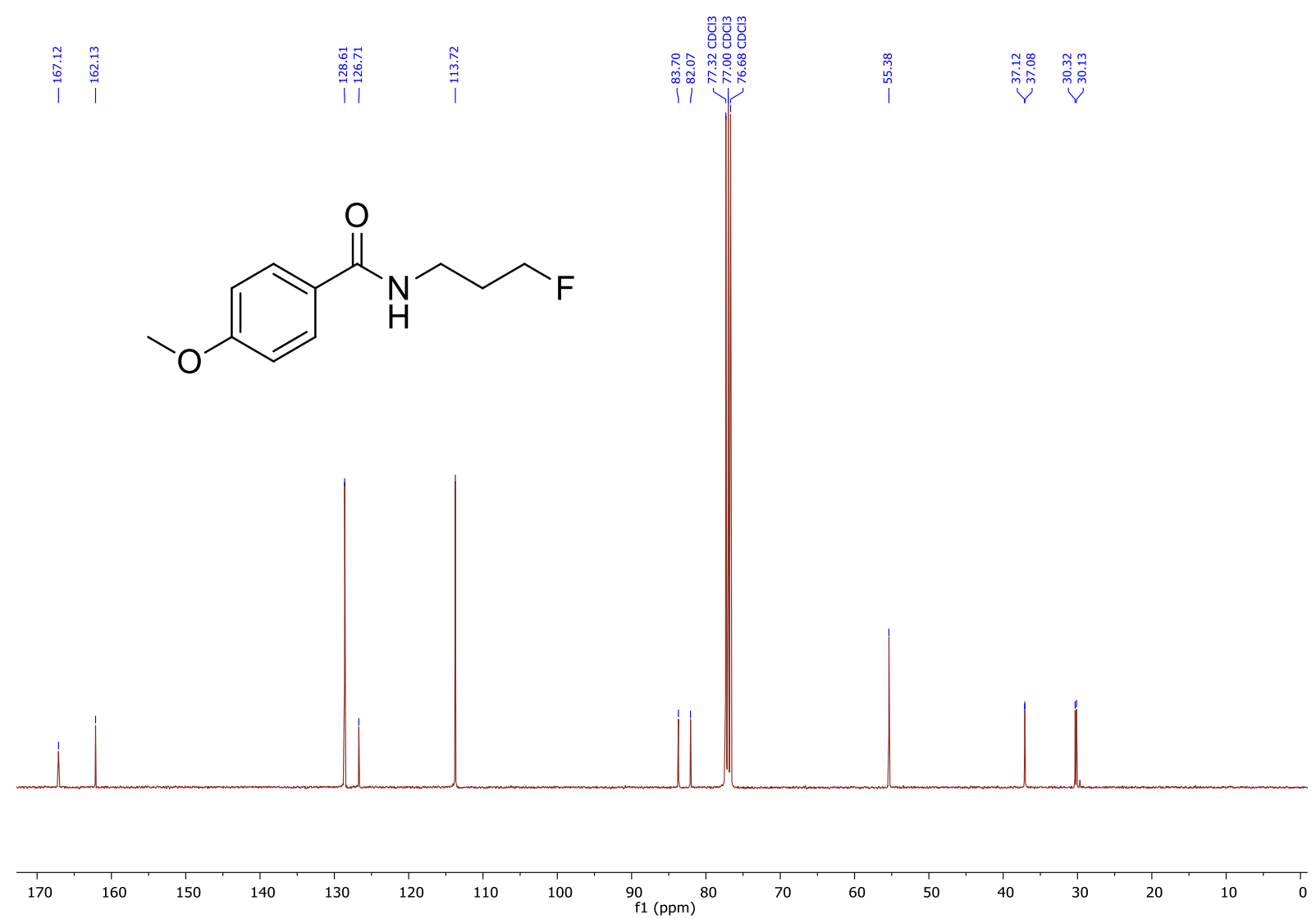

${ }^{19}$ F-NMR (376 MHz, CDCl3)<smiles>COc1ccc(C(=O)NCCCF)cc1</smiles>

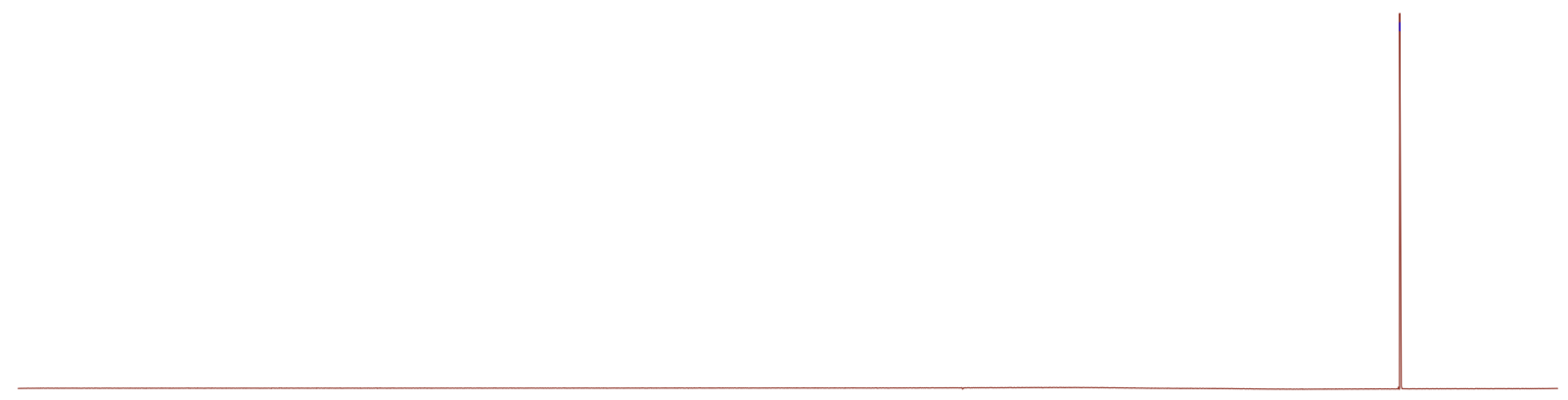

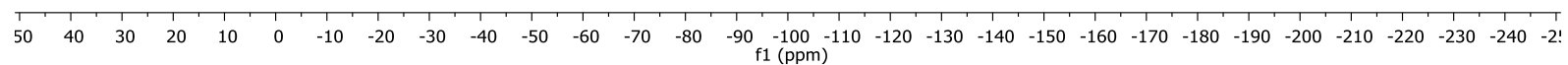
IR 


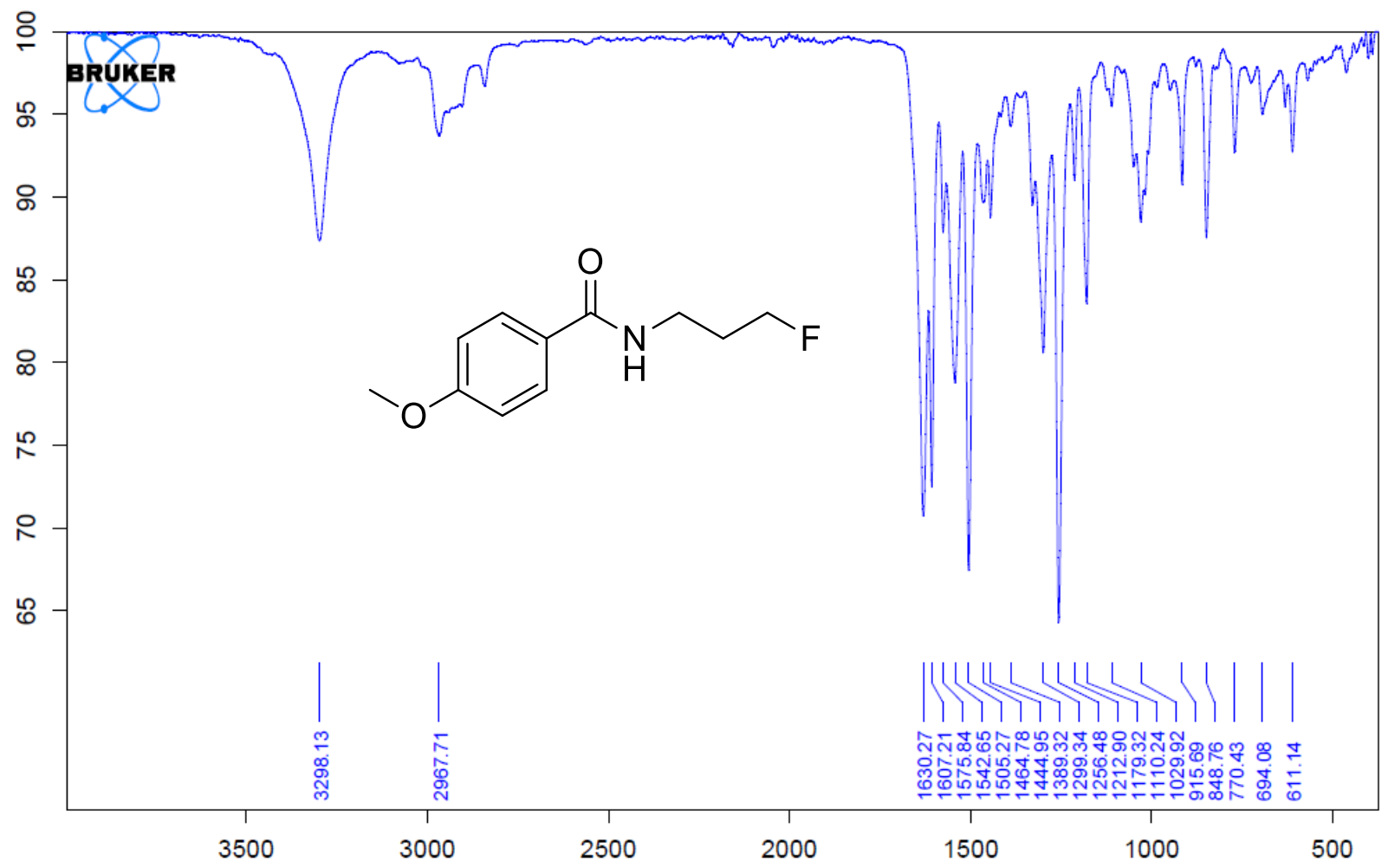

(E)- $N$-(5-Fluoro-1-phenylpent-1-en-3-yl)-4-methoxybenzamide (3h)

${ }^{1}$ H-NMR (400 MHz, Acetone-d6)

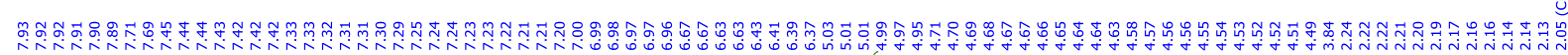

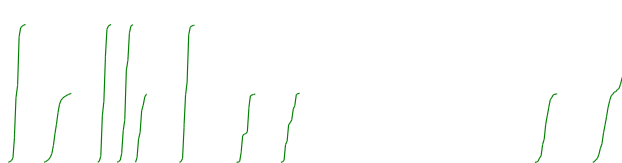<smiles>COc1ccc(C(=O)NC(/C=C/c2ccccc2)CCF)cc1</smiles>

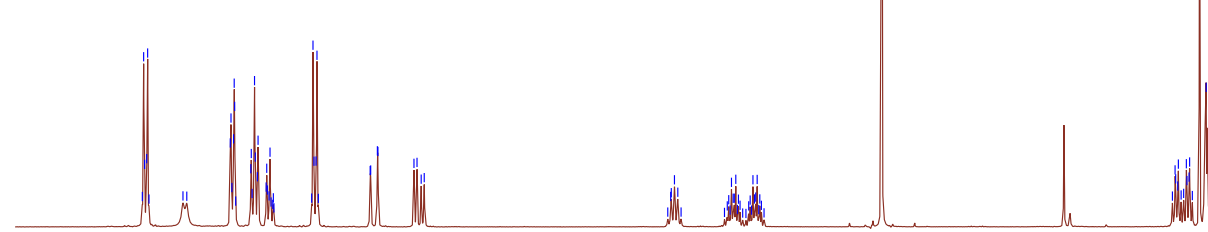

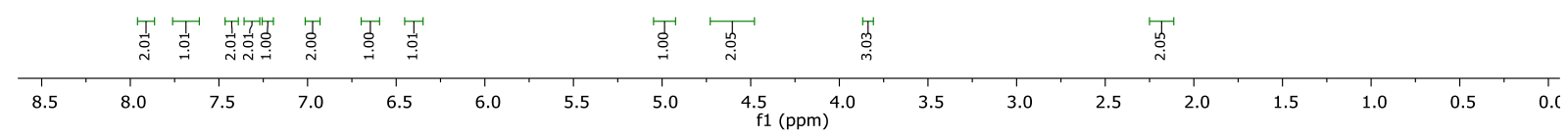

${ }^{13}$ C-NMR (101 MHz, Acetone- $\left.d_{6}\right)$ 


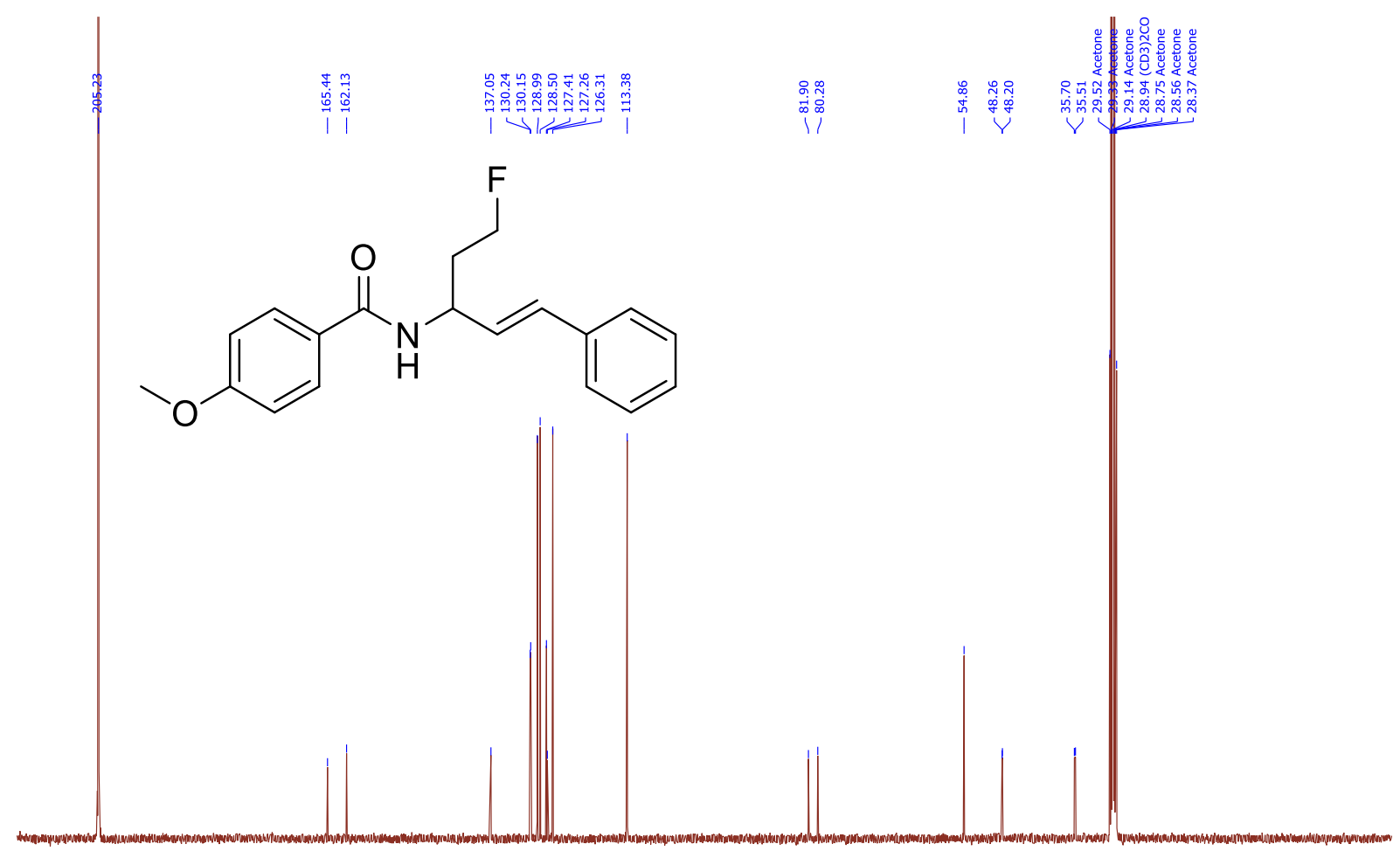

$\begin{array}{lllllllllllllllllllllll}210 & 200 & 190 & 180 & 170 & 160 & 150 & 140 & 130 & 120 & 110 & \begin{array}{l}100 \\ \mathrm{f} 1(\mathrm{ppm})\end{array} & 90 & 80 & 70 & 60 & 50 & 40 & 30 & 20 & 10 & 0 & -10\end{array}$ ${ }^{19}$ F-NMR (376 MHz, Acetone-d6)<smiles>COc1ccc(C(=O)NC(/C=C/c2ccccc2)CCF)cc1</smiles>

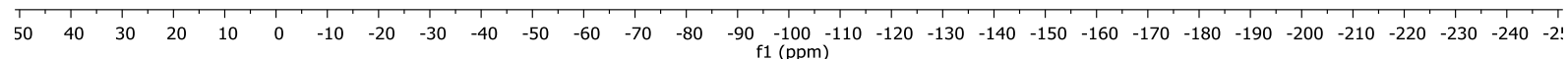
IR 


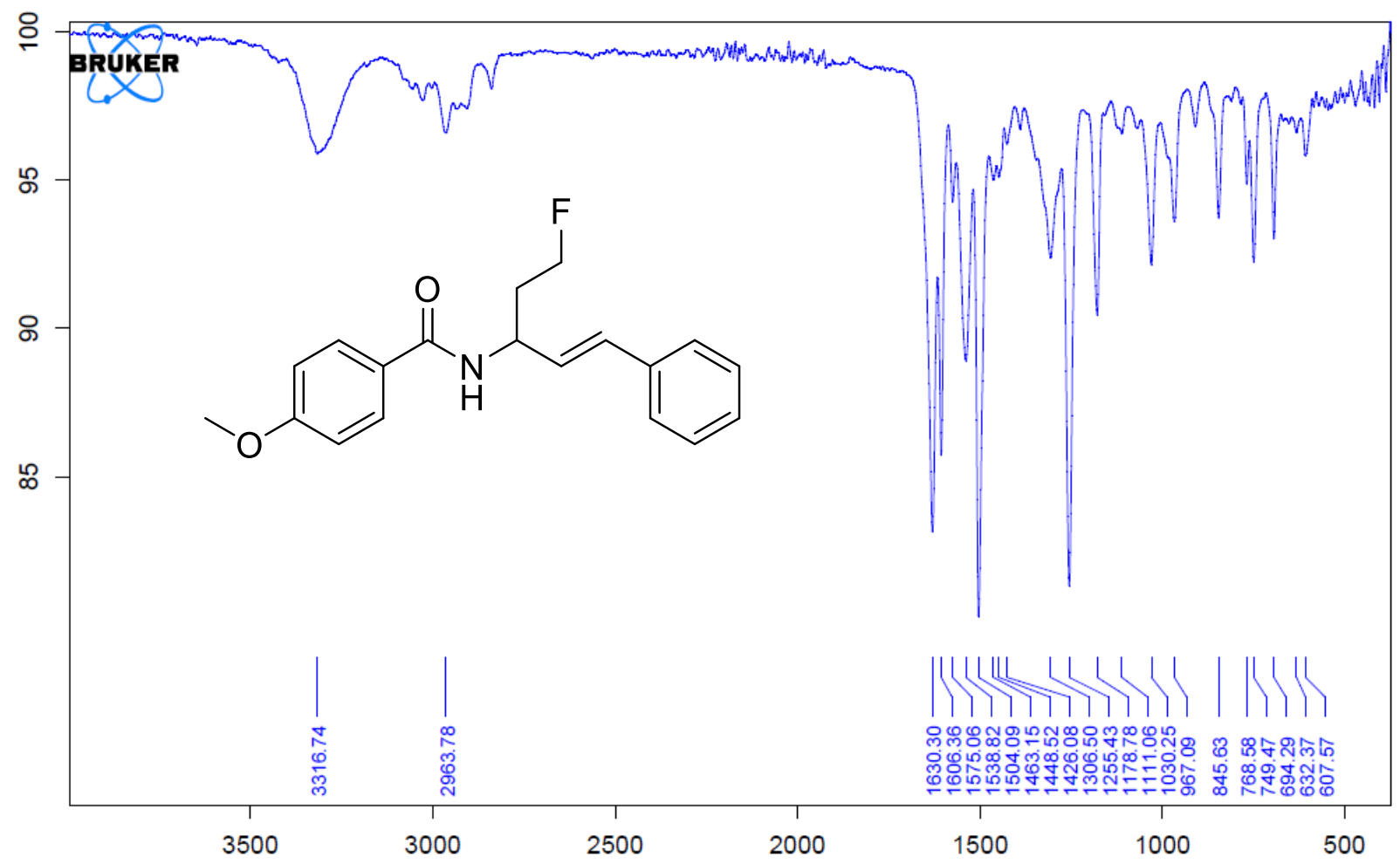

$N$-(3-Fluoro-1-(2,4,6-trimethoxyphenyl)propyl)-4-methoxybenzamide (3i)

${ }^{1} \mathrm{H}-\mathrm{NMR}$ (400 MHz, $\mathrm{CDCl}_{3}$ )

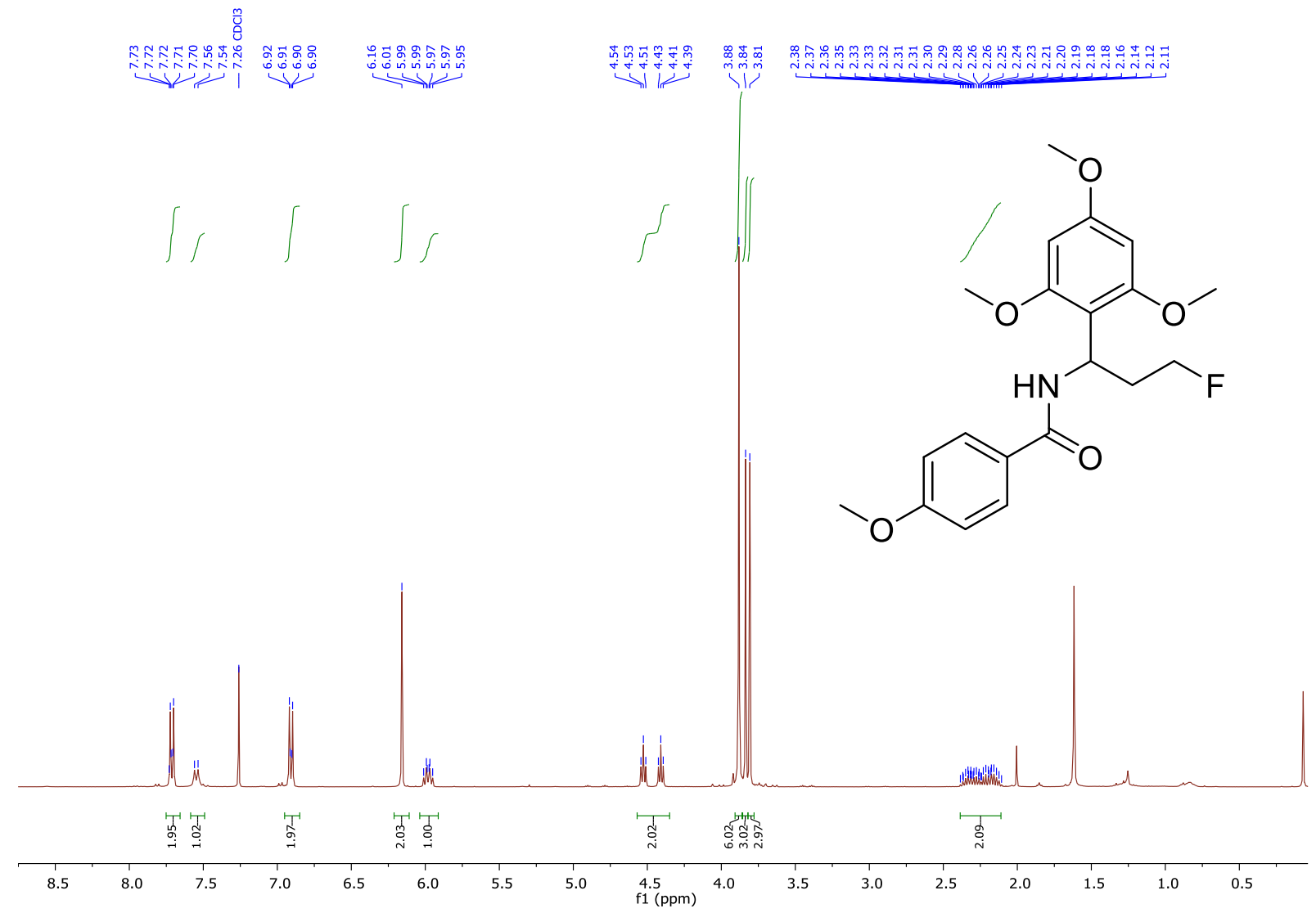

${ }^{13}$ C-NMR (101 MHz, CDCl3) 


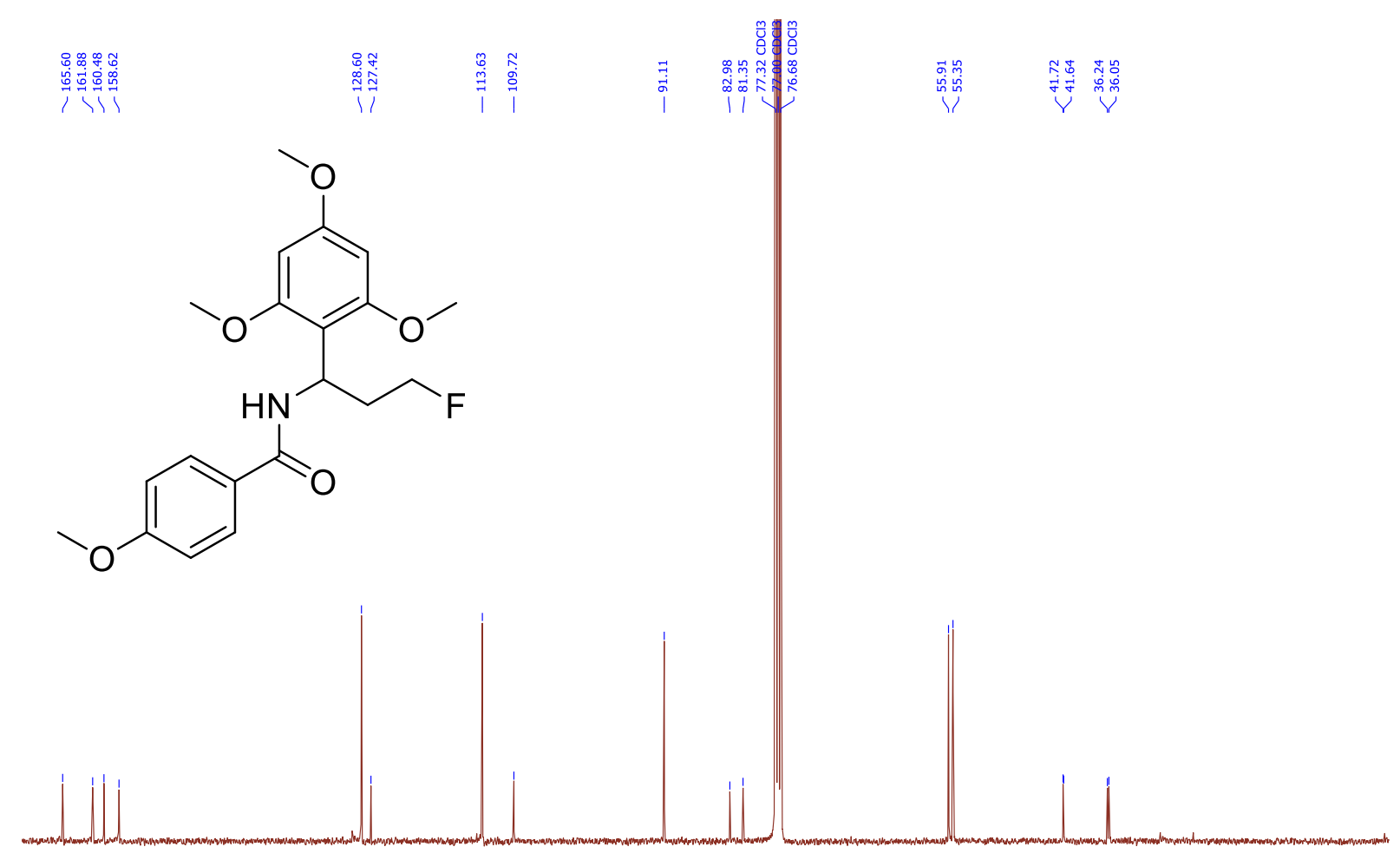<smiles>COc1ccc(C(=O)NC(CCF)c2c(OC)cc(OC)cc2OC)cc1</smiles>

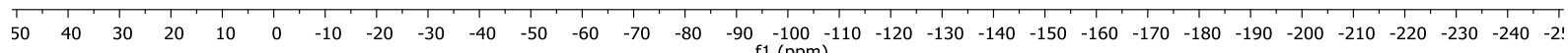
IR 


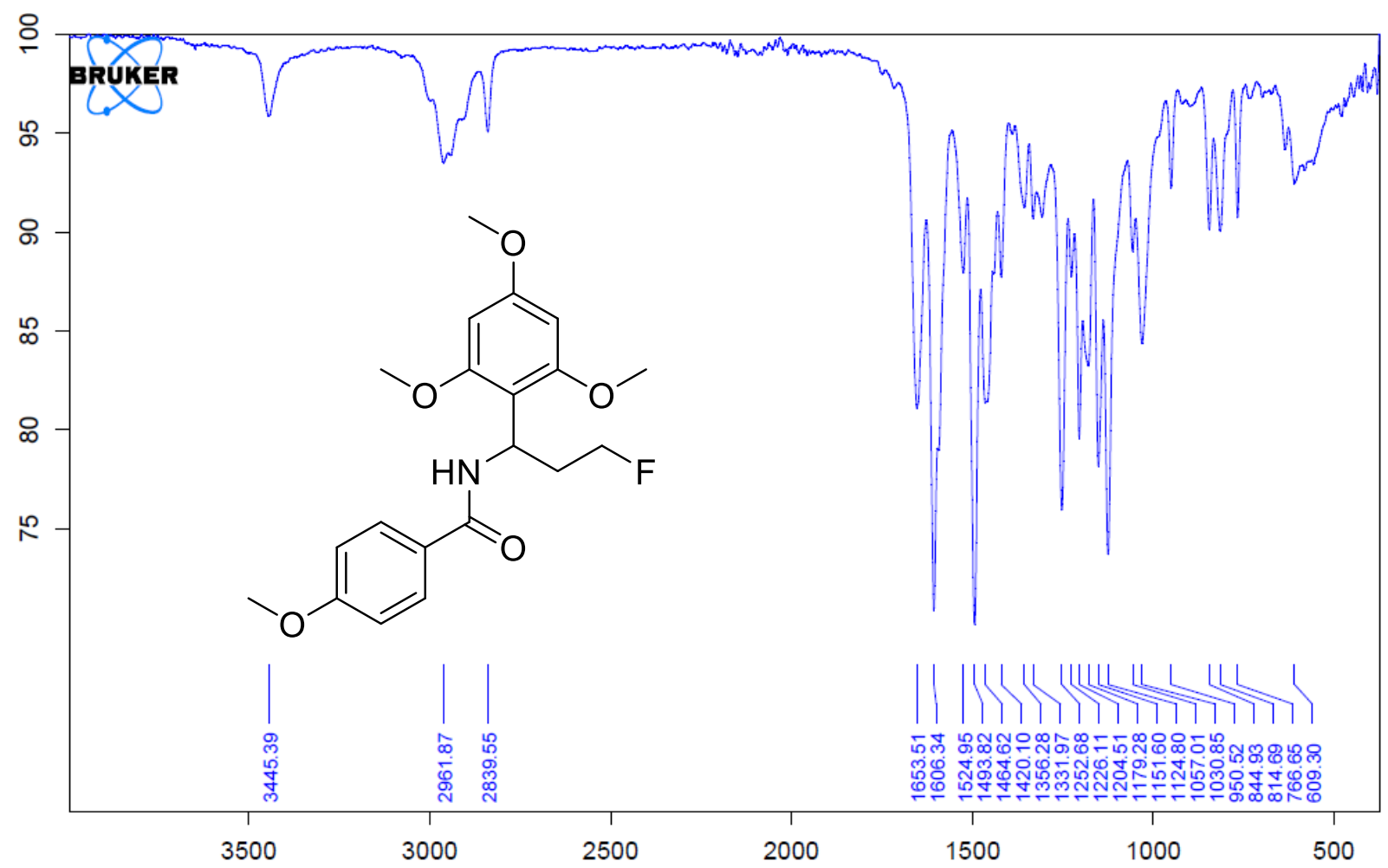

$N$-(1-(2,4-Dimethoxyphenyl)-3-fluoropropyl)-4-methoxybenzamide (3j) ${ }^{1}$ H-NMR (400 MHz, Acetone- $d_{6}$ )

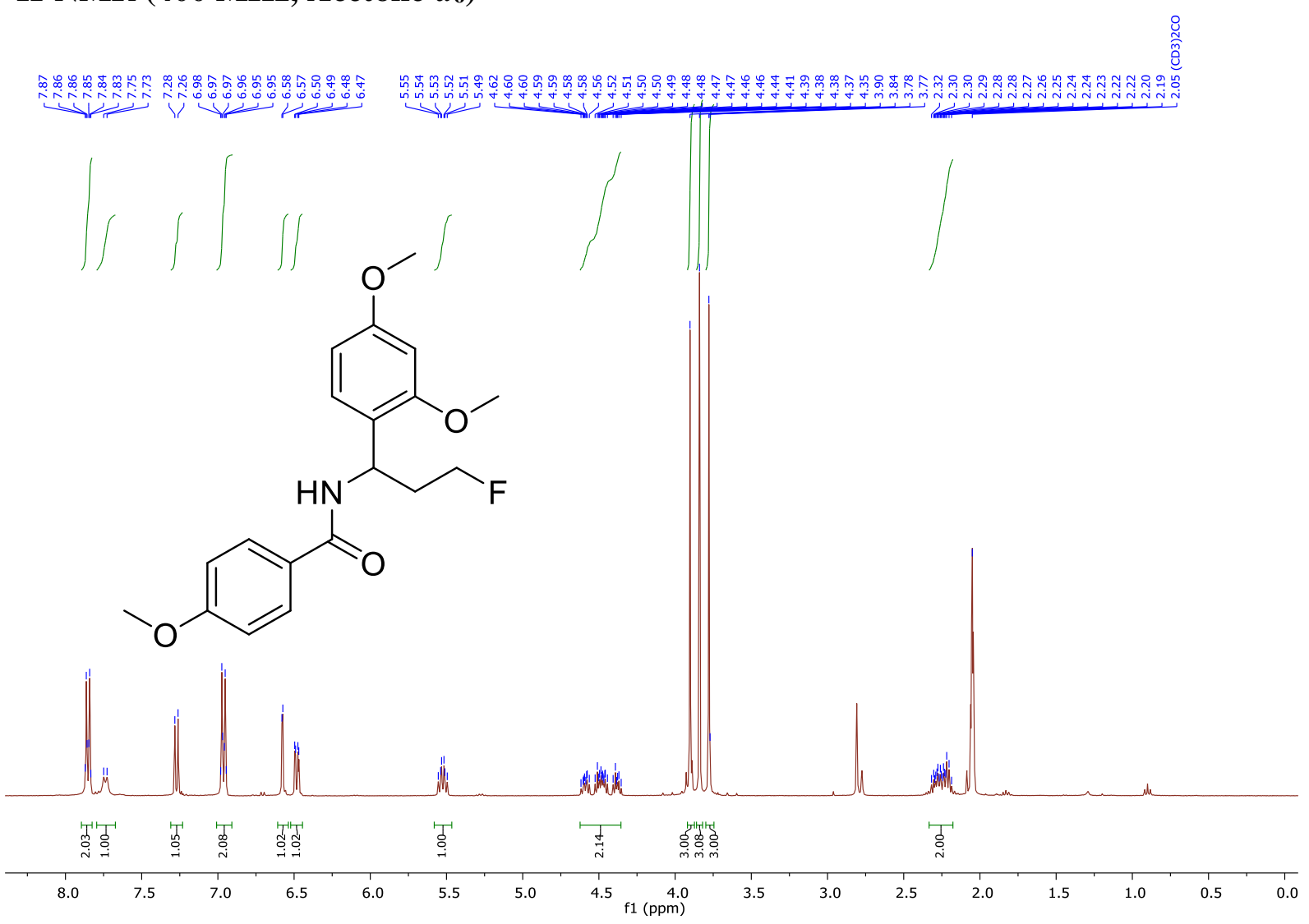

${ }^{13}$ C-NMR (101 MHz, Acetone- $\left.d_{6}\right)$ 


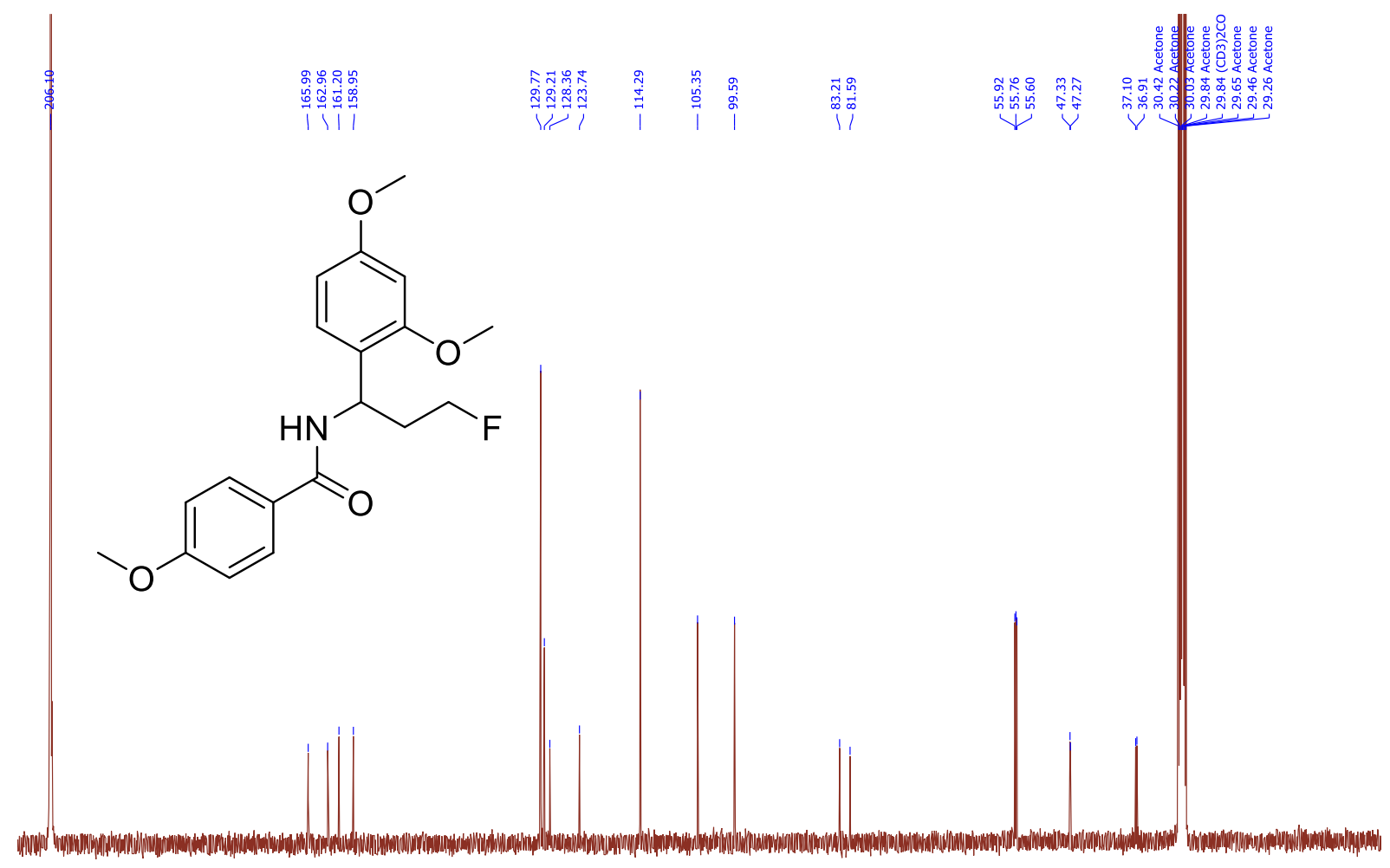

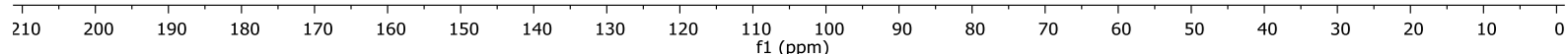

${ }^{19}$ F-NMR (376 MHz, Acetone-d6)<smiles>COc1ccc(C(=O)NC(CCF)c2ccc(OC)cc2OC)cc1</smiles>

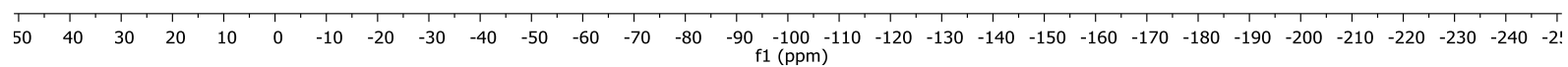
IR 


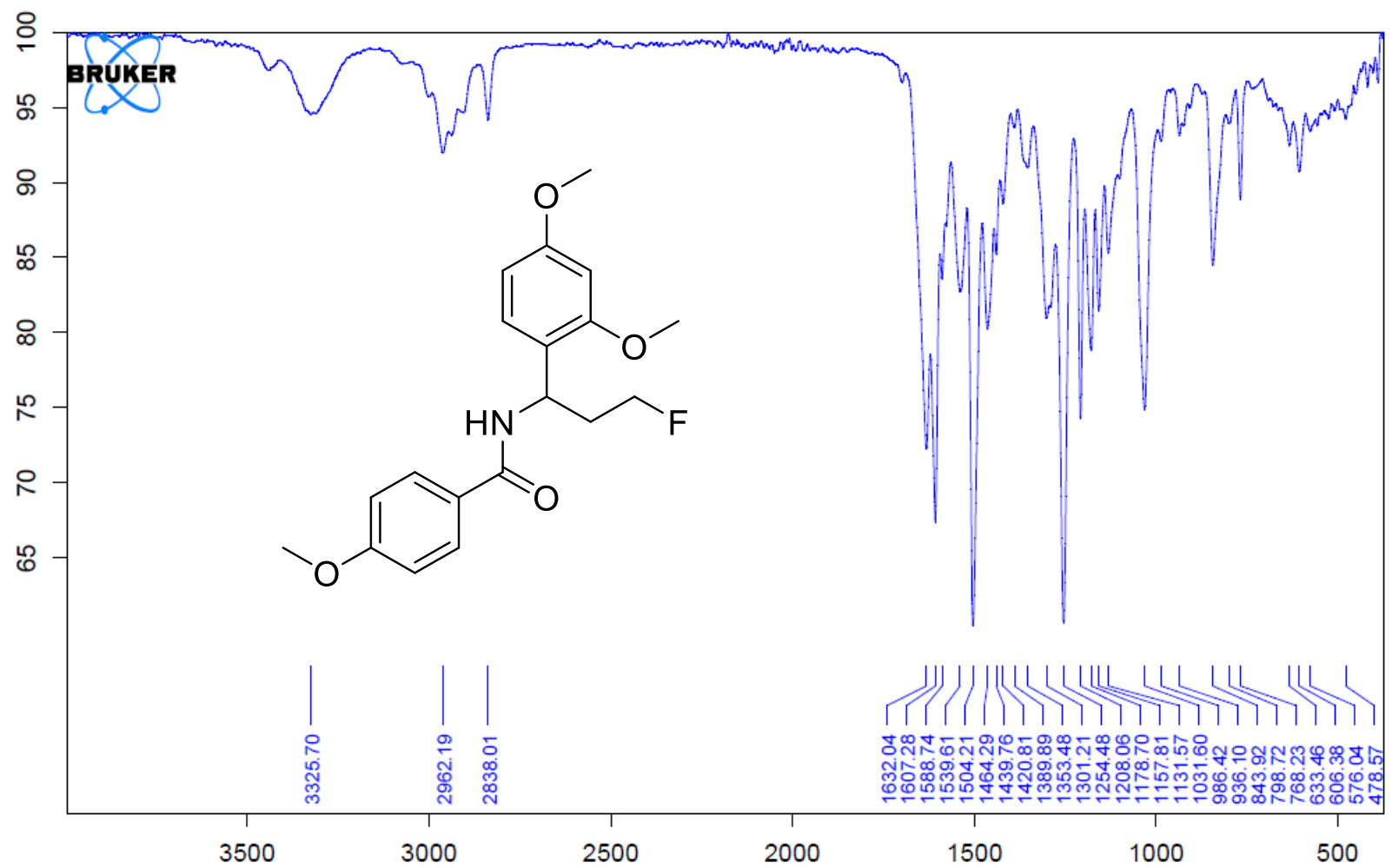

$N$-(3-Fluoro-1-(1H-pyrrol-2-yl)propyl)-4-methoxybenzamide (3k)

${ }^{1} \mathrm{H}-\mathrm{NMR}\left(400 \mathrm{MHz}, \mathrm{CDCl}_{3}\right)$

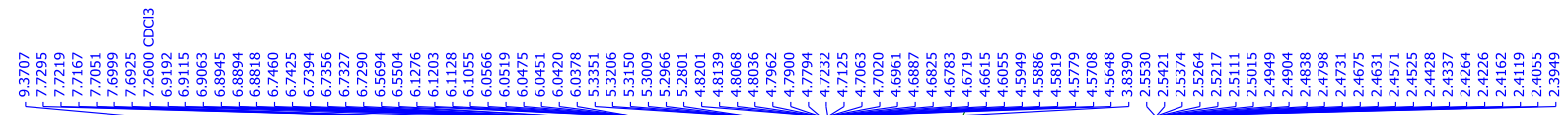

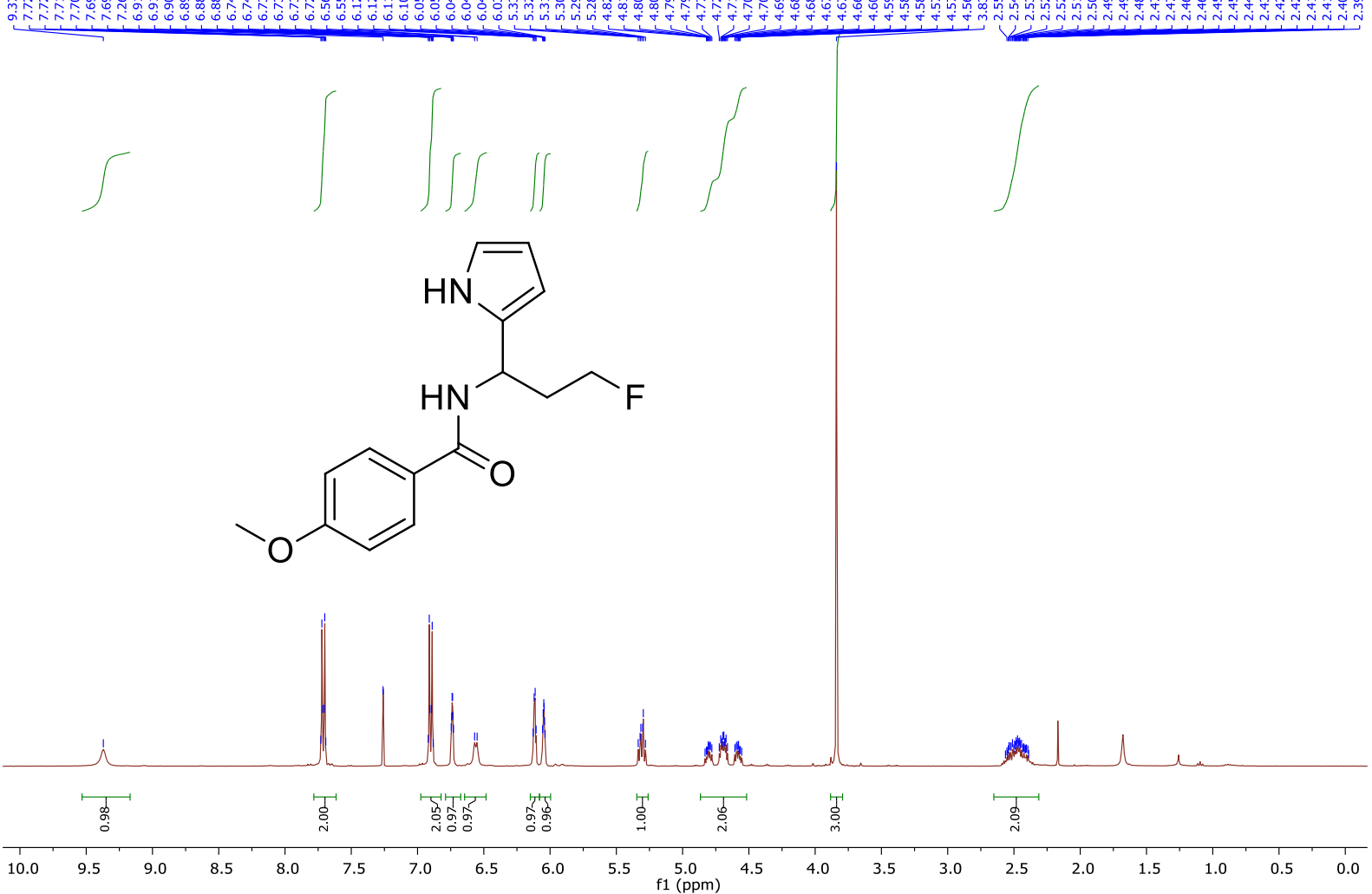

${ }^{13} \mathrm{C}-\mathrm{NMR}\left(101 \mathrm{MHz}, \mathrm{CDCl}_{3}\right)$ 


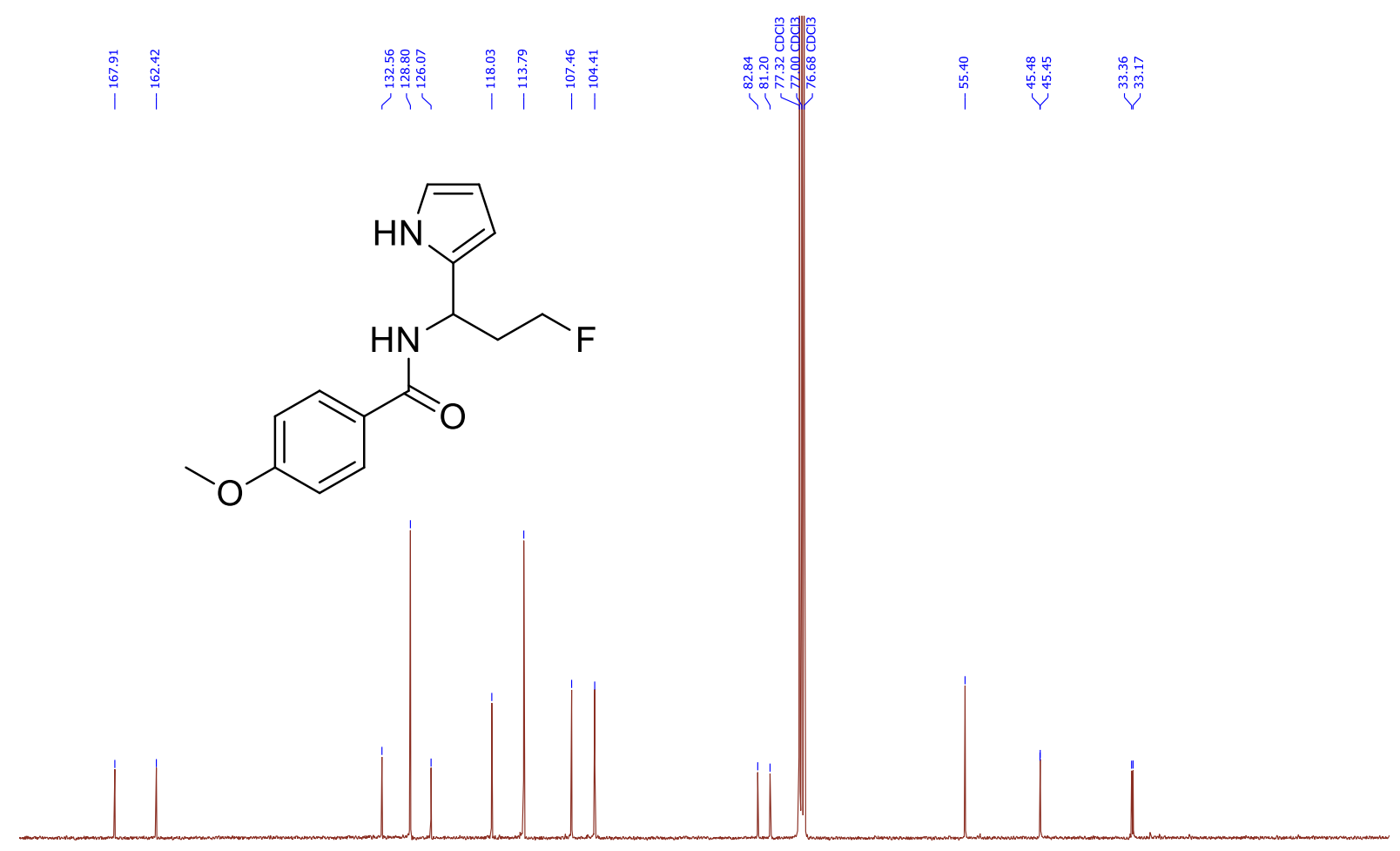<smiles>COc1ccc(C(=O)NC(CCF)c2ccc[nH]2)cc1</smiles>

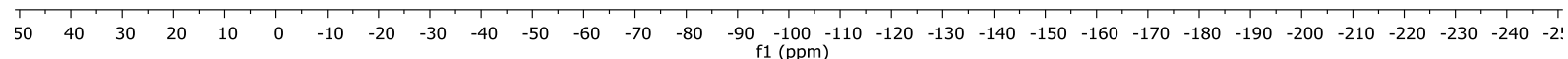
IR 


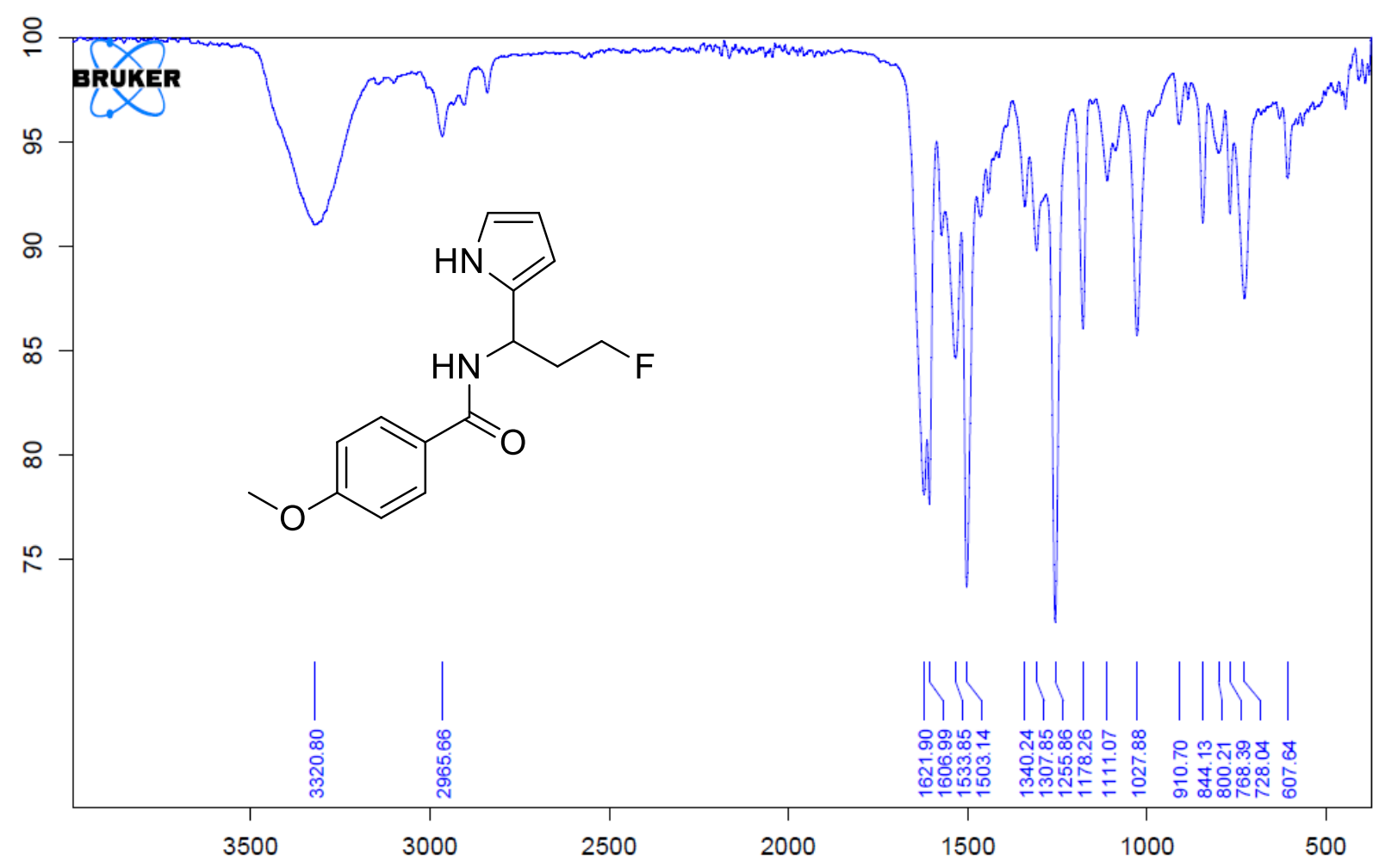

$N$-(3-Fluoro-1-(1H-indol-3-yl)propyl)-4-methoxybenzamide (3I) ${ }^{1} \mathrm{H}-\mathrm{NMR}$ (400 MHz, $\left.\mathrm{CDCl}_{3}\right)$

尊
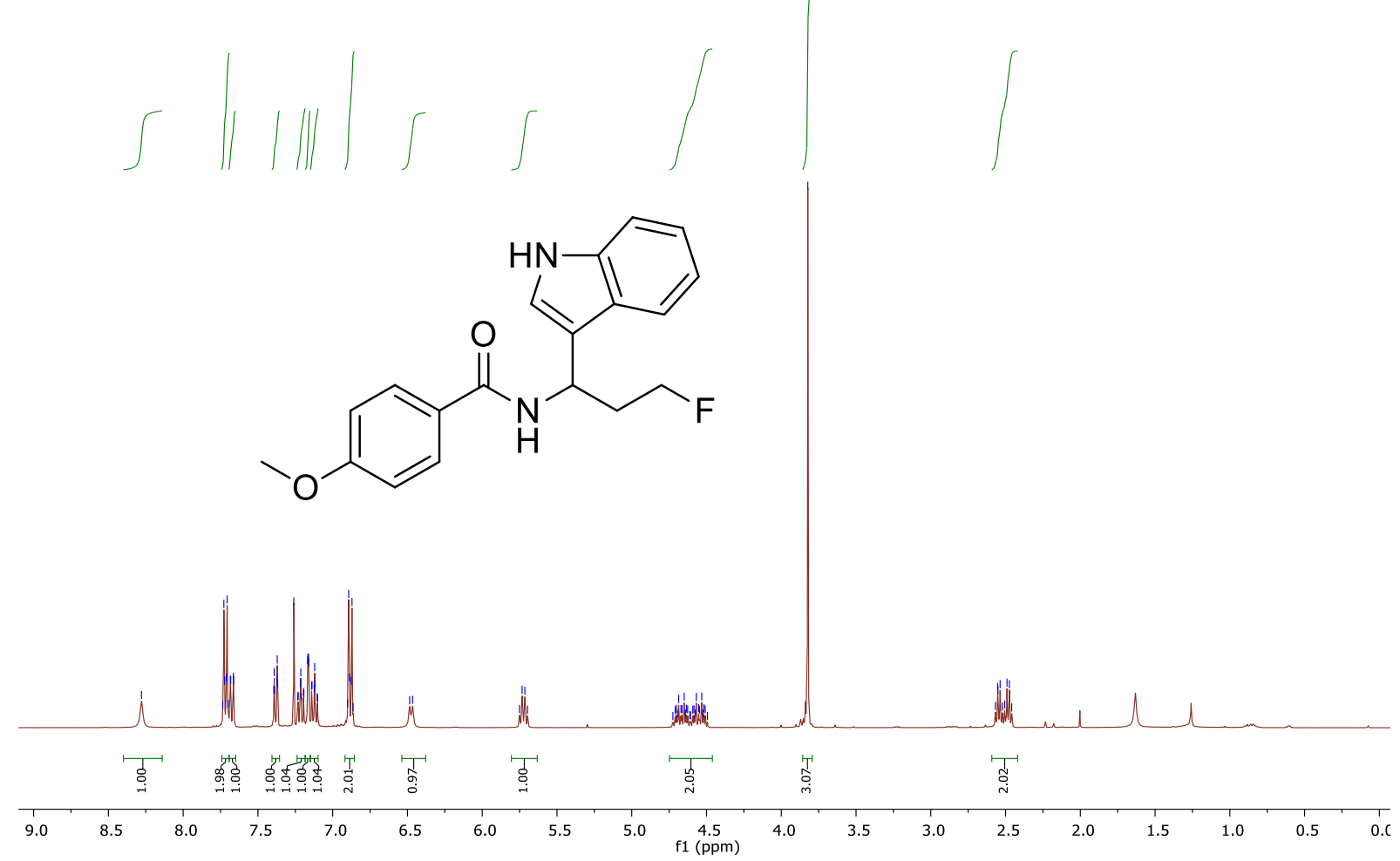

${ }^{13}$ C-NMR (101 MHz, CDCl3) 

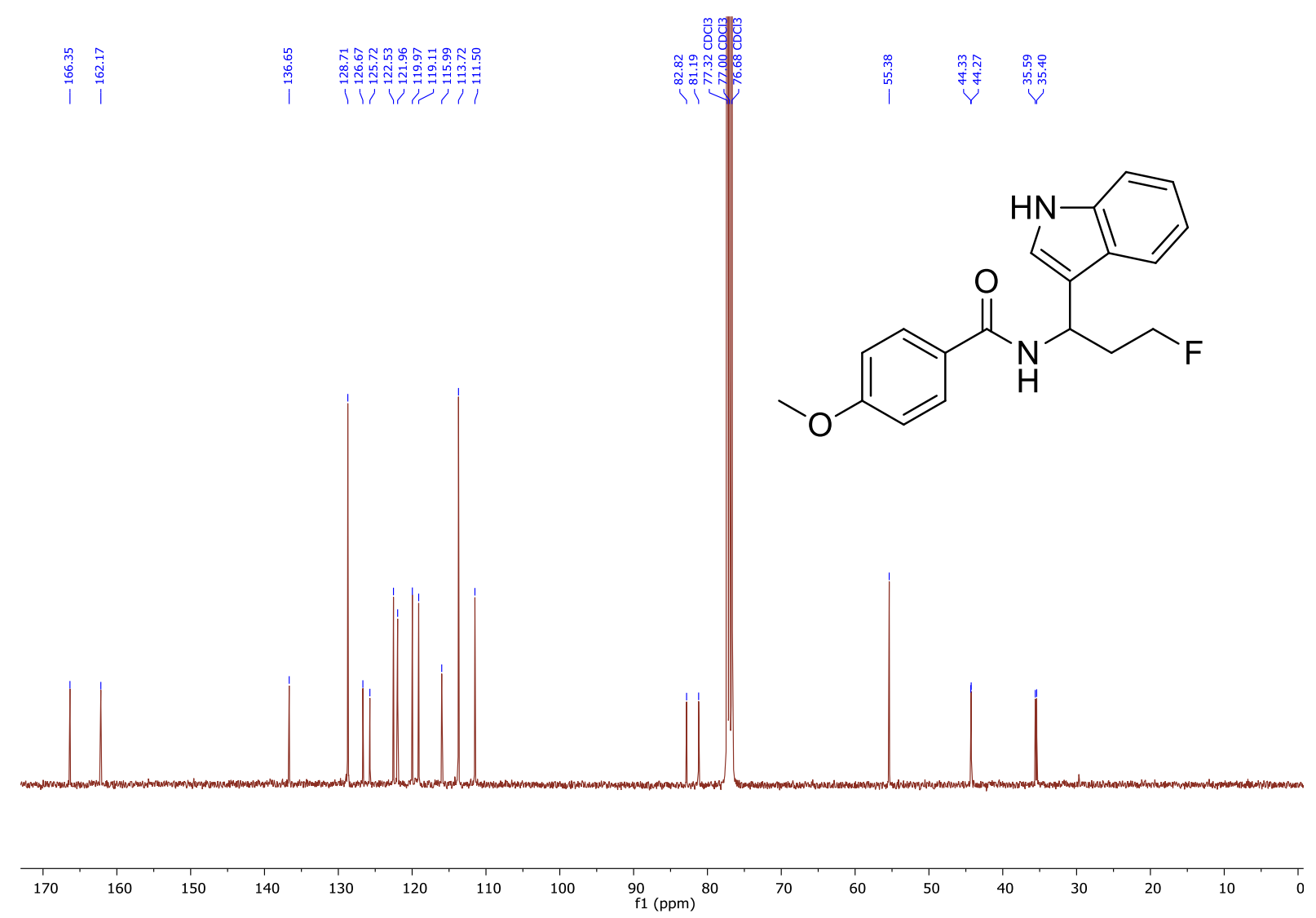

${ }^{19}$ F-NMR (376 MHz, CDCl3)

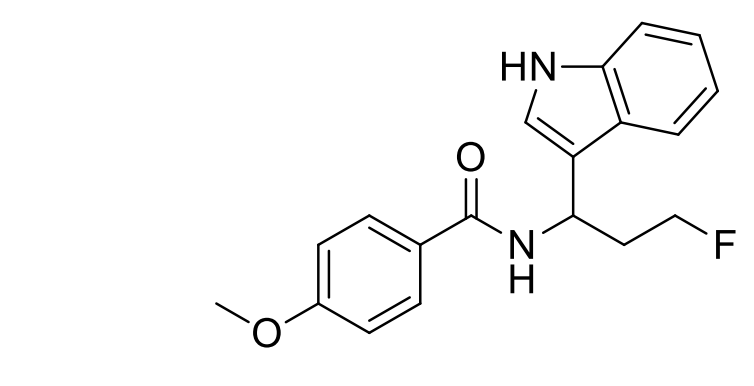

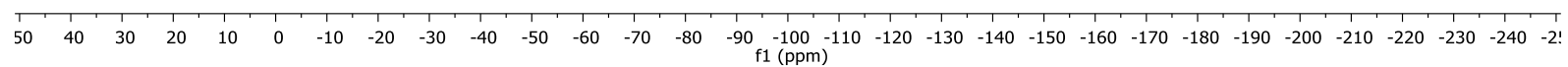
IR 


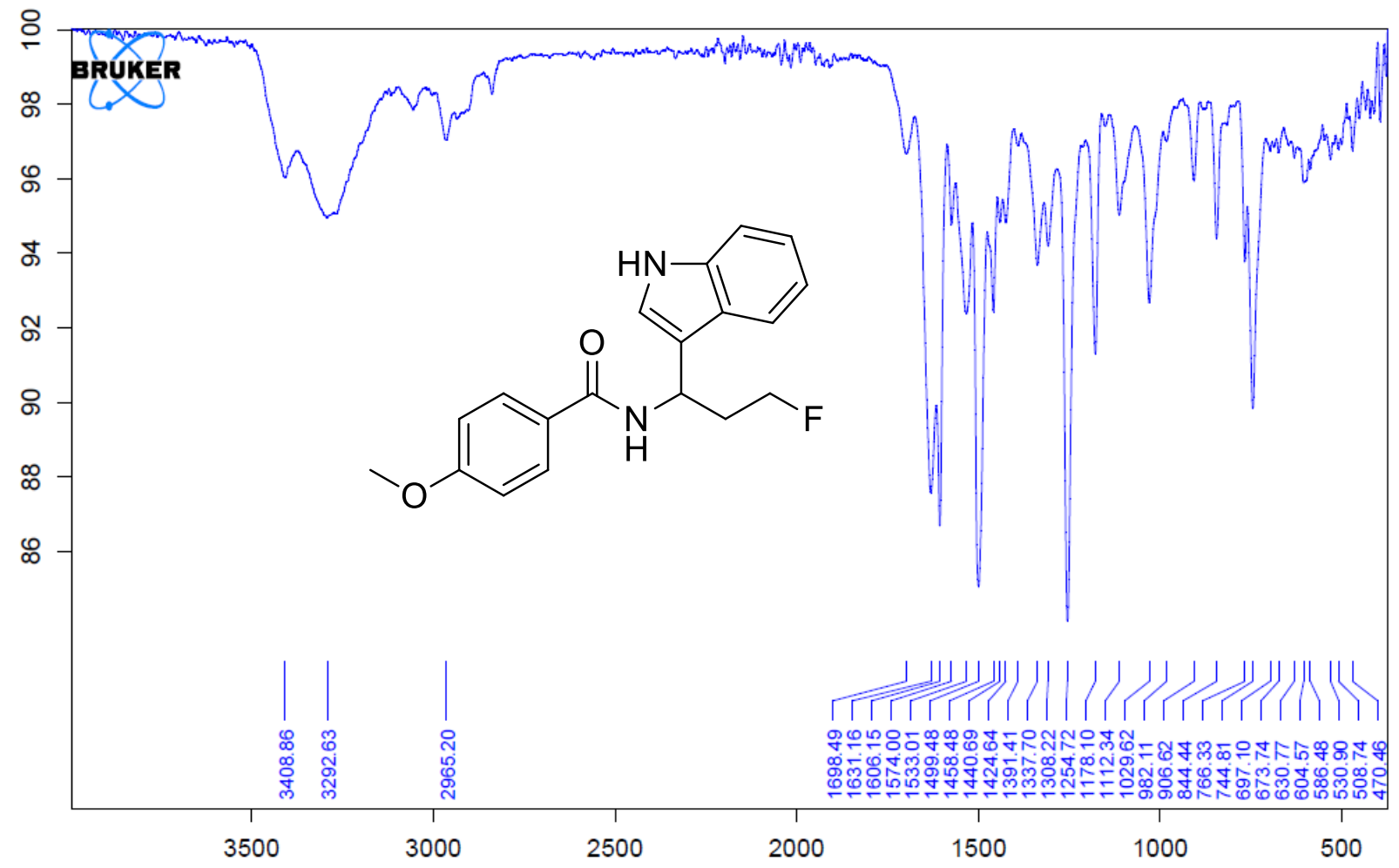

$N$-(3-Fluoro-1-(1-methyl-1H-indol-3-yl)propyl)-4-methoxybenzamide (3m)

${ }^{1}$ H-NMR (400 MHz, Acetone- $d_{6}$ )

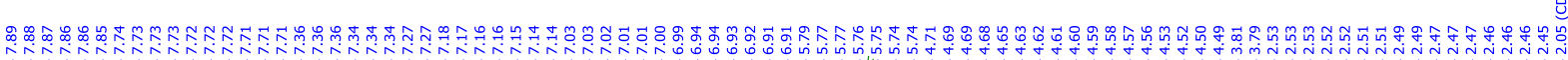

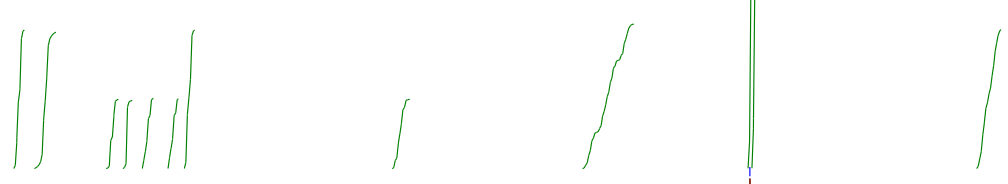<smiles>COc1ccc(C(=O)NC(CCF)c2cn(C)c3ccccc23)cc1</smiles>

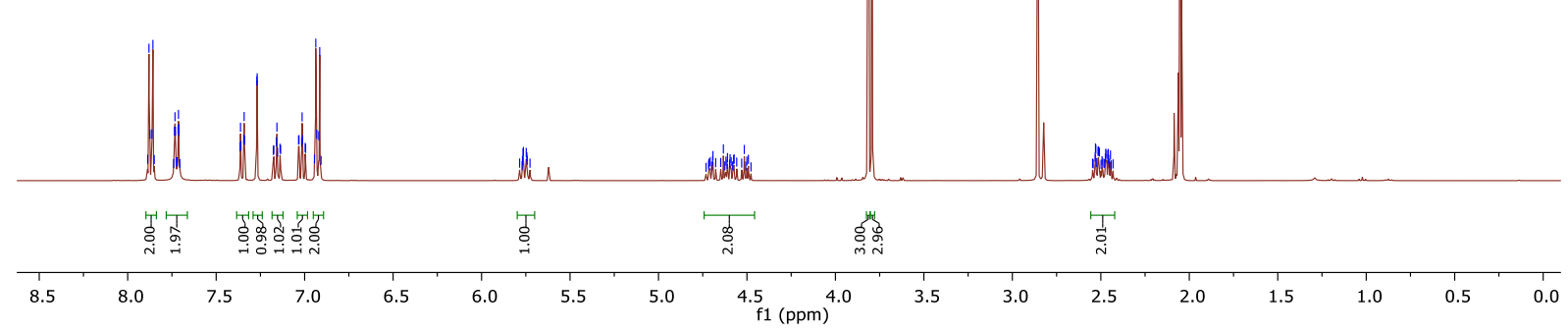

${ }^{13} \mathrm{C}-\mathrm{NMR}$ (101 MHz, Acetone- $\left.d_{6}\right)$ 


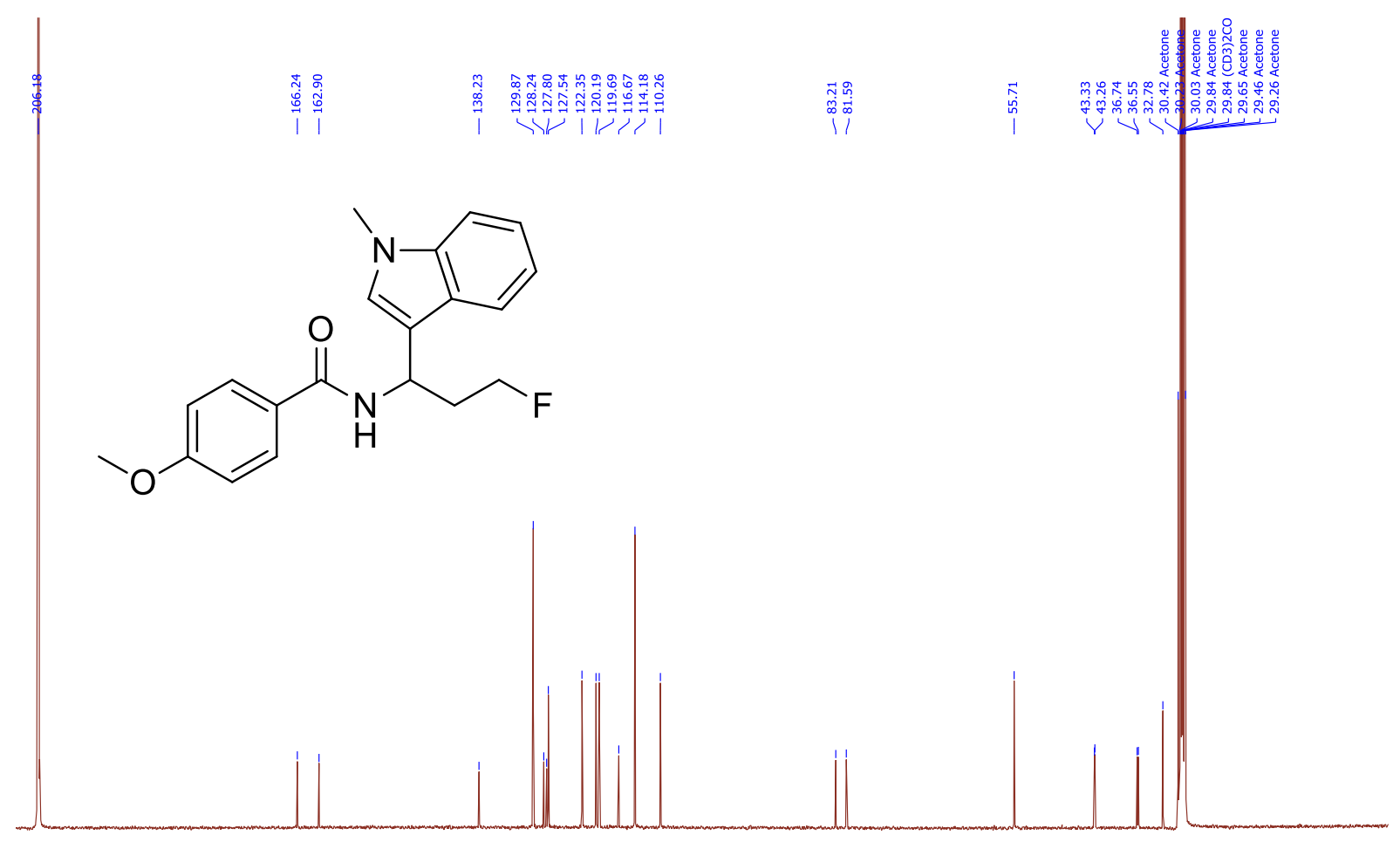

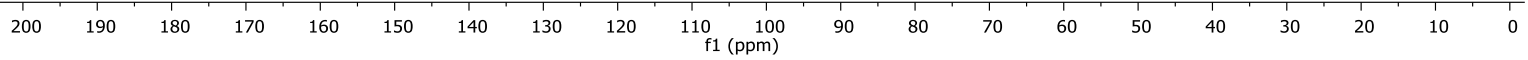

${ }^{19}$ F-NMR (376 MHz, Acetone-d6)<smiles>COc1ccc(C(=O)NC(CCF)c2cn(C)c3ccccc23)cc1</smiles>

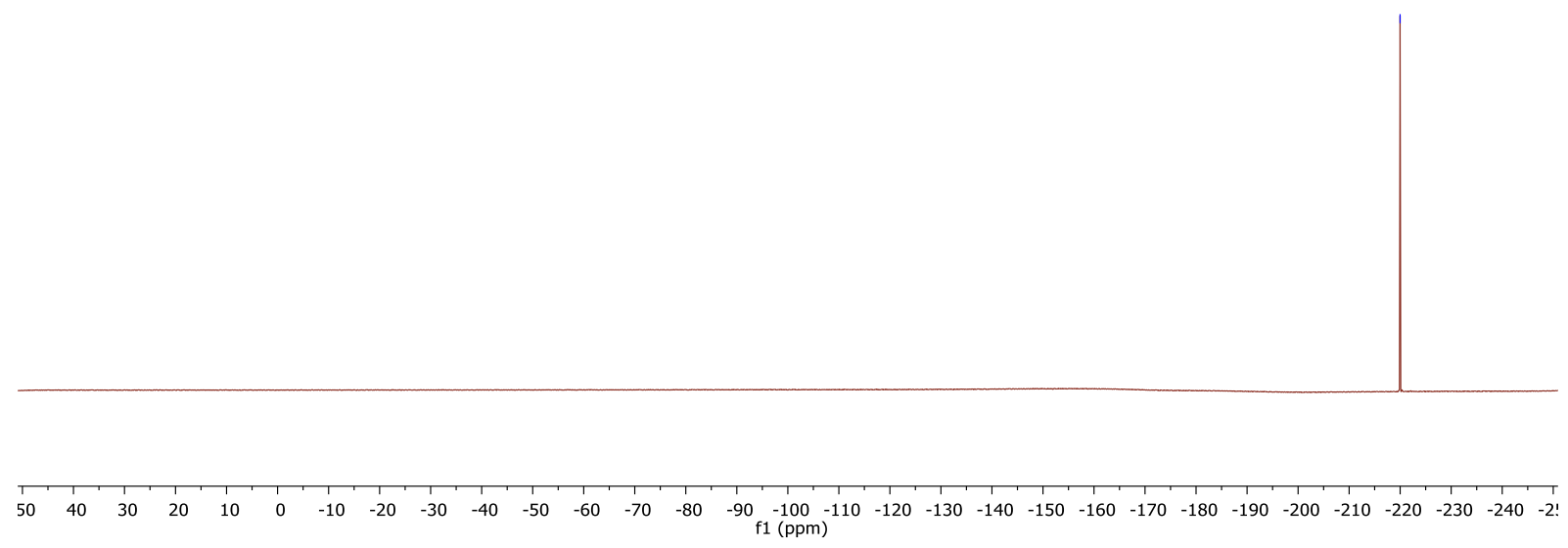

IR 


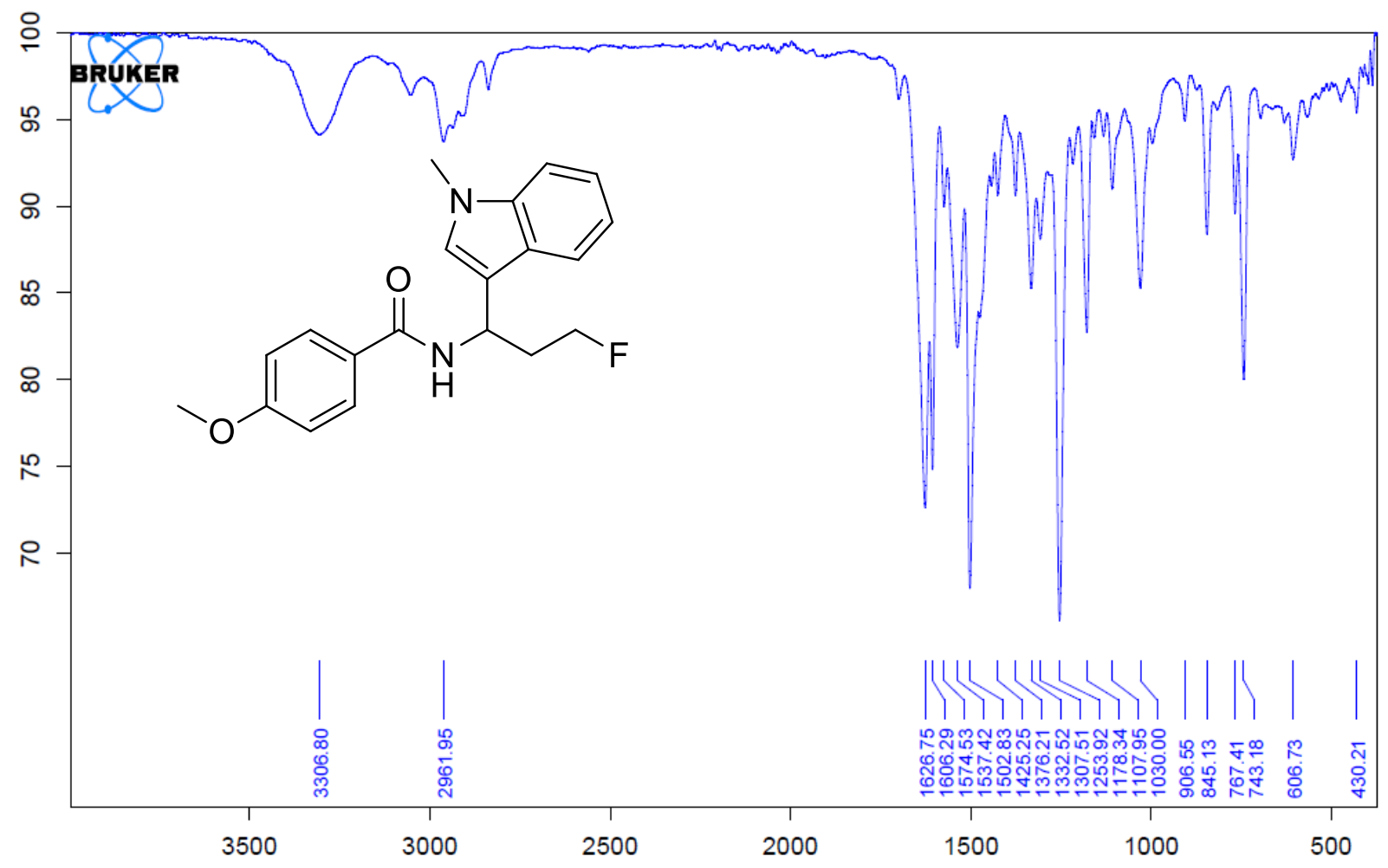

$N$-(3-Fluoro-1-(2-phenyl-1H-indol-3-yl)propyl)-4-methoxybenzamide (3n) ${ }^{1}$ H-NMR (400 MHz, CDCl 3 )

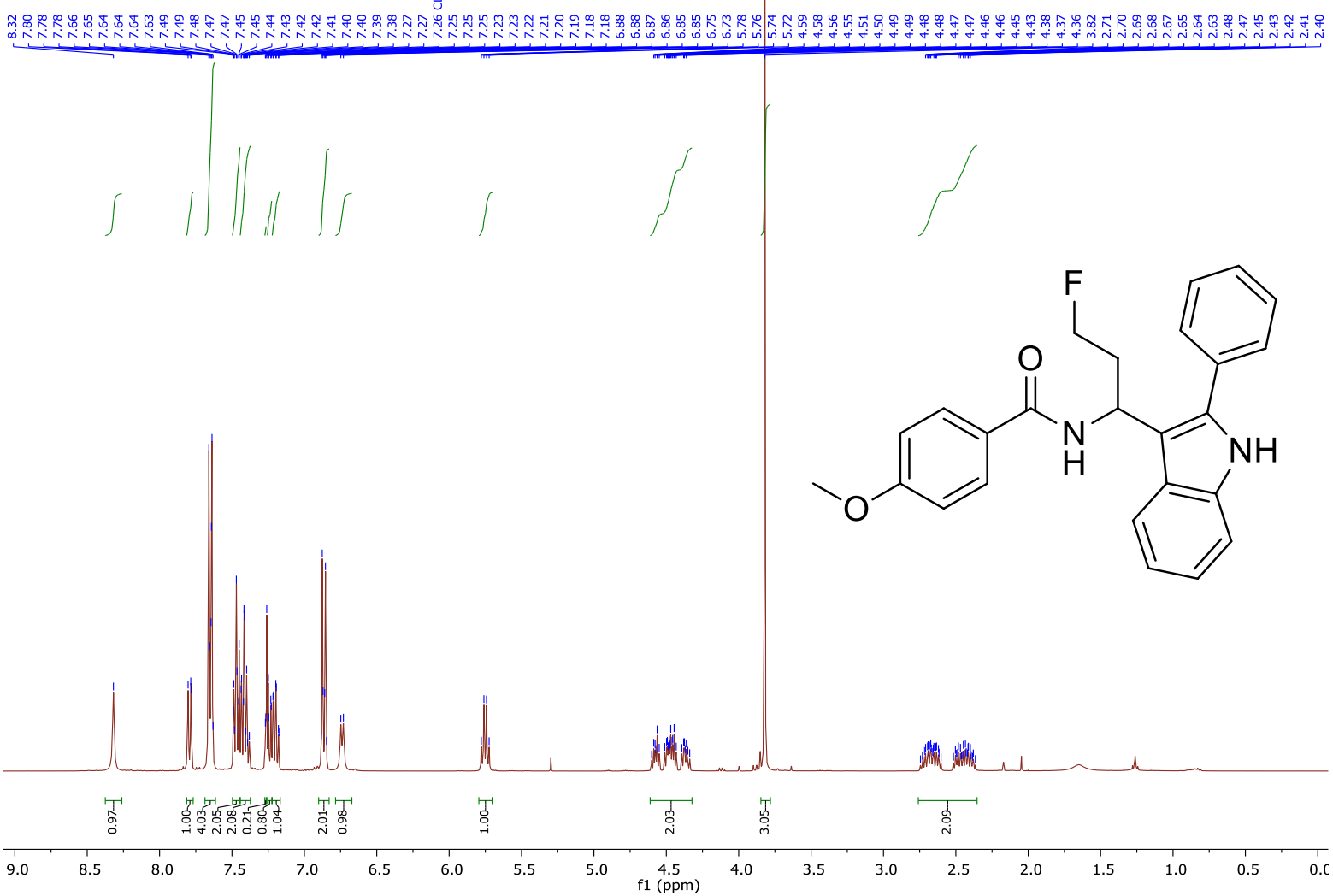

${ }^{13} \mathrm{C}-\mathrm{NMR}\left(101 \mathrm{MHz}, \mathrm{CDCl}_{3}\right)$ 

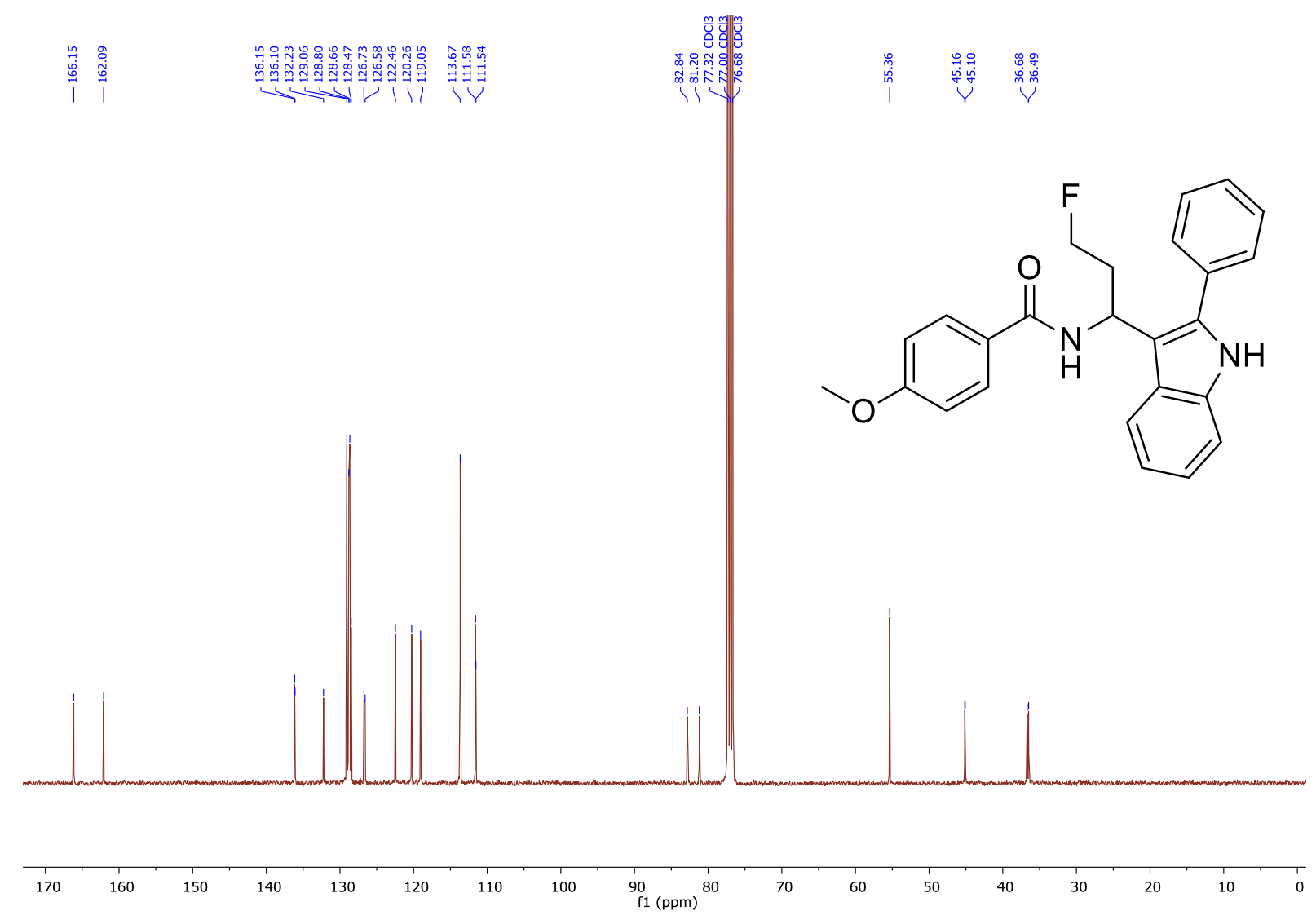

${ }^{19}$ F-NMR (376 MHz, CDCl3)<smiles>COc1ccc(C(=O)NC(CCF)c2c(-c3ccccc3)[nH]c3ccccc23)cc1</smiles>

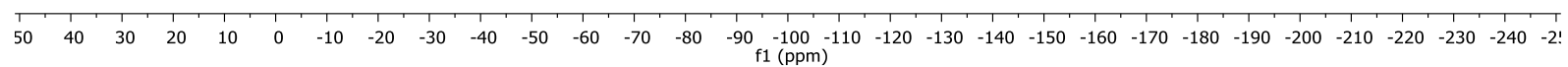
IR 


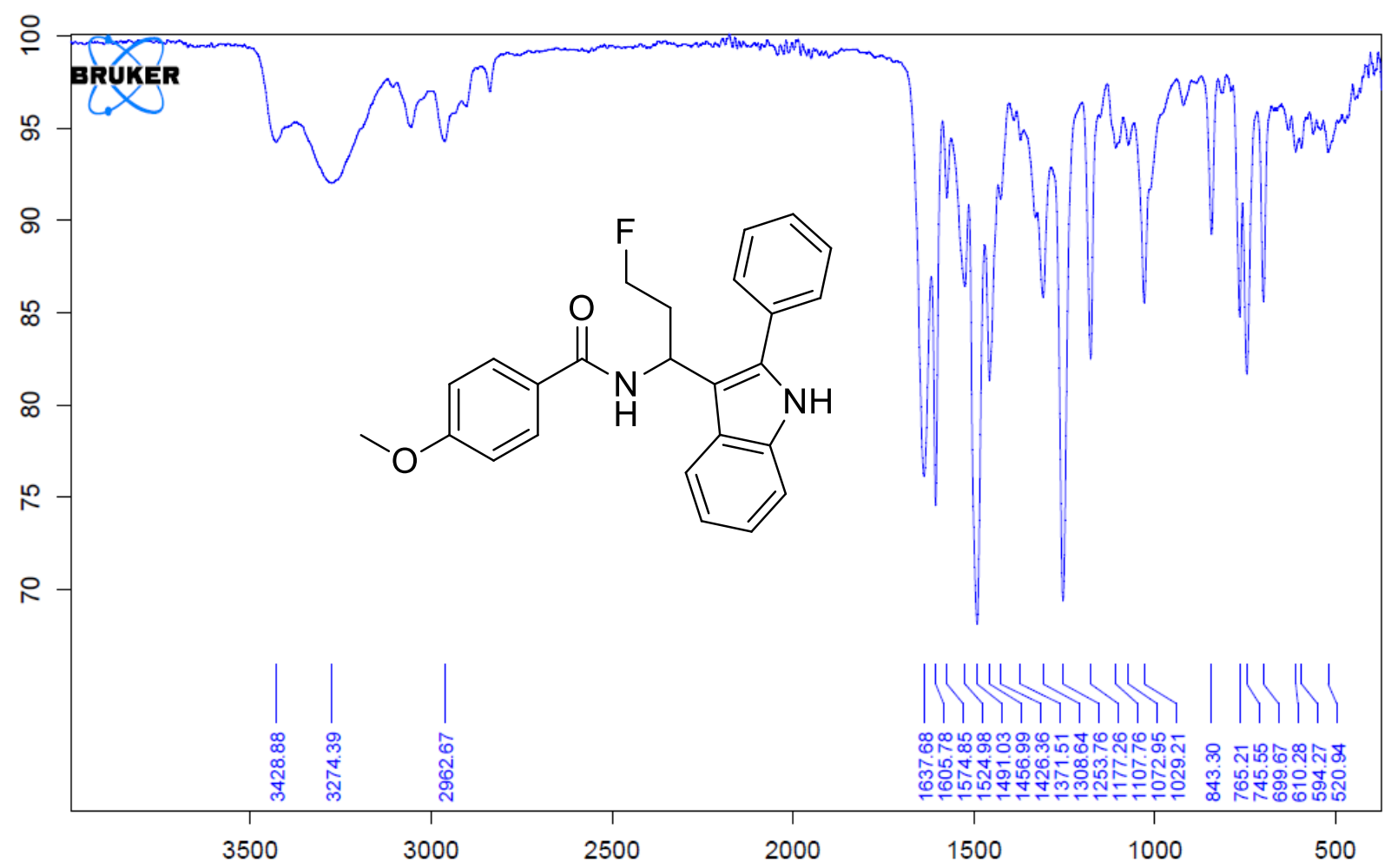

$N$-(3-Fluoro-1-(3-methyl-1H-indol-2-yl)propyl)-4-methoxybenzamide (3o)

${ }^{1}$ H-NMR (400 MHz, $\mathrm{CDCl}_{3}$ )

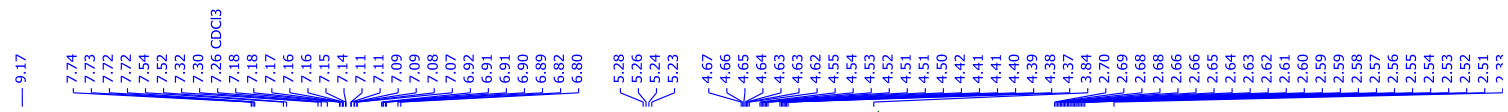

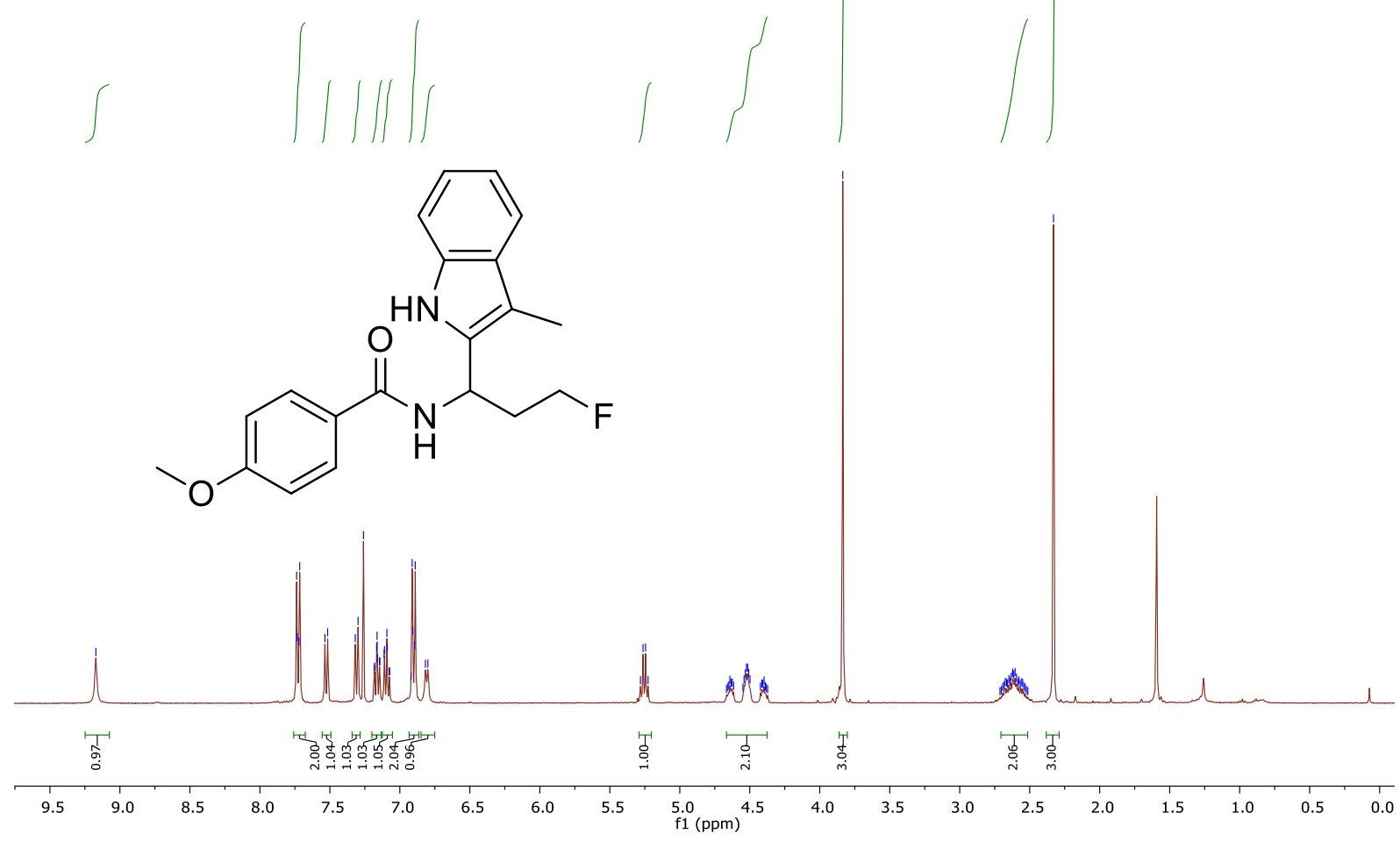

${ }^{13}$ C-NMR (101 MHz, Acetone-d6) 

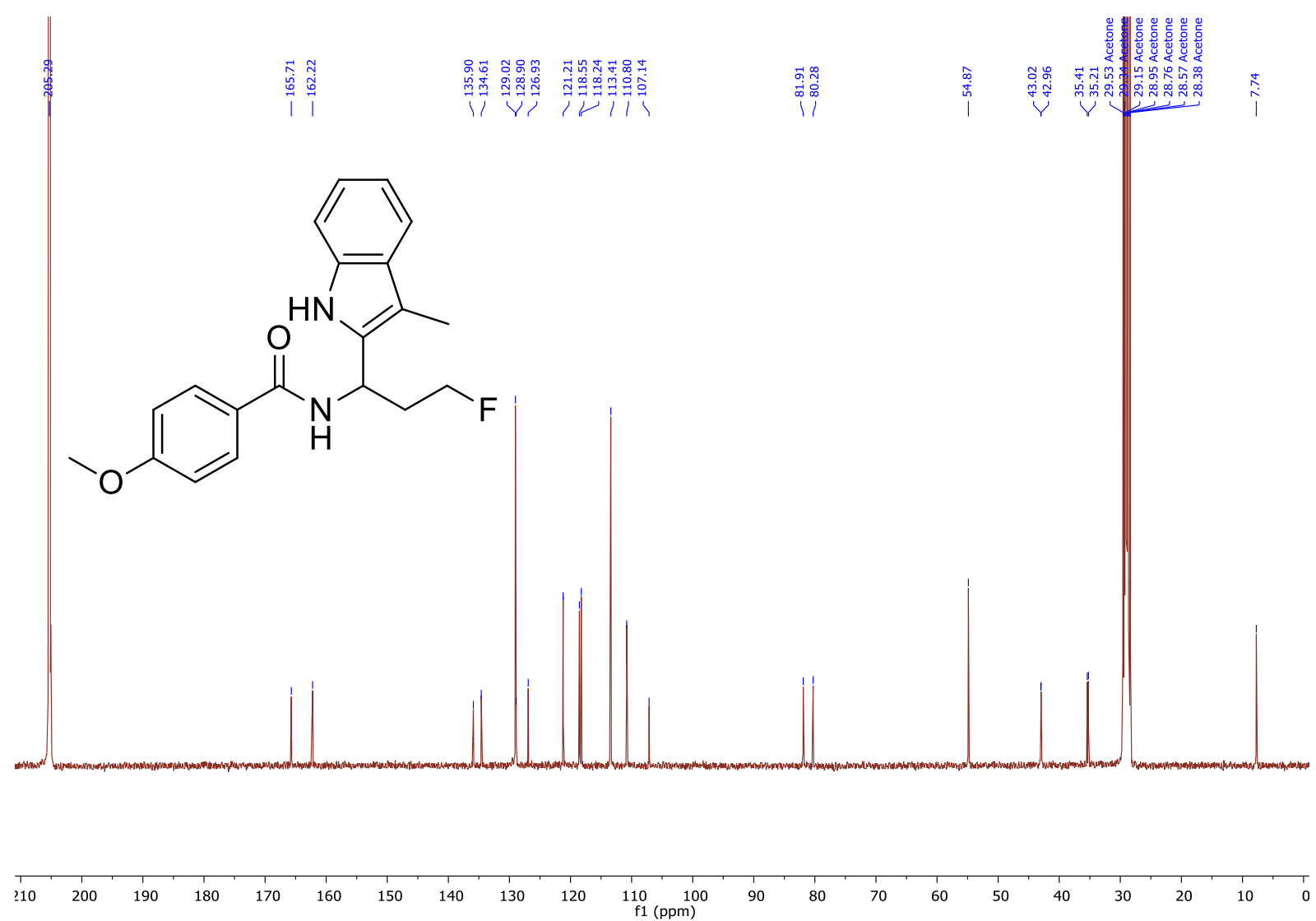

${ }^{19}$ F-NMR (376 MHz, $\mathrm{CDCl}_{3}$ )<smiles>COc1ccc(C(=O)NC(CCF)c2[nH]c3ccccc3c2C)cc1</smiles>

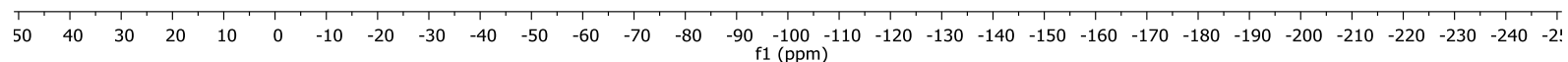
IR 


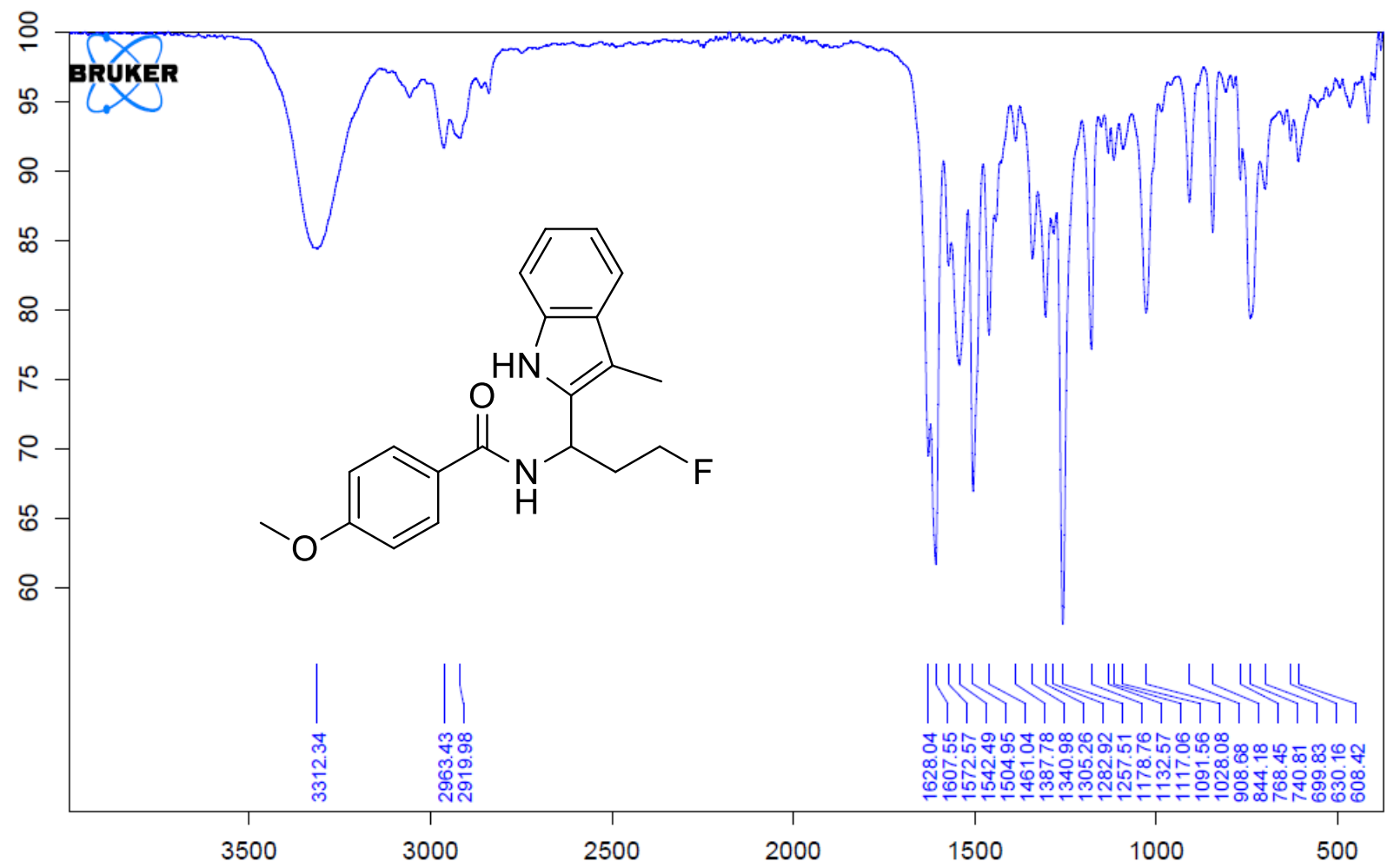

$N$-(3-Fluoro-1-(3-methyl-1H-indol-1-yl)propyl)-4-methoxybenzamide (3p) ${ }^{1} \mathrm{H}-\mathrm{NMR}$ (400 MHz, $\mathrm{CDCl}_{3}$ )

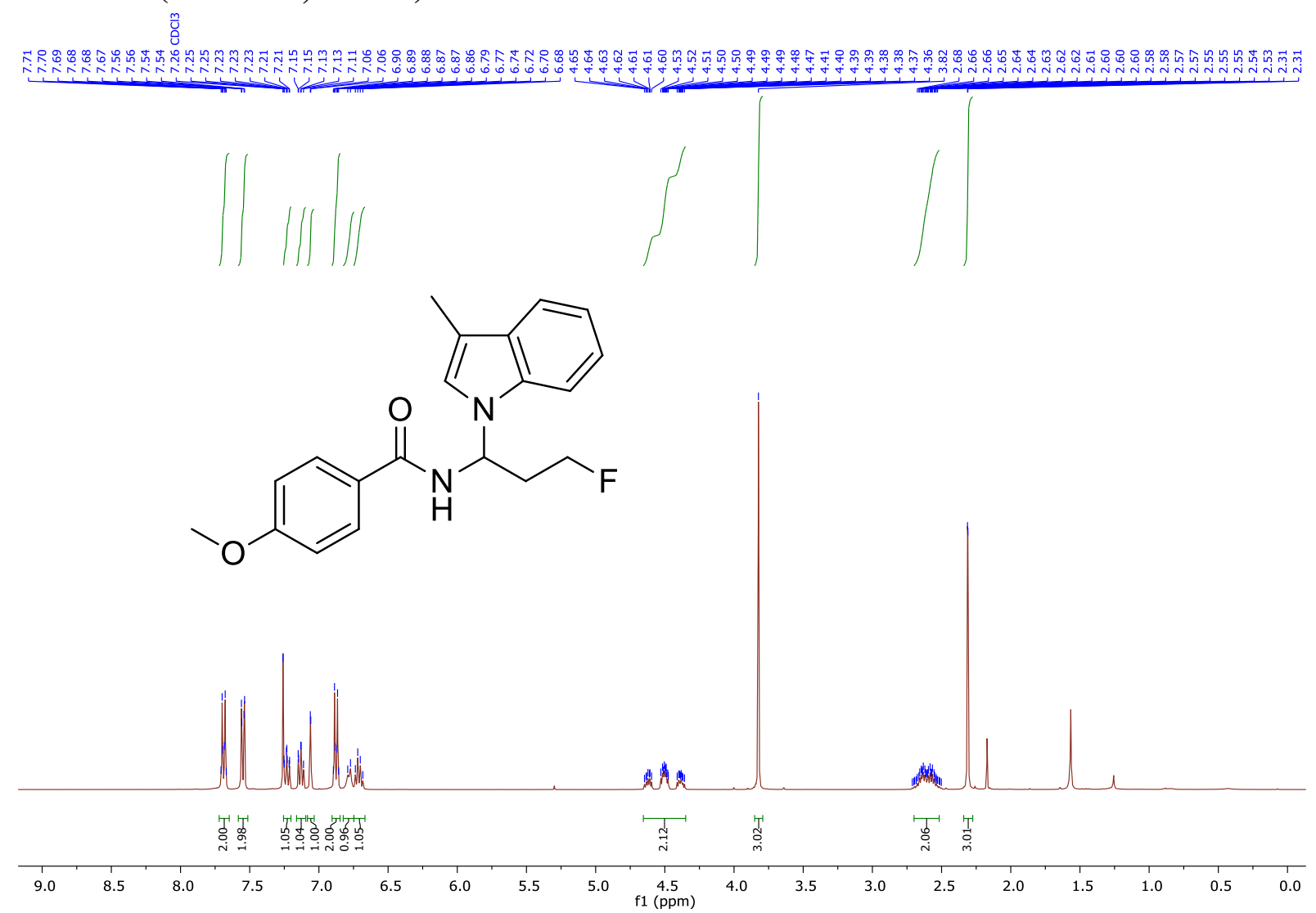

${ }^{13} \mathrm{C}-\mathrm{NMR}$ (101 MHz, CDCl3) 

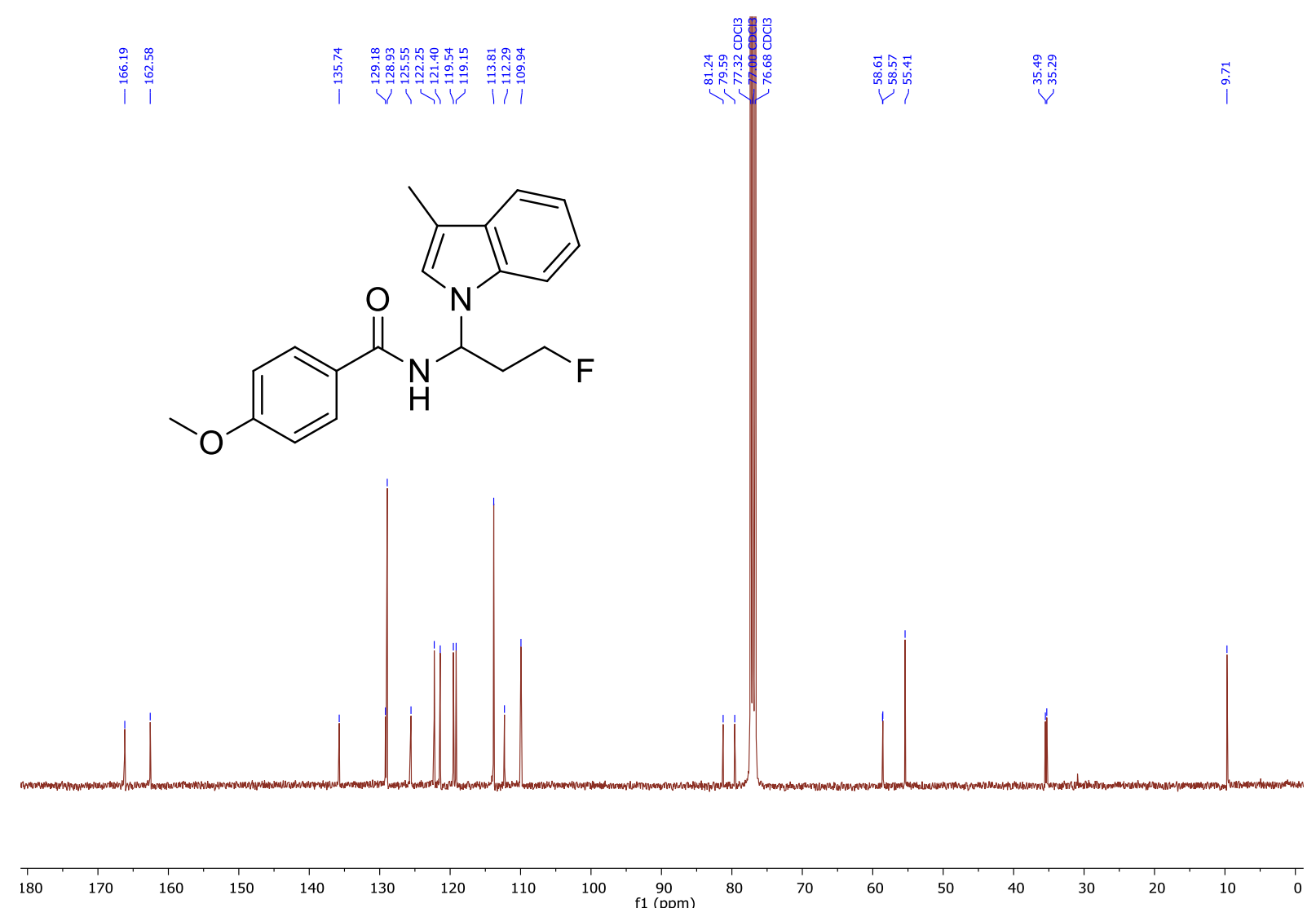

${ }^{19}$ F-NMR (376 MHz, CDCl3)

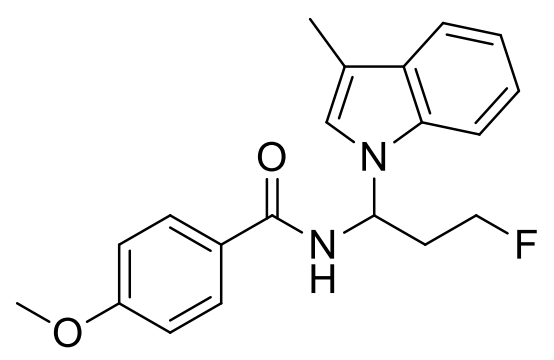

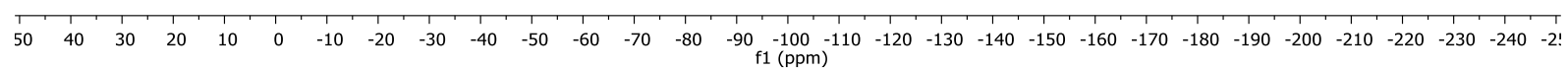
IR 


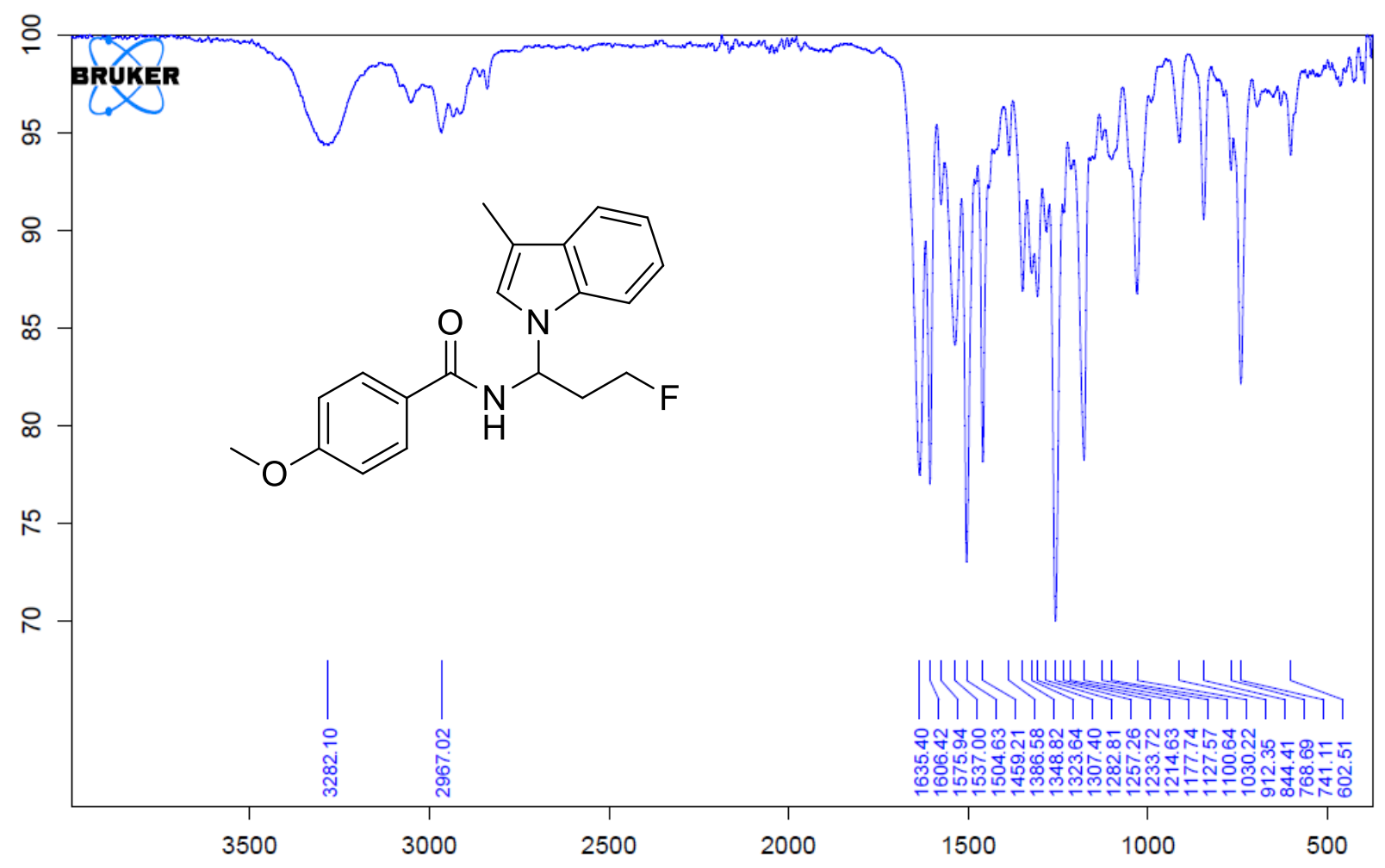

$N$-(3-Fluoro-1-(4-fluoro-1 $H$-indol-3-yl)propyl)-4-methoxybenzamide (3q) ${ }^{1}$ H-NMR (400 MHz, Acetone- $d_{6}$ )

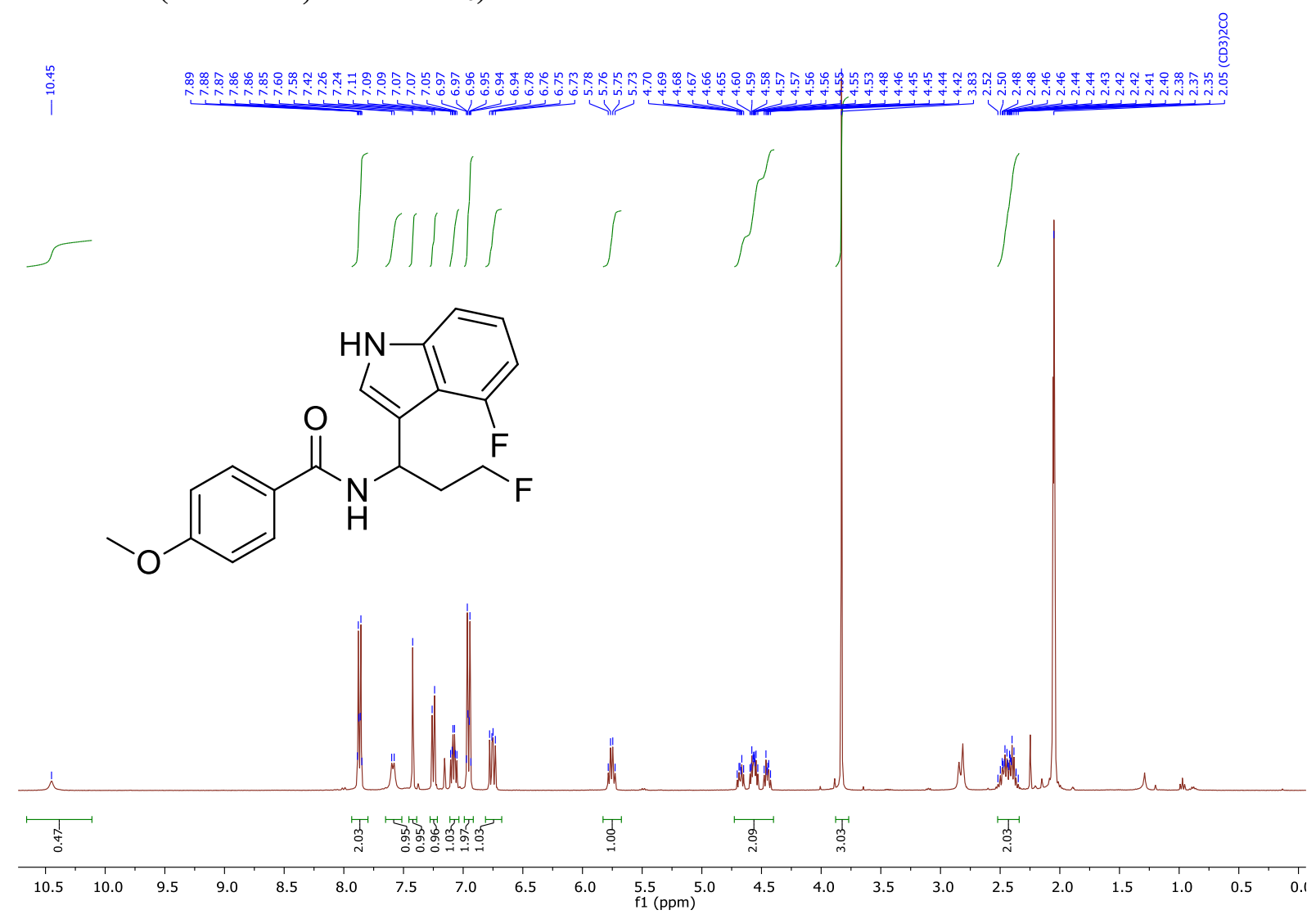

${ }^{13}$ C-NMR (101 MHz, Acetone- $d_{6}$ ) 


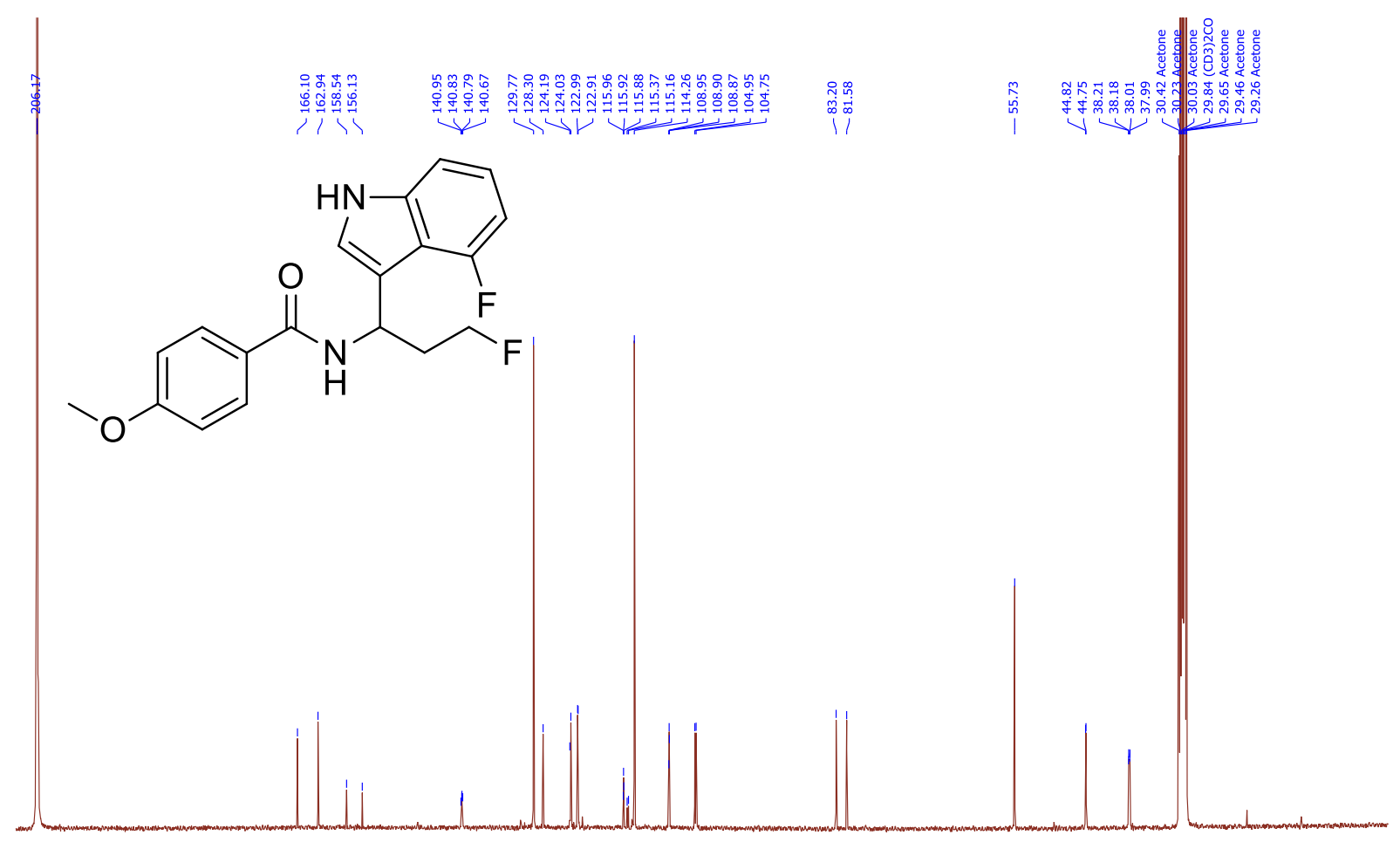

$\begin{array}{lllllllllllllllllllll}200 & 190 & 180 & 170 & 160 & 150 & 140 & 130 & 120 & \underset{1}{110} \begin{array}{r}100 \\ (\mathrm{ppm})\end{array} & 90 & 80 & 70 & 60 & 50 & 40 & 30 & 20 & 10 & 0\end{array}$

${ }^{19}$ F-NMR (376 MHz, Acetone-d6)

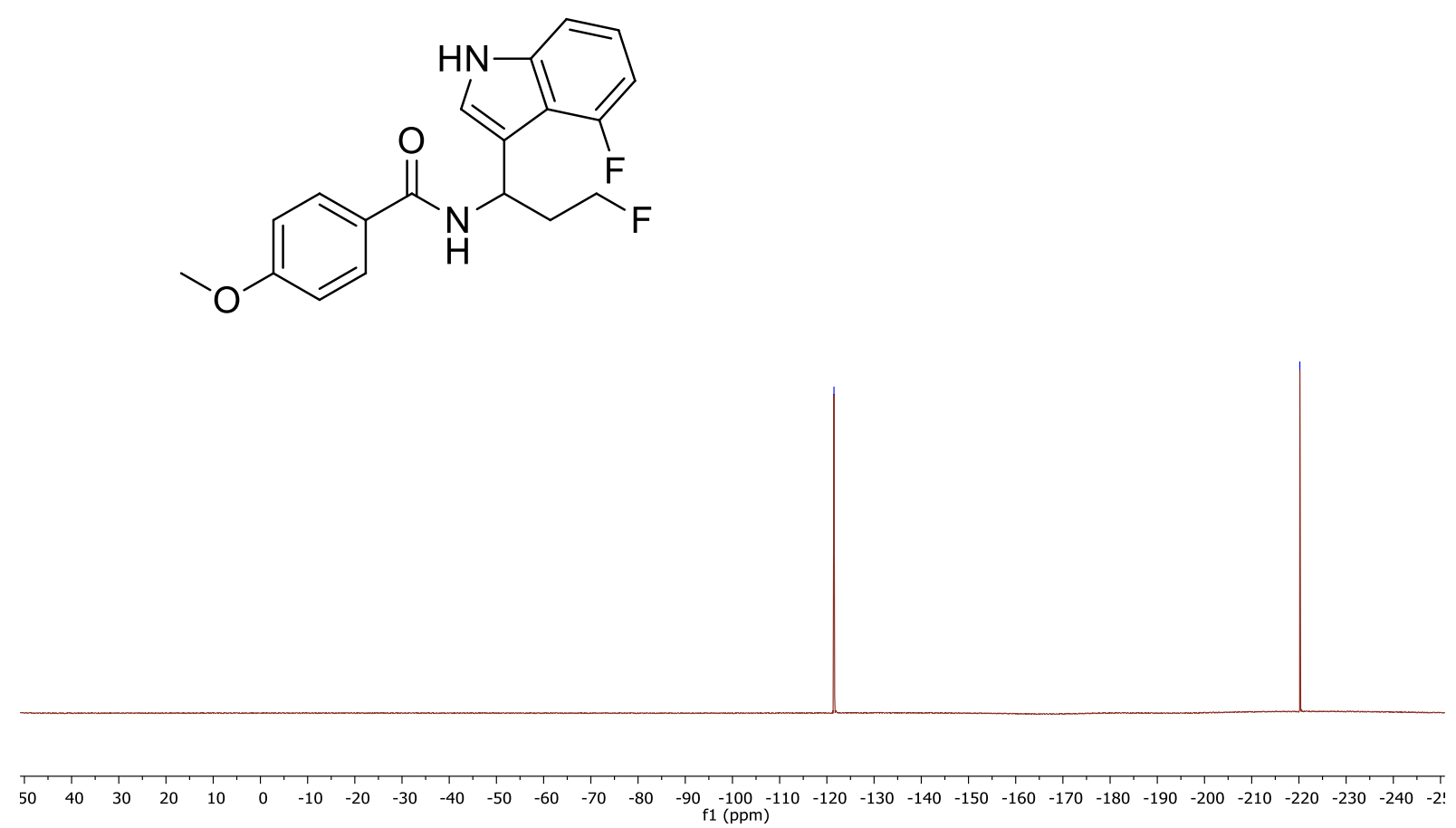

IR 


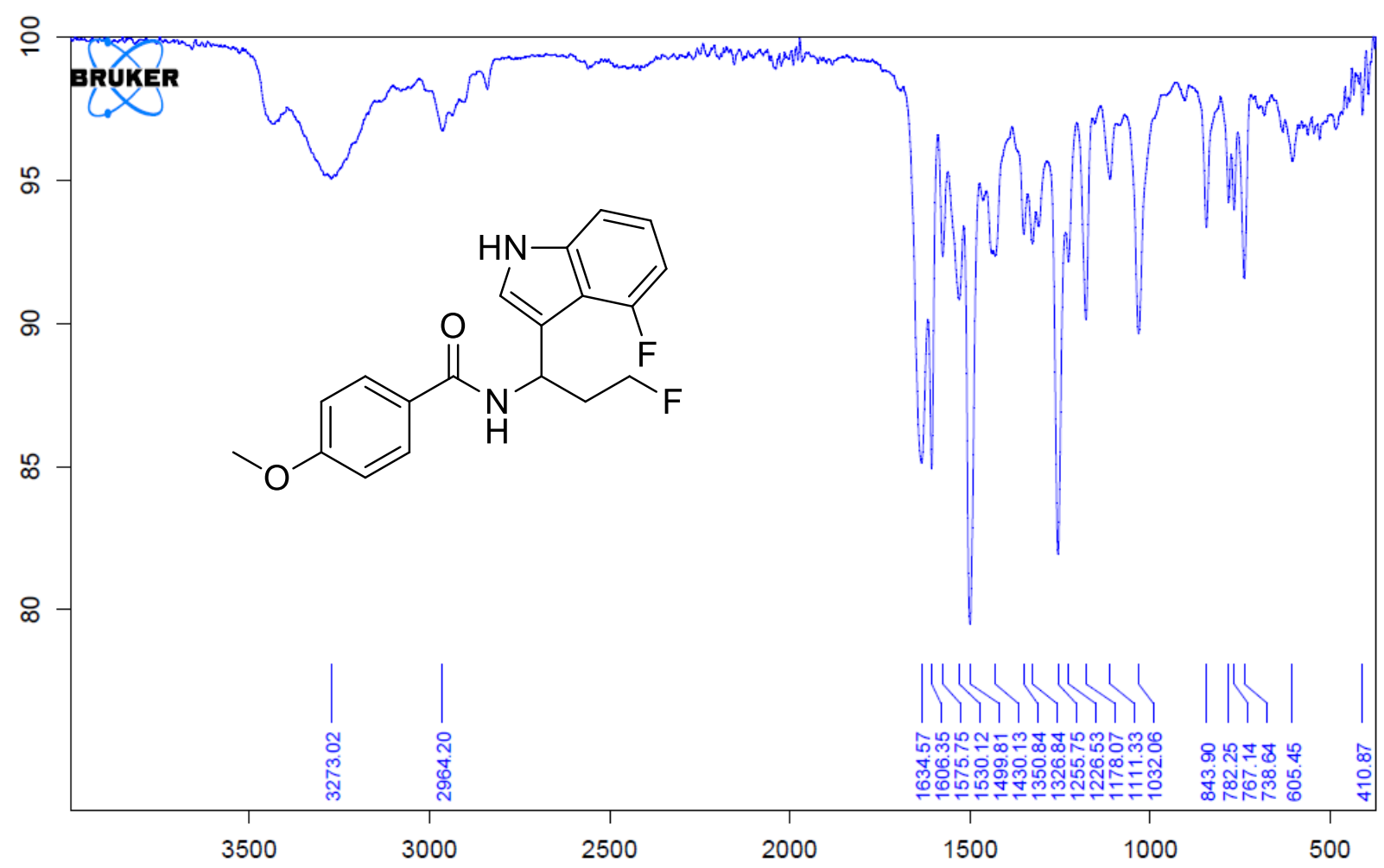

$N$-(3-Fluoro-1-(5-methoxy-1H-indol-3-yl)propyl)-4-methoxybenzamide (3r) ${ }^{1}$ H-NMR (400 MHz, Acetone-d $d_{6}$ )

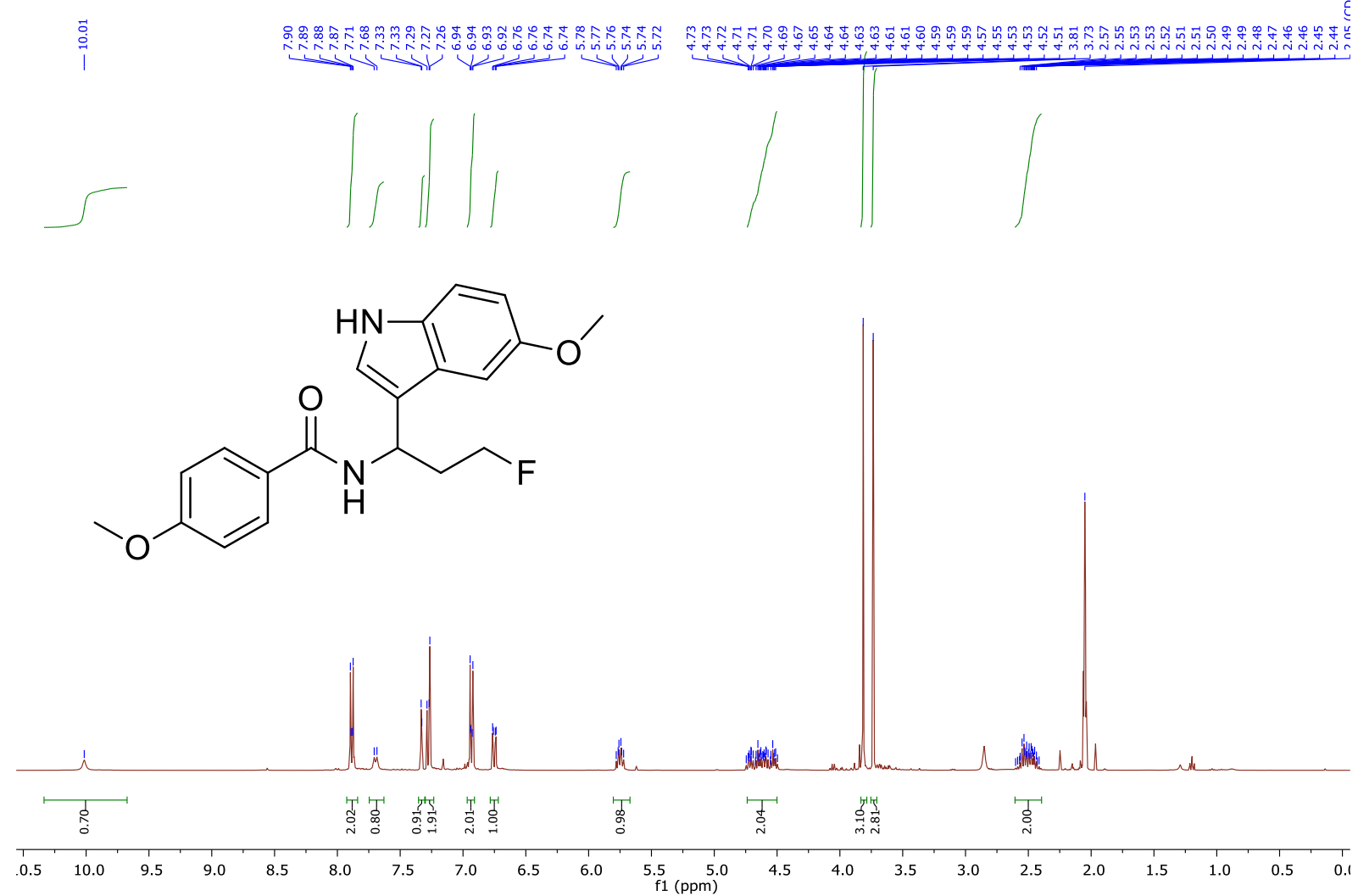

${ }^{13}$ C-NMR (101 MHz, Acetone-d6) 


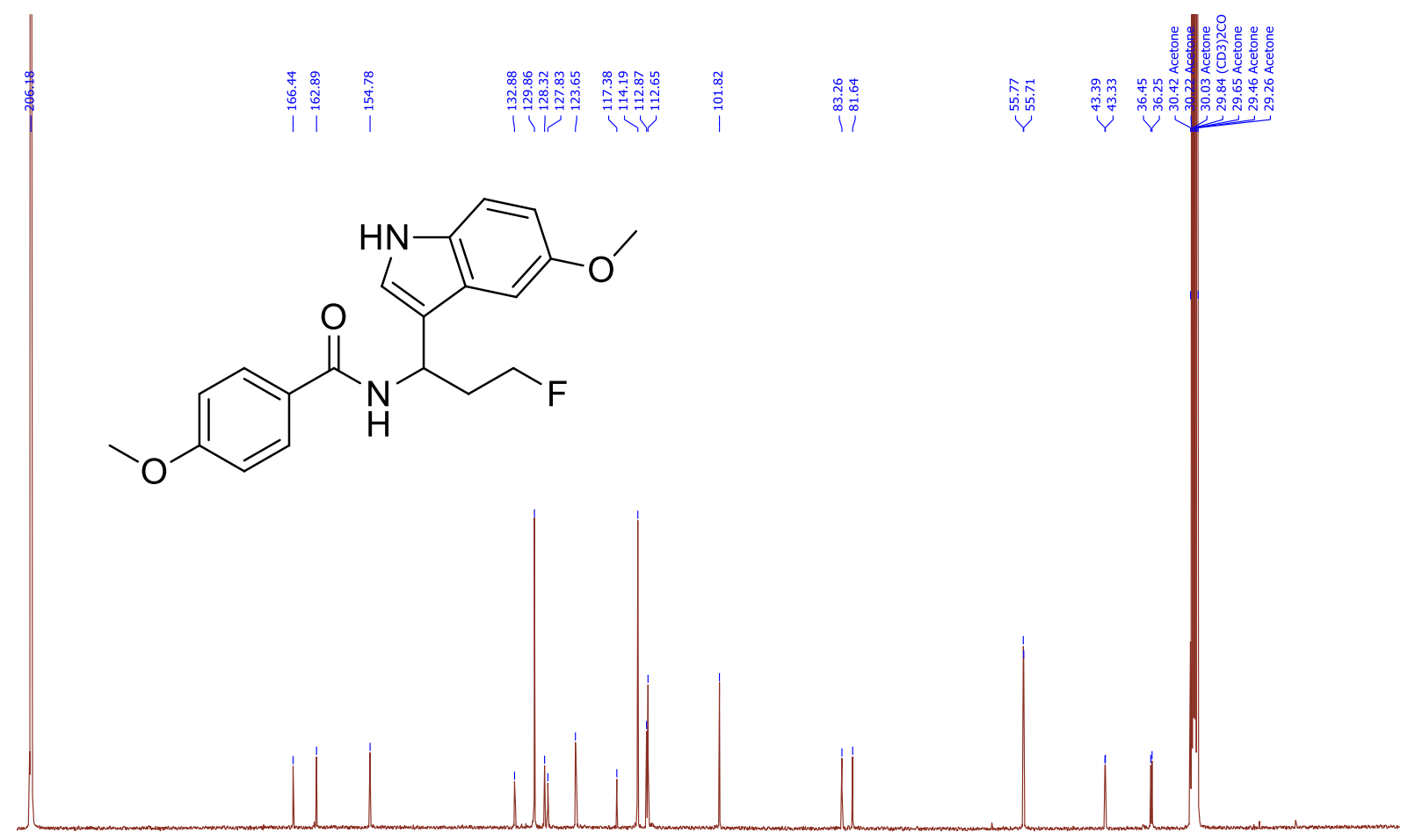

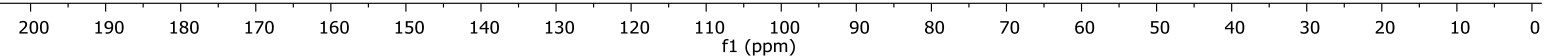

${ }^{19}$ F-NMR (376 MHz, Acetone-d6)<smiles>COc1ccc(C(=O)NC(CCF)c2c[nH]c3ccc(OC)cc23)cc1</smiles>

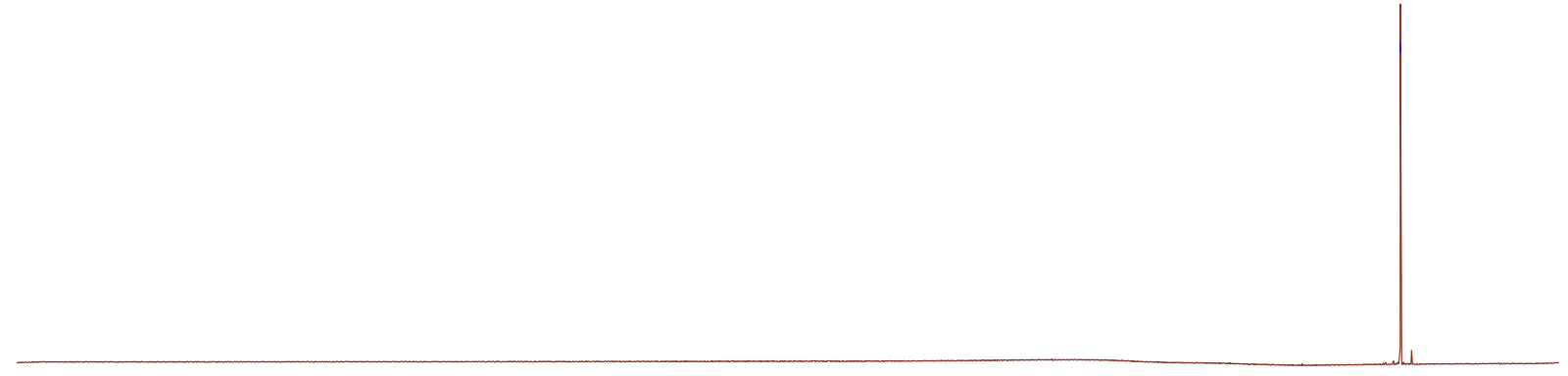

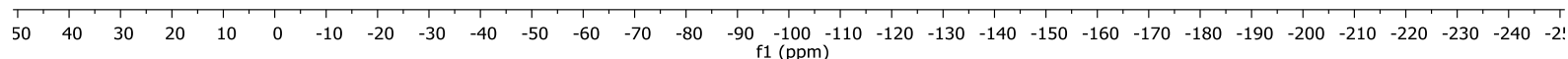
IR 


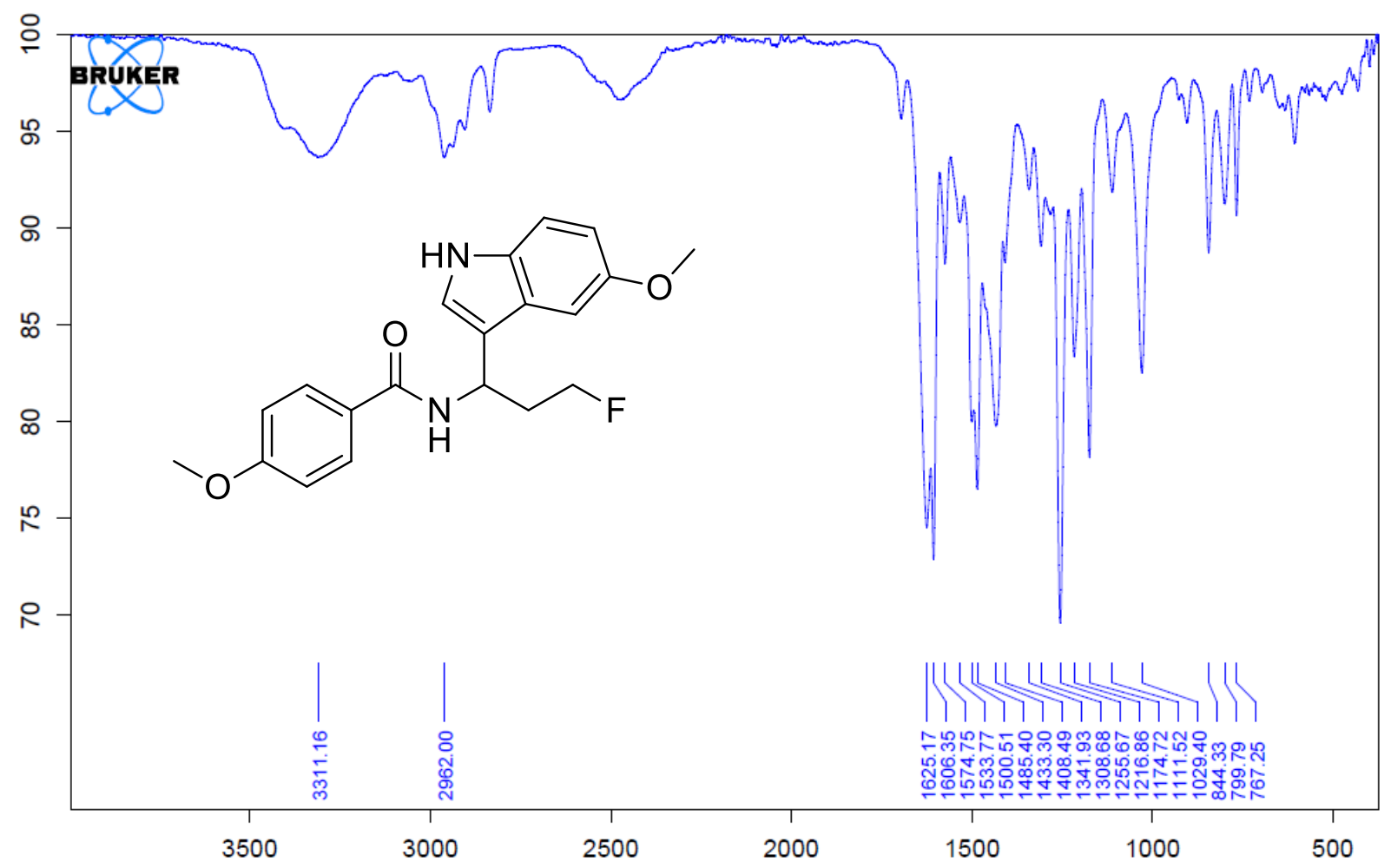

$N$-(3-Fluoro-1-(5-methyl-1H-indol-3-yl)propyl)-4-methoxybenzamide (3s) ${ }^{1} \mathrm{H}-\mathrm{NMR}$ (400 MHz, $\left.\mathrm{CDCl}_{3}\right)$

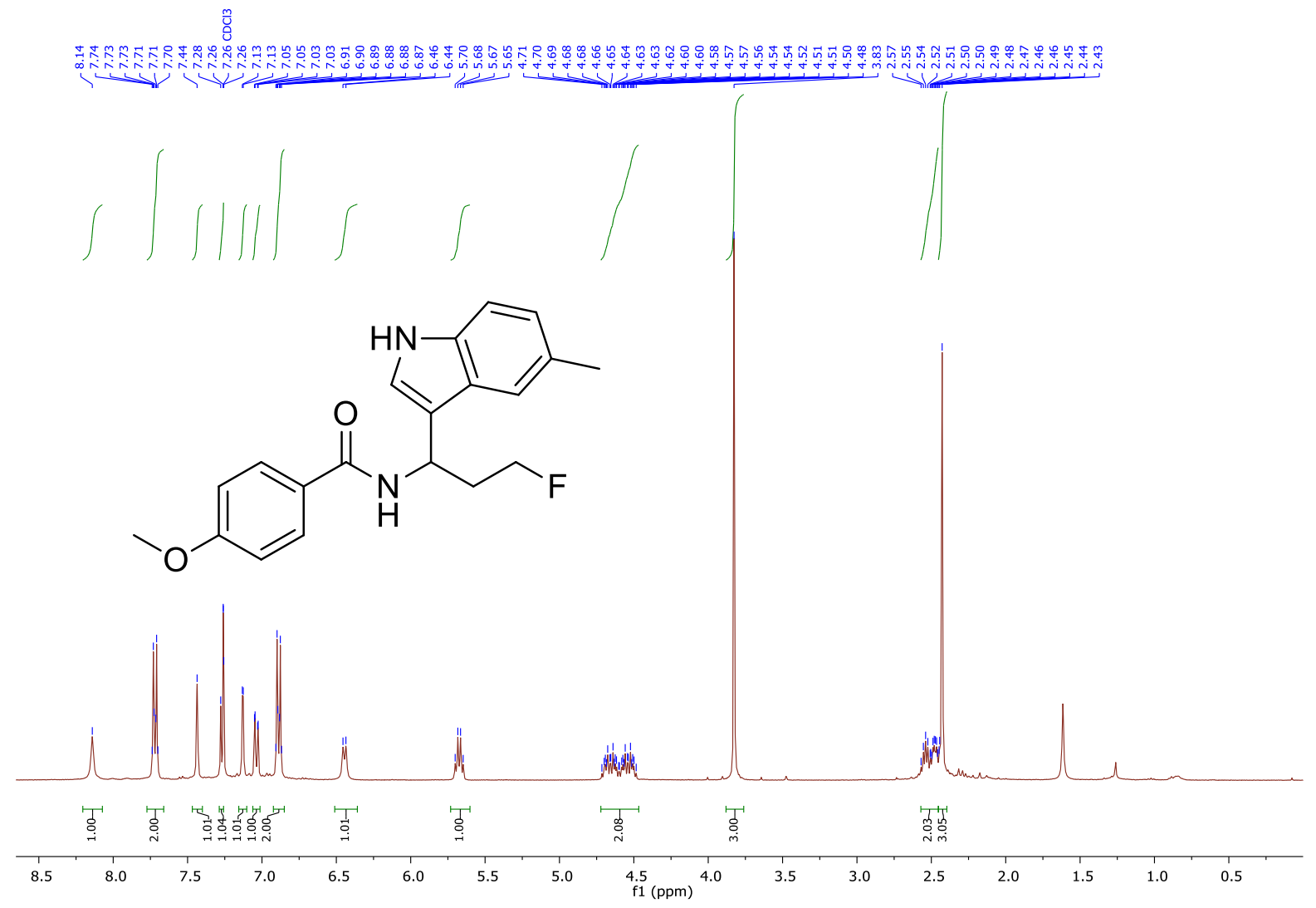

${ }^{13}$ C-NMR (101 MHz, CDCl3) 

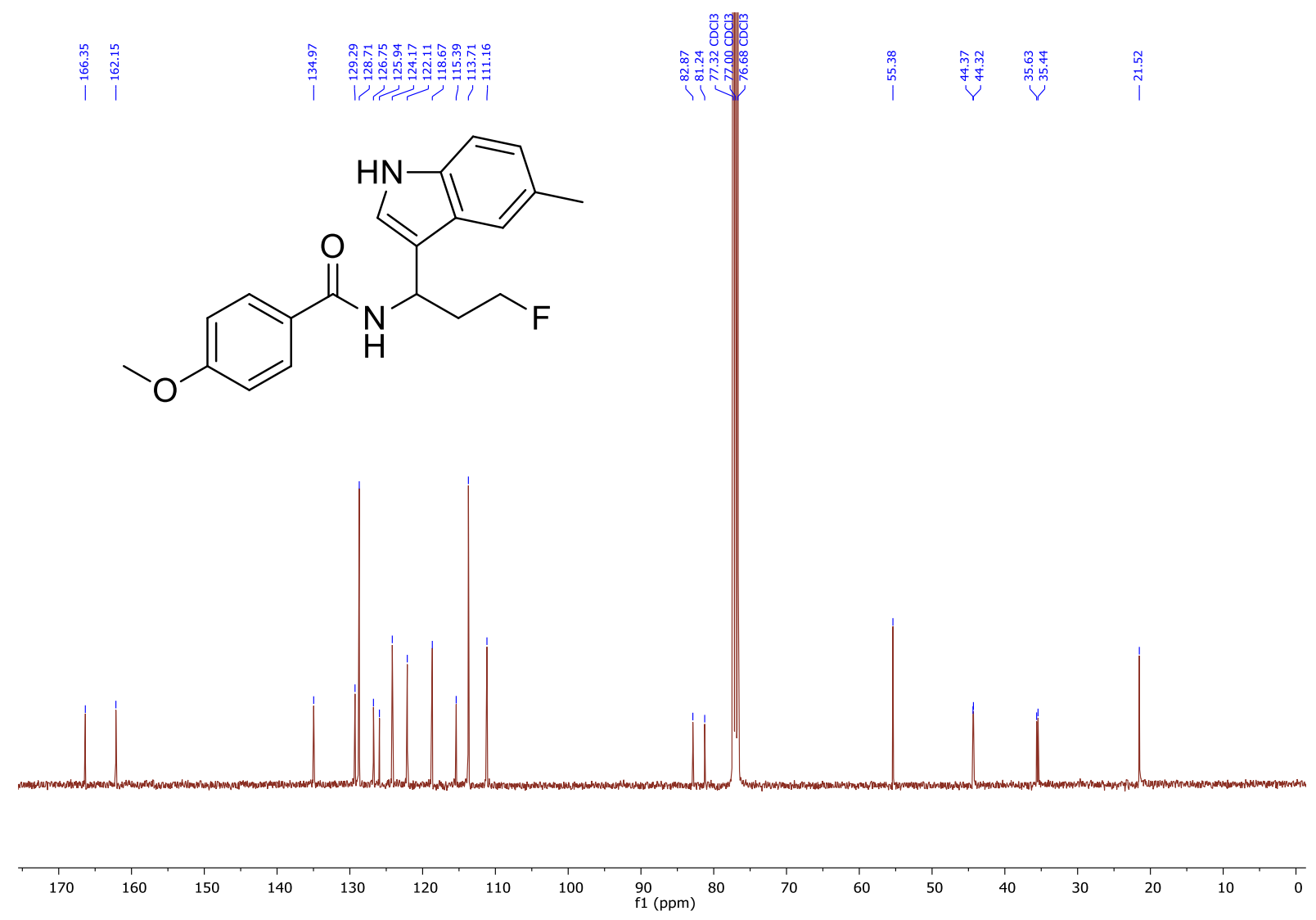

${ }^{19}$ F-NMR (376 MHz, CDCl 3$)$<smiles>COc1ccc(C(=O)NC(CCF)c2c[nH]c3ccc(C)cc23)cc1</smiles>

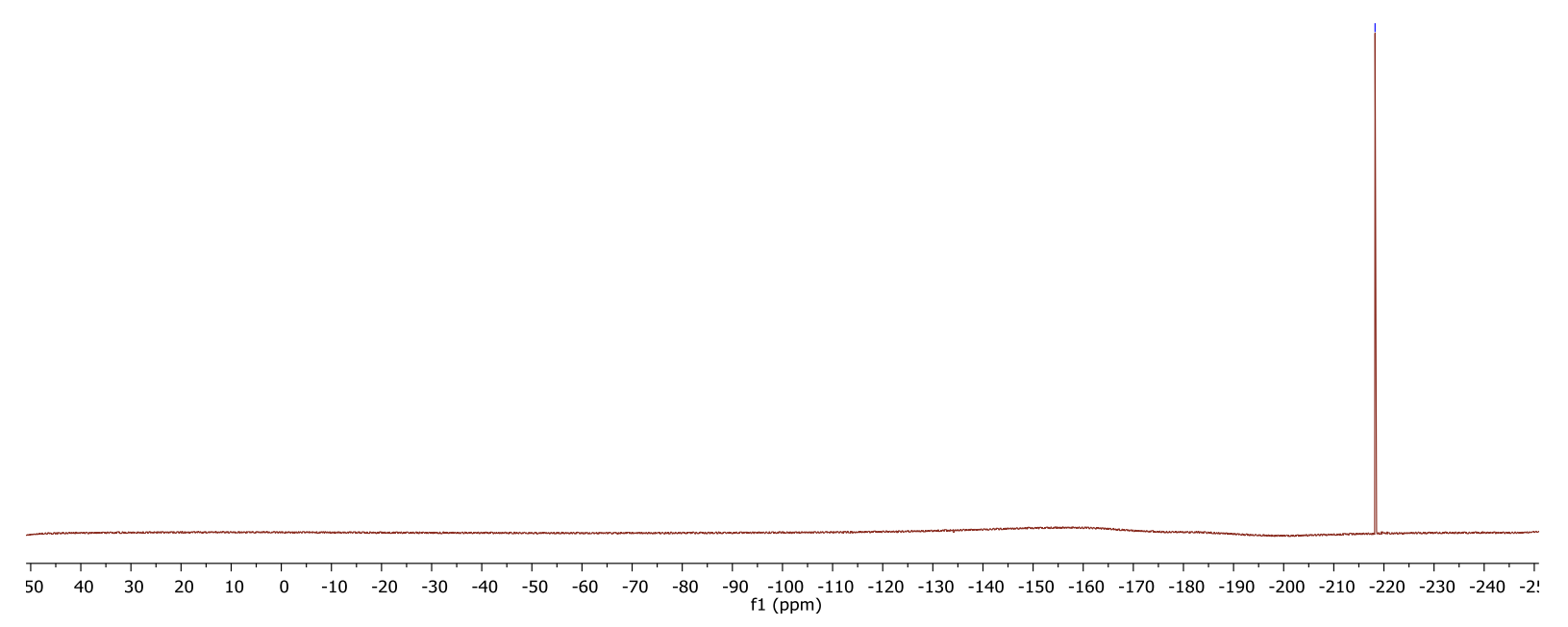

IR 


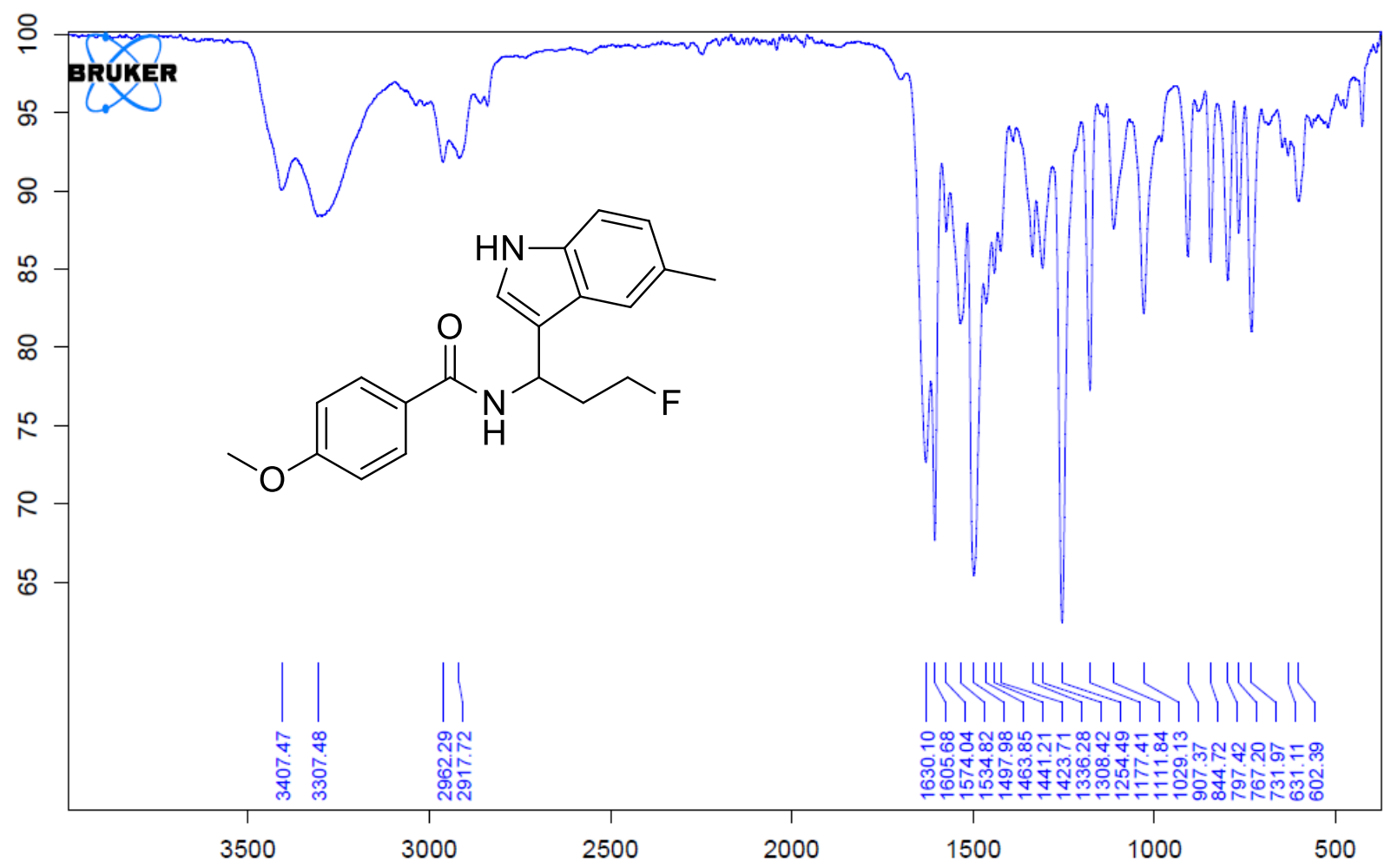

$N$-(1-(5-Chloro-1H-indol-3-yl)-3-fluoropropyl)-4-methoxybenzamide (3t) ${ }^{1} \mathrm{H}-\mathrm{NMR}$ (400 $\mathrm{MHz}, \mathrm{CDCl}_{3}$ )

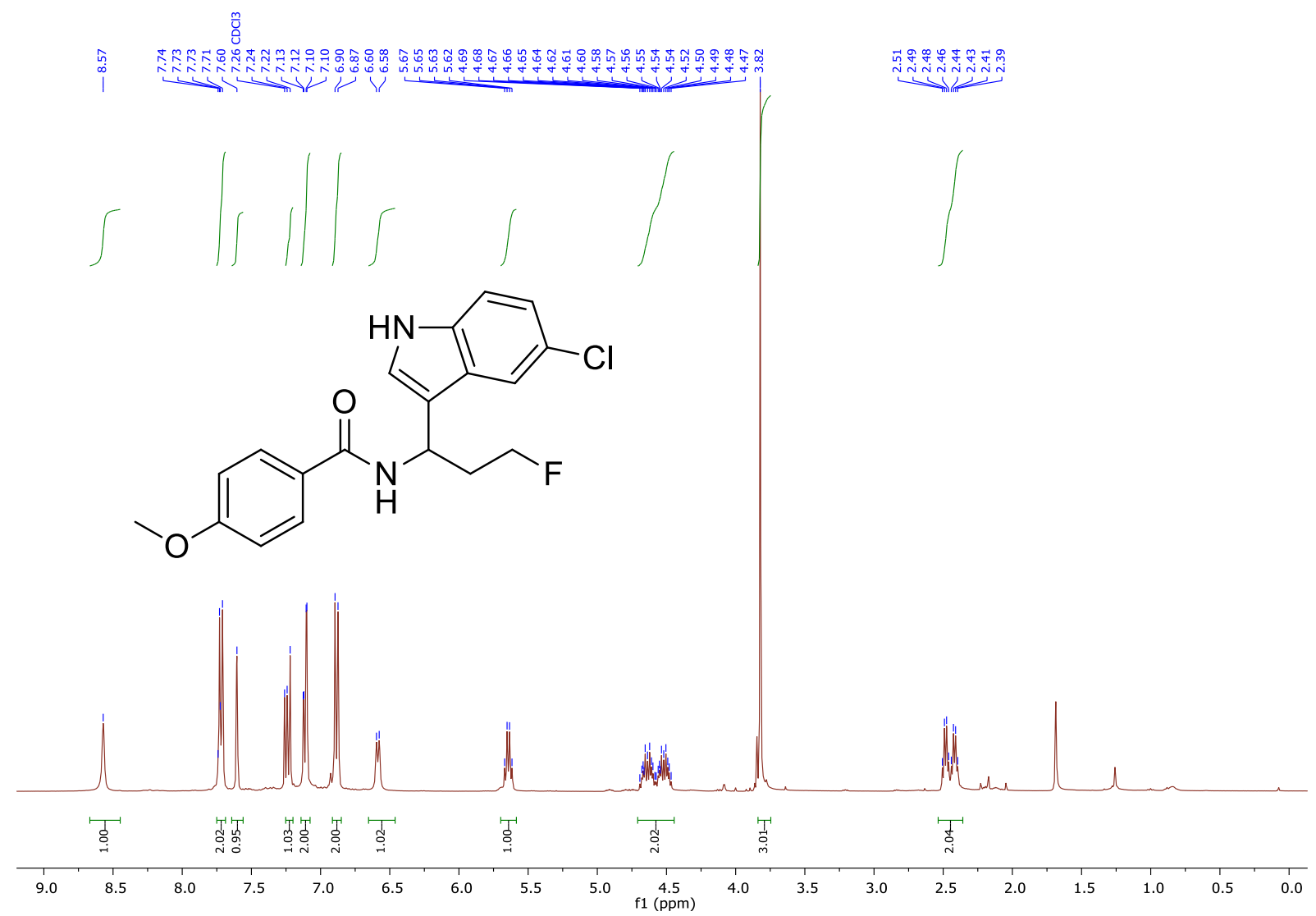

${ }^{13} \mathrm{C}$-NMR (101 MHz, $\mathrm{CDCl}_{3}$ ) 

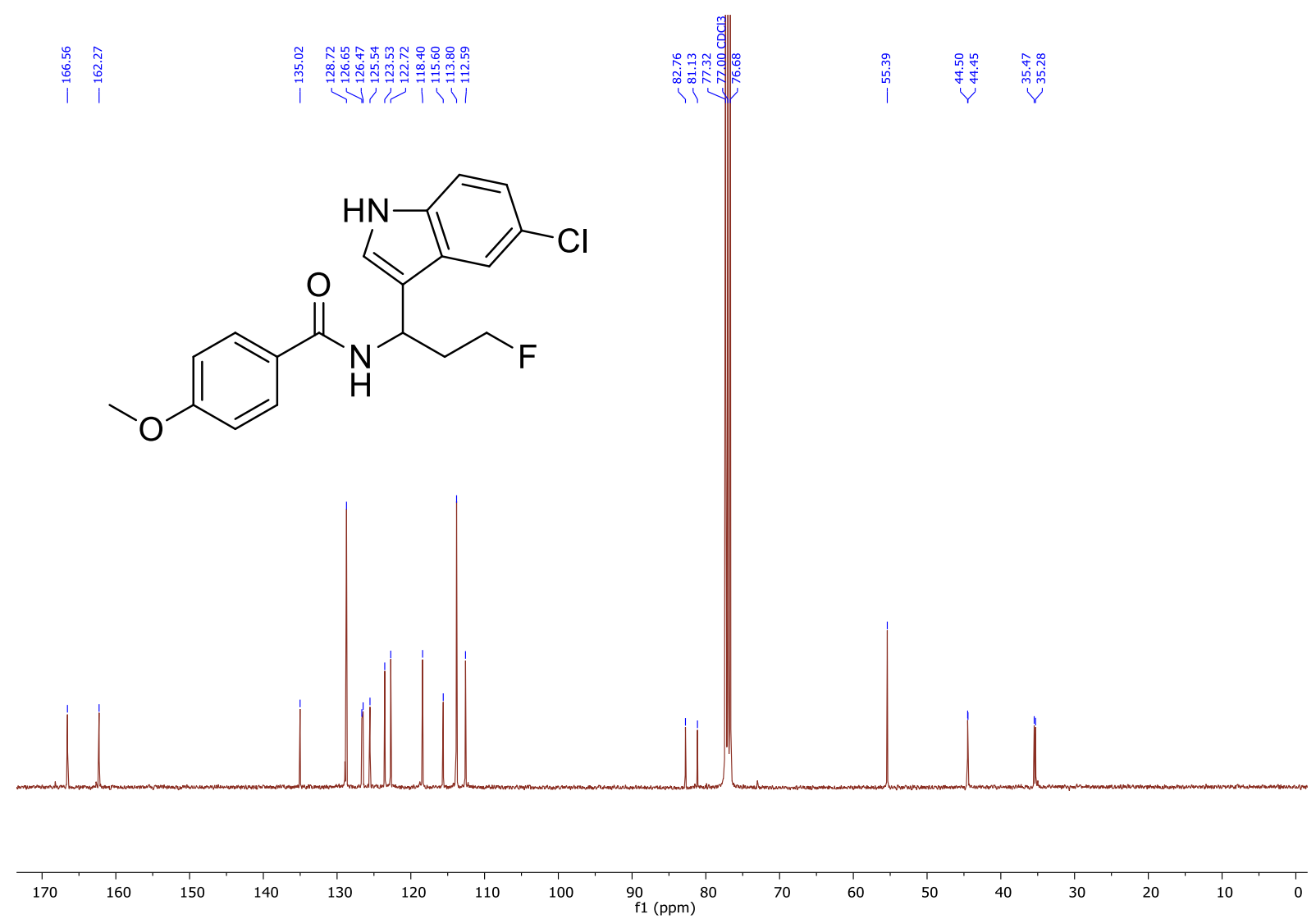

${ }^{19}$ F-NMR (376 MHz, CDCl3)<smiles>COc1ccc(C(=O)NC(CCF)c2c[nH]c3ccc(Cl)cc23)cc1</smiles>

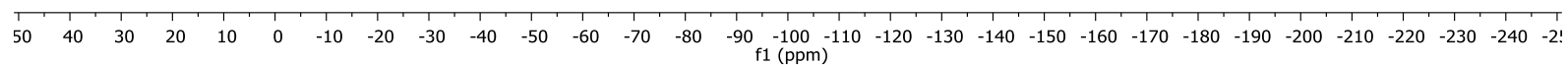
IR 


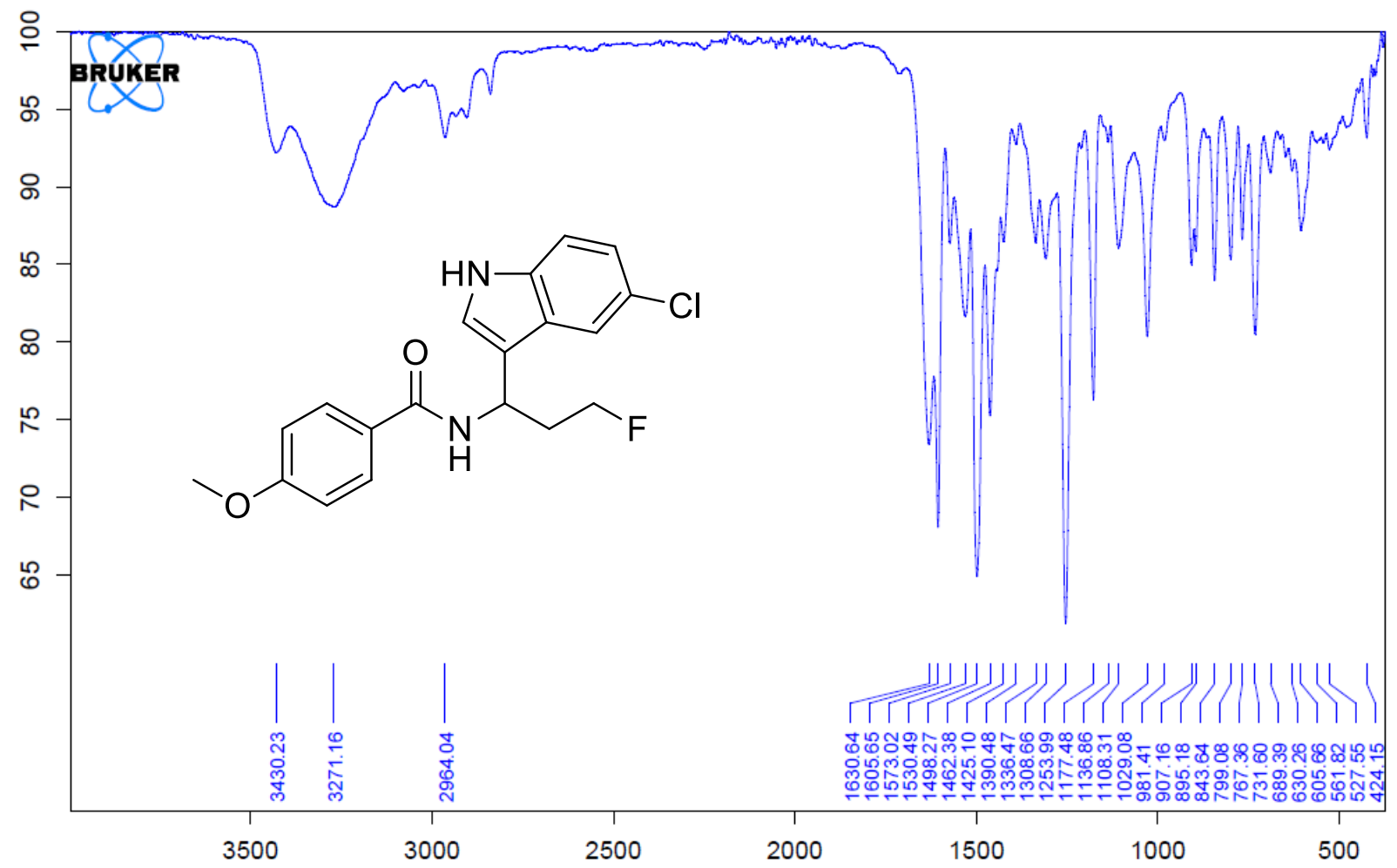

Methyl 3-(3-fluoro-1-(4-methoxybenzamido)propyl)-1H-indole-5-carboxylate (3u) ${ }^{1}$ H-NMR (400 MHz, Acetone-d6)

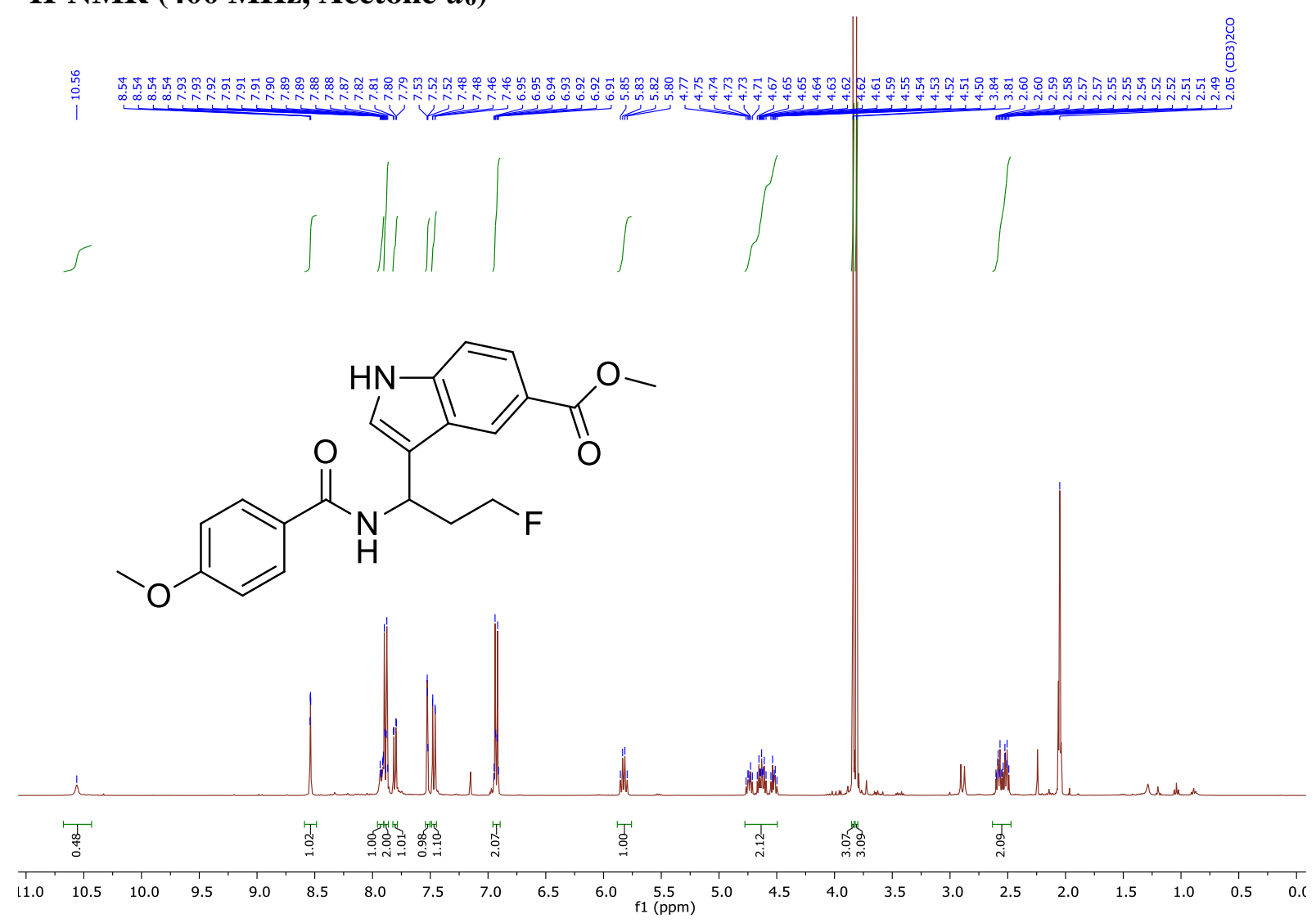

${ }^{13}$ C-NMR (101 MHz, Acetone-d6) 


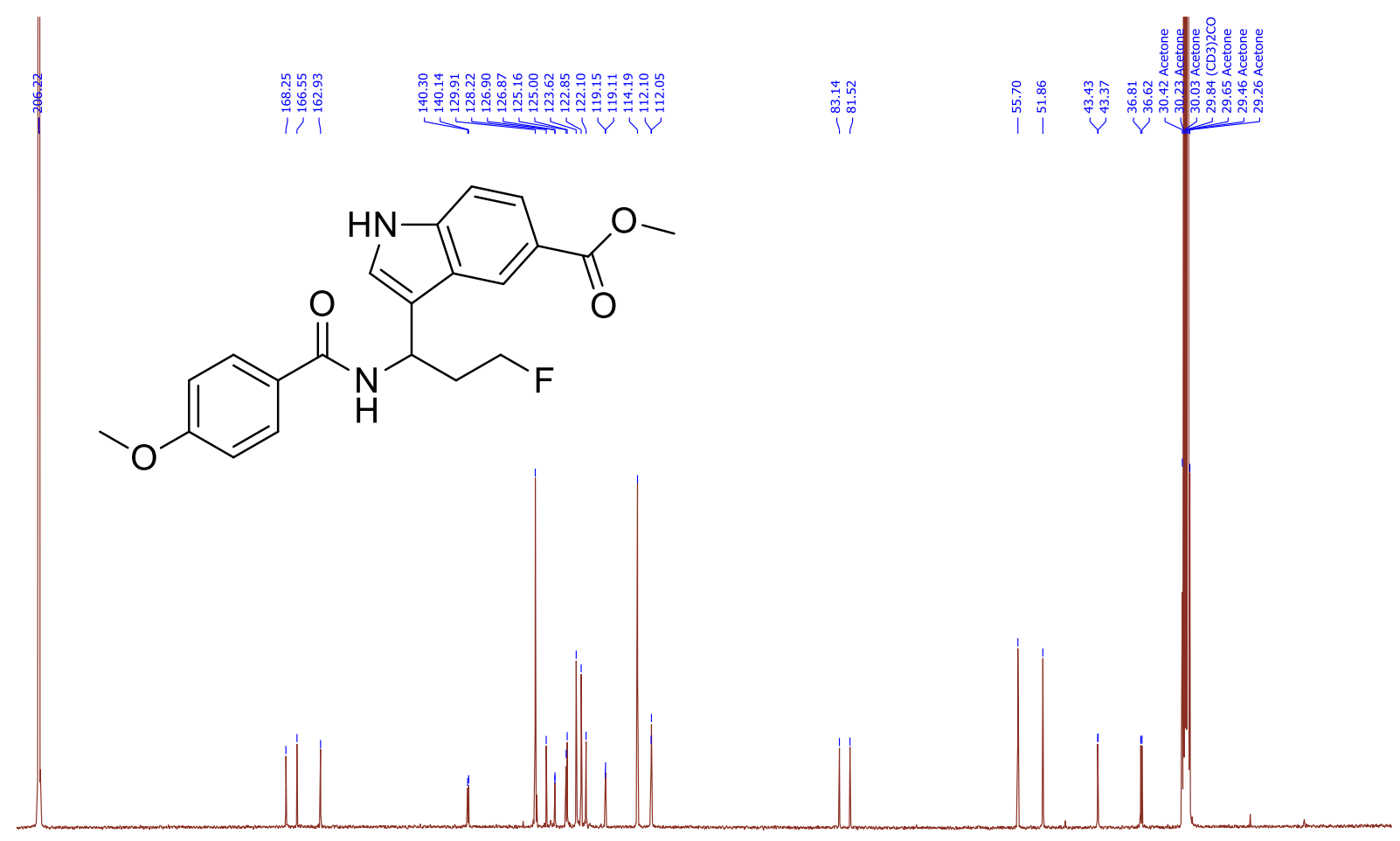

$\begin{array}{llllllllllllllllllllll}1 & 100 & 190 & 180 & 170 & 160 & 150 & 140 & 130 & 120 & \begin{array}{c}110 \\ \mathrm{f} 1(\mathrm{ppm})\end{array} & 90 & 80 & 70 & 60 & 50 & 40 & 30 & 20 & 10 & 0\end{array}$

${ }^{13} \mathrm{C}-\mathrm{NMR}\left(151 \mathrm{MHz}\right.$, Acetonitrile-d3, $\left.70{ }^{\circ} \mathrm{C}\right)$
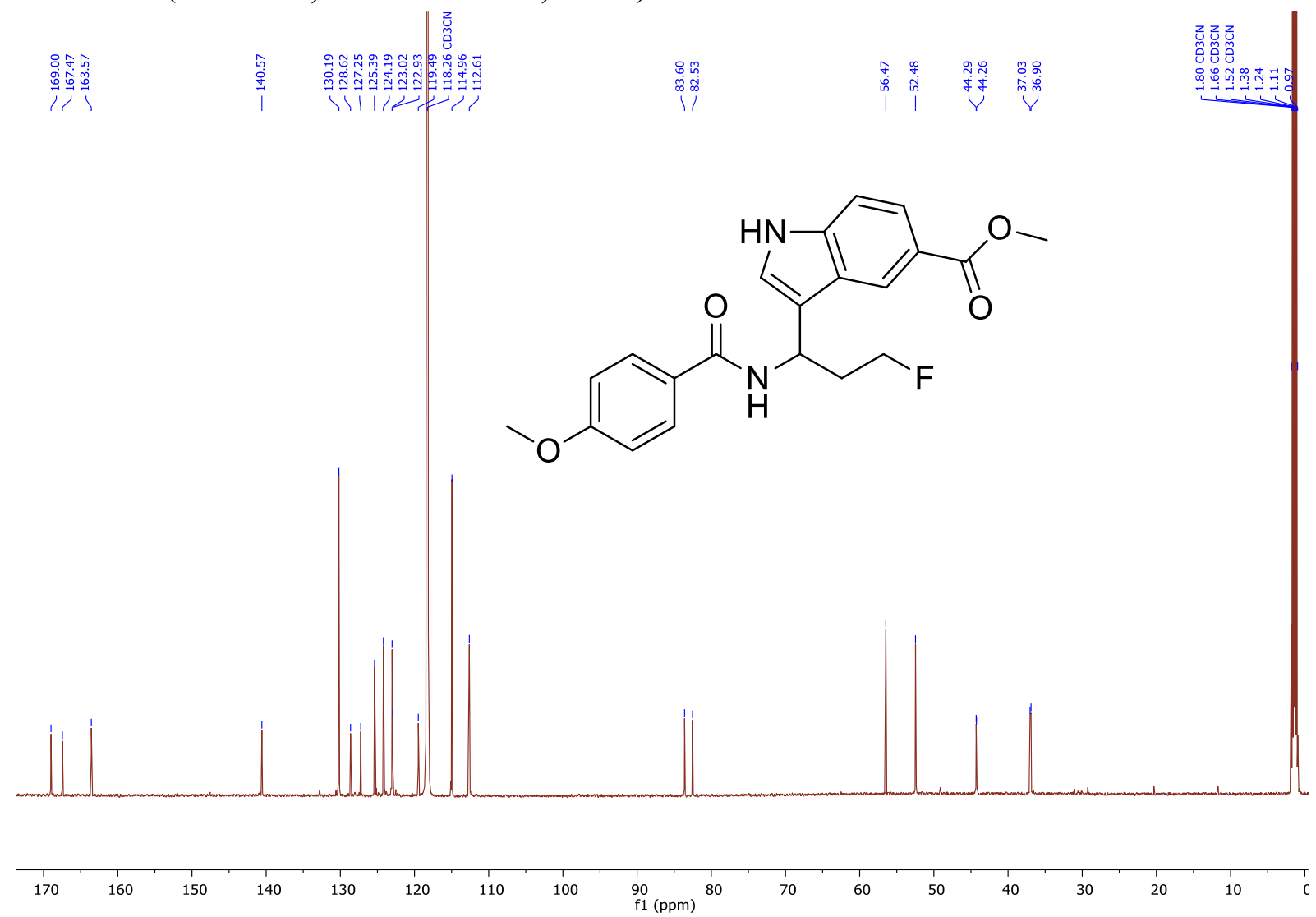

${ }^{19}$ F-NMR (376 MHz, Acetone-d6) 
<smiles>COC(=O)c1ccc2[nH]cc(C(CCF)NC(=O)c3ccc(OC)cc3)c2c1</smiles>

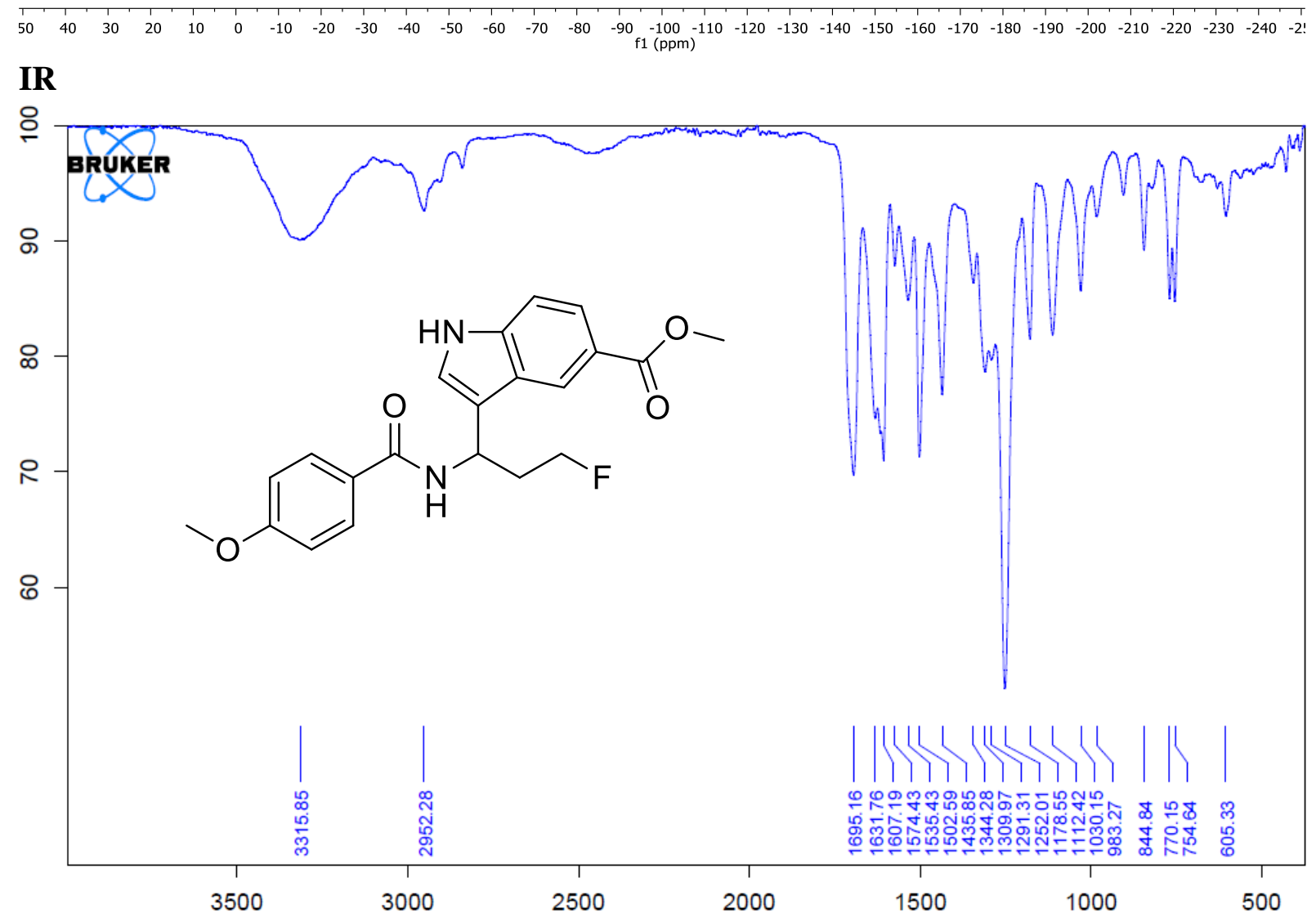

$N$-(3-Fluoro-1-(6-methyl-1H-indol-3-yl)propyl)-4-methoxybenzamide (3v) ${ }^{1}$ H-NMR (400 MHz, Acetone-d6) 


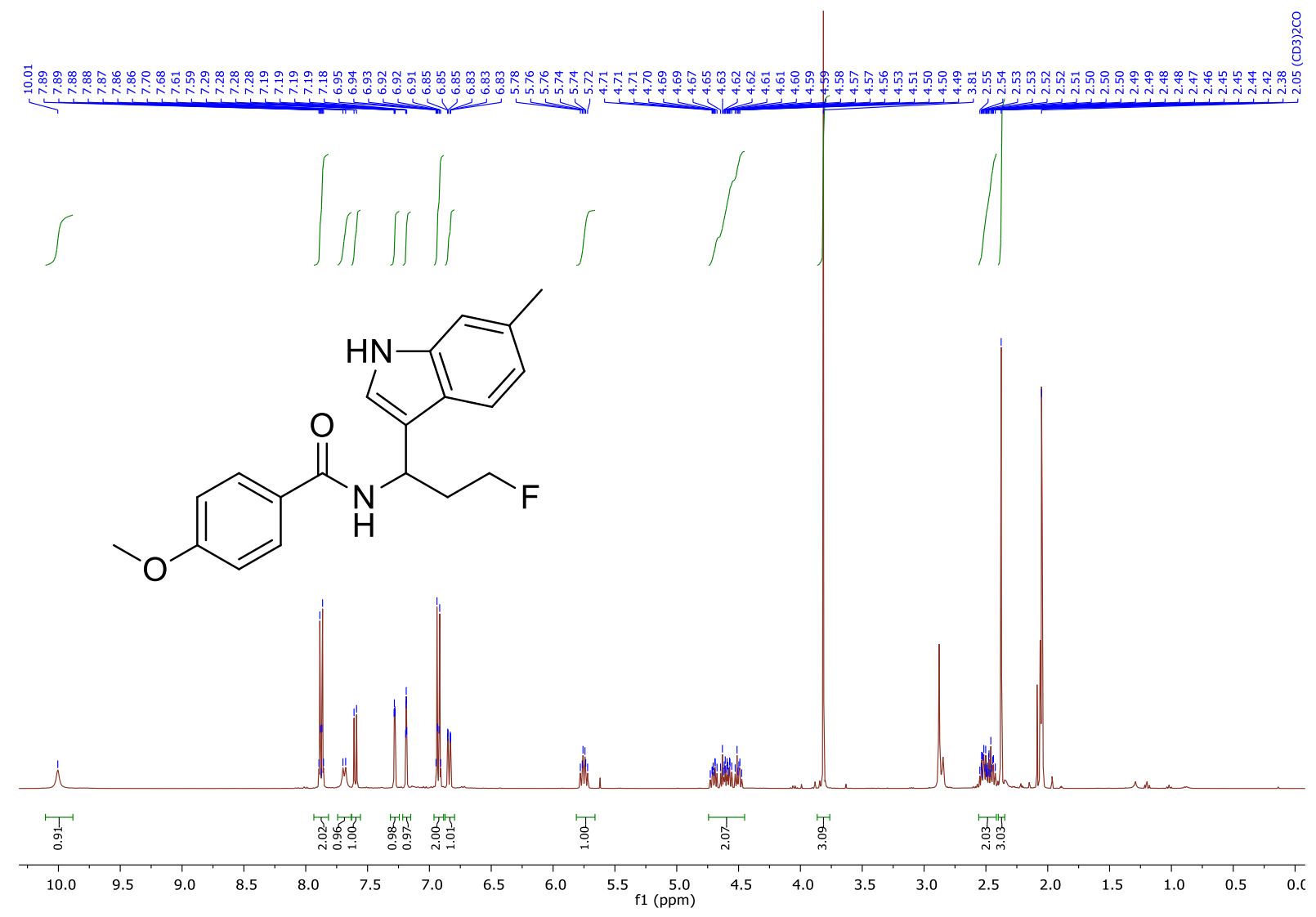

${ }^{13}$ C-NMR (101 MHz, Acetone-d6)

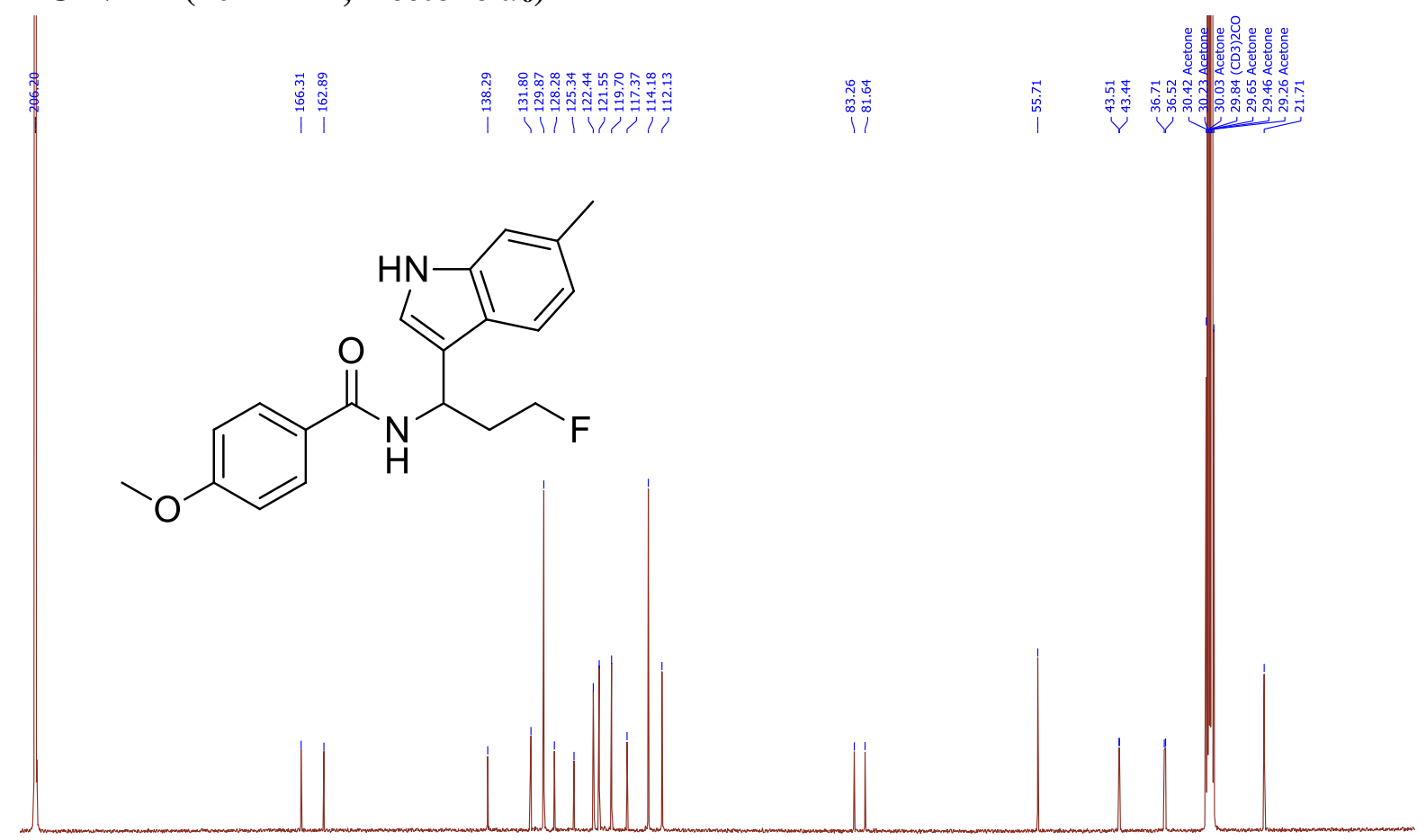

$\begin{array}{llllllllll}200 & 190 & 180 & 170 & 160 & 150 & 140 & 130 & 120 & 110 \underset{\mathrm{f} 1(\mathrm{ppm})}{10}\end{array}$

${ }^{19}$ F-NMR (376 MHz, Acetone-d6) 
<smiles>COc1ccc(C(=O)NC(CCF)c2c[nH]c3cc(C)ccc23)cc1</smiles>

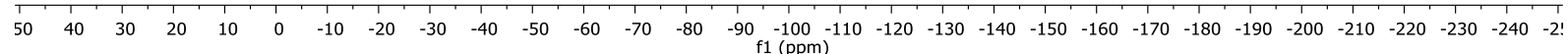

IR

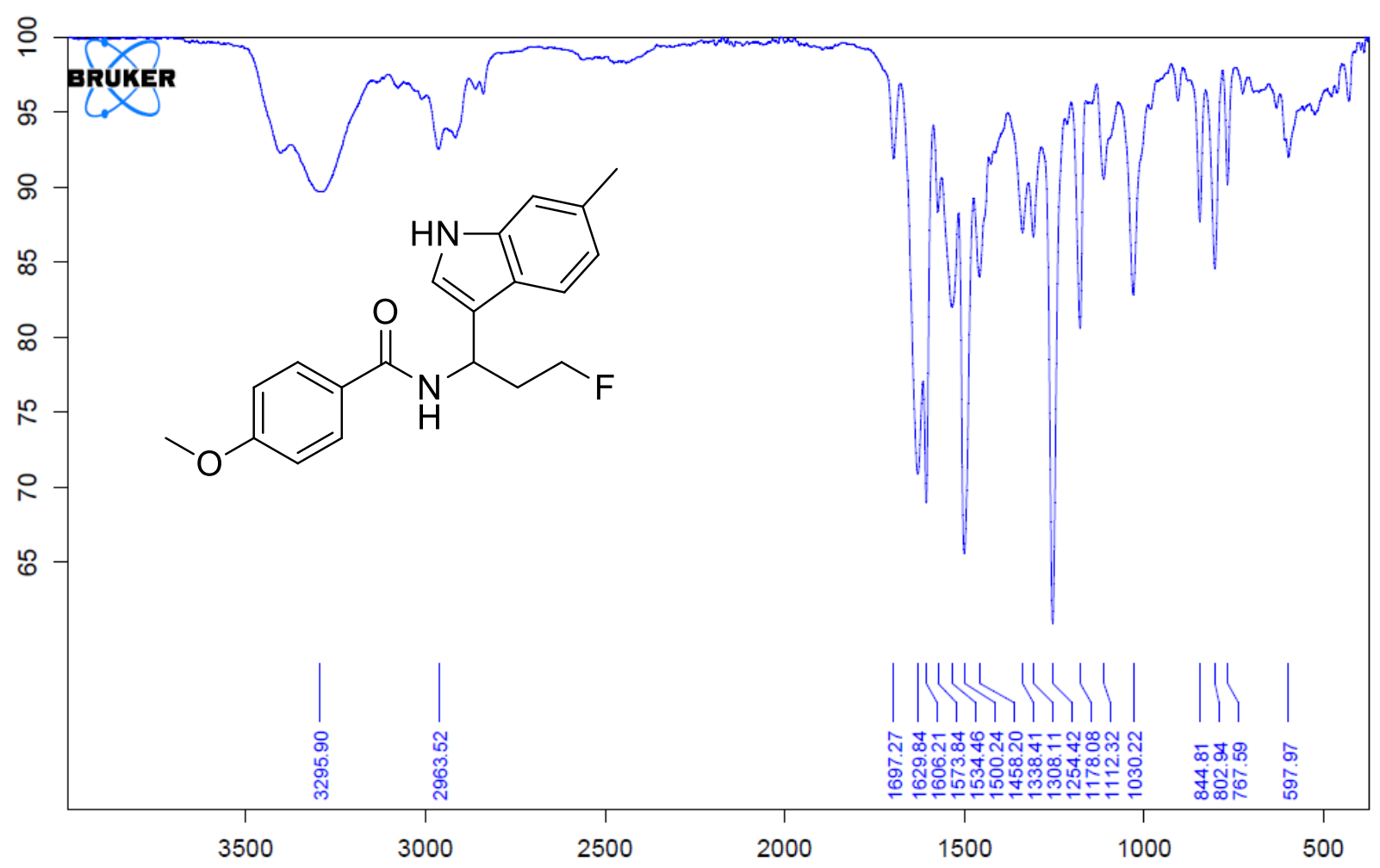

$N$-(3-Fluoro-1-(6-(trifluoromethyl)-1H-indol-3-yl)propyl)-4-methoxybenzamide (3w) ${ }^{1} \mathrm{H}-\mathrm{NMR}$ (400 MHz, $\mathrm{CDCl}_{3}$ ) 


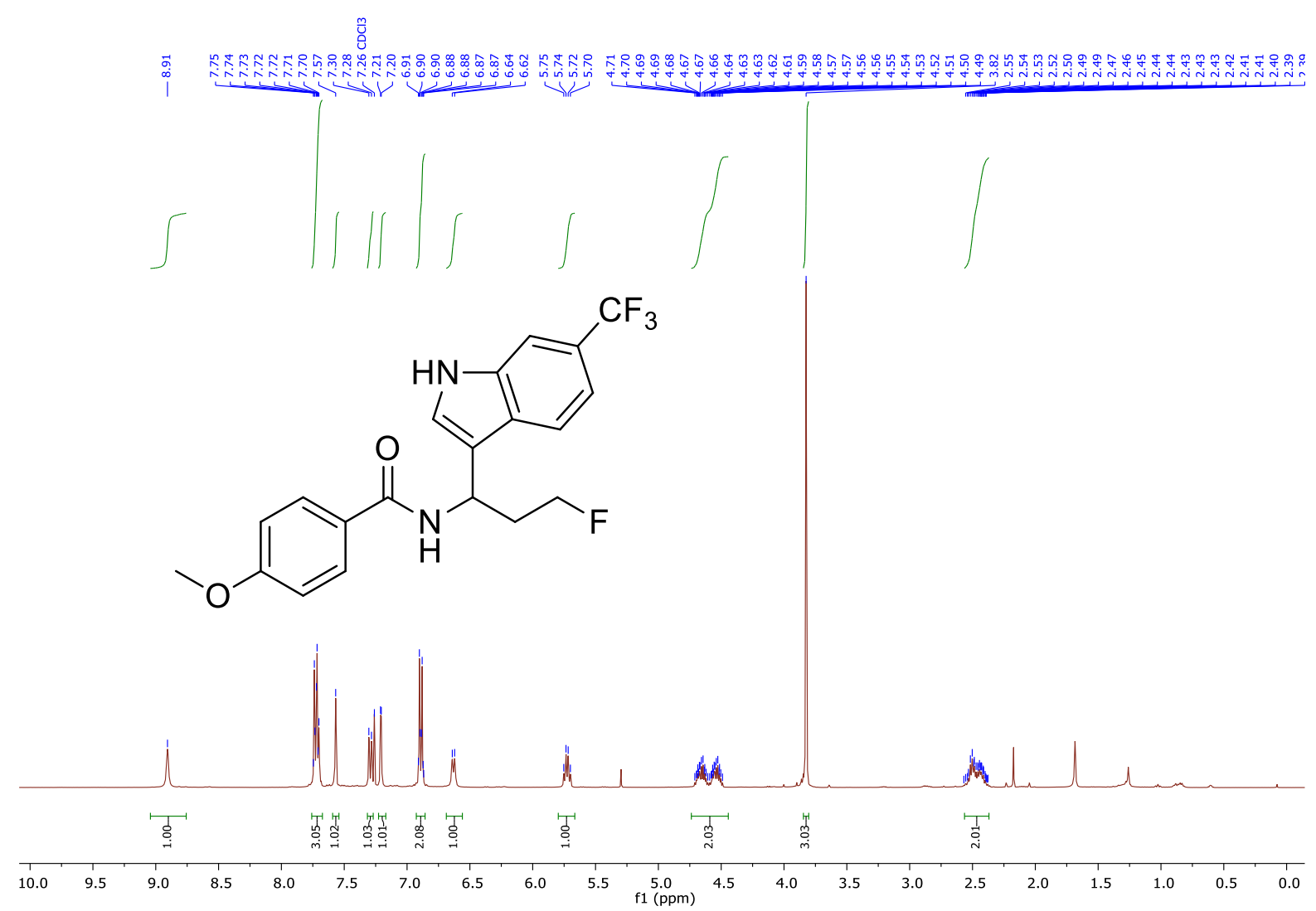

\section{${ }^{13} \mathrm{C}-\mathrm{NMR}$ (101 MHz, $\left.\mathrm{CDCl}_{3}\right)$}
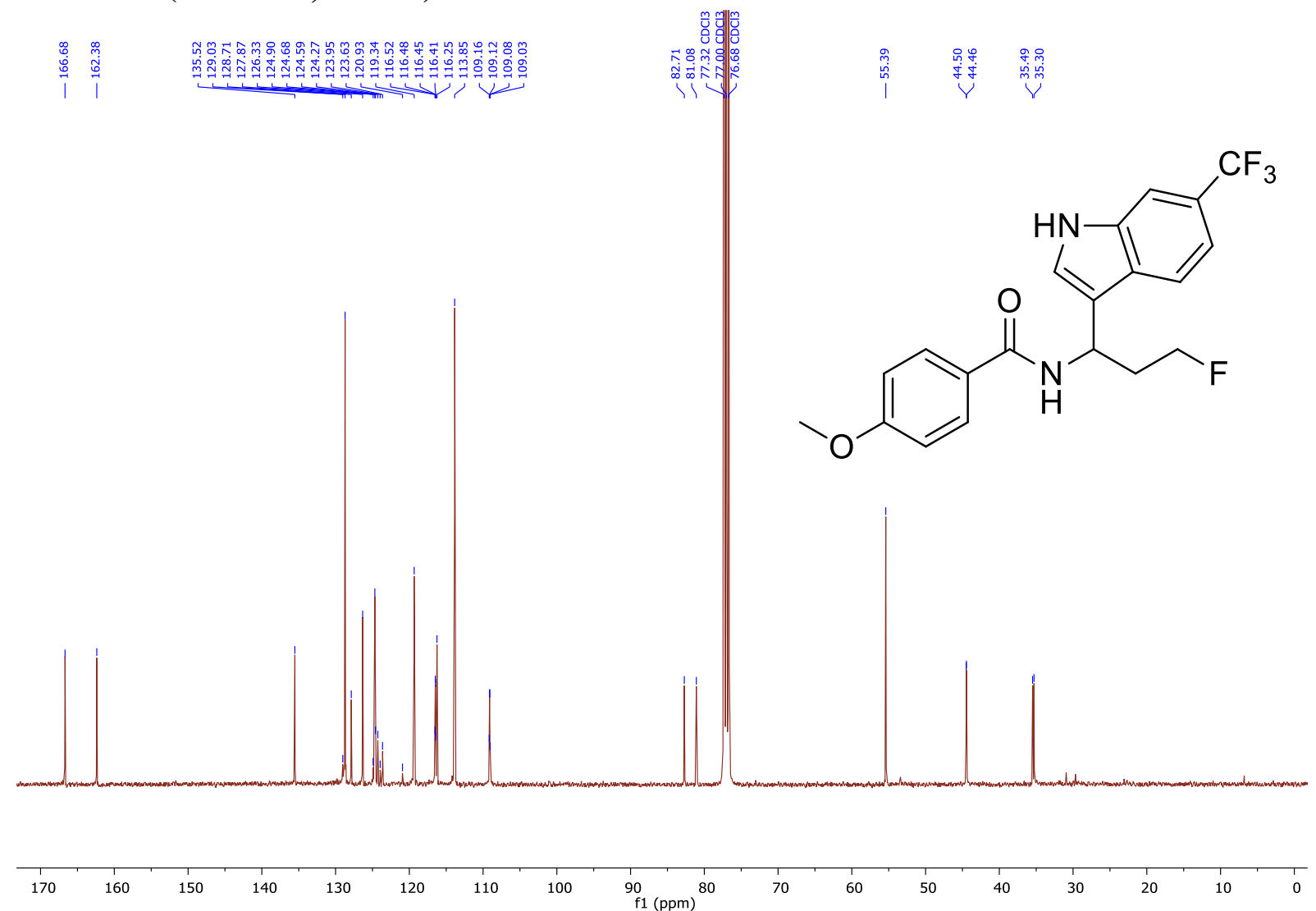

${ }^{19}$ F-NMR (376 MHz, CDCl3) 


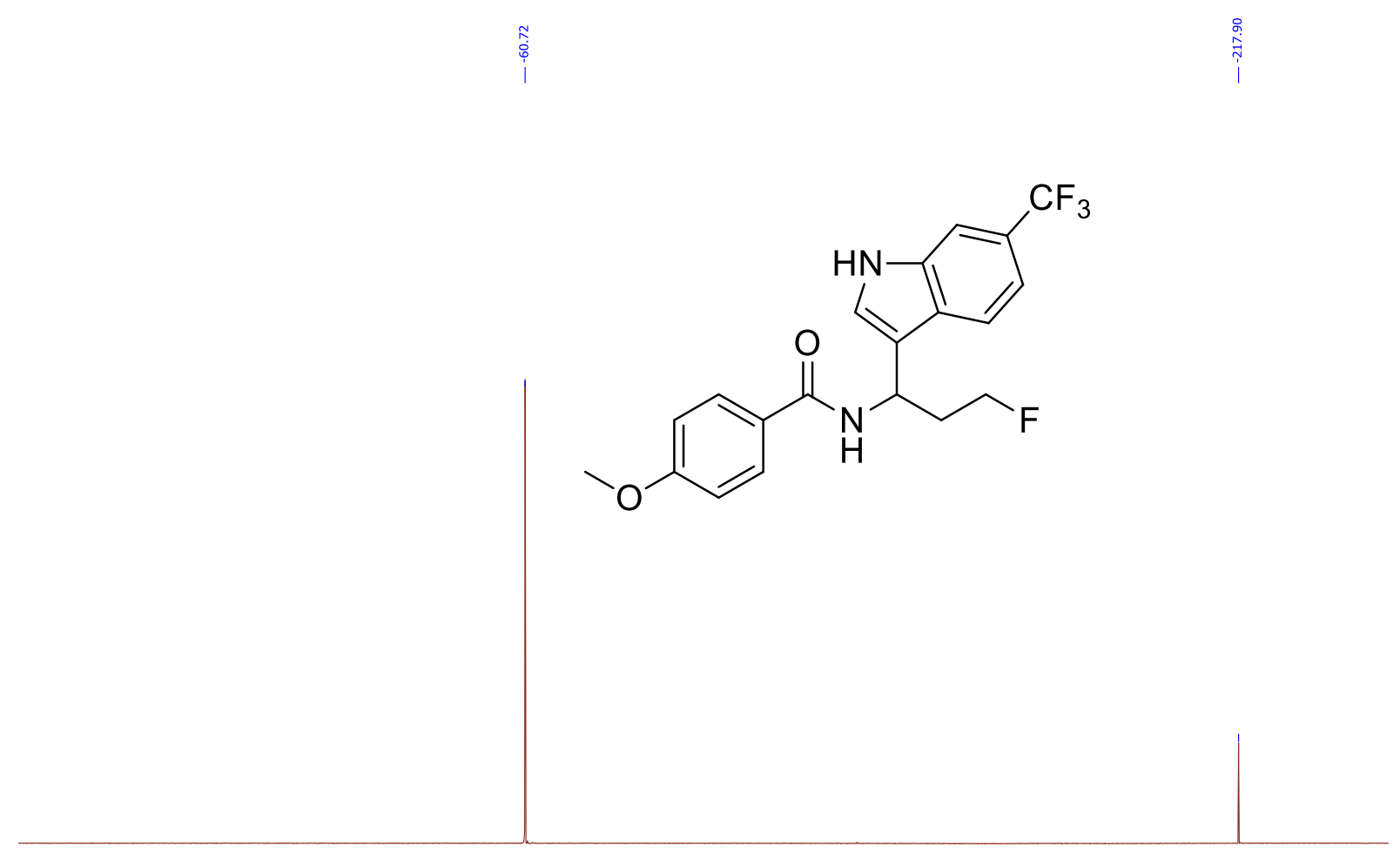

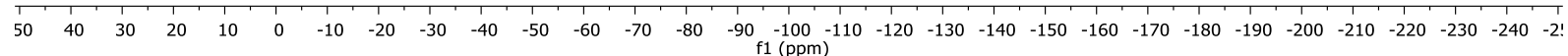

IR

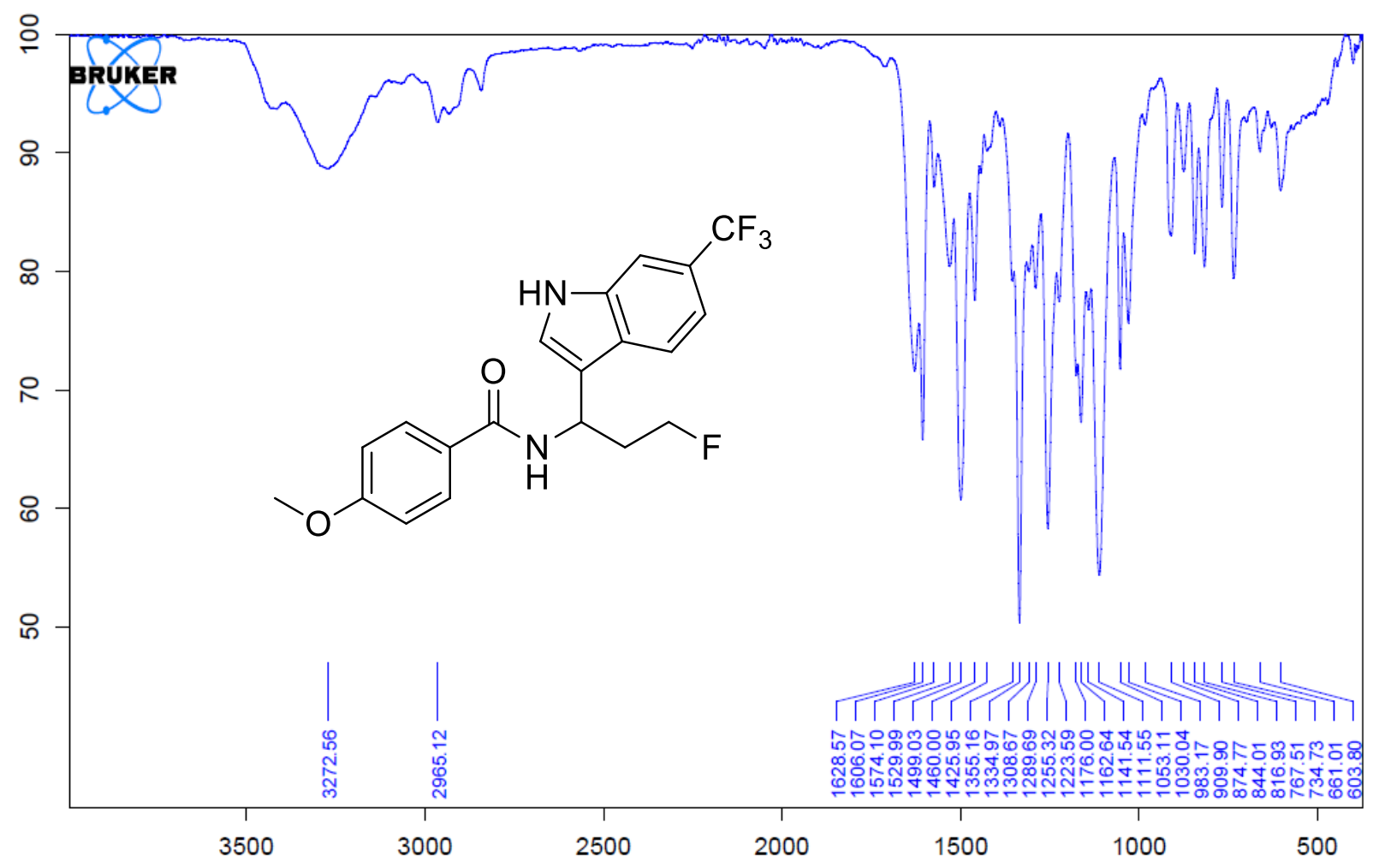

$N$-(1-(6-Bromo-1H-indol-3-yl)-3-fluoropropyl)-4-methoxybenzamide (3x)

${ }^{1} \mathrm{H}-\mathrm{NMR}\left(400 \mathrm{MHz}, \mathrm{CDCl}_{3}\right)$ 


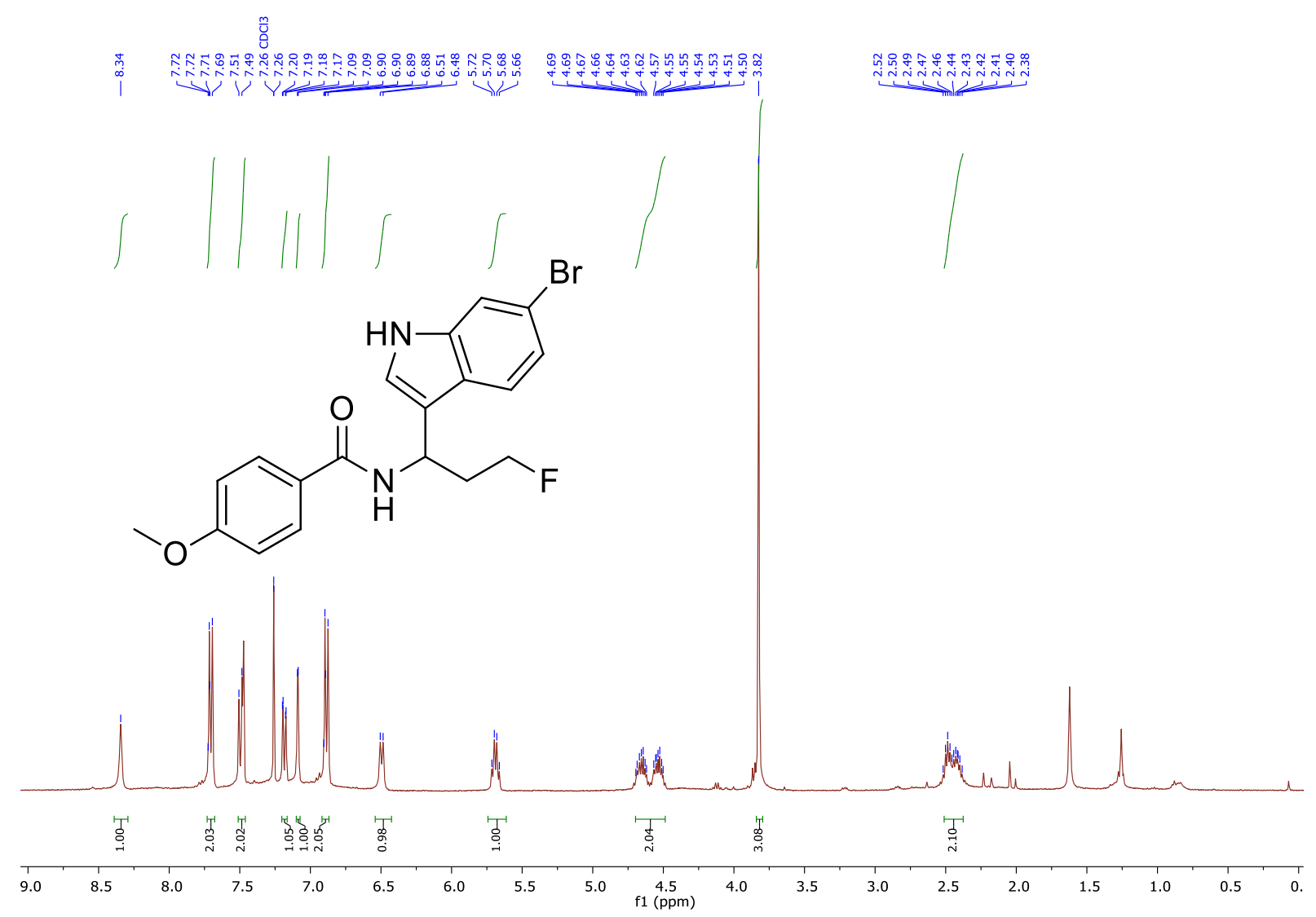

${ }^{13} \mathrm{C}-\mathrm{NMR}\left(101 \mathrm{MHz}, \mathrm{CDCl}_{3}\right)$

is

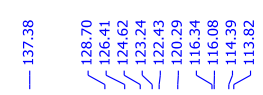<smiles>COc1ccc(C(=O)NC(CCF)c2c[nH]c3cc(Br)ccc23)cc1</smiles>

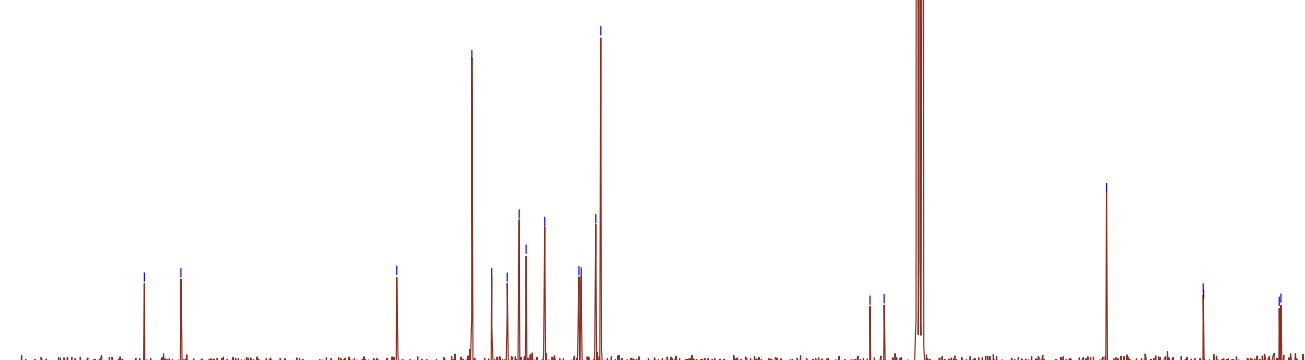

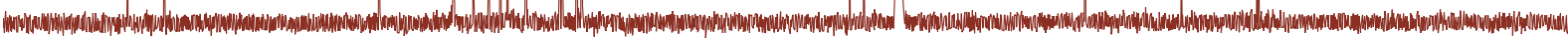

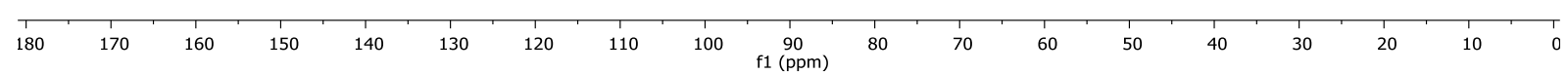

${ }^{19}$ F-NMR (376 MHz, $\mathrm{CDCl}_{3}$ ) 
<smiles>COc1ccc(C(=O)NC(CCF)c2c[nH]c3cc(Br)ccc23)cc1</smiles>

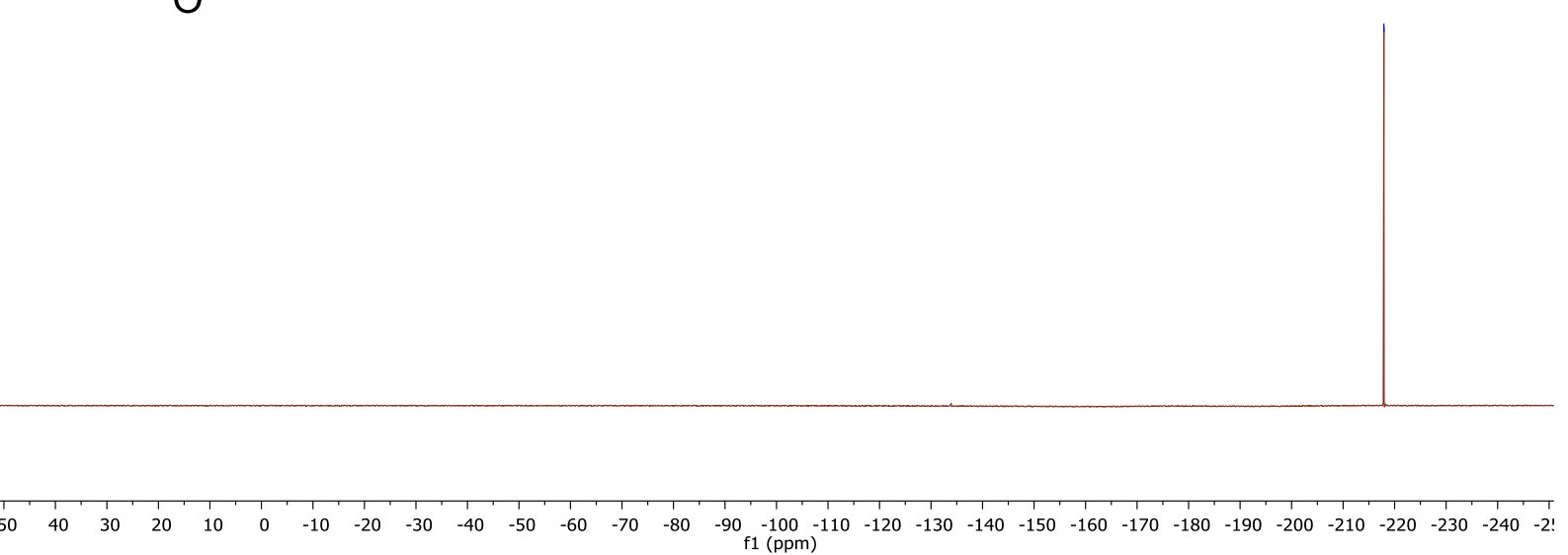

IR

은

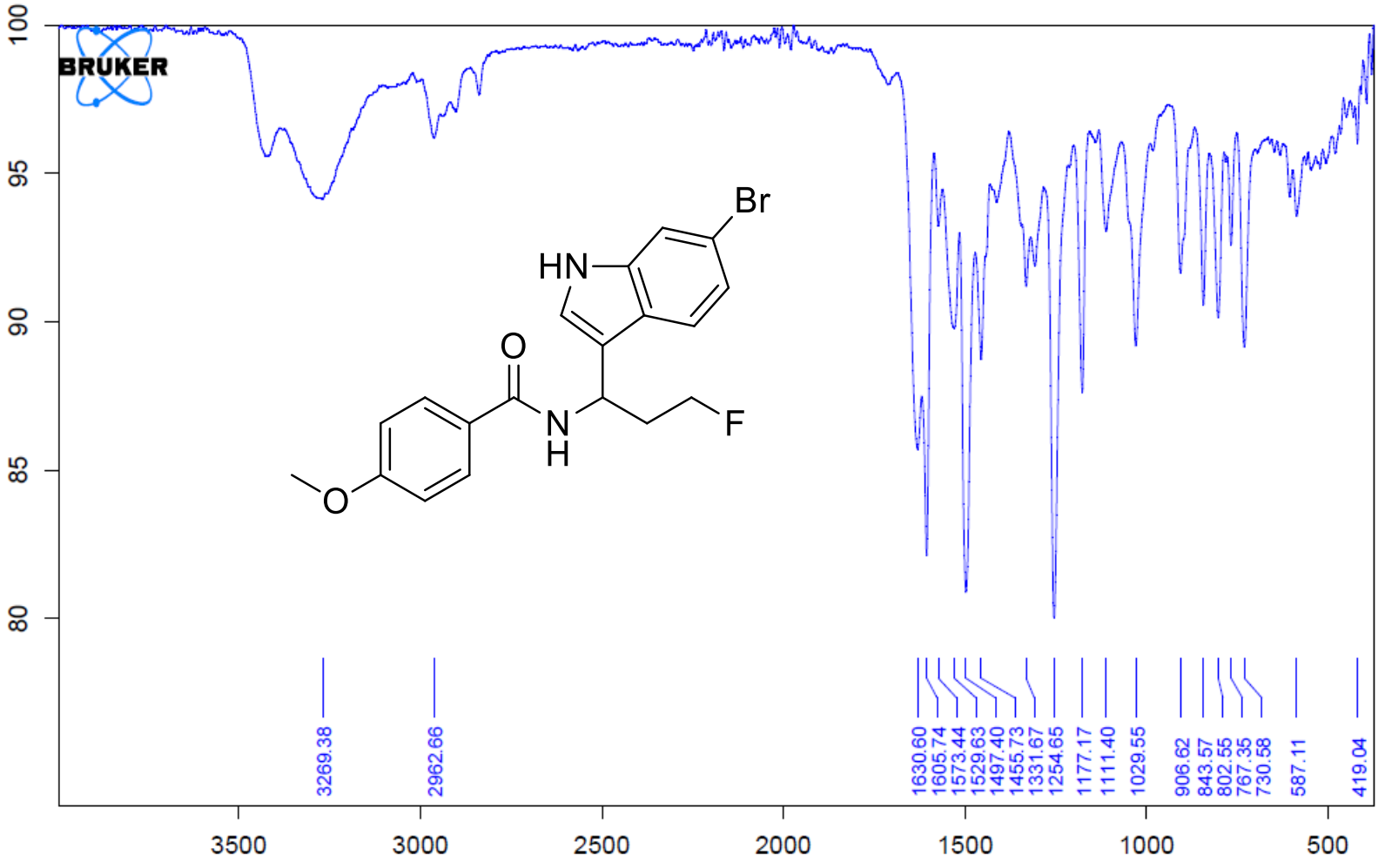

$N$-(3-Fluoro-1-(7-methyl-1H-indol-3-yl)propyl)-4-methoxybenzamide (3y) ${ }^{1}$ H-NMR (400 MHz, Acetone-d6) 


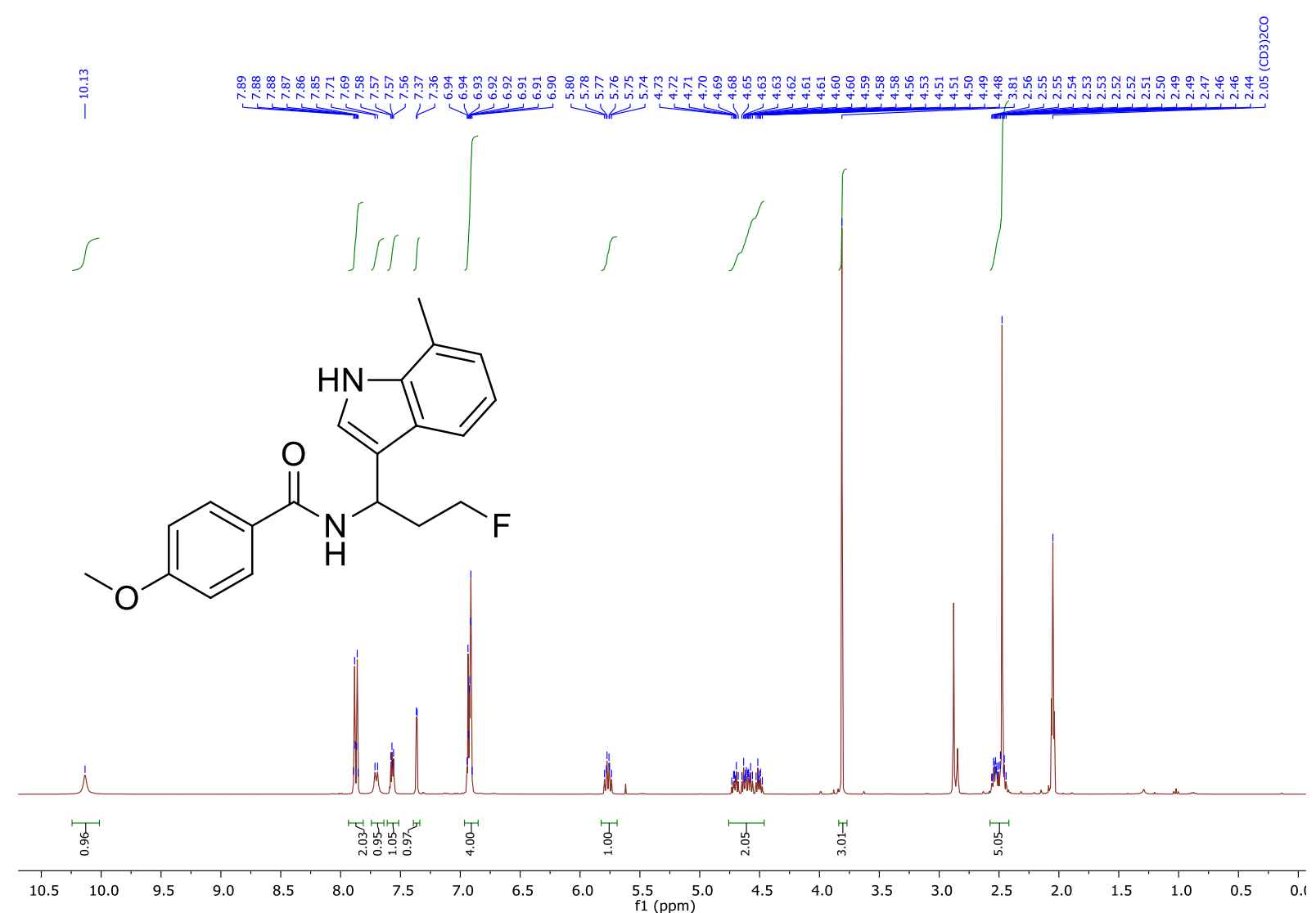

${ }^{13}$ C-NMR (101 MHz, Acetone-d6)

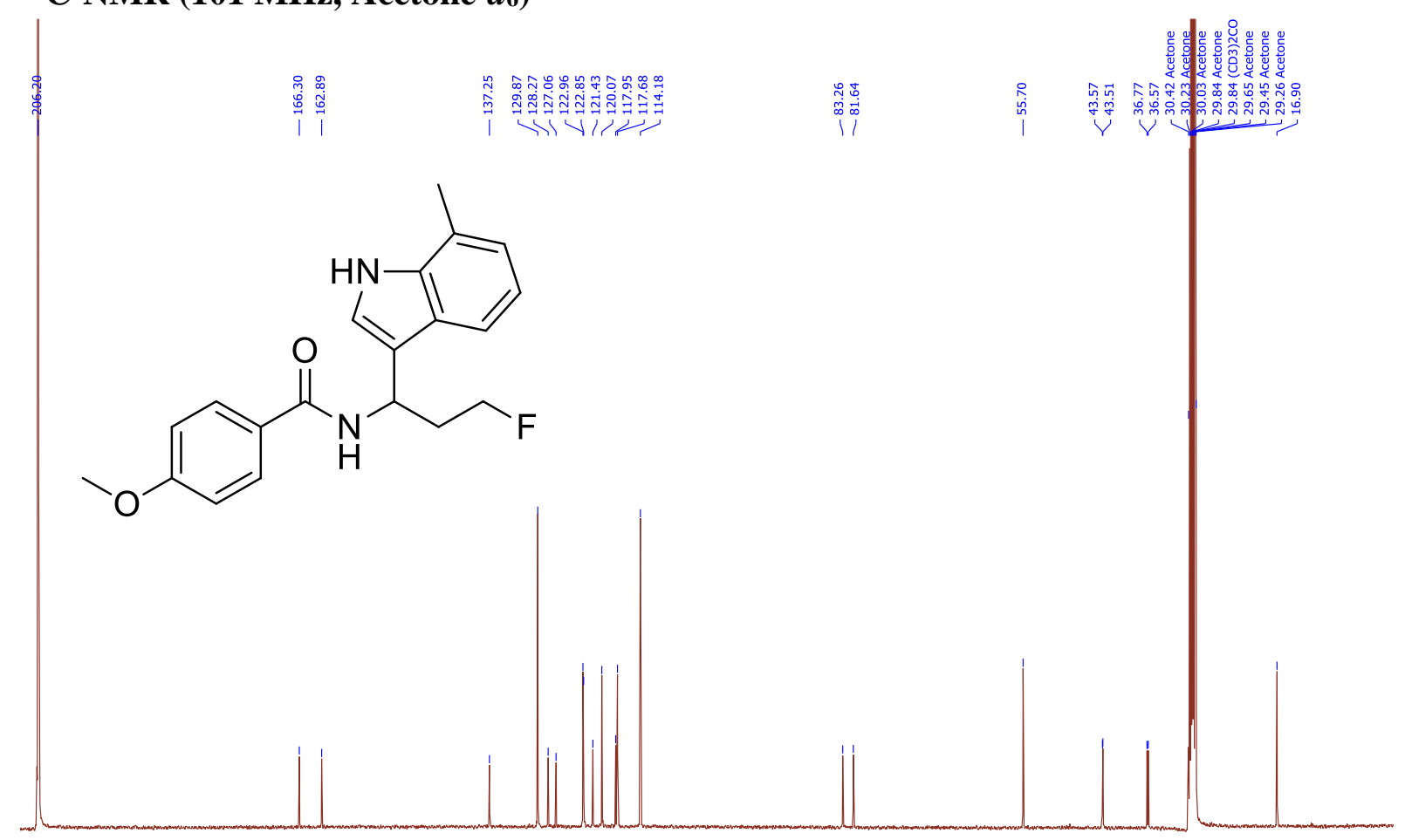

$\begin{array}{llllllllll}200 & 190 & 180 & 170 & 160 & 150 & 140 & 130 & 120 & \begin{array}{c}110 \\ \mathrm{f} 1(\mathrm{ppm})\end{array}\end{array}$

${ }^{19}$ F-NMR (376 MHz, Acetone- $d_{6}$ ) 
<smiles>COc1ccc(C(=O)NC(CCF)c2c[nH]c3c(C)cccc23)cc1</smiles>

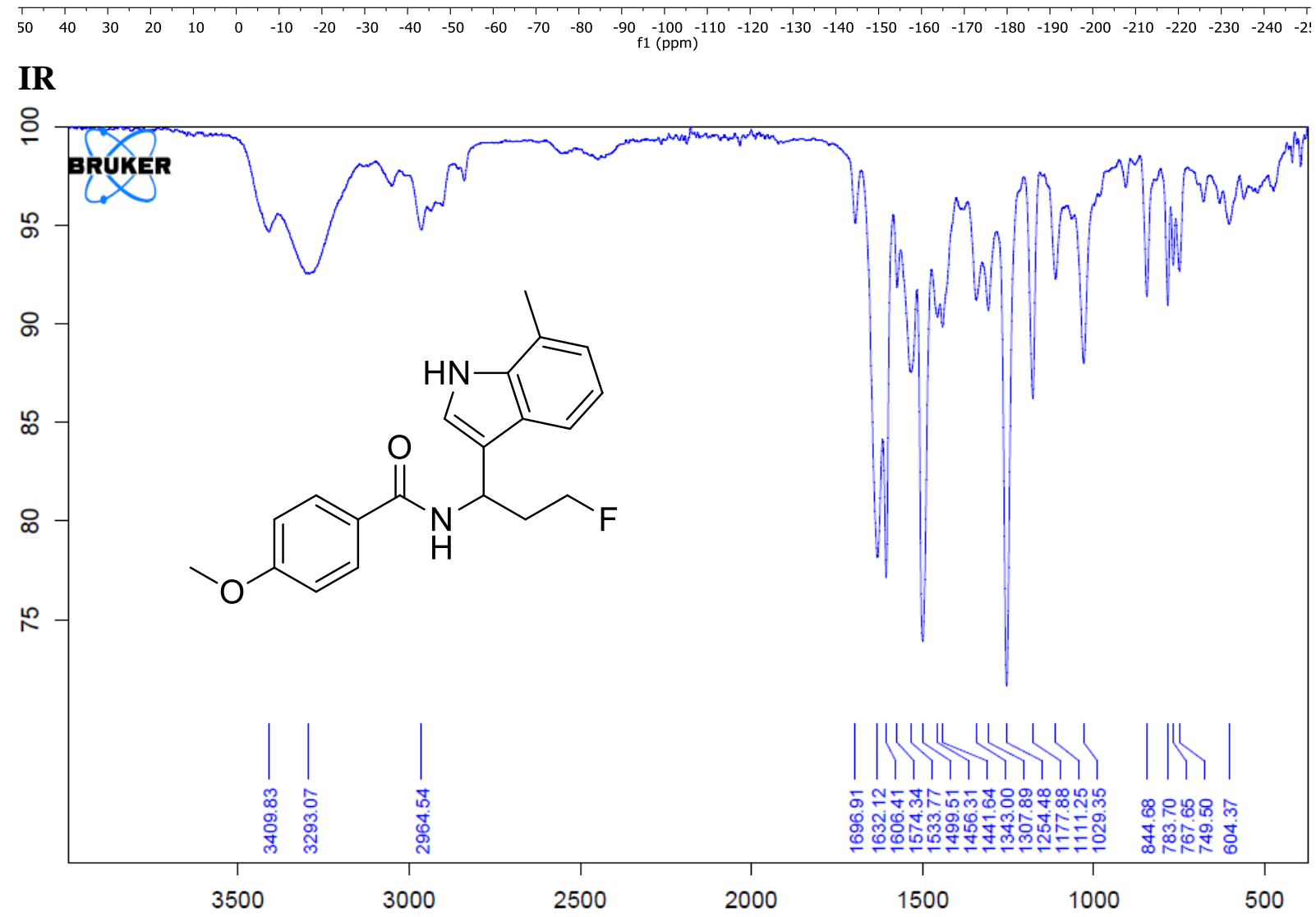

$N$-(3-Fluoro-1-hydroxybutyl)-4-methoxybenzamide (5a)

${ }^{1}$ H-NMR (400 MHz, Acetone- $d_{6}$ ) 


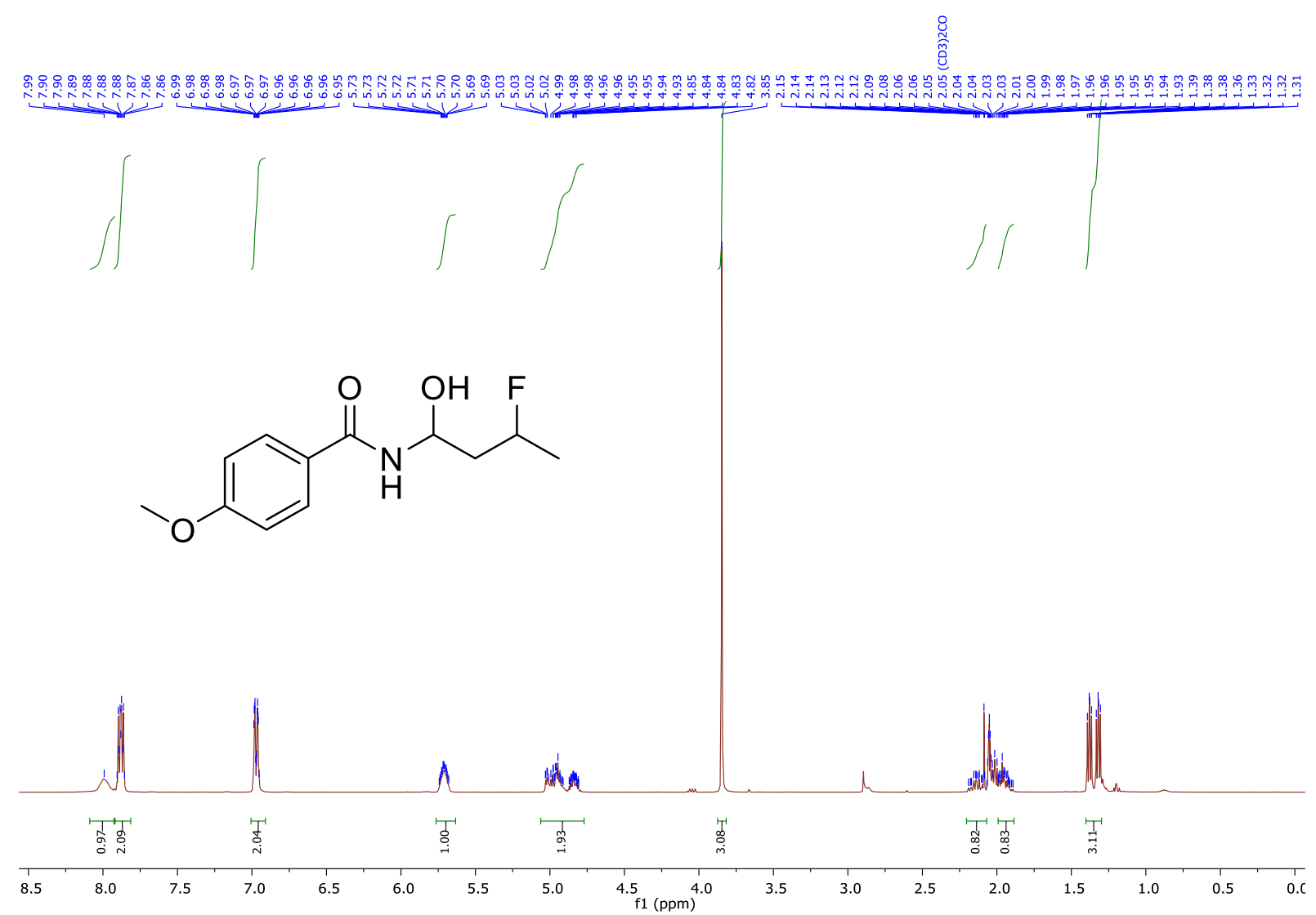

${ }^{13}$ C-NMR (101 MHz, Acetone-d6)

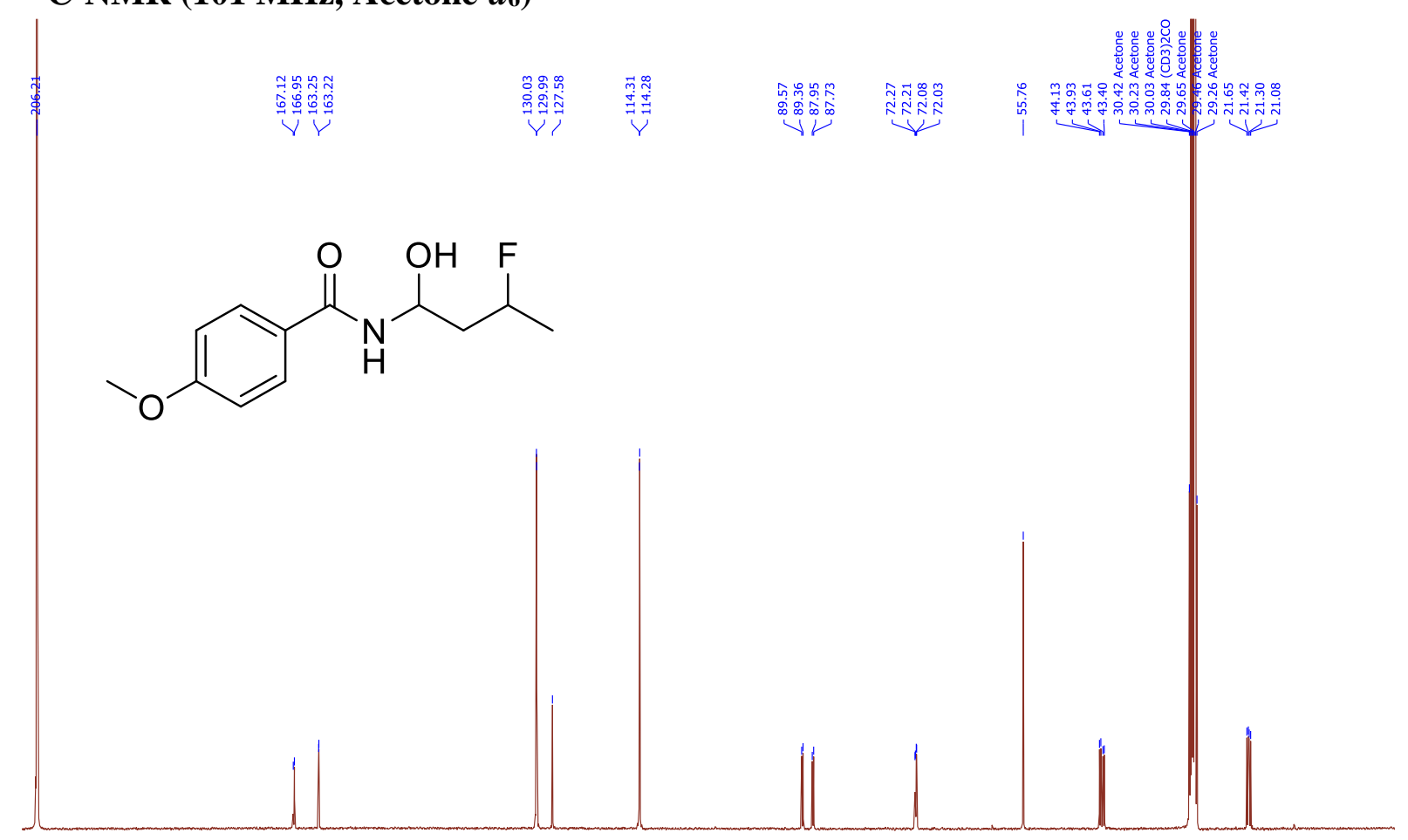

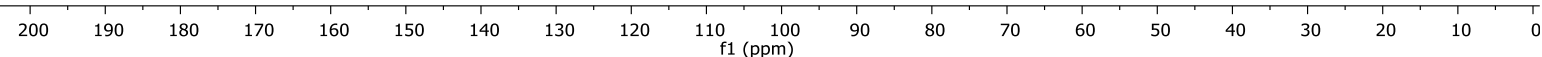

${ }^{19}$ F-NMR (376 MHz, Acetone-d6) 
<smiles>COc1ccc(C(=O)NC(O)CC(C)F)cc1</smiles>

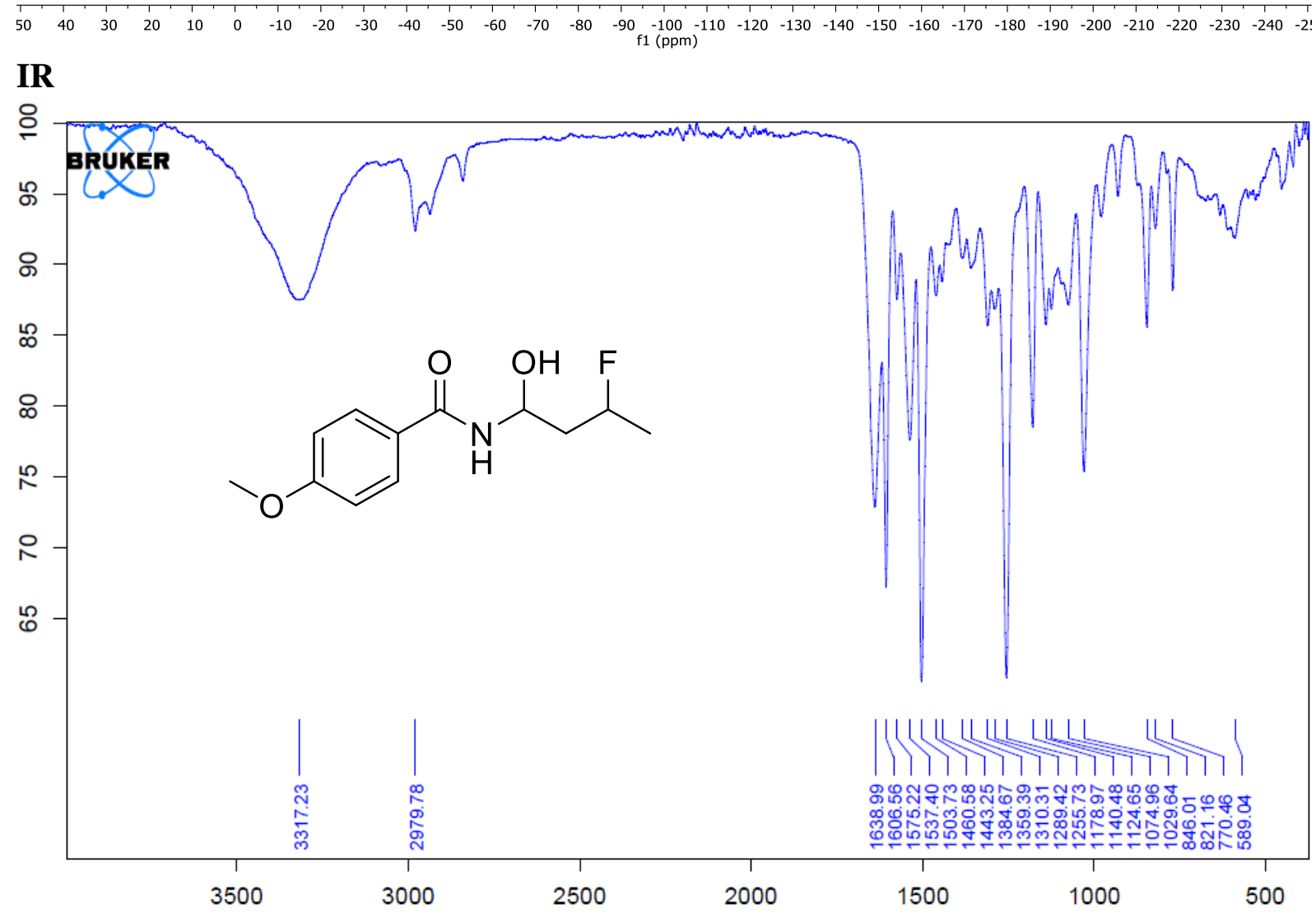

$N$-(3-Fluoro-3-phenylpropyl)-4-methoxybenzamide (5b)

${ }^{1}$ H-NMR (400 MHz, $\mathrm{CDCl}_{3}$ ) 


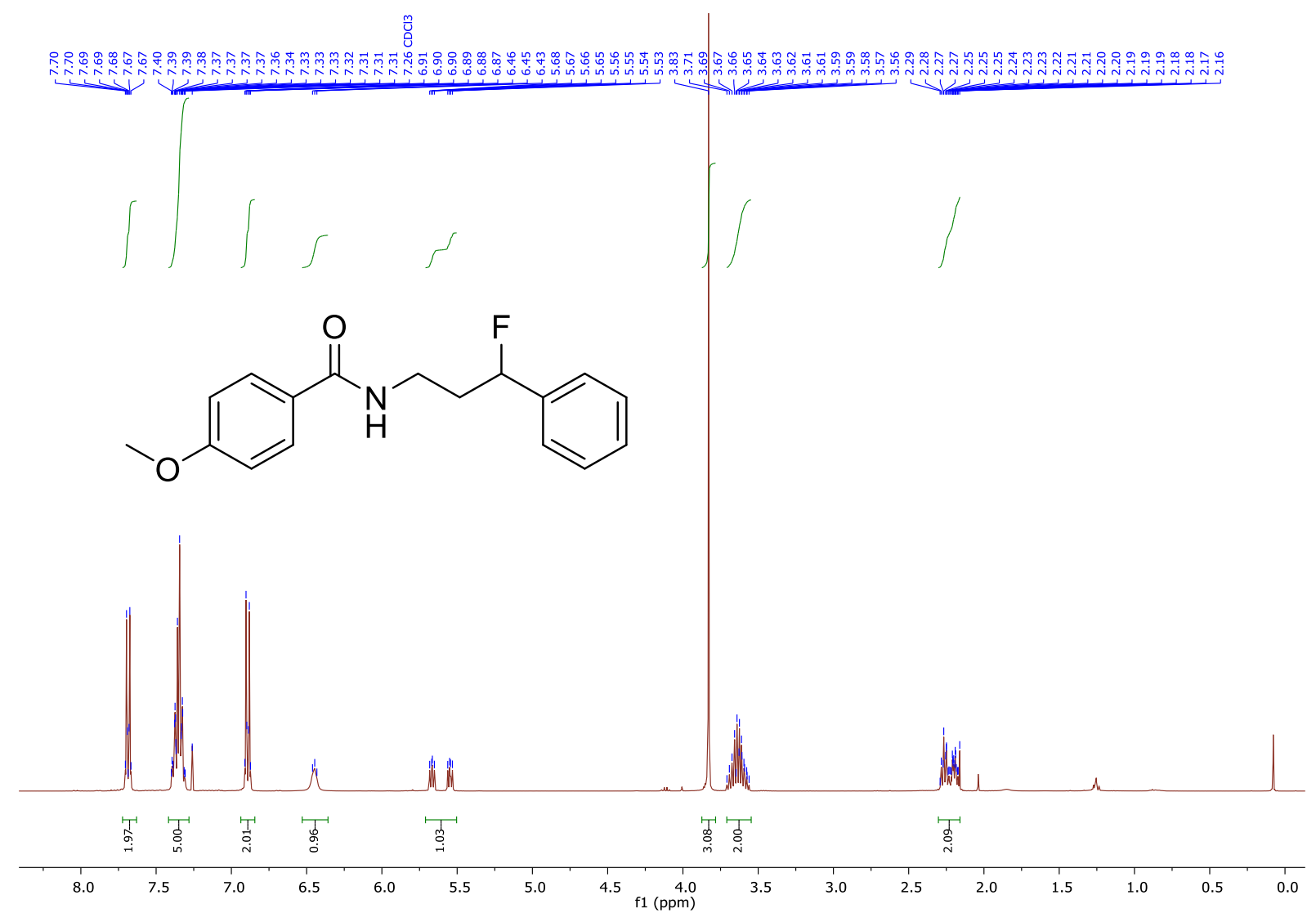

${ }^{13}$ C-NMR (101 MHz, CDCl3)

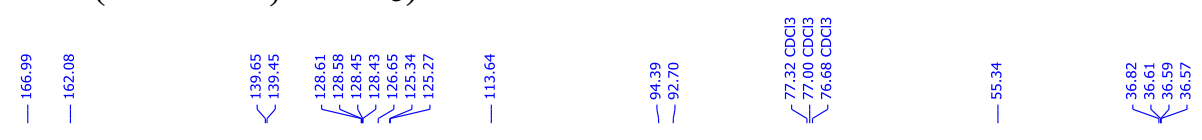<smiles>COc1ccc(C(=O)NCCC(F)c2ccccc2)cc1</smiles>
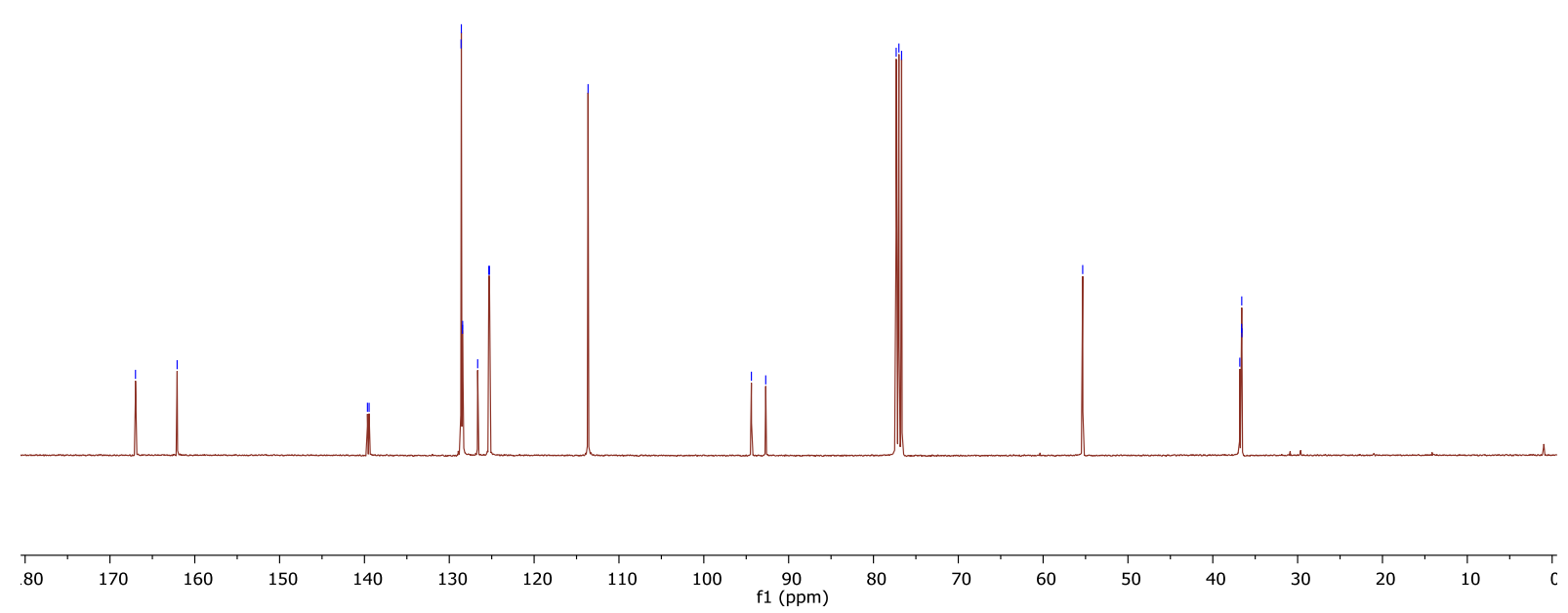

${ }^{19}$ F-NMR (376 MHz, CDCl 3 ) 
<smiles>COc1ccc(C(=O)NCCC(F)c2ccccc2)cc1</smiles>

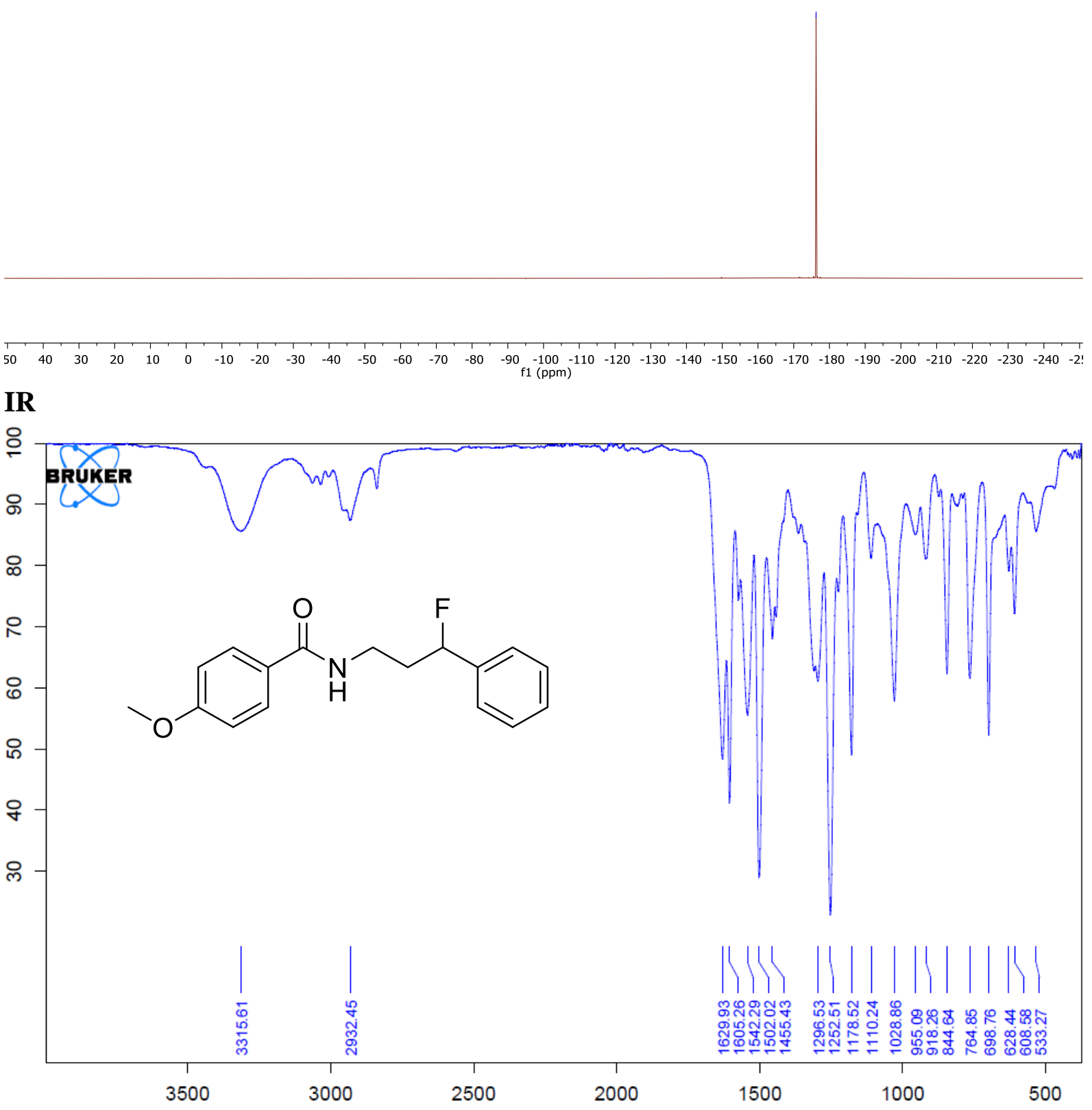

tert-Butyl 3-fluoro-4-((4-methoxybenzamido)methyl)pyrrolidine-1-carboxylate (5c) ${ }^{1} \mathrm{H}-\mathrm{NMR}$ (400 MHz, $\mathrm{CDCl}_{3}$ ) 


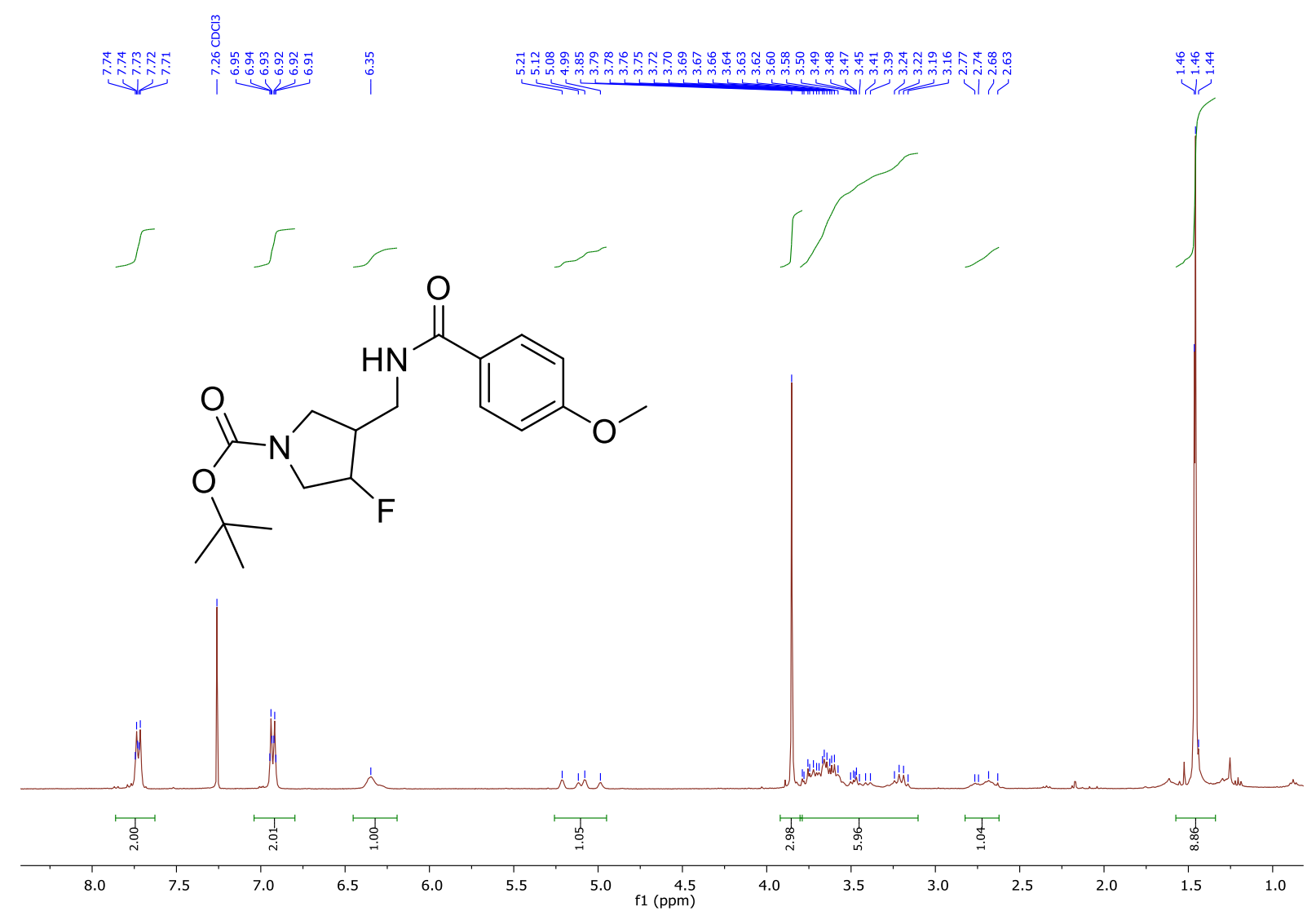

${ }^{1} \mathrm{H}-\mathrm{NMR}\left(400 \mathrm{MHz}, \mathrm{CD}_{3} \mathrm{CN}, 70^{\circ} \mathrm{C}\right)$

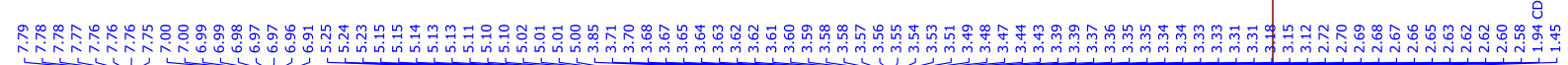
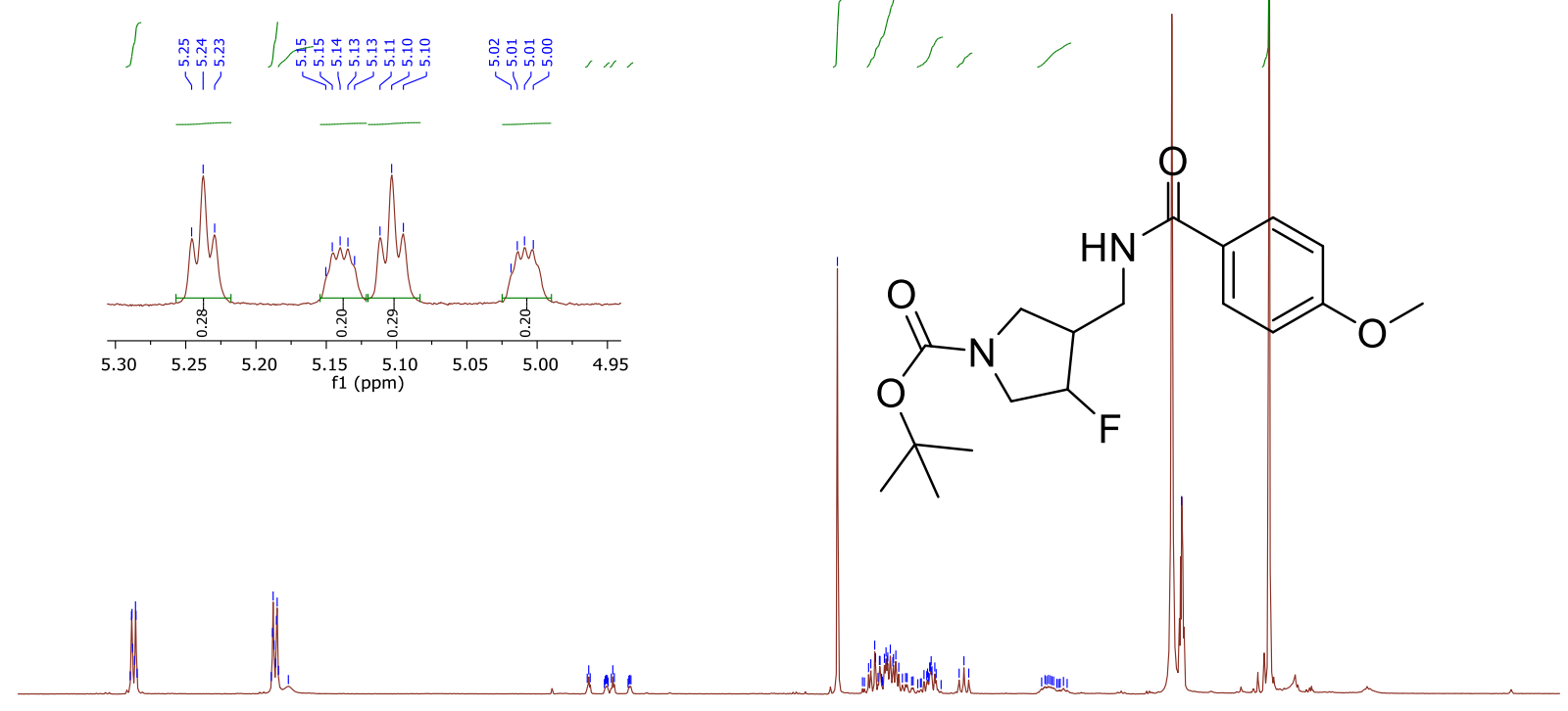

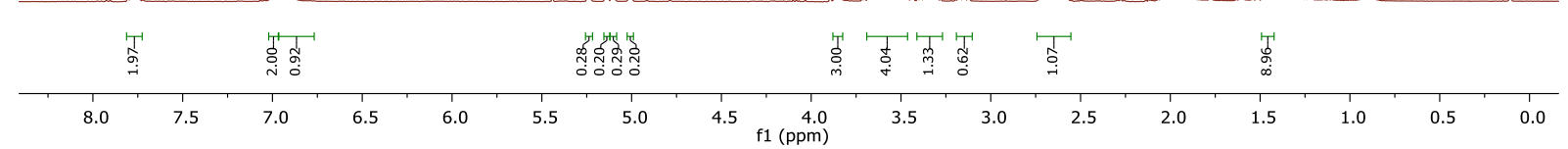

${ }^{13} \mathrm{C}-\mathrm{NMR}\left(101 \mathrm{MHz}, \mathrm{CDCl}_{3}\right)$ 

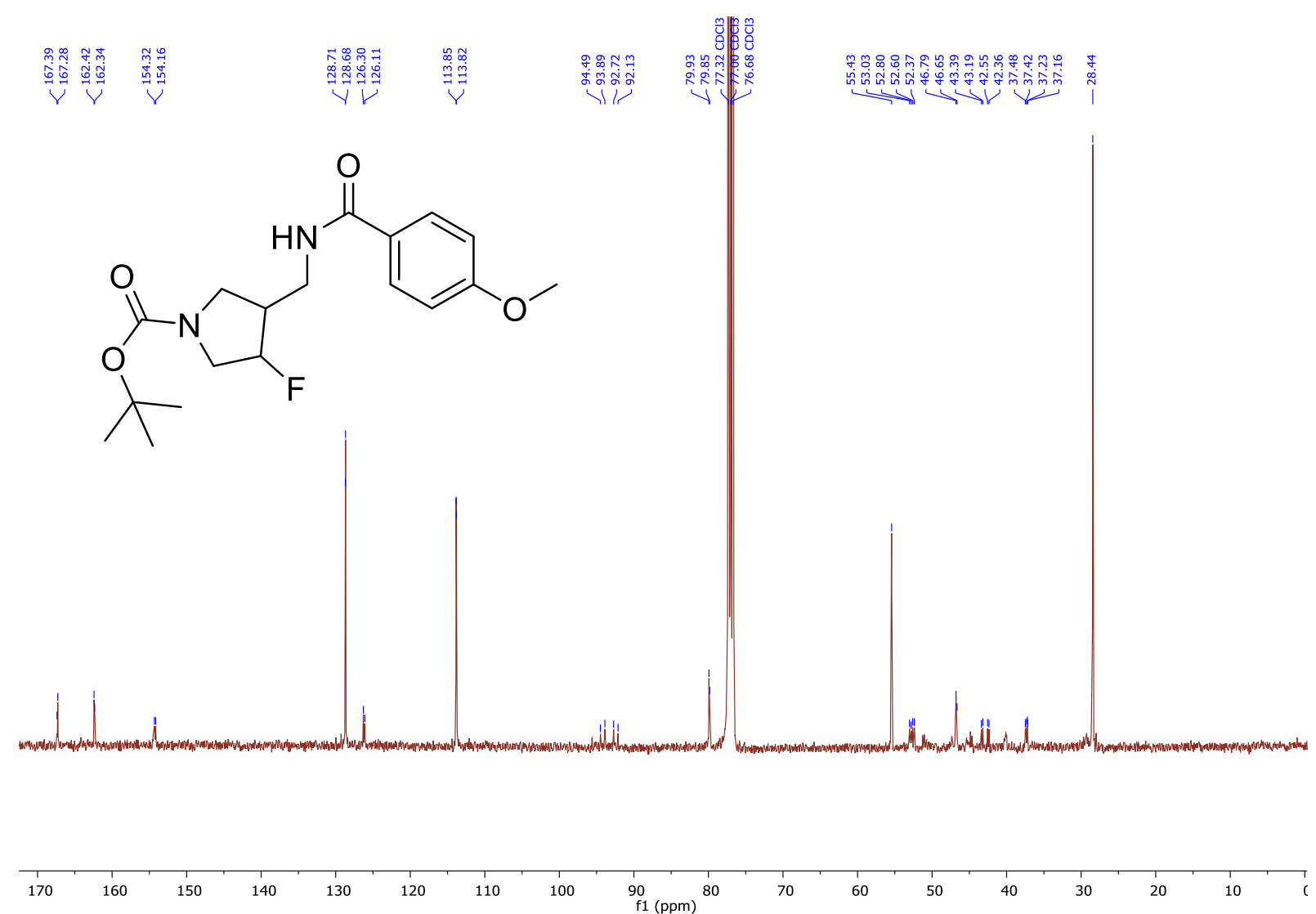

${ }^{19}$ F-NMR (376 MHz, CDCl3)

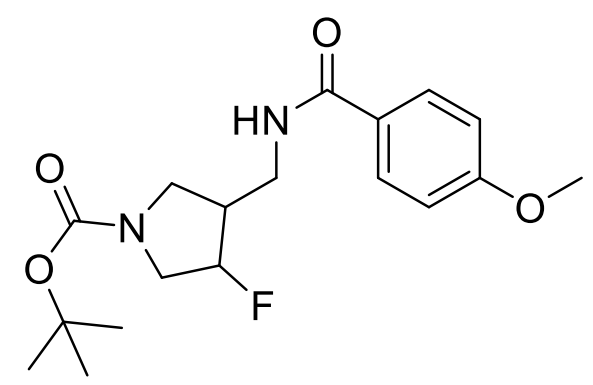

\begin{tabular}{llllllllllllllllllllllllllllllllllllllllllll}
\hline 50 & 40 & 30 & 20 & 10 & 0 & -10 & -20 & -30 & -40 & -50 & -60 & -70 & -80 & -90 & -100 & -110 & -120 & -130 & -140 & -150 & -160 & -170 & -180 & -190 & -200 & -210 & -220 & -230 & -240 & $-2 !$
\end{tabular} IR 


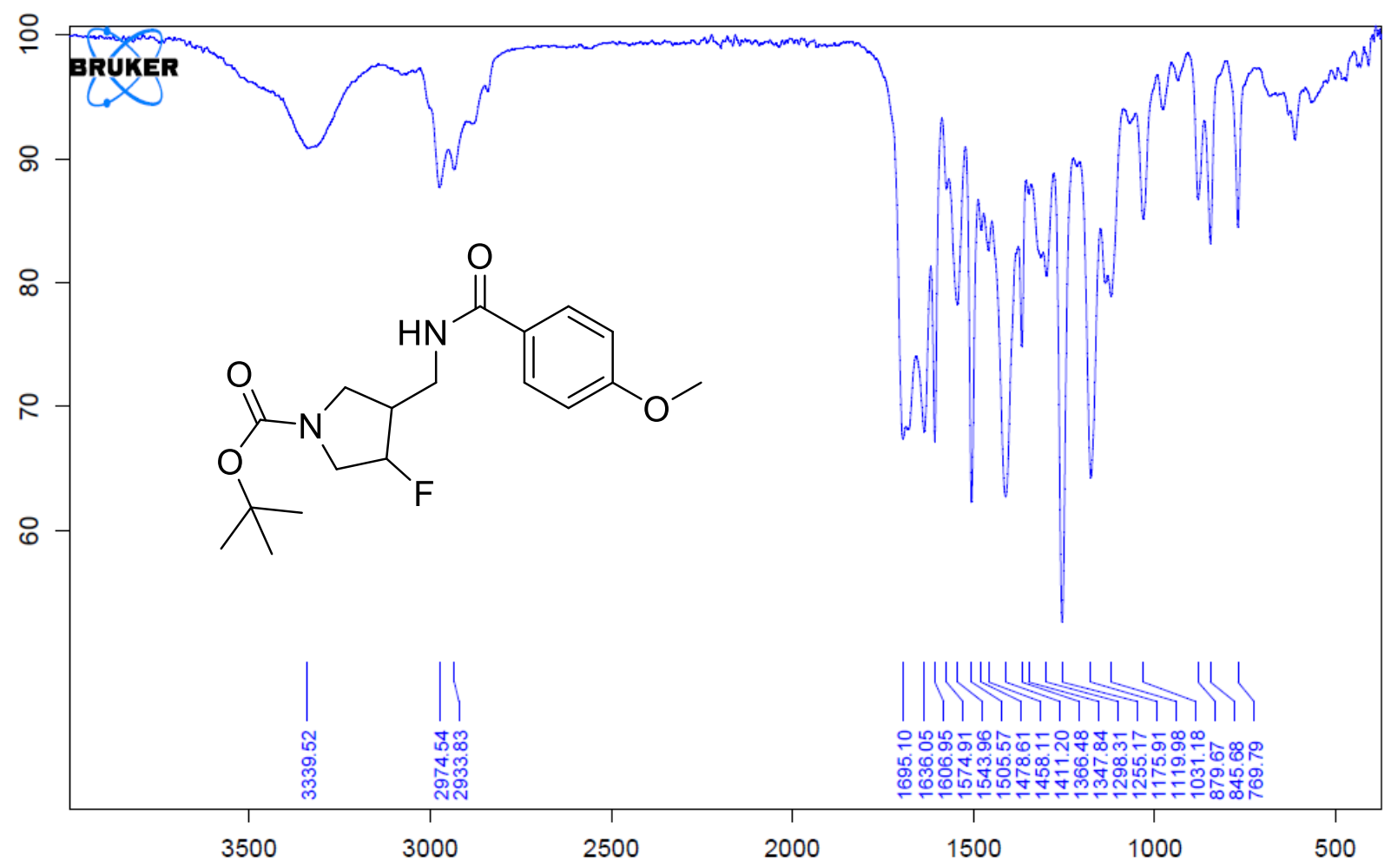

4-Methoxy- $N$-(3,3,3-trifluoro-1-hydroxypropyl)benzamide (5d).

${ }^{1}$ H-NMR (400 MHz, Acetone- $d_{6}$ )

|

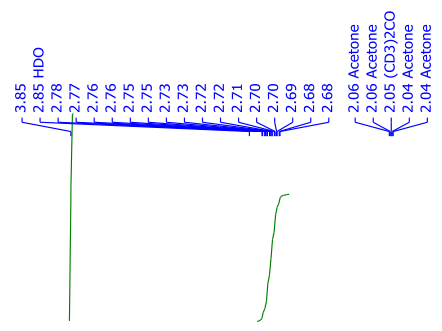

(N)

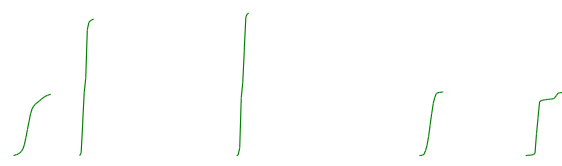

117771717

웅

$1500 \quad 1000$

500

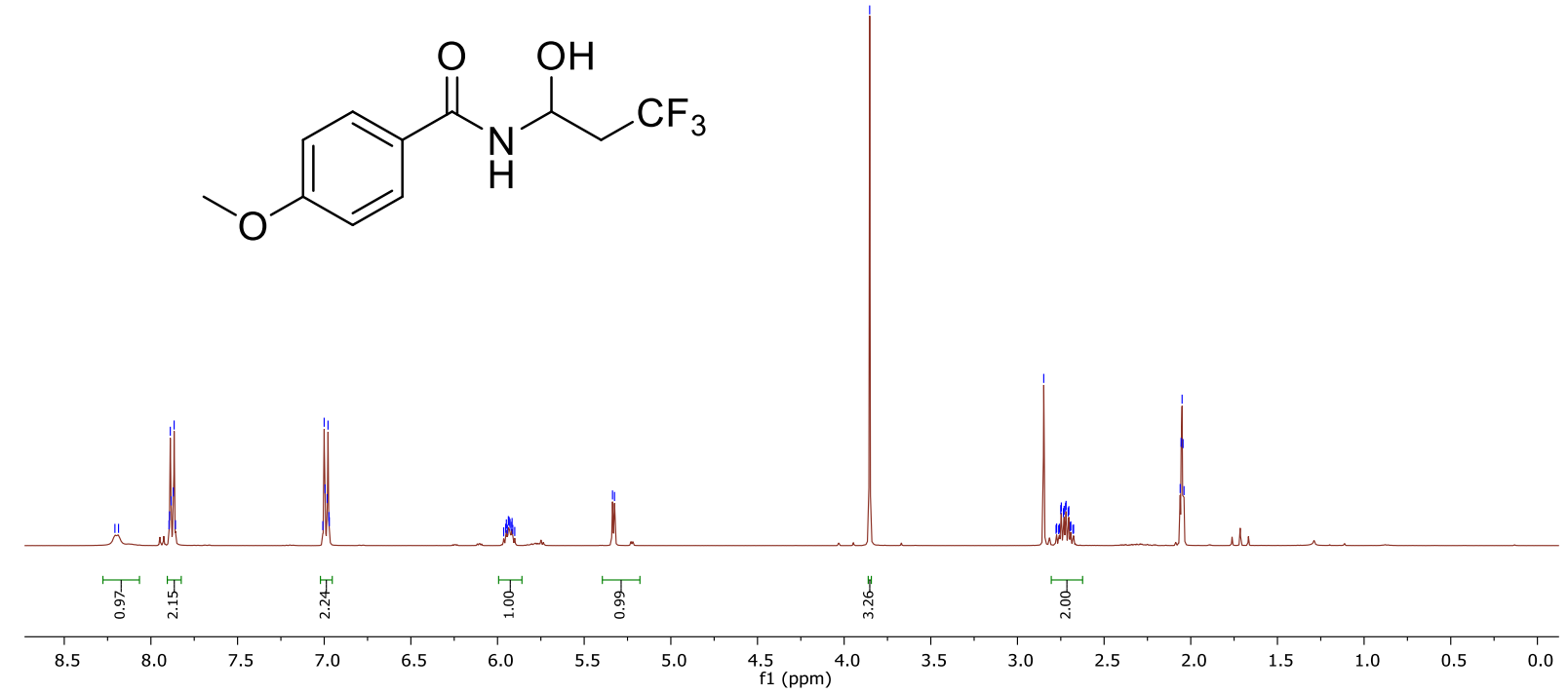

${ }^{13}$ C-NMR (101 MHz, Acetone- $\left.d_{6}\right)$ 

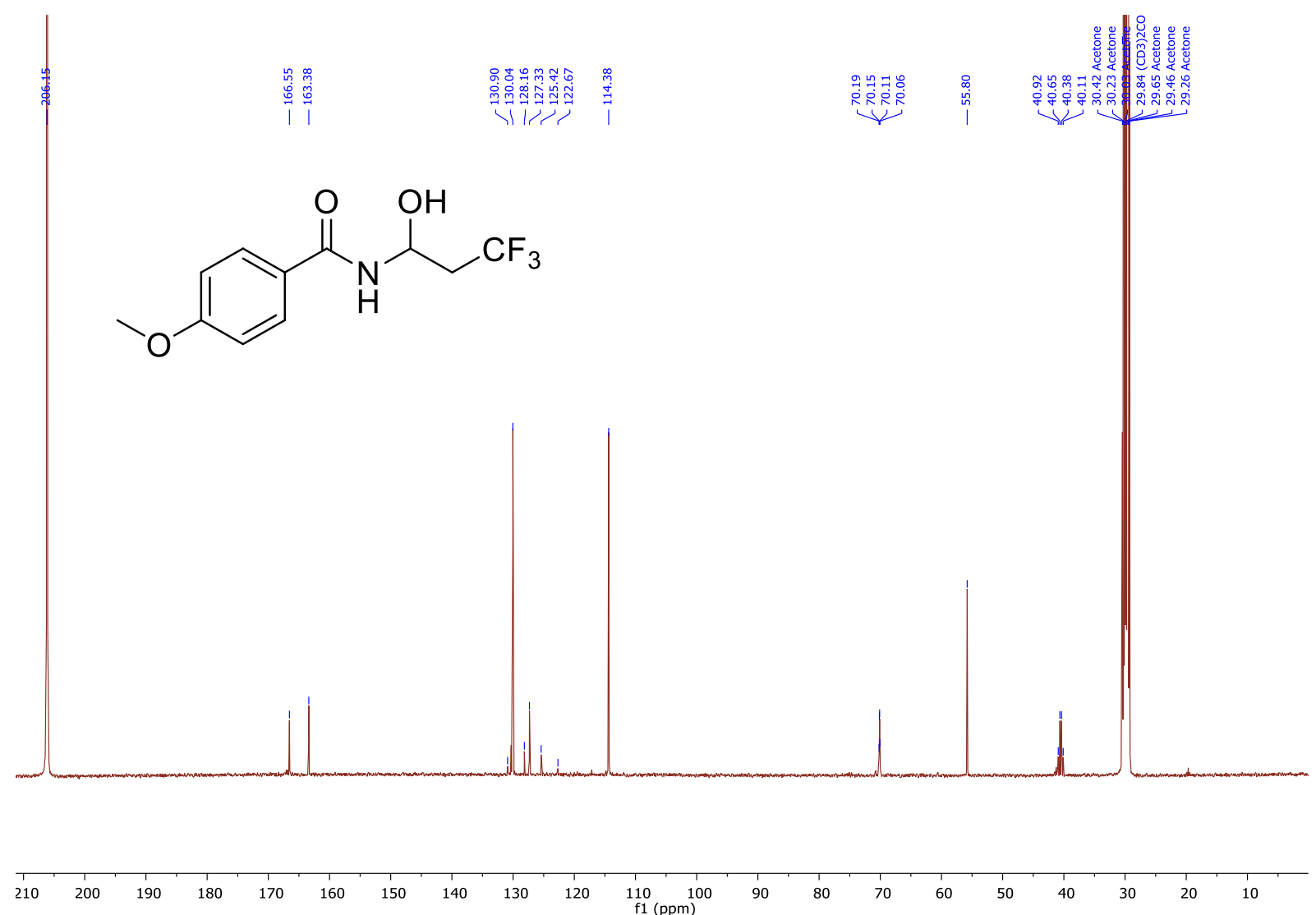

${ }^{19}$ F-NMR (376 MHz, Acetone-d6)

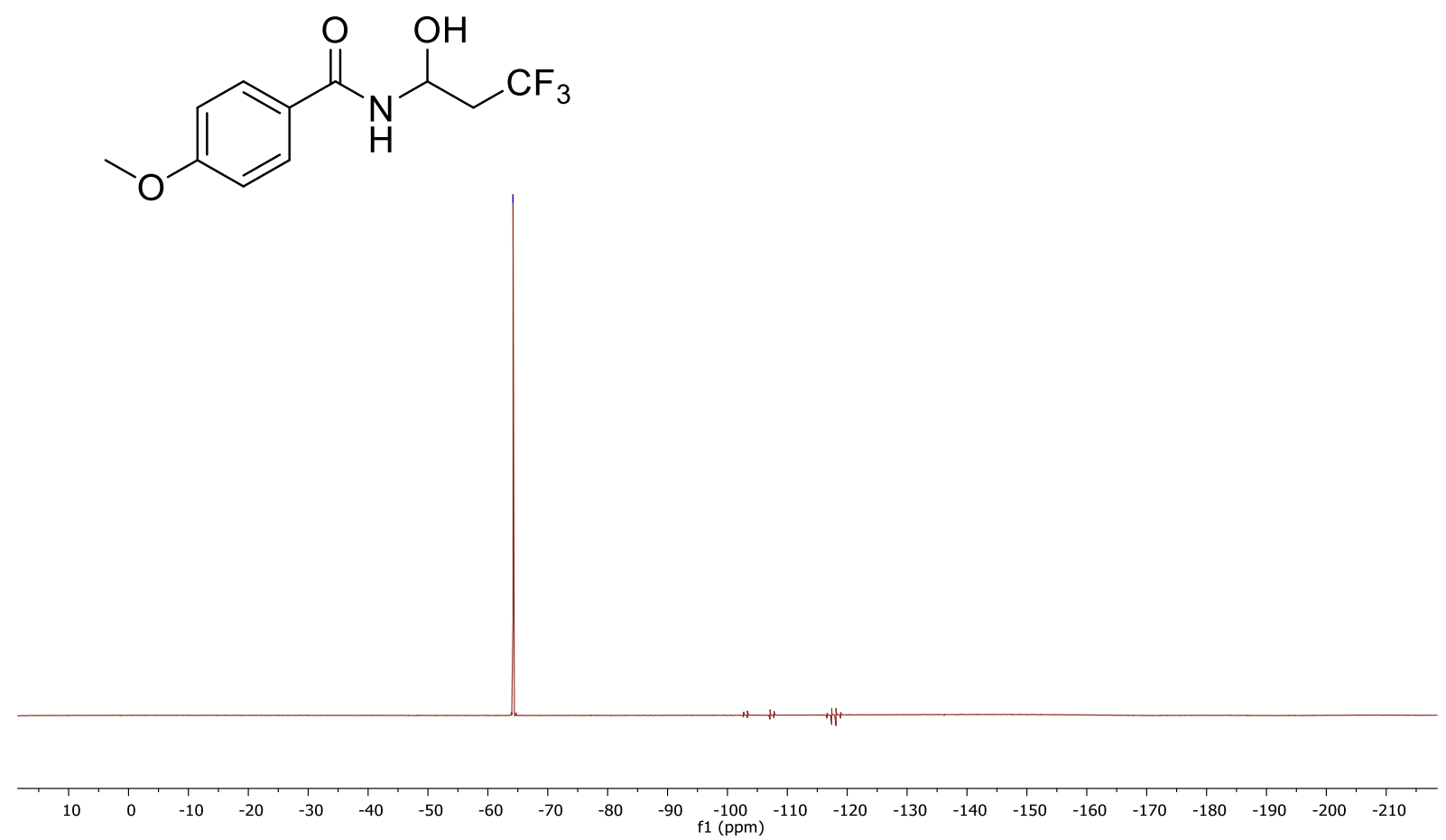

IR 


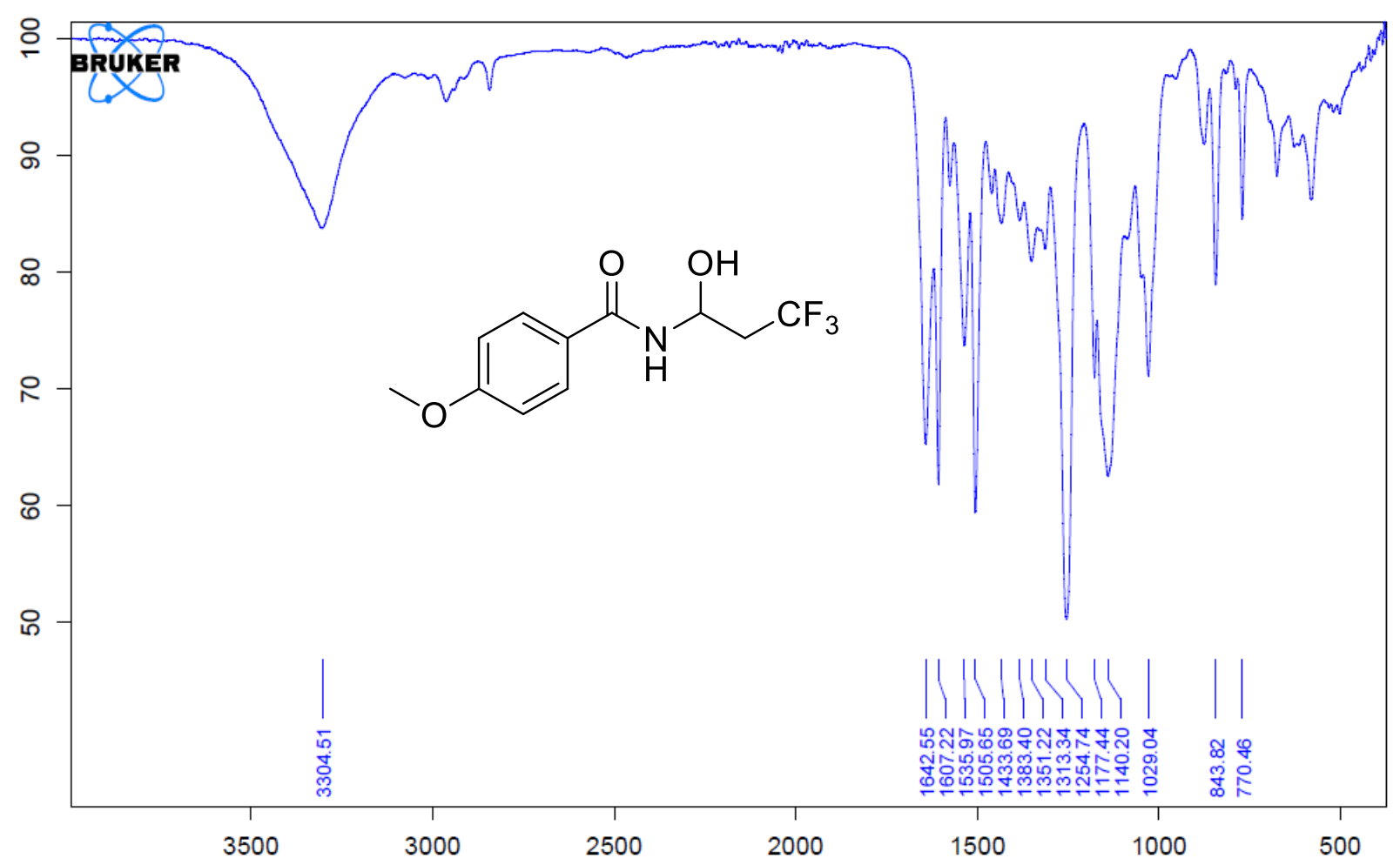

$N$-(4-Fluorobutyl)-4-methoxybenzamide (5ea)

${ }^{1} \mathrm{H}-\mathrm{NMR}$ (400 MHz, $\mathrm{CDCl}_{3}$ )
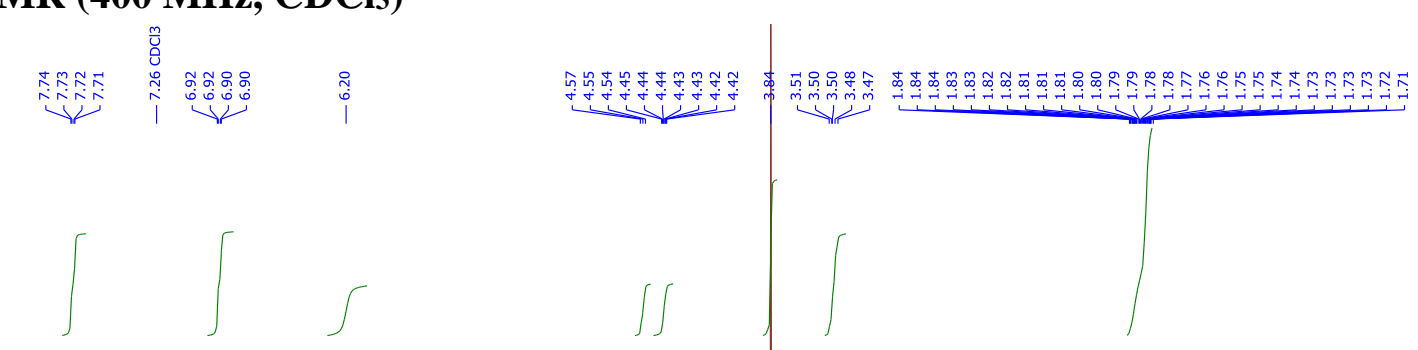<smiles>COc1ccc(C(=O)NCCCCF)cc1</smiles>

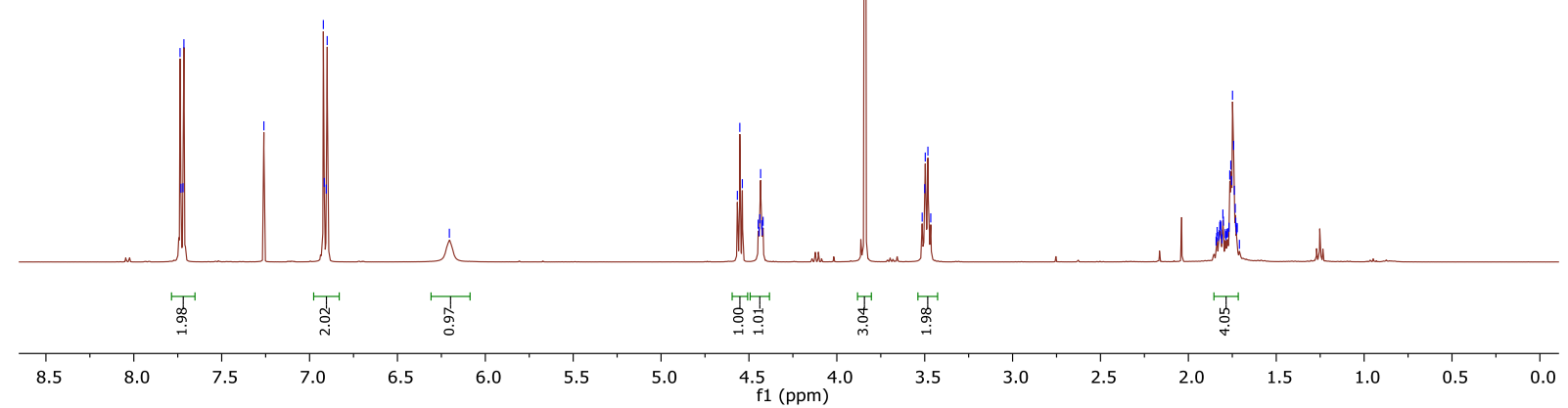

${ }^{13} \mathrm{C}-\mathrm{NMR}$ (101 MHz, $\left.\mathrm{CDCl}_{3}\right)$ 

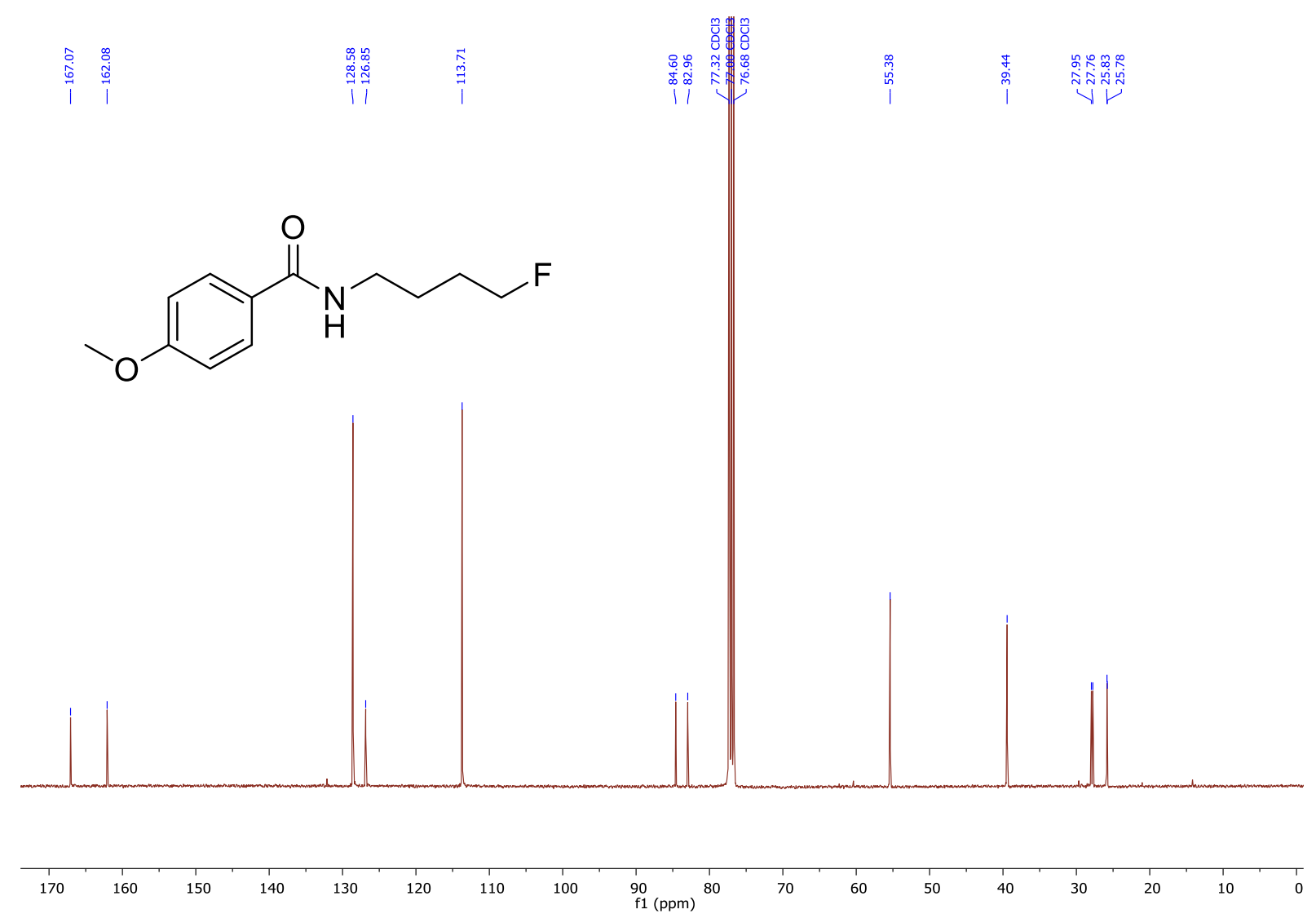

${ }^{19}$ F-NMR (376 MHz, CDCl3)<smiles>COc1ccc(C(=O)NCCCCF)cc1</smiles>

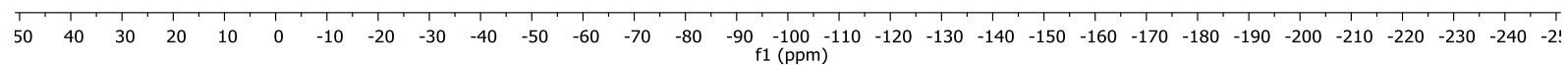
IR 


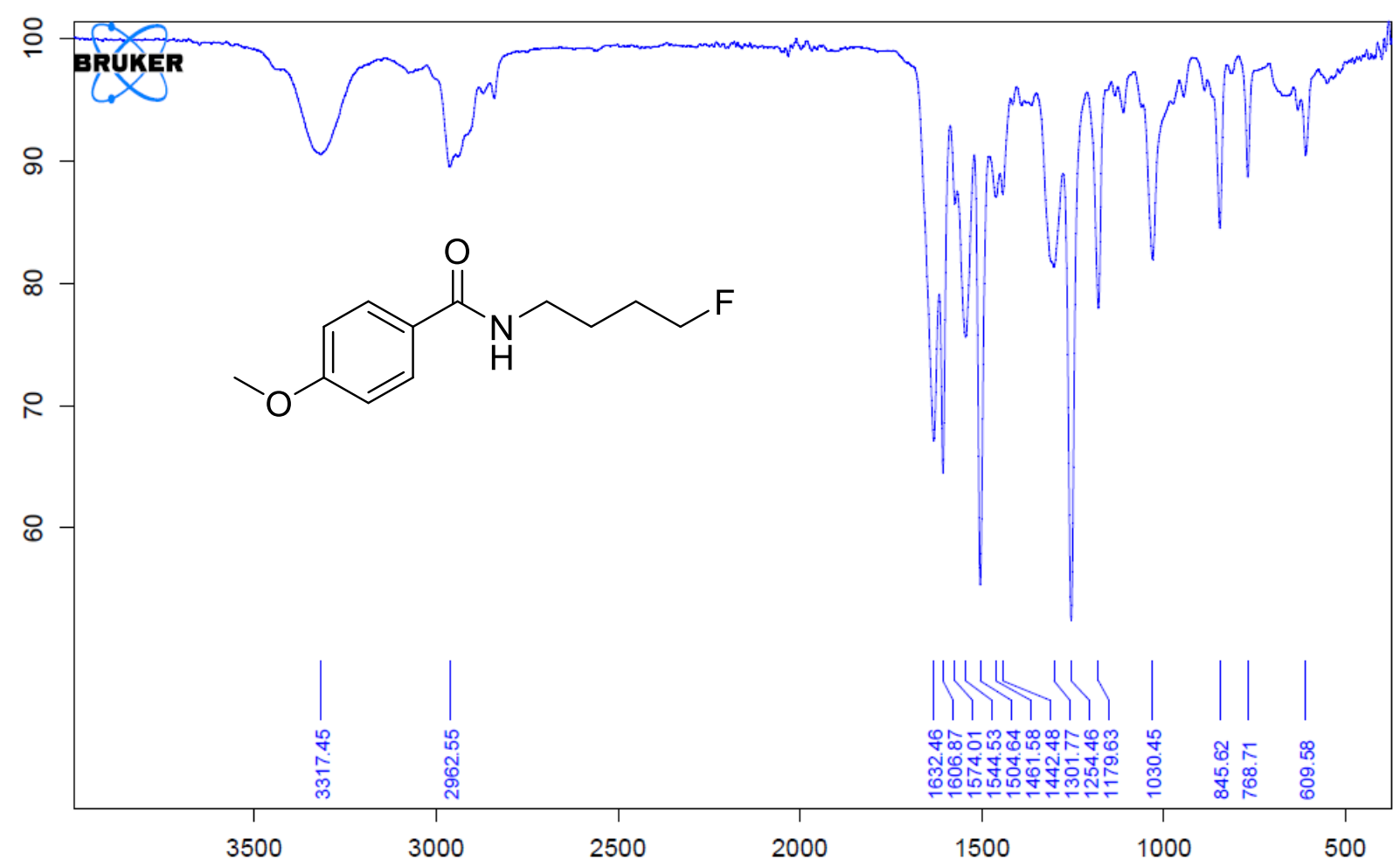

$N$-(4-Fluoro-1-(1H-indol-3-yl)butyl)-4-methoxybenzamide (5eb)

${ }^{1} \mathrm{H}-\mathrm{NMR}$ (400 MHz, $\mathrm{CDCl}_{3}$ )

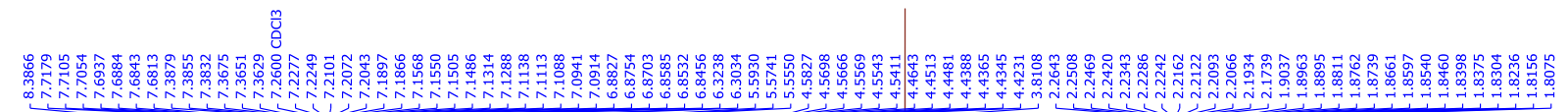

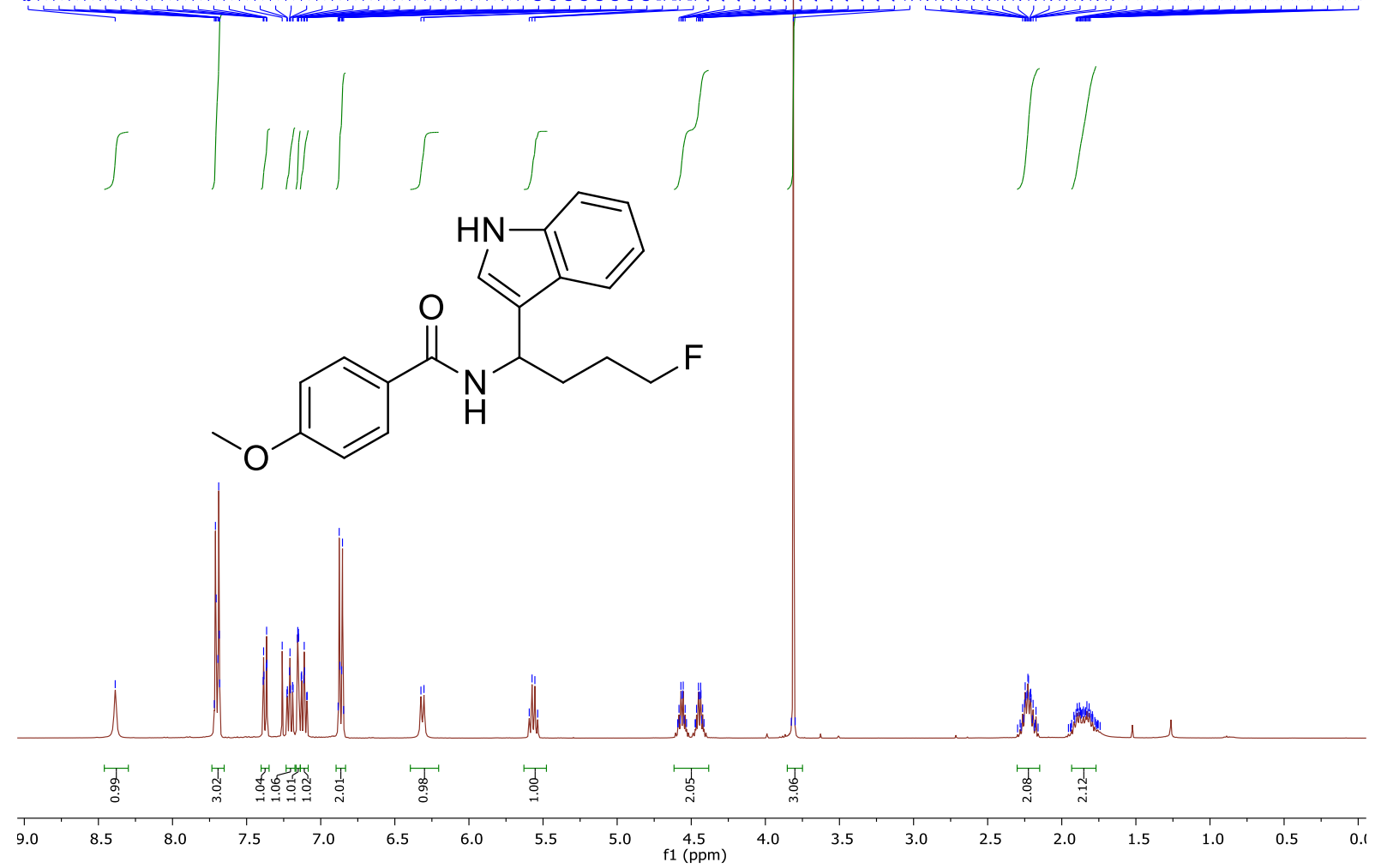

${ }^{13} \mathrm{C}-\mathrm{NMR}$ (101 MHz, $\left.\mathrm{CDCl}_{3}\right)$ 

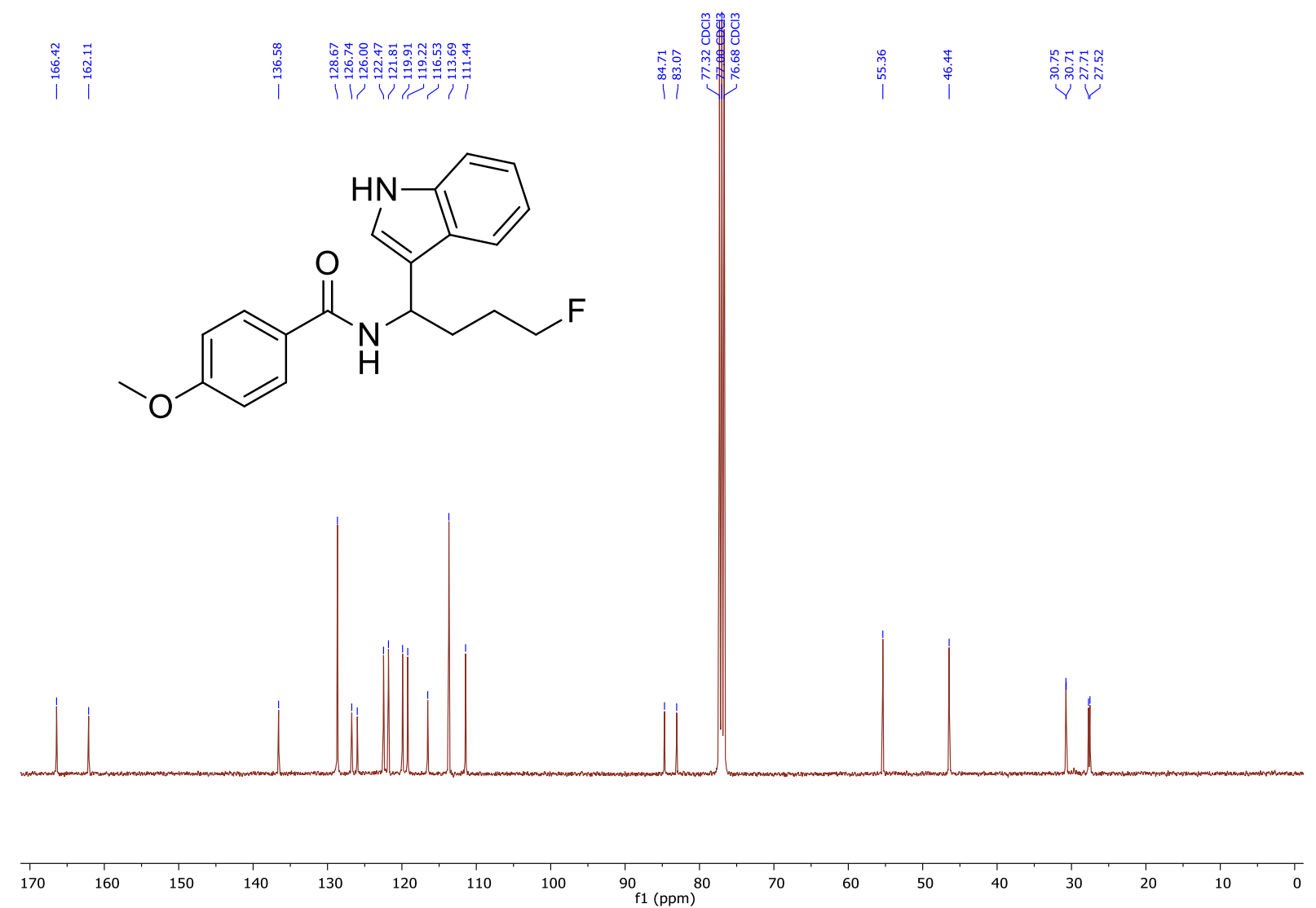

${ }^{19}$ F-NMR (376 MHz, CDCl3)

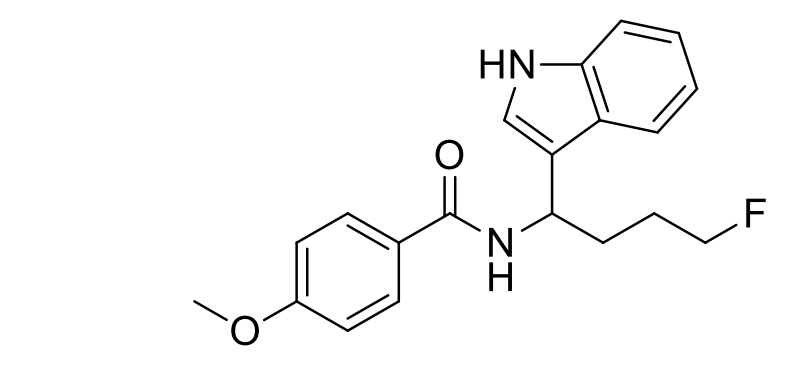

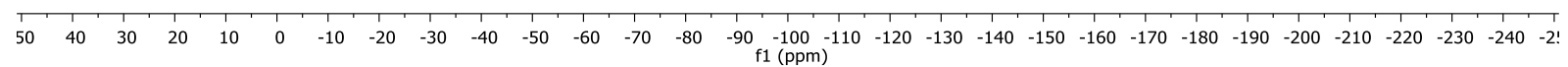
IR 


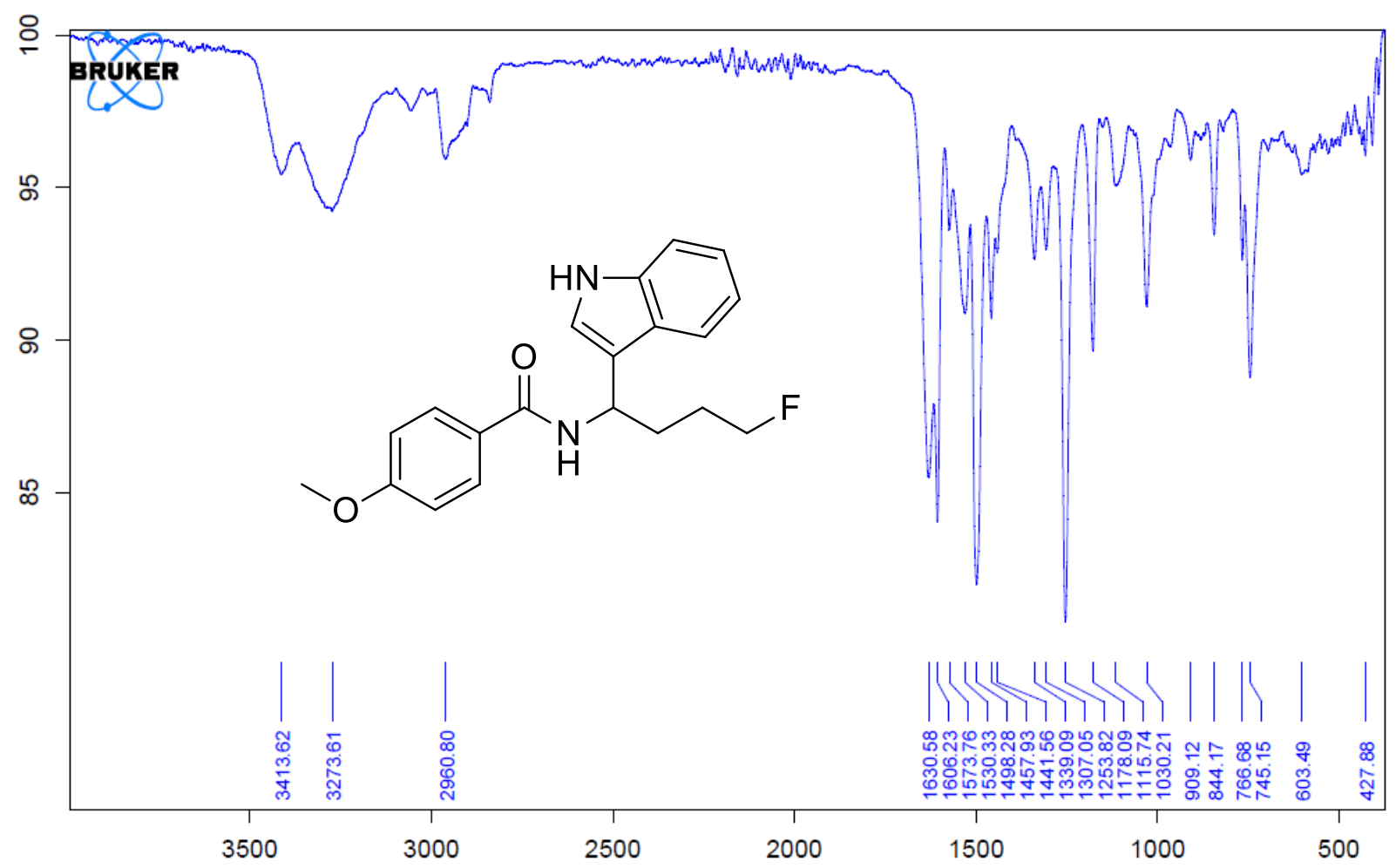

$N$-(4-Fluoro-1-(1H-pyrazol-1-yl)butyl)-4-methoxybenzamide (5ec) ${ }^{1}$ H-NMR (400 MHz, Acetone- $d_{6}$ )

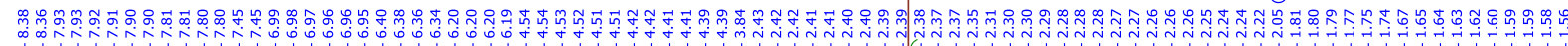<smiles>CCCCCCC(C)C(=O)c1ccc(OC)cc1</smiles>

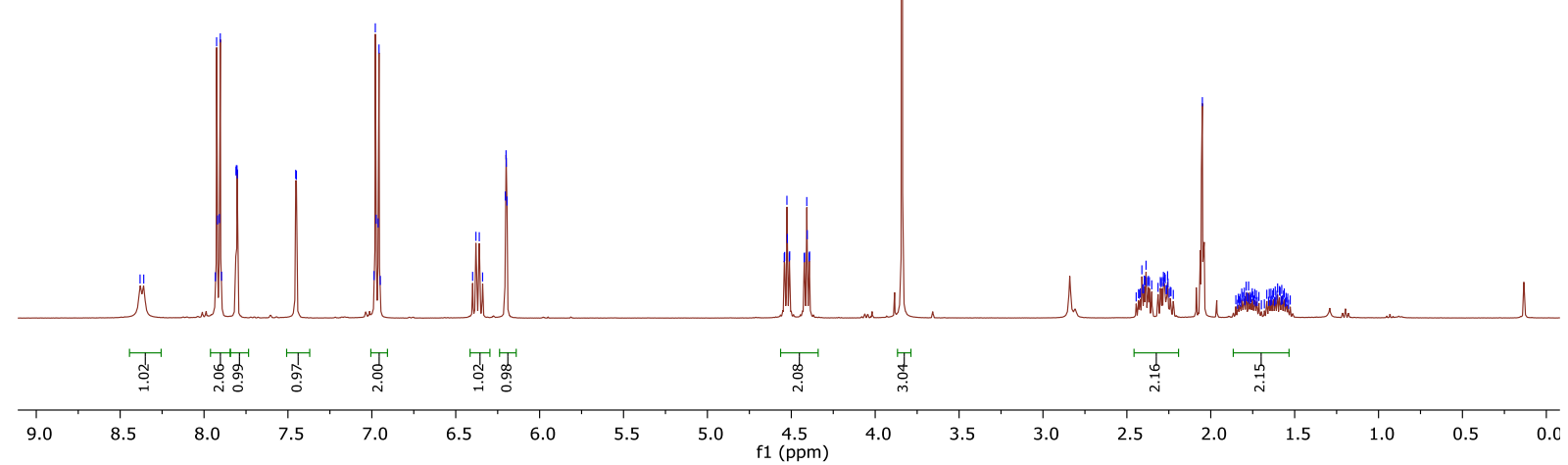

${ }^{13}$ C-NMR (101 MHz, Acetone-d6) 


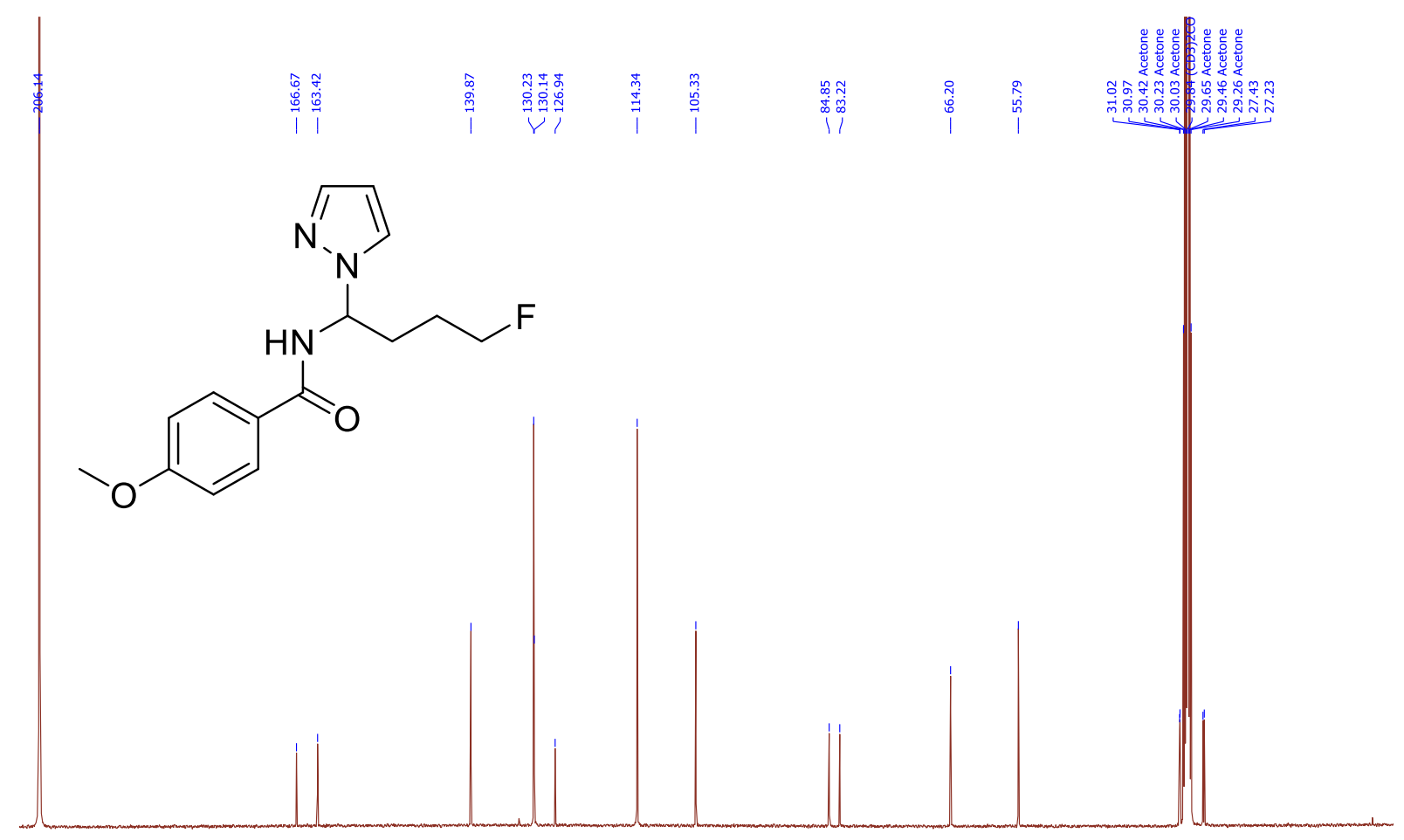

$\begin{array}{llllllllllllllllllll}200 & 190 & 180 & 170 & 160 & 150 & 140 & 130 & 120 & \begin{array}{c}110 \\ \mathrm{f} 1(\mathrm{ppm})\end{array} & 90 & 80 & 70 & 60 & 50 & 40 & 30 & 20 & 10 & 0\end{array}$

${ }^{19}$ F-NMR (376 MHz, Acetone-d6)<smiles>COc1ccc(C(=O)NC(CCCF)n2cccn2)cc1</smiles>

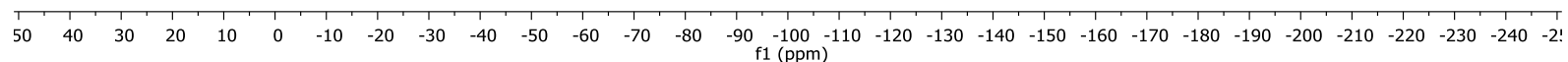
IR 


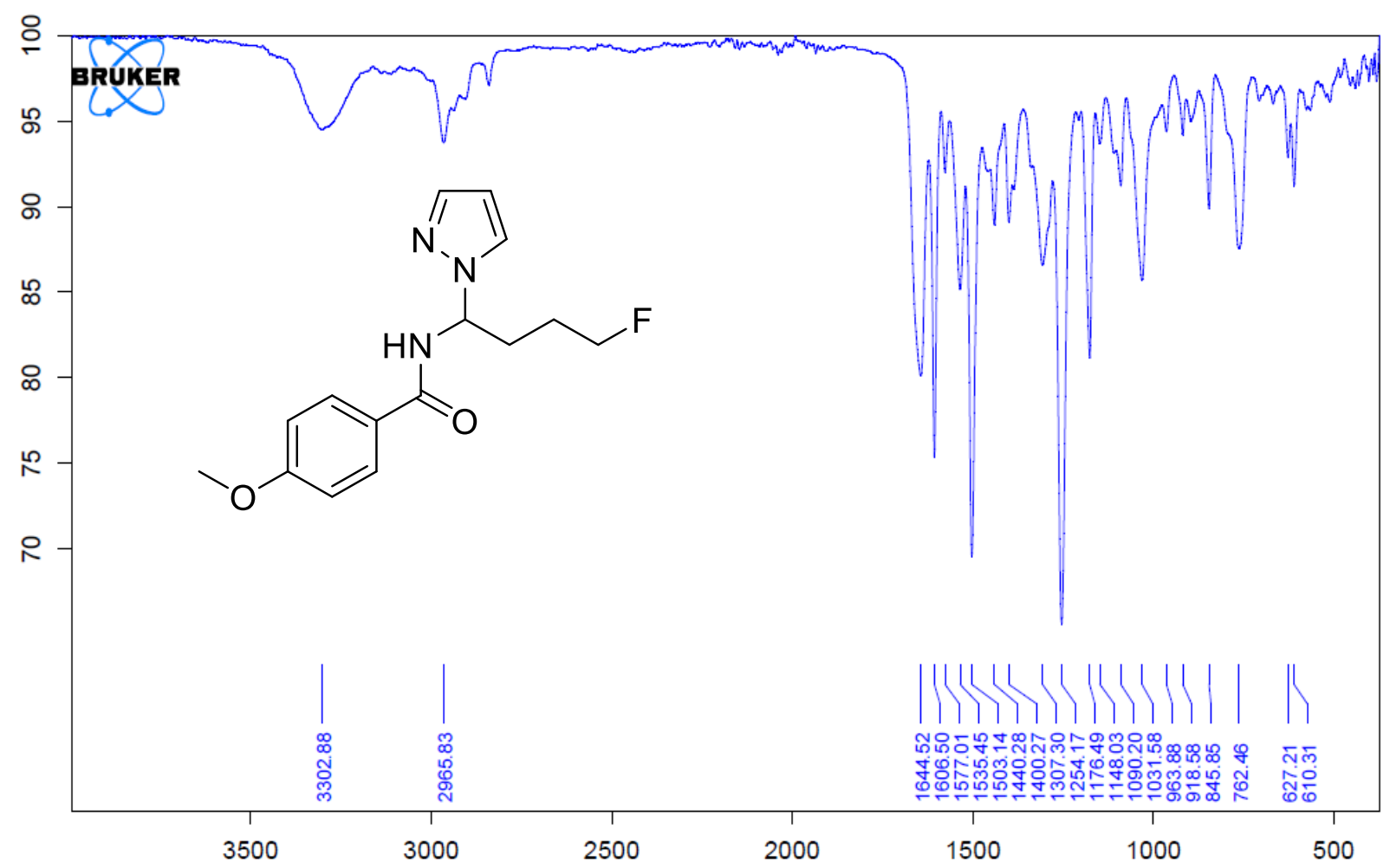

$N$-(4-Fluoro-3-phenylbutyl)-4-methoxybenzamide (5f)

${ }^{1}$ H-NMR (400 MHz, $\mathrm{CDCl}_{3}$ )

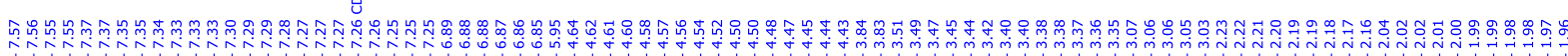

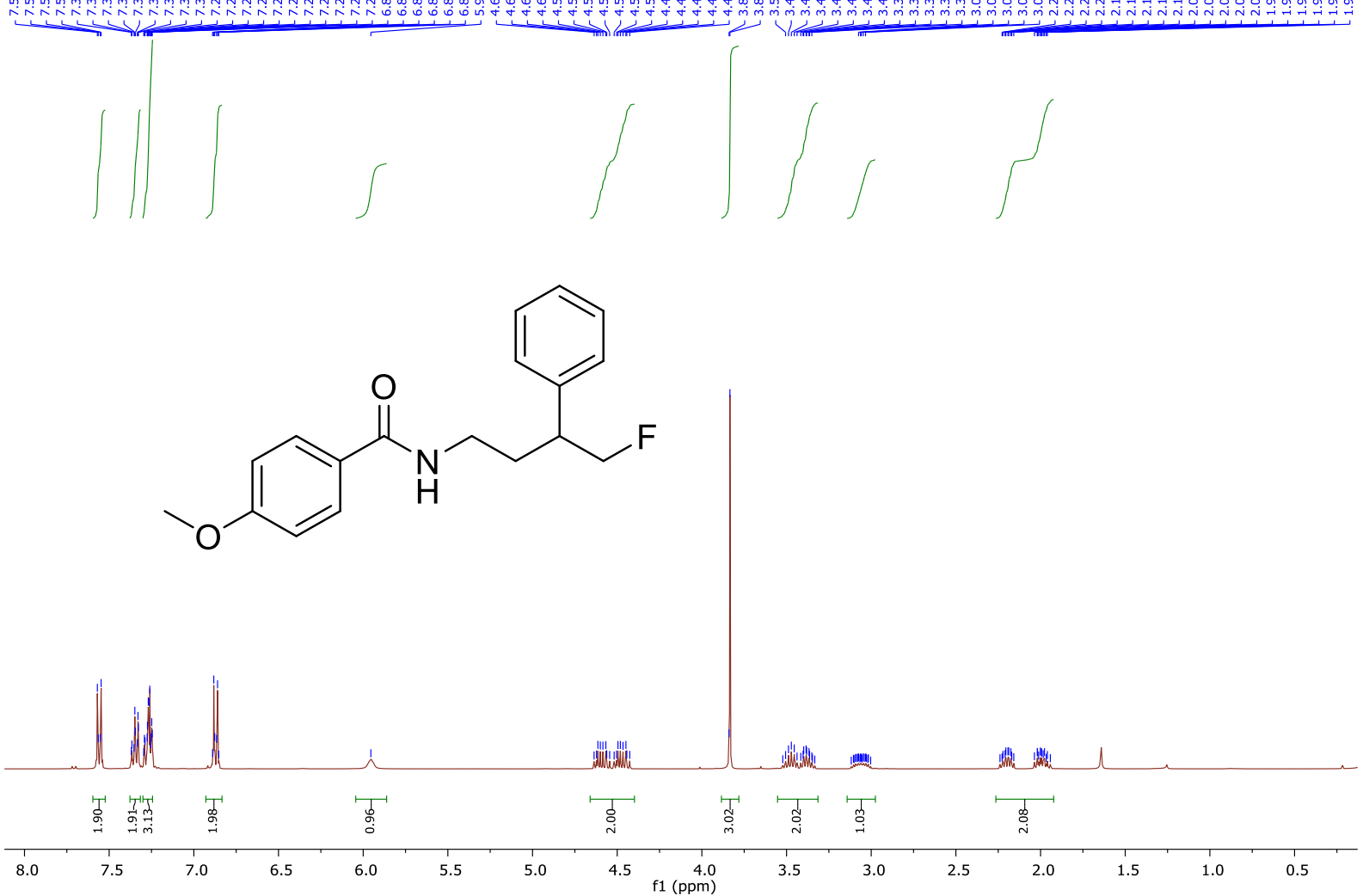

${ }^{13} \mathrm{C}-\mathrm{NMR}\left(101 \mathrm{MHz}, \mathrm{CDCl}_{3}\right)$ 

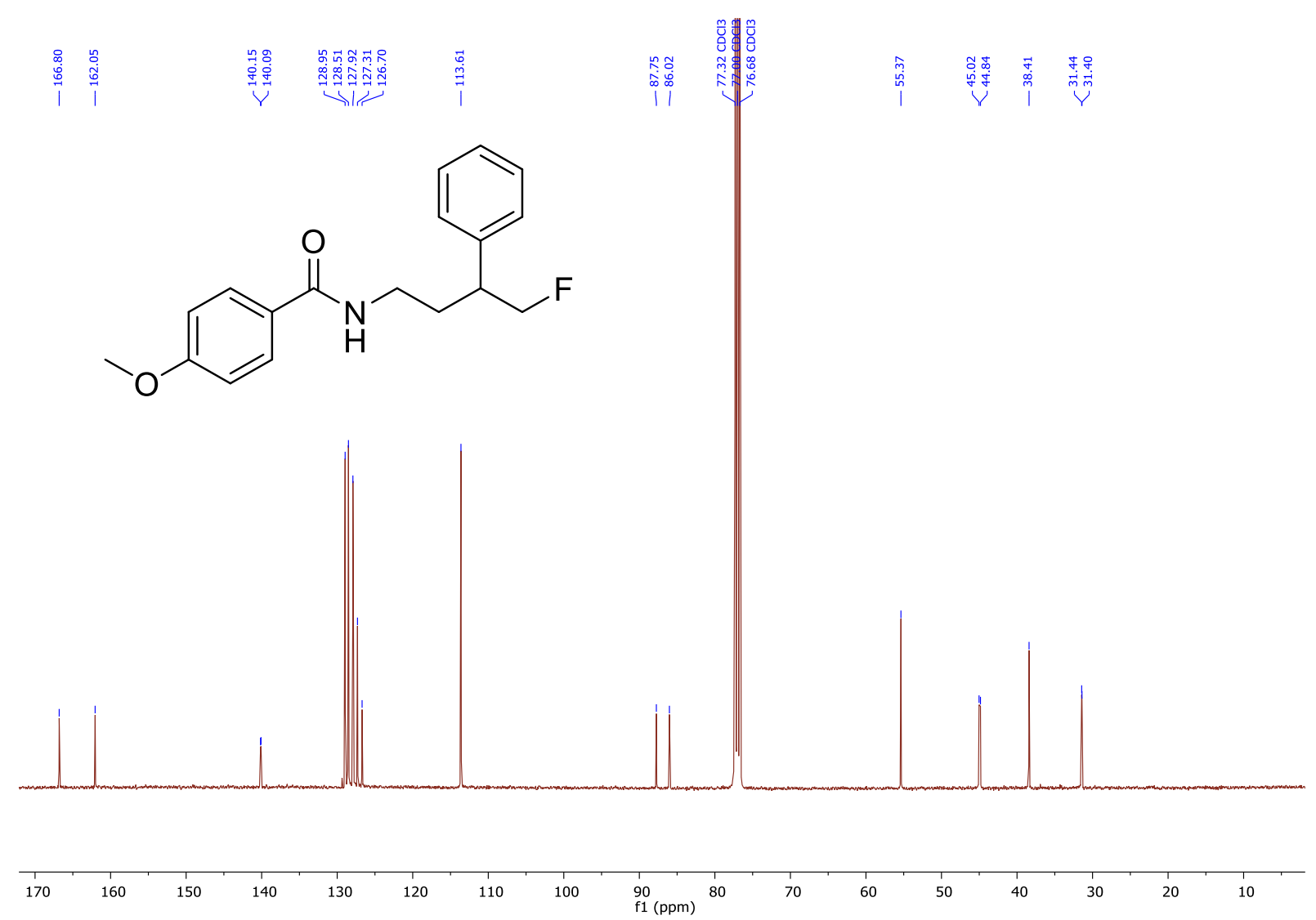

${ }^{19}$ F-NMR (376 MHz, CDCl3)<smiles>COc1ccc(C(=O)NCCC(CF)c2ccccc2)cc1</smiles>

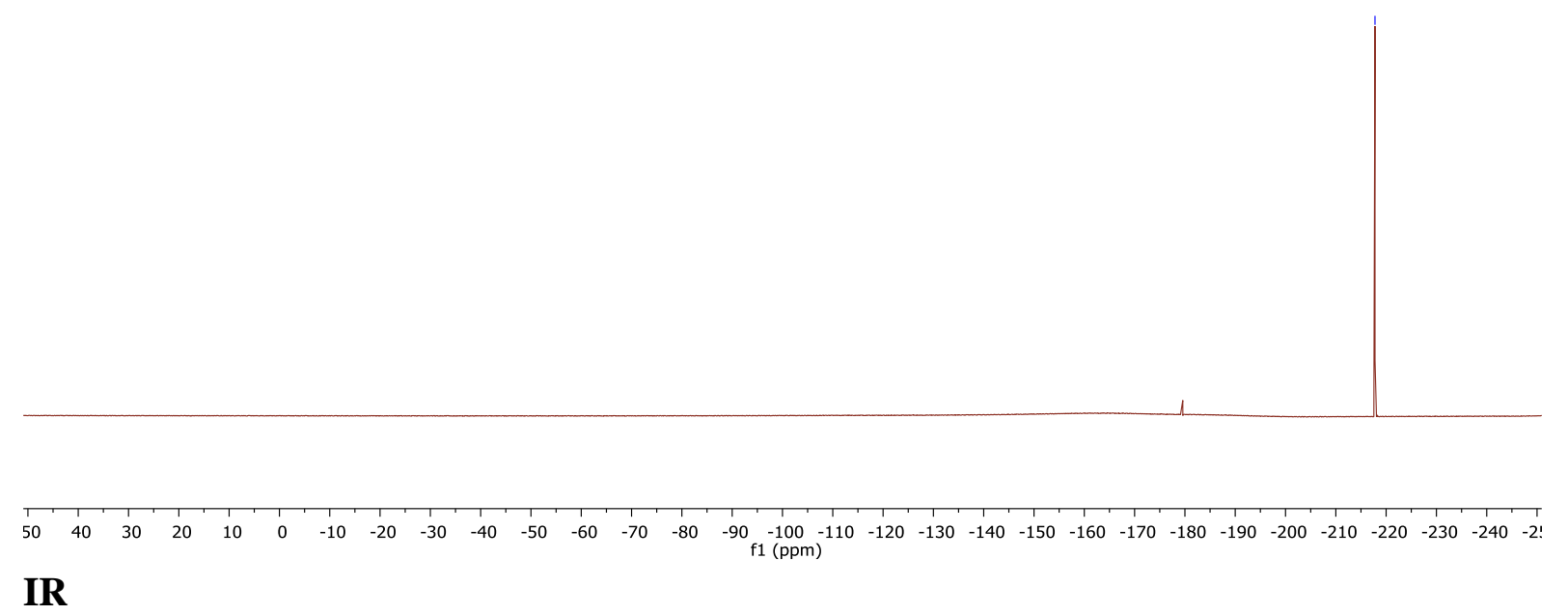




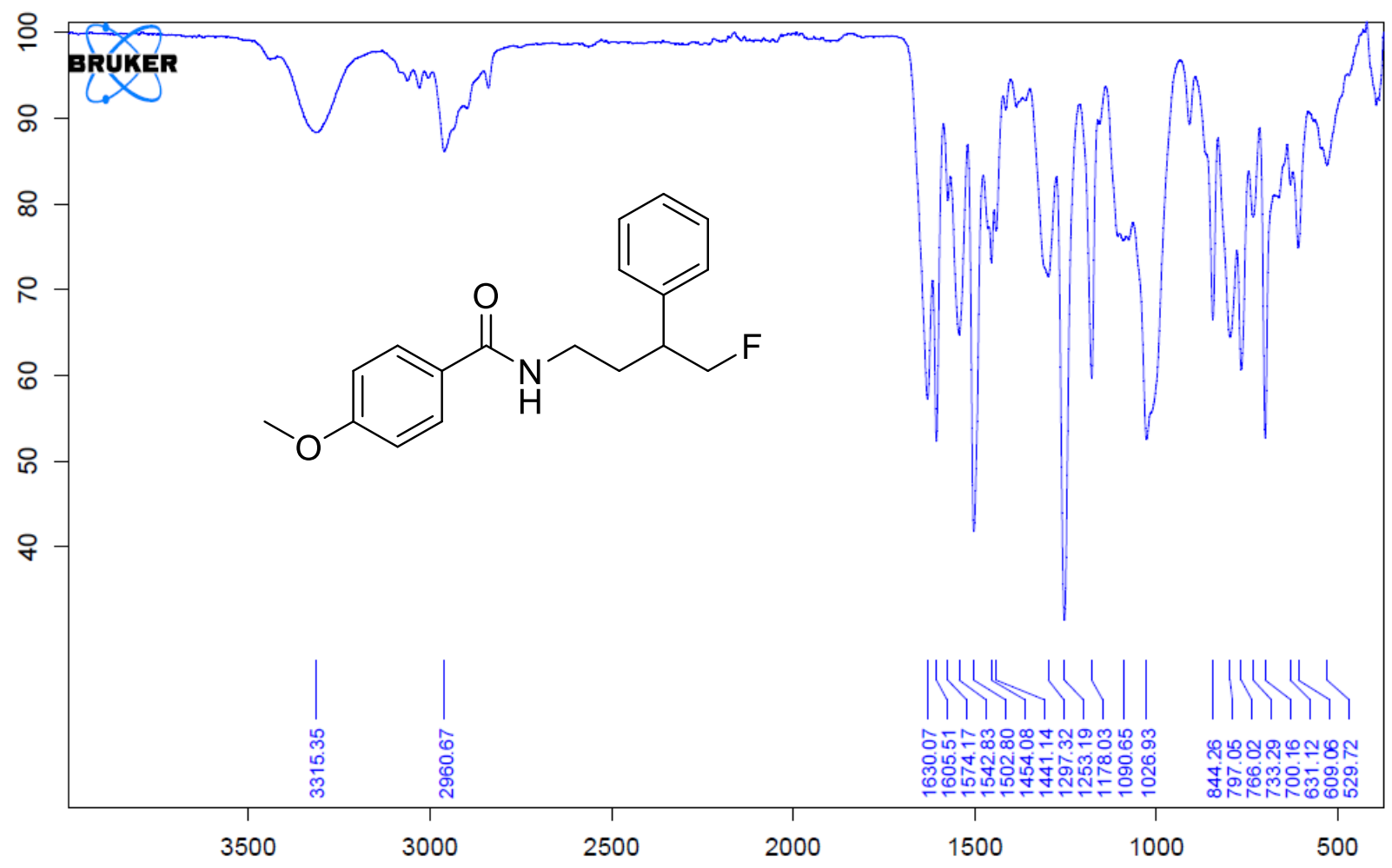

3-Fluoro-3-phenylpropan-1-ol (7)

${ }^{1} \mathrm{H}-\mathrm{NMR}\left(400 \mathrm{MHz}, \mathrm{CDCl}_{3}\right)$

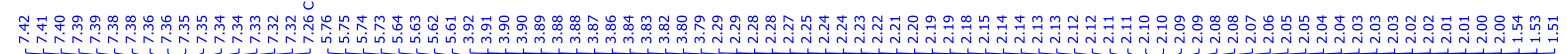<smiles>OCCC(F)c1ccccc1</smiles>

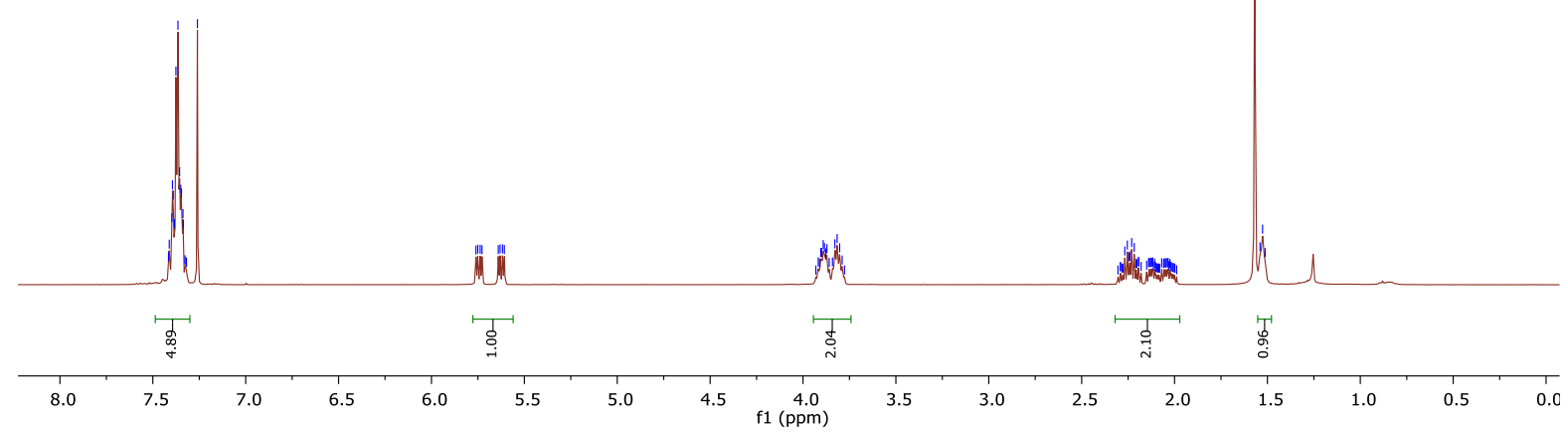

${ }^{13}$ C-NMR (101 MHz, $\left.\mathrm{CDCl}_{3}\right)$ 


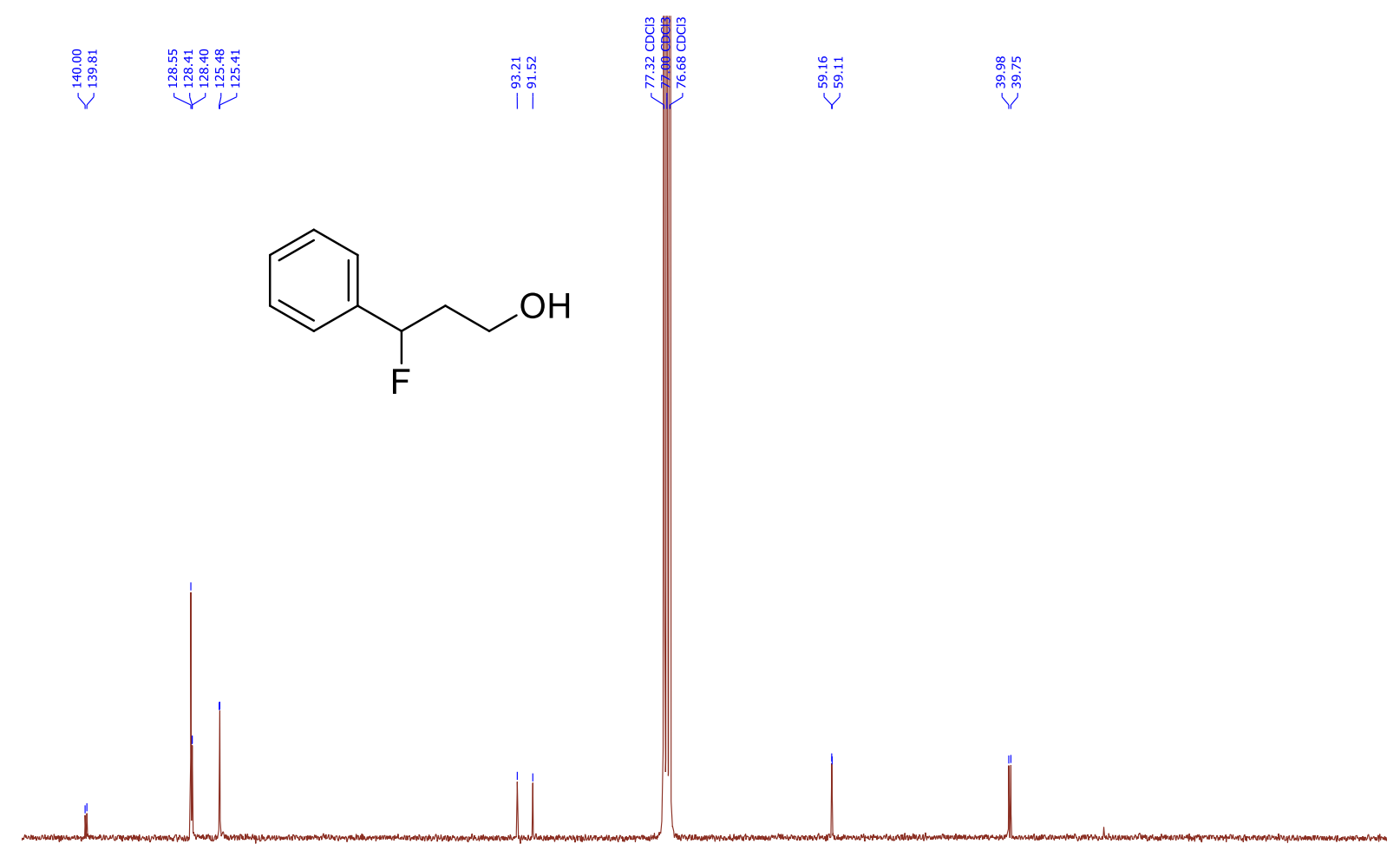

$\begin{array}{llllllllllllllllllllllllllllllllllll}145 & 140 & 135 & 130 & 125 & 120 & 115 & 110 & 105 & 100 & 95 & 90 & 85 & 80 & \begin{array}{c}75 \\ \mathrm{f} 1(\mathrm{ppm})\end{array} & 65 & 60 & 55 & 50 & 45 & 40 & 35 & 30 & 25 & 20 & 15 & 10 & 5 & 0\end{array}$

${ }^{19}$ F-NMR (376 MHz, CDCl3)<smiles>OCCC(F)c1ccccc1</smiles>

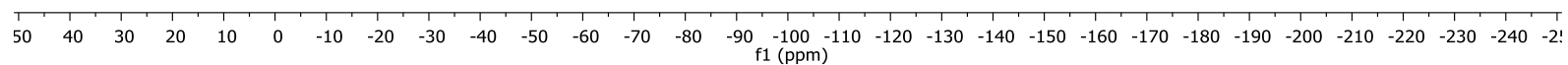
IR 


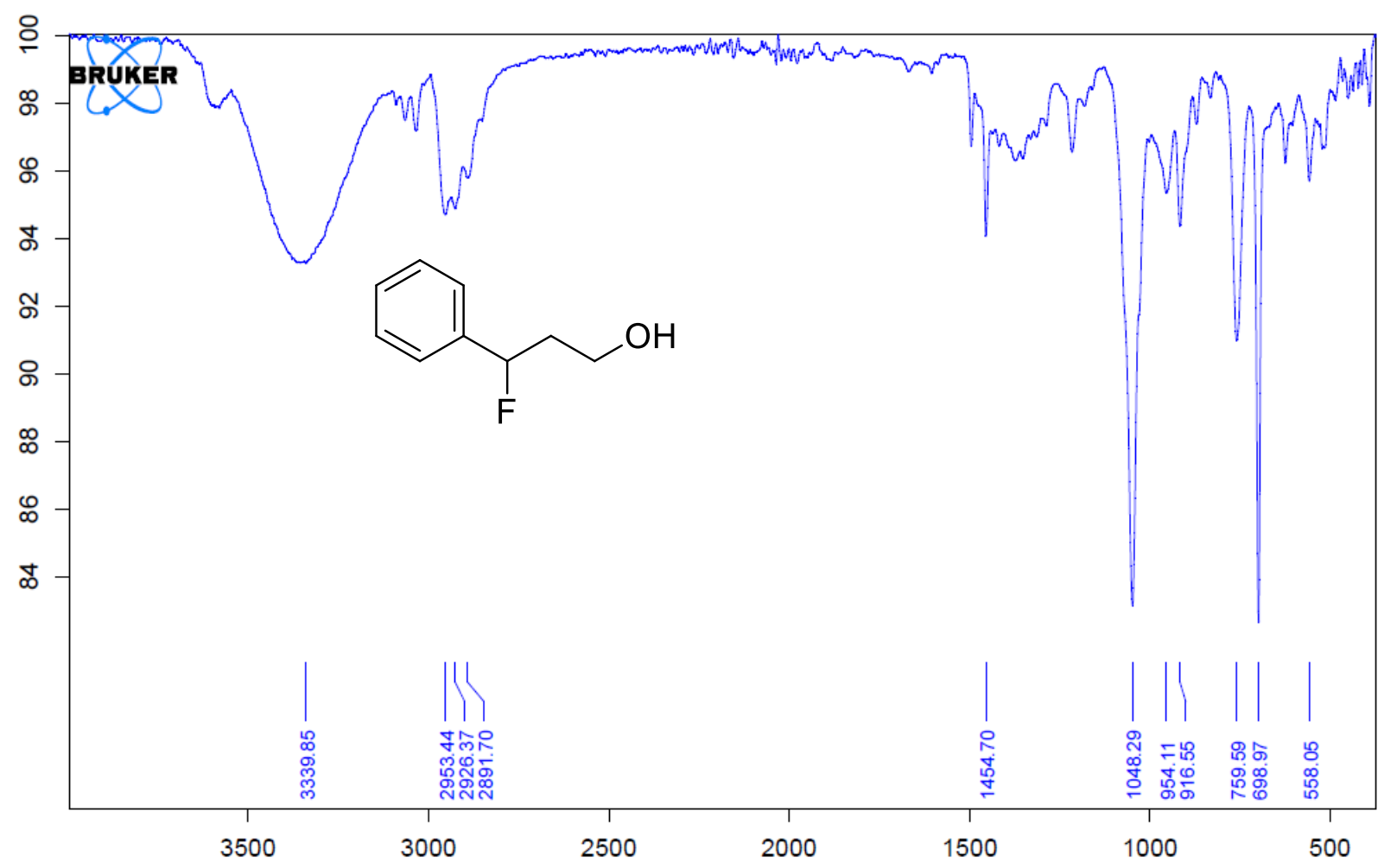

$N$-(1-Hydroxy-3-((2,2,6,6-tetramethylpiperidin-1-yl)oxy)propyl)-4-methoxybenzamide (8) ${ }^{1} \mathrm{H}-\mathrm{NMR}$ (400 MHz, $\left.\mathrm{CDCl}_{3}\right)$

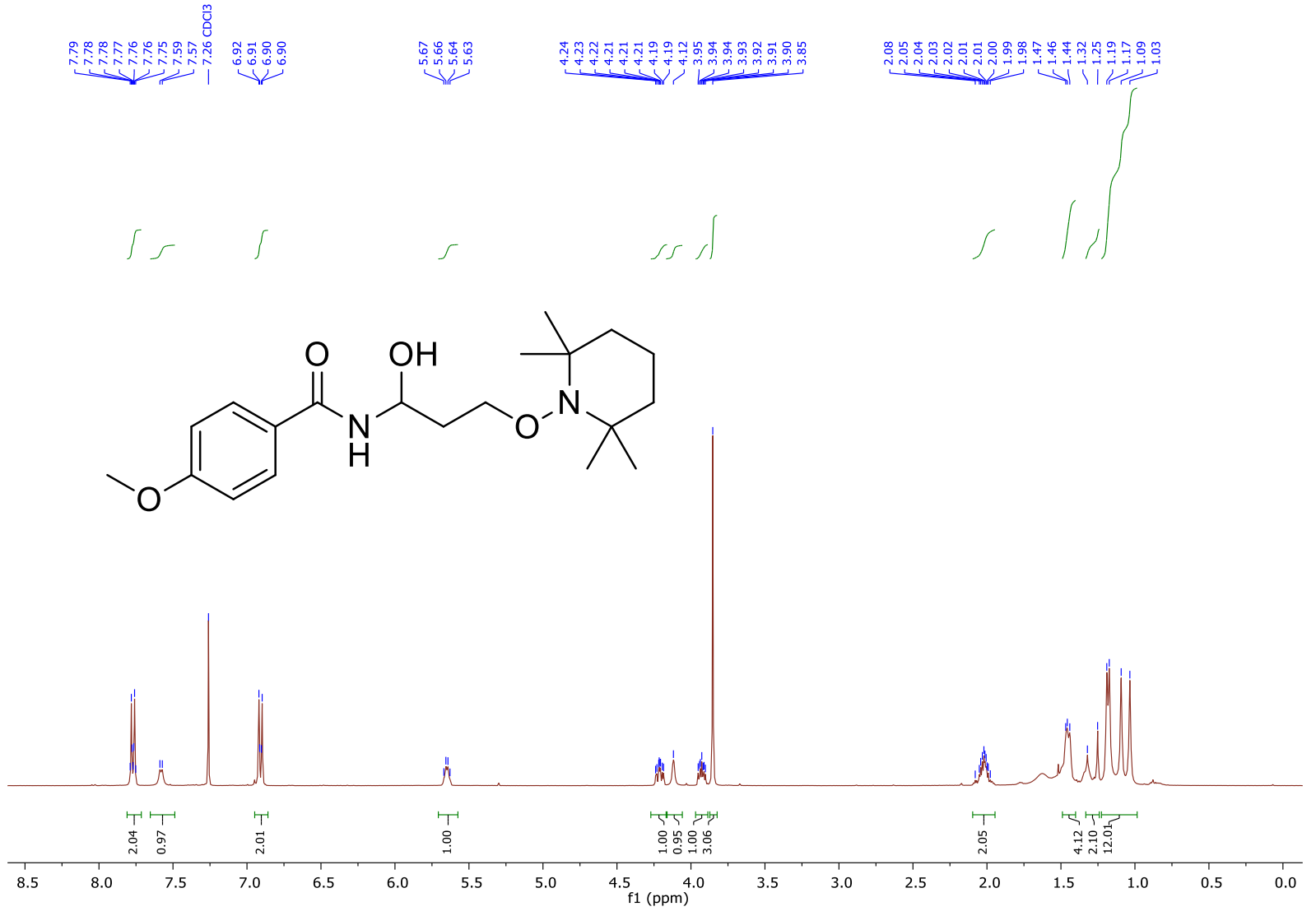

${ }^{13} \mathrm{C}-\mathrm{NMR}\left(101 \mathrm{MHz}, \mathrm{CDCl}_{3}\right)$ 

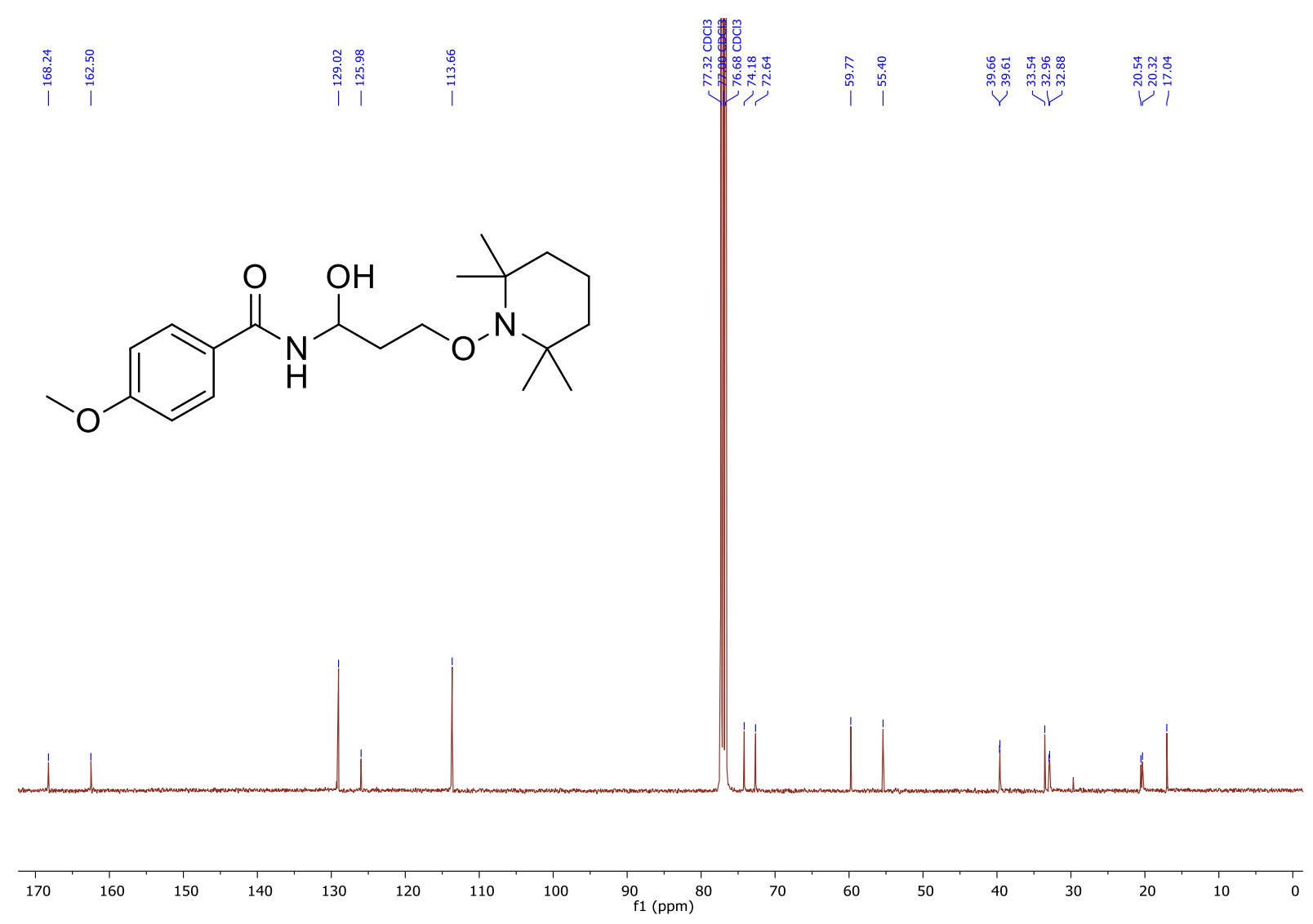

IR

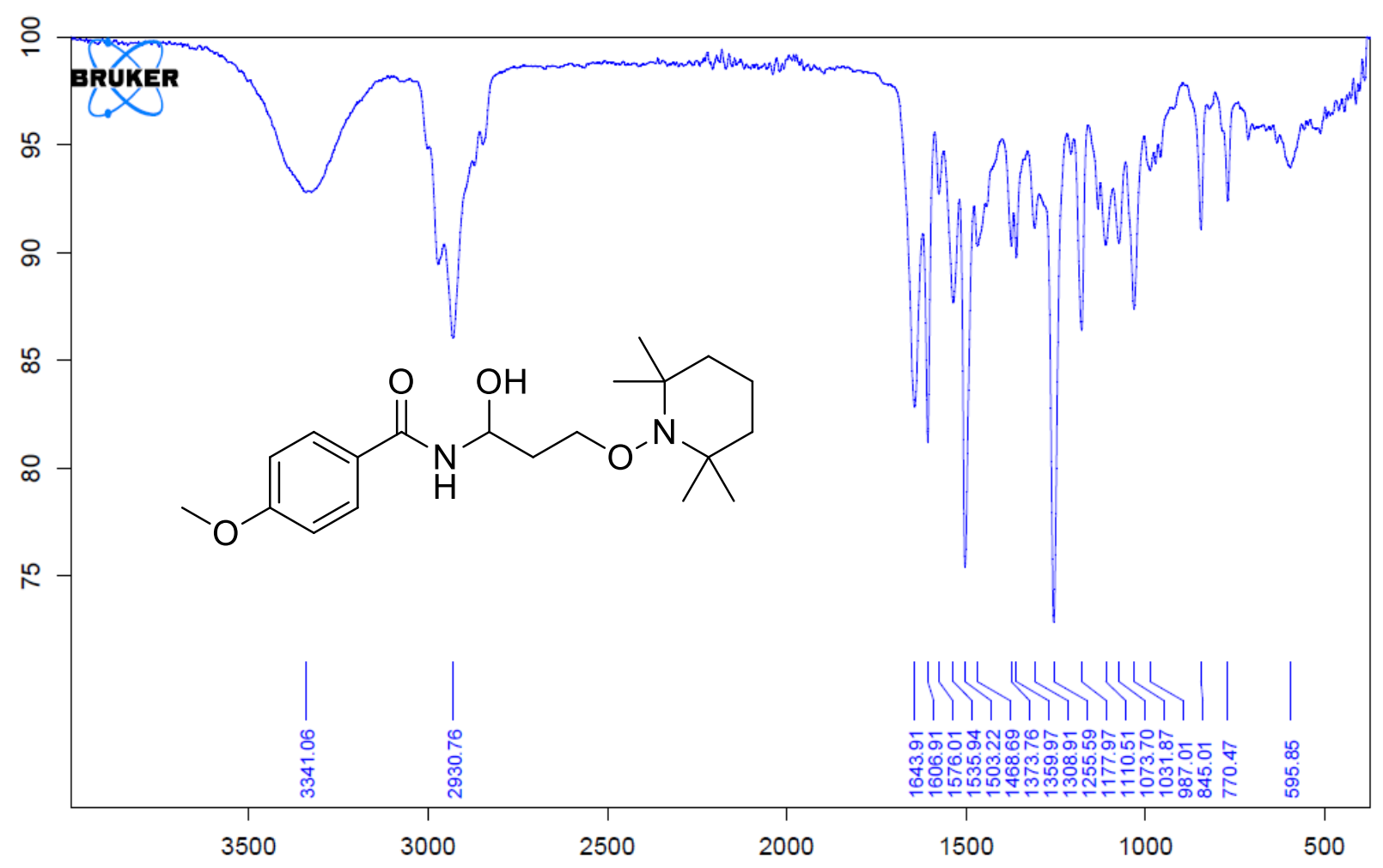

